\title{
A MONOGRAPH OF THE FERN GENUS BOLBITIS \\ (Lomariopsidaceae)
}

\section{E. HENNIPMAN}

Rijksherbarium, Leiden

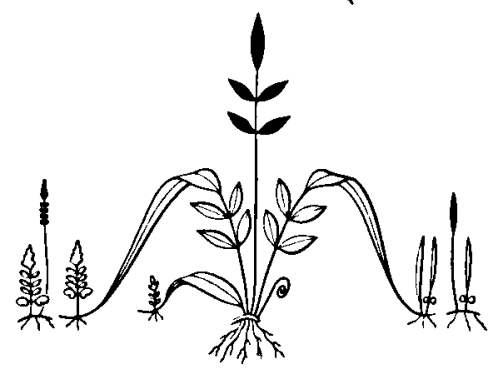


Published with financial support from the Netherlands Organization for the Advancement of Pure Research (Z.W.O.).

ISBN 90.6021.405.6.

No part of this book may be reproduced in any form, by print, photoprint, microfilm or any other means, without written permission from the publisher.

Printed in the Netherlands by Intercontinental Graphics.

Photoset in Malta by Interprint (Malta) Ltd. 


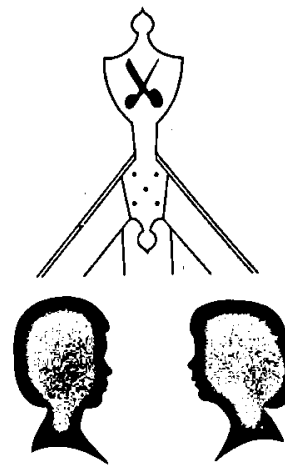

Aan Alexander en Annemieke 


\section{CONTENTS}

\section{GENERAL PART}

1. Introduction and summary $\ldots \ldots \ldots \ldots \ldots \ldots$

2. Acknowledgements .................. 2

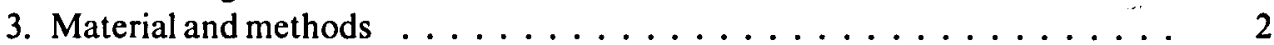

4. Taxonomic history of the genus $\ldots \ldots \ldots \ldots \ldots$

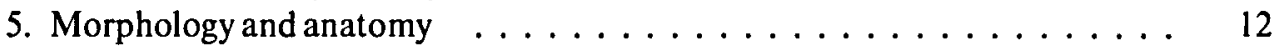

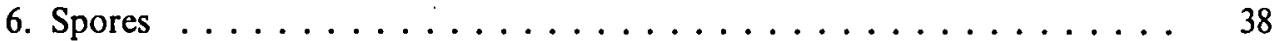

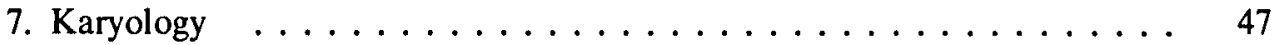

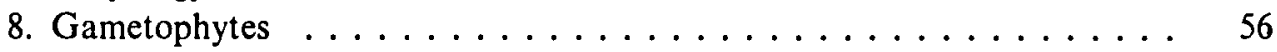

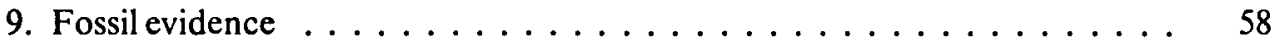

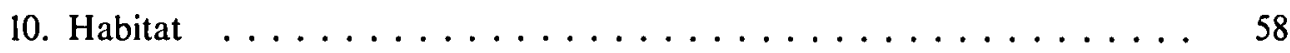

11. Juvenile leaves . . . . . . . . . . . . . . . . . . . 59

12. Juvenile leaves and phylogeny in Bolbitis . . . . . . . . . . 73

13. Retention of juvenile characters and past diversification of ferns . . . . . 82

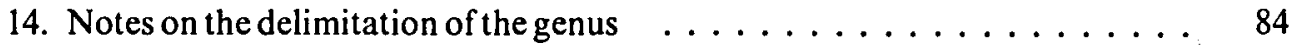

15. The systematic position of the genus $\ldots \ldots \ldots \ldots \ldots \ldots$

16. Subdivision of the genus $\ldots \ldots \ldots \ldots \ldots \ldots \ldots$

17. Notes on specific delimitation $\ldots \ldots \ldots \ldots \ldots \ldots \ldots \ldots \ldots \ldots \ldots$

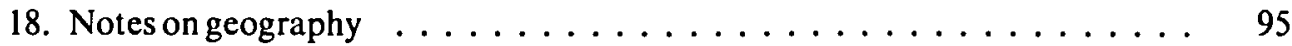

19. References in previous chapters . . . . . . . . . . . . . 99

Plates . . . . . . . . . . . . . . . . . . . . . . . . . 103

\section{TAXONOMIC PART}

1. Notes on the presentation of data $\ldots \ldots \ldots \ldots \ldots \ldots \ldots$

2. Bolbitis ....................... 123

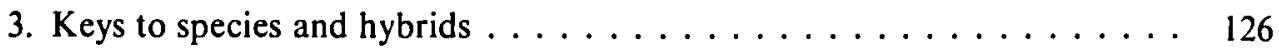

4. Descriptions

Ser. Alienae ......................... 132

Ser. Bipinnatifidae . . . . . . . . . . . . . . . . . . . 144

Ser. Bolbitianae . . . . . . . . . . . . . . . . . . 147

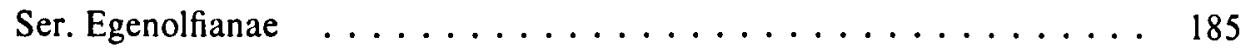

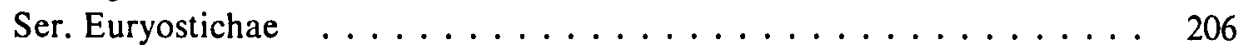

Ser. Heteroclitae . . . . . . . . . . . . . . . . 220

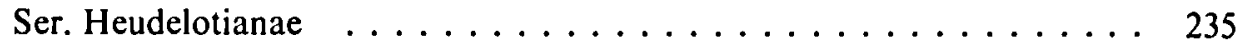

Ser. Lindigianae . . . . . . . . . . . . . . . . . . 240 
Ser. Portoricenses . . . . . . . . . . . . . . . . . 242

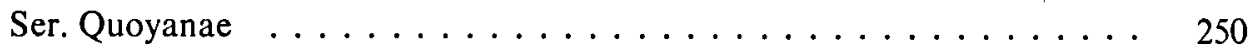

Species incertae sedis . . . . . . . . . . . . . . . . . 259

Hybrids . . . . . . . . . . . . . . . . . . . 284

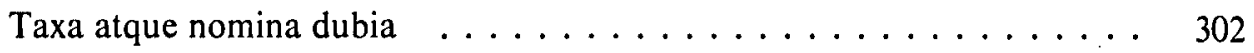

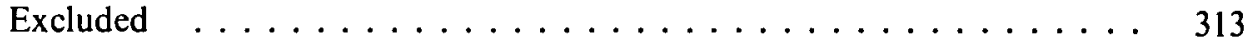

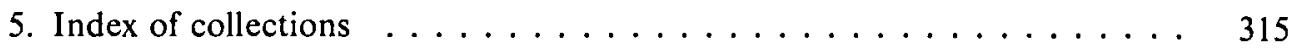

6. Index to taxonomic names $\ldots \ldots \ldots \ldots \ldots \ldots \ldots \ldots \ldots$ 
General Part 


\section{INTRODUCTION AND SUMMARY}

The present study deals with systematic and taxonomic problems in the acrostichoid fern genus Bolbitis (Lomariopsidaceae). The idea came from Prof. Holttum, Kew, in view of his intended revision of the lomariopsidoid ferns for Flora Malesiana.

Originally the study was confined to the Asian representatives. However, various problems remained unsolved after the study of these taxa alone, and it was decided to monograph the genus.

The taxonomy of the genus Bolbitis was seriously misunderstood in the past-parallel evolution obscuring true relationships-and a rather intricate synonymy has resulted. A brief historical sketch of its taxonomic adventures is worked out.

The gross-morphological criteria used for the discrimination of the species by Copeland (who revised the Asian representatives in 1928) and Ching (who revised ser. Egenolfianae in 1931) could not be applied satisfactorily to the ample material at hand. Therefore, several features of the sporophyte were analyzed in detail, including rhizome- and petiole-anatomy, venation pattern and spore-morphology. The results are described and discussed.

Further, the possible occurrence of hybridization and polyploidization was investigated. For this purpose a collection of different species was taken into cultivation and their mitotic and/or meiotic chromosome number and behaviour recorded. A limited number of species could also be studied in the field. From several species significant information on the morphology of the juvenile leaf stages could be obtained.

As could be demonstrated the delimitation of the genus as given especially by Ching (in Christensen, 1934) is largely correct, though he included the species with anastomosing veins only. A number of Ching's species, however, are excluded and referred to non-lomariopsidoid genera. Also, in a few cases, species from other genera are here included in the genus for the first time. As most of the taxa under discussion were formerly included in Leptochilus, this genus was critically investigated as well. The results will be published separately. The genus Bolbitis as here defined and emended includes the genera Egenolfia and Edanyoa.

Fourty-four species (one of which new) and 13 hybrids ( 12 of which new) are recognized, based on morphological criteria. The venation pattern and the spores provide important characters. In some species infraspecific categories are listed. Identification keys to all taxa are included. The genus is subdivided into 10 series which accommodate a total of 34 species; another ten species are separately listed mostly in view of their suspected hybrid origin.

Hybrids could be detected because of structural irregularities, especially in spores, but usually also as to other features. Putative hybrids between cytotypes of one species are not separately listed. Generic and/or specific delimitation differs considerably from that given by Ching (in Christensen, 1934), Christensen (1934), Holttum (1954), and Copeland (1960); the latter eventually recognized 97 different species in four different genera (Stenosemia dimorpha included).

Notes are given on specific delimitation. Data are supplied which stress the evo- 
lutionary importance of polyploidization, hybridization, vegetative propagation, and the retention of juvenile characters, factors that may explain the often fascinating diversity found within many species.

The study of ontogenetic leaf stages (which may be very different from adult ones) has received great attention after it was understood that at least part of the variation as found in the adult sterile leaves recalls features found in the juvenile leaves. The relation between ontogeny and phylogeny is shortly discussed. Some facts are given which support my impression that the retention of juvenile characters may have played an important role during the diversification within the genus.

Some general considerations about the probably great general significance of the retention of juvenile characters for the past diversification of the leptosporangiate ferns are included. It is postulated that the recognition of this neglected phenomenon may add considerably to our understanding also of the larger groups of leptosporangiate ferns.

\section{ACKNOWLEDGEMENTS}

It is a pleasure to express my gratitude to Prof. Dr. C. G. G. J. van Steenis, who appointed me as a pteridologist at the Rijksherbarium, and under whose stimulating directorship the present study was largely completed. I had the privilege that Prof. Dr. R. E. Holttum took an interest in my work; he has been (and still is!) very helpful in various ways.

Specific subjects were discussed with staff members at Leiden, which include Dr. R. C. Bakhuizen van den Brink (nomenclature) who also prepared the Latin diagnoses, Dr. Ding Hou (karyology), Dr. P. W. Leenhouts (general), Mr. J. Muller (spore-morphology), and Dr. W. Vink (editing).

Technical assistance was received from Miss Ruth van Crevel (illustrations), Mr. A. K. Groenewegen and staff (loans of specimens), Mr. B. N. Kieft (photography), Mr. D. N. F. Kiehl (proof-reading), Miss A. M. Kuenen (microtechnique, photography), Mr. A. Mulder (cultivation), Miss E. E. van Nieuwkoop (typing), Mr. E. Vijsma (drawings; lay-out of plates), and Mr. L. Vogelenzang and staff (bibliography). S.E.M.photographs could be made due to the valued co-operation with the Netherlands Organization for Applied Scientific Research TNO (Miss R. N. Hooftman), and the Geological Institute, Leiden (Mr. H. Kammeraat).

Several correspondents from overseas sent living plants of quite a few species. Amongst them is Mr. M. G. Price who sent all the Philippine plants recorded here, and to whom I am especially indebted.

Prof. Dr. I. Manton (Leeds) and Dr. T. G. Walker (Newcastle-upon-Tyne) permitted me to include a few unpublished records on chromosome numbers.

Dr. L. R. Atkinson (Amherst, Mass.) kindly put at my disposal the notes on gametophytes of Bolbitis species prepared by the late Dr. A. G. Stokey and herself.

Although several European herbaria (Berlin, Brussels, Kew, London, and Paris) could be visited personally, a considerable number of specimens were received on loan from these and other herbaria for quite a period, due to the kind co-operation of their Directors. My stay at Berlin was financed by the Netherlands Organization for the Advancement of Pure Research (Z.W.O.).

Constructive criticism on (parts of) the manuscript was received from Mr. G. J. de Joncheere, Prof. Dr. Č. Kalkman, Prof. Dr. K. U. Kramer (Zürich), and Prof. Dr. C. G. G. J. van Steenis.

I tender my sincere thanks to these persons and to all others who took an active interest in my work.

\section{MATERIAL AND METHODS}

\section{Material}

The far greater part of the specimens studied is located in the following herbaria (the abbreviations refer to the standard abbreviations of the Index Herbariorum): 


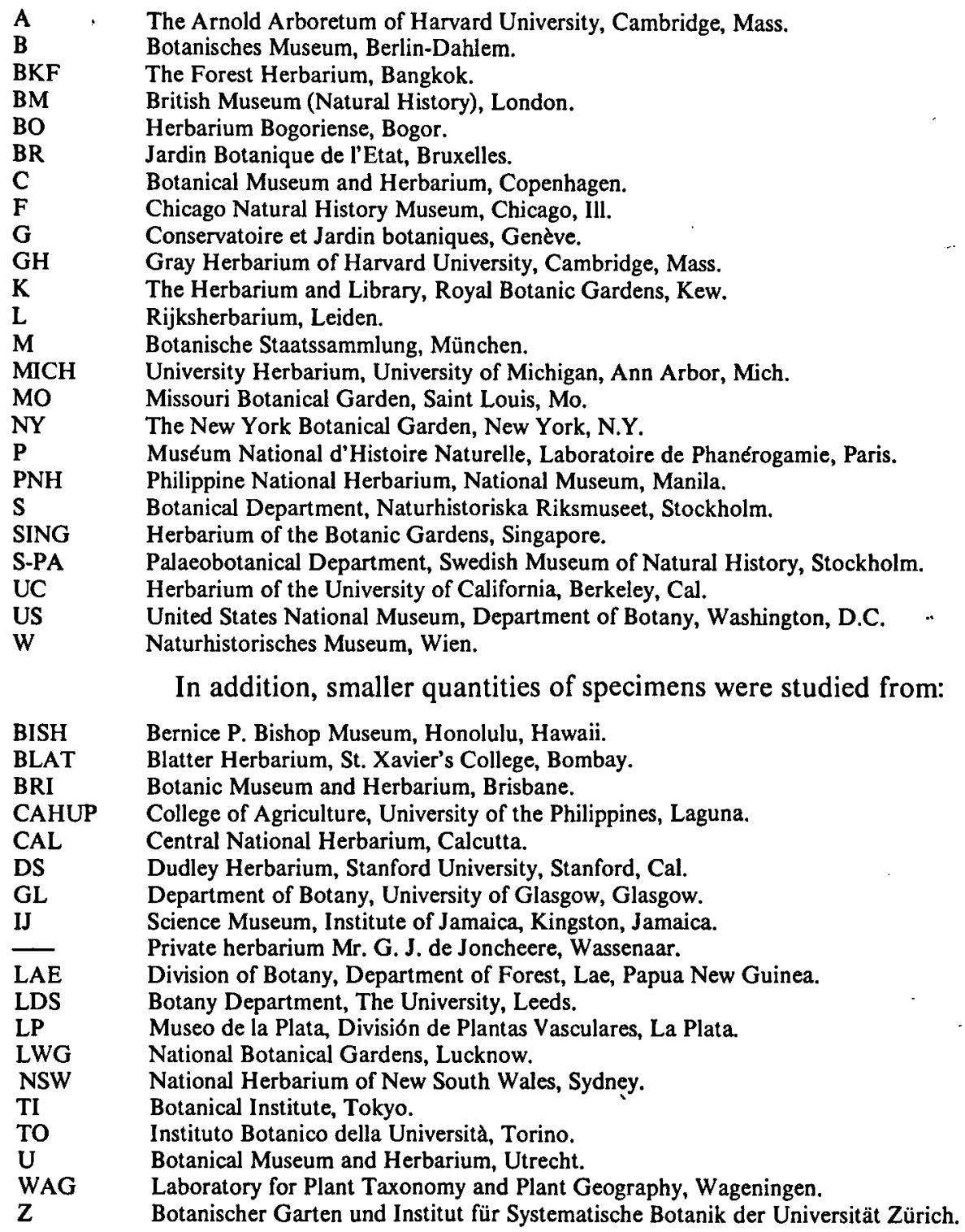

Some taxa were studied in the field during a botanical expedition to Thailand, 1965/1966; living material of several species was cultivated at the Botanic Garden, Leiden.

A considerable part of the specimens studied comprise so-called duplicates. Phanerogamists sometimes object to studying duplicates as they are said not to provide anything new. This may sound sensible when large Angiosperms (trees!) are concerned. However, when studying Bolbitis specimens, it was found very informative to see duplicates as they give often a better idea of the usually large variation between 
specimens from a particular site. They further afford a better chance for studying characters not always present on all sheets(e.g. rhizome, mature spores).

\section{Methods}

\section{Scales}

Scales were brushed with a strong solution of a detergent (Kodak, Photo-Flo 200) in water (Kramer, 1957), and after washing in water embedded in glycerine jelly.

\section{Rhizome and petiole}

To obtain sections of the rhizome and petiole from herbarium material, pieces of petiole and rhizome were softened in polivinyl lactophenol (Edward Gurr, Ltd.; for details see Van Brummelen, 1967) for 24 hours at $60^{\circ} \mathrm{C}$. After washing in distilled water the material was sectioned with a slide microtome (Reichert) using razor blades. Sections were embedded in glycerine jelly. By these methods the (presumed) natural condition of the material could be reconstructed rather easily.

For anatomical studies of living material, small pieces of material were preserved in F.A.P.A. (formaline 40\%: 5; glacial acetic acid: 2.5 ; propionic acid: 2.5 ; alcohol $50 \%$ : 90), for 24 hours or longer. A routine histological paraffine procedure was followed. Sections were stained with saffranine followed by fast green.

\section{Venation pattern}

For the present study it became necessary to develop a method which made it possible to obtain a large number of magnified illustrations of the venation pattern in a short time. The method applied was based on the idea that liquid-stored cleared parts of the leaves show sufficient contrast between the veins and the tissues in between, for using these parts as negatives (without grains!) in an enlarger. For this purpose a special jar of similar size as the negative holder of an enlarger, holding the material in a flat position, was devised. The method used did not make it necessary to stain the xylem elements for contrast (as is usually done). Besides, many patterns of any magnification could be recorded on photographic paper in a short time.

\section{a. Clearing of (parts of) the leaves}

1. Boil the herbarium material during 5 minutes in water to which a few drops of a detergent are added.

2. Fix in F.A.P.A. during 24 hours at room temperature.

3. Replace F.A.P.A. by chloro-lactophenol (chloralhydrate: 1, lactophenol: 1) to which a small amount of hydroxyperoxide $\left(\mathrm{H}_{2} \mathrm{O}_{2}\right)$ is added. Store for 2 or 3 days at $60^{\circ} \mathrm{C}$. The amount of hydroxyperoxide depends on the quality of the herbarium 
material. In well-dried specimens it can be ommitted. When too much hydroxyperoxide is added the cleared material can suffer from air bubbles which then have to be evacuated.

4. Replace by pure chloro-lactophenol. Store for 1 to 3 days at $60^{\circ} \mathrm{C}$.

5. Rinse in several changes of distilled water.

6. Proceed through graded alcohol to alcohol $50 \%$ in which it can be stored until used for photography.

The material may be still of a light brown colour after this treatment. This does not affect its use, provided there is reasonable contrast between the veins and the remaining parts.

7. Dry the material and remount it on the herbarium sheet.

\section{b. Projection photography of cleared parts}

The cleared parts were put into a jar made of plexiglass of similar sizes as the negative holder of a Liesegang 'Raja' enlarger. Representative areas up to $9 \mathrm{~cm}$ long were recorded on photographic paper (Agfa-Gevaert BH 1).

The square jar (see fig. 1) consists of a basal part with supports situated at the inside corners, apart from a cover which is resting upon the supports and is provided with vertical flanges somewhat remote from the margin. The supports of the basal part are hookshaped (following the corners) and composed of two 'steps', each $3 \mathrm{~mm}$ tall and $6 \mathrm{~mm}$ broad, the lowest one of which faces the jar's interior. The cover rests on the lowest step of the supports at each corner, thus leaving a narrow slit along the margin between the supports and between the outside margin of the cover and the inside wall of the basal part. Addition or removal of liquid through these slits is easy as the flanges on the cover prevent possible excessive liquid to spread over the cover. The slits also allow long materials to project through the slits beyond the jar. The actual jar which contains the liquid (evacuated alcohol $50 \%$ ) is thus only $3 \mathrm{~mm}$ tall.

Cleared parts were arranged in the jar filled with some liquid, and with the cover removed. Usually several objects could be arranged side by side. Flattening of the material was readily obtained using thin pieces of glass of different sizes (the different kinds of object slides do well). When fixing the cover one has to avoid the inclusion of large air bubbles. After the adjustment of the right amount of liquid the jar is ready for use in the enlarger. The equipment of the enlarger devised for frames of $6 \times 9 \mathrm{~cm}$ was applied. To prevent too much spreading of light through the jar during magnification, the jar's outsides were painted black.

Good prints showing (details of) the venation pattern could then be readily obtained. Representative photographs are given in plate 1 . For the present study the standard magnification of the venation pattern was four times.

In spite of its liquid contents the jar appeared to be quite handy in use.

\section{c. Drawings}

For publication in the Taxonomic Part, a selection of the photographs were exposed to transmitted light, and the venation pattern copied on translucent paper. Afterwards the drawings were reduced five times. 


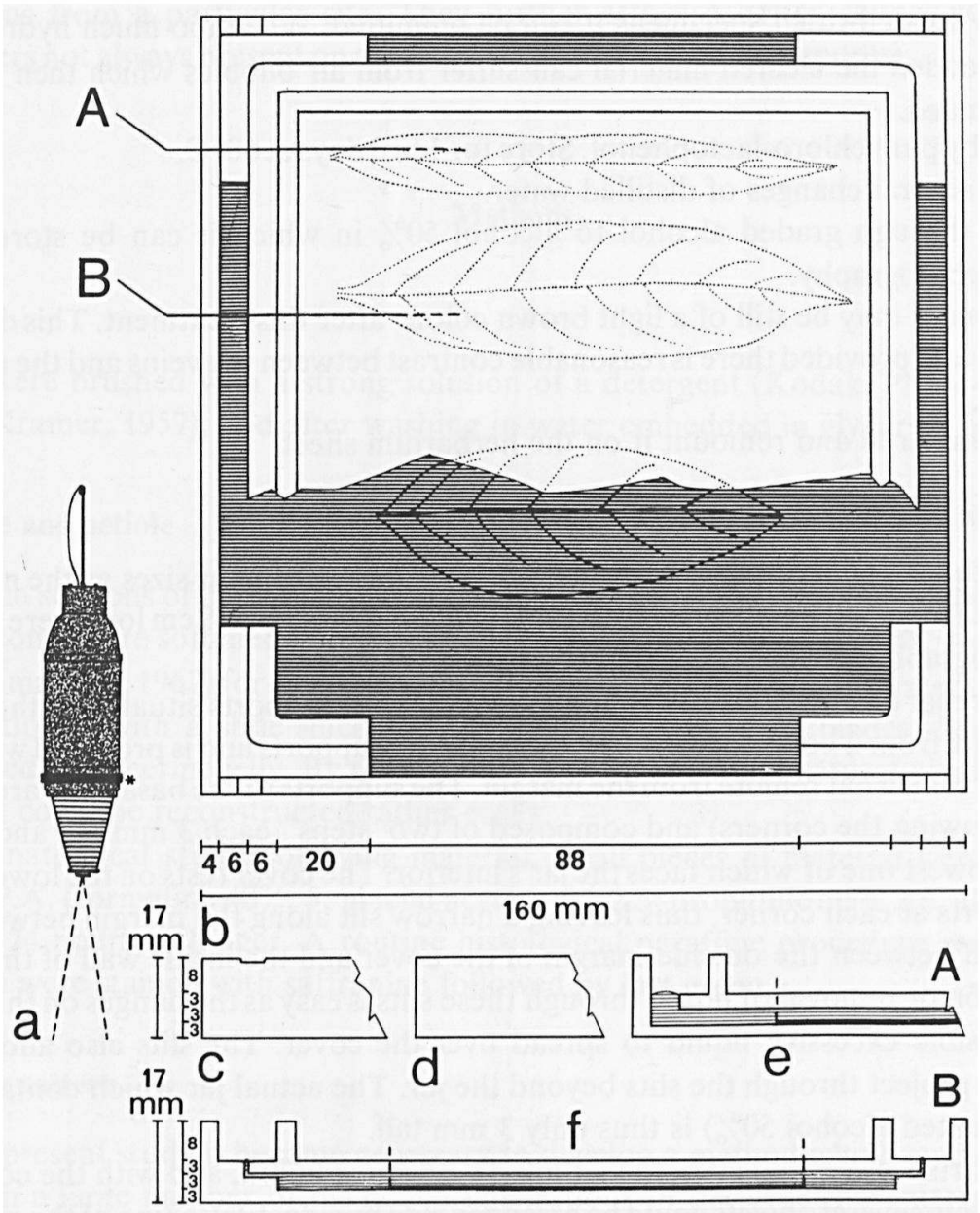

Fig. 1. Schematic drawings of the jar used for projection photography of cleared pinnae or laminas.-a. enlarger, the asterisk indicates the location of the jar in the enlarger; $b-d$. different views of the jar; $b$. view from above; c. part of frontal view; d. part of lateral view; e, f. cross-sections at A and B resp. of fig. b. For further information see text.

\section{Spores}

Spores of herbarium material were transferred to glycerine jelly after wetting with alcohol $96 \%$. A representative selection of the material of each species was studied. In addition, some samples of different species were weakly acetolyzed following routine procedures.

The different types of spores as seen with the light microscope were also studied with a Cambridge S.E.M. (scanning electron microscope). For this purpose spores were directly taken from the herbarium material, fixed to a standard aluminium specimen 
stub with double-stick Scotch tape, and coated with evaporated gold shortly before examination at 10 or $20 \mathrm{kV}$.

Cross-sections of sporangia and spores were obtained from F.A.P.A.-fixed (sporangia) or from dried material (spores) after sectioning in ice in a kryostate at $-30^{\circ} \mathrm{C}$. The sections, mounted on a specimen stub of the S.E.M., were dried at room temperature prior to coating.

\section{Chromosomes}

Manton (1950) gives an ample discussion about the techniques that can be applied to record the mitotic and meiotic chromosome number. The following technique is basically similar:

1. Pretreat with alpha-bromonaphtalen in distilled water (ABN) during 24 hours at $5^{\circ} \mathrm{C}$; only applied when necessary.

2. Fix in Carnoy's solution (alcohol 100\%: 3, glacial acetic acid: 1) for at least 5-10 minutes at room temperature. In this solution it can stay for one or two days at room temperature, or much longer at $-20^{\circ} \mathrm{C}$.

3. Hydrolyze in a solution of equal parts of absolute hydrochloric acid and absolute alcohol for one to several minutes at room temperature.

4. Wash in two changes of Carnoy's solution.

5. Stain in acetocarmine for at least 10 minutes at room temperature.

6. Squash a selected piece of material under a cover slip. Remove excessive dye and seal with paraffine. A sufficient spreading of the chromosomes was sometimes difficult to achieve. The application of a mixture of pectinase and cellulase (Kawano, 1965) did not improve the results markedly.

7. Photograph the suitable plates using phase contrast microscopy when necessary.

Vouchers of the author's counts are at Leiden, those of Manton and Walker-which are published here for the first time-at $\mathrm{K}$ and the BM, respectively. The subsequent numbers of Walker's collections of $B$. quoyana have all been included. The morphological heterogeneity of the vouchers excludes the possibility of their being parts of one clone.

\section{Microscopy}

The preparations were studied with a binocular microscope (Leitz, 'Greenough') and/ or a light microscope (Leitz, 'Laborlux'). Photographs of microscopic preparations were made with a photomicroscope (Leitz, 'Ortholux').

\section{Drawings of outlines of the leaves}

The outline drawings in the Taxonomic Part were all made at natural size from dried specimens, then reduced five times. 


\section{Cultivation}

The cultivation of tropical lowland ferns in a greenhouse with only limited facilities in a temperate area is a matter of trial and error. I have persisted in my attempts to grow living material in view of the presumed contribution of cytological, morphological, and anatomical studies to the taxonomy of the present genus.

The best results with the cultivation of sporophytes were obtained when the plants were grown in large terraria or in small houses of polyethylen within the greenhouses.

The specimens were potted in rocky soil composed of variously large pieces of lavarock, apart from a mixture of about equal parts of chopped Sphagnum, coarse sand, and black peat.

During the winter period growth was slow or even absent. Sometimes the temperature in the greenhouses did not rise above $17^{\circ} \mathrm{C}$ for several weeks. Then measures had to be taken to prevent the atmosphere from becoming saturated which causes rapid moulding and rotting. Considerable changes in temperature between day-time and night-time, as well as between sunny and cloudy outside weather conditions, are regarded the most important factors causing untimely death of the specimens.

The majority of the specimens showed good or excellent growth during the summer period. Then most of the specimens could be vegetatively propagated in order to get sufficient material for carrying the species through the winter.

The Leiden greenhouses suffer from American cockroaches which were reasonably controlled by inundating the terraria at intervals during periods of good growth, or by trapping specimens in brown flasks with a narrow neck containing old fruit.

Spores of only a few species could be sown on sterilized soil, following routine procedures. Growth of the gametophytes is very slow; the cultures are easily affected by growth of bryophytes and fungi.

It is noted that in cultivation the plants may show aberrances which are not formed in the wild. A specimen of Bolbitis crispatula var, copelandii formed bipinnatifid and bipinnate leaves in cultivation, whereas the leaves of all the wild specimens of this taxon are simply pinnate.

\section{TAXONOMIC HISTORY OF THE GENUS}

The genus Bolbitis comprises acrostichoid ferns, i.e. ferns with separate fertile leaves the underside of which is fully covered with sporangia possessing a vertical annulus. The earliest authors, who used the shape of the sporangium, the shape of the sorus; and the presence or absence of an indusium as the main principles for classification, included the acrostichoid ferns all into the genus Acrostichum (e.g. Swartz, 1806; Willdenow, 1810). Kaulfuss (1824) placed two species with free veins in the genus Gymnogramma, a genus he created to accommodate the ferns with sporangia mainly or exclusively on the veins, and lacking an indusium.

The genus Bolbitis was established by Schott (1834) for a part of Acrostichum with a 
creeping rhizome and anastomosing veins. He included 'B. serratifolia, virens, diversifolia, flagellifera, repanda, etc.'. At the same time Schott accommodated a free-veined species in the genus Egenolfia (here united with Bolbitis) which included Polybotrya sensu Blume (1828). The genus Polybotrya was restricted by Schott to its present circumscription. The recognition of the Asian genus Egenolfia, separate from the American genus Polybotrya, is illustrative for the quality of his work. It was not until Ching (1931; in Christensen, 1934) that this classification was accepted.

Presl(1836) who used mainly anatomical characters and especially the venation pattern, merged Egenolfia with Polybotrya, both having free veins. The other species were placed in the genera Poecilopteris, Campium, and the two subgenera of Gymnopteris.

Smith(1841: 150) too listed the species with free veins under Polybotrya, the others composed the genus Cyrtogonium (a homotypic synonym of Bolbitis) and the minor part of Gymnopteris.

Fée (1845) was the first to monograph the acrostichoids. He based his work on the material present in the large herbaria in continental Europe; some of Wallich's species were not taken into consideration. Using the venation pattern and the leaf architecture as the more important characters, he divided the acrostichoids into 18 genera. The species of the genus Bolbitis in its present construction were classed in the genera Polybotrya, Heteroneuron (which included Presl's genera Campium and Poecilopteris, as well as $H$. raddianum = Lomagramma guianensis), Gymnopteris, and Leptochilus (one species). Later Fée (1850-1852) recognized the genus Egenolfia. Presl (1851), giving additions and emendations to the Tentamen (1836), placed the species in Anapausia (which was a subgenus of Gymnopteris in the Tentamen), Poecilopteris, Campium, and Heteroneuron (in a much narrower sense than Fée, 1845). Presl inferred that the features of the venation pattern would add much to the understanding of the classification: '..., darf man der Hoffnung Raum geben, dass man endlich in das geheimnisvolle Dunkel dieser wundervoll organisierten Gewächse eindringen wird.'

The systems proposed by Fée and Presl were in part artificial, and not convincing to Hooker (1861, opp. pl. 88): 'Notwithstanding the valuable labours of M. Fée and his excellent writings and figures of the Acrostichaceae, no group of Ferns requires more careful study and revision than this does. Species are, assuredly, too much multiplied, I fear on very slight grounds: and the multitude of Genera only serve to puzzle and perplex the student as well as the practical Pteridologist himself.'

Hooker (1864: 194) (and also Baker, in Hooker \& Baker, 1865-1868) reverted to the classical conception and massed all acrostichoids into the genus Acrostichum which he subdivided into the subgenera Egenolfia, Heteroneuron, Gymnopteris, and Leptochilus; of these the three last also contained representatives of one or more other genera. Baker accommodated the species in the subgenera Egenolfia, Gymnopteris (in part), and Chrysodium (species which Hooker included in Gymnopteris and Heteroneuron).

J. Smith (1875) reinstated Egenolfia as a genus. The species with anastomosing veins were placed in the genera Poecilopteris and Gymnopteris (in part).

Classical authors used to place all or most of the acrostichoids as a separate group either at the beginning (J. Smith, 1875) or at the end of their classification (e.g. Hooker, 
1864) of the 'Polypodiaceae' (leptosporangiate ferns with a vertical annulus). Christ (1897) and Diels (in Engler \& Prantl, 1899), however, referred all the acrostichoids with free veins again to Polybotrya, those with anastomosing veins to the genus Gymnopteris which included quite a variety of unrelated ferns as well, and both these unnatural genera were placed in the Aspidiinae (Polypodiaceae-Aspidieae) also comprising dryopteroid and tectarioid ferns. Underwood (1903) accommodated the present species of Bolbitis in Egenolfia and a heterogeneous Anapausia, including both in the PolypodiaceaeDryopterideae, a group containing dryopteroid, thelypteroid and tectarioid ferns. Christensen (1904) followed Underwood, but correctly used the generic name Leptochilus for the assemblage named Anapausia by Underwood. He thought the genus Leptochilus to include mainly Old World species. Only a dozen species were said to occur in America which he referred to sect. Bolbitis, at the same time supplying a revision; the others he assembled later(1906) in sect. Anapausia and were regarded as 'essentially different'. Leptochilus guianensis (= Lomagramma guianensis) was included in sect. Bolbitis with misgivings; it possibly should be regarded 'a peculiar subgenus or even genus'. In the Index (Christensen, 1906) the species were placed in Leptochilus sect. Bolbitis and sect. Anapausia, and in sect. Egenolfia of Polybotrya. Both genera were included in the Polypodiaceae-Aspidieae.

Schumann (1915) followed Christensen, but regarded the Leptochilus species with pinnate leaves, such as Leptochilus heteroclitus $(=B$. heteroclita $)$, as ex-indusiate derivatives of thelypteroid ('dryopteroid') ferns, whereas the species with simple leaves were thought to represent derivatives of Polypodium species. Although she based herself on but little material, her key is of interest as it also mentions features of the spores. Bower $(1917,1928)$ largely agreed with Schumann.

Copeland (1928) attempted to clear up the heterogeneous assemblage called Leptochilus by Christensen and Gymnopteris by Diels. He referred the American species of Leptochilus sensu Christensen to the genus Bolbitis which he thought to be derived from Phlebodium (polypodioid). These were not included in his revision. The greater part of the Old World species of Leptochilus were, together with representatives of other genera, transferred to the genus Campium, and thought to have evolved from Old World polypodioid ancestors with 'definite sori'. Campium linnaeanum (a dwarf of $B$. heteroclita) was regarded as the most primitive element of the genus. Other Old World species of Leptochilus sensu Christensen were classified in the genera Leptochilus, Christiopteris, Lomagramma, Hemigramma, and Quercifilix, a view now generally accepted.

Christensen (1931) agreed with Copeland to keep the American and the Old World species generically separate. He retained Leptochilus sect. Anapausia for the American species and accepted the genus Campium though in a somewhat different circumscription as he suggested to exclude from Campium the simple-leaved species that Schumann considered of polypodioid origin. According to Christensen the genus so construed was of thelypteroid affinity, and closely related to, or even congeneric with the genus Egenolfia. As regards the American species, Christensen thought Leptochilus alienus $(=B$. aliena), the type species of Leptochilus sect. Anapausia, to be derived from Tectaria, 'and it is also possible that certain Asiatic species, such as $L$. [Leptochilus] diversifolius $[=B$. 
sinuata], have their nearest relatives among the species of Tectaria.' This shows Christensen's doubts as to the systematic position of these acrostichoids as dealt with by Copeland (the pinnate fern Leptochilus diversifolius being included by Copeland in the genus Campium).

Ching (1931) monographed the genus Egenolfia. He recognized nine species and added one variety. The genus was divided into two sections. Later (in Christensen, 1934), he referred Christensen's Leptochilus sect.Anapausia and part of Copeland's genus Campium to Bolbitis, thus largely delimiting the genus as circumscribed here. This I regard as an important advance. Christensen himself, in the same paper, also added several new combinations in Bolbitis, which shows his interest in the acrostichoids. He also stimulated Ching to make further studies in this group, as is suggested by Ching's later publication (1932). Ching apparently also attempted to monograph the genus Bolbitis; I found Ching's annotations on sheets present in the herbaria at Paris and Kew (including type sheets of several unpublished species).

Christensen (1938) regarded the genera Bolbitis and Egenolfia as probably related to the Polypodiaceae-Dryopterideae (i.e. to the dryopteroid and thelypteroid ferns).

Copeland (1947) qualified his earlier treatment of the acrostichoids (Copeland, 1928) as 'inexcusably bad' (which may be true as far as the genus Campium is concerned) and further agreed with Christensen (1938) as to the relationship of the genus Bolbitis.

Kramer (1954), following a suggestion of Holttum (1937), transferred Lomagramma guianensis, a paraphysate species, to Bolbitis; it is here excluded.

Manton \& Sledge (1954) identified $B$. singaporensis Holttum as a hybrid between Egenolfia appendiculata $(=B$. appendiculata $)$ and $B$. diversifolia $(=B$. sinuata $)$.

Iwatsuki (1959) revised the Japanese species and, following a suggestion of Christensen (1931), united the genera Egenolfia and Bolbitis. He divided the genus into four sections.

Nayar \& Kaur (1964a, 1964b) published an elaborate but uncritical paper on the taxonomy of the species occurring in India based on but a few collections. In addition, they published a number of papers dealing with the morphology of these acrostichoids (for references see Kaur, 1974). They kept the genera Bolbitis and Egenolfia separate, also because of supposed differences in the shape of the perispore of the spores which was refuted by the present author (Hennipman, 1970). Kaur (1974) thought the genus Bolbitis (excluding Egenolfia) the most primitive member of the Lomariopsidaceae (including Elaphoglossum) which she regarded related to the tectarioid ferns.

Earlier studies on the acrostichoids (Hennipman, 1965, 1976) revealed the proper taxonomic alliance of Leptochilus neglectus (F. M. Bailey) C. Chr. (=Pteridoblechnum neglectum (F. M. Bailey) Hennipman), and Leptochilus acuminatus White \& Goy (= Pteridoblechnum acuminatum (White \& Goy) Hennipman). Detailed information on Bolbitis macrophylla (Kunze) Maxon \& Morton (= Thelypteris macrophylla (Kunze) Morton) was made known to Mr. Morton, who accordingly transferred the species to its proper place (Morton, 1971).

As a result of floristic work by Christ, Copeland, Maxon \& Morton, and Holttum (for references see the Taxonomic Part), a considerable number of new species were 
described during this century. Copeland described 18 new species and one new genus which belong to the present genus; of these only one species is here retained.

\section{MORPHOLOGY AND ANATOMY}

\section{Introduction}

Representatives of the present genus have been studied in detail by Schumann (1915), Bower (1917), and Nayar and collaborators (for references see Kaur, 1974).

Schumann studied Leptochilus heteroclitus $(=B$. heteroclita) and Leptochilus cuspidatus $(=B$. repanda) from living material at Munich. Bower studied the vascular organisation in the rhizome of $B$. ('Leptochilus') nicotianifolia and B. repanda ('Leptochilus cuspidatus'). The Indian representatives have been dealt with by Nayar et al. The works of the Indian authors contain much interesting and in part new information as was confirmed during the present study. However, their material represents only three of the ten series recognized. The names of the taxa used in their morphological studies are those as given in a kind of elaborate checklist of the Indian members (Nayar \& Kaur, 1964a, 1964b). The latter publications are based on but very few collections, whereas the nomenclature of part of the species listed is incorrect; their taxonomic concepts differ much from those presented here.

It is further much regretted that their works include some distinctly erroneous observations such as those on the spores of Bolbitis, which consequently led them to recognize the genus Egenolfia as distinct (see Hennipman, 1970). The value of their publications is also marred by the fact that part of the material attributed by them to Bolbitis belongs to other genera. For instance, the bipinnate juvenile leaves reported to occur in certain Bolbitis species (of which I studied the juvenile leaves myself) do not belong to the present genus but presumably to Microlepia species (Nayar \& Kaur, 1965b: pl. 2 facing p. 155). Further, a special kind of venation pattern, generally called diplodesmic, reported by them (Nayar \& Kaur, 1966) to be found in the fertile leaf of $B$. heteroclita (' $B$. subsimplex'), actually does not occur in the reference sheet cited (kindly sent on loan), nor for that matter in the genus. They also described certain species as 'high-climbing' whereas in reality true Asian Bolbitis species do not conform to this mode of life. These erroneous observations play an important role in their ideas regarding the phylogeny of the lomariopsidoid ferns: 'From the evolutionary point of view, Bolbitis possibly is the more primitive genus in the family. It exhibits a tendency towards evolution of high climbing forms with special venation to supply sporangia in the fertile leaves (B. subsimplex); this might lead on to the high-climbing genera, as well as towards forms with a short, creeping, dorsiventral rhizome with simplification of venation pattern, leading to genera like Egenolfia.'

Speaking about classification in general, Holttum (1967) stated that there is still much confusion "because the right kind of morphological knowledge is not all available, and because no one has yet discerned which kinds of such knowledge are most signifi- 

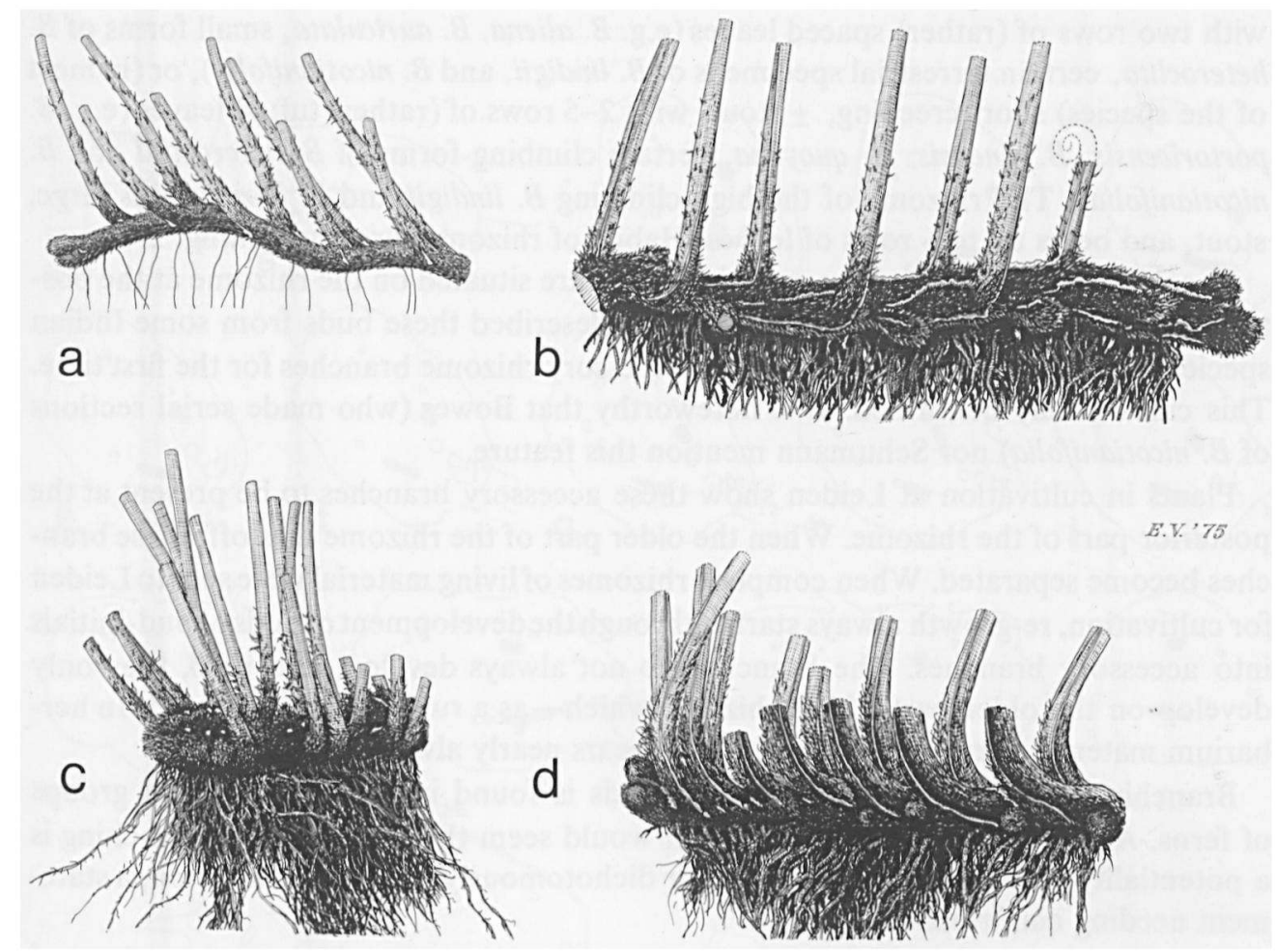

Fig. 2. Habits of rhizomes drawn from living material showing amongst other things aerophores and (in fig. b and d) accessory buds, $\times 3 / 5$. - a. Bolbitis heteroclita; b. B. heudelotii; c. B. sinensis; d. B. sinuata (a. Hennipman 4085 ; b. H. 4118 ; c. H. 4046; d. H. 4052).

cant in particular cases ... 2 The present chapter has been written in view of my agreement with that statement.

My observations on the rhizome and petiole have bèn taken from cross-sections of herbarium material of all the species of which adequate material was at hand, as well as from cross-sections of living material pickled in the field or cultivated. In several species from different series (except ser. Lindigianae) serial sections were studied in order to obtain information on the spatial arrangement of the vascular system. Indument, spores, and venation patterns were studied from all the species. Of only a few species gametophytes were obtained in cultivation.

\section{Rhizome}

In most of the species the rhizome is creeping, usually on rocks or in rocky soil; in a few species it is either low- or-in some American species-high-climbing. The shape of the rhizome shows variation. The creeping rhizome may be long-creeping, slender, 
with two rows of (rather) spaced leaves (e.g. B. aliena, B. auriculata, small forms of $B$. heteroclita, certain terrestrial specimens of $B$. lindigii, and $B$. nicotianifolia), or (in most of the species) short-creeping, \pm stout, with 2-5 rows of (rather) tufted leaves (e.g. $B$. portoricensis, $B$. sinensis, $B$. quoyana, certain climbing forms of $B$. heteroclita and $B$. nicotianifolia). The rhizome of the high-climbing $B$. lindigii and $B$. bernoullii is large, stout, and bears up to 6 rows of leaves. Habits of rhizomes are given in fig. 2 .

In some species conspicuous spherical buds are situated on the rhizome at the posterior side of the leaf-bases. Nayar \& Kaur described these buds from some Indian species, and reported development into accessory rhizome branches for the first time. This can be fully confirmed. It is noteworthy that Bower (who made serial sections of $B$. nicotianifolia) nor Schumann mention this feature.

Plants in cultivation at Leiden show these accessory branches to be present at the posterior part of the rhizome. When the older part of the rhizome dies off, these branches become separated. When complete rhizomes of living material were sent to Leiden for cultivation, re-growth always started through the development of buds or bud-initials into accessory branches. The branches do not always develop, and if so, they only develop on the older part of the rhizome which-as a rule-is not collected. In herbarium material, therefore, the rhizome appears nearly always unbranched.

Branching due to the development of buds is found in several unrelated groups of ferns. According to Dickason (1946) it would seem that this mode of branching is a potentiality added to the unbranched or dichotomously branched condition, a statement needing confirmation.

The rhizome consists of parenchymatous ground-tissue in which the vascular and in many species also sclerenchyma strands are situated. In association with the leaves, a sheath of relatively thin-walled, often dark-coloured sclerenchyma cells may occur a few cells below the epidermis. Aerophores also associated with the leaves are present dorsally and laterally; in these places the sheath of sclerenchyma is interrupted.

The stele is a dorsiventral dictyostele (incorrectly called solenostele by Nayar \& Kaur, 1965a), a characteristic of all lomariopsidoid ferns (Holttum, 1946), with dorsally (and laterally) very wide overlapping leaf-gaps. The ventral part consists of a (rather) broad, entire, gutter- or ribbon-shaped strand. Root traces arise from this ventral strand (either in two irregular rows, or irregularly from all over the strand) \pm laterally and ventrally. The dorsal part consists of one to five cylindrical or strap-like strands. In case the rhizome bears two rows of leaves, leaf traces originate from the lateral sides

Fig. 3. Rhizome anatomy. a-m. Bolbitis auriculata; a. schematic habit of rhizome, $\times 1 ; \mathbf{b}-\mathrm{m}$. cross-sections as indicated in fig. a, $\times 4 .-n-u$. cross-sections of rhizomes, $\times 4$ (except for fig. $r-u$, which are $\times 2$ ); $n$. Bolbitis heudelotii; o. B. heteroclita; p. B. heteroclita (triploid); q. B. oligarchica; r. B. lindigii; s. B. portoricensis; $\mathrm{t}, \mathrm{u}$. B. bernoullii.-ae = aerophore, bt $=$ bud trace, bu $=$ rhizome bud, $\mathrm{db}=$ dorsal bundle, $1 \mathrm{t}=$ lateral bundle, $r=$ root; $r_{1}, r_{2}=$ subsequent roots; $\mathrm{rt}_{1}, \mathrm{rt}_{2}=$ subsequent root traces, $\mathrm{vb}=$ ventral bundle; for further explanation see text (a-m. Louis 12196, BM; n. Hennipman 4118, L; o. Mann s.n., US 329566; p. Hennipman 4069, L; q. Scamman 7683, GH; r. Alston 7836, BM; s. Alston 5826, BM; t, u. Philipson \& Idrobo 1796, BM). 

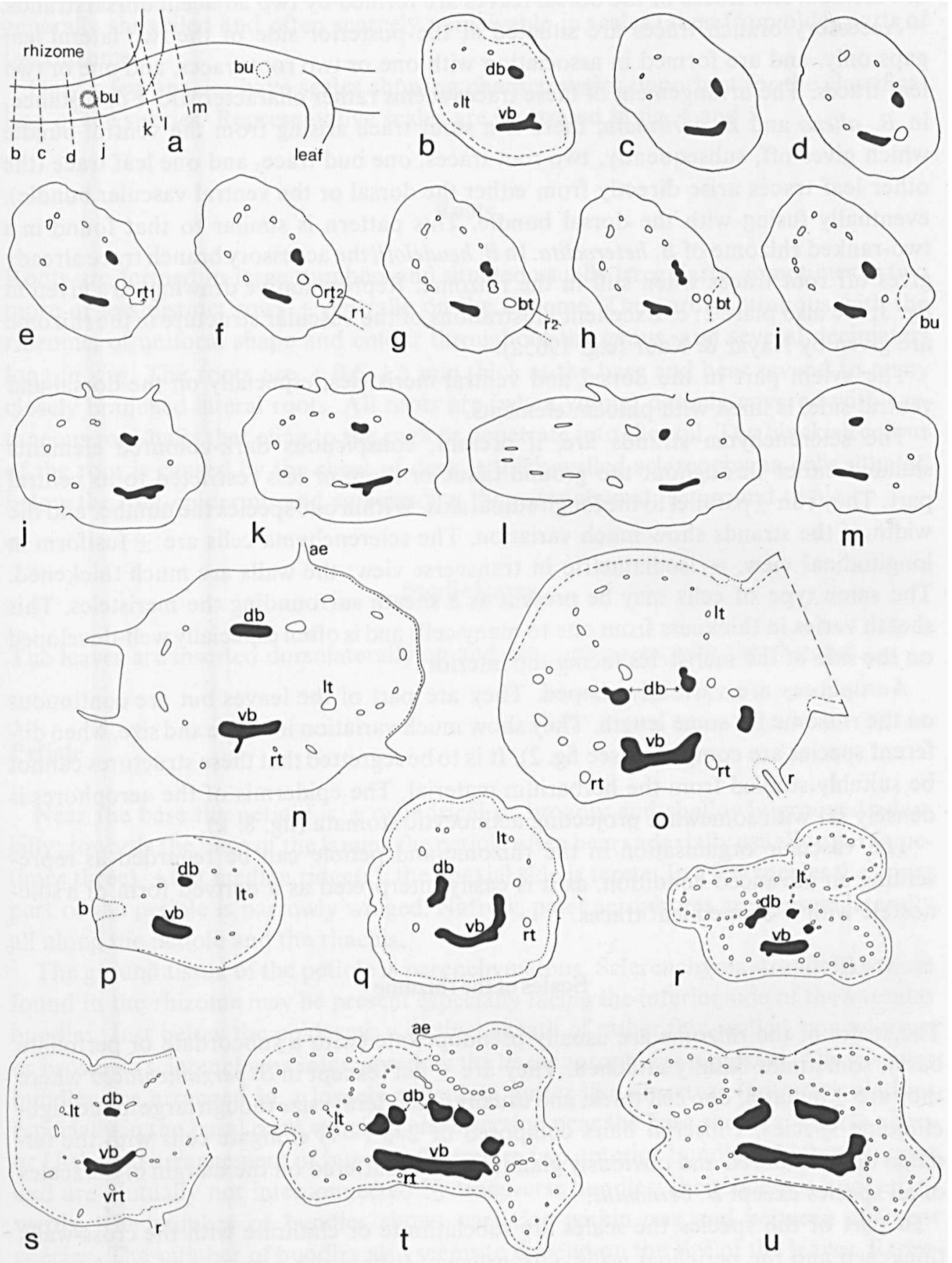
of both the ventral and the single dorsal strand. When more than two rows of leaves are formed, leaf traces of the dorsal leaves are formed by two adjacent dorsal strands.

Accessory branch traces are situated at the posterior side of the two lateral leaf gaps only, and are formed in association with one or two root traces, and one or two leaf traces. The arrangement of these traces seems rather characteristic. For instance, in $B$. aliena and $B$. auriculata, there is a stout trace arising from the ventral bundle which gives off, subsequently, two root traces, one bud trace, and one leaf trace (the other leaf traces arise directly from either the dorsal or the ventral vascular bundle), eventually fusing with the dorsal bundle. This pattern is similar to that found in a two-ranked rhizome of $B$. heteroclita. In $B$. heudelotii the accessory branch trace already gives off root traces when still in the rhizome. Representative drawings are given in fig. 3; see also plate 2: c. Excellent illustrations of the vascular structure in the rhizome are given by Nayar \& Kaur (e.g. 1965a).

The xylem part in the dorsal and ventral meristeles, especially on the dorsal and ventral side, is lined with phloem elements.

The sclerenchyma strands are, if present, conspicuous dark-coloured elements situated either throughout the ground tissue or more or less restricted to its central part. They run \pm parallel to the longitudinal axis. Within one species the number and the width of the strands show much variation. The sclerenchyma cells are \pm fusiform in longitudinal view, \pm isodiametric in transverse view; the walls are much thickened. The same type of cells may be present as a sheath surrounding the meristeles. This sheath varies in thickness from one to many cells and is often especially well-developed on the side of the meristeles facing the interior.

Aerophores are variously shaped. They are part of the leaves but are continuous on the rhizome for some length. They show much variation in shape and size, when different species are compared (see fig. 2). It is to be regretted that these structures cannot be suitably studied from the herbarium material. The epidermis of the aerophores is densely set with somewhat projecting actinocytic stomata (fig. 8: k).

The vascular organisation in the rhizome and petiole can be regarded as representing an advanced condition, as it is easily interpreted as a derived form of a solenostele with U-shaped leaf traces.

\section{Scales of the rhizome}

The scales of the rhizome are usually pseudopeltate (with a subcordate or perfoliate base), sometimes basally attached. They are \pm flat (except in $B$. pergamentacea where they are \pm bullate), one cell thick, and usually of moderate size though large in the highclimbing species. Uniserial hairs composed of 2-3 (-7) elongate cells with the terminal dark-coloured and obviously glandular, are scattered on the margin of the scales of all species except $B$. bernoullii.

In part of the species the scales are subclathrate or clathrate with the cross-walls thickened and the periclinal walls \pm translucent (often with a mother-of-pearl shine), in other species the scales are opaque throughout. 
Scales are best studied from the apical region of the rhizome. They grow from a basal meristem. Mature glandular hairs are best studied from developing scales, as they are generally shrivelled and often scarcely perceivable in scales taken from older parts of the rhizome.

Only a few species have scales showing characteristics important for the classification of the species. Representative scales are illustrated in fig. 4 and 5.

\section{Roots}

Roots are formed in large numbers and situated usually irregularly, sometimes in two more or less distinct rows \pm ventrally on the rhizome. They are continuous with the rhizome, of uniform shape and colour throughout the genus, and several decimeters long in vivo. The roots are $\pm 0.5-1.5 \mathrm{~mm}$ thick at the base and bear several to many closely branched lateral roots. All roots are (when young) densely covered with castaneous root-hairs that cling to the rock or penetrate into the soil. The blackish colour of the root is caused by the sheet of dark \pm thick-walled sclerenchyma cells situated below the root-epidermis and surrounding the parenchymatous ground tissue.

\section{Sterile leaves}

The leaves are inserted dorsolaterally on and are continuous with the rhizome.

\section{Petiole}

Near the base the petiole is \pm cylindrical or broadly and shallowly grooved adaxially; towards the base of the lamina the petiole often bears adaxially usually one (sometimes three) \pm flat median ridge(s); the abaxial side is terete. In a few species the upper part of the petiole is narrowly winged. Narrow, paler aerophores are present laterally all along the petiole and the rhachis.

The ground tissue of the petiole is parenchymatous. Sclerenchyma strands like those found in the rhizome may be present especially facing the interior side of the vascular bundles. Just below the epidermis a distinct sheath of rather thin-walled, concolorous or brownish sclerenchyma interrupted by the linear aerophores is present. The vascular bundles are arranged in a loosely reticulate gutter-like structure (with reticulations especially in the basal part) which in cross-section near the base shows a thorse-shoeor U-shaped arrangement of bundles. There are two anterior bundles which are largest and are mutually not interconnected by transverse bundles; their xylem is hooked inwards. The number of bundles shows variation within one and between different species. The number of bundles also seems to depend on the size of the leaves. Representative illustrations are given in fig. 6 . 

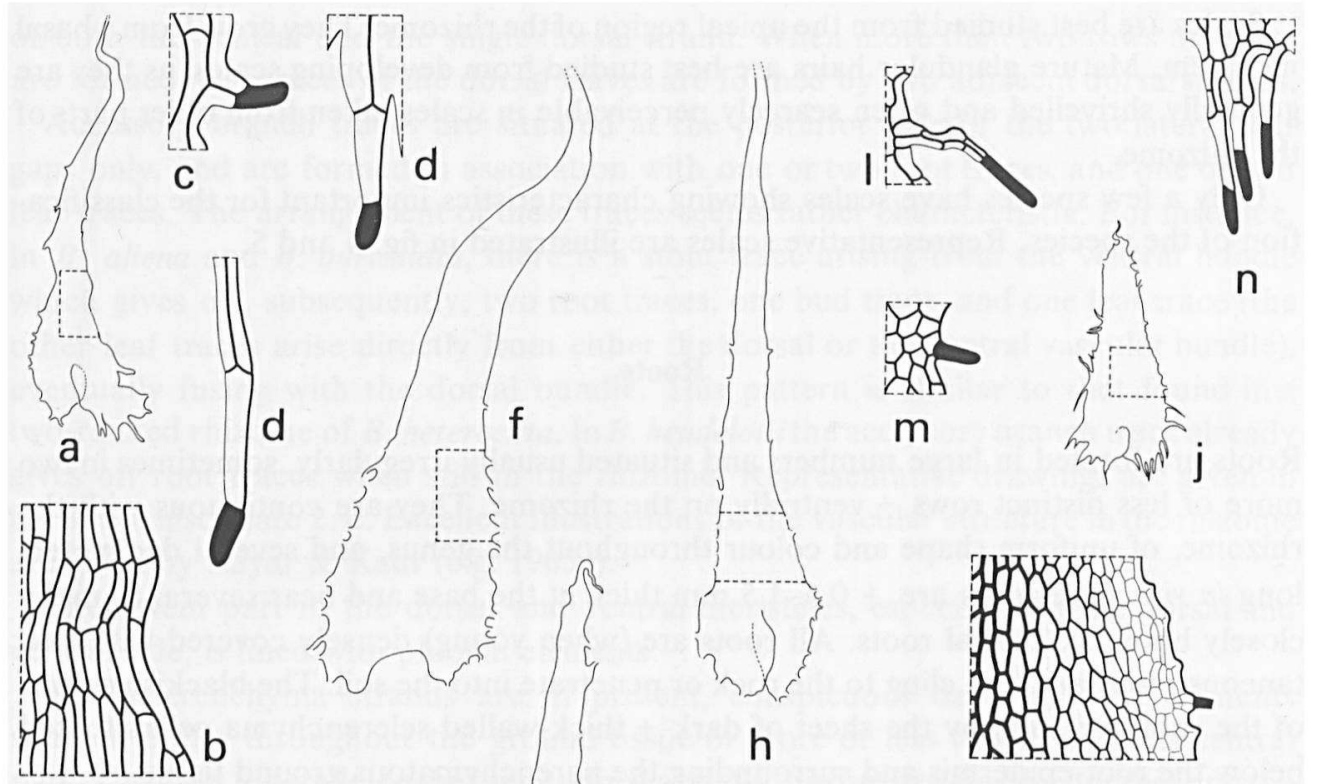

h

k
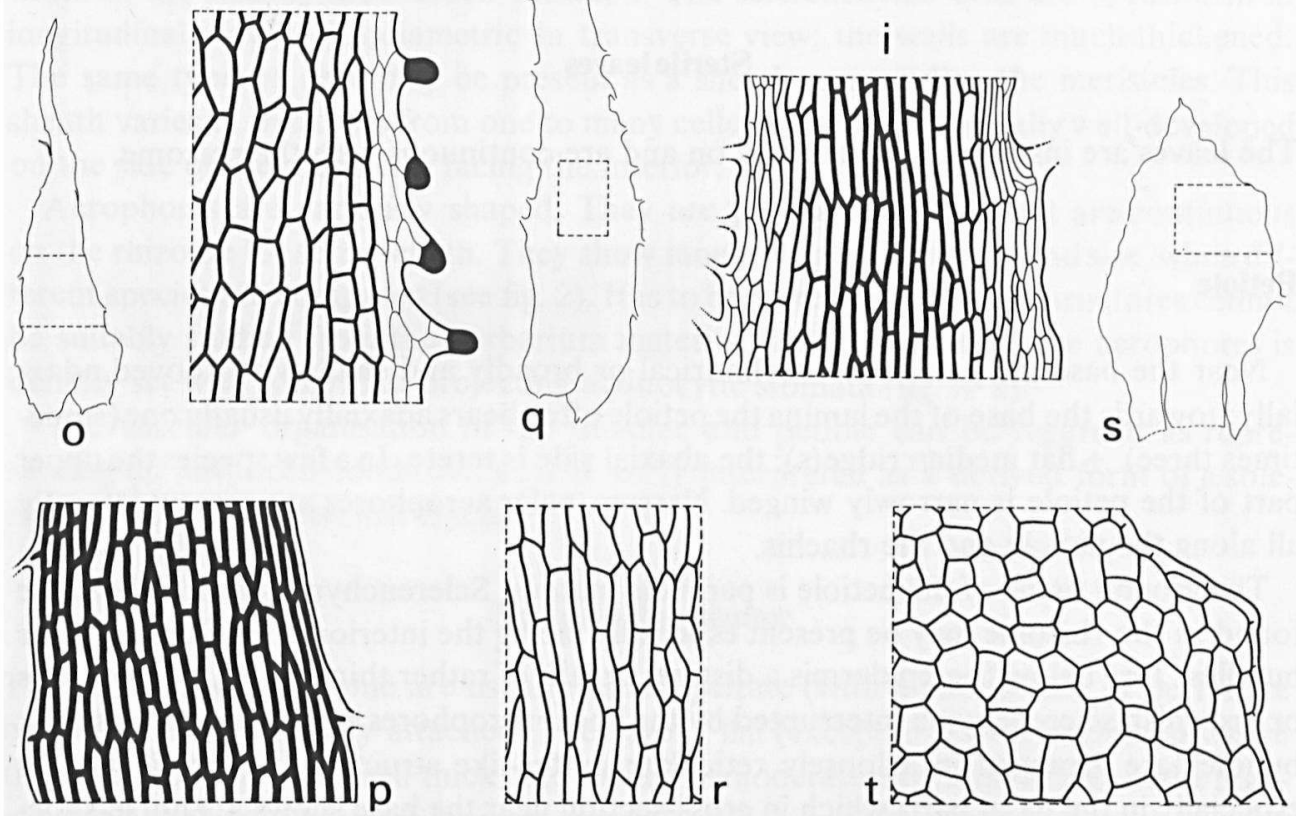

Fig. 4. Scales; outlines $\times 10$, details $\times 40$, marginal hairs $\times 45 .-a-e$. Bolbitis aliena; $f, g$. B. bipinnatifida; h, i. B. acrostichoides; j-n. B. rhizophylla; o, p. B. gaboonensis; q, r. B. nicotianifolia; s, t. B. pergamentacea (a-e. Valeur 535, K; f, g. Jeffrey 759, K; h, i. Hepper 1476, K; j-n. Hennipman 4066, L, from living material ex Price s.n., L; o, p. Zenker 2456, S-PA; q, r. Maxon 9316, US; s, t. Tonduz 13353, G). 


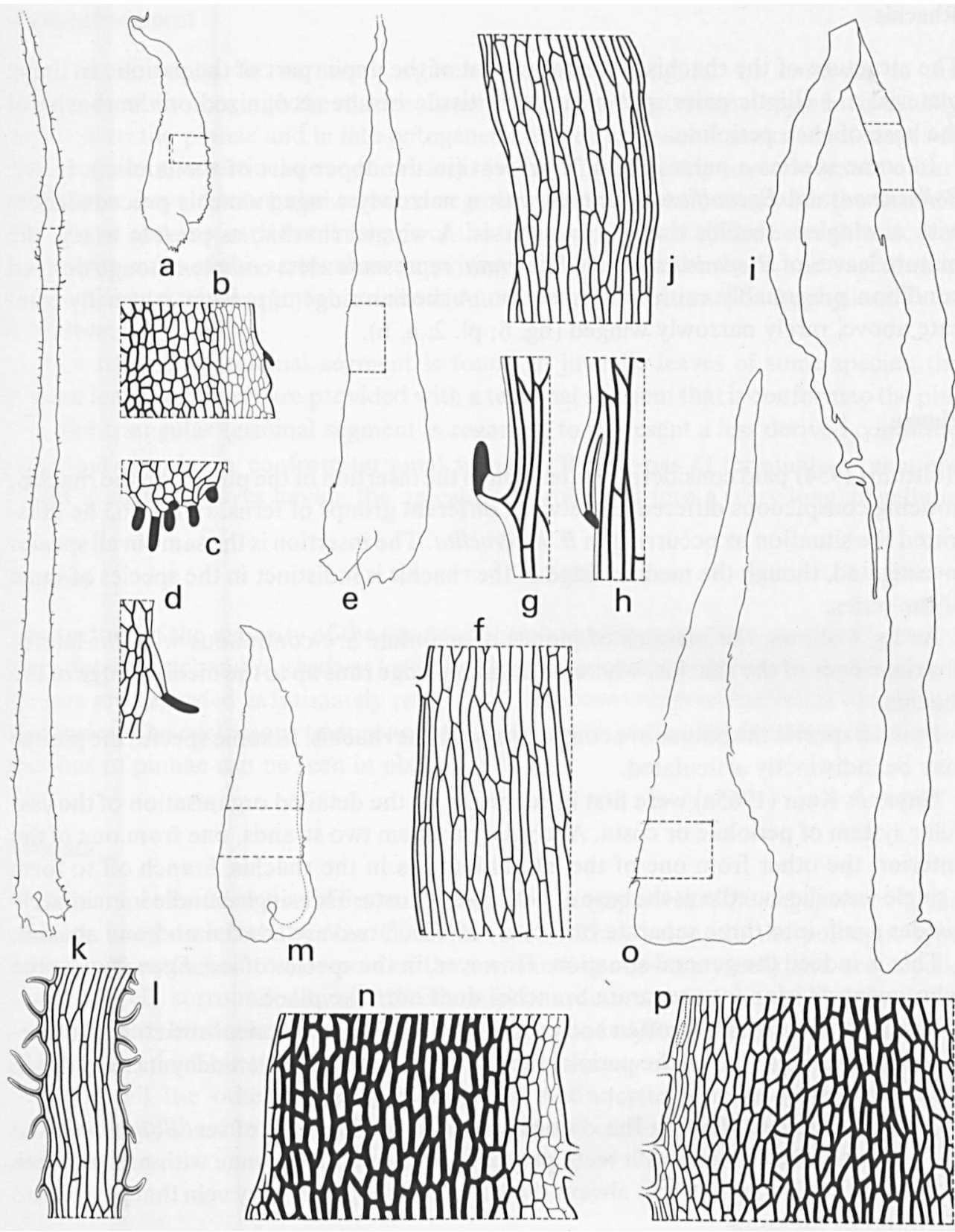

Fig. 5. Scales; outlines $\times 10$, details $\times 40$, marginal hairs $\times 45 .-\mathrm{a}-\mathrm{d}$. Bolbitis heteroclita; e-h. B. heudelotii; i, j. B. lindigii ; k, 1. B. bernoullii; m, n. B. quoyana; o, p. B. portoricensis (a-d. Hennipman 4085, L, from living material ex Price 303, L; o-h. H. 4118, L, from living material ex Van Bruggen; i, j. Brade 37l, BM; k, 1 . Philipson \& Idrobo 1796, BM; m, n. Pleyte 452, BO; o, p. Salvin \& Goodman 280, K). 


\section{Rhachis}

The structure of the rhachis is similar to that of the upper part of the petiole. In living material a \pm elliptic paler spot of aerating tissue can be recognized on the rhachis at the base of each petiolule.

In some species a narrow wing is present (in the upper part of the lamina). In ser. Bolbitianae and Egenolfianae leaves with a narrowly winged rhachis precede leaves with a wingless rhachis during ontogenesis. A winged rhachis, as present in e.g. the mature leaves of $B$. semicordata and $B$. serrata, represents a less complex though derived condition presumably caused by retention. A median ridge, if present, is usually truncate above, rarely narrowly winged (fig. 6; pl. 2: a, b).

\section{Pinnae}

Holttum (1954) paid considerable attention to the insertion of the pinnae on the rhachis, noticing conspicuous differences between different groups of ferns. In fig. 263 he illustrated the situation as occurring in $B$. heteroclita. The insertion is the same in all species investigated, though the median ridge of the rhachis is indistinct in the species of some of the series.

As fig. 6 shows, the margins of pinnae or petiolule are continuous with the lateroabaxial ridges of the rhachis, whereas the pinna-ridge runs up to the median ridge of the rhachis.

In most species the pinnae are continuous with the rhachis. In some species the pinnae may be indistinctly articulated.

Nayar \& Kaur (1965a) were first in reporting on the detailed organisation of the vascular system of petiolule or costa. According to them two strands, one from one of the anterior, the other from one of the lateral bundles in the rhachis, branch off to form a single vascular bundle at the base of petiolule or costa. This single bundle immediately divides again into three separate branches, of which two are adaxial and one abaxial.

This is indeed the general situation. However, in the species of ser. Egenolfianae the subsequent division into separate branches does not take place.

The base of the pinna is often somewhat swollen. The anatomical structure is otherwise identical with that of the petiole, though aerophores and sclerenchyma strands are absent.

Teeth and spines. During the ontogenesis of several species of ser. Bolbitianae and Egenolfianae lobed pinnae with teeth precede lobed or entire pinnae with narrow teeth or spines. In these species it is always the basal acroscopic tertiary vein that passes into the tooth or the spine.

Teeth are found in the sinuses of the pinnae of several species outside the genus, for instance in Pleocnemia (tectarioid). As during ontogenesis leaves with teeth in the sinuses precede leaves with spines (e.g. B. appendiculata), the margin with spines therefore may represent a more complex condition than the margin with teeth. 


\section{Terminal segment}

In most species the terminal segment, whether triangular or conform to the pinnae, is continuous with the rhachis. In ser. Bolbitianae the terminal segment is usually conform to the pinnae and in late ontogenetic as well as in some mature leaf-stages of some species \pm subarticulate to the rhachis (see fig. 7). The early juvenile leaves of ser. Bolbitianae have a triangular terminal segment which is continuous with the rhachis. The shape and the variation in shape of the terminal segment of the heteroblastic leaf series of species of this series show surprising similarities to the shape and variation in shape of the terminal segment as found in normal and transitional bathyphylls of Ieratophyllum.

As a triangular terminal segment is found in juvenile leaves of some species, the mature leaves of which are provided with a terminal segment that is conform to the pinnae, the triangular terminal segment is regarded to represent a less derived condition than that showing a conform terminal segment. Both types of terminal segment are found in mature leaves having the apical part prolonged into a (very long) flagellum.

\section{Texture}

The texture of the majority of the species is(firmly) herbaceous. Two species, $B$. sinuata (ser. Heteroclitae) and B. rivularis (ser, Quoyanae), have a coriaceous texture. These two species are regarded as intimately related to herbaceous representatives of the respective series. The coriaceous texture apparently represents a parallel development. Crosssections of pinnae can be seen in plate $3: a, b$.

\section{Stomata}

From six species from five series, and from $B . \times$ singaporensis ('B. singaporensis') the stomata were described by Van Cotthem (1968). They belong to the polocytic type (guard cells attached to one pole of the surrounding subsidiary cell) and are acyclic (stomatal cells surrounded by guard cells only) in structure. According to Van Cotthem, the length of the stomata varies from 35-65 $\mu \mathrm{m}$. I found the stomata of all the species to be polocytic. This type of stoma is also found in many other leptosporangiate ferns including all the other lomariopsidoid ferns. Actinocytic stomata-present on the aerophores of the rhizome-have not been reported earlier from isosporous leptosporangiate ferns.

\section{Indument}

The indument of the leaves comprises three different kinds of excrescences. When young the (basal part of the) petiole and rhachis are covered with spreading scales rather like those on the rhizome although generally with the cross-walls somewhat less thick- 

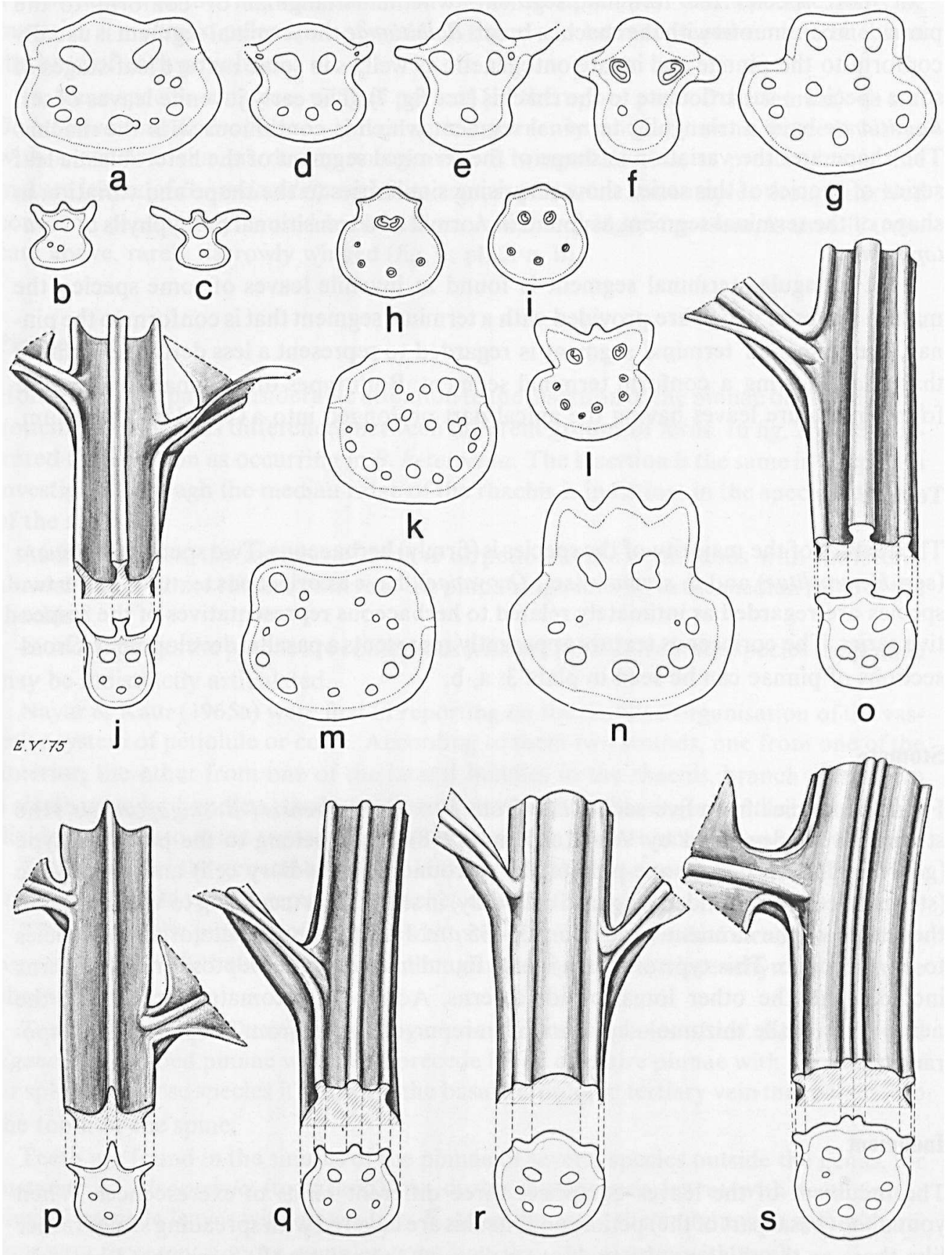
ened. Smaller, almost orbicular appressed scales of the same structure or with a fimbriate margin occur throughout on the (young) petiole and rhachis. The laminar parts of the circinnate leaves of all species are usually densely covered with 2-5-celled uniseriate glandular hairs. See fig. 8. Old leaves may have lost all or most of their indument.

\section{Leaf shape}

Within the genus various types of leaf shapes occur. The leaf shape (including that of juvenile leaves) shows much plasticity. On the other hand similar types of leaf shape may occur in series which are otherwise very different, for instance as regards features of the rhizome and venation pattern. Thus a pinnatipartite leaf is found in B. fuviatilis (ser. Alienae) and in B. hastata (ser. Portoricenses).

The variation in leaf shape as found in some series is schematically illustrated (fig. 9) and shortly discussed. In the illustration the solid-line arrows indicate the sequence of the leaves during ontogenesis, the broken-line arrows point to shapes that are here regarded as more derived in a semophyletic sense. The shape of juvenile leaves is indicated if known and/or relevant. The shape of the mature leaves should be evaluated preferably in relation to that of the juvenile leaves. See therefore also chapter 12.

Ser. Alienae and Lindigianae.-Apart from leaf shape ser. Alienae is very homogeneous; the monotypic ser. Lindigianae is possibly closely related to the first series. The different types of leaf shape found in these two series can be easily related to those as found in B. aliena with deltoid basal pinnae. Apart from other considerations this idea is based on the similarities of part of the juvenile leaves of $B$. auriculata and $B$. lindigii with those as presumed for $B$. aliena.

As regards $B$. fluviatilis there seems to be little doubt that it sprung from $B$. auriculata. The restricted distribution of $B$. fluviatilis supports its derived character. The \pm entire mature leaves of part of the material of $B$. fuviatilis (origin: Congo Basin) resembles that of certain juvenile leaves of $B$. auriculata. Such plants are obviously derived from $B$. auriculata. However, in an other part of $B$. fluviatilis (origin: islands in the Gulf of Guinea) the plants may have pinnatipartite leaves that are generally also larger than those of the plants discussed above. An ample collection of mature plants from Fernando Poo with pinnatipartite leaves shows that entire juvenile leaves precede the

Fig. 6. a-s. Cross-sections through the upper part of petioles (fig. a, d, h, k) and the lower part of rhachises (all others), $\times$ 7. $-\mathrm{j}, \mathrm{o}-\mathrm{s}$. Adaxial views on rhachises and on junction of rhachis with pinnae, X 7.-a. Bolbitis auriculata; b, c. B. aliena; d. B. gaboonensis; e, f. B. oligarchica; g. B. hemiotis; h, i. B. rhizophylla; j. B. sinensis; k. B. bernoullii; 1 . B. sinuata; m. B. lindigii; n. B. portoricensis; o. B. virens; p. B. appendiculata ssp. appendiculata; q. B. heteroclita; r. B. heudelotii; s. B. quoyana. Fig. h-j, l, o-s drawn from living material (a. Halle 4231, L; b, c. Proctor \& Mullings 21999, U; d. Zenker 2456, L; e, f. Standley 33952, US; g. Jermy 2639, L; h, i. Hennipman 4066, L; j. H. 4046, L; k. Purpus s.n., vi-1913, M;1. Hennipman 4052, L; m. Buchtien 2220, G; n. Donnell Smith 2721, US; o. Hennipman 4047, L; p. H. 4048, L; q. H. 4085, L; r. H. 4118, L; s. H. 4147, L). 


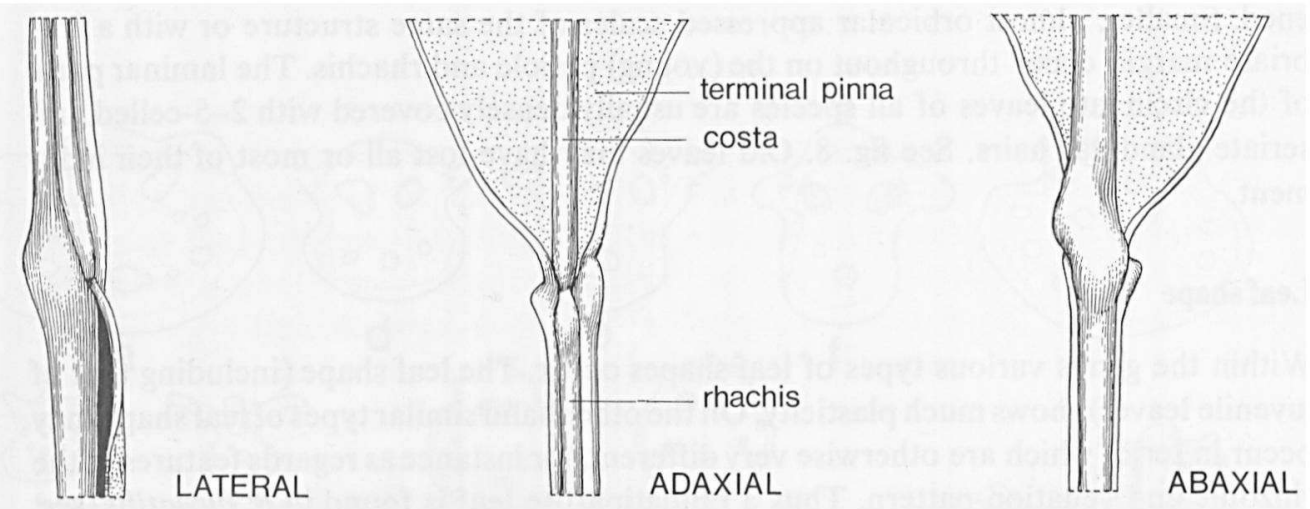

Fig. 7. Different views on the junction between rhachis and terminal segment (terminal pinna) in Bolbitis virens, $X 4$ (Hennipman 4047, L, drawn from living material ex Van Beusekom 2606, L).

pinnatipartite ones during ontogenesis. Therefore, it seems probable that plants with pinnatipartite leaves are derivatives of plants with entire leaves.

$B$. lindigii starts life as a terrestrial fern. As such its rhizome structure and leaf shape are much like those of $B$. aliena. The mature condition as shown in high-climbing forms with a coarse rhizome bearing several rows of leaves with many pinnae is habitually much different. The leaf shape (with many pinnae) as found in the latter form is regarded as more complicated and is presumably also more derived.

Ser. Euryostichae.-Most of the species have usually pinnate leaves; the number of pinnae per leaf varying from 2-10. A simple leaf is found in B. gaboonensis, and in part of the material of $B$. nicotianifolia, B. pergamentacea, and B. hemiotis. Characteristics of the shape of the pinnae of $B$. hemiotis (asymmetrical pinna base), $B$. oligarchica (ternate leaves), and $B$. pergamentacea (rhomboid terminal segment) are derived if compared with those of $B$. nicotianifolia.

Ser. Heteroclitae.-The shape of the juvenile leaves of $B$. heteroclita indicates how the derived condition of a pinnate leaf with a terminal segment that is conform to the lateral pinnae is related to a condition, where a pinnate leaf has a triangular terminal segment. In the Taxonomic Part the wealth of variations in leaf shape of $B$. heteroclita is amply discussed. See also fig. 13, 20, 60: a-s.

$B$. sinuata. The various types of leaf shape as found in this species are similar to (part of the) mature leaf shapes found in $B$. heteroclita. The available material shows that variation may be determined by differences in ploidy as well. The leaf shapes of the diploid specimens from the Philippines (fig. 61: e, g) may well be regarded as more derived if compared with those found in specimens from outside the Philippines, as the sinuata margin of the segments of the Philippine diploid is likely to represent a retentive condition. The (auto)triploid specimen from the Philippines (fig. 61: c, d) 
forms entire leaves much different from the simple and pinnate leaves of the Philippine diploid, but on the other hand agreeing in detail with the simple leaves formed by the diploid and (auto)triploid specimen from Singapore. The only difference between the latter two specimens and the triploid Philippine specimen is to be found in the pinnate leaves. Those of the Philippine triploid are often irregularly lobed in the basal part of the terminal segment and have more or less adnate pinnae, whereas the leaves of the specimens from Singapore are perfectly regular with sessile or shortly petiolulate pinnae.

Ser. Portoricenses. $-B$. portoricensis is obviously the central species. This widely distributed fern varies much in leaf shape. The leaf shape of $B$. hastata, a Mexican endemic, is related to that of $B$. portoricensis; both have a similar venation pattern. The shape (and size) of its leaves is similar to that of certain forms of the not closely related $B$. fluviatilis. B. pandurifolia is found at the southern limit of the distribution of $B$. portoricensis. Its leaves are entire or ternate. A leaf shape with only two pinnae can be regarded to be simpler than that found in mature $B$. portoricensis. On the other hand, other aspects determining the shape (for instance, the shape of the terminal segment which is conform to the pinnae) can be interpreted as more complex. Juvenile leaves could not be investigated in either B. hastata or B. pandurifolia. This is regretted as one should like to know for instance if entire juvenile leaves precede the pinnatifid ones during development of the mature leaf of $B$. hastata (as is the case in B. fluviatilis).

Ser. Quoyanae.-In B. quoyana the leaf shape shows considerable variation (fig. 69: a-f). The small forms of this species are certainly derived. The leaf of $B$. taylorii comprises a proportionally large terminal segment and a winged rhachis (in the upper part). Some juvenile leaves of $B$. quoyana (for instance those of Price 2255, L) match some mature leaves of $B$. taylorii in detail.

$B$. rivularis, which has a \pm coriaceous leaf texture, is polymorphic (see fig. 71). The shape of its terminal segment of pinnate leaves is not always easy to describe in terms of 'triangular' or 'conform' to the pinnae'.

Discussion.-The variation in leaf shape as shown by (the ontogenetic stages of the) species of this genus is very striking. The drawings illustrate that the mature entire leaf can be regarded as representing a derived condition. This shape is found as a parallel development in different, unrelated series. Obviously such a leaf shape was acquired rather easily during diversification of species with pinnate leaves, a conclusion which was also reached by Tryon (1966). An entire leaf is a dominant feature in several other groups of unrelated ferns (e.g. Vittariaceae and to a lesser extent Polypodiaceae). A pinnatipartite leaf as found e.g. in B. fluviatilis (ser. Alienae) possibly represents an even more derived condition than does the entire leaf. It remains to be demonstrated if plants with this kind of leaf shape can revert, by divergent evolution, to a plant, of which the mature leaves are similar to the entire leaf except for being pinnate. 


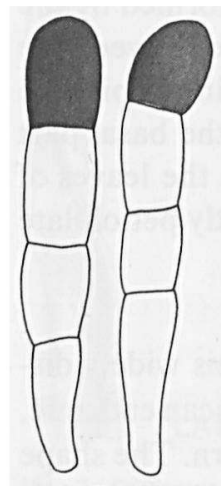

a

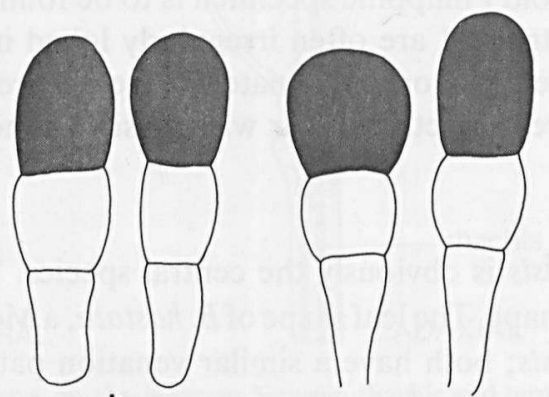

b

C

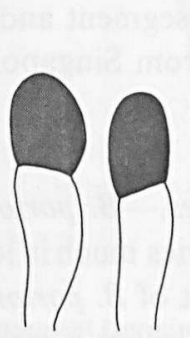

d

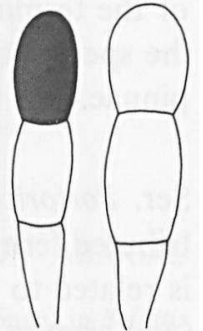

e

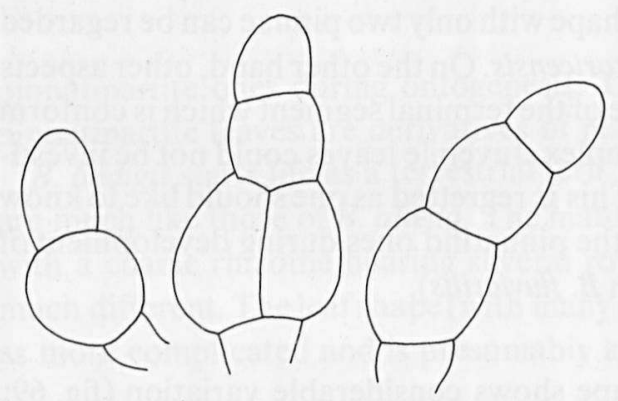

f

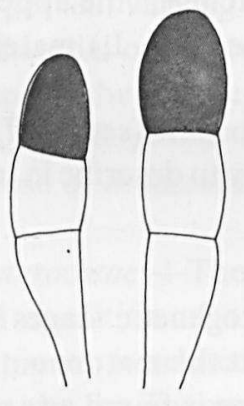

i

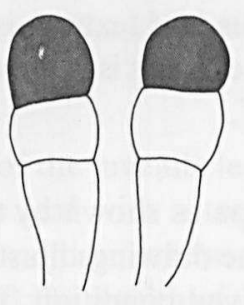

j

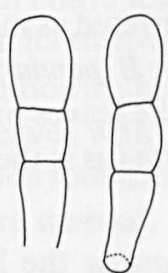

$\mathrm{g}$

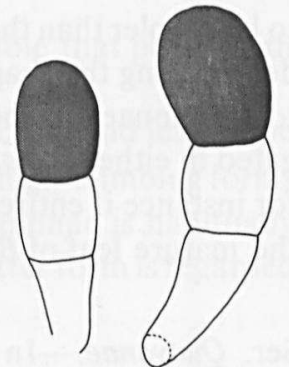

$\mathrm{h}$

Fig. 8. a-j. Leaf epidermal hairs, $\times 180$; all except fig. $d$ and $g$ drawn from living material or material preserved in F.A.P.A. a. Bolbitis bipinnatifida; b. B. subcrenata; c. B. rhizophylla; d. B. nicotianifolia; e. B. heteroclita; f. B. heudelotii; g. B. lindigii; h. B. portoricensis; i. B. quoyana; j. B. repanda, tetraploid (a. Kew 301-73, 04045 ex Whitehead 45, K; b. Hennipman 4142, L, ex Jarrett 600, K; c. H. 4068, L; d. LePrieur 17, P; e. Hennipman 4085, L; f. H. 4118, L; g. Gascoyne-Cecil 88, L; h. Kew 407-74, 03190 ex Jermy 10987, BM; i. Hennipman 4147, L; j. H. 4023, L). - k. Bolbitis heudelotii, actinocytic stoma from the aerophores at the petiole base, $\times 180$ (Hennipman 4118, L). 
From the study of the juvenile and mature leaves in Bolbitis it is postulated that the ancestral form had a (bi-?)pinnate non-flagellate leaf with a triangular terminal segment, and triangular basal pinnae.

\section{Venation pattern}

The venation pattern of the fern leaf has been studied in detail by many pteridologists, including Von Ettingshausen (1865), Mettenius in his 'Farngattungen' (for references see Christensen, 1906), and Goebel (1922). Presl (1851) used this feature extensively in his classification of the genera, a system now generally regarded obsolete in many instances.

The venation pattern, like some other features such as the soral arrangement and the leaf shape, is subject to parallel evolution. Thus the venation pattern of $B$. crenata is about similar to that of the meniscioid thelypteroid ferns. Schumann (1915: fig. 36) who was much praised by Bower (1928), illustrated a progressive series of venation patterns comprising a meniscioid Thelypteris species and some Bolbitis species which she thought closely related. Another fine example of parallelism is displayed by the venation patterns of B. (Leptochilus) heteroclita and Pteridoblechnum (Leptochilus) acuminatum (White \& Goy) Hennipman.

Within the present genus different types of venation occur. A considerable number of species has been founded mainly or exclusively upon characteristics in the venation pattern.

The venation pattern is difficult to describe or to keep in mind. For a proper weighing of the taxonomic relevance of properties of the venation pattern it became therefore necessary to develop a quick and easy method to obtain (enlargements showing) the details of the pattern. The variation in venation pattern of one specimen was traced by studying that of basal, central, and upper parts of the segments (pinnae as well as laminas) of different leaves from a single rhizome. The venation of the pinnae and laminas illustrated were all drawn from central parts of mature pinnae and laminas respectively, unless stated otherwise.

In all the species the secondary veins run parallel; typical differences are therefore expressed by the tertiary etc. veins only. The venation in the pinnate leaves is anadromic as the lowermost lateral vein of a pinna or pinnule is inserted at the acroscopic side.

The following types of venation pattern are recognized:

1. The sagenioid venation. Veins anastomosing in a reticulate pattern, with \pm isodiametric or elongate, angulate areoles and generally without recurrent included free veins (the so-called sagenioid venation pattern). This type is widespread in the genus and is a characteristic for ser. Alienae (e.g. fig. 34: e, f), Heteroclitae (e.g. fig. 60: u-z), Lindigianae (fig. 63: 1, m), and Quoyanae (e.g. fig. 69:j-s). The arrangement of the areoles may show slight differences. In $B$. lindigii the areoles are more uniform than is usual in ser. Heteroclitae; the pattern does not change when going from the costa towards the margin of the pinna. In B. fluviatilis (with simple leaves) 

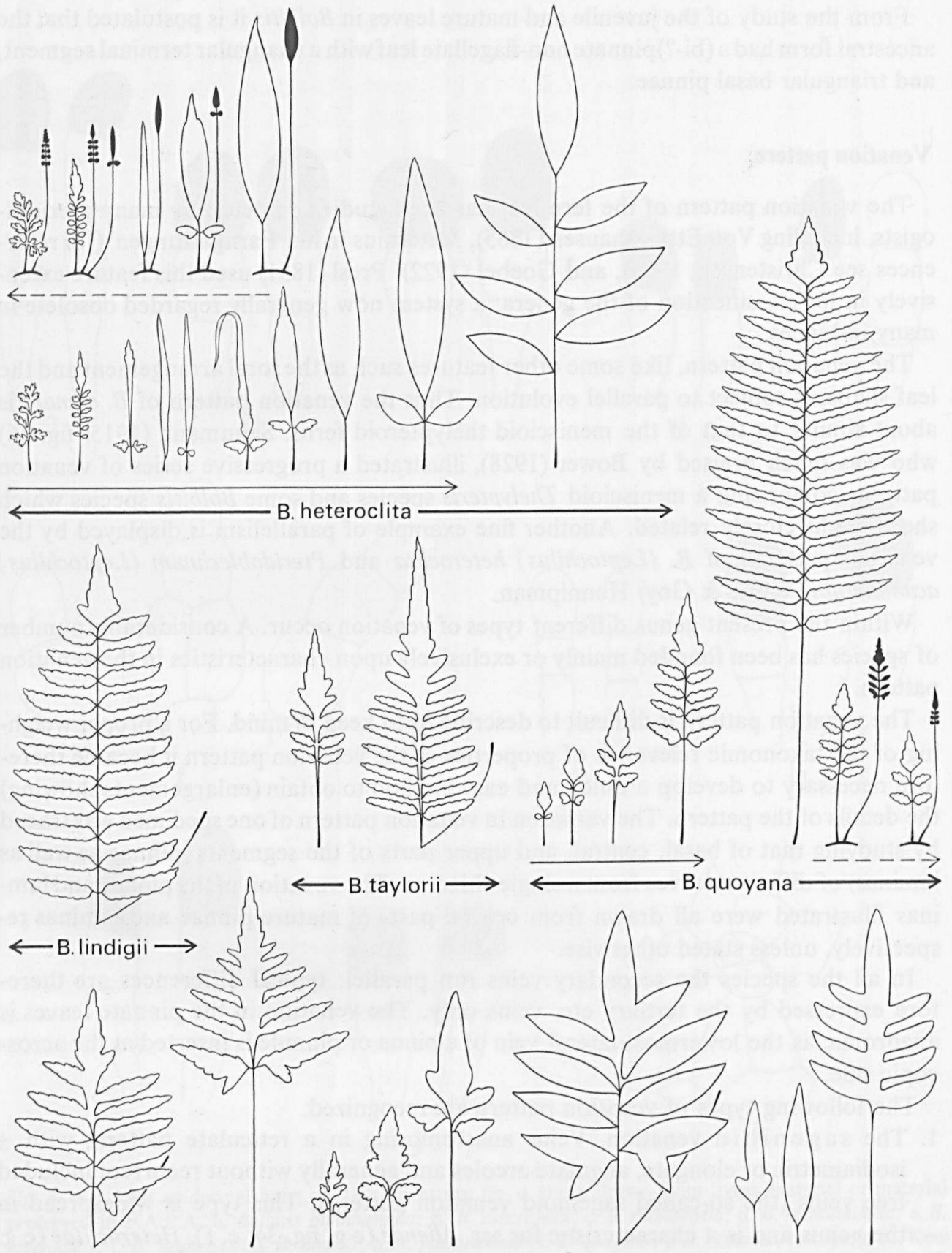

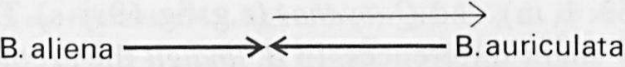

B. fluviatilis $\longrightarrow$

Fig. 9. Leaf shapes in some taxa of Bolbitis. The solid-line arrow indicates the sequence of the leaves during ontogenesis; the broken-line arrow indicates plants with increasingly more derived leaf shapes. 
the areoles gradually decrease in size towards the margin (fig. $36: \mathrm{h}, \mathrm{i}$ ); it is the only species where the secondary veins are difficult to perceive.

In ser. Bipinnatifidae included free excurrent veins are present in part of the areoles (fig. 37: e-g).

2. The Bolbitian a e type of venation. Veins anastomosing to form at least a costal areole, otherwise without or with, usually excurrent, included free veins. This type of venation shows much variation; it is found in ser. Bolbitianae. The occurrence of excurrent included free veins in the areoles of several species is regarded as indicating a derived condition if compared with a reticulate pattern. In a few specimens of $B$. acrostichoides (see fig. 38: $f$ ) and $B$. serratifolia (see fig. 45: $d$, c) the venation pattern is (locally) sagenioid (type 1). In $B$. angustipinna the venation pattern as seen between two adjacent secondary veins may be asymmetrical (see fig. 40: e, f).

Within the Asian species of ser. Bolbitianae a considerable variation in the venation pattern is found. A simple type is present in $B$. crispatula (fig. $40: \mathrm{h}, \mathrm{i}$ ) and $B$. semicordata (fig. 43: $\mathrm{g}-\mathrm{i}$ ) whereas a very intricate regular network showing excurrent included free veins is found in other species, for instance in B. costata (fig. 41: d-f). The latter type of pattern is not at all reminiscent of the sagenioid pattern sometimes displayed by others. All these types can, however, be interpreted as variations of an ancestral one if the ontogenesis of the patterns as shown by different juvenile leaves is considered. See chapter 12 .

3. The pattern with $\mathrm{fre}$ e ve in s. This is found in ser. Egenolfianae. The evidence from the study of juvenile and mature leaves of different species of ser. Bolbitianae, in combination with characteristics of ser. Egenolfianae, indicates that the pattern probably represents a derived condition.

4. The reticulate venation with recurrent (and excurrent) included free veins. This type is found in ser. Euryostichae, Heudelotianae, and Portoricenses. The venation of $B$. portoricensis is essentially regular and it probably evolved from divergent evolution and not by (recent) hybridization. This is not necessarily true for the type of venation shown by ser. Euryostichae; it was found that presumed hybridization between different cytotypes of one species (B. sinuata) or between two species with a (slightly different) reticulate venation may produce an almost identical condition; see fig. 10, and the venation pattern of Leptochilus $\times$ trifidus in fig. 85: v, w.

5. The irregular venation. Detailed analysis of the venation of a specimen nearly always results in finding incidental irregularities. These are regarded as analogous to for instance the occurrence of incidental aborted spores in the spore output of a sexual diploid, and have therefore not been given much attention. On the other hand it was found that in known or suspected hybrids or alloploids the venation may be structurally irregular, e.g. in $B$. lonchophora (fig. 76: c-g), B. repanda $(-3 \mathrm{n}$ and $-4 \mathrm{n}$; fig.78: $\mathrm{c}-\mathrm{i}$ ), $B . \times$ singaporensis (fig. 85: a-d), etc. Besides, a number of taxa which were thought to be of hybrid origin because of other properties like the presence of aborted spores and the irregular leaf shape $(B . \times$ boivinii, $B$. angustipinna $\times$ sinensis), often show a very irregular type of venation pattern as well. By 'irregular' is meant here the condition in a sterile leaf, where marked differences exist between 


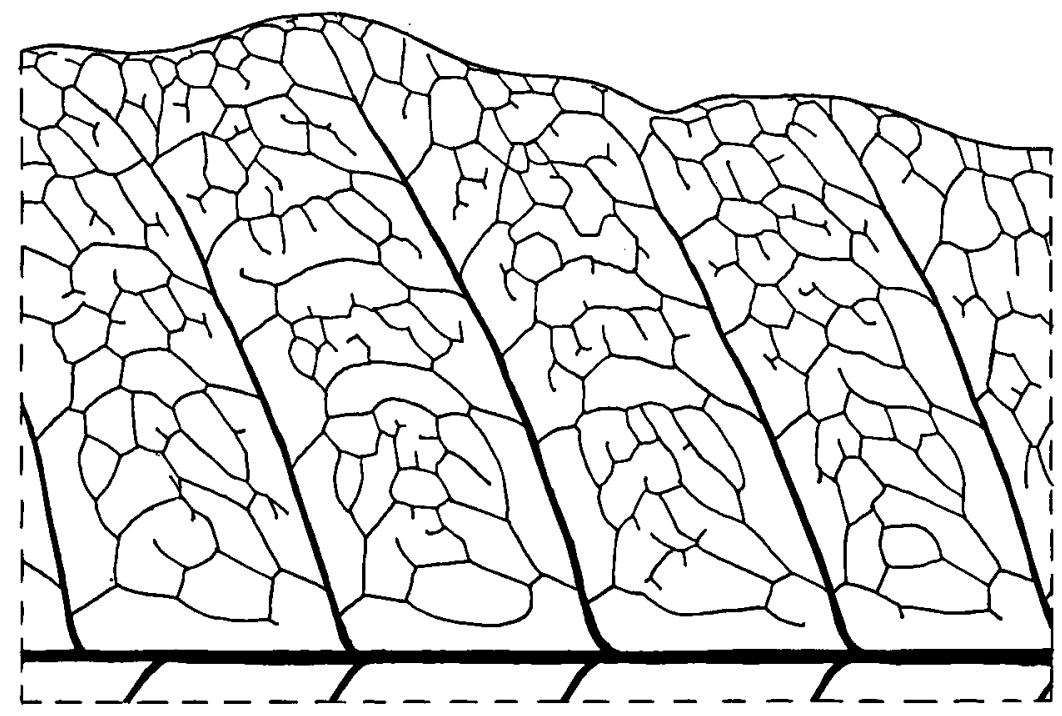

Fig. 10. Bolbitis sinuata, irregular venation pattern, $\times 2$ (Williams 2055, UC; drawn from dried material).

the venation of the various areas included by the secondary veins (or parts of that area in case an intricate type is involved). Irregularities in the venation are here regarded as an important indication for the hybrid status of a certain plant. On the other hand hybrids do not necessarily have an irregular type of venation. In some (B. $\times$ sinuosa; fig. 85: $\mathrm{f}, \mathrm{h}, \mathrm{j}, \mathrm{l}, \mathrm{n}, \mathrm{p}$ ) it may be perfectly regular.

In a number of cases a very irregular pattern was found in specimens which were typical as to other features. Thus one of the autotriploid specimens of $B$. heteroclita in cultivation shows many irregularities in its pattern. And so does the venation of many collections belonging to species with an intricate network. Examples are included in the patterns illustrated for the species.

In some irregular intricate patterns pieces of veins not connected with other veins may be found within an areole. See the asterisks in fig. 85: v, w.

Discussion. - The ferns with the types of venation patterns as listed above can be regarded as derivatives of a hypothetical predecessor with a free venation. Juvenile leaves of such an ancestral fern would all show an open venation.

The venation pattern of the contracted laminas (i.e. laminas which are less divided and often also smaller than those found in related taxa) as present in many a modern fern shows much variation. The contraction of the leaf obviously resulted in a vast array of venation patterns. These patterns may have free veins (e.g. the type with closely placed parallel veins) or may comprise variously intricate networks. All these different types, whether simple of intricate, are derived in a semophyletic sense. The pattern of juvenile leaves of ser. Bclbitianae - as well as that of many other leptospor- 
angiate ferns having mature leaves with a complex venation pattern-may have existed in the juvenile leaves of the ancestral fern.

The same kind of processes that led to the contraction of parts of the leaf, may also cause the retention of certain leaf stages in ferns which acquired a complex venation and a (more or less) contracted lamina. The resulting neotenic fern may then have leaves With a venation that might be very similar to that of the juvenile leaves of the ancestral fern. But in spite of this, the neotenic fern is more derived than the fern showing the more complex type of venation from which it originated. The open types of venation found in small or moderately sized ferns (Polypodiaceae, Grammitidaceae, Hymenophyllaceae, etc.) should be interpreted also in view of this hypothesis.

Bower (1923) regarded the ferns with an open type of venation pattern as primitive, as the study of fossil ferns showed that ferns with an open type of venation preceded those with a more intricate type. Bower (p. 96) also applied this to the modern ferns: 'Instances might be multiplied which indicate that among living Ferns there has been frequent progression within narrow circles of affinity from the more primitive open to the more advanced reticulate venation.' This idea which is still generally accepted seems, however, not necessarily correct in view of both the differences in the venation pattern of heteroblastic leaf stages and the general occurrence of retentive stages.

The idea that ferns with a simple morphological expression are also primitive is reflected in classifications. The present genus represents a good example, as the species with an open type of venation (ser. Egenolfianae) have been formerly (and still are) given generic recognition, whereas the species showing a more complex venation were assembled in another genus which was considered more derived.

As regards the types of complex venation patterns Bower (p. 96) remarked: 'Beyond the fact that these complex venations are characteristic for the most part of relatively late and advanced types of Ferns, their detailed study cannot be used as a consistent or trustworthy basis for the phyletic seriation of the Filicales at large. This conclusion, which robs the subject of much of its interest, naturally follows from the fact now demonstrated in so many isolated genera that a closed venation has originated repeatedly in distinct stocks. It is in fact a widely homoplastic character, determined in great measure by physiological necessity or convenience.'

I agree with Bower as regards the homoplastic nature of the intricate types of venation. But the same may be true for patterns showing free veins only. Better than to disregard the venation one should first attempt to understand the different types of venation as occurring in juvenile and mature leaves of species forming a natural group.

The study of the venation patterns as shown by a particular sporophytic phase is here considered of great importance, as such data known for ser. Bolbitianae have added to the understanding of the diversification of this group. Another result from such a study might be a better knowledge of the intricate venation patterns as present in modern isosporous ferns.

As regards the ancestral type of venation in the genus Bolbitis (which is presumably also the ancestral type of venation of the lomariopsidoid ferns), ser. Bolbitianae - a coherent group of species-furnishes significant information. From the variation in the 
venation of $B$. acrostichoides and $B$. serratifolia it is inferred that the sagenioid type of venation represents the original type within ser. Bolbitianae. The species of this series with mainly free excurrent veins (B. crispatula) as well as the species showing a venation consisting of an intricate network with excurrent included free veins, can easily be derived from the species with a more sagenioid type of venation; these different species obviously resulted from divergent evolution, and, accordingly, represent a derived element within this series. In view of the interpretation of the simple type of venation within ser. Bolbitianae, it seems possible that the free venation as shown by ser. Egenolfianae represents a derived condition as well.

The two other types of venation are the 'true' sagenioid venation and that with recurrent (and excurrent) included free veins. A sagenioid venation is found in series from all over the tropics, including tropical America, tropical Africa eastward to Madagascar and the Mascarenes, northeast India to eastern Malesia and the Pacific, and the Bonin Islands, but excluding southern India and Ceylon. Groups with a venation showing recurrent included free veins are restricted to Africa and America.

In $B$. portoricensis included free veins are found only in the late juvenile leaf stages with a reticulate venation and in the adults. As earlier leaf stages show a \pm reticulate venation only, and as such a pattern is found in mature specimens of the related ser. Bipinnatifidae, it seems likely that the mature pattern of $B$. portoricensis (with included free veins) represents a derived condition.

Morphological evidence suggests that divergent evolution in ferns with a sagenioid venation pattern may lead to species having a free venation or a venation with several types of included free, ex- and/or recurrent veins.

The venation pattern as found in ser. Euryostichae could have resulted also from hybridization between species, one of which has a sagenioid venation.

\section{Bulbils}

Three quarters of the species have bulbil(s) on the sterile (and fertile) laminas. The bulbils in the genus Bolbitis are usually \pm globular, sometimes elongate, persistent structures, situated adaxially on the lamina.

Bulbils are covered with scales like those on the rhizome though smaller. Crosssections of the subterminal bulbil of $B$. repanda and $B$. sinuata show a \pm orbicular structure with mostly parenchyma and vascular tissue (plate $3: a, b)$. The vascular system comprises a single strand which is continuous with a (the) rhachis bundle. In large bulbils this strand gives off lateral bundles that serve the (primordia of) roots and leaves. In most species leaves and roots are formed already on the globular bulbil. In $B$. auriculata and $B$. gaboonensis the development of the bulbil into a short rhizome occurs prior to the formation of leaves and roots.

The different localisations of the bulbil:

1. The subterminal bulbil is situated subterminally (and sublaterally) on an entire lamina, or subterminally on the terminal (and lateral) segments of a (bi)pinnate leaf. Thus on an entire lamina a conspicuous subterminal bulbil which is inserted later- 
ally on the rhachis at the junction with a secondary vein, as well as (primordia of) bulbils which are inserted subterminally on a secondary vein at a junction with a tertiary vein, usually can be recognized. In pinnate leaves, primordia of bulbils are usually present subterminally on the pinnae as well. They are likewise inserted where the costa and one of the secondary veins of the pinna meet. Certain leaf stages with but few pinnae and a relatively large terminal segment may show subterminal bulbils on the pinnae and the terminal segment, apart from several bulbils situated sublaterally on the terminal segment. The illustration in the original publication of Cyrtogonium rivulare $(=B$. rivularis) is a good example.

In most of the species only one subterminal bulbil is found on the terminal segment, whereas several remotely placed bulbils occur on the long-flagelloid terminal part of the terminal segment of $B$. portoricensis (fig. 68: c). Some leaves of a normally triploid specimen of $B$. heteroclita kept in cultivation formed a multitude of closely set bulbils on the flagelloid terminal part of the terminal segment. Wild specimens invariably bear one subterminal bulbil only.

The subterminal bulbils of the leaves of certain plants grown in cultivation in terraria, for instance those of $B$. repand $a$ and $B$. heteroclita $\times$ sinensis, all developed into small plants. These plants, situated subterminally on pinnae and terminal segment, reach the ground only after senescence of the leaf on which they are formed.

2. The te r m in al bulbil. In B. rhizophylla (see fig. 52: d, e) and B. novoguineensis (see fig. 74: $\mathrm{k}, \mathrm{l})$ the bulbil is always situated terminally, or almost so, as in B. rhizophylla an inconspicuous portion of the lamina is incidentally present distally from the bulbil. A (primordium of a) terminal bulbil is present on the pinnae of $B$. novoguineensis (fig. 74: $\mathrm{m}$ ) but generally absent on those of $B$. rhizophylla.

Part of the material attributed to $B$. crispatula var.copelandii has the bulbil situated at the pseudo-articulation of the rhachis just below the lamina of the terminal segment. In another part of this material the terminal segment is scarcely developed (obviously aborted). Incidentally the terminal segment is not developed at all; as a result the situation of the bulbil is terminal (Hennipman 3632, L; Tagawa et al. T 1910, L).

3. The axill a ry bulbil. Distinct, almost spherical or elongate structures are present in the axils of (part of) the pinnae (rarely on the petiolule somewhat away from the rhachis) of $B$. hemiotis (fig. 56: a) and B. oligarchica (fig. 56: e, f). Axillary bulbils occur incidentally in B. auriculata. In one specimen of the latter species(Ledermann 689 , B) the bulbils of the lowermost axils had developed into elongate structures up to $7 \mathrm{~mm}$ long, still devoid of roots or leaves, a condition reminiscent of that of B. gaboonensis.

4. The bulbil of $B$. gemmifera is situated on the $\mathrm{rh} \mathrm{ach}$ is in the basal part of the terminal segment and generally opposite the laminar part of the usually unequal base. The shape of the terminal segment as well as the exact place where the bulbil is inserted varies, as illustrated in fig. $74 ; \mathrm{a}, \mathrm{c}$, and d. In relation to this unique position of the bulbil it should be noted that morphological characteristics afford evidence that $B$. gemmifera probably has originated from hybridization between $B$. 
acrostichoides and $B$. auriculata. The first species always has a distinct subterminal bulbil on a terminal segment which is conform to the pinnae; the second is generally devoid of bulbils, though axillary bulbils are incidentally present. The shape of the leaves of these species and B. gemmifera are illustrated in the Taxonomic Part.

The distribution of the types of bulbils throughout the genus.-Bulbils are absent from ser. Heudelotianae and Lindigianae. In ser. Alienae (axillary) bulbils are incidentally present in $\boldsymbol{B}$. auriculata only. In ser. Euryostichae axillary bulbils are present in two species, a subterminal bulbil in one, whereas bulbils are lacking in the other two species. In all other series the subterminal (or terminal) bulbil is present in (nearly) all of the species (and its specimens).

Marchal (1965) studied a considerable number of bulbiferous ferns including some Bolbitis species. She recognized apical, axillary, subapical, and laminar bulbils. From my observations it seems impractical to discriminate between the latter two types of bulbils. The bulbil of $B$. gemmifera was not included in her studies.

\section{Fertile leaves}

The fertile leaves are erect, generally of the same architecture, anatomy, and of similar size as the sterile ones occurring in conjunction. They stand more erect, have a longer petiole, a smaller lamina which is often proportionally shorter, and a more coriaceous texture. The shape of the fertile segments is usually about the same (though much contracted) as in the sterile ones, but sometimes different. The margin of the fertile segments often lacks the prominent marginal projections found in the sterile ones; bulbils are less prominent or even absent.

Shape of the pinnae.-In many species the shape of the fertile pinnae is the contracted version of the sterile one. Some deviating shapes are found as well.

The fertile pinnae of $B$. appendiculata ssp. appendiculata are mostly contracted; the sporangia are inserted exclusively on the veins (in specimens from mainland Asia) or all over the lower surface (e.g. in specimens from the Philippines). However, within a restricted area in the borderland of Burma and Thailand plants with the contracted type of fertile pinna are found next to plants with typical sterile leaves but with (bi) pinnate fertile ones. The fertile pinnae of these are moniliform with the sporangia restricted to the lower surface of semicircular patches of lamina. Between this type (on which Polybotrya helferiana was based) and the typical one all intermediates are found (fig. 49: $\mathrm{k}-\mathrm{p}$ ). In ssp. vivipara var. vivipara the fertile leaves lack laminar tissue; the sporangia are inserted on the costa (and costules) and exposed in all directions (plate 3: e). In var. neglecta a narrow strip of laminar tissue is present along the costa on which the sporangia are inserted; they are exposed to both the upper and lower sides.

Within ser. Bolbitianae the shape of the fertile pinnae shows much variation. Some taxa have contracted fertile pinnae, those of others are either linear, or not fully acrostichoid and so-called intermediate.

The shape of the fertile leaf is usually similar to that of the preceding sterile one. 
It is not rare in cultivation that a sterile leaf of a shape as found in juvenile ones, occurs in association with a fertile leaf of similar shape, whereas the sterile and fertile leaves formed on the full-grown plant are quite different. This, and the common occurrence of so-called intermediate leaves point to the important part physiological factors may play in the determination of the fertility.

Intermediate leaves.- Leaves that are in part fertile and in part sterile are here called 'intermediate' leaves. They do not rarely occur and show much variation as to the sporangial arrangement, also in one species (see fig. 11). A few examples will illustrate this.

In B. portoricensis, the specimen Linden 1751 (P) has a fertile leaf with its lowermost pinnae, the basal part of its central pinnae, and the lowermost lobes of its terminal segment sterile, otherwise being completely acrostichoid; in l'Herminier s.n. (P) the leaf shows irregularly shaped areas with sporangia between the secondary veins of the pinnae in the basal part of the lamina, the leaf being more regularly acrostichoid towards the apex; in Rivoire s.n. (P) one of the fertile leaves displays a pteridoid arrangement of sporangia.

In B. heteroclita one of the several intermediate leaves found has large fertile pinnae all showing a perfectly regular distribution of the sporangia exclusively on the veins (E. Smith $1696, \mathrm{~K}$ ). This hemionitioid arrangement of the sporangia is rather obligate in B. acrostichoides, an old species in ser. Bolbitianae, as well as in some other taxa of this series. In several species of other series the sporangia are also inserted on the veins.

In $B$. quoyana one out of several different intermediate leaves shows the sporangia situated towards the margin of the pinna only.The linear'sorus' is in some places interrupted. As a result irregularly shaped 'sori' can be distinguished which are in part 'protected' by the marginal lobes the lateral margins of which face each other. See fig. 11: j.

The venation pat tern.-The venation largely recalls that of the sterile leaves, although free included veins and small areoles are less frequent or (largely) lacking. The fertile leaves of $B$. semipinnatifida, $B$. heudelotii, and $B$. portoricensis are as a rule almost devoid of free included veins which are always present in the sterile leaves. The fertile pinnae of $B$. rhizophylla sometimes have locally some costal areoles which are always lacking in the sterile ones.

Incorrectly, Nayar et al. (see Kaur, 1974) reported a special kind of venation pattern (diplodesmic venation) occurring in the fertile segments of the present genus.

$\mathrm{S}$ por a n i ia.-Insertion of the sporangia is variable. In some groups the sporangia are situated all over the lower surface (e.g. ser. Alienae, plate 1: a), in other taxa they are present on the tertiary veins only (e.g. B. acrostichoides, plate $1:$ c; B. sinensis) or both conditions occur within one taxon (e.g. B. appendiculata ssp.appendiculata).

Sporangia are formed in dense masses. During maturation of the fertile leaf sporangia of different age occur together without definite sequence (the so-called 'mixed sorus' of Bower, 1928). In a cross-section of a mature pinna (plate 3: c-e) the sporangia are nearly all of the same developmental stage and irregularly piled together, often three above each other. In spite of the basipetal development of the fertile leaf, the 


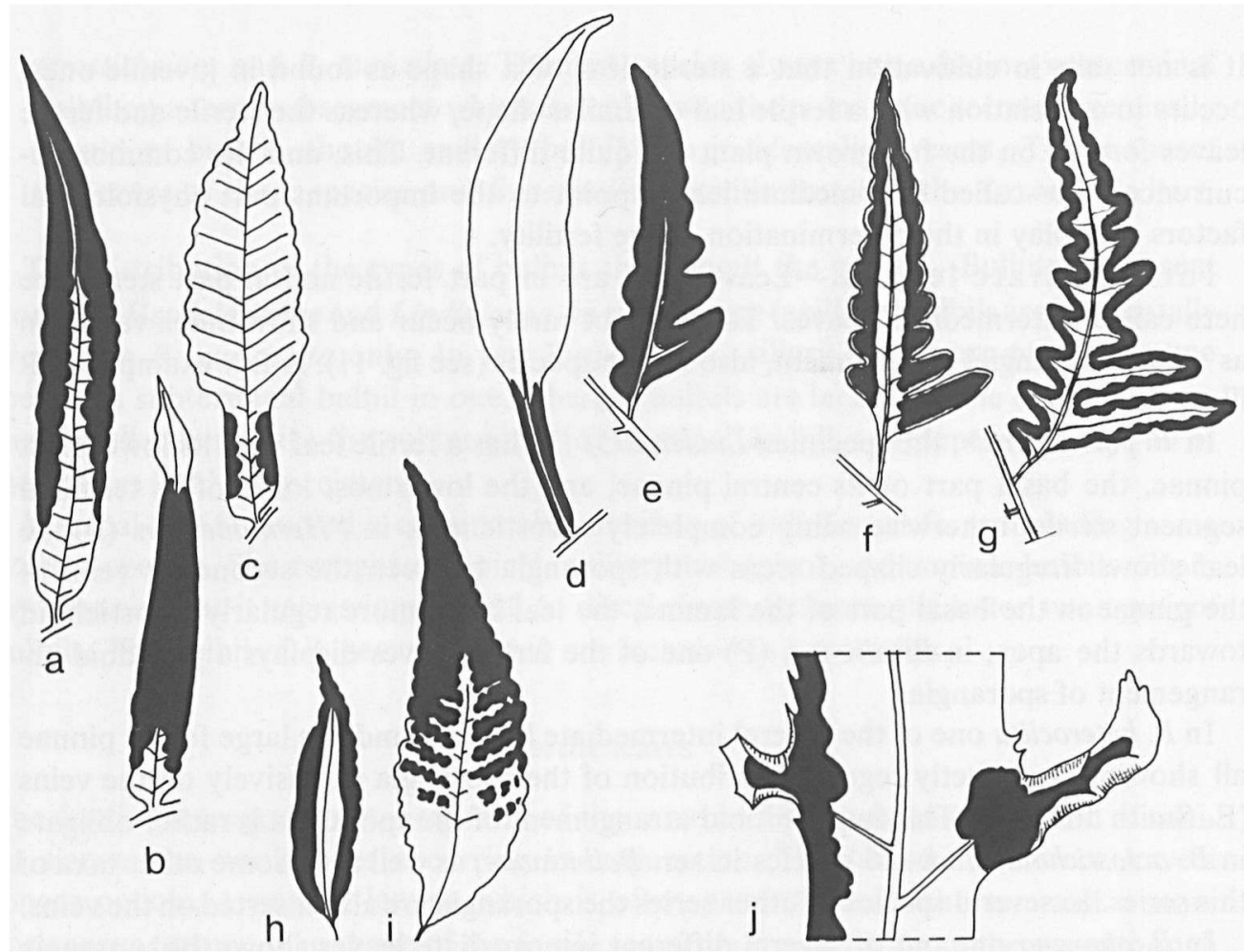

Fig. 11. Abaxial view of (parts of) so-called intermediate pinnae, all $\times 1 / 2$ except for fig. $j$ which is $\times 6 .-$ a-c. Bolbitis serratifolia, pinnae from the lower (fig. a), central (fig. b) and upper (fig. c) part of the same side of a leaf of which all pinnae from the other side are completely sterile. d-g. B. portoricensis; d. central pinnae; o-g. lowermost pinnae; fig. $f$ has been drawn from a leaf of which all other pinnae including the other lowermost pinna are completely fertile. h, i. B. heteroclita, central pinnae.j. B. quoyana, central part of central pinna (a-c. Stübel 690, B; d. Skinner s.n., K; e. Proctor 19262, U; f. Anonymus 6477 bis, US; g. Rivoire s.n., P; h. Levinge s.n., G; i. Clarke 11964 A, BO; j. Herb. Filic. Luerssen no. D, P).

sporangia of all parts are all mature at about the same time. When in cultivation two or three fertile leaves were formed in succession it was not rarely found that all leaves reach maturity at about the same time.

Sporangia are long-stalked, broadly elliptic when seen from the flat side, 1-1.5 times as long as wide, provided with a vertical annulus which is interrupted at the insertion of the stalk (plate 3: d). For further details see Nayar \& Kaur (1965a). The sporangia were not studied in detail. From incidental observations it was found that within one species the stalk may vary much as to its length and colour (B. appendiculata), and the head as to its size (B. sinensis).

The ancestral condition.-In the genus Bolbitis a considerable range of different intermediate leaves is found, and some of these are characteristic for certain taxa. Part of these have misled the systematists, e.g. those of Jenkinsia undulata $(=B$. virens $\mathrm{f}$. virens $)$ and Meniscium deltigerum $(=B$. virens var. deltigera $)$. 
Intermediate leaves do not give uniform evidence regarding a supposed ancestral soral condition. Some show a pteridoid arrangement of sporangia, which represents a derived condition. In others the sporangia may be situated exclusively or mainly on the veins. Schumann (1915), who cultivated a few species at Munich, studied the ontogeny of the fertile leaves of Leptochilus cuspidatus $(=B$. repanda) and Leptochilus heteroclitus $(=B$. heteroclita). As generally the sporangia develop first on the veins, and as sporangia are situated mainly on the veins in intermediate leaves ('Mittelformen') of these species she concluded that the species investigated were derivatives from ferns 'bei denen die Sporangien dem Verlauf der Adern folgen', which may be correct for the present genus. Incidentally marginal 'sori' are found. Such a condition as present in the specimen of $B$. quoyana illustrated can be easily interpreted as an incomplete pteridoid arrangement. Indusiate sori have never been found in the ample material of different intermediate leaves.

When trying to reconstruct the original condition of the sporangial arrangement in Bolbitis, we note that distinct submarginal sori are a generic characteristic of the monotypic lomariopsidoid genus Thysanosoria from New Guinea. This genus is based on two collections (from adjacent localities) agreeing in detail with those of Lomariopsis kingii (Copel.) Holttum (Holttum, in preparation) except for having round sori on small marginal lobes of the fertile pinnae. Their sporangia are situated on a narrow cone-shaped receptacle placed at right angles to the pinna-lamina, somewhat apart from the marginal lobe. Because of this sporangial arrangement, Holttum postulates that an indusiate (!) sorus at a vein-ending might be the original fertile state of the group of lomariopsidoid ferns. This seems a far-fetched conclusion and to me Thysanosoria should be regarded as a deparioid derivative (Christensen, 1938: 'peculiar derivative') of Lomariopsis.

Within the non-acrostichoid fertile leaves found in Bolbitis the hemionitidoid arrangement of the sporangia is best represented. Such an arrangement is also found in such 'old' species as B. acrostichoides. However, the non-indusiate hemionitidoid arrangement might represent a secondary development as well. If so, it is postulated that the acrostichoid condition was already present prior to the differentiation of the ancestral group into the modern genera of Lomariopsidaceae.

Notes on the acrostichoid condition.-It is now generally understood that the acrostichoid condition has developed in quite different lines independently. Further, that the tendency to become acrostichoid (or pteridoid) can be perceived in the major groups of isosporous ferns.

Copeland (1929: 381) and Ching (1958) have pointed out advantages of an erect disposition of the fertile leaves of dimorphic ferns. '... the fertile fronds, on longer stipes than the sterile stand quite erect and high in the middle of the plant to insure better light and consequently a better chance of dispersal of its spores in the dry air ...' (Ching, 1958: 133).

The acrostichoid condition is not always 'complete'; and so-called intermediate leaves, which are in part fertile, in part sterile, may occur. On the other hand, the acrostichoid condition may be characteristically 'intermediate' or unstable in other ferns. 
Sledge (1972) noted that in the acrostichoid fern Quercifilix zeilanica (Houtt.) Copel. round sori may occur on the basal lobes of the fertile lamina. This, and the fact that this fern hybridizes easily with Tectaria decurrens (Presl) Copel. induced him to sink Quercifilix in Tectaria.

As species of the present genus have been formerly included in the genus Leptochilus, I have checked all of its species as conceived by Ching (1933). It will be demonstrated (Hennipman, in preparation) that the acrostichoid arrangement in Leptochilus shows (a characteristic) instability in several species. Thus in Leptochilus minor Fée one can sometimes recognize individual elongate sori situated parallel to the rhachis of the narrow fertile leaf, a situation similar to that found in Colysis poilanei $\mathrm{C}$. Chr. \& Tardieu-Blot. Further, I traced a specimen of Colysis hemionitidea (Wall.) Presl which consisted of a \pm acrostichoid fertile leaf of a type as found in Leptochilus species, apart from a typical monomorphic fertile leaf (with round and elongate sori between the secondary veins), occurring together on a single rhizome! I agree with Ching (1933) that the genera Leptochilus and Colysis are otherwise similar, and there seems no objection to unite these genera formally.

The true affinity of acrostichoids is sometimes difficult to ascertain. In Leptochilus acuminatus White \& Goy the linear arrangement of sori may be 'broken' showing two rows of variously elongate sori parallel to the costa. The identity of this fern became manifest only after an intermediate leaf became available showing a woodwardioid arrangement of sori in an atavistic part of this leaf. The species is transferred to the genus Pteridoblechnum (Hennipman, 1976).

The facts indicate that the acrostichoid condition may become manifest in any group at any time. The observations on the fertile leaves of Leptochilus and Colysis reveal that such a condition might not necessarily be the result of a gradual change in leaf shape and soral insertion as is generally believed. The acrostichoid condition is sometimes difficult to weigh taxonomically, as potentially important features like those pertaining to the sori are lacking, whereas the vegetative features of the non-acrostichoid potential relatives are not (yet) always fully known.

\section{SPORES}

\section{Introduction}

Although spores as seen with the light microscope were described as early as the last century (e.g. Fée, 1845), the study of the morphology of the spores has been rather neglected until recently. For a survey see Lugardon $(1972,1974)$ and Wagner (1974).

As regards the spores of the genus Bolbitis, Erdtman (1957) supplied a brief description and an illustration of the spores of $B$. turrialbae $(=B$. pergamentacea $)$ and Polybotrya appendiculata $(=B$. appendiculata). Nayar \& Kaur (1965c) described the spores of mainly the Indian species. These authors included features of the perispore ('perine') to keep the genera Bolbitis and Egenolfia (here united) separate. This, and my interest in the structural properties of the spore walls, induced me to study the spores of several 
species with both the light and the electron microscope (Hennipman, 1970). It was shown that the spores do not provide evidence justifying the treatment of Egenolfia as a separate genus. Erdtman \& Sorsa (1971) reported the light microscopical observations on 21 species of the genus (including the species formerly referred to Egenolfia). Their data were largely taken from the publications of Nayar \& Kaur.

The structure of the spores of the ferns as seen with the electron microscope was first described by Lugardon (1965) for Blechnum spicant, by Pettitt (1966) for Asplenium adiantum-nigrum, by myself (op. cit.) for four species of Bolbitis, and by Lugardon (1972, 1974) for a considerable number of ferns including also eusporangiate ferns. Lugardon's excellent work is of general interest.

As regards the detailed structure of the spores of Bolbitis I demonstrated that the exospore ('exine') of several species does not show noteworthy structural differentiation. Further, that the perispore ('perine") is not a simple but a much differentiated structure. Besides, the perispore of closely related species may be markedly different, whereas the spatial distribution of the perispore in almost mature sporangia of different species shows surprising variation. During the present study spores of all taxa were studied with the light microscope. Representatives of the different spore types recognized were studied with the scanning electron microscope (S. E. M.). In the descriptions of the spores I have usually mentioned the general characteristics only. For the detailed structure the reader is referred to the S. E. M. photographs.

The present chapter includes some observations on certain aspects of the sporogenesis, including those obtained from material in which abnormal spores are formed.

The term 'spores' stands for the eventual shape of the divisional products of the sporemother-cell (S. M. C.) and includes the perispore. I now prefer to use the terms exospore and perispore instead of exine and perine, respectively. These terms are applied as defined by Lugardon (1972).

\section{Results}

\section{Types of spores}

Spores as seen with the light microscope are monolete, biconvex, and provided with a variously shaped brown perispore. The exospore is thin, structurally not differentiated, and shows a short, straight laesura which is rarely forked at one end. The perispore as seen from cross-sections studied with the S. E. M. is composed of two more or less distinct elements which have been named 'inner' and 'outer' perispore. The types of spores as set out below are primarily based on differences in the shape of the outer perispore, which can also be recognized with the light microscope.

TYPE A.-Spores with the outer perispore reticulate and cristate, the inner perispore closed and the crests of adjacent spores in a \pm mature sporangium continuous. The inner surface of the inner perispore is smooth and does not show connections with the exospore; the outer surface of the inner perispore is \pm smooth, though its fine sculpture is somewhat scabrous because of the occurrence of minute, irregular, 
wart-like excrescences. The crests of the outer perispore are continuous with the plasmodial remains on the inner wall of the almost mature sporangium. This reticulate cristate perispore occurs in $B$. appendiculata.

Within this species two main subtypes of perispore can be distinguished. In the first subtype the outer perispore consists of crests only. The crests are generally \pm perpendicular to the inner part of the perispore, and are reticulately arranged, showing in the meshes included crests which end free and gradually decrease in height distally (ssp. appendiculata; plate $4: \mathrm{a}, \mathrm{b} ; 5$ ). In the other subtype the outer part of the perispore covers the inner part completely (ssp. vivipara). Between its crests the outer part of the perispore is apparently either adnate to the inner part of the perispore (var. vivipara; plate 4: c, d) or continuous with it (var. neglecta; plate 4: e, f).

Atypical specimens of ssp. appendiculata from southern India (autopolyploids?) show large spherical spores with a low-cristate perispore the outer surface of which shows thread-like irregularities (plate 10: a, b).

TYPE B.-Spores with the outer part of the perispore closed, cristate or cristate-undulate, the inner part closed, and adjacent spores in an almost mature sporangium not connected, or connected by a few thread-like structures, or with partly continuous crests (plate 6-8).

In a cristate-undulate perispore, the inner perispore is of the same shape as the exospore; its inner surface is smooth, whereas the outer surface \pm densely set with spine- or wart-like excrescences and scattered threads (not septae as communicated earlier by me) which connect the outer surface of the inner perispore with the inner surface of the outer perispore. The outer part of the outer perispore is cristate-undulate and the proximal folds may be adnate to the inner part of the perispore. The inner surface of the outer perispore is smooth except for thread-like structures usually running to the inner perispore or rarely connecting the lateral sides of a particular fold. The outer surface of the outer perispore is either about smooth or densely or sparsely beset with minute, spine- or wart-like excrescences (plate 6, 7).

A cristate perispore is similar but lacks folds in its outer perispore. The crests of the outer perispore are \pm perpendicular to the inner one (plate 8).

Although some species are characterized by either a cristate or a cristate-undulate perispore, the spores of several other species display both types of perispore as well as the intermediates.

This type is very common in the genus. It is found in a few variants relating to the density of folds or crests.

TYPE C.-Spores with the outer part of the perispore undulate, the inner part of the perispore closed, membrane-like, and adjacent spores in an almost mature sporangium not connected.

The inner part of the perispore adheres closely to the exospore. Its outer surface is smooth and in a few places, as it seems, connected with the proximal sinuses of the undulations of the other perispore. 
The inner surface of the outer perispore is smooth; its outer surface is also smooth or minutely scabrous. The perispore of adjacent spores are not interconnected in mature sporangia.

This type is found in all species of ser. Bolbitianae (plate 9). The shape of the outer part of the perispore varies from shallowly to densely undulate; in one species (B. semicordata; plate 9: d, e) it may be verrucate.

TYPE D.-Spores with the outer part of the perispore closed and undulate, the inner part of the perispore present as a multitude of small, irregular, solitary or aggregated warts or pustules on the exospore; adjacent spores in an almost mature sporangium not connected. Outer perispore similar to that described above, generally situated at a considerable distance from the exospore.

This type is found in the monotypic ser. Heudelotianae only (plate 10: C).

With the light microscope it is not possible to discriminate between the types Cand D. It should be noticed that ser. Bolbitianae and ser. Heudelotianae are not closely related.

Erdtman \& Sorsa (1971) erroneously reported the absence of a perispore in the type material of Bolbitis longiflagellata $(=B$. spec. dub.). The sporangia of the type specimen are immature. A perispore was found in another collection of this taxon (see the Taxonomic Part), which is in accordance with Tardieu-Blot (1966). Stratification of the spore wall as published by Erdtman (1957) for Bolbitis turrialbae (= B. pergamentacea) could not be confirmed.

The variation in the perispore of the present genus as demonstrated earlier by me with the electron microscope (Hennipman, 1970) is not unique. For instance, Wood (1973) demonstrated a similar variation in Thelypteris s.l., and Tryon \& Tryon (1973) in cheilanthoid ferns, both with the use of the S.E.M. A.F. Tryon (1971) in a very interesting study found even exceptional variation in the shape of the spores of Thelypteris palustris $s .1$., which she thought to indicate a more complex relationship than that supposed till now.

The differences between the perispores of different species in Bolbitis provide important taxonomic characters. For instance, the presence of a distinct type of perispore in ser. Bolbitianae adds significant weight to its taxonomic recognition.

\section{Notes on variations in shape of the perispore}

The shape of the perispore in a plant with normal spores shows but little variation. This variation mainly applies to the density of the crests or folds, the height of the crests, etc. A more surprising heterogeneity was occasionally also observed.

a. The spiny perispore. In some species with a cristate-undulate perispore plants were found with part of their spores with a densely spiny perispore as seen with the light microscope. When studied with the S.E.M. the outer perispore appeared not to be smooth but perforated and to consist of low spines and narrow crests. In one collection sporangia with all of its spores with a spiny perispore occurred next to sporangia 
with typical spores. Occasionally sporangia are found with both types of spores and including the intermediate condition (see plate 6: c).

Occasionally a spiny perispore was found in a tetraploid specimen of $B$. repanda and in a specimen of $B$. sinensis (Rock 2913, US; see $B$. sinensis note 2a).

b. In a few specimens of species with a cristate perispore(e.g. B. fluviatilis), spores with an undulate or cristate-undulate perispore were traced.

c. An observation which seems worth mentioning regards the differences between the spore output of different specimens belonging to one gathering, collected at the same site. I had the privilege to study all specimens of a considerable number of collections prior to the distribution of duplicates. Not rarely the quality of the spores of different specimens was very different. In some cases autoploidy is inferred.

d. Nayar \& Kaur (1963: 94) concluded that a branched or trilete laesura, as occasionally found by them in the spores of $B$. costata, B. presliana, Egenolfia helferiana $(=B$. appendiculata ssp. appendiculata), and Egenolfia sinensis $(=B$. sinensis), might indicate a comparatively primitive nature of these species. Although I have not paid attention to the abnormal laesuras, I suffice to say that my studies are not in support of their conclusion.

Size

The spores of a certain plant, and of different collections of one species, show variation in size. Examples are given in the Taxonomic Part.

Differences in the average size of the spores of different species are slight. For details see Erdtman \& Sorsa (1971). The average spore size is hard to ascertain from herbarium specimens. Proportionally few sheets have mature spores. Exact measuring is made difficult by the presence of a perispore. In some species noteworthy differences were found in the size of the spores of individual plants. These anomalies are discussed in the notes on the species, when necessary.

In some cases conspicuous variation was found in the size of the spores of the same or different collections. This may reflect, though not necessarily so(Britton, 1972), genetical differences (Manton, 1950). On the other hand, A. F. Tryon (1971) found that mature spores of Thelypteris palustris var. pubescens could vary up to one-third in length in a single sporangium.

\section{Number of spores per sporangium}

The number of spores in a sporangium of pickled or cultivated material was invariably found to be 64. Sporangia of herbarium material were only investigated when apogamy was suspected (in which case the sporangia should regularly contain 32 spores only).

Abnormal spores (including aborted S.M.C.'s)

For a taxonomist it is important to know the appearance of abnormal spores and to what extent this kind of spores contributes to the total spore production of a certain leaf. 
Therefore, different types of abnormal spores are described. In case the spore output of a plant shows a mixture of normal and abnormal spores, as was frequently found in the Bolbitis specimens investigated, it is often impossible, and when possible it may be time-consuming, to find out if this mixture has possibly resulted from e.g. apogamous reproduction.

Abnormal spores are formed as a result of certain genetic and metabolic conditions of the plant.

a. The genetic condition.-The fertile leaves of the cultivated material were as a rule first studied during meiosis of the S.M.C.'s, and later again as the remaining part of the leaf showed mature sporangia. The shape of the mature spores largely reflects the pairing behaviour of the chromosomes during meiosis. When there is no pairing at all, as found in primary hybrids (e.g. B. heteroclita $\times$ rhizophylla) and sometimes also in autotriploids of both $B$. heteroclita and $B$. sinuata, spore-formation is already prevented early during sporogenesis. As a result the sporangia are eventually filled with 16 or so (mostly) shrivelled bodies enveloped by perisporial substances, usually located in the central part of the sporangium. When almost complete pairing takes place during sporogenesis (e.g. B. sinensis), the sporangium is eventually filled with 64 shrivelled or normally-shaped spores (the latter with a \pm typical perispore), which are equally distributed throughout the sporangium. All intermediate types of abnormal spores, and of spore-distribution in the sporangium, are represented. For instance, the abnormal spores were not rarely found to be clustered in the central part of the sporangium and enveloped by a (sometimes very regularly shaped) 'perispore' (plate 10: $d-f$ ).

Part of the spores formed by a certain leaf may be small, the size being obviously related to the amount of cytoplasm that becomes associated with the different nuclear parts resulting from an abnormal meiosis. In some primary hybrids minute spherical hyaline bodies were found in the mature sporangium.

b. The metabolic condition.-The fact that qualitatively different spore outputs were found in a single plant in cultivation (B. repanda, $4 \mathrm{n}$ ) induced me to study the spore output of a single fertile leaf during development of the sporangia. The few earliest sporangia reaching mature size were empty. Sporangia formed at a later stage, i.e. the great majority, contained 64 normal spores. Those that matured subsequentlystill a considerable part-were in part filled with abnormal spores or even aborted S.M.C.'s, and the last formed were all filled with abnormal spores or aborted S.M.C.'s.

The fertile leaf first formed in a specimen of $B$. sinensis at the end of the summer after three years of cultivation showed but slight irregularities during meiosis. A few of the chromosomes were sometimes found to be univalents. The resulting spores were mostly shrivelled, in part normally shaped.

The degree of abnormality of the spores is not necessarily a good indication of the degree of relationship. The spore output of an autotriploid specimen may show (mostly) aborted S.M.C.'s only, whereas the diploid hybrid between two different species may show shrivelled spores. 
External factors have an influence on the metabolic condition of the plant (Manton \& Sledge, 1954) and thus also on the processes involving sporogenesis.

The 'Fehlschlag' of spores caused by a sudden change in the weather conditions in temperate areas is well known. Braithwaite (1964: 301) reported the complete abortion of almost all sporangia in an Asplenium polyploid in South Africa, which in the previous year had produced an abundance of good spores. Manton \& Sledge found that a high proportion of newly transplanted adult plants from other latitudes failed to pair their chromosomes successfully when sporangia were first produced in cultivation. A varia-
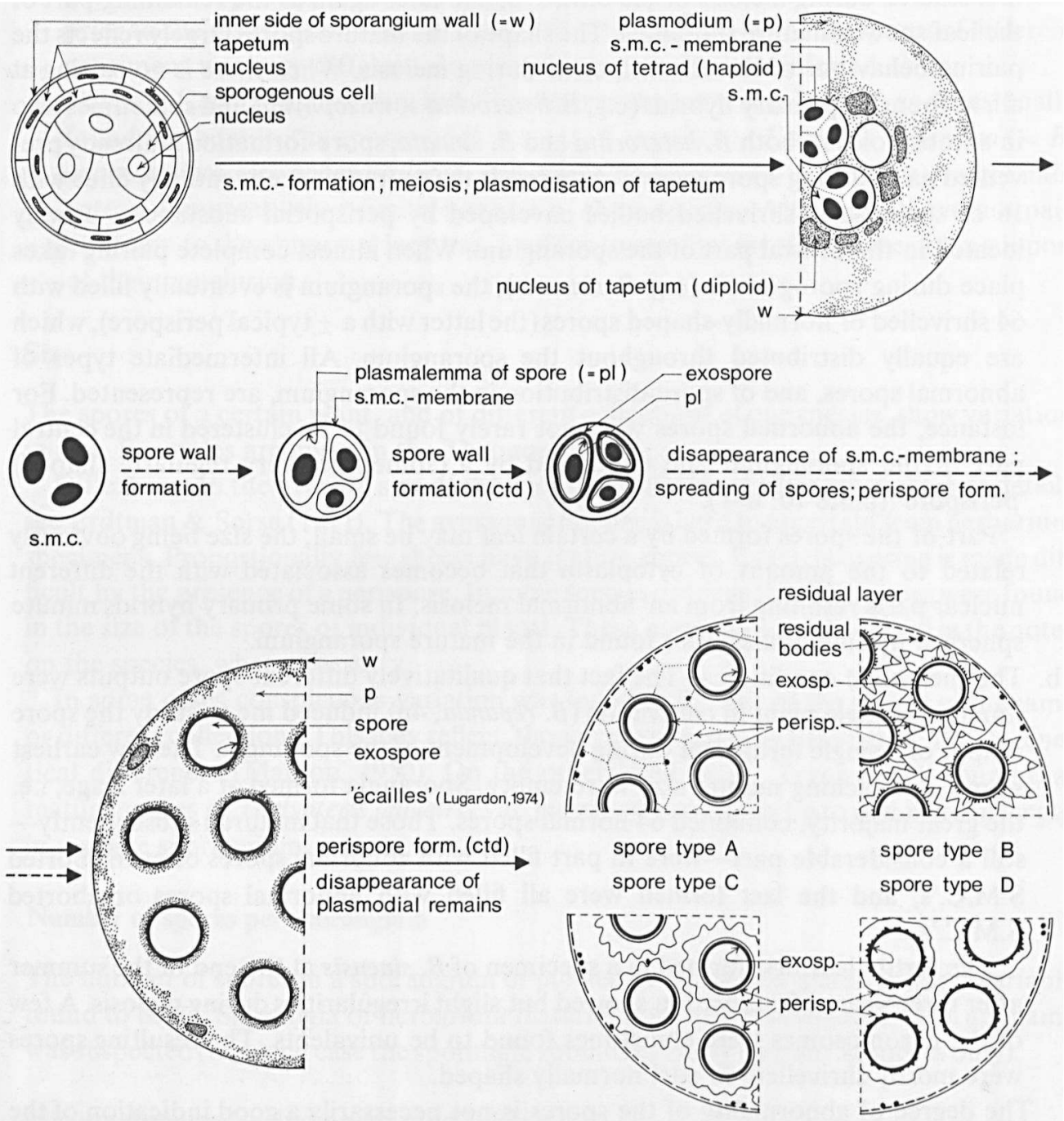

Fig. 12. Schematic illustration of supposed sporogenesis in Bolbitis. The diagram illustrating the start of the perispore formation (left hand bottom figure) is based on data from Lugardon (1974) and the present author (unpublished). See also text and Plate 12: a, b. 
tion in spores as found in cultivated specimens of $B$. repanda and B. sinensis may be caused by the suboptimal conditions in the greenhouses where they were cultivated.

In some cases it was found that the spores of a single sporangium looked in part perfectly normal, in part abnormal. Such a condition can only be explained in view of the genetical differences between meio-spores.

\section{Notes on sporogenesis}

Some aspects of sporogenesis could be studied with the light microscope from the cultivated material that was primarily used for chromosome counts.

During sporogenesis in plants producing normal spores a developmental stage occurs in which the spores are arranged in 16 cross-tetrads (Erdtman, 1945) in the central part of the sporangium within the tapetal plasmodium. At this stage the exospore of the spores looks already mature, and each tetrad is enveloped by a thin hyaline membrane (called here the S.M.C.-membrane) which is best demonstrated using phase-contrast microscopy. In a slightly older sporangium the spores are distributed regularly throughout the sporangium, whereas their exospores show inconspicuous hyaline deposits; an S.M.C.-membrane cannot be recognized anymore. In the oldest stages of the sporangium, the spores are similarly distributed in the sporangium and are enveloped by a perispore. Intermediate stages as regards the earliest stages of perispore formation were only rarely found.

The significance of the S.M.C.-membrane during sporogenesis is also inferred from the study of abnormal spores. The sporogenesis in plants eventually producing shrivelled spores is similar to that of normal spores until the stage in which the S.M.C.-membrane cannot be perceived anymore. This means that abnormal spores with abnormal nuclear contents and often with conspicuous rounded lagging chromosomes show a typical shape and structure when still in a cross-tetrad arrangement and enveloped by a S.M.C.-membrane. See pl. 11: b; fig. 31: b. Shrivelled spores (pl. 11: a; fig. 31: a), however, were never found surrounded by a S.M.C.-membrane; they always do show (inconspicuous) perisporial deposits. These data suggest that the disappearance of the S.M.C.-membrane coincides with the end of exospore formation, with the start of perispore formation, and with the spreading of the spores throughout the tapetal plasmodium in the maturing sporangium. The formation of the perispore after the formation of the exospore during sporogenesis has also been suggested by others (for instance Erdtman, 1969, Lugardon, 1974).

A perispore or perisporial deposit was always found whether the spores are haploid, diploid, or abnormal, clustered, etc. The shape of the perisporial deposit that eventually envelopes a cluster of abnormal spores, or a S.M.C. which retained its spherical shape, might even be rather regular as is typical for a true perispore. Perispore formation does not seem to be exclusively correlated with the production of normal spores.

The structure of the perispore or perisporial material does not show structural differences when studied with the light microscope. 
In my previous publication on the spores I demonstrated \pm spherical, small, acetolysis-resistant so-called 'residue bodies' in the residual material inside the mature sporangium of $B$. contaminans ( $=$ B. angustipinna). Residual bodies which were also found by Lugardon (1974, 'globules') have been found frequently by me during the study of the perispore (also of other genera) with the S.E.M., and their presence may be related to the formation of the outer perispore (Hennipman, in preparation).

The tapetal origin of the perispore is also inferred from the S.E.M. observations that the perispore of the spores in sporangia of $B$. appendiculata and $B$. sinensis is continuous with the residual material on the inner wall of a mature sporangium and including these residual bodies.

The exospore and the perispore, two apparently quite different structures, are both involved in the construction of the (dia)spore.

In fig. 12 supposed sporogenesis is schematically presented.

\section{The fine structure of the spores and its significance for classification}

From the results of my previous work on the fine structure of Bolbitis spores I anticipated that this kind of data about the structure of the spore-walls might provide evidence leading to a better delimitation of especially the higher taxa of Pteridophytes (Hennipman, 1970).

Lugardon's $(1972,1974)$ studies on the spores with the electron microscope fully confirm this. His studies are of particular interest as they provide basic general knowledge about the structure of the spores in different groups of both eu- and leptosporangiate ferns. Lugardon was able to demonstrate a perispore in all the different types of spores he studied. Formerly a perispore was supposed to be lacking in several groups of ferns. In case the perispore-which is always structurally different from the exospore when studied with the transmission electron microscope-is adherent to the exospore, it cannot be perceived with the light microscope, also as the optical qualities of both the exospore and the perispore might be identical. Within the ferns Lugardon recognized three main types of perispore to which I do not assign much systematic weight because of the differences of the perispore as found in otherwise related groups. I am convinced, however, that the eventual significance for classification of the differences within the often heterogeneous and complex structures, called 'perispore', can only be known when more information about its genesis within the different groups has become available.

Because of differences in the complexity of the exospore Lugardon recognized five different types which in the present author's view are easily reduced to three: that found in the eusporangiate ferns (the most complex), that found in the Osmundaceae and the Gleicheniaceae, and that found in the remaining leptosporangiate ferns (the simplest).

Lugardon (1974: 222) further emphasized the limitations of the light microscope for the recognition of the structure of the exospore '... les couches que la plupart des auteurs ont distingué dans cette paroi n'ont qu'un très lointain rapport avec sa structure réelle'. And this largely holds for the descriptions and drawings of Bolbitis spores by Erdtman (1957) and Nayar et al. 


\section{KARYOLOGY}

The existing knowledge of the chromosome number of Bolbitis species is scanty. There is, for instance, not a single record in literature of the chromosome number of the commonest Asian (B. heteroclita) and American (B. portoricensis) species. The haploid number as far as reported is based on $n=41$. This number is also found in the other genera of lomariopsidoid ferns whereas in Lomariopsis also an aneuploid number lower than 41 is known (Roy \& Manton, 1966).

I have attempted to study a fair number of different plants of several species of the present genus to find out to what extent polyploidy and hybridization might contribute to the often surprising heterogeneity of the (vegetatively propagating) species. Therefore, material was assembled of Asian representatives. A good start to cultivation was given by Prof. Holttum, who had brought several specimens of the present genus to Kew, collected by him during his trips to Asia, and material of which he kindly sent to Leiden. Additional material was received from several correspondents, especially from Mr. M. G. Price.

Table 1 includes all plants successfully grown in cultivation in Leiden, as well as a number of unpublished records kindly made available to me by Dr. T. G. Walker (Newcastle-upon-Tyne) and Dr. I. Manton (Leeds). Different morphological forms of one species have been given fancy names (added between quotation marks to the specific name) which have no status according to the Code. When the study of the chromosomes at meiosis was completed, the fertile leaf was as a rule not removed until maturation of the greater part of the sporangia. Then the spores were studied and a voucher prepared. Literature records have been included when reliable evidence regarding the identity of the material was available. Species are cited in the same sequence as in the Taxonomic Part. All voucher specimens are in Leiden. Living duplicate specimens obtained by vegetative multiplication can be forwarded on request.

\section{Notes}

Ser. Alienae.

B. aliena. Tetraploid. The specimen investigated by Dr. Walker was taken from a very shady place on limestone.

$B$. auriculata. Tetraploid. This count is not al all a surprise in view of the notorious variation found in herbarium specimens.

\section{Ser. Bolbitianae.}

B. crispatula var. copelandii. Diploid. Fertile leaves were formed following a period of good growth. The spores of the fertile leaf from which the meiotic chromosome number was obtained were for the greater part shrivelled.

$B$. virens f. virens. Diploid. Pl. 12: b. In spite of excellent vegetative growth of both plants, fertile leaves were only occasionally formed during cultivation. Although both collections originate from the same mountain (Doi Suthep), the shape of their fertile leaves is somewhat different. The fertile pinnae formed by the specimen of Van 


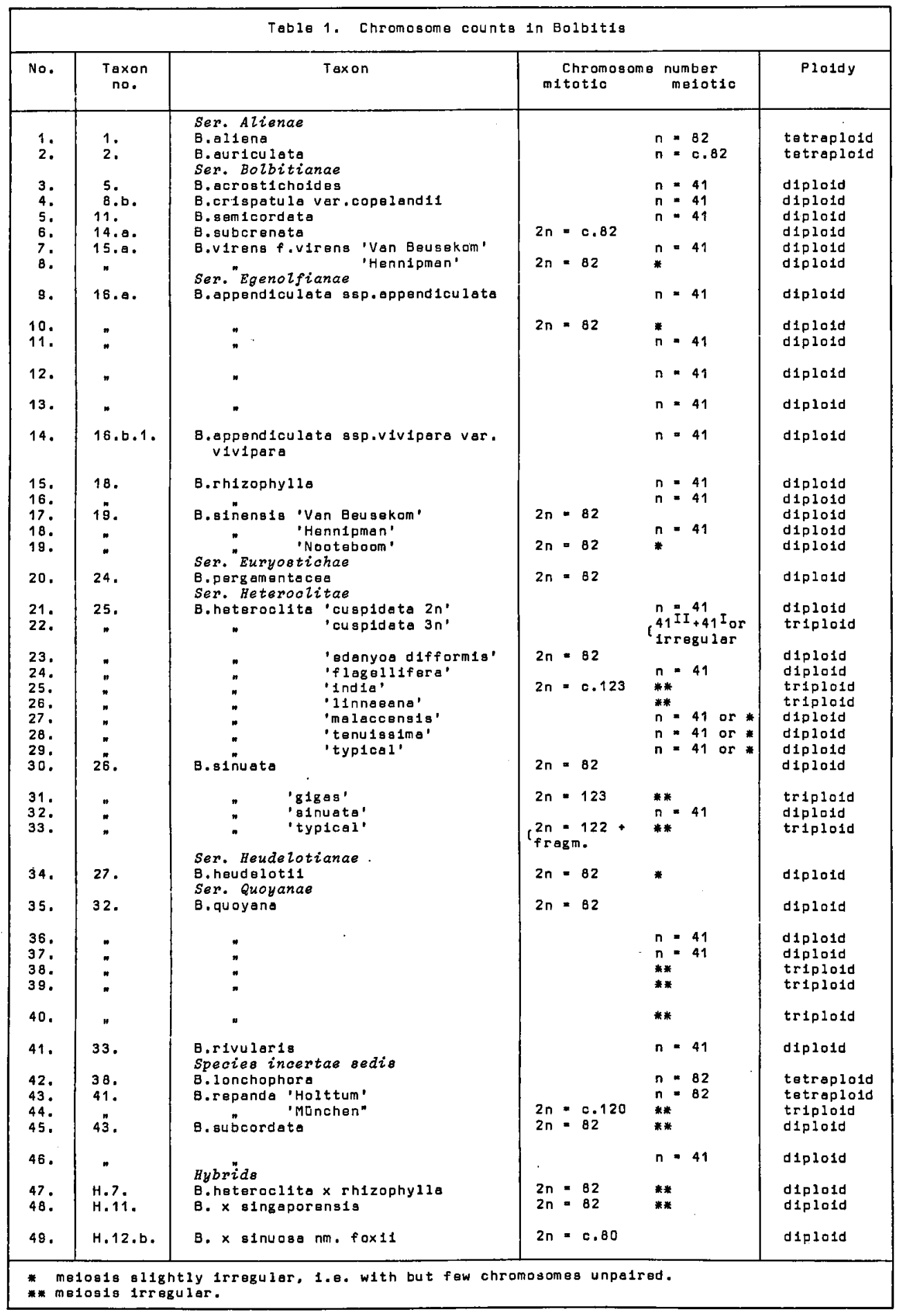




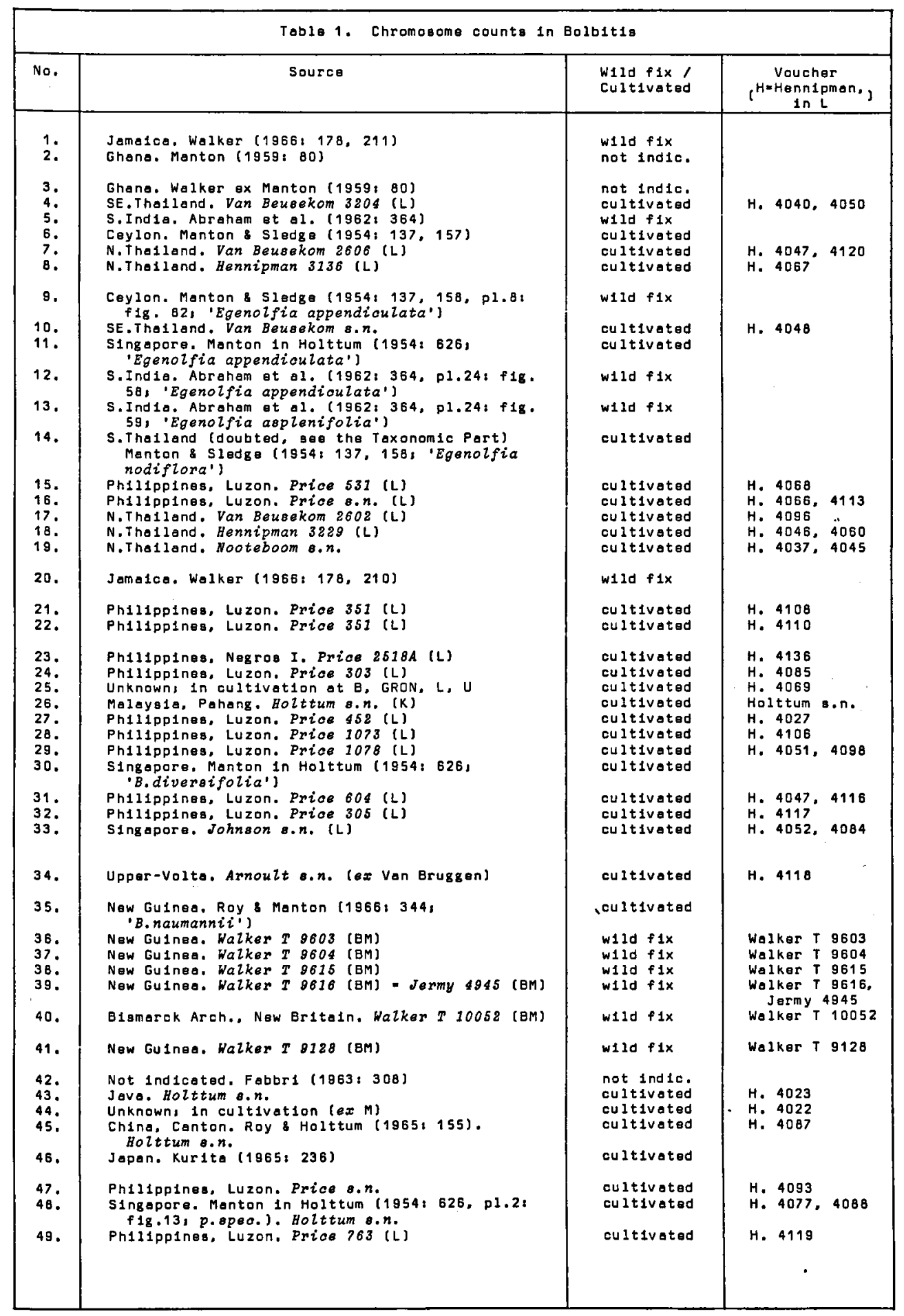


Beusekom are narrow and fully acrostichoid (sporangia all over the lower surface) whereas those of Hennipman's are much wider and \pm hemionitidoid (sporangia restricted to the veins). The spores formed by the fertile leaves from which the meiotic counts were obtained are for the greater part abnormal.

\section{Ser. Egenolfianae.}

B. appendiculata ssp. appendiculata. Diploid. Although meiosis was seemingly regular, the greater part of the spores that were eventually formed appeared shrivelled.

B. rhizophylla. Diploid. Pl. 11: f; fig. 31: f. Vegetative growth was excellent in cultivation. Only few fertile leaves were formed the spores of which were for the greater part abnormal.

B. sinensis. Diploid. Pl. 12: d. Growth was very slow in cultivation. In both plants a fertile leaf was formed only once. Spores of both for the greater part abnormal.

\section{Ser. Euryostichae.}

B. pergamentacea. Diploid. Ser. Euryostichae comprises plants similar to certain species of Tectaria $(\mathrm{n}=40)$. The chromosome number given by Dr. Walker further justifies its inclusion in the present genus.

\section{Ser. Heteroclitae.}

B. heteroclita. Diploid and triploid. The plants enumerated below are all 'distinct' as regards the shape of the mature leaves formed when the plants show good growth. Many of such plants have been formerly described as separate species. The similarities in the shape of the juvenile leaves and the leaves formed on bulbils of these plants is manifest.

$B$. heteroclita 'typical'. Diploid. Fig. 13: k. A large number of variously shaped juvenile leaves preceded typical leaves during cultivation.

$B$. heteroclita 'flagellifera'. Diploid. Fig. 13: $\mathrm{m}$. The leaf is typical except for its potentiality to form a very long flagellum provided with usually irregularly spaced bulbils. Fertile leaves were only occasionally formed.

B. heteroclita 'india'. Triploid. Fig. 13: 1. Plants in cultivation at the Botanic Gardens of Berlin, Groningen, and Utrecht are all similar and obviously have the same origin. They are coarse plants the leaves of which form distinct flagella with conspicuous buds. This form occurs in the wild in northeastern India. As the cultivated plant is similar to typical specimens of $B$. heteroclita, it is presumably an autotriploid.

Fig. 13. Cultivated material of Bolbitis heteroclita. a, b. B. heteroclita 'malaccensis', plants, $\times 1 / 5$; c. ibid., venation pattern of sterile pinna, $\times 4 / 5 ; \mathrm{d}-\mathrm{f}$. B. heteroclita 'tenuissima', sterile and fertile leaves, $\times 1 / 5 ; \mathrm{g}$. $B$. heteroclita 'cuspidata $3 n^{\prime}$, plant, $\times 1 / 5$; h. ibid., venation pattern of sterile pinna, $\times 4 / 5$; i. $B$. heteroclita 'cuspidata $2 n$ ', plant, $\times 1 / 5 ;$ j. $B$. heteroclita 'linnaeana', plant, $\times 1 / 5 ; \mathrm{k}$. B. heteroclita 'typical', leaf, $\times 1 / 5$; 1. B. heteroclita 'india', plant, $\times 1 / 5 ; \mathrm{m}$. B. heteroclita 'flagellifera', plant, $\times 1 / 5$ (a. Hennipman 4027, L; b, c. H. 4092 , L; d, e. H. 4106, L; f. H. 4090, L; g, h. H. 4110, L; i. H. 4039, L; j. Holttum s.n., K; k. Hennipman 4098 , L; 1. H. 4069, L; m. H. 4085, L; for chromosome numbers see table 1). 


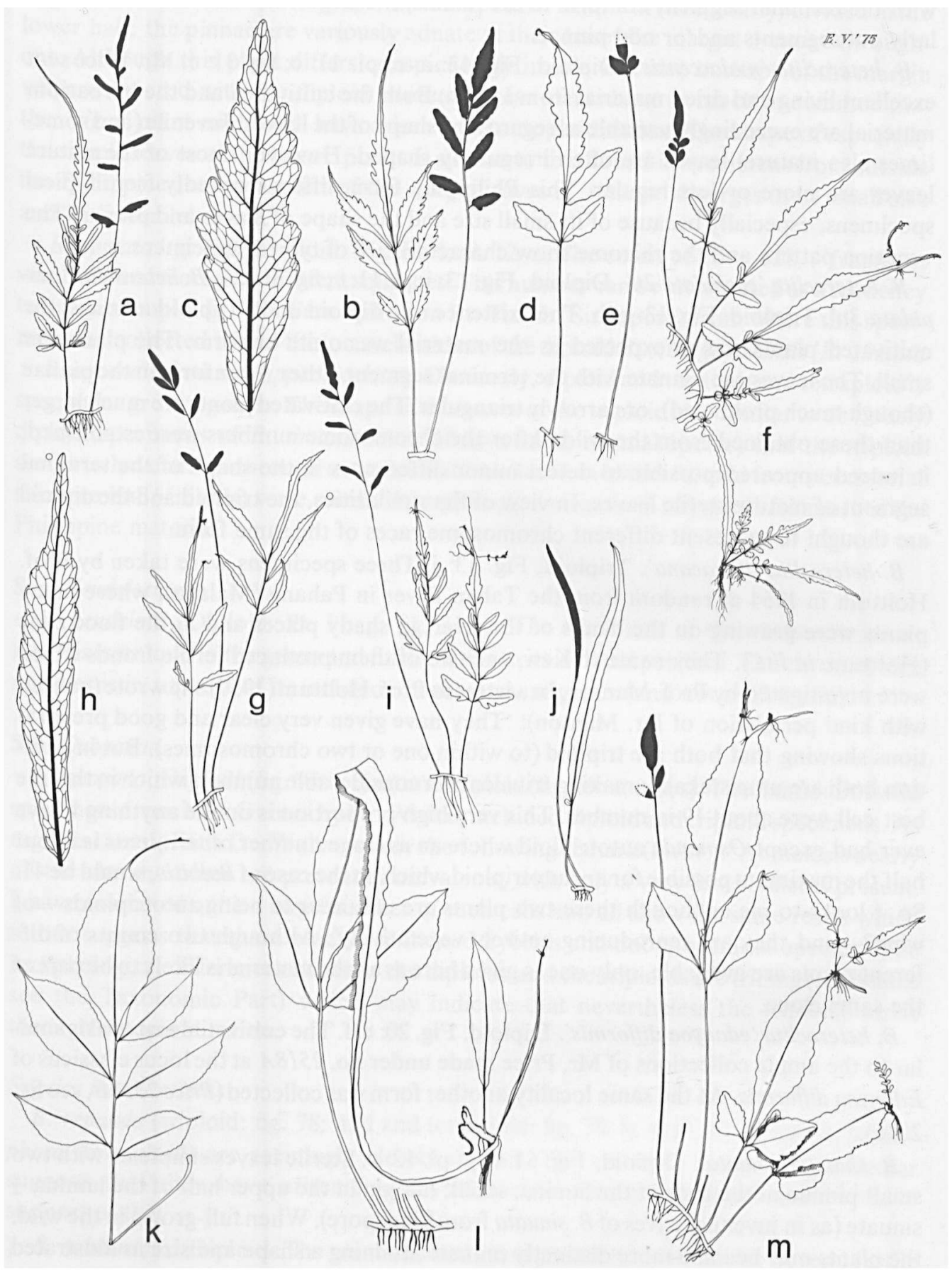


B. heteroclita 'tenuissima'. Diploid. Fig. 13: d-f. Rather small plants. Leaves pinnate with the terminal segment conform to the pinnae, the largest leaves often with irregularly cut segments and/or odd pinnae.

B. heteroclita 'malaccensis'. Diploid. Fig. 13: a-c; pl. 11: c; fig. 31: c. Mr. Price sent excellent living and dried material from Luzon. Both the cultivated and the herbarium material are exceedingly variable as regards the shape of the leaves. Juvenile (and sometimes also mature) leaves are often irregularly shaped. However, most of the mature leaves are more or less regular. This Philippine form differs markedly from typical specimens, especially because of its small size and the shape of leaves and pinnae. The venation pattern and the rhizome show characteristics of typical specimens.

$B$. heteroclita 'cuspidata $2 n$ '. Diploid. Fig. 13: i; pl.11: e; fig. 31: e.-B. heteroclita 'cuspidata $3 n$ '. Triploid. Fig. 13: g, h. The existence of a diploid and a triploid amongst the cultivated plants was unexpected as the material was quite uniform. The plants are small. The leaves are pinnate with the terminal segment either \pm conform to the pinnae (though much prolonged), or narrowly triangular. The cultivated plants are much larger than those obtained from the wild. After the chromosome numbers were established, it indeed appeared possible to detect minor differences in the shape of the terminal segment of mature sterile leaves. In view of the similarities, the triploid and the diploid are thought to represent different chromosome races of the same form.

$B$. heteroclita 'linnaeana'. Triploid. Fig. 13: j. Three specimens were taken by Prof. Holttum in 1954 at random from the Tahan River in Pahang (Malaya), where many plants were growing on the banks of the river, in shady places and in the flood zone (Holttum, in litt.). They came to Kew, and two of them produced fertile fronds which were investigated by Prof. Manton. In a letter to Prof. Holttum (1957) she wrote (quoted with kind permission of Dr. Manton): 'They have given very clear and good preparations showing that both are triploid (to within one or two chromosomes). But in addition both are unmistakably making trivalents in considerable numbers which in the one best cell were about 19 in number. This very high proportion is unlike anything I have ever had except Osmunda autotriploid where an average number of trivalents is about half the maximum possible for an autotriploid which, in the case of Bolbitis, would be 41 . So it looks to me as though these two plants are very near to being autotriploids-of what?-and they are reproducing entirely vegetatively'. Although two counts of different plants are available, only one is given here as all the material is likely to be part of the same clone.

B. heteroclita 'edanyoa difformis'. Diploid. Fig. 20: e, f. The cultivated material is similar to the ample collections of Mr. Price made under no. 2518A at the locus classicus of Edanyoa difformis. At the same locality another form was collected (Price 2518B, see fig. 20: o).

B. sinuata 'sinuata'. Diploid, Fig. 61: e, g; pl. 12: c. Sterile leaves simple or with two small pinnae at the base of the lamina, small; margin in the upper half of the lamina \pm sinuate (as in juvenile leaves of $B$. sinuat $a$ from Singapore). When full-grown in the wild, the plants may become more distinctly pinnate attaining a shape and size as illustrated in fig. 61: a. 
B. sinuata 'gigas'. Triploid. Pl. 11: d; fig. 31: d. The cultivated and the wild material (fig. $61 ; \mathrm{c}, \mathrm{d}$ ) are similar. The sterile leaves are either simple or (irregularly) divided in the lower half; the pinnae are variously adnate at their base; the margin \pm entire throughout. Although this plant differs conspicuously in size and also in shape of the margin when compared with the diploid 'sinuata', these plants have so much in common that the 'gigas' plant might well have arisen from autoploidization. The shape of especially the simple leaves of the 'gigas' plants is similar to that found in specimens from outside the Philippines, whereas that of the simple leaves of 'sinuata' diverges in its small size and its 'juvenile' shape.

B. sinuata 'typical'. Triploid. Fig. 61: 1; pl. 12: a. The count of $2 n=122+$ fragment was obtained from five different squashes. This number can be interpreted as a tendency towards aneuploidy. The Fern Valley on Bt. Timah (Singapore) from where this specimen was collected is also the locus classicus of the diploid $B . \times$ singaporensis $(B$. sinuata $\times B$. appendiculata ssp. appendiculata). Obviously, diploid and triploid (and tetraploid?) specimens grow together. The shape of the leaves of the triploid is so similar to obviously diploid specimens of $B$. sinuata from Malaya (with normal spores) that the triploid seems to have arisen through autoploidization. The first leaves formed from accessorial rhizome bud(-initials) were small, the upper part sinuate as is typical for most of the Philippine material.

\section{Ser. Heudelotianae.}

B. heudelotii. Diploid. Pl. 11: g; fig. 31: g. The small size of the chromosomes at meiosis is unique in the genus and further adds to its isolated position. The chromosome number is in support of its inclusion in the present genus (and not in Tectaria).

Ser. Quoyanae.

B. quoyana. Diploid (fig. 69: b, f, m) and triploid (fig. 69: a, e, p, s). The fixations of wild specimens from New Guinea by Dr. Walker included diploid and triploid specimens. As regards the triploids Dr. Walker made the following remark (in litt.): 'At meiosis nearly all the chromosomes are present as univalents, with only a very few bivalents present. I have examined quite a large number of such cells and all show the same result which rather suggests that these plants are indeed hybrids and not apogamous species.' The morphological differences between the diploids and the triploids are trifling(for details see the Taxonomic Part) which may indicate that nevertheless the triploids arose through autoploidization.

\section{Species incertae sedis}

B. repanda (triploid: fig. 78: a, d and tetraploid: fig. 78: b, c; pl. 12: e) and B. lonchophora (tetraploid) are seemingly closely related. The venation pattern in particular suggests that they have arisen from hybridization. The chromosome number is in support of this.

B. subcordata. Diploid. The plant showed good growth in cultivation. Several fertile leaves were formed. They all showed irregular pairing of chromosomes at meiosis and 
mature sporangia of all were filled with mainly aborted S.M.C.'s. Possibly a hybrid. The shape of the pinnae of the voucher includes a distinct basal acroscopic lobe and is strongly reminiscent of cultivated material of $B . \times$ singaporensis. The morphology of the former hybrid gives some indication that $B$. subcordata arose through hybridization. The regular meiosis $(n=41)$ reported by Kurita (1965) is not in support of this.

\section{Hybrids}

B. $\times$ sinuosa nm. foxii. Diploid. Growth slow; up to the present (1976) only sterile leaves have been formed.

B. heteroclita $\times$ rhizophylla. Diploid. Growth excellent. For a considerable time the plants remained rather small and sterile showing abundant vegetative propagation (also subterminally on the pinnae). After a considerable period of sunny weather in early summer of 1971, much larger sterile leaves as well as two large fertile ones were formed rather suddenly. There was no pairing of chromosomes at meiosis, sporangia showing aborted S.M.C.'s only.

B. $\times$ singaporensis. Diploid. Growth good. Fertile leaves were formed regularly. There was no pairing of chromosomes at meiosis, sporangia containing aborted S.M.C.'s only. The morphology of this hybrid is of a kind that a relationship with $B$. sinuata does not seem obvious even should it be known that Bolbitis species are involved.

\section{Discussion and conclusions}

Chromosome numbers are given for 49 plants: 41 belong to 16 species from 7 series, 5 to 3 species of the group Species incertae sedis, and 3 to 3 hybrids. Out of 46 plants belonging to 19 species, $33(72 \%)$ were diploids, $9(19 \%)$ were triploids, and $4(9 \%)$ were tetraploids. These data confirm the idea of the common occurrence of hybridisation and polyploidisation as obtained from the study of herbarium material.

From table 2 it can be seen that the majority of the polyploid specimens are from ser. Heteroclitae and ser. Quoyanae. The taxonomy of the species of these series is notoriously difficult.

All the triploids are supposed to be autotriploids, as their morphology shows but trifling differences if compared with the diploids. The diploids (and triploids) of especially $B$. heteroclita are strikingly different from each other and include also several distinct dwarfs. During the cultivation of these forms similarities in their juvenile leaves became manifest. There is no correlation between the ploidy and the shape of the plants of $B$. heteroclita investigated. Many of such distinct forms have formerly been described as separate species. See also chapter 17.

Although quite a number of counts are available for series Bolbitianae and Egenolfanae, only diploids were found.

The present investigation confirms the haploid chromosome number of Bolbitis species to be 41 or 82 . In one species (B. sinuata) a weak indication of aneuploidy was found. Within the lomariopsidoid ferns aneuploidy was earlier demonstrated, as said before, by Roy \& Manton (1966) in Lomariopsis. 


\begin{tabular}{|c|c|c|c|c|c|}
\hline \multirow[t]{2}{*}{ Table 2 . } & \multicolumn{5}{|c|}{$\begin{array}{l}\text { Distribution of presently known chromosome } \\
\text { numbers according to series, species in- } \\
\text { certae sedis, and hybrids. }\end{array}$} \\
\hline & $\begin{array}{l}\text { total of } \\
\text { counts }\end{array}$ & $\begin{array}{c}\text { number of } \\
\text { species }\end{array}$ & $2 n$ & $3 n$ & $4 \mathrm{n}$ \\
\hline \multicolumn{6}{|l|}{ SERIES } \\
\hline Alienae & 2 & 2 & - & - & 2 \\
\hline Bolbitianae & 6 & 5 & 6 & - & - \\
\hline Egenolfianae & 11 & 3 & 11 & - & - \\
\hline Euryostichae & 1 & 1 & 1 & - & - \\
\hline Heteroclitae & 13 & 2 & 8 & 5 & - \\
\hline Heudelotianae & 1 & 1 & 1 & - & - \\
\hline Quoyanae & 7 & 2 & 4 & 3 & - \\
\hline \multicolumn{6}{|l|}{ SPECIES } \\
\hline B. repanda & 2 & 1 & - & 1 & 2 \\
\hline B. lonchophora & 1 & 1 & - & - & - \\
\hline \multirow[t]{2}{*}{ B. subcordata } & 2 & 1 & 2 & - & - \\
\hline & $\overline{46}$ & $\overline{19}$ & $\overline{33}$ & $\overline{9}$ & 4 \\
\hline \multirow[t]{2}{*}{ HYBRIDS } & 3 & - & 3 & - & - \\
\hline & $\overline{49}$ & $\overline{19}$ & $\overline{36}$ & $\overline{9}$ & 4 \\
\hline
\end{tabular}

The chromosomes are rod-shaped and well discernable with the light microscope. They show variation in size, place of the centromere, and occurrence of satellites.

Several plants showed considerable variation in the pairing behaviour of the chromosomes belonging to different S.M.C.'s in different sporangia. Some S.M.C.'s showed bivalents only, whereas others showed a various number of univalents.

Apogamy was not found in any of the specimens. Manton \& Sledge(1954) estimated that $c .10 \%$ of the ferns of Ceylon were apogamous. Abraham et al. (1962) stated $8 \%$ for pteridophytes in southern India.

Several observations were made implying that, though meiosis was (largely) regular, the greater part of the spores eventually formed was abnormal and mostly shrivelled 
(B. crispatula, B. appendiculata ssp. appendiculata, B. rhizophylla (both plants), B. sinensis (both plants), $B$. heteroclita 'typical', 'flagellifera', 'tenuissima', and 'malaccensis'). Whether this is (solely) caused by external factors remains to be demonstrated.

\section{GAMETOPHYTES}

Gametophytes of the present genus have been described by Nayar \& Kaur (for references see Nayar \& Kaur, 1971) and Atkinson (1973). These authors studied a few species from several series and described the gametophytes as cordate or strap-like, consisting of a relatively thick and wide midrib and two (overlapping) wings of one layer of cells; the (ill-defined) meristem being present in the anterior sinus. Hairs were reported to be generally absent (Nayar \& Kaur, 1965b), although in some species marginal hairs were said to occur. Atkinson reported that the sex organs of all lomariopsidoid gametophytes belong to the so-called advanced type. She further compared the gametophyte of $B$. repanda which is subcordate with (un) branched marginal glandular hairs, with that of $B$. portoricensis (' $B$. cladorrhizans') which is strap-like with an illdefined meristem and devoid of hairs.

Gametophytes of three species were at my disposal; they are here briefly described. Atkinson's material of $B$. repanda proved to have the same origin as mine.

1. The mature gametophytes of $B$. angustipinna are cordate or elongate, measuring up to 5 by $4 \mathrm{~mm}$. The sinus is narrow and the wings somewhat overlap apically. Hairs are absent. Material: Hennipman 3637 (L). See fig. 14: a-c.

2. Mature gametophytes of $B$. heteroclita are usually elongate with a (sub)cordate apex measuring up to 10 by $4 \mathrm{~mm}$. The apical part bears $2-5$-celled glandular hairs especially on the margin, but also scattered on both the upper and the lower surfaces. The hairs are either similar to those occurring on the young sporophytic leaves (which have only one terminal glandular cell) or consist of 2-3 cells all of which are glandular. Small glandular scales occur on the larger gametophytes. Material: Price 1277 (L), kindly cultivated for the present study by Mr. M. G. Price at Laguna, Philippines. See fig. 14: $d-j$.

3. Mature diploid gametophytes of the allotetraploid B. repanda are cordate or rather elongate with a cordate apex. Cordate gametophytes are up to 10 by $6 \mathrm{~mm}$ with non-overlapping wings and a narrow sinus. Few-celled glandular hairs are present on the anterior margin and on the upper and the lower surfaces. Gametophytes that are 9 months old are elongate and measure up to $15 \mathrm{by} 10 \mathrm{~mm}$, the meristem firmly appressed to the substratum and the adjacent part of the non-overlapping wings spreading. Glandular hairs like those described for the gametophytes of $B$. heteroclita as well as few-celled scales are present in the apical part, both on the margin as well as on upper and lower surfaces. See fig. 14: k-o.

My observations largely agree with those of Nayar \& Kaur and Atkinson. The latter also reported the occurrence of branched hairs on the gametophytes of $B$. repanda. This observation is likely to be erroneous as such hairs were not found in the ample 


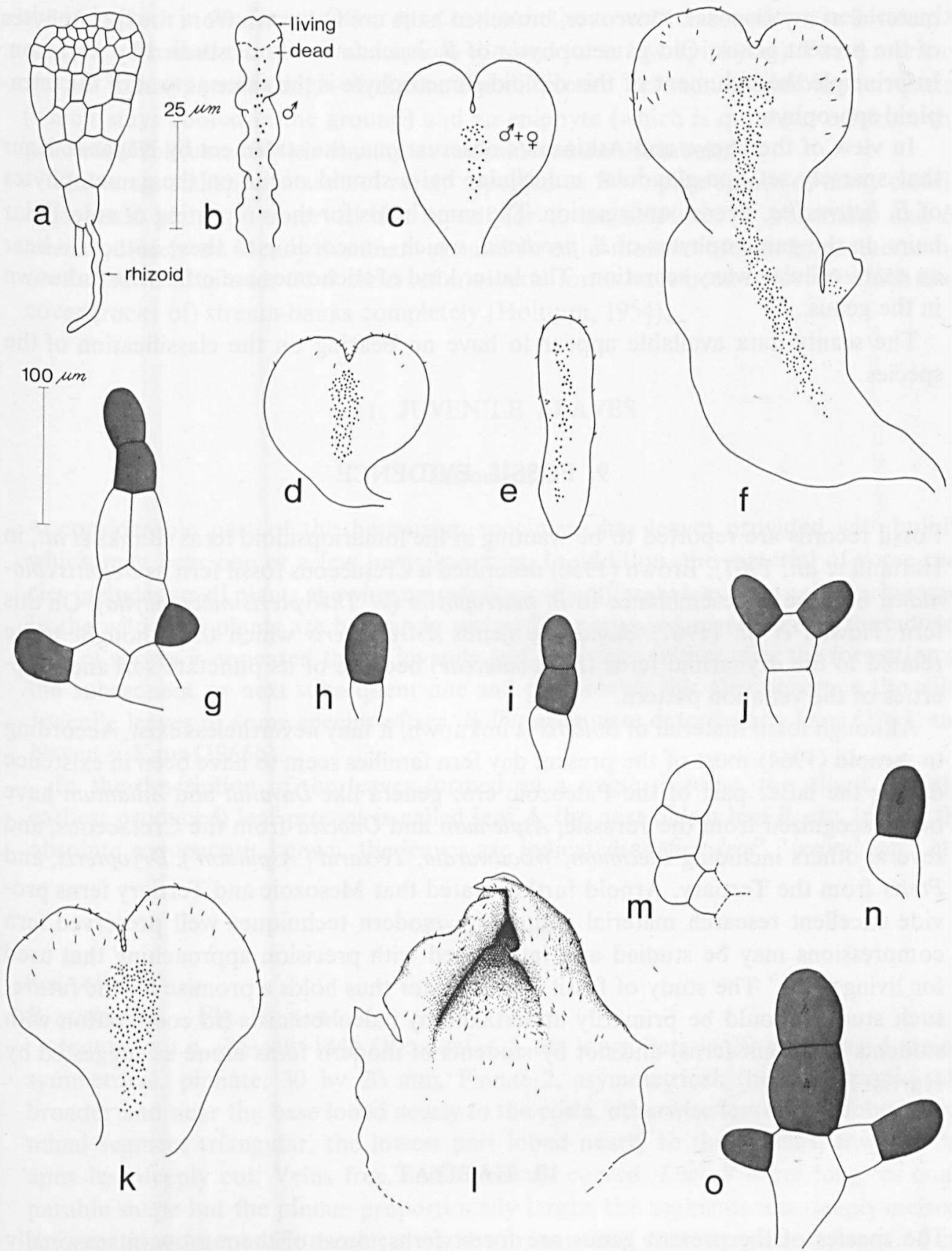

Fig. 14. Gametophytes. a-c. Bolbitis angustipinna; cultivated from spores of Hennipman 3637, L; b, c, X6. d-j. B. heteroclita, cultivated in the Philippines by Mr. Price from spores of Price 1277, L; d-f. habits, X6; g-j. glandular indument. - k-0. B. repanda, tetraploid, cultivated from spores of Hennipman 4023, L; K. habit of a 3 months old gametophyte, $\times 6 ; 1-0$. a 9 months old gametophyte; 1 . habit, $\times 3 ; \mathrm{m}-0$. glandular indument. 
material at my disposal. Moreover, branched hairs are unknown from the sporophytes of the present genus. Old gametophytes of $B$. repanda were not studied by Atkinson. In principle the indument of this diploid gametophyte is the same as that of the tetraploid sporophyte.

In view of the above and Atkinson's observations, the statement by Nayar \& Kaur that sparsely set non-glandular unicellular hairs should occur on the gametophytes of $B$. heteroclita, needs confirmation. The same holds for their reporting of unicellular hairs on the gametophytes of $B$. presliana, which-according to these authors-bear an extra-cellular waxy secretion. The latter kind of trichomes are otherwise unknown in the genus.

The scanty data available appear to have no bearing on the classification of the species.

\section{FOSSIL EVIDENCE}

Fossil records are reported to be wanting in the lomariopsidoid ferns (Banks et al., in Harland et al., 1967). Brown (1950) described a Cretaceous fossil fern as Bolbitis coloradica because of resemblance to $B$. macrophylla (= Thelypteris macrophylla). On this fern Tidwell et al. (1967) based the genus Astralopteris which they thought to be related to the drynarioid ferns (Polypodiaceae) because of its punctate sori and properties of the venation pattern.

Although fossil material of Bolbitis is unknown, it may nevertheless exist. According to Arnold (1964) most of the present day fern families seem to have been in existence during the latter part of the Paleozoic era; genera like Davallia and Adiantum have been recognized from the Jurassic, Asplenium and Onoclea from the Cretaceous, and several others including Blechnum, Woodwardia, Tectaria ('Aspidium'), Dryopteris, and Pteris from the Tertiary. Arnold further stated that Mesozoic and Tertiary ferns provide excellent research material and: 'With modern techniques well preserved fern compressions may be studied and interpreted with precision approaching that used for living ferns.' The study of fossil Pteridophytes thus holds a promise for the future; such studies should be primarily undertaken by paleobotanists (in cooperation with students of recent ferns) and not by students of modern ferns alone as suggested by Harris (1973).

\section{HABITAT}

The species of the present genus are forest ferns; most of them grow in seasonally dry forest, others in everwet habitats. One species ( $B$. heudelotii) is aquatic and grows also outside the forest belt. They mostly occur at low altitudes; a few species are found above $1500 \mathrm{~m}$.

The greater part of the species prefer rocks, especially near rivers in more or less 
shaded places in the forest. Other species root in the ground. Some otherwise terrestrial species are sometimes also found as low-climbers (or as low-epiphytes). Two American species are apparently high-climbers. The difference between a climbing terrestrial (which stays rooted in the ground) and an epiphyte (which is quite cut off from the ground) (Holttum, 1954) is usually not considered on the labels.

The terrestrial species are not rarely reported to form pure stands of many closely aggregated plants. I observed this myself for B. angustipinna, B. crispatula, and $B$. sinensis, which are locally dominant ground ferns in monsoon forest in northern and northeastern Thailand. Of $B$. heteroclita several forms have been reported which may cover (rocks of) stream-banks completely (Holttum, 1954).

\section{JUVENILE LEAVES}

\section{Introduction}

A considerable part of the herbarium specimens has leaves provided with bulbils which may bear one or a few juvenile leaves. In addition, the material of some species includes small plants showing morphologically different leaves on a single rhizome. In the wild such plants are but rarely met as I experienced myself. From the cultivation of spores it appeared that a juvenile leaf may soon wither after the formation of the subsequent or next subsequent one and presumably this also occurs in the wild. Juvenile leaves of some species of ser. Bolbitianae were described by Devi (1963) and Nayar \& Kaur (1965b).

In the description of the leaves formed on a single rhizome, the oldest (i.e. the earliest produced) leaf present is called leaf $A$, the next oldest leaf $B$, etc. In case the absolute sequence is known, the leaves are indicated as 'first leaf', 'second leaf', etc.

\section{Observations}

Ser. Alienae.

B. auriculata. -Fig. 15: a-e.

Anonymus s.n., 25-viii-1846 (P). Leaf A $5 \mathrm{~cm}$ long. Petiole $20 \mathrm{~mm}$ long. Lamina symmetrical, pinnate, 30 by $20 \mathrm{~mm}$. Pinnae 2, asymmetrical, their basiscopic side broader and near the base lobed nearly to the costa, otherwise less deeply lobed. Terminal segment triangular, the lowest part lobed nearly to the rhachis, towards the apex less deeply cut. Veins free, some endings curved. Leaf $B 6 \mathrm{~cm}$ long, of comparable shape but the pinnae proportionally larger, the segments less deeply incised, the venation pattern with areoles along the primary and secondary veins. Leaf $C 10 \mathrm{~cm}$ long. Petiole $45 \mathrm{~mm}$ long. Lamina 55 by $30 \mathrm{~mm}$, of about the same shape as leaf $B$, but the segments less deeply cut, the veins anastomosing as in the adult stage. Bulbils absent.

Anonymus s.n., 29-viii-1846 (P). Leaves $A$ (7 cm long) and $B(10 \mathrm{~cm}$ long) otherwise 


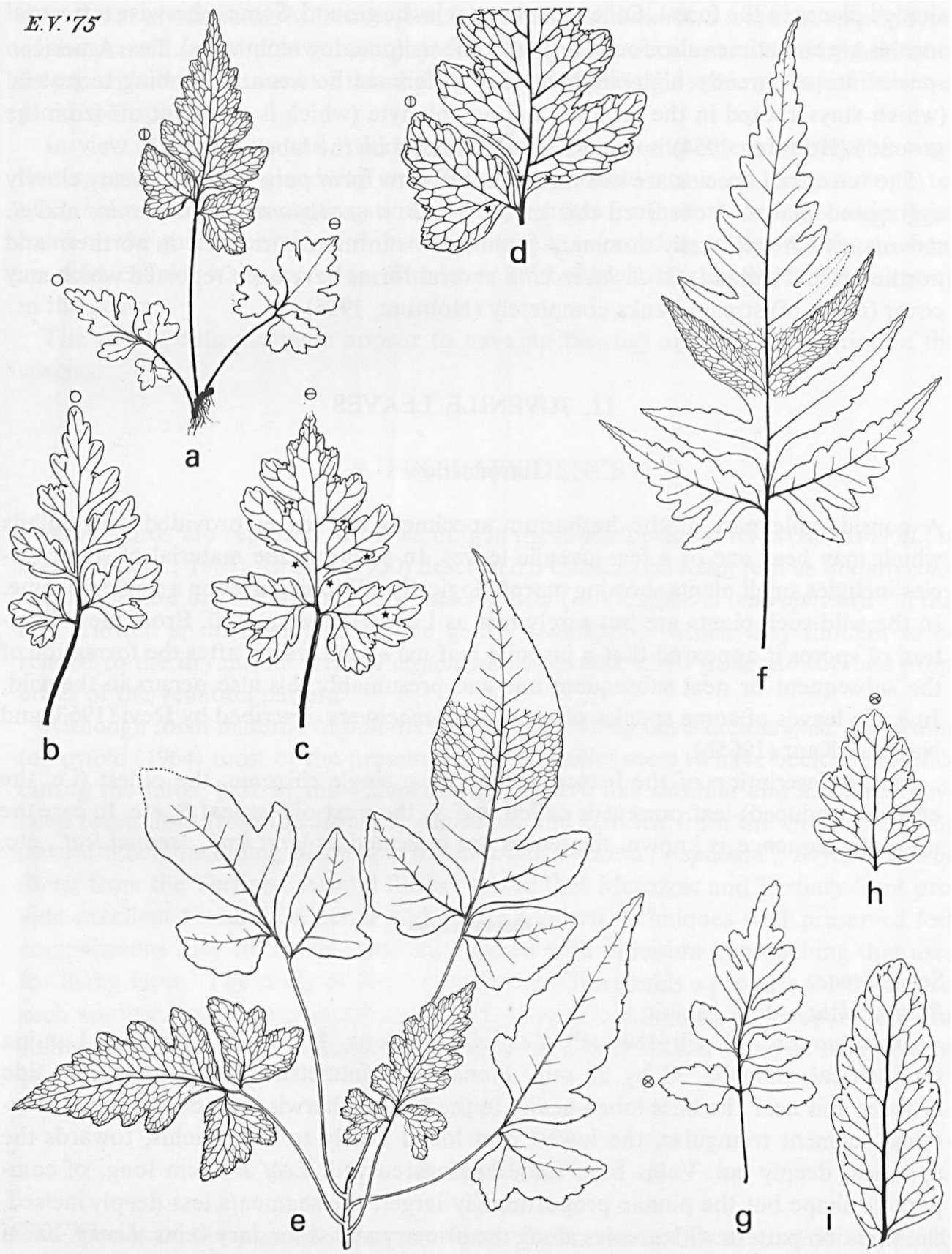

Fig. 15. Juvenile leaves. a-e. Bolbitis auriculata. a. plant with 3 leaves, $\times 1 / 2$; b-d. details of fig. a, $\times 1$; e. plant with 5 leaves, $\times 1 / 2$; asterisks indicate recurrent veins (a-d. Anonymus s.n., 28-viii-1946, P; e. Anonymus s.n., 25-viii-1946, P).-f. Bolbitis aliena, $\times 1 / 2$ (Proctor \& Mullings 21999, U). - g-i. Bolbitis bipinnatifida. g, $\times 1 / 2$; h, i. pinnae, $\times 1$ (g, h. Hennipman 4144, L; i. Brauer 12, B). 


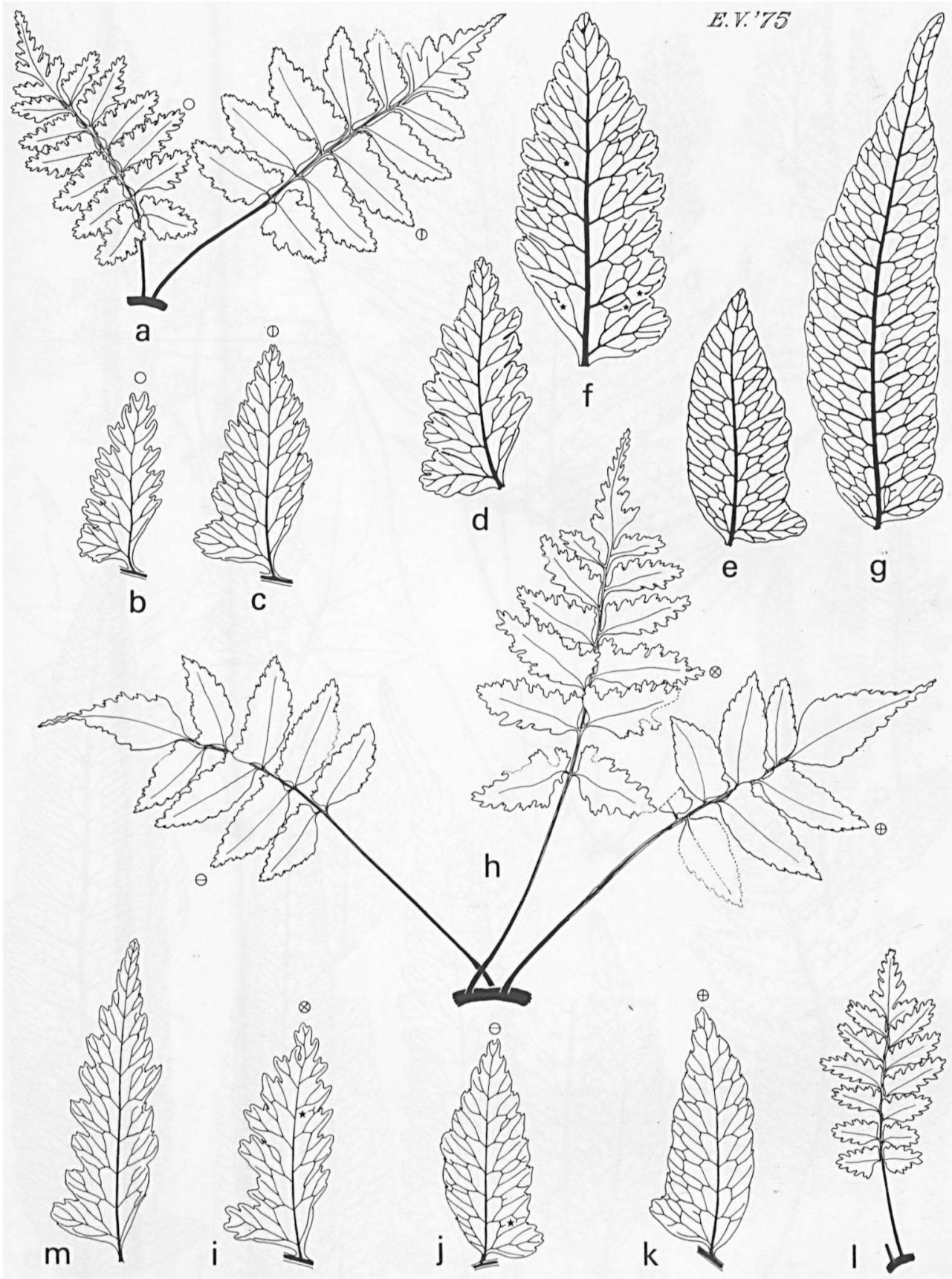

Fig. 16. Juvenile leaves of Bolbitis acrostichoides. a. plant with 2 leaves, $\times 1 / 2 ; \mathrm{b}$, c. pinnae of fig. a, $\times 1$; d. pinna of plant 1 leaf $n, \times 1$; e. ibid. of leaf $n+1, \times 1 ; f, g$. pinnae of different leaves of plant $2, \times 1 ; h$. plant with 3 leaves, $\times 1 / 2$; i-k. pinnae of fig. $h, \times 1 ; 1 . \times 1 / 2$; m. pinna, $\times 1$; asterisks indicate recurrent veins (a-g. Mugnier Sérand s.n., P; h-k. Mugnier Sérand 3, P; 1. Cremers 307, P; m. Schweinfurth 2235, P). 


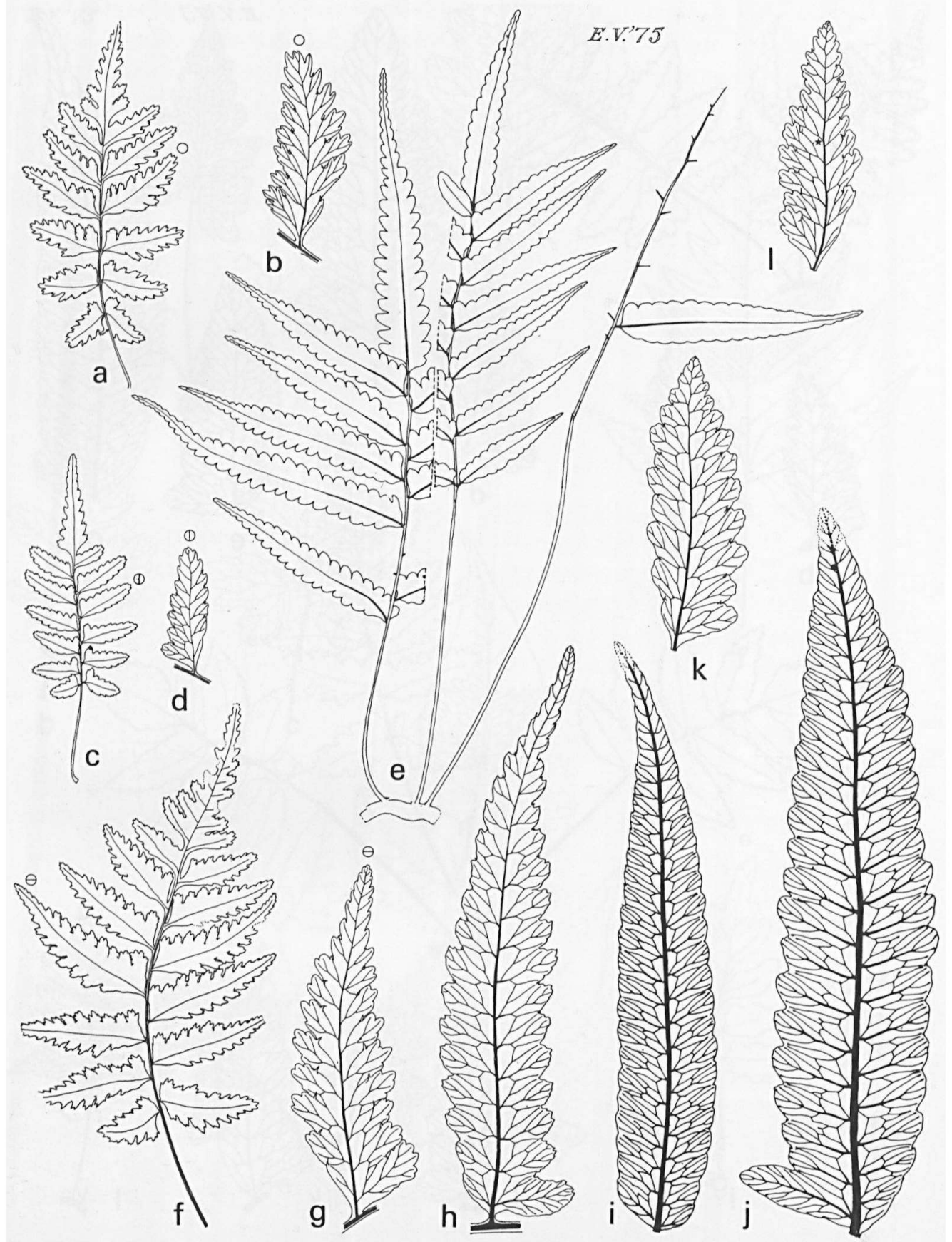

Fig. 17. Juvenile leaves of Bolbitis angustipinna. a. $\times 1 / 2$; b. pinna of fig. a, $\times 1$; c. $\times 1$; d. pinna of fig. c, $\times$ 1 ; e. plant with 3 leaves, $\times 1 / 2$; f. $\times 1 / 2$; g. pinna of fig. $f, \times 1$; h. pinna, $\times 1$; i. pinna of leaf $n+1$ of plant $1, \times 1 ; j$. pinna of leaf $n$ of plant $1, \times 1 ; k$, l. pinnae of different leaves of a single plant, $\times 1$ (a-j. Hennip$\operatorname{man} 3361$, L; k, 1. H. 4146, L). 


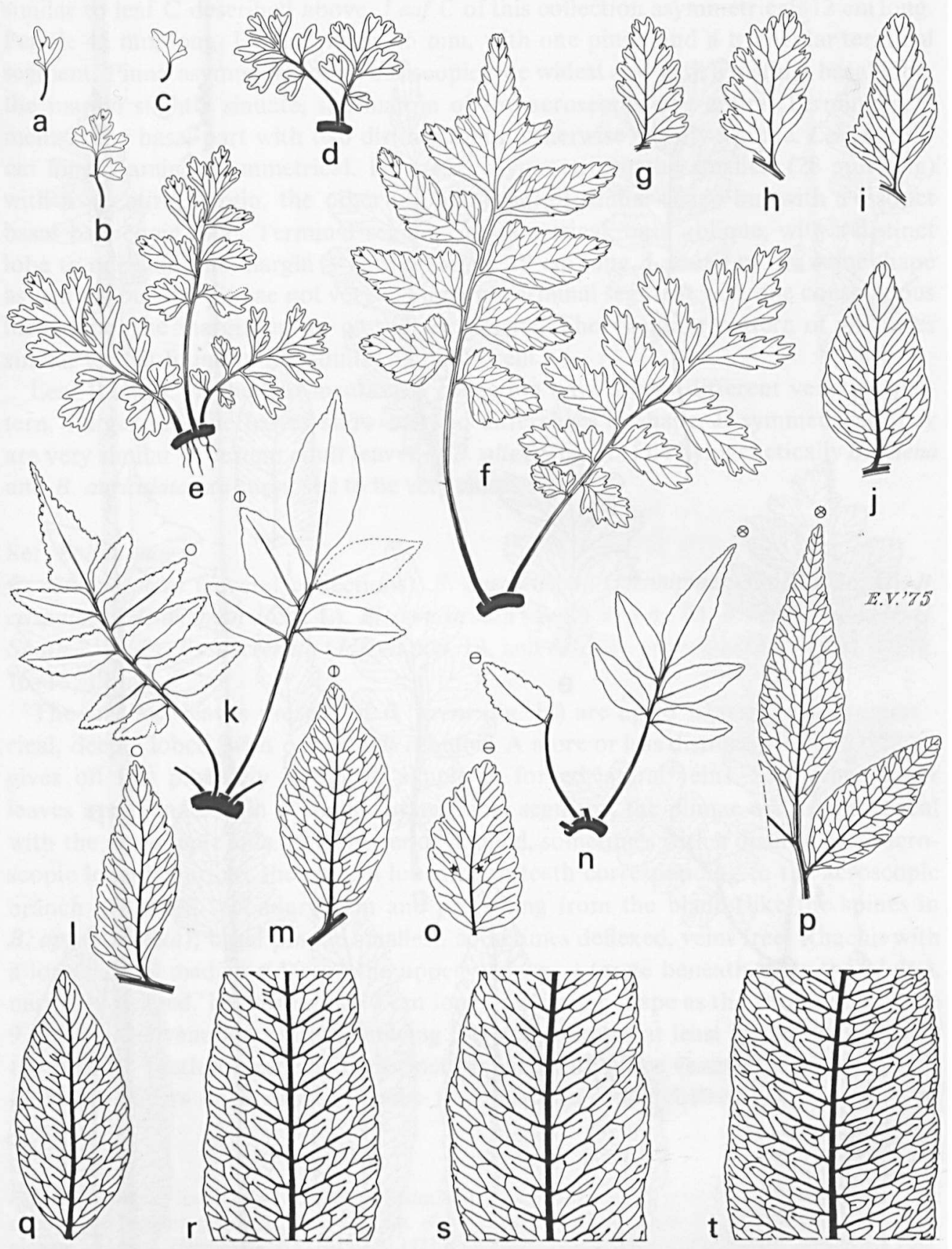

Fig. 18. Juvenile leaves of Bolbitis virens. a. first leaf, $\times 1 ; b-f . \times 1 ; g-j .4$ pinnae of 4 subsequent leaves of a single plant, $\times 1$; $k$. plant with 2 leaves, $\times 1 / 2 ; 1$, m. pinnae of fig. $k, \times 1 ; n$. plant with 2 leaves, $\times 1 / 2$; o, p. pinnae of fig. $n, \times 1$; q-t. pinnae, $\times 1$; asterisks indicate recurrent veins $(a-c, g-j, n-p, r-t$. Hennipman $3159, \mathrm{~L} ; \mathrm{d}-\mathrm{f}, \mathrm{k}-\mathrm{m}, \mathrm{q} . H .3320, \mathrm{~L})$. 


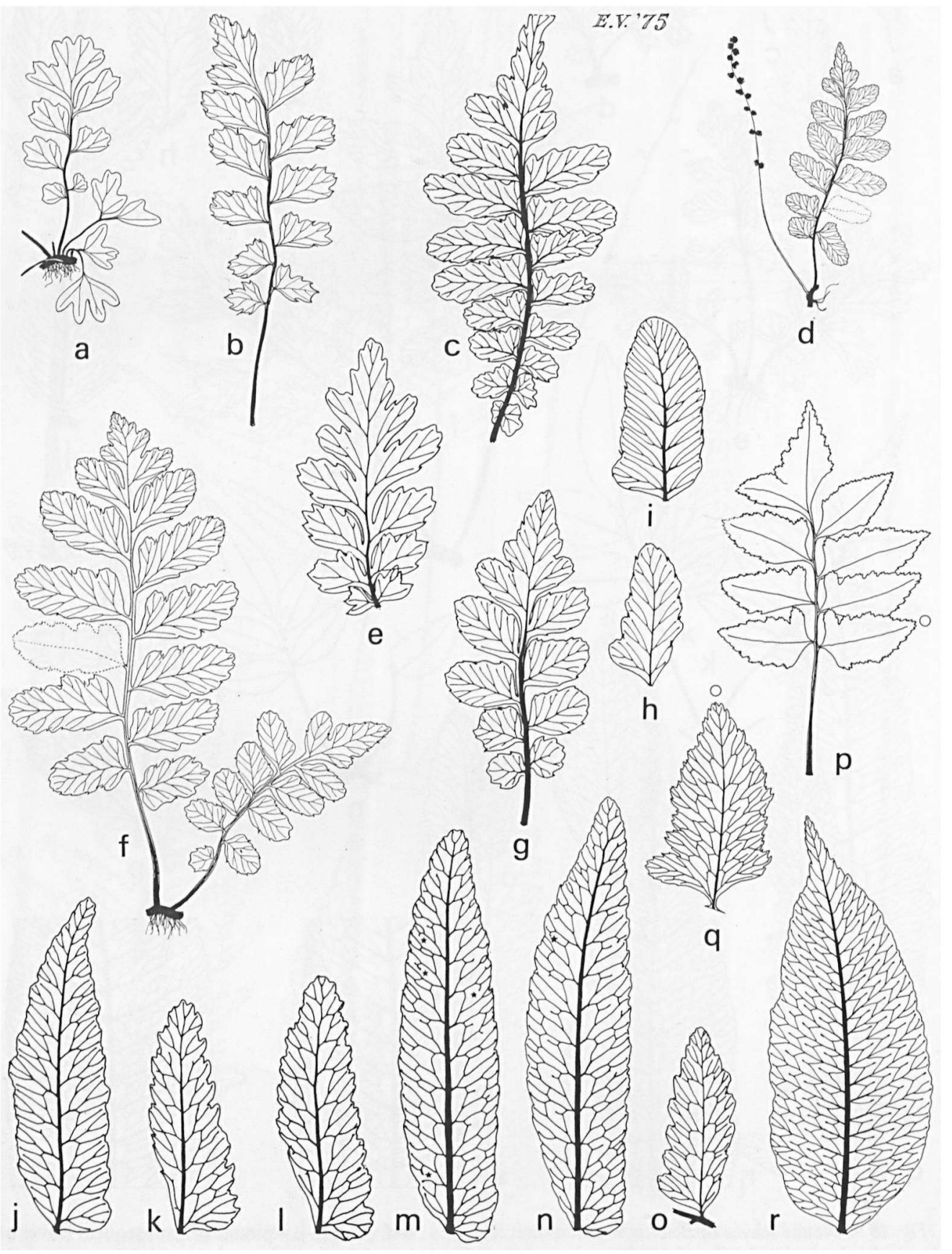


similar to leaf $C$ described above. Leaf $C$ of this collection asymmetrical, $12 \mathrm{~cm}$ long. Petiole $45 \mathrm{~mm}$ long. Lamina 75 by $45 \mathrm{~mm}$, with one pinna and a triangular terminal segment. Pinna asymmetrical, its basiscopic side widest and with a distinct basal lobe, the margin slightly sinuate, the margin of the acroscopic side entire. Terminal segment in the basal part with two distinct lobes, otherwise slightly sinuate. Leaf $D$ c. 13 $\mathrm{cm}$ long. Lamina asymmetrical. Pinnae 2, asymmetrical, the smallest ( $28 \mathrm{~mm}$ long) with a \pm entire margin, the other ( $38 \mathrm{~mm}$ long) of similar shape but with a distinct basal basiscopic lobe. Terminal segment asymmetrical, base oblique, with a distinct lobe to one side, the margin \pm entire. Leaf $E 19 \mathrm{~cm}$ long. Lamina of the same shape as leaf $\mathrm{D}$, but the pinnae not very dissimilar. Terminal segment with one conspicuous basal lobe, the margin entire or slightly sinuate. The venation pattern of all leaves similar to that found in the adults; bulbils absent.

Leaf $B$ and $C$ of the first-mentioned collection have a very different venation pattern. Larger juvenile leaves show marked differences in shape. If symmetrical, they are very similar to certain adult leaves of $B$. aliena (fig. 15:f). Phylogenetically $B$. aliena and $B$. auriculata are supposed to be very close.

\section{Ser. Bolbitianae}

B. acrostichoides (several collections), B. angustipinna (Hennipman 3361, 3536, L), B. crispatula (Hennipman 3632, L), B. semicordata (Perrottet s.n., L), B. serratifolia (H. H. Smith 2522, ?), B. subcrenata (Jarrett 600, L), and B. virens (several collections).-Fig. 16-18; 19: j-r.

The smallest leaves present of $B$. virens (fig. 18) are up to $10 \mathrm{~mm}$ long, asymmetrical, deeply lobed, with or without a bulbil. A more or less distinct, flexuous rhachis gives off few pinnately arranged, simple or forked lateral veins. Somewhat larger leaves are pinnate with a triangular terminal segment; the pinnae are asymmetrical with the acroscopic side much better developed, sometimes with a distinct basal acroscopic lobe or auricle, the margin lobed, with teeth corresponding to the acroscopic branch of forked secondary vein and projecting from the blade (like the spines in B. appendiculata); basal pinnae smallest, sometimes deflexed, veins free. Rhachis with a longitudinal median ridge on the upper surface, \pm terete beneath (as in the adults), narrowly winged. Leaves up to $10 \mathrm{~cm}$ long of a similar shape as the above, with up to 9 pinnae; the venation pattern showing few anastomoses at least in the basal part of the pinnae. Teeth are especially distinct on parts with a free venation. The latter type of leaves occurs on a single rhizome together with \pm equal-sized pinnate leaves of

Fig. 19. (Juvenile) leaves. a-d. Bolbitis appendiculata ssp. appendiculata. a. plant with 4 leaves, $\times 1 ;$ b. $\times 1$; c. $\times 1 / 2$; d. mature plant, $\times 1 / 2$. -e-i. ssp. vivipara. e. $\times 1 ; \mathrm{f}$. plant with 2 leaves, $\times 1 / 2 ; g . \times 1 ; \mathrm{h}, \mathrm{i}$. pinnae, $\times 1$ (a, b. Hennipman 3855, L; c. H. 3527, L; d. Hansen \& Smitinand 12205, C; e-h. Hennipman 3488, L; i. H. 3320, L).-j. Bolbitis crispatula, pinna, $\times 1$ (Hennipman 3632, L).-k-n. Bolbitis semicordata, pinnae, $\times 1$; asterisks indicate recurrent veins (Anonymus, L 908.316-446, L).-0. Bolbitis serratifolia, pinna, $\times 1$ (Smith s.n.,?).-p-r. Bolbitis subcrenata. p. $\times 1 / 2$; q. pinna of fig. p, $\times 1$; r. pinna of the same plant, $\times 1$ (p-r. Hennipman 4142, ex Jarrett 600). 
quite different shape. These different leaves resemble the adults by having a relatively longer petiole, a firmer texture, far less ( 3 or 4$) \pm$ symmetrical pinnae with an entire or crenate margin, a conform terminal segment which is \pm articulated to the rhachis, and a more intricate venation pattern (with thicker veins) having costal areoles with included excurrent free veins.

A single rhizome of a specimen of $B$. virens sometimes also bears a leaf which is morphologically intermediate between these two types. Such leaves show much variation. For instance, they may have asymmetrical pinnae (as found in the preceding leaf) with an entire margin (as found in the next subsequent leaf), or may have symmetrical pinnae (as found in the next subsequent leaf) with a venation pattern as simple as that of the preceding leaf. It is interesting to see that the degree of complexity demonstrated by a certain feature on a certain juvenile leaf, is not strictly correlated with that as displayed by another feature on the same leaf.

Marked differences between subsequent or next-subsequent leaves also occur in single plants of $B$. acrostichoides (fig. 16) and $B$. subcrenata (in which the number of pinnae per leaf is not significantly different in subsequent or next subsequent dissimilar leaves), and $B$. angustipinna (in which this kind of juvenile leaves may be up to $45 \mathrm{~cm}$ long); see fig. 19: p-r and fig. 17, respectively. The adult leaf of the latter species generally bears a large number of pinnae. Separate juvenile leaves of $B$. semicordata and $B$. serratifolia show similar morphological differences. The little material available of $B$. crispatula var. copelandii indicates that two successive leaves may be conspicuously different as regards the shape of the pinnae and the size of the leaves. All young juvenile leaves of the species of this series have many, mostly 3-celled, glandular hairs like those found in young adults. Bulbils are present in all juvenile leaves except the smallest, but not in those of $B$. crispatula.

\section{Ser. Egenolfianae.}

B. appendiculata ssp. appendiculata.-Fig. 19: a-d.

Hennipman $3835(\mathrm{~L})$. Leaf $A$ (= first leaf?) $8 \mathrm{~mm}$ long. Petiole $3 \mathrm{~mm}$ long. Lamina simple, asymmetrical, 5 by $6 \mathrm{~mm}$, with three lobes of unequal size. Veins pinnately arranged. Leaf $B 12 \mathrm{~mm}$ long. Petiole $6 \mathrm{~mm}$ long. Lamina symmetrical, 6 by $9 \mathrm{~mm}$, with 4 c. $3 \mathrm{~mm}$ long lobes. Leaf $C$ incomplete, the terminal part lacking. Petiole $7 \mathrm{~mm}$ long. Lamina with two alternately placed, \pm symmetrical pinnae, the lowermost 7 by $6 \mathrm{~mm}$, distinctly lobed, the other smaller. Leaf $D 20 \mathrm{~mm}$ long. Petiole $5 \mathrm{~mm}$ long. Lamina symmetrical, widest about the middle, 15 by $12 \mathrm{~mm}$, pinnate with a deeply. lobed triangular terminal segment. Pinnae 4, asymmetrical, the two lowest the smallest. Leaf $E 30 \mathrm{~mm}$ long. Petiole $8 \mathrm{~mm}$ long. Lamina 22 by $13 \mathrm{~mm}$, otherwise similar to that of leaf $D$ but with a less deeply cut terminal segment and with small basal pinnae. Larger juvenile leaves of the same architecture, but the lamina with more pinnae, the pinna-margin with teeth, the venation pattern more elaborate. Bulbils absent in the earliest juvenile leaves. The smallest leaf is slightly asymmetrical and entire. The basal pinnae of the smallest pinnate leaf are larger than those present on the later formed juvenile leaves which are very similar to the adults.

The pinnate juvenile leaves have teeth on the pinna-margin which project from the 


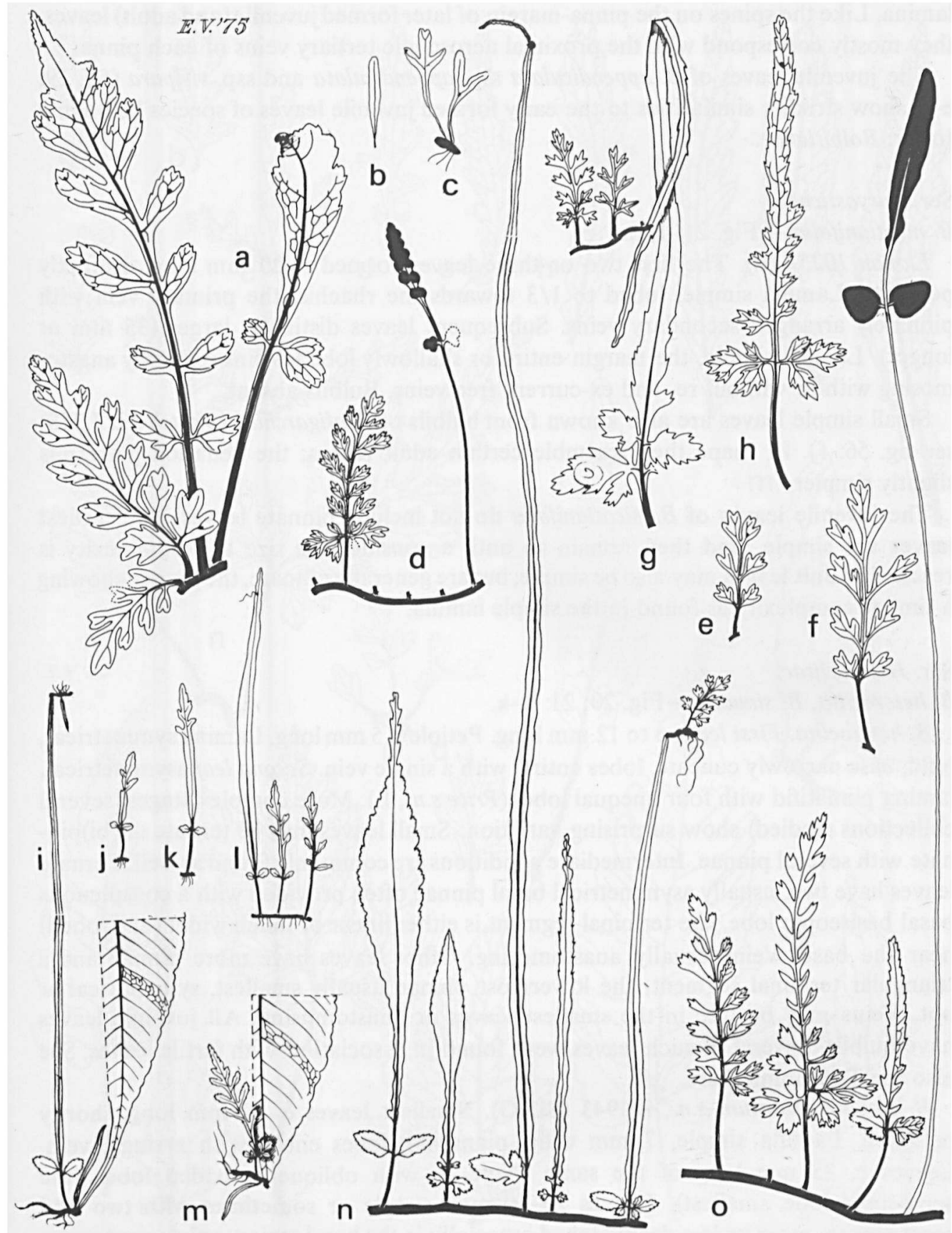

Fig. 20. (Juvenile) leaves of Bolbitis heteroclita.-a. plant with 4 leaves, $\times 1$; b. first leaf, $\times 1$; c. second and third leaf, $\times 1$; d. mature plant, $\times 1$ ('Edanyoa difformis'); e, f. $\times 1$ (ibid.); g, h. $\times 1 ; \mathrm{i}-\mathrm{n} . \times 1 / 2 ; 0$. mature plant, $\times 1$ (aff. 'Edanyoa difformis') (a. Hennipman 4085, culta, L; b, c. Price 1277, culta, L; d. Price 2518A, L; e, t. Hennipman 4i $14 \overline{3}, \overline{\mathrm{L}}$ ( = Price 2518A, culta); g, h. Edaño PNH 21374, MICH; i. LeRoy Topping 718, US; j, 1. LeRoy Topping 444, US; k. ibid., GH; m. Elmer 18068, L; n. Jacobs 7950, L; o. Price 2518B, L, loc. class. of 'Edanyoa difformis'). 
lamina. Like the spines on the pinna-margin of later formed juvenile (and adult) leaves, they mostly correspond with the proximal acroscopic tertiary veins of each pinna.

The juvenile leaves of $B$. appendiculata ssp. appendiculata and ssp. vivipara (fig. 19: e-i) show striking similarities to the early formed juvenile leaves of species belonging to ser. Bolbitianae.

\section{Ser. Euryostichae.}

B. nicotianifolia.-Fig. 21 : $1, \mathrm{~m}$.

Ekman 10257 (S). The first two or three leaves formed c. $20 \mathrm{~mm}$ long, distinctly petiolate. Lamina simple, lobed to $1 / 3$ towards the rhachis, the primary vein with pinnately arranged secondary veins. Subsequent leaves distinctly larger $(35 \mathrm{~mm}$ or longer). Lamina simple, the margin entire or shallowly lobed. Veins regularly anastomosing with or without re- and ex-current free veins. Bulbils absent.

Small simple leaves are also known from bulbils of B. oligarchica (Skutch 2927, US; see fig. 56: f). In shape they resemble certain adult leaves; the venation pattern is slightly simpler.

The juvenile leaves of $B$. nicotianifolia do not include pinnate leaves. The earliest leaves are simple, and they remain so until a considerable size and complexity is reached. Adult leaves may also be simple, but are generally pinnate, the pinnae showing a similar complexity as found in the simple lamina.

\section{Ser. Heteroclitae.}

B. heteroclita, B. sinuata.-Fig. 20; 21: a-k.

$B$. heteroclita. First leaf up to $12 \mathrm{~mm}$ long. Petiole $c .5 \mathrm{~mm}$ long. Lamina symmetrical, bifid, base narrowly cuneate, lobes entire, with a single vein. Second leaf asymmetrical, lamina pinnatifid with four unequal lobes (Price s.n., L). More complex stages (several collections studied) show surprising variation. Small leaves may be ternate or (bi)pinnate with several pinnae. Intermediate conditions are commonly found as well. Ternate leaves have two, usually asymmetrical basal pinnae often provided with a conspicuous basal basiscopic lobe, the terminal segment is either linear or much wider (and lobed) near the base. Veins usually anastomosing. Other leaves have more pinnae and a triangular terminal segment, the lowermost pinnae usually smallest, symmetrical or not. Veins may be free in the smallest leaves or anastomosing. All juvenile leaves have bulbils. Several of such leaves were found in association with fertile leaves. See also the Taxonomic Part.

B. sinuata. Holttum s.n., ii-1943 (SING). Smallest leaves $c$. $20 \mathrm{~mm}$ long, shortly petiolate. Lamina simple, $7 \mathrm{~mm}$ wide, pinnatifid, lobes entire with a single vein. Leaves $c .25 \mathrm{~mm}$ long of the same shape or with obliquely divided lobes (the acroscopic lobe smallest). Leaves $25-50 \mathrm{~mm}$, simple or sometimes with two odd basal pinnae, more or less deeply lobed especially in the basal region; veins anastomosing. Larger leaves agreeing with the adult simple leaf though with a less complex venation pattern. All leaves are \pm coriaceous short-petiolate or sessile. The large juvenile leaves have bulbil initials. The smallest leaf of $B$. sinuata agrees in shape with 


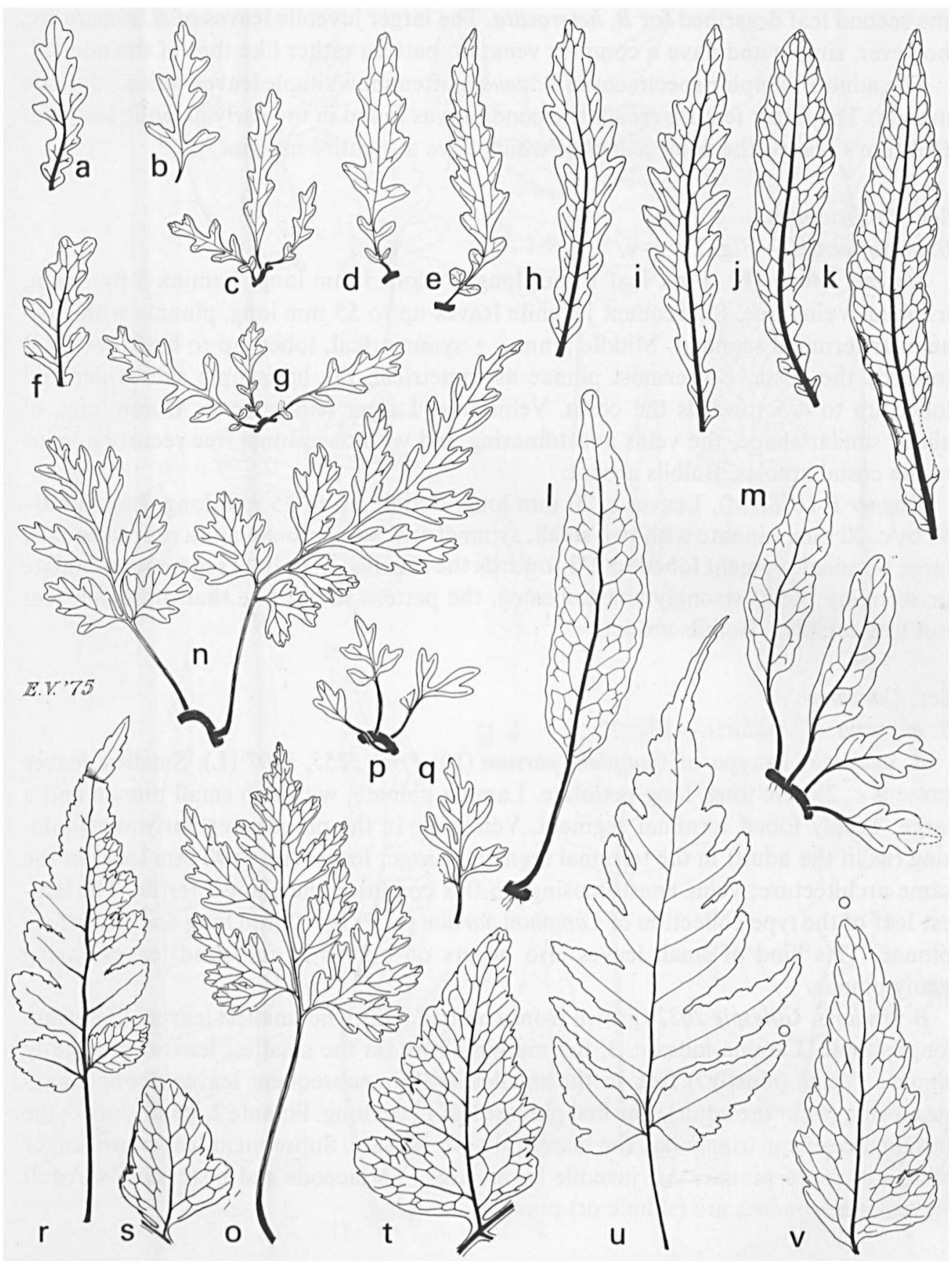

Fig. 21. Juvenile leaves. a-k. Bolbitis sinuata, $\times 1$ (Holttum s.n., SING 20804).-1, m. Bolbitis nicotianifolia, $\times 1($ Ekman 10257b, S).-n-v. Bolbitis portoricensis. n-r. $\times 1$; s. basal pinna of a leaf subsequent to the leaf of fig. r, $\times 1$; t. $\times 1$; u. $\times 1 / 2$; v. basal pinna of fig. u, $\times 1$ (n-q. Sintenis 5464b, B; r, s. M. Wagner s.n., M; t-v. Foumier s.n., 1885, P). 
the second leaf described for $B$. heteroclita. The larger juvenile leaves of $B$. sinuat $a$ are, however, simple and have a complex venation pattern rather like that of the adult.

The adult Philippine specimens of $B$. sinuata often show simple leaves with a \pm sinuate margin. The latter feature recalls the condition as found in the early juvenile leaves of Holttum's plants, the adult leaves of which have a \pm entire margin.

\section{Ser. Portoricenses.}

B. portoricensis.-Fig. 21: $\mathrm{n}-\mathrm{v}$.

Sintenis $5464 b$ (B). First leaf $8 \mathrm{~mm}$ long. Petiole $3 \mathrm{~mm}$ long. Lamina 5 by $3 \mathrm{~mm}$, trilobed. Veins free. Subsequent juvenile leaves up to $55 \mathrm{~mm}$ long, pinnate with a triangular terminal segment. Middle pinnae \pm symmetrical, lobed up to halfway $(-2 / 3)$ towards the costa. Lowermost pinnae asymmetrical, the basiscopic side wider and lobed up to $4 / 5$ towards the costa. Veins free. Larger leaves up to $70 \mathrm{~mm}$ long, of about similar shape, the veins anastomosing and with occasional free recurrent veins in the costal areoles. Bulbils absent.

Wagner $i-1858$ (M). Leaves c. $70 \mathrm{~mm}$ long. Petiole up to $25 \mathrm{~mm}$ long. Lamina 50 60 by c. $20 \mathrm{~mm}$, pinnate with two small, symmetrical basal pinnae and a proportionally large terminal segment lobed to $2 / 3$ towards the rhachis; margin of segments crenulate or serrulate. Veins strongly anastomosing, the pattern rather like that of adult leaves but less complex. Bulbils absent.

\section{Ser. Quoyanae.}

B. quoyana, B. rivularis.-Fig. 22.

B. quoyana; isotype of Campium parvum (L); Price 2255, 3007 (L). Smallest leaves present $c .25 \mathrm{~mm}$ long, long-petiolate. Lamina pinnate, with two small pinnae and a large, deeply lobed terminal segment. Veins free in the pinnae, regularly anastomosing (as in the adult) in the terminal segment. Larger leaves (up to $90 \mathrm{~mm}$ long) of the same architecture; veins anastomosing. In this condition the plant is fertile. The largest leaf of the type collection of Campium parvum (at P) is $150 \mathrm{~mm}$ long and bears four pinnae. This kind of small leaves also occurs on bulbils of full-sized leaves having many pinnae.

B. rivularis. Gillespie 2622 (grown from a bulbil ?, US). The smallest leaves(12-70 mm long) simple. Lamina index $c .3$, the margin entire (in the smallest leaves) or slightly sinuate. Veins (mostly?) free in the smallest leaf, in subsequent leaves strongly anastomosing (as in the adult). The first pinnate leaf $12 \mathrm{~cm}$ long. Pinnate 2, small, entire; the terminal segment triangular, the margin slightly sinuate. Subsequent leaves are longer and have more pinnae. All juvenile leaves are \pm coriaceous and bear bulbils. Adult leaves of $B$. rivularis are (simple or) pinnate.

\section{Species incertae sedis.}

B. repanda.-Fig. 23: a-f.

Hennipman 4139 (L), a tetraploid specimen in cultivation. First leaf up to $15 \mathrm{~mm}$ long. Petiole 5-7 mm long. Lamina simple, as long as wide, the base broadly acute, otherwise 


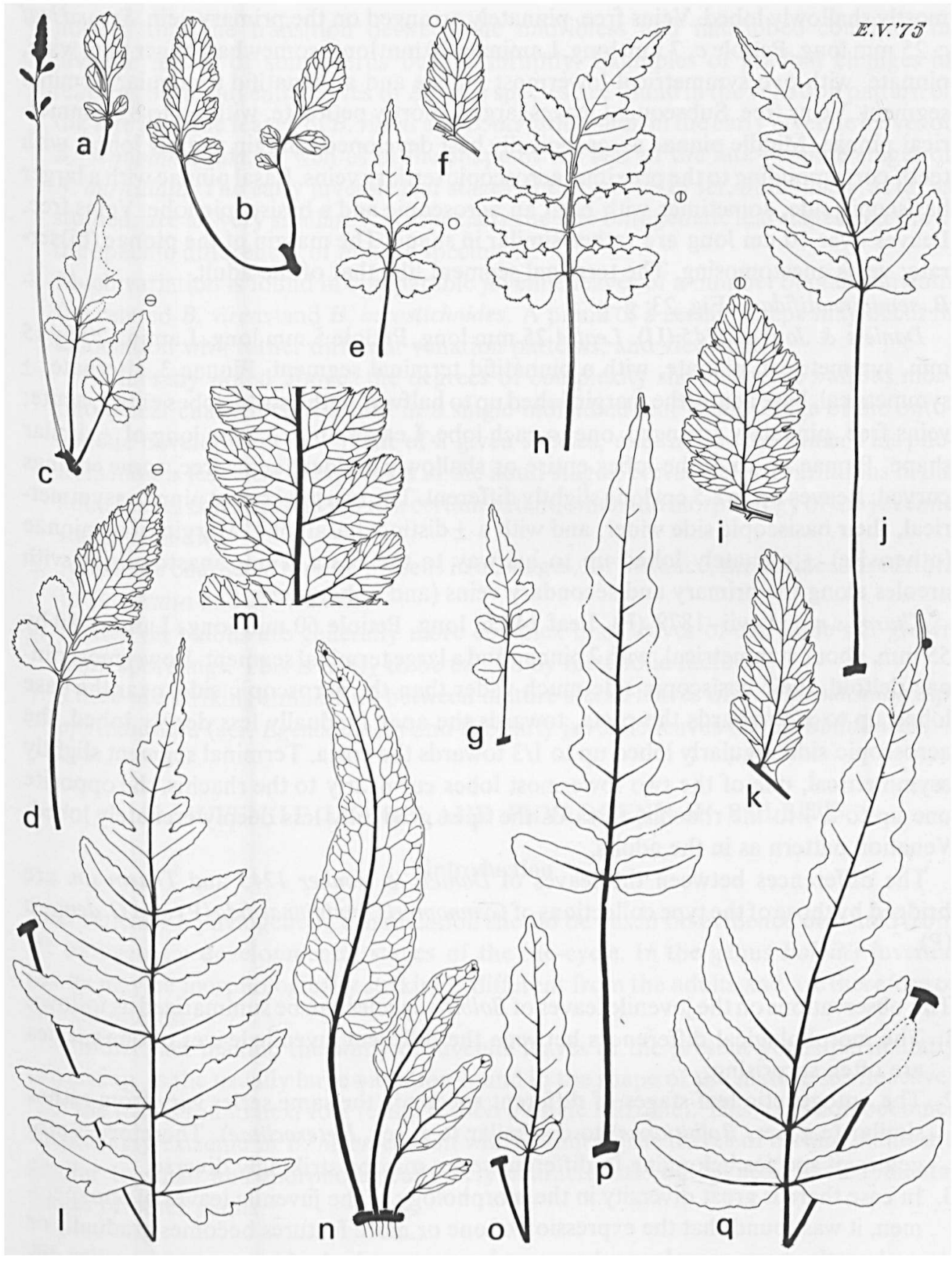

Fig. 22. (Juvenile) leaves. a-m. Bolbitis quoyana. a. $\times 1$; b. plant with 2 leaves, $\times 1$; c. mature plant, $\times 1 / 2$ ('Campium panum'); d. sterile leaf of fig. c, $\times 1$; e. $\times 1 / 2$; f. pinna of fig. e, $\times 1 ; g, h . \times 1 / 2$; i. pinna of fig. $h, \times 1 ; j . \times 1 / 2 ; k$. pinna of fig. j, $\times 1 ; 1 . \times 1 / 2 ; \mathrm{m}$. venation pattern of central part of a central pinna, $\times 1$ (a-d. Schlechter 16163, L, isotype of Campium parrum; e, f, h, i. Price 3007, L; g, j-m. Price 2255, L).$\mathrm{n}-\mathrm{q}$. Bolbitis rivularis. n. plant, $\times 1$; 0-q. $\times 1 / 2$ (Gillespie 2622, UC). 
mostly shallowly lobed. Veins free, pinnately arranged on the primary vein. Second leaf c. $25 \mathrm{~mm}$ long. Petiole c. $7 \mathrm{~mm}$ long. Lamina c. $20 \mathrm{~mm}$ long, somewhat longer than wide, pinnate, with two symmetrical lowermost pinnae and a pinnatifid triangular terminal segment. Veins free. Subsequent leaves larger, shortly petiolate, with up to 8 asymmetrical pinnae. Middle pinnae acroscopically best developed, margin acutely lobed, with teeth corresponding to the proximal acroscopic tertiary veins. Basal pinnae with a larger basiscopic side, sometimes with both an acroscopic and a basiscopic lobe. Veins free. Leaves over $10 \mathrm{~cm}$ long are rather similar in shape. The margin of the pinnae (bi)serrate; veins anastomosing. The terminal segment like that of the adult.

B. semipinnatifida.-Fig. 23: g-p.

Daniëls \& Jonker 1245 (U). Leaf A $25 \mathrm{~mm}$ long. Petiole $5 \mathrm{~mm}$ long. Lamina 20 by 15 $\mathrm{mm}$, symmetrical, pinnate, with a pinnatifid terminal segment. Pinnae 3, alternate, \pm symmetrical, base acute, the margin lobed up to halfway to the costa, lobes entire, acute; veins free, pinnately arranged, one to each lobe. Leaves up to $3.5 \mathrm{~cm}$ long of \pm similar shape. Pinnae up to 4 , the lobes entire or shallowly divided; veins free, some endings curved. Leaves up to $8.5 \mathrm{~cm}$ long slightly different. The two lowermost pinnae asymmetrical, their basiscopic side wider, and with a \pm distinct basal lobe. Margin of all pinnae (otherwise) \pm crenately lobed up to halfway to the costa. Veins anastomosing with areoles along the primary and secondary veins (and in between).

Thurm s.n., 31-vii-1879 (P). Leaf $14 \mathrm{~cm}$ long. Petiole $60 \mathrm{~mm}$ long. Lamina 80 by $55 \mathrm{~mm}$, about symmetrical, with 2 pinnae and a large terminal segment. Lowermost pinnae deltoid, their basiscopic side much wider than the acroscopic side, near the base lobed up to $3 / 4$ towards the costa, towards the apex gradually less deeply lobed, the acroscopic side regularly lobed up to $1 / 3$ towards the costa. Terminal segment slightly asymmetrical, one of the two lowermost lobes cut nearly to the rhachis, the opposite one up to $3 / 4$ to the rhachis, towards the apex gradually less deeply crenately lobed. Venation pattern as in the adults.

The differences between the leaves of Daniëls \& Jonker 1245 and Thurm s.n. are bridged by those of the type collections of Gymnopteris semipinnatifida $(\mathrm{P})$ and G. dentata (P).

The observations on the juvenile leaves of Bolbitis species can be summarized as follows:

1. The morphological differences between the different juvenile leaves of one species are often surprising.

2. The ontogenetic leaf stages of different species in the same series vary from rather similar (e.g. ser. Bolbitianae) to dissimilar (e.g. ser. Heteroclitae). Therefore, ontogenies of species belonging to different series may be strikingly diverse.

3. In case there is great diversity in the morphology of the juvenile leaves of one specimen, it was found that the expression of one or more features becomes gradually or \pm abruptly more complex; subsequent leaves on a single rhizome may be quite different in various respects. Abrupt changes may be observed e.g. in the shape of the terminal segment and the pinnae of juvenile leaves of $B$. virens and $B$. acrostichoides, and in the venation pattern of early juvenile leaves of $B$. auriculata. Wagner(1952) 
noticed that the transition between the midribless and midribbed condition in juvenile leaves of some ferns occurs abruptly. Examples of gradual changes in features of the juvenile leaves of Bolbitis species are found in the venation pattern of the late juvenile leaves of $B$. virens and $B$. acrostichoides, in the early juvenile leaves of $B$. semipinnatifida, as well as in the shape of the leaf of the late juvenile stages of $B$. auriculata. The early juvenile leaf stages of the species of ser. Bolbitianae, as far as studied, are all very similar. It is especially the late ontogenetic leaf stages that show the specific differences of mature specimens.

4. Much variation is found in comparable juvenile leaves of a number of plants in both the related $B$. virens and $B$. acrostichoides. A pinna of a certain shape may occur in association with rather different venation patterns, and vice versa.

As already noted above, the degrees of complexity shown by the various morphological characters as found in a single individual leaf at any stage of the ontogenetic development in a plant of a given species, may not be the same. This phenomenon is less clearly expressed in the adult stages. Nevertheless, variations in the adult leaves can be predicted to a certain extent, when the morphology of the juvenile stages is known.

5. A specific character may be present in all stages, for instance, the coriaceous texture in $B$. sinuata and $B$. rivularis.

6. Leaves on bulbils are generally more complex than leaves of the same size grown from sporelings. This is likely to be caused by metabolic factors.

7. There are striking similarities between mature sterile leaves of $B$. appendiculata ssp. appendiculata (ser. Egenolfianae) and the early juvenile leaves of ser. Bolbitianae.

\section{JUVENILE LEAVES AND PHYLOGENY IN BOLBITIS}

\section{Introduction}

Theoretically a phylogenetic classification should be based on evidence obtained from all ontogenetic developmental stages of the life-cycle. In the genus Bolbitis juvenile plants may be morphologically strikingly different from the adults and are therefore of special interest.

Another fact making the study of juvenile leaves of the present genus particularly promising, is the usually large variation found in the shape of the mature sterile leaves (i.e. the leaves found next to a fertile leaf on a single rhizome). This variation becomes surprisingly extreme in $B$. heteroclita in which adult sterile leaves of certain plants are similar to small and morphologically very characteristic ontogenetic developmental stages of other plants.

The fact that subsequent stages of a certain life-cycle may be morphologically strikingly different has fascinated especially zoologists. It inspired Haeckel (1866) to state that ontogeny is the recapitulation of phylogeny. Thus phylogeny is the mechanical cause of ontogeny. Haeckel's law has received great attention. However, several authors have 


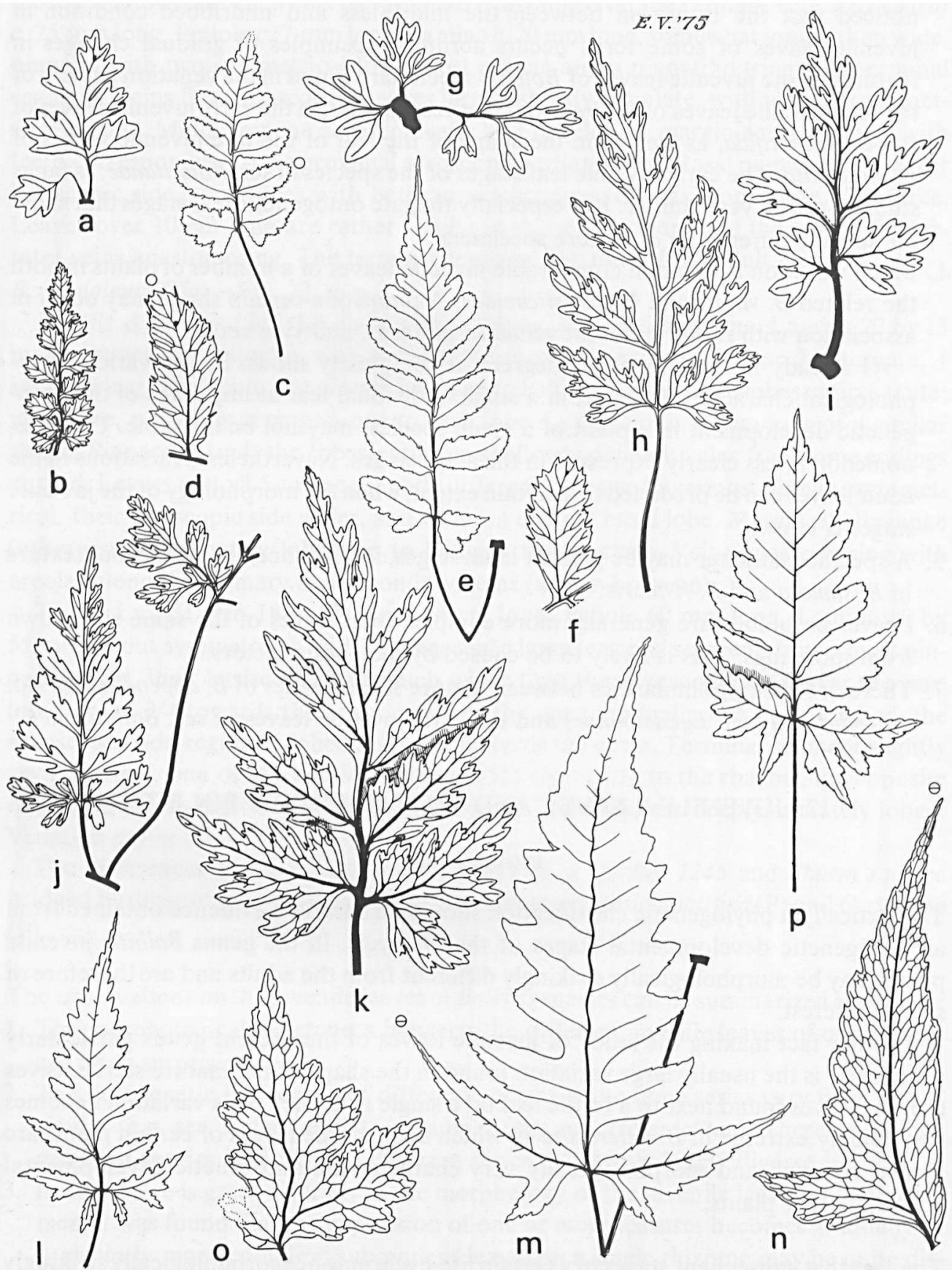

Fig. 23. Juvenile leaves. a-f. Bolbitis repanda. a. first and second leaf, $\times 1$; b, c. $\times 1 / 2$; d. pinna of fig. c, $\times$ 1 ; e. $\times 1 / 2$; f. pinna of fig. e, $\times 1$ (Hennipman 4139, tetraploid, drawn from cultivated material ex Holttum, Java). - g-p. Bolbitis semipinnatifida. g. plant with 3 leaves, $\times 1 ; \mathrm{h}, \mathrm{i}, \mathrm{k} . \times 1$; j. plant with 2 leaves, $\times 1$; m. $\times 1 / 2$; n. basal pinna of fig. m, $\times 1$; o. basal pinna, $\times 1$; p. $\times 1 / 2(\mathrm{~g}-\mathrm{i}, \mathrm{k}$. Daniëls \& Jonker 1245, U; j, 1-n. Le Prieur s.n., P, type of Gymnopteris semipinnatifida; o. ibid., P, type of Gymnopteris dentata; p. Thurm s.n., P). 
- often independently - objected to this theory by stating just the reverse. They include the zoologists Boas (1881, cited by Diels, 1906), Garstang (1922), Koltzoff (1936, cited by Takhtajan, 1959), Hardy (1954), and De Beer (1951), and the botanists Diels (1906) and Takhtajan (1959).

Diels's interesting book on precocious fructification was written following a trip to Australia. He adhered to the view that fructification is the result of quantitative changes in the physiology and not connected with a certain developmental stage. "Aus allem geht mit Bestimmtheit hervor, dass in den tropischen und subtropischen Ländern bei vielen Arten das Verhältnis zwischen vegetativer Entfaltung und generativer Reife unbeständig ist, d.h. dass irgend welche Abhängigkeit des Blühens von einer bestimmten Phase des vegetativen Wachtstums nicht besteht' (p. 19). He accepted Boas's idea of neoteny to indicate the situation by which 'das Tier auf einer Entwicklungsstufe geschlechtsreif wird, auf welcher seine übrigen Organe-alle oder wenige-nicht die volle Ausbildung erreicht haben und dann überhaupt diese Ausbildung nicht erreichen' (p. 115), and pointed out that certain retentive ontogenetic stages, when fertile, might become the roots ('Phylembryonen', p. 110) of a new phylogeny. Garstang said it more concisely: 'Ontogeny does not recapitulate Phylogeny: it creates it.' He and De Beer stressed the fact that the real phylogeny (of Metazoa) has never been a direct succession of the adult forms, but a succession of ontogenies or life-cycles. Thus, new characters may appear at all stages of ontogeny.

Zimmermann (e.g., 1959) paid considerable attention to the significance of the ontogeny of a plant for the understanding of its phylogeny. He used the term 'Hologenese' to indicate the history of a plant in terms of its past life-cycles. As regards Haeckel's law, he is of the opinion that the juvenile stages of a plant often recapitulate the ancestor. In his first edition of 'Die Phylogenie der Pflanzen' (1930) he roughly estimated that Haeckel's law would be true for $80 \%$. Neoteny is mentioned in the second edition of his book (1959) as an (obviously rare) case of heterochrony. Neoteny as circumscribed by Diels affords a means by which an organism is able 'to escape from specialization' (Hardy) or to 'despecialize' (Takhtajan). According to Takhtajan (who took the idea of neoteny from Koltzoff) it is very probable that the neotenic forms with their simpler phaenotype but with their complex genotype, retain evolutionary plasticity, as they have lost the burden of specialization.

Little is known about the significance of neoteny (s.1.) for the phylogeny of the Pteridophytes. Diels gave examples of how neoteny might have played a role in the creation of some ferns, including Phylloglossum and some Bolbitis (Gymnopteris) species. Unfortunately, the plants that Diels thought to represent different neotenic forms of a single Bolbitis species, belong to different species. Williams (1927) pointed out that the stele of Antrophyum cannot be properly explained by reduction; he convincingly demonstrated that it can be regarded much better as an arrested development, the arrest having come into operation at an early stage. Van Steenis (1948) communicated Dr. Donk's view on the identity of Holttumiella Copeland, which was originally assigned to the lindsaeoid ferns. Donk regarded Holttumiella simply as a neotenic Taenitis to which it was formally reduced by Holttum (1968). Kramer (1957: 128) noticed that Haeckel's law cannot be well applied to the different leaf shapes occurring in certain Lindsaea species: 'simply 
pinnate leaves appear in the development of a single plant before the bipinnate ones, but in a phylogenetic sense they are very probably more derived.' Devi(1963) and Nayar \& Kaur (1965b) reported an indument exclusively confined to the juvenile leaves of ser. Bolbitianae. Devi discussed the possible phylogenetic significance of this finding accepting Haeckel's ideas. In the stimulating book 'The Phylogeny and Classification of the Ferns' (Jermy et al., eds., 1973), based on papers read at the International Symposium on Ferns at London, neoteny is only mentioned by De la Sota as a 'rare phenomenon' (p. 231) to explain the foliar dichotomy of Dipteris; Wagner (ibid., p. 249) still listed recapitulation as one of the criteria for the determination of primitive states.

\section{General observations and results}

How genetic changes may affect the ontogenetic developmental stages of the predecessor is theoretically figured in fig. 24. In this model the ontogenetic developmental stages of adult sporophytic generations are indicated by horizontal lines. The most primitive fern illustrated is the one whose adult stage is the sum total of $n$ different ontogenetic stages (i.e. the whole of its ontogeny). Mutations in this fern may result in a progeny with a slightly different ontogenesis. The latter's ontogeny may show, if compared with that of its parent, a loss or an increase of development(al stages). The adult progenitors are of course capable of radiating in all directions. The scheme illustrates schematically only some of the numerous theoretical possibilities. The real ontogenies of related ferns are of course much more complicated. The scheme just aims to provide one possible mechanism to understand the complex diversity found in the genus.

Although the term neoteny can be restricted to the phenomenon of a sexual maturity in a juvenile phase (in the axolotl, the classical example, neoteny is even facultative (!): De Beer, 1951), it is often also used in a broader sense (Diels, 1906; De Beer, 1951; Takhtajan, 1959) in which it covers the retention of every juvenile character by the adult. The difference between neoteny as thus defined and De Beer's gerontomorphosis (i.e. the phylogenetic change as a result of modifications affecting 'characters already adult') is a gradual one in view of the scheme presented above.

The probable significance of neoteny for the past diversification in the genus Bolbitis as seen from the juvenile leaves:

Ser. Alienae.

$B$. auriculata and $B$. aliena.

The similarities between the late juvenile leaves of $B$. auriculata and mature leaves of $B$. aliena can be interpreted differently. Gerontomorphosis in a fern like $B$. aliena may have resulted in a fern like $B$. auriculata with a more complex leaf shape. Theoretically, however, the reverse may be true in case of a genetic change in a fern like $B$. auriculata causing the retention of a juvenile stage with a symmetrical leaf but with already a reticulate venation. 
Ser. Bolbitianae and Egenolfianae.

The species of series Bolbitianae show marked differences between subsequent or next-subsequent juvenile leaves of a certain stage of development of the juvenile plants. Possibly part of the ontogenesis of the ancestor has disappeared. The diversity of the juvenile leaves shows great resemblances in the different species. The differences in the ontogeny of the species investigated become manifest in the more complex juvenile leaves. Especially, with particular reference to the shape and the number of the pinnae, and the venation pattern. The first leaves grown from bulbils resemble late juvenile leaves of sporelings.

The early formed juvenile leaves are morphologically very similar to adult leaves of B. appendiculata ssp. appendiculata. However, they lack spines on the margin of the pinnae. This difference does not seem to be important, as the teeth present in the juvenile leaves can be regarded as homologous with the teeth as found in the juvenile leaves of B. appendiculata ssp. appendiculata.

The surprising similarities between the leaves of $B$. appendiculata ssp. appendiculata and the juvenile leaves found in some species of ser. Bolbitianae can be explained by assuming that the shape of the leaves of the former taxon resulted from retention of juvenile characters as found in ser. Bolbitianae. It is noteworthy that presumably ser. Bolbitianae and Egenolfianae are genetically closely related in view of the occurrence of hybrids between these series. The simple type of (juvenile) leaf shape found in B. appendiculata ssp. appendiculata may well have been formed by retention. The shape of the spores and the complex anatomy found in the base of the pinnae are in favour of the supposition that ssp. appendiculata has a derived genome in spite of its simple leaf shape.

From the study of the ample material of juvenile and mature specimens of ser. Bolbitlanae it seems justified to present the following hypothesis regarding the past speciation in this series. For this purpose some characteristics of the series are recapitulated.

As far as known from the study of mature specimens, ser. Bolbitianae is homogeneous. For instance, all its members have a characteristic type of spores. Cytologically they appear uniform. All species are (largely) confined to seasonally dry forests. The pantropical distribution of the series is the widest in the genus.

Leaving two critical species out of further consideration (i.e. B. salicina which is African, and $B$. serrata which is American), this series consists of eleven species. The one American representative is very similar to the one African species. The other nine species are confined to Asia (from S. India and Ceylon eastwards to Formosa, the Philippines, and Celebes; see fig. 28). The species with a venation pattern best matching that as found in the American and African species is B. angustipinna, the most widespread species in Asia. The venation pattern of $B$. scalpturata, a somewhat critical species, is usually only slightly different. A markedly deviating venation is found in the other Asian representatives. In the small-sized $B$. semicordata (endemic to S. India) and $B$. crispatula (with two local varieties) it is much simpler, in $B$. virens, $B$. subscrenata, and especially $B$. costata it is much more complicated than that of $B$. angustipinna. 
The venation patterns of $B$. semicordata and $B$. crispatula should be regarded to represent a primitive condition when following Haeckel, as this kind of patterns precedes the more complex types as found in the adults of other species. The other species with a more complex network should then be derived. This supposition, however, seems unlikely as it would mean that a fern like B. semicordata should have spread as far as Africa and America giving rise to \pm similar plants much different from their ancestor. Further, it so happens that the spores of $B$. semicordata (and B. costata) are slightly but conspicuously different from those of all other species of the group, which have very uniform spores.

Another possible explanation presented here starts with the assumption that a species with a venation pattern as found in $B$. acrostichoides and $B$. serratifolia once also inhabited (parts of) Asia. This seems realistic as some of the species, including the widespread $B$. angustipinna, have a venation agreeing with that of these species. The Ceylonese plants of $B$. angustipinna in particular show a pattern reminiscent of the patterns as found in specimens of $B$. acrostichoides from eastern Africa. A hypothetical scheme of the evolution of the venation patterns in this series is given in fig. 24 (B. scalpturata is not included). $B$. semicordata has small asymmetrical pinnae and a narrowly winged rhachis, features also found in the early juvenile leaves. The venation shows included excurrent free veins resembling certain late juvenile stages as found in $B$. virens. The venation of $B$. semicordata is likely to be the result of the retention of such a pattern in species like $B$. angustipinn $a, B$. virens, $B$. subcrenata, or $B$. costata. If so, the (somewhat atypical) spores of $B$. semicordata may represent a derived condition as well. $B$. crispatula, with small symmetrical pinnae, is very near to $B$. angustipinna, and this type obviously arose by retention from a late juvenile stage of $B$. angustipinna.

The venation of $B$. virens shows resemblances to that of $B$. angustipinna, and it seems possible that $B$. virens arose from the latter species because of a genetic change causing a different venation pattern already in rather early ontogenetic stages. The venation patterns of $B$. subcrenata and $B$. costata have much in common with that of $B$. virens. $B$. costata has the most complex type of venation of the series, and has slightly deviating spores in addition.

The various types of venations as found in early and mature leaves of the species in this series can be easily understood when it is accepted that all the Asian species of ser. Bolbitianae are derivatives of a species with a venation as found in B. acrostichoides and $B$. serratifolia. Presumably, the genetic changes leading to speciation have been only slight, as the early juvenile leaves are hardly affected; only the later juvenile stages show differences in venation, number and shape of the pinnae. The species showing the extreme conditions in the venation patterns are also divergent in other features (spores, size, etc.). If this hypothesis is correct, one may even boldly suggest that a considerable

Fig. 24. Schematic illustration of probable past evolution of the Asian representatives of ser. Bolbitianae. The length and width of the pinnae represent the average length and width of sterile central pinnae of the respective species, based on 30 or more (Bolbitis semicordata: 15) leaves of each species. The venation pattern as far as given is schematic. Broken-line illustrations are hypothetical. For further explanation see the text. 


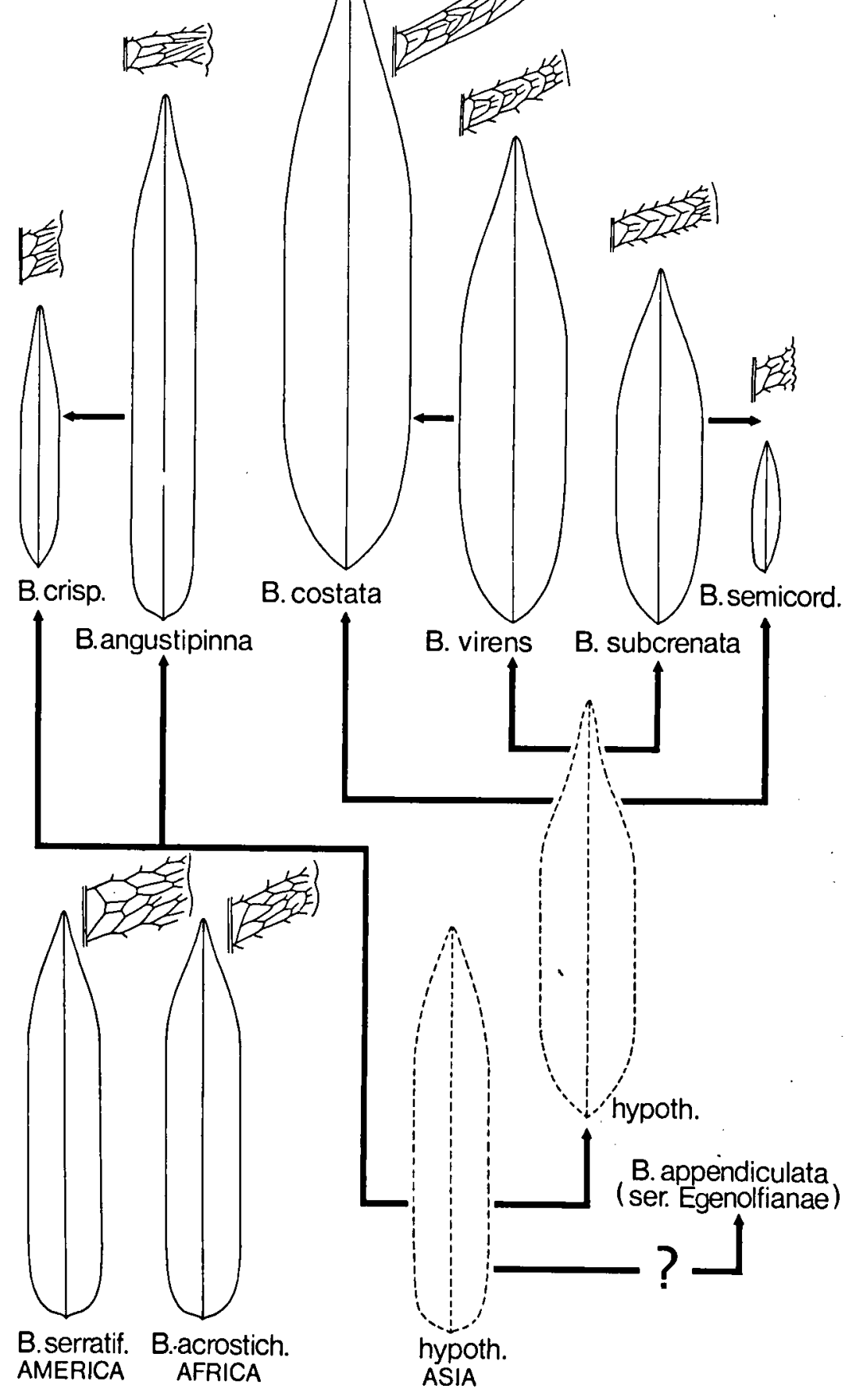


genetic change in a representative of ser. Bolbitianae may cause the retention of still earlier stages resulting in an adult with free veins and pinnate leaves, the pinnae of which are asymmetrical. Such a fern may be very similar to B. appendiculata ssp. appendiculata (ser. Egenolfianae)! On the other hand, gerontomorphosis in a fern like B. serratifolia or $B$. acrostichoides may result in a fern with an intricate venation pattern (as realized in $B$. costata). It is fascinating to see that in this series both the morphologically simple and complex species can be regarded as representing derived conditions.

A comparative analysis of all the different juvenile leaves of all the species is of importance as it may give additional evidence regarding the significance of retention of juvenile characters when trying to unravel the evolution in this group in the past.

Ser. Euryostichae.

B. nicotianifolia.

Only the smallest leaf stages show \pm marked differences. Obviously part of the juvenile stages as present in the ancestral fern has been 'lost' during evolution. The venation pattern-basically similar in all representatives of this group-is very complex.

Adults of ser. Euryostichae are usually pinnate, but adults with simple leaves are known from all species but one. The latter adults are likely to represent a derived condition, as their sterile leaves are similar to the simple sterile juvenile leaves that precede the adult pinnate ones.

Ser. Heteroclitae.

The ontogenies of $B$. heteroclita and $B$. sinuata are different. They only share the shape (not the texture) of the smallest juvenile leaves. The juvenile leaves of $B$. sinuata show but slight morphological variation. Obviously, the genetic changes leading to the origin of $B$. sinuata caused a loss of juvenile stages. This, and the coriaceous texture of the juvenile leaves is here regarded to represent a derived condition. A parallel development is seen in ser. Quoyanae (B. rivularis).

The astonishing variation in the morphology of the leaves as found in $B$. heteroclita (see fig. 9, 13, 20, and the Taxonomic Part) indicates that when (slight) genetic differences cause retention of characters, morphologically very different populations (or species) may be formed. It is supposed that the pinnate condition as found in large mature specimens was present prior to the other types of leaves found in mature specimens of this species.

Ser. Portoricenses.

B. portoricensis.

The two collections studied show considerable differences which are of a kind also found in the juvenile leaves of $B$. auriculata and B. semipinnatifida. It is noteworthy that free included veins occur rather late during ontogenesis.

The leaf shape and the venation pattern of certain stages are also similar to the juvenile leaves, as far as known, from B. bipinnatifida (fig. 15: $\mathrm{g}-\mathrm{i}$ ). The juvenile leaves of the latter species have a reticulate venation pattern except for the incidental occur- 
rence of short re-(!) and excurrent free veins. The mature leaves of B. bipinnatifida have a characteristic pattern with short included excurrent veins. The similarities observed in the juvenile leaves of that species and $B$. portoricensis have parallels in the adult leaves which have, however, a different type of venation (without and with many included free veins, respectively). The study of the juvenile leaves has been a considerable help in properly weighing these differences for taxonomic purposes.

\section{Ser. Quoyanae.}

$B$. quoyana and $B$. rivularis.

The ontogeny of $B$. rivularis shows simple leaves in all juvenile stages; the venation is like that as found in adults (except in the smallest leaf). This, and the coriaceous texture are regarded to represent derived conditions.

Part of the juvenile leaves of $B$. quoyana are similar in size, shape, and, almost so, in venation, to certain mature leaves of $B$. taylorii, and the latter may well have come into existence by the retention of a juvenile condition.

\section{Species incertae sedis.}

\section{B. repanda.}

The adult condition of the tetraploid studied is established through gradual changes occurring in the (successive) leaves. The first leaf formed shows greater complexity than that in $B$. heteroclita, one of its putative parents.

B. semipinnatifida.

The collections studied have very diverse juvenile leaves. The differences between the juvenile leaves are of a kind also seen in $B$. auriculata. The adult condition of the venation results from gradual changes.

\section{Discussion}

From the observations on the juvenile leaves in the genus it has become likely that retention of juvenile characters might have been a factor of importance in creating the present diversification. According to Stebbins (1950) it is probable that genetic changes acting relatively late in ontogeny are less likely to disorganise the whole process of development than those which alter the earlier stages. 'The mutations established will therefore usually be those which affect development at the latest possible stage for the modification of the mature structure in the direction of a selectively advantageous shape. That part of the ontogeny which is less modified by the sum total of these late-acting genes will show embryonic similarity' (p. 492).

As becomes clear also from the juvenile leaves studied in ser. Bolbitianae, it is especially the late stages and not exclusively the latest stage, that are affected (for instance in leaf shape). This and other observations suggest that Haeckel's law is only of limited value for the determination of phylogenetic relationships. A theory about fern diversification taking into consideration all stages of ontogeny, therefore, constitutes a better approach to the understanding of fern phylogeny. 
In spite of the fact that Zimmermann (e.g. 1959) largely adhered to the Biogenetical Law, he also mentioned facts which I regard as strong arguments against the validity of this theory. Firstly, in Haeckel's law the juvenile stages of the modern organisms are compared with the adult stage of the ancestor. There is little in this context that justifies a comparison between different elements of two ontogenies. Besides, if true, all of the ancestors of modern leptosporangiate ferns would be of very limited size. Secondly, only a part of the morphological features show special characteristics in their juvenile leaves. Thus, in ser. Bolbitianae the juvenile leaves, if compared with adult ones have, for instance, a deviating leaf shape and texture, but are similar as regards other features (e.g. the rhizome scales). Therefore, the features of a juvenile leaf or stage may be often better studied semophyletically and not phylogenetically.

If retention of juvenile characters commonly takes place, one has to reconsider the morphological features in terms of primitive and derived. For instance, a free venation is generally regarded to represent the more primitive condition if compared with an anastomosing venation. One should realize, however, that the latter pattern is that as found in the adults; the juvenile stages of such ferns may have a venation that is less, if at all, anastomosing. In case the juvenile condition is retained during diversification, a simple morphology is not necessarily an indication for a primitive genome.

The interpretation of the morphology of a fern is further complicated by the fact that a similar leaf shape, e.g. the pinnate condition, may be found in different ontogenetic developmental stages of a particular plant as already noticed by Holttum (1954) for leaves of $B$. heteroclita. Pinnate leaves from different parts of the ontogeny may have a different complexity as regards venation pattern, texture, etc. In principle both pinnate conditions are liable to retention.

The observations on the juvenile leaves have a great bearing also on classification. They not only confirmed the classification as based on the study of adults but also suggested the relationship of several other taxa.

Finally it can be pointed out that within the lomariopsidoid ferns as defined by Holttum (1946) morphologically very different expressions in a single plant are also found in mature Teratophyllum (with bathyphylls and acrophylls). Also, the similarities between mature Elaphoglossum and Lomariopsis palustris (Hook.) Mett. are striking. It seems of interest to study their juvenile leaves as the systematic position of Elaphoglossum is ambiguous. The relationship of the derivatives of Elaphoglossum (Copeland, 1947), Microstaphyla, and Peltapteris to each other and to Elaphoglossum, may become better known when the juvenile leaves of Elaphoglossum species, e.g. E. dimorphum (Hook. \& Grev.) Moore are studied. As far as studied, Microstaphyla and Peltapteris easily fit in Elaphoglossum (as already advocated by Christensen, 1906) and the recognition of these dwarfs as separate genera seems just a matter of convenience.

\section{RETENTION OF JUVENILE CHARACTERS AND PAST DIVERSIFICATION OF FERNS}

Amongst the modern isosporous leptosporangiate ferns great differences in morphological complexity exist, not only between the various groups (families) of a higher 
order, but also between species of e.g. the same genus. In this connection one may mention the morphologically simple structure of small dichotomously branched leaves as found in a number of hymenophyllaceous ferns, as against the complex architecture of the large fronds as displayed by the Cyatheaceae.

Though the phenomenon of reduction is widely known and accepted, and therefore generally referred to, it is nevertheless not uncommon to find that modern research is still afflicted with the idea that leaves with a dichotomous venation or branching system (as known from fossils) are in principle primitive.

The preceding chapter contains arguments that make it plausible to postulate that a simple morphology of the leaf as found in some representatives of the genus Bolbitis, does not necessarily mean that the taxa bearing such leaves, are primitive as well. It is therefore tempting to ascertain whether a more general and wider interpretation may be attributed to this finding.

In order to circumscribe more precisely the arguments that should be used for defining the identity of a fern in terms of primitive and derived, it seems useful to recapitulate the following two phenomena encountered when studying the ontogeny of the pteridophytes:

1. The adult sporangiferous leaf is often preceded by a series of juvenile sterile leaves that have-especially in the early developmental stages-a completely different morphology, not only if compared with the full-grown stage, but also when a comparison is made of such leaves inter se. Such observations are not limited to leaf shape, but may also apply to e.g. venation, relative size of a single organ, and glandularity.

2. Fertility of the individual may be reached at various stages in the ontogeny. Dwarfing is known from many groups (incl. Cyatheaceae!). Diels (1906) already observed that there exists no strict correlation between the morphological development of a plant and its capacity to become fertile.

The results of the present study make it possible to connect the above two phenomena. In Bolbitis we know dwarfed specimens that in the sterile stage show a leaf morphology strikingly similar to that of the juvenile stages of other full-grown plants. It looks as if such different forms may be found even within one population of a certain species.

What does this observation mean to the question as to how evolution progressed in ferns generally? In this connection it is interesting to notice that is now generally believed that the leptosporangiate ferns evolved under influence of and during the evolution of seed plants (A. R. Smith, 1972). The pteridophytes must have been crowded out to a large extent during the development of these plants. However, at the same time the seed plants themselves created numerous new habitats several of which with only limited conditions for growth. Also because of properties of the spores (which are generally produced in large quantities and easily dispersed) and the genome (high chromosome numbers allow pre-adaptation to many environments, see Klekowski \& Baker, 1966) it is not surprising to find that many ferns are epiphytes or terrestrials in rain forest. The habitat of the first mentioned has good conditions for light but the 'soil' conditions are poor, whereas the situation is just the reverse in the 
second. Ferns are also found commonly in rock crevices, and even on (Bolbitis!) and below rocks.

In so-called 'poor' habitats there might have been a strong selection for ferns being able to complete their life-cycle. And it seems likely that under these circumstance mutations that caused (also) a retention of the juvenile stages had better chances to become established than those affecting the adult stage. Such 'juvenile-looking' progenitors may show a very simple morphological development as to one or more features which are nevertheless expressions of a derived genome.

Although I agree with Wagner (1952) that the small ferns showing foliar dichotomy are derived, foliar dichotomy itself did not necessarily arise de novo as Wagner stated. In my opinion it may be also possible that foliar dichotomy is caused by the retention of juvenile properties. The idea that the simply built small ferns represent either an evolutionary end-point (Wagner) or a primitive condition (what is the classical conception) is unlikely. These simple ferns are the result of a derived genome and may represent starting points for further divergent evolution; in a genus like Platycerium divergent evolution is evident.

It is likely that retention of juvenile characters has played a part in the development of, for instance, Hymenophyllaceae and Polypodiaceae. In view of Williams's work (1927) on the vittarioid ferns it seems further of interest to find out in how far the so-called primitive characters, e.g. the simple type of vascular organisation in part of the lindsaeoid ferns, the free venation of small ferns, and the simple leaf shape, cannot be better regarded as representing a derived condition caused by the retention of characteristics of the juvenile leaves. Kramer (1957) already suggested that the simple type of vascular organisation in the lindsaeoid ferns should represent a derived stage. It must be added here that if retention affects one organ only, it may be difficult to recognize.

The remarks given in this chapter should be regarded as a plea for a comparative study of the juvenile leaves of the isosporous leptosporangiate ferns as well; it is the present author's opinion that such studies may reveal the vital importance of retention of juvenile characters as one of the phenomena that induced the present-day morphological diversity. See also fig. 25.

\section{NOTES ON THE DELIMITATION OF THE GENUS}

'But the point upon which pteridologists appear to differ most, and upon which the only agreement seems to be an agreement to differ, is the definition of genera and their limits.'

J. Smith, Genera Filicum, 1875, p. 5.

Bolbitis, an acrostichoid genus in the old sense, has not escaped that most serious problem that confronts systematists when studying the ferns in general. Convergent evolution or parallelism, 'the tendency of separate evolutionary lines to produce similar characteristics' (Wagner, 1966: 165) is particularly well-expressed, not only in 


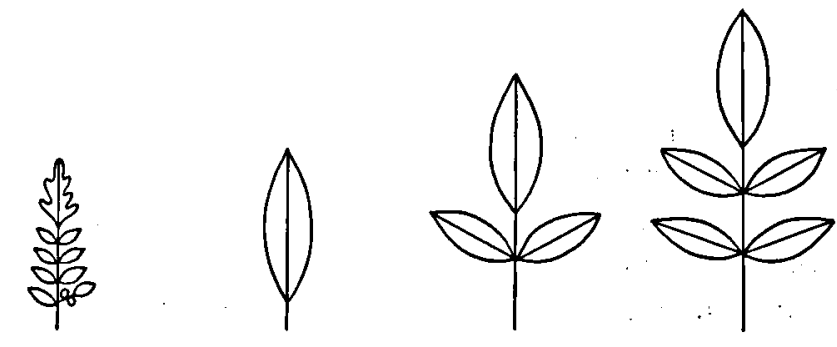

A

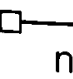

n

B
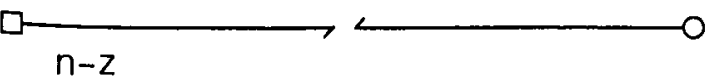

C
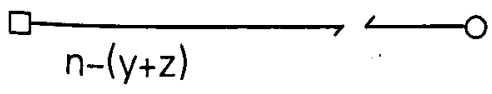

$D$ ar+a3

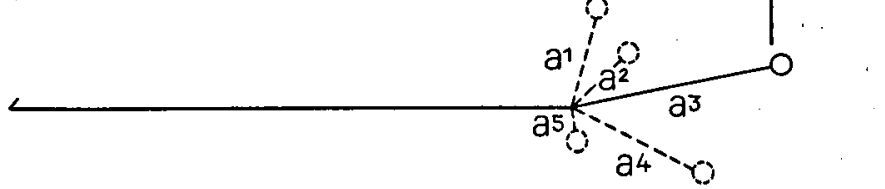

$E \underset{n-(n-a)}{-}$

$\mathrm{F}$

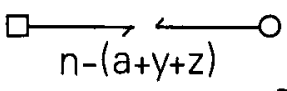

G

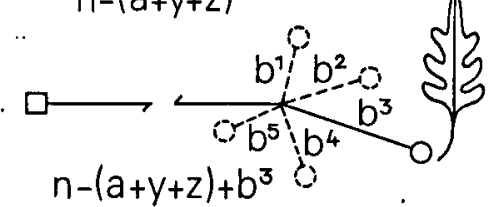

Fig. 25. Schematic illustration of supposed relation between ontogenesis and diversification. A represents a fern the ontogenesis of which comprises $n$ different stages. Genetic changes in a population with ferns like A may on one hand affect the latest ontogenetic stages of fern A (ferns B and C) or on the other hand (also) early ontogenetic stages of fern A (fern E). Ferns B and C are retended, i.e. their adult shape is reminiscent of ontogenetic developmental stages of their supposed predecessor. Progressed evolution of ferns like A may result in a heterogeneity including fern $D$. Fern $F$ arose after the loss of both early and late developmental stages in populations of ferns like A. Ferns like $G$ resulted from progressive evolution of ferns like $\mathrm{F}$.

In this diagram $a^{1}-a^{5}$ and $b^{1}-b^{5}$ of ferns $D$ and $G$ resp., represent evolutionary potentialities leading to more complex ferns. 
the shape of the leaves and the venation pattern, but also in the soral location and arrangement. The acrostichoid condition has clearly evolved in definite lines not otherwise related. As a result of the classical tendency to lay great stress on soral conditions for classification, the 'Acrosticheae' of most classical authors were heterogeneous, and the resulting confusion has been an obstacle to the proper delimitation and classification of the genus Bolbitis in the past (see chapter 4). It is hoped that the present study will be conductive to a better understanding of the genus.

My work on the genus shows Ching's and Christensen's concept of it (though in a narrower sense, excluding ser. Egenolfianae) as given by these authors in Christensen's Third Supplement (1934) to be largely correct. As far as the delineation of the genus is concerned, Ching's and Christensen's work is therefore considered of great importance.

Nevertheless, several more or less recent publications are illustrative of the difficulty to evaluate morphological features in view of the decision if a taxon properly belongs to Bolbitis.

Meniscium macrophyllum Kunze is an American fern of which ample material was studied in detail by Maxon \& Morton (1938). They concluded that the species did not fit into Dryopteris (s.l.; including the thelypteroid ferns), and that it must be included in Bolbitis (B. macrophylla (Kunze) Maxon \& Morton) because of characteristics of the venation pattern and the fertile parts. My studies of the leaf indument, petiole anatomy, and the spores indicated, however, that it represents a genuine thelypteroid fern, to which it was transferred later by Morton (1971).

Soon after starting the study of the acrostichoids, it was shown that an insufficiently known acrostichoid fern attributed to the present alliance was a blechnoid fern for which the genus Pteridoblechnum was created (Hennipman, 1965).

Recently, it became clear (Hennipman, 1976) that Leptochilus acuminatus White \& Goy, which was thought to be related to species of the present genus, also fits in Pteridoblechnum. Its proper systematic position became known largely by surprise: a so-called intermediate leaf showed indusiate blechnoid sori atavistically. The venation pattern of this fern falls within the range of that found in $B$. heteroclita (to which White \& Goy thought their species related), and represents a fine example of parallel development.

Some other ferns, referred to the present genus by experienced pteridologists, are here identified as representatives of the genera Cyclodium, Colysis, and Lomagramma (for details see the excluded species in the Taxonomic Part).

On the other hand, following a hint given by Prof. Holttum, it became clear that Stenosemia dimorpha (Copeland, 1955; = B. quoyana) fits the genus as presently construed.

From the above it will not surprise the reader that species of Bolbitis have been assigned to 23 different genera in the past, resulting in a somewhat intricate synonymy.

Studies of other Asian acrostichoid ferns now in progress give strong indication that some significant changes in the taxonomy of part of this group can be anticipated. 


\section{THE SYSTEMATIC POSITION OF THE GENUS}

The genus Bolbitis is nowadays generally referred to the lomariopsidoid ferns for which the family name Lomariopsidaceae, given by Alston (1956), is available. In the sense of Holttum (1946, and onwards), the genus Elaphoglossum should be included in this alliance, whereas Ching (1940) and Pichi Sermolli (1968) accommodated this genus in the monotypic family Elaphoglossaceae. The great resemblance of Elaphoglossum to (juvenile leaves of) Lomariopsis is in support of Holttum's classification. The inclusion of the genus Lomagramma in this group has never been questioned, also as the venation pattern is very much like that found in part of Bolbitis. The former genus deviates from all others because of the presence of paraphyses amongst the sporangia. Whether this feature has a bearing on its taxonomic position cannot be ascertained until these organs are better understood. Thysanosoria is here regarded as an atavistic Lomariopsis, as serious doubts can be expressed whether a characteristic of the fertile leaf alone in this group of ferns is sufficient for recognizing a separate genus.

Holttum $(1946,1949)$ first recognized this group following excellent work on most of its genera (unfortunately not of the present genus). The 'dorsiventral rhizome with its broad root-bearing ventral vascular strand, the acrostichoid condition, in most cases the simply pinnate fronds with free veins, and the perispore' (1949: 290) were regarded by him as distinctive common characters. He later (1954) also paid some attention to the shape of the rhachis and the way of insertion of the pinnae, which can be considered important characteristics as well.

There are several opinions about the interrelationship within the lomariopsidoid ferns and the relationship of the lomariopsidoid ferns to the other groups of leptosporangiate ferns.

With reference to the relationship of Bolbitis to the other lomariopsidoid ferns, Holttum $(1946,1949)$ considered Lomariopsis the 'central' genus. From his elegant observations on the bathyphylls (a kind of juvenile leaves) of Teratophyllum he concluded that a rhizome bearing two rows of leaves, and leaves with a free venation should be interpreted as representing the primitive condition. As regards the venation pattern it is elucidated elsewhere that the free venation in Bolbitis can also be considered as representing a derived condition. The leaves of ser. Egenolfianae and ser. Bolbitianae show conspicuous similarities to the bathyphylls and acrophylls of Teratophyllum, which might be an indication of the relationship between these taxa (and the related genera Arthrobotrya, Lomariopsis, and Elaphoglossum). The affinity of the other groups in Bolbitis to these genera is far less obvious. The position of Lomagramma needs further study. Pichi Sermolli (1969) thought Egenolfia, Edanyoa, and Bolbitis (here united) as not very closely related to the other genera of his Lomariopsidaceae (Elaphoglossum excluded) and assigned these two groups to two different tribes. The interrelationships between the lomariopsidoid ferns are still insufficiently understood. The respective genera show conspicuous biological differences (for details, see Holttum, 1946).

There is little agreement regarding the relationship of the lomariopsidoid ferns to other groups of ferns. Some of the different opinions of rather recent years are the 
following. Christensen (1938) thought the genus an acrostichoid representative of probably the tectarioid derivative of his Polypodiaceae-Dryopteridoidae. Copeland (1941) was still quite pertinent as to the relationship of Bolbitis (in a very artificial conception comprising the American representatives only) to Phlebodium (a genus of polypodioid ferns of quite different affinity): 'As to the real Bolbitis, I merely repeat myself, that it is derived from Phlebodium, and I have yet to see any refutation of the evidence by those who ignore it. Its resemblance to Campium [which comprises in Copeland's conception exclusively Old World species of mainly Bolbitis], however complete, is none the less casual, the result of divergent evolution' (p. 168). He later (1947) corrected his view and agreed with Christensen. Holttum related the lomariopsidoid ferns to the dennstaedtiaceous ferns (a very large assemblage of non-polypodioid ferns) 'probably on a line near Davallia' (1946: 146). Nayar \& Kaur(1965b) thought the genus systematically rather isolated, possibly 'remotely related to the 'RumohraPolystichum line of evolution in Copeland's Aspidiaceae' (p. 152). In another publication (Nayar \& Kaur, 1965c) they favoured a tectarioid ancestry of the group, which was elaborately set forth again by Kaur (1974). These authors based themselves mainly on similarities between certain lomariopsidoid ferns and Tectaria species which are, however, quite different as to the indument of the young leaves and the chromosome number. At present I cannot give any positive indication as to the systematic position of the lomariopsidoid ferns. Absence of a detailed study of Bolbitis bernoullii, which possibly originated from intergeneric hybridization with a non-lomariopsidoid genus (Polybotrya?), as well as of Leptochilus $\times$ trifidus (possibly a cross between $B$. sinuata and Leptochilus decurrens) precludes further speculation at present.

\section{SUBDIVISION OF THE GENUS}

Iwatsuki (1959), basing himself almost exclusively on Japanese species, was the first to unite Egenolfia and Bolbitis. He divided the emended genus into four sections; two are based on species here referred to $B$. appendiculata, one is based on B. laxireticulata (a dubious species from Japan), whereas all other species are included in sect. Bolbitis.

When using characters from the venation pattern, the perispore, and the rhizome, it is easy to fit the majority of the species into ten series. The remaining species, nearly all regarded as of hybrid origin, have been kept separate as species incertae sedis. Distribution maps of the series are given in fig. 27-30.

The series are mainly defined on characters as present in the adult stages. The often scarce and incomplete data available on juvenile forms or even the complete absence of such information concerning quite a number of species in the genus made it not always possible to compare the ontogeny of the constituent species in a series, or of the series mutually.

Group 1. Ser. Alienae, Lindigianae, Quoyanae, and Heteroclitae. The venation pattern generally shows a regular network of \pm isodiametric or elongate, angulate areoles without included (ex-and/or) recurrent free veins. 
Ser. Alienae is a very natural group. It occurs terrestrially in America and throughout tropical Africa to Madagascar and the Mascarenes. It seems intimately related to the monotypic American ser. Lindigianae, a high-climber. The leaf architecture and the venation pattern of ser. Alienae show marked resemblances to that of ser. Quoyanae which occurs in eastern Malesia, the Pacific, and the Bonin Islands.

The venation pattern and leaf architecture of the species of ser. Heteroclitae differ from those of the other members of Group 1. The mature pinnate leaves of $B$. heteroclita and $B$. sinuata have a terminal segment that is conform to the pinnae (triangular in the others). Such a difference is not necessarily of much significance; both types of leaves are found, for instance, in ser. Portoricenses.

Group 2. Ser. Portoricenses, Heudelotianae, and Euryostichae. The venation generally shows a part of the areoles to have re- and excurrent included free veins.

Ser. Portoricenses (America) is characterized also by its scales of the rhizome. The group appears a natural one in spite of differences in shape of the leaves of its members. Ser. Heudelotianae (Africa) is unique because of its habitat requirements and the shape of the perispore. Both are likely to represent a derived condition.

Ser. Euryostichae (America and Africa). The American species are very homogeneous. The only African species deviates, amongst other things, in the localisation of the bulbil. This group puzzled me for some time as it shows several features (venation pattern, leaf shape, petiole anatomy, spores) which show similarities to some species of Tectaria. However, the chromosome number as found in this series $(n=41)$ differs from that of Tectaria $(n=40)$, and so does the indument as found on (young) leaves.

The systematic position of ser. Bipinnatifidae is of interest. Its venation pattern is very close to that of the series listed in Group 1. On the other hand, the adult plants of $B$. bipinnatifida have a number of characteristics in common with $B$. portoricensis (ser. Portoricenses) including rather large basally attached scales, a leaf with a proportonally large terminal segment, and teeth in the sinuses of the pinnae. Further, the scanty material of the juvenile leaves of these species shows conspicuous and surprising similarities. Apart from a similar outline, the juvenile leaves may have an almost identical venation pattern (reticulate without or with but few included free veins). The relationship of ser. Bipinnatifidae to ser. Portoricenses seems a reality in spite of its type of venation. To this alliance may also belong B. umbrosa (Species incertae sedis) which has leaves with a reticulate venation without free included veins. The proper position of this species will become better known after complete material of the rhizome has become available.

The remaining series Bolbitianae and Egenolfianae do not fit the two groups mentioned above.

Ser. Bolbitianae. This pantropical series is conspicuous because of the shape of the perispore and the venation pattern. One species (B. crispatula) shows costal areoles only. The group is especially characterized by its undulate perispore. The undulate condition of the perispore possibly represents a derived condition in ser. Heudelotianae, 
and almost certainly so in a few specimens of $B$. fluviatilis (a derivative of B. auriculata), normally with a cristate perispore. Therefore, the undulate perispore as found in ser. Bolbitianae may likewise represent a derived condition.

$B$. acrostichoides and $B$. serratifolia are regarded to represent the most primitive species. Amongst specimens of both species a similar type of venation pattern may be found as is typical of the series of Group 1. This seems in favour of a relationship between ser. Bolbitianae and this group.

Ser. Egenolfianae is unique because of its free venation. Therefore, this Asian group of species has been generally regarded as primitive. Its species show variation as to the localisation of the bulbil, features of the rhizome, petiole anatomy, spores, etc. The venation pattern is superficially nearest to that of $B$. crispatula (ser. Bolbitianae). Besides, certain mature leaves of $B$. appendiculata ssp. appendiculata are surprisingly similar to the juvenile leaves of species in ser. Bolbitianae, which may indicate relationship.

In view of earlier publications it was unexpected to find that these two groups, formerly given generic rank, may be closely related.

Further research will show in how far the idea is justified to treat the series of Group 1, those of Group 2 (including ser. Bipinnatifidae), and ser. Bolbitianae and Egenolfianae, as the three sections of the present genus. See also table 3.

\begin{tabular}{|c|c|c|c|c|}
\hline \multicolumn{5}{|c|}{$\begin{array}{l}\text { The series of Bolbitis arranged according to properties of their spores and } \\
\text { venation patterns. Squares indicate groups of probably related series. }\end{array}$} \\
\hline$\underbrace{\text { PERINE }}_{\text {VENATION }}$ & $\begin{array}{l}\text { smooth } \\
\text { undulate } \\
\text { (spore type D) }\end{array}$ & $\begin{array}{l}\text { smooth } \\
\text { cristate-undulate } \\
\text { (spore type B) }\end{array}$ & $\begin{array}{l}\text { reticulate } \\
\text { cristate } \\
\text { (spore type A) }\end{array}$ & $\begin{array}{l}\text { smooth } \\
\text { undulate } \\
\text { (spore type c) }\end{array}$ \\
\hline velns free & & \multirow[t]{2}{*}{ Egenolftanae } & \multirow[t]{2}{*}{ Egenolfianae } & \multirow[b]{2}{*}{ Bolb1t1anae } \\
\hline bolbitianae type & . & & & \\
\hline $\begin{array}{l}\text { reticulate } \\
\text { without } \\
\text { recurrent } \\
\text { veins }\end{array}$ & & $\begin{array}{l}\text { Allenae } \\
\text { Quoyanae } \\
\text { Heteroclitae } \\
\text { Lindiglanae }\end{array}$ & & \\
\hline & & Bipinnatifidae & & \\
\hline $\begin{array}{l}\text { with recurrent } \\
\text { veins }\end{array}$ & Heudelotianae & $\begin{array}{l}\text { Portoricenses } \\
\text { Euryostichae }\end{array}$ & & \\
\hline
\end{tabular}

\section{Hybrids and infrageneric classification}

It is generally believed that hybrids between taxa are an expression of the genetic relationship between the parents. On the other hand, one should not give much weight to the absence of hybrids between taxa prior to experimental proof. During the present study I came across a considerable number of hybrids or hybrid derivatives. 
Those of which the parents can be postulated with reasonable certainty and of which the parents belong to different series are:
B. angustipinna $\times$ sinensis:
Bolbitianae $\times$ Egenolfianae
$B$. appendiculata $\times$ virens: Egenolfianae $\times$ Bolbitianae
B. $\times$ boivinii:
B. cadieri:
B. fluviatilis $\times$ heudelotii:
Alienae $\times$ Euryostichae
$B$. heteroclita $\times$ rhizophylla:
Bolbitianae $\times$ Heteroclitae
B. interlineata:
Alienae $\times$ Heudelotianae
B. lonchophora:
Heteroclitae $\times$ Egenolfianae
Bolbitianae $\times$ Heteroclitae
B. nicotianifolia $\times$ portoricensis:
Egenolfianae $\times$ Heteroclitae
B. presliana:
B. repanda:
B. semipinnatifida:
B. $X$ singaporensis:
Euryostichae $\times$ Portoricenses Bolbitianae $\times$ Egenolfianae Egenolfianae $\times$ Heteroclitae Alienae $\times$ Euryostichae Egenolfianae $\times$ Heteroclitae

This list shows that seven of the ten series are involved in this kind of hybridization. Hybridization occurs most ( 9 out of a total of 13 cases) between species of ser. Bolbitianae, Egenolfianae, and Heteroclitae. This indicates a relationship between ser. Bolbitianae and Egenolfianae, and of both to ser. Heteroclitae, which is in accordance with the remarks given above. There are no hybrids listed between species of these series and other series. See, however, also below.

Three other hybrids have parents belonging to species of which the series are classified in Group 1 and 2. There are hybrids between species of ser. Alienae with both ser. Euryostichae $(2 x)$ and ser. Heudelotianae $(1 x)$.

The first two hybrids and the hybrid between species of ser. Portoricenses and Euryostichae further confirm the inclusion of the latter series in the present genus. See also above.

Several other hybrids or hybrid derivatives are worth mentioning:

1. B. gemmifera probably arose from hybridization between $B$. auriculata (ser.Alienae) and a representative of either ser. Bolbitianae or ser. Euryostichae.

2. Because of properties of the venation pattern $B$. humblotii seems to have been derived from hybridization between species here referred to series listed in Group 1 and Group 2 respectively.

3. $B$. longiflagellata may have originated through hybridization between a species of ser. Alienae and B. humblotii (see 2).

4. B. subcordata could be the result of hybridization between species here referred to ser. Bolbitianae and ser. Quoyanae.

5. Probably $B$. laxireticulata is a hybrid between a species from ser. Egenolfianae and $B$. subcordata (see 4).

These hybrids further indicate the genetic relationship between the respective series.

6. B. bernoullii arose from a cross between $B$. lindigii (ser. Lindigianae) and a species belonging to a différent genus (Polybotrya?). 
7. Leptochilus $\times$ trifidus probably is a hybrid between $B$. sinuata (ser. Heteroclitae) and Leptochilus decurrens.

The study of the latter two taxa seems promising in so far that it may be the key to trace the proper systematic position of the present genus (and of the Lomariopsidaceae).

\section{NOTES ON SPECIFIC DELIMITATION}

\section{Introductory remarks}

Manton's (1950) publication on the cytology and evolution of the pteridophytes initiated a new era of taxonomic research. She convincingly demonstrated, further elaborated by herself and later (co-)workers in this field (for references see Lovis, 1973), the common occurrence of hybridity, auto-and alloploidy, apospory, and apogamy, as well as the consequential importance of these phenomena for phylogeny and taxonomy.

Formerly, when hybridization was considered to be non-existent, or extremely rare in ferns, hybrids were not recognized as such in the vast majority of cases. They were described as species, and being intermediate, thought to be 'links' in the (gradual) course of evolution. Manton and others elucidated the origin of new species by the process of hybridization, auto- and alloploidization. So-called reticulate relationships were found to exist in many groups of ferns. The Appalachian Aspleniums are a famous example (for details see Wagner, 1954).

Generally hybrids, alloploids and also autoploids (as was only more recently shown) are apparently so commonly formed that they may constitute a dominant element amongst the ferns of a certain area. Walker (1966) estimated the amount of polyploids in tropical ferns at $60 \% ; 53 \%$ of the British ferns are polyploids (Manton, 1953).

As to apogamy, several hybrids and also a considerable number of 'good' species are known to reproduce in that way and it is thought to be widespread in ferns (Manton, 1950). Evans (1969) boldly suggested that many, if not all taxa that are considered 'sterile hybrids', have the capacity of apogamous reproduction, albeit on a (very) low level; also that in principle the same possibility exists in sexually reproducing species.

Last but not least, modern cytogenetic research made clear that certain recognized morphological characters are correlated with karyological differences, whereas others, although frequently used for specific taxonomic differentiation in the past, are not. The latter characteristics are now regarded as the obvious result of divergent evolution and/ or habitat conditions, and are taxonomically treated accordingly.

Cytogenetic research has mainly advanced in the ferns of North-temperate regions; Manton originally based her studies on mainly North-European taxa. However, ample information has been published more recently on tropical ferns (for references see Walker, 1973) indicating clearly that the same phenomena as recognized in temperate ferns are also found in tropical ones. As a consequence the difficulties experienced in taxonomy when investigating the limited number of ferns of Europe or the U.S.A. is not an isolated case. 
In the present study attention has been given to apply these methods of research to the various taxa of the genus Bolbitis. Although these investigations were-perforce-relatively limited, sufficient data were obtained to state with confidence that hybridization, allo- and autoploidization are common phenomena in this group too. An indication of apogamy could not be detected.

The impossibility of further research in this field and the absence of karyological data on quite a few species in the genus, is regretted. However, it must not be forgotten that, when even in e.g. Europe difficulties are experienced to come to satisfactory and final conclusions in cytogenetic research, such difficulties become tenfold when working with tropical groups. The number of species is of quite a different order, live material can only be obtained to any extent by organizing (costly) expeditions and provided that sophisticated methods of cultivation can be applied. The attempt made to obtain significant information regarding the karyology of the ferns under study was an important reason why the present study took as long as it did. The data submitted, although far from complete, must be regarded as the best obtainable under the circumstances.

One may wonder whether there is any sense in starting a systematic study of a group of tropical acrostichoid ferns which are notoriously plastic in their leaf shape, if mainly herbarium material is at hand. The answer is related to the extent in which such factors as hybridization and polyploidization, precocious fructification, etc. can be reliably recognized from herbarium material.

The detection of hybrids and polyploids in herbarium material. - Hybrids and alloploids may be traced because of the following properties:

1. Spores. Hybrids generally have aborted spores. However, hybrids may be able to reproduce apogamously by normally shaped mito-spores. In that case the number of sporangia that produce 32 normally shaped spores may vary in different taxa from all to merely an incidental sporangium.

The number of spores per sporangium is usually difficult to estimate in herbarium material (Kramer, 1957), as I experienced also myself.

On the other hand, normal species may have at least some abortive spores (Wagner \& Lim Chen, 1965), or may produce abnormal spores only, for reasons set forth elsewhere (see chapter 6). Alloploids produce normal spores. Spores of autoploids are either normal or abnormal, the frequency of abnormal spores being dependent on the ploidy level (Wagner \& Lim Chen, 1965).

2. Instability. When the herbarium taxonomist traces extreme variation in shape of different plants thought to belong together, he should try to find out by studying the spores if such plasticity might be the result of hybridization. Some hybrids may reach a mature stage which resembles ontogenetic stage (s) of the parents involved, resulting in an excessive variation.

3. Intermediacy. Hybrids generally tend to bridge the differences between their parents. The hybrid is a 'compromise' (Wagner, 1968: 118). Few exceptions are known from this rule. However, I found it very difficult or even impossible to identify a certain expression of a hybrid as representing the exact intermediate condition. What is the intermediate 
condition of the venation pattern when that of the two parent species is very different? I doubt if anyone would have been able to predict that the venation pattern as found in B. $\times$ singaporensis (fig. 85: a-d) is intermediate between that of B. appendiculata $\mathrm{ssp}$. appendiculata (fig. 49: q-d') and B. sinuata (fig. 61: $\mathrm{k}-\mathrm{m}$ )! The same holds for the localisation of the bulbil in $B$. gemmifera ( \pm at the base of the terminal segment of the sterile leaf) (fig. 74 ; a, c, d), a presumed hybrid derivative of either $B$. acrostichoides or $B$. gaboonensis (both with subterminal bulbils) and $B$. auriculata (usually without, rarely with axillary bulbils).

4. Structural irregularity. Wagner (1962) brought together the 'sporadic observations' (p. 87) on ferns in which there is deviation from normal symmetry, either morphologically or anatomically. Wagner found that these irregularities were associated with known or suspected hybridity and called them structural irregularities. The feature that is irregularly expressed is generally one in which the parents are highly different. It is best known from the irregularly shaped leaves as found in some hybrids. Von Giesenhagen (1918) related the irregular leaves of Pteris otaria Beddome, a hybrid between Pteris biaurita and Pteris multiaurita (Walker, 1958), with suboptimal nutrient conditions; this explanation is rejected by Wagner with good reasons.

Wagner suggested that structural irregularities might be more common than actually observed by him, which is confirmed by the present investigations.

In conclusion, it is my opinion that the herbarium taxonomist has good possibilities to trace reticulate relationship especially when he has ample, preferably including some live material at his disposal.

Hybridization and evolution. - There is some difference of opinion regarding the role of hybridization in evolution. Wagner (1968: 122) concluded after perusing numerous monographs 'that the majority of species in most groups are allopatric and divergent, i.e. "normal" species produced by ordinary processes of mutation, recombination, selection and isolation'. Wagner (1970), admitting that tetraploids have the capacity for evolution, regarded the main pillars for plant evolution to be populations of 'normal' species, i.e. diploid, sexual, and outbreeding. The fact that 'normal' species with normal life cycles still prevail today should according to him be a strong indication for such plants to be responsible for 'the primary thrust of evolution'. Apogamous ferns and alloploids are regarded by him as 'evolutionary noise' that vanishes while the diploid continues to evolve. This idea is not quite shared by others. For instance, Walker (1973) included hybridization and polyploidization as mechanisms in his hypothesis on the evolution of the different chromosome numbers as found in Dennstaedtia and the tree ferns. Stebbins (1971) speaking about Angiosperms thought it likely that polyploidization has been important in the diversification of genera and species within families (but not in the origin of families and orders themselves). De Wet \& Stalker (1974:695) commented on Wagner (1970): 'In fact, "normal" species may be diploid or polyploid, annual or perennial, sexual or facultatively apomictic, and inbreeding or outbreeding. What makes a species normal is its ability to strike a balance between releasing variability and maintaining adaptive gene combinations.' In the present paper a distinct 
minority of the species is regarded to be of hybrid origin. In view of the opinions mentioned above their significance for evolution is as yet not ascertainable.

\section{Concluding remarks}

Apart from reticulate relationship I have also paid attention to the shape of the juvenile leaves (see chapter 11). Knowledge of the juvenile leaves enabled me to properly identify material in spite of precocious fructification.

Different plants belonging to one species were found to behave differently when in cultivation. This is not only true for plants with different cytotypes. For instance, the plant of B. sinensis which was collected by Dr. H. P. Nooteboom on Doi Chieng Dao (diploid; Thailand) developed into a rather small plant which regularly formed fertile leaves. The specimens collected by myself from Doi Inthanon (diploid; Thailand) grew out into large plants which but seldom formed a fertile leaf. On the other hand certain diploid and triploid dwarfs of $B$. heteroclita are morphologically almost indistinguishable. Habitat conditions may further determine the shape of the plant considerably.

I have tried to classify the material in categories that can be morphologically recognized. Sometimes it appeared easy to draw lines in material showing continuous variation when studied superficially, also as important new characters became available after my detailed studies of some features. Most of the common species are, however, extremely variable, and could, moreover, not be divided into infraspecific catergories. This variation is also illustrated by the characteristics of a number of spores studied from each of the species (see the Taxonomic Part). Species that could be subdivided into lower categories happened to belong to series in which polyploids are not (yet?) known. It also became clear that many of the morphologically distinct plants described during the last decades as new species could be interpreted as undoubted representatives of longstanding species when taking full account of the phenomena mentioned in this chapter.

\section{NOTES ON GEOGRAPHY}

Distribution maps have been compiled for most of the taxa recognized. They represent a historical element which might help in understanding the present diversity. As I do not feel qualified to weigh properly the present theories which deal with the origin and fate of the continents, I confine myself to mentioning a few characteristics of the distribution patterns of the series which seem to be of interest for plant geographers. See table 4 and fig. 26-30.

In the genus ser. Bolbitianae has the largest number of species; it is the only series that is pantropical. The majority of its species are confined to Asia. B. serratifolia is the most widespread American species, also occurring on the so-called Brazilian shield. The African $B$. acrostichoides is a close relative, but also resembles $B$. angustipinna, the most widespread Asian species of this series.

Ser. Euryostichae and Alienae inhabit both Africa and America. The only African rep- 


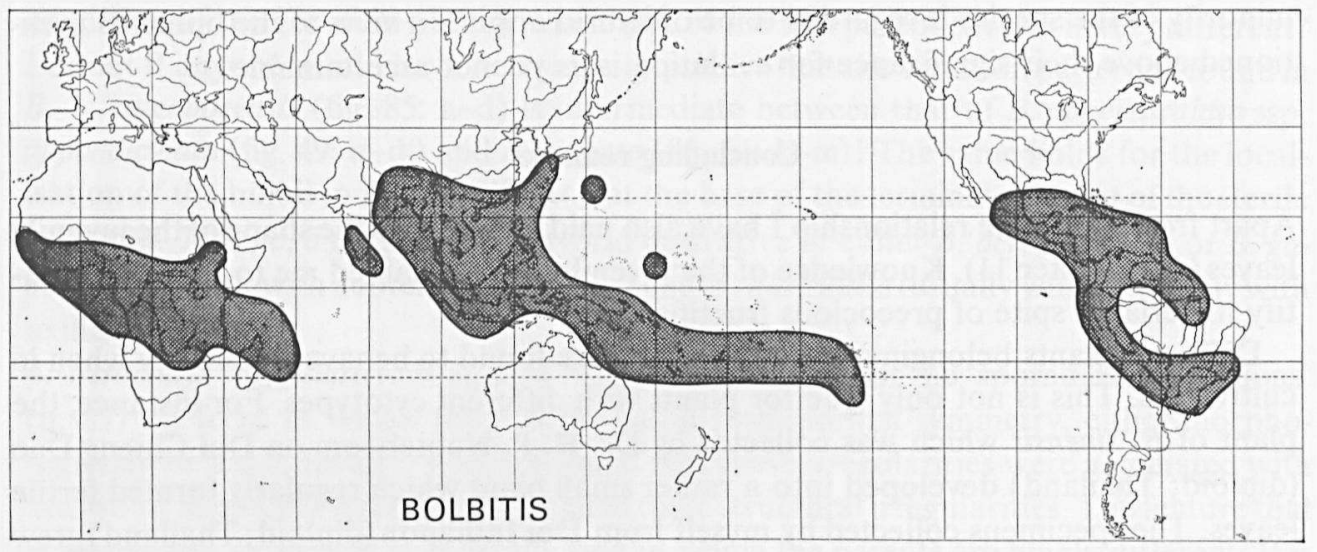

Fig. 26. Distribution of Bolbitis.

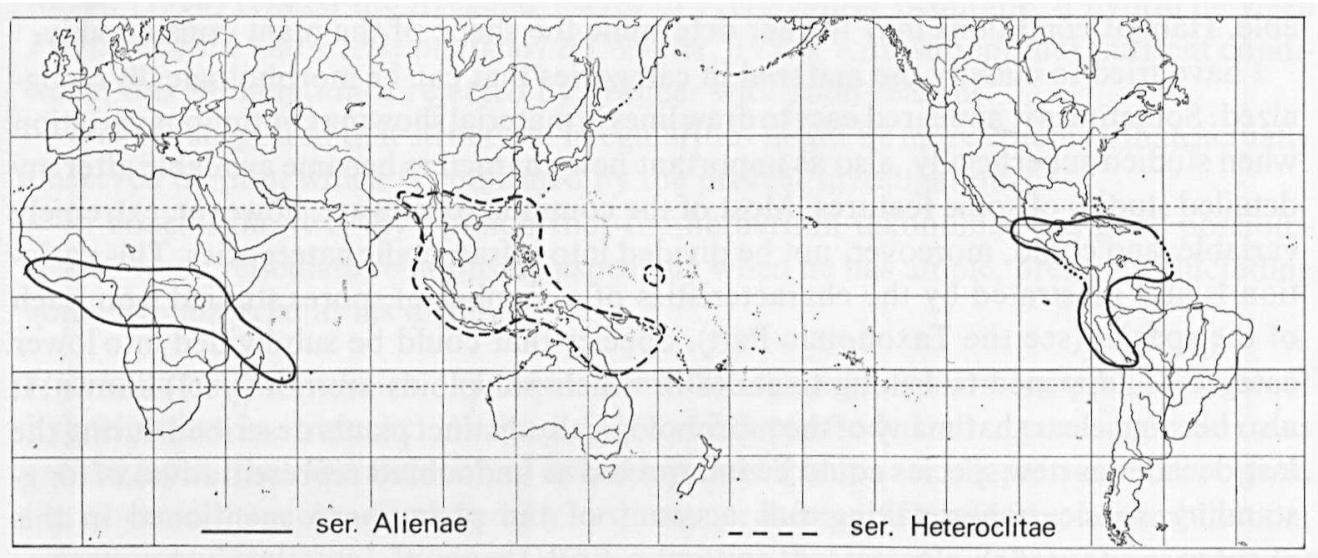

Fig. 27. Distribution of ser. Alienae and ser. Heteroclitae.

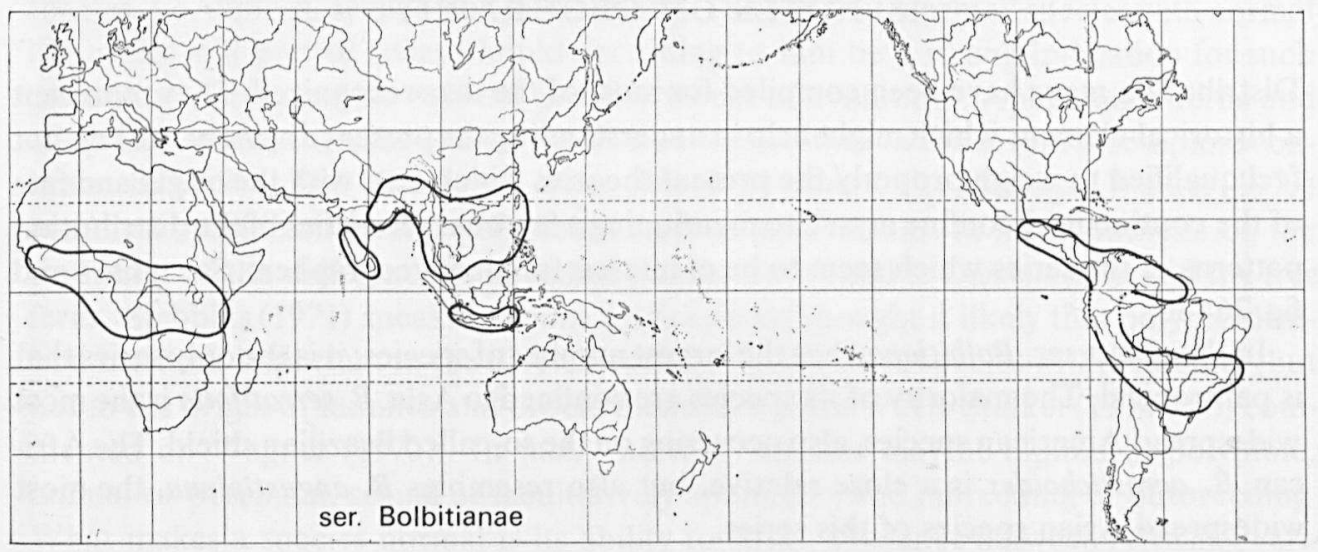

Fig. 28. Distribution of ser. Bolbitianae. 


\begin{tabular}{|c|c|c|c|c|c|}
\hline \multirow[b]{2}{*}{ Serles } & \multicolumn{4}{|c|}{ Number of species } & \multirow[b]{2}{*}{ Notes } \\
\hline & Total & Africa & America & $\begin{array}{l}\text { Asia } \\
\text { Pacific }\end{array}$ & \\
\hline Bolbitianae & 11 & 2 & 2 & 7 & $\begin{array}{l}\text { Including southern India and } \\
\text { Ceylon; not in New Guinea and } \\
\text { the Pacific }\end{array}$ \\
\hline Allenae & 3 & 2 & 1 & - & \multirow{5}{*}{$\begin{array}{l}\text { Also in Madagascar and the } \\
\text { Mascarenes } \\
\text { The African specles confined to } \\
\text { the Congo Basin } \\
\text { Seychelles only } \\
\text { Not in Madagascar and the Mas- } \\
\text { carenes } \\
\text { Andean }\end{array}$} \\
\hline Euryostichae & 5 & 1 & 4 & - & \\
\hline Bipinnatifidae & 1 & 1 & - & - & \\
\hline Heudelotianae & 1 & 1 & - & - & \\
\hline Lindigianae & 1 & - & 1 & - & \\
\hline Portoricenses & 3 & - & 3 & - & \multirow{4}{*}{$\begin{array}{l}\text { Not in southern India and } \\
\text { Ceylon } \\
\text { Eastern Malesia to the Pacific; } \\
\text { Bonin Is. }\end{array}$} \\
\hline Egenolfianae & 4 & - & - & 4 & \\
\hline Heteroclitae & 2 & - & - & 2 & \\
\hline Quoyanae & 3 & - & - & 3 & \\
\hline Species incertae sedis & 10 & 3 & 2 & 5 & \multirow{3}{*}{ " } \\
\hline Total no. of species & 44 & 10 & 13 & 21 & \\
\hline Total no. of series & 10 & 5 & 5 & 4 & \\
\hline
\end{tabular}

resentative of ser. Euryostichae is confined to the Congo Basin. As regards ser. Alienae, $B$. auriculata is of interest as it occupies a large area in Africa, also occurring in Madagascar and the Mascarenes. Phylogenetically this species seems very close to the American $B$. aliena, differing mainly in leaf architecture (a feature liable to variation in other groups), indicating that speciation in this group is possibly very slow indeed.

Endemic to America are ser. Lindigianae and ser. Portoricenses. Ser. Lindigianae is possibly related to ser. Alienae; the only species-presumably a high-climber-is restricted to the Andes. One of the three species of ser. Portoricenses is widely distributed, the two other species are restricted to rather small areas in Mexico and South America respectively.

Two monotypic series are strictly African. Ser. Bipinnatifidae is confined to the Seychelles, whereas ser. Heudelotianae is the most widespread African taxon, obviously due to habitat tolerance. These two series are possibly related to the American ser. Portoricenses.

As to the Asian elements of the genus it is of interest to note that ser. Egenolfianaelike the Asian part of ser. Bolbitianae - covers a large area including southern India and Ceylon but not New Guinea and the Pacific. On the other hand, ser. Heteroclitae and Quoyanae are absent from southern India and Ceylon, but do occur in eastern Malesia and the Pacific. Ser. Quoyanae is restricted to eastern Malesia, the Pacific, and the Bonin Is., whereas the distribution of ser. Heteroclitae includes mainland Asia from northeastern India to Japan, the whole of Malesia, and the western part of the Pacific. (A dis- 


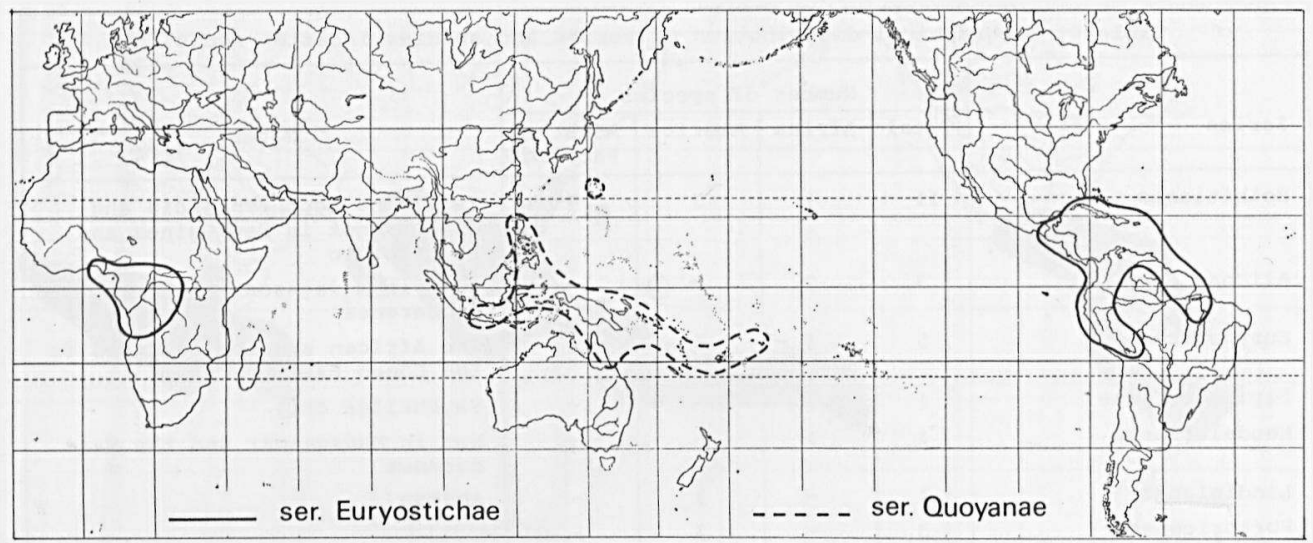

Fig. 29. Distribution of ser. Euryostichae and ser. Quoyanae.

tribution pattern like that found in ser. Quoyanae is also known for Santalum(Santalaceae) and Metrosideros (Myrtaceae)-Pers. common. Dr. M. M. J. van Balgooy).

A pantropical distribution is displayed by the group (Group 1 as described in chapter 16) representing the species with a reticulate venation pattern, i.e. those of ser. Alienae, Heteroclitae, Lindigianae, and Quoyanae. If we consider ser. Bolbitianae to represent (possibly together with ser. Egenolfianae) another group, there are two groups in the genus with a pantropical distribution (which may be regarded as an indication of age). It is difficult to find out which of these two groups is the oldest. It is, however, of interest to notice that the venation pattern of ser. Bolbitianae can be regarded as derived from a reticulate pattern. Besides, the distribution of the first group in Asia is much more widespread than that of the other.

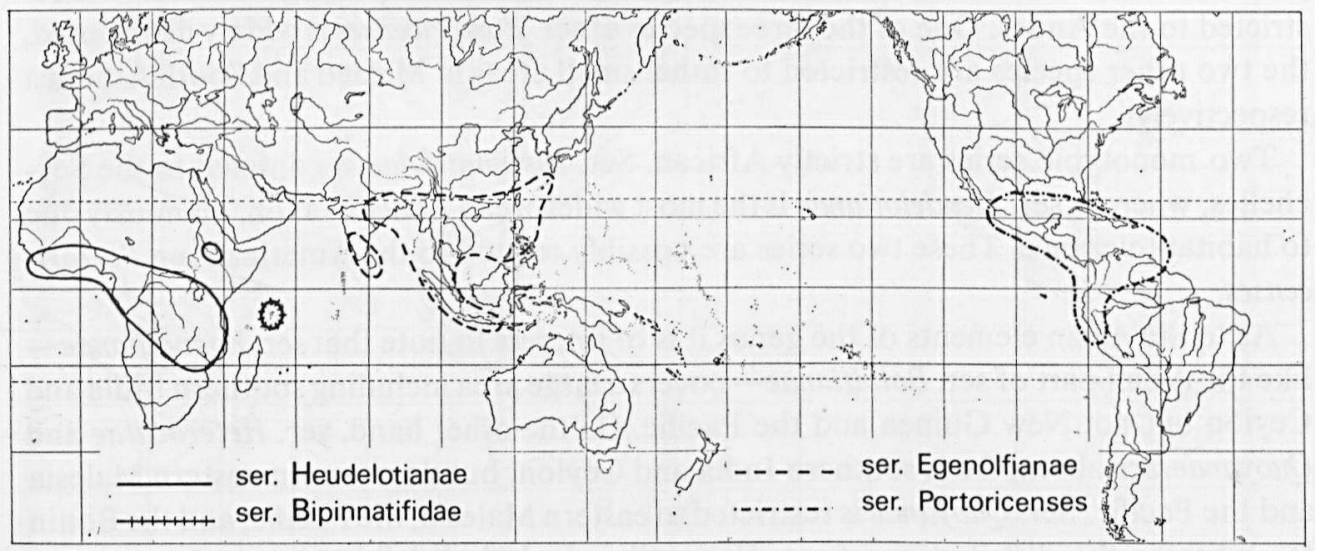

Fig. 30. Distribution of ser. Bipinnatifidae, ser. Egenolfianae, ser. Heudelotianae, and ser. Portoricenses. 
The morphological distinctness of both the ser. Heteroclitae and ser. Quoyanae from their nearest relatives (ser. Alienae and ser. Lindigianae) may suggest that these Asian series arose earlier than did the Asian part of ser. Bolbitianae from the African element. Whether the 'western' distribution of ser. Bolbitianae in Asia is indeed in support of this idea remains unsolved; besides, we do not know how possible differences in habitat preferences (ser. Heteroclitae and ser. Quoyanae presumably prefer a wetter habitat than does ser. Bolbitianae) could also determine the present distribution.

Ser. Euryostichae, Alienae, and Bolbitianae have closely related American and African species. This allows the assumption of a possible relationship between ser. Heudelotianae and ser. Bipinnatifidae (African) and ser. Portoricenses (American). Except for the pantropical ser. Bolbitianae, all Asian series are endemic.

Series with reticulate venation pattern occur throughout the distribution area of the genus, whereas the one series with free veins is exclusively Asian (ser. Egenolfianae). As stated elsewhere the great number of species of ser. Bolbitianae in Asia is possibly the product of relatively recent speciation. Also because of certain similarities in the ontogenies of ser. Bolbitianae and Egenolfianae it seems possible, although contrary to the commonly accepted idea that a free venation is more primitive than an anastomosing pattern, that ser. Egenolfianae is the result of recent speciation likewise.

\section{REFERENCES IN PREVIOUS CHAPTERS}

Abraham, A., C. A. Ninan \& P. M. Mathew. 1962. Studies on the cytology and phylogeny of the Pteridophytes. VII. Observations on one hundred species of South Indian ferns. J. Indian Bot. Soc. 41: 339-421, pl. 15-34.

ALsTon, A. H. G. 1956. The subdivision of the Polypodiaceae. Taxon 5: 23-25.

ARNold, C. A. 1964. Mesozoic and Tertiary fern evolution and distribution. Mem. Torrey Bot. Club 21: 5866.

AtKinson, L. R. 1973. The gametophyte and family relationships. In: Jermy, A. C., et al.: 73-90.

Banks, H. P., M. G. Collett, F. N. GNAuck \& N. F. Hughes. 1967. Pteridophyta 2. In: Harland, W. B., et al. (eds.). The fossil record: 233-245.

BEER, G. R. DE. 1951. Embryos and ancestors. Rev. ed.

BLUME, C. L. 1828. Enumeratio plantarum Javae et Insularum adjacentium. Fasc. II. Filices.

BOWER, F. O. 1917. Studies in the phylogeny of the Filicales. VI. Ferns showing the 'acrostichoid' condition, with special reference to dipterid derivatives. Ann. Bot. 31: 1-39, 2 pl.

- 1923. The Ferns (Filicales). 1. Analytical examination of the criteria of comparison.

1928. The Ferns (Filicales). 3. The leptosporangiate ferns.

Braithwaite, A. F. 1964. A new type of apogamy in ferns. New Phytol. 63: 293-305, pl. 18-20.

BRITTON, D. M. 1972. Spore ornamentation in the Dryopteris spinulosa complex. Canad. J. Bot. 50: 16171621, pl. 1-4.

BROWN, R. W. 1950. Cretaceous plants from southwestern Colorado. U.S. Geol. Surv. Prof. Paper 221-D: $45-60$.

BRUMmELEN, J. VAN. 1967. A world-monograph of the genera Ascobolus and Saccobolus (Ascomycetes, Pezizales). Persoonia 1, Suppl.: 5-260, $17 \mathrm{pl}$.

CHING, R. C. 1931. On the genus Egenolfia Schott. Bull. Fan Mem. Inst. Biol. 2: 297-317.

1932. The genus Lomagramma in America. Amer. Fern. J. 22: 15-18. 
Ching, R. C. 1933. The studies of Chinese ferns. X. Microsorium, Colysis, Leptochilus. Bull. Fan Mem.

Inst. Biol. 4: 293-354.

- 1940. On natural classification of the family 'Polypodiaceae'. Sunyatsenia 5: 201-268.

- 1958. The fern genus Plagiogyria on the mainland of Asia. Acta Phytotax. Sin. 7: 105-154, pl. 28-40.

Christ, H. 1897. Die Farnkräuter der Erde.

Christensen, C. 1904. On the American species of Leptochilus sect. Bolbitis. Bot. Tidsskr. 26: 283-297. 1906. Index Filicum.

1931. Asiatic Pteridophyta collected by Joseph F. Rock 1920-1924. Contr. U.S. Nat. Herb. 26: vi +

265-337 + vii-xii, pl. 13-29.

- 1934. Index Filicum supplementum tertium pro annis 1917-1933.

- 1938. Filicinae. In: Verdoorn, F. (ed.), Manual of Pteridology: 522-550.

Copeland, E. B. 1928. Leptochilus and genera confused with it. Philip. J. Sc. 37: 333-416, 32 pl.

- 1929. The fern genus Plagiogyria. Philip. J. Sc. 38: 377-417, 15 pl.

- 1941. Comment on natural classification of the family Polypodiaceae by R. C. Ching. Sunyatsenia 6: $159-177$.

1947. Genera Filicum. Chronica Botanica Co.

1955. New Philippine ferns. XI. Philip. J. Sc. 84: 161-165, 2 pl.

1960. Fern flora of the Philippines 2.

Cotthem, W. van. 1968. Vergelijkend-morfologische studie van de stomata bij de Filicopsida. Proefschrift, Rijksuniversiteit Gent.

Devi, S. 1963. The juvenile leaves of Bolbitis. Proc. Indian Acad. Sc. sect. B, 58: 267-273.

Dickason, F. G. 1946. A phylogenetic study of the ferns of Burma. Ohio J. Sc. 46: 73-141.

Diels, L. 1899. Polypodiaceae. In: Engler, A. \& K. Prantl, Die Natürlichen Pflanzenfamilien i 4:139-339.

- 1906. Jugendformen und Blütenreife im Pflanzenreich.

ERdTMan, G. 1945. Pollen morphology and plant taxonomy. V. On the occurrence of tetrads and dyads.

Svensk Bot. Tidskr. 39: 286-297.

— 1957. Pollen and spore morphology/plant taxonomy. Gymnospermae, Pteridophyta, Bryophyta.

(Illustrations).

- 1969. Handbook of palynology, morphology, taxonomy, ecology. An introduction to the study of pollen grains and spores.

— \& P. Sorsa (with special chapters by J. PetTitT and B. Gullv\&G). 1971. Pollen and spore morphology/plant taxonomy. Pteridophyta. (Text and additional illustrations).

ETTINGSHAUSEN, C. voN. 1865. Die Farnkräuter der Jetzwelt.

Evans, A. M. 1969. Problems of apomixis and the treatment of agamic complexes. BioScience 19: 708-711.

FABBri, F. 1963. Primo supplemento alle Tavole chromosomiche delle Pteridophyta di Alberto Chiarugi. Caryologia 16: 237-335.

FÉe, A. L. A. 1845. Deuxième Mémoire: Histoire des Acrostichées.

— 1850-1852. Genera Filicum (Cinquième Mémoire sur la famille des Fougères).

GaRSTANG, W. 1922. The theory of recapitulation: A critical restatement of the biogenetic law. J. Linn.

Soc. Zool. 35: 81-101.

GiesenhaGeN, K. vON. 1918. Über einen seltsamen Farn der Flora von Ceylon. Flora 111-112: 294-316.

GoEBEL, K. 1922. Gesetzmässigkeiten im Blattaufbau. Bot. Abh. 1: 1-78.

HAECKel, E. 1866. Generelle Morphologie der Organismen. Allgemeine Grundzüge. 1. Allgemeine Anatomie der Organismen; 2. Allgemeine Entwicklungsgeschichte der Organismen.

HARDY, A. C. 1954. Escape from specialization. In: Huxley, J., et al.(eds.), Evolution as a process: 122-142.

HARRIs, T. M. 1973. What use are fossil ferns? In: Jermy, A. C., et al.: 41-44.

Hennipman, E. 1965. Pteridoblechnum, a new genus of blechnoid ferns from Australia. Blumea 13: 397-403.

- 1970. Electron and light microscopical observations on the perine of the spores of some Bolbitis species (Filices). Acta Bot. Neerl. 19: 671-680.

— 1976. A second species of Pteridoblechnum (Blechnaceae) formerly referred to Leptochilus acuminatus

C. T. White \& Goy. Proc. R. Soc. Qd 87: 95-98.

HoltTUM, R. E. 1937. The genus Lomagramma. Gard. Bull. Sing. 9: 190-221.

- 1946. A revised classification of leptosporangiate ferns. J. Linn. Soc. Bot. 53: 123-186.

- 1949. The classification of ferns. Biol. Rev. 24: 267-296.

- 1954. A revised flora of Malaya. 2. Ferns of Malaya.

- 1967. Comparative morphology, taxonomy and evolution. Phytomorphology 17: 36-41.

- 1968. A re-definition of the fern-genus Taenitis Willd. Blumea 16: 86-95. 
Holtrum, R. E. 1977? Lomariopsis group. Flora Malesiana ser. II, 1, 4. In preparation.

HoOKeR, W. J. 1861. A second century of ferns.

- 1864. Species Filicum. 5.

\& J. G. BAKER. 1865-1868. Synopsis Filicum.

IWATsUKI, K. 1959. Taxonomic studies of Pteridophyta IV. 4. Emendation of Bolbitis, with special reference to the Far Eastern species. Acta Phytotax. Geobot. 18: 44-59.

Jermy, A. C., J. A. Crabbe \& B. A. Thomas. 1973. The phylogeny and classification of the ferns. J. Linn. Soc. Bot. 67, Suppl. 1.

KAULFUSS, G. F. 1824. Enumeratio Filicum.

KAUR, S. 1974. The family Lomariopsidaceae (Filicopsida) and its probable ancestors. J. Linn. Soc. Bot. 68: $153-162$.

KaWANo, S. 1965. Application of pectinase and cellulase in an orcein squash method. Bot. Mag. Tokyo 78: 36-42.

KLEKOWSKI Jr, E. J., \& H. G. BAKER. 1966. Evolutionary significance of polyploidy in the Pteridophyta. Science 153: 305-307.

KRAmer, K. U. 1954. A contribution to the fern flora of French Guiana. Acta Bot. Neerl. 3: 481-494.

1957. A revision of the genus Lindsaea in the New World with notes on allied genera. Acta Bot. Neerl. 6: $97-290$.

KURTA, S. 1965. Chromosome numbers of some Japanese ferns (4). J. Jap. Bot. 40: 234-244.

Lovis, J. D. 1973. A biosystematic approach to phylogenetic problems and its application to the Aspleniaceae. In: Jermy, A. C., et al.: 211-228, pl. 1 \& 2.

Lugardon, B. 1965. Structure des parois de la spore de Blechnum spicant (L.) Roth. Pollen et Spores 7: 409$428,7 \mathrm{pl}$.

- 1972. La structure fine de l'exospore et de périspore des filicinées isosporés. I. Géneralités. Eusporangiées et Osmundales. Pollen et Spores 14: 227-261.

1974. La structure fine de l'exospore et de périspore des filicinées isosporées. II. Filicales. Commentaires. Pollen et Spores 16: 161-226.

Manton, I. 1950. Problems of cytology and evolution in the Pteridophyta.

1953. The cytological evolution of the fern flora of Ceylon. Symp. Soc. Exp. Biol. 7 (Evolution): 174 185.

1954. Cytological notes on one hundred species of Malayan ferns. In: Holttum, R. E., A revised flora of Malaya. 2. Ferns of Malaya: 623-627, pl. 1-3.

1959. Cytological information on the ferns of West Tropical Africa. In: Alston, A. H. G., The ferns and fern-allies of West Tropical Africa: 75-81.

\& W. A. SLEDGE. 1954. Observations on the cytology and taxonomy of the Pteridophyte flora of Ceylon. Phil. Trans. R. Soc. Lond. ser. B, 238: 127-185, pl. 3-14.

Marchal, M. 1965. Le bourgeonnement epiphylle spontané des fougères tropicales. Adansonia 5: 239-270.

MAXON, W. R., \& C. V. MORTON. 1938. The American species of Dryopteris, subgenus Meniscium. Bull. Torrey Bot. Club 65: 347-376.

Morron, C. V. 1971. The proper disposition of Meniscium macrophyllum Kunze. Amer. Fern J. 71: 1720.

NAYAR, B. K., \& S. KaUR. 1963. Spore morphology of some species of Lomariopsidaceae. Pollen et Spores 5: $87-94$

- \& 1964a. Ferns of India. XI. Bolbitis. Bull. Nat. Bot. Gard. Lucknow 88; 1-74.

- 1964b. Ferns of India. XIII. Egenolfia. Bull. Nat. Bot. Gard. Lucknow 100: 1-38.

\& 1965a.. Studies on the fern genera Bolbitis and Egenolfa. I. Morphology of the sporophytes. J. Linn. Soc. Bot. 59: 127-140, 2 pl.

\& 1965b. Studies on the fern genera Bolbitis and Egenolfia. II. The gametophytes and juvenile sporophytes. J. Linn. Soc. Bot. 59: 141-154, 2 pl.

- \& 1965c. Spore morphology of the Lomariopsidaceae. J. Palynol. 1: 10-26.

- 1966. Morphology of the fertile leaves of the Lomariopsidaceae, with special reference to the venation. New Phytol. 65: 221-239, pl. 9 \& 10.

PE - 1971. Gametophytes of homosporous ferns. Bot. Rev. 37: 295-396.

Petritr, J. M. 1966. Exine structure in some fossil and recent spores and pollen as revealed by light and electron microscopy. Bull. Br. Mus. Nat. Hist. A. Geol. 13: 221-257, pl. 1-21.

Pichi Sermolu, R. E. G. 1968. Adumbratio Florae Aethiopicae. 15. Elaphoglossaceae. Webbia 23: 209246, map 1 \& 2. 
Pichi Sermolli, R. E. G. 1969. Adumbratio Florae Aethiopicae. 18. Lomariopsidaceae. Webbia 23: 379-396, map 5.

PresL, C. B. 1836. Tentamen Pteridographiae. 1851. Epimeliae botanicae.

Roy, S. K., \& R. E. HoltTUM. 1965. Cytological observations on ferns from southern China. Amer. Fern J. 55: $154-158$.

- \& I. MANTON. 1966. The cytological characteristics of the fern subfamily Lomariopsidoideae sensu Holttum. J. Linn. Soc. Bot. 59: 343-347, pl. 1 \& 2.

SCHOTT, H. 1834-1836. Genera Filicum.

SchumanN, E. 1915. Die Acrosticheen und ihre Stellung im System der Farne. Flora 108: 201-260.

SLEDGE, W. A. 1972. The tectarioid ferns of Ceylon. Kew Bull. 27: 407-424.

SMITH, A. R. 1972. Comparison of fern and flowering plant distribution with some evolutionary interpretations for ferns. Biotropica 4: 4-9.

SMITH, J. 1841. An arrangement and definition of the genera of ferns. J. Bot. (Hooker) 4: 147-198, pl. $17 \& 18$.

1875. Historia Filicum.

SOTA, E. DE LA. 1973. On the classification and phylogeny of the Polypodiaceae. In: Jermy, A. C., et al: 229-244.

StebBins Jr, G. L. 1950. Variation and evolution in plants.

- 1959. The role of hybridization in evolution. Proc. Am. Phil. Soc. 103: 231-251.

- 1971. Chromosomal evolution in higher plants.

Steenis, C. G. G. J. van. 1948. General considerations. Flora Malesiana ser. I, 4: xiii-lxix.

SwarTZ, O. 1806. Synopsis Filicum.

Takhtajan, A. 1959. Die Evolution der Angiospermen.

TARDIEU-BLOT, M. L. 1966. Sur les spores de fougères Malgaches: Filicales (fin), Marattiales, Ophioglossales (1). Pollen et Spores 8: 75-122.

Tidwell, W. D., S. R. Rusforth \& J. L. ReVEAL. 1967. Astralopteris, a new Cretaceous fern genus from Utah and Colorado. Brigham Young Univ. Geol. Stud. 14: 237-240, pl. 1-6.

TRYON, A. F. 1971. Structure and variation in spores of Thelypteris palustris. Rhodora 73: 444-460.

Tyron Jr, R. M. 1966. Evolution in the leaf of living ferns. Mem. Torrey Bot. Club 21: 73-85.

\& A. F. TRYON. 1973. Geography, spores and evolutionary relations in the cheilanthoid ferns. In:

Jermy, A. C., et al.: 145-153, pl. 1-3.

UNDERWOOD, L. M. 1903. A summary of our present knowledge of the ferns of the Philippines. Bull. Torrey

Bot. Club 30: 665-684.

WAGNER Jr. W. H. 1952. Types of foliar dichotomy in living ferns. Amer. J. Bot. 39: 578-592.

- 1954. Reticulate evolution in the Appalachian Aspleniums. Evolution 8: 103-118.

- 1962. Irregular morphological development in hybrid ferns. Phytomorphology 12: 87-100.

1966. Modern research on evolution in the ferns. In: Jensen, W. A. \& L. G. Kavaljian (eds.), Plant

biology today, 2nd ed.: 164-184.

- 1968. Hybridization, taxonomy and evolution. In: Heywood, V. H. (ed.), Modern methods in plant taxonomy: 113-138.

1970. Biosystematics and evolutionary noise. Taxon 19: 146-151.

1973. Some future challenges of fern systematics and phylogeny. In: Jermy, A. C., et al.: 245-256. 1974. Structure of spores in relation to fern phylogeny. Ann. Mo. Bot. Gard. 61: 332-353.

- \& K. Lim ChEN. 1965. Abortion of spores and sporangia as a tool in the detection of Dryopteris hybrids. Amer, Fern. J. 55: 9-29.

WALKER, T. G. 1958. Hybridization in some species of Pteris L. Evolution 12: 82-92.

- 1966. A cytotaxonomic survey of the Pteridophytes of Jamaica. Trans. R. Soc. Edinb. 66: 169-237, pl. 1-5.

1973. Evidence from cytology in the classification of ferns. In: Jermy, A. C., et al:: 91-110.

WET, J. M. J. DE, \& H. T. STALKER. 1974. Gametophytic apomixis and evolution in plants. Taxon 23:689697.

WILLDENow, C. L. 1810. Caroli a Linné species plantarum. 4.

Williams, S. 1927. A critical examination of the Vittarieae with a view to their systematic comparison.

Trans. R. Soc. Edinb. 55: 173-217, 3 pl.

Woon, C. C. 1973. Spore variation in Thelypteridaceae. In: Jermy, A. C., et al.: 191-202, pl. 1-4.

Zimmermann, W. 1930. Die Phylogenie der Pflanzen. Ein Uberblick über Tatsachen und Probleme.

— 1959. Die Phylogenie der Pflanzen. Ein Überblick über Tatsachen und Probleme. 2. Aufl. 


\section{Plates}



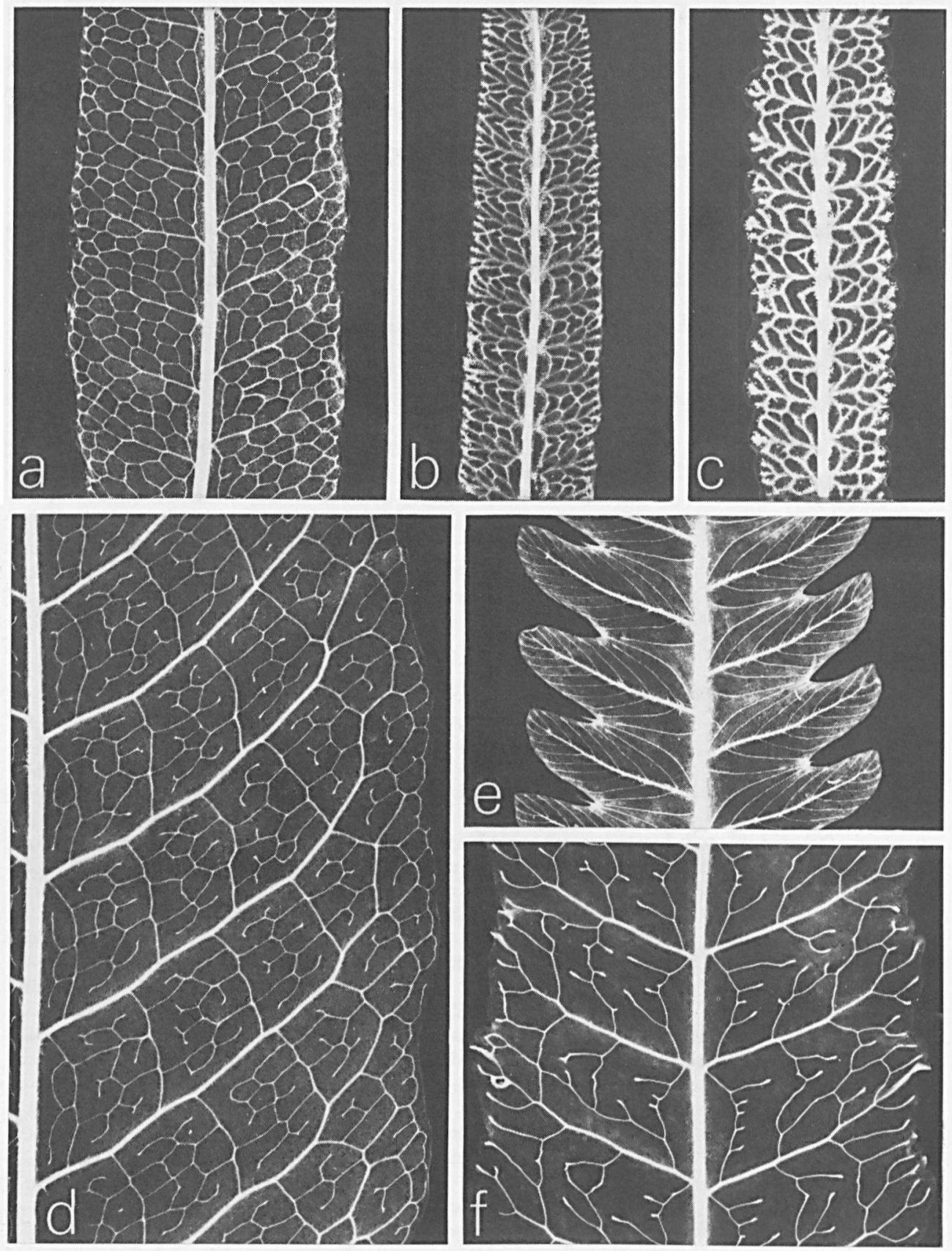

Plate 1 

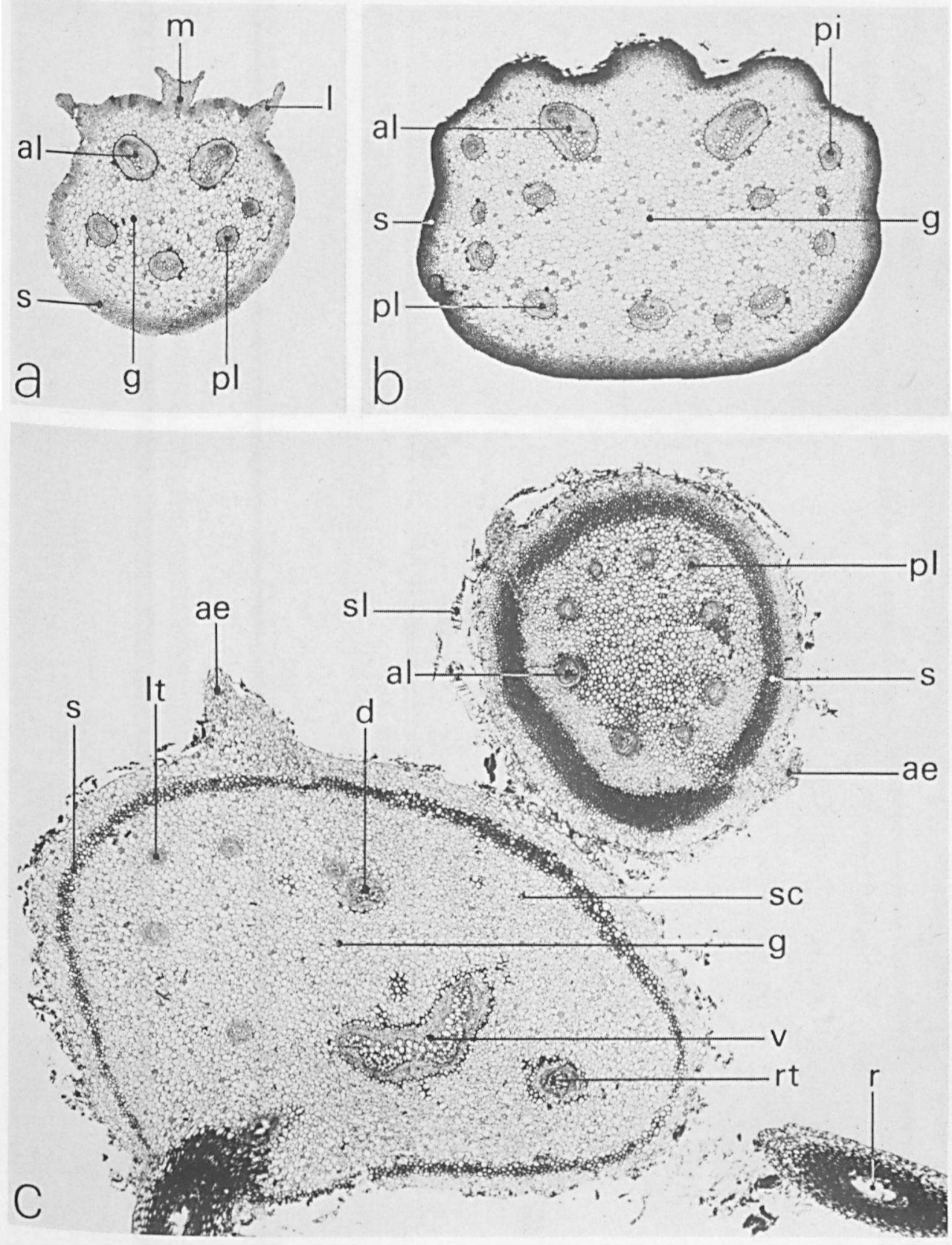

Plate 2 

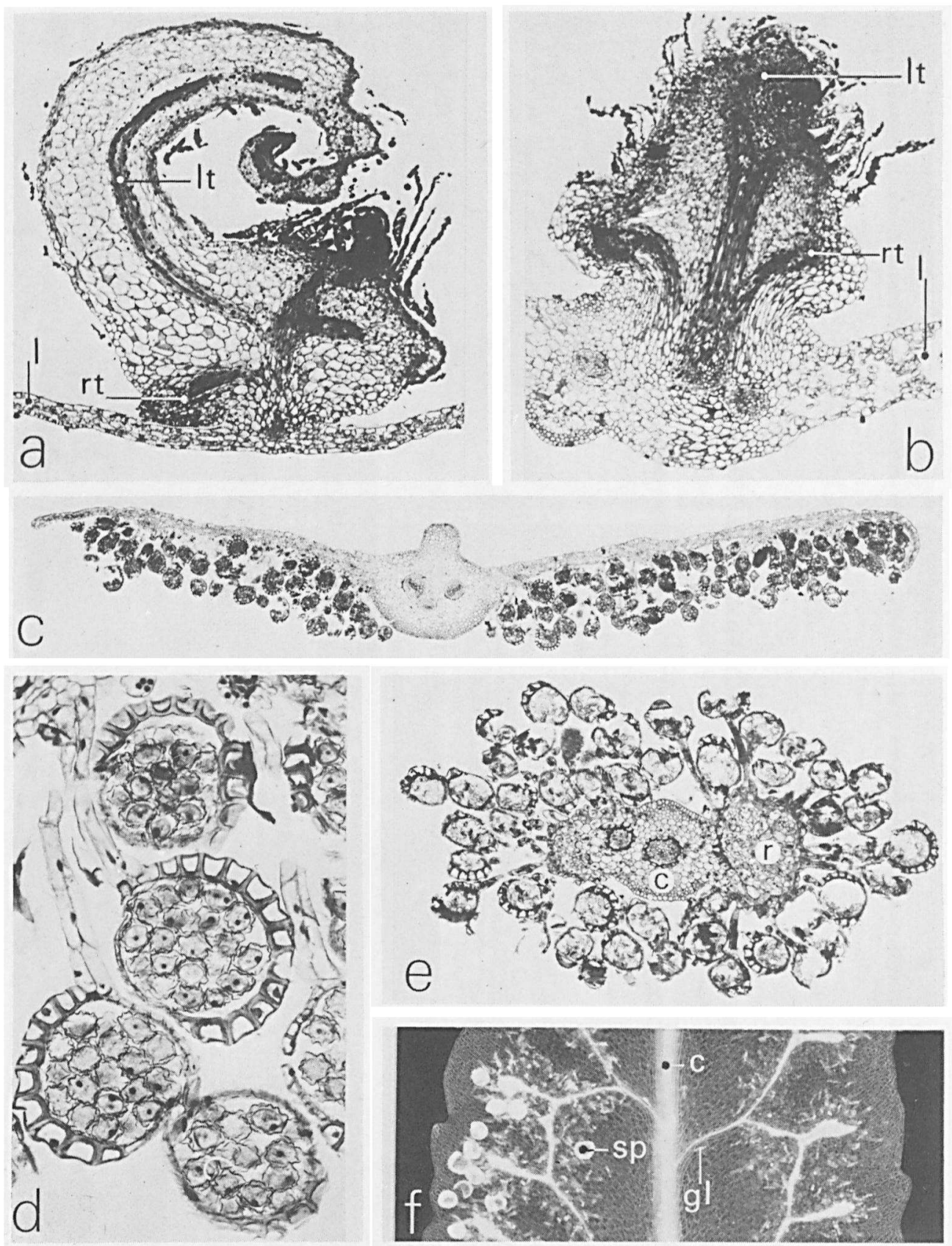

Plate 3 

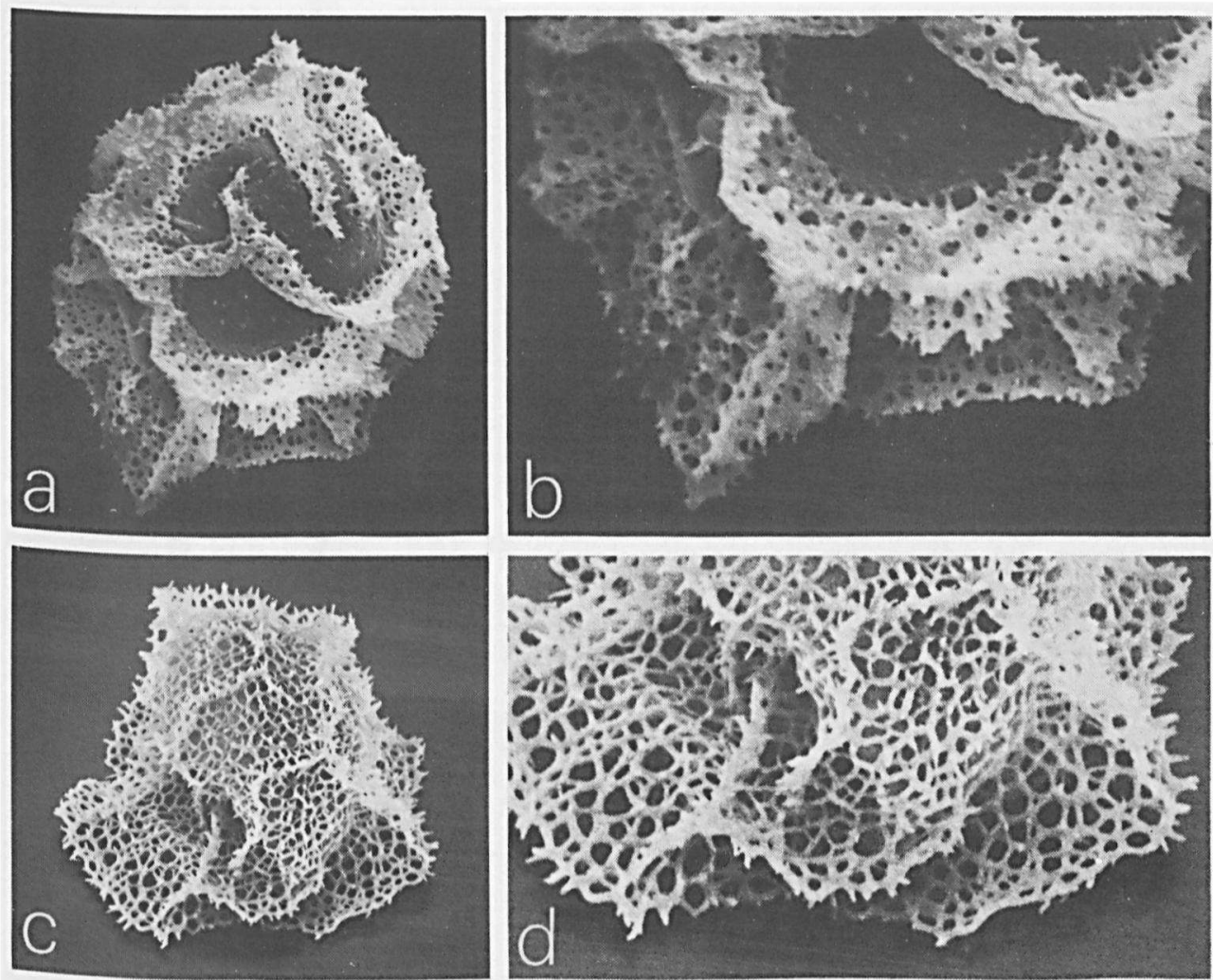

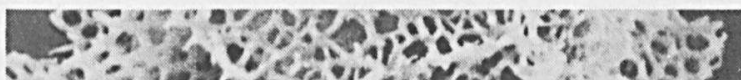

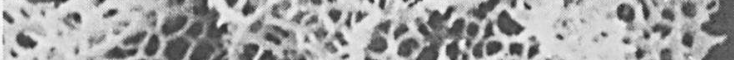

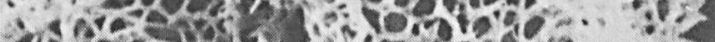

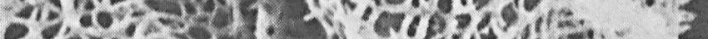

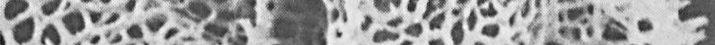
i. P.

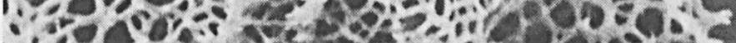

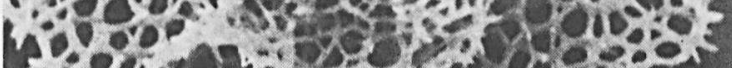

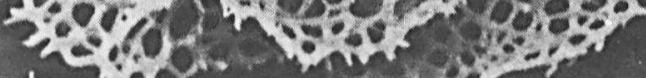

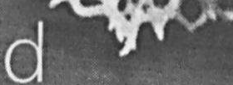

$$
\operatorname{lig}_{2} v^{2}-v^{2}
$$
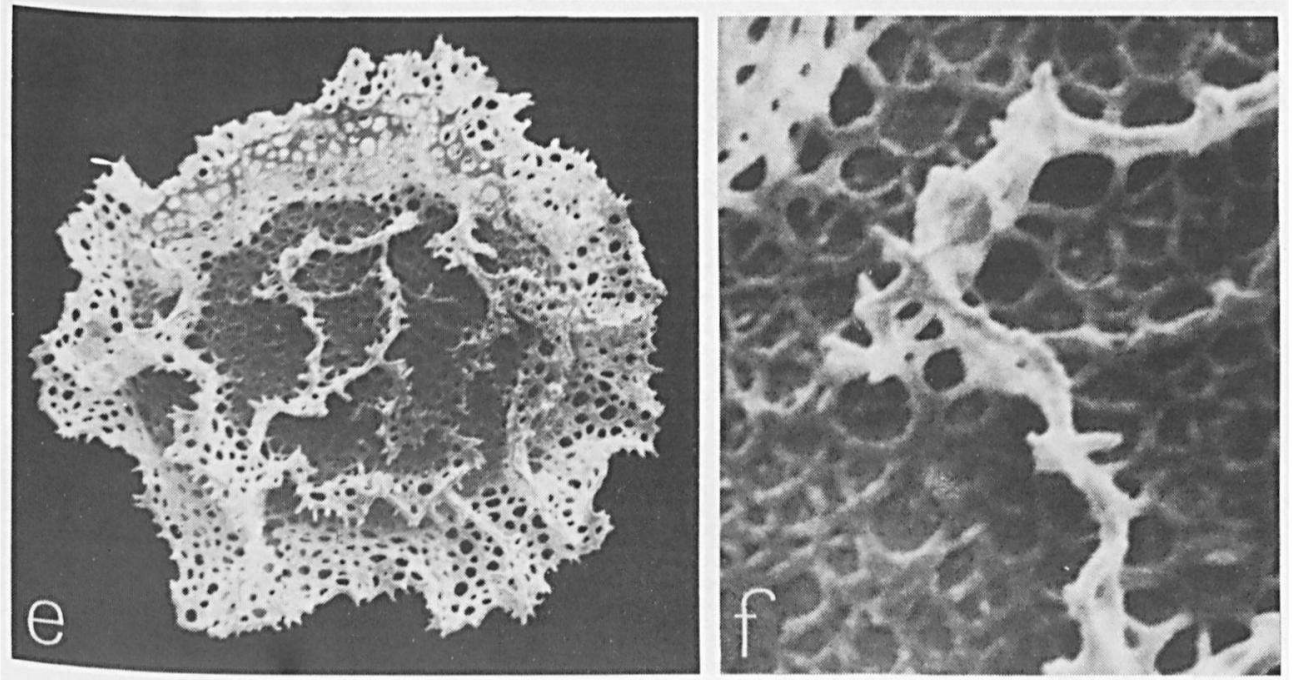

Plate 4 


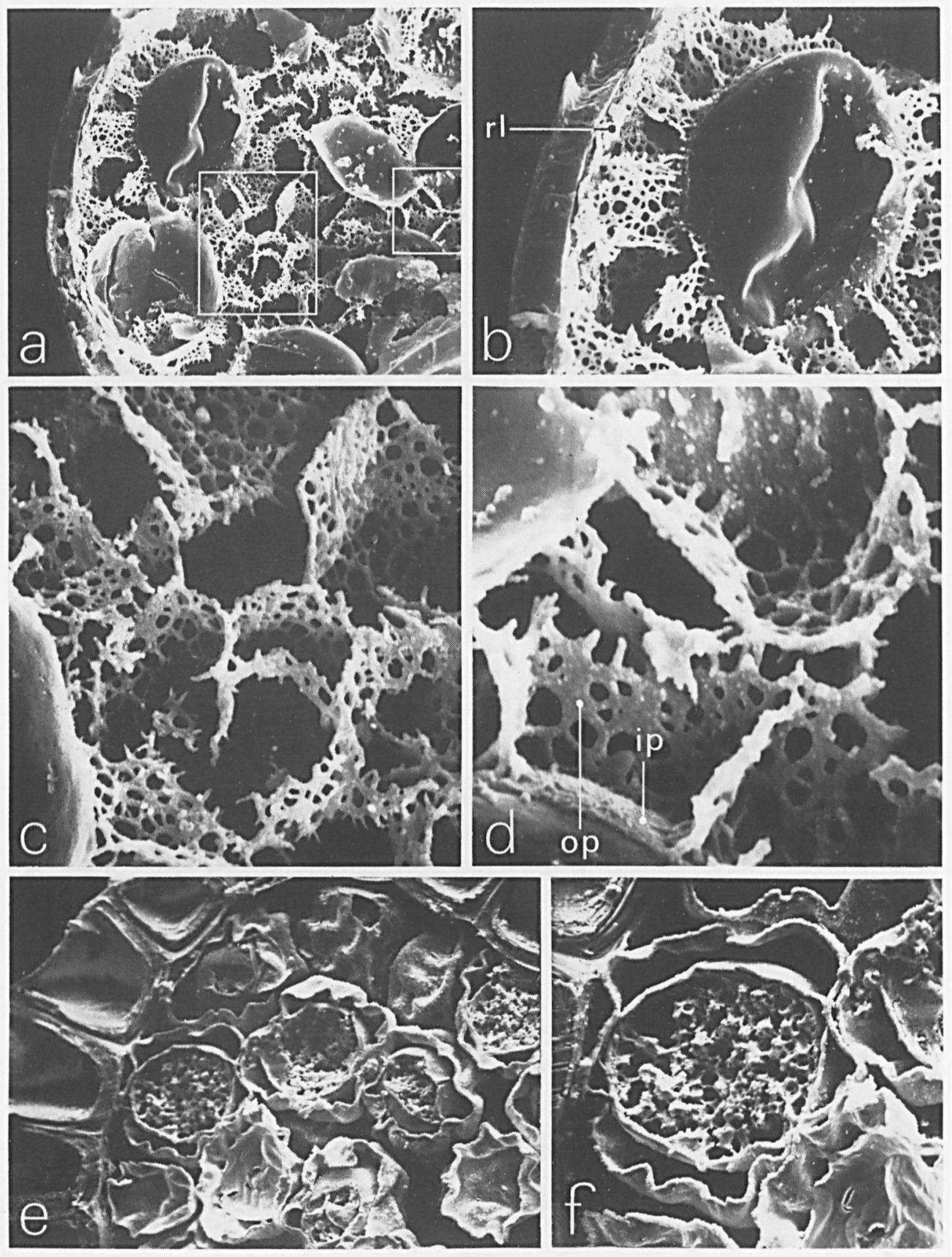

Plate 5 

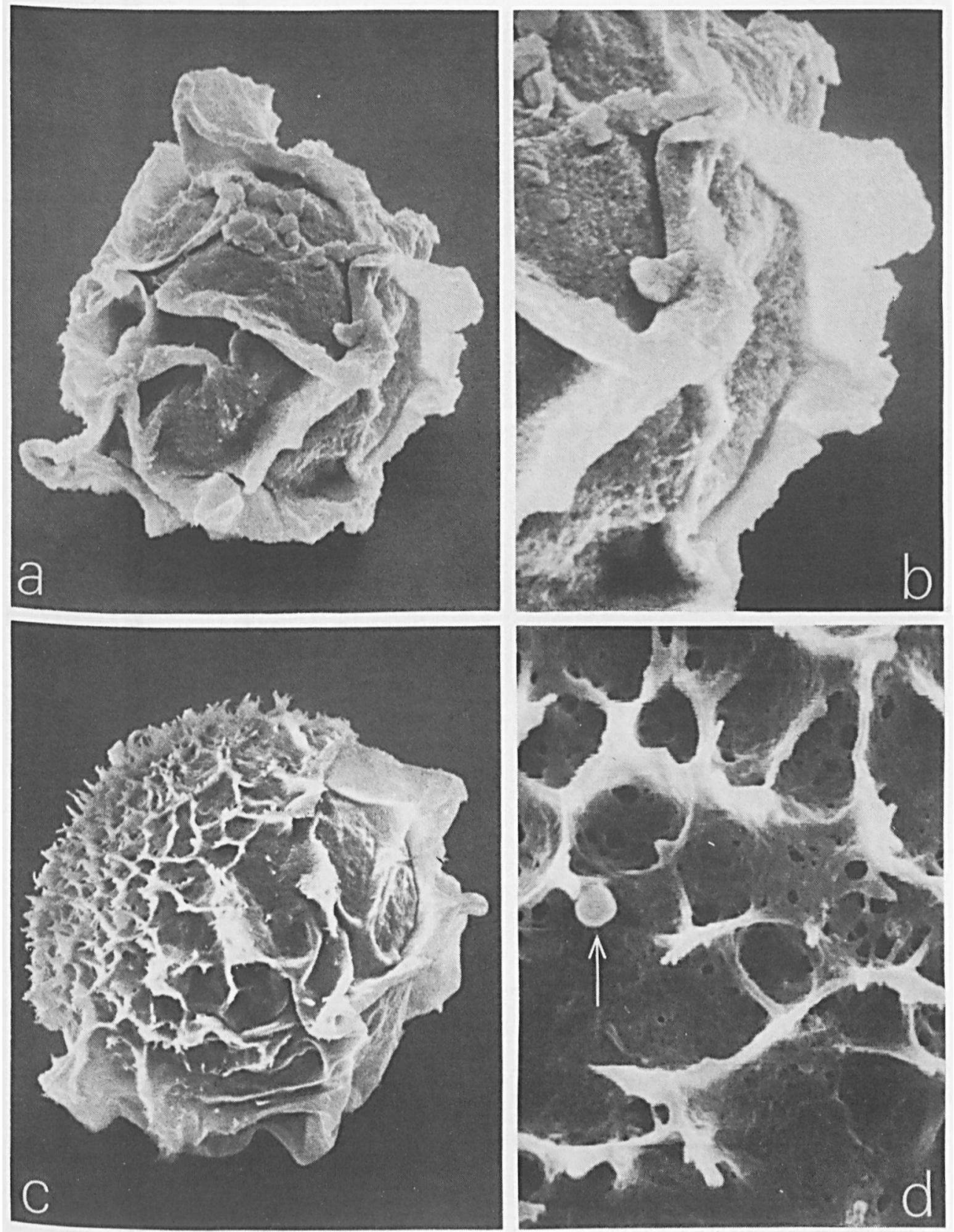

Plate 6 

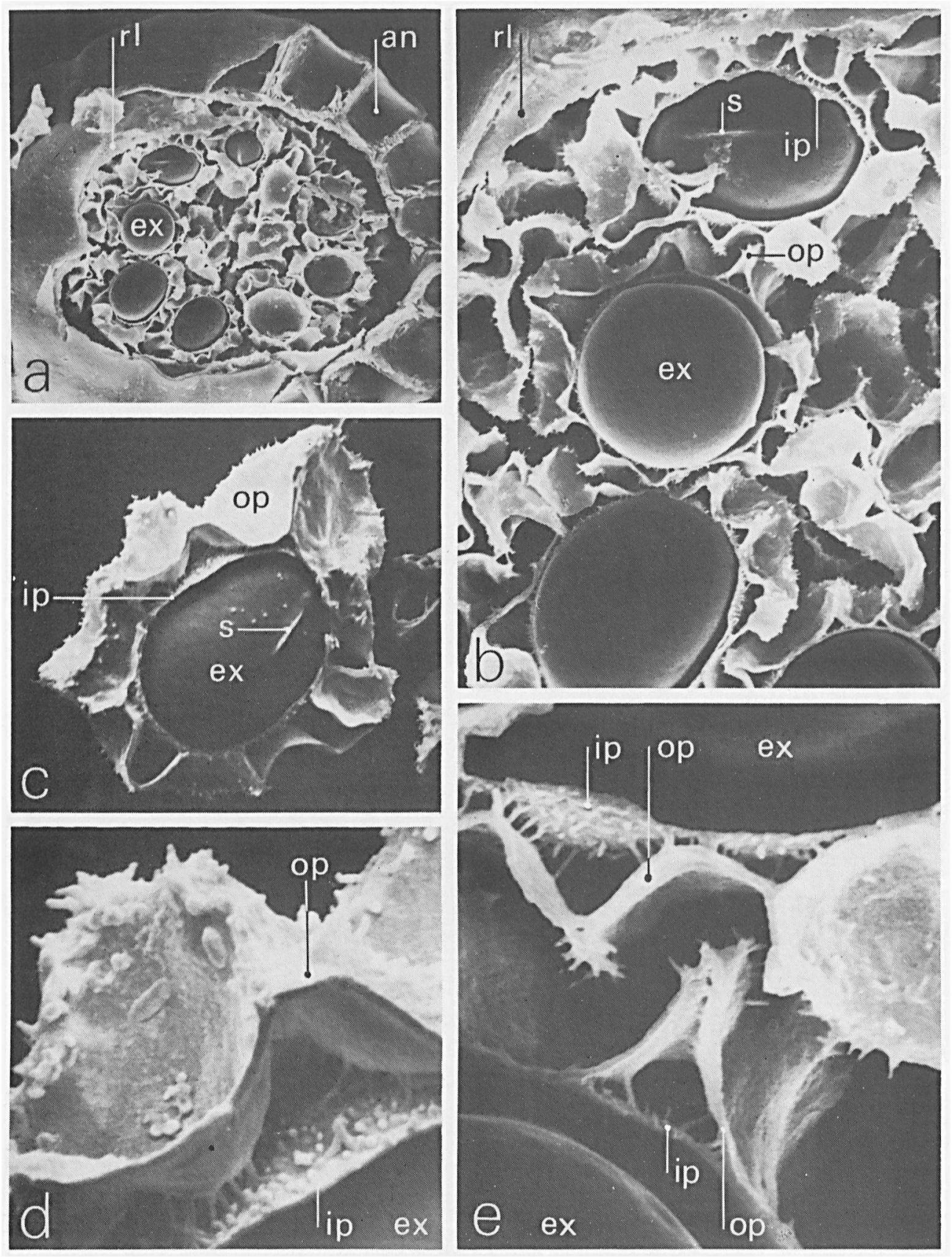

Plate 7 

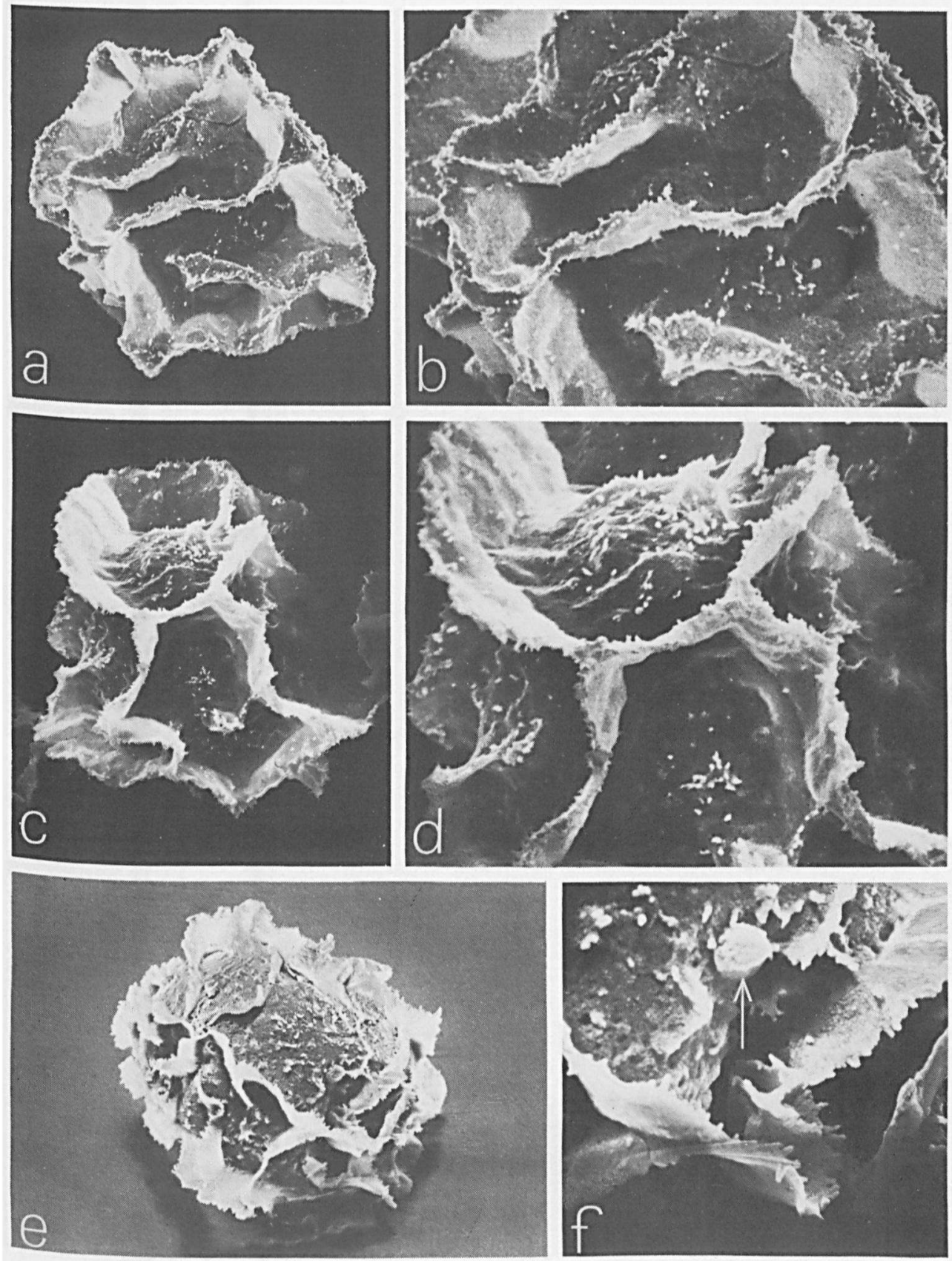

Plate 8 

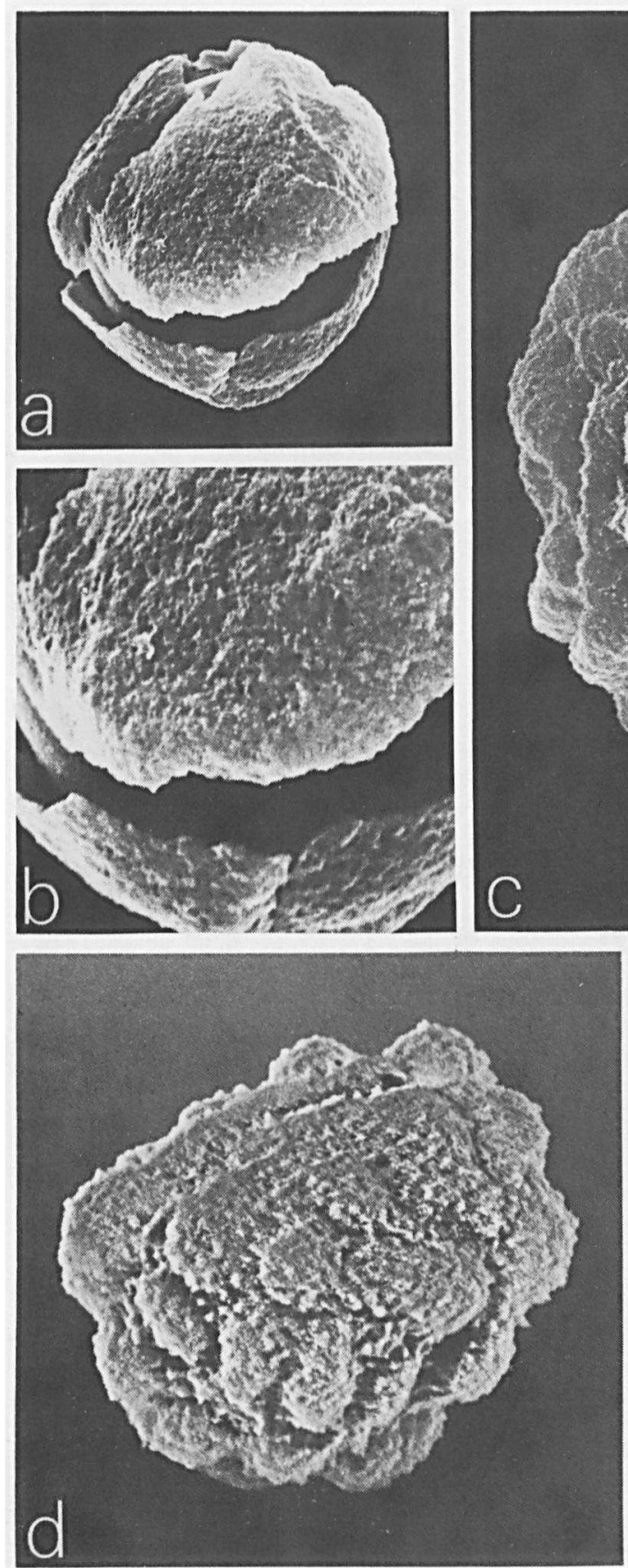
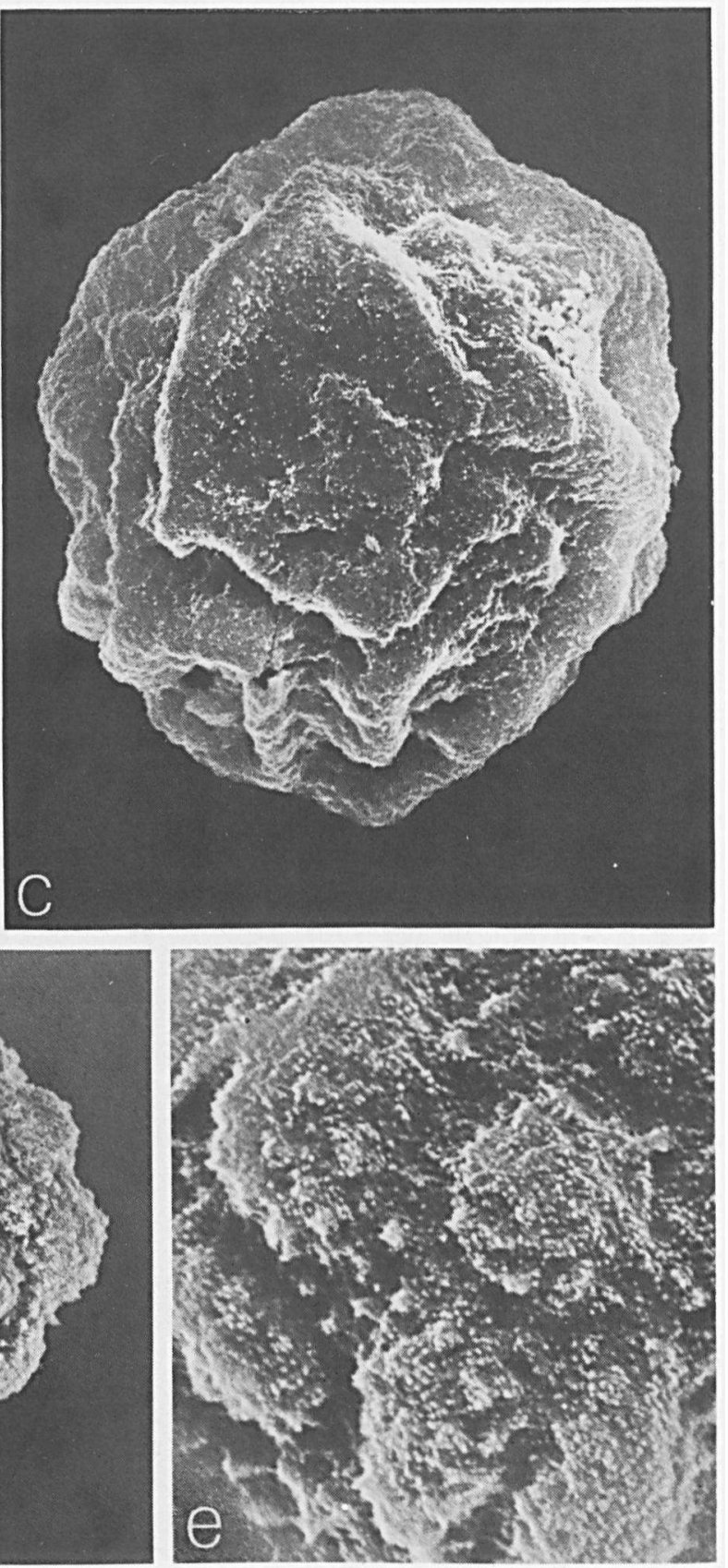

Plate 9 

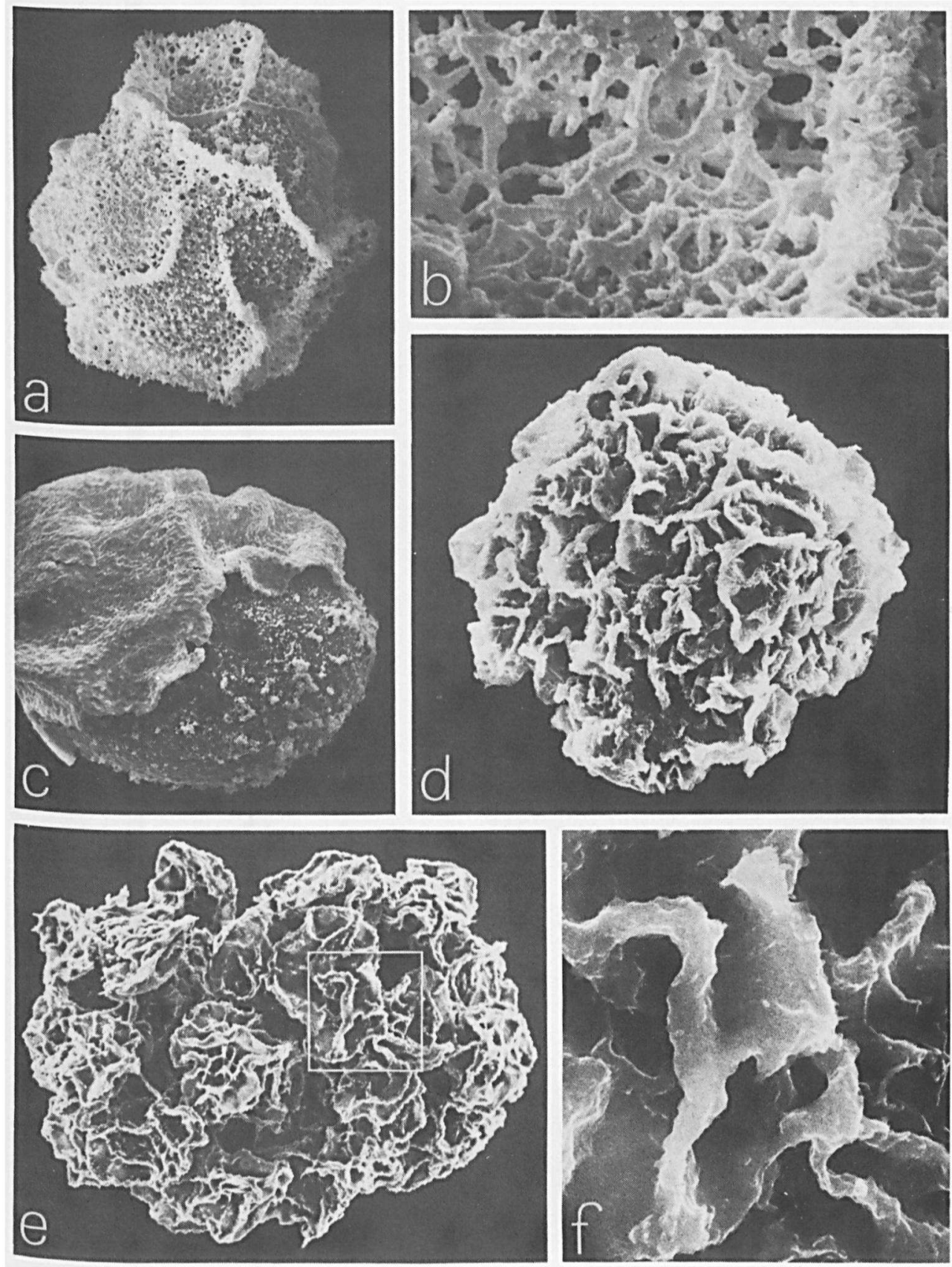

Plate 10 

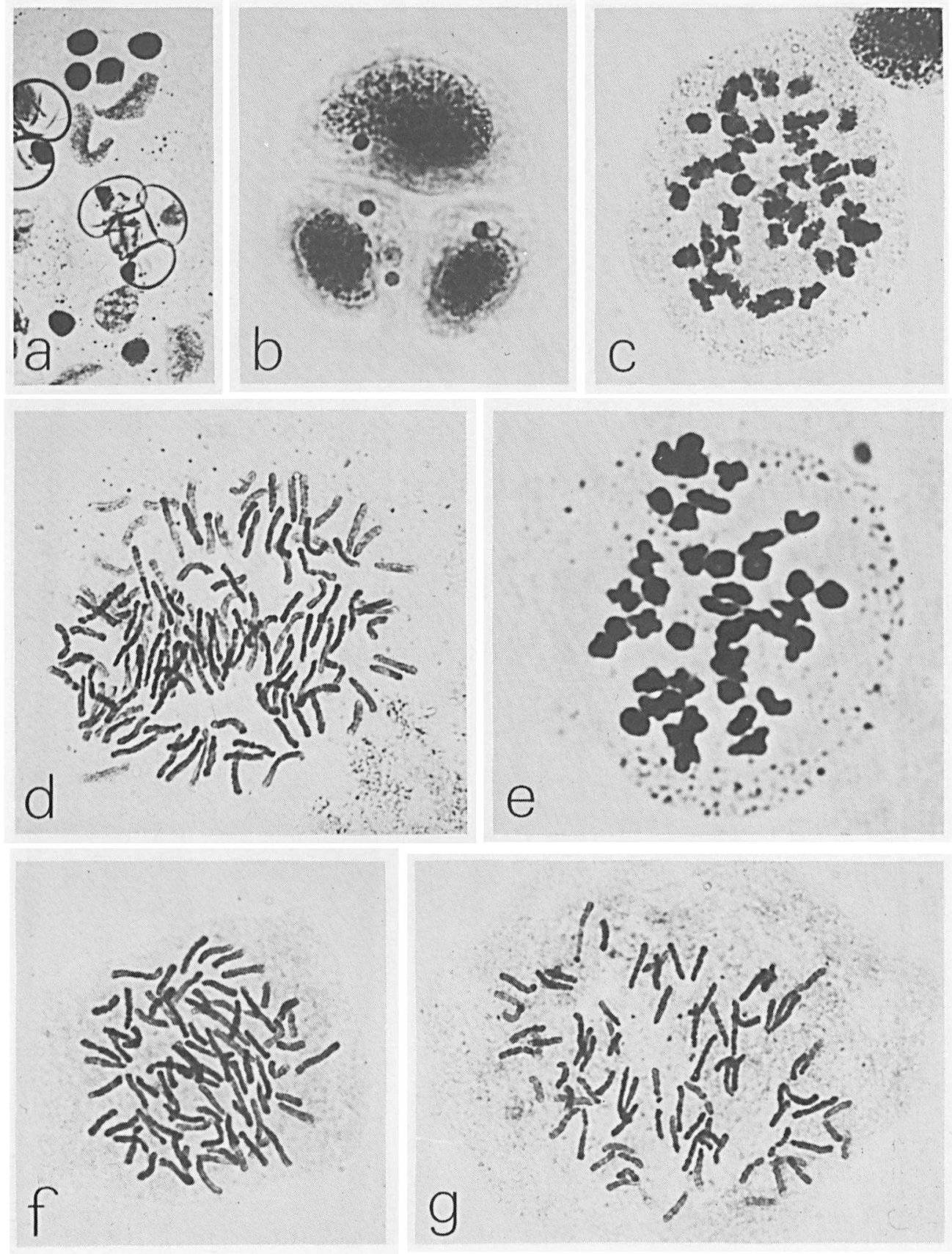

Plate 11 

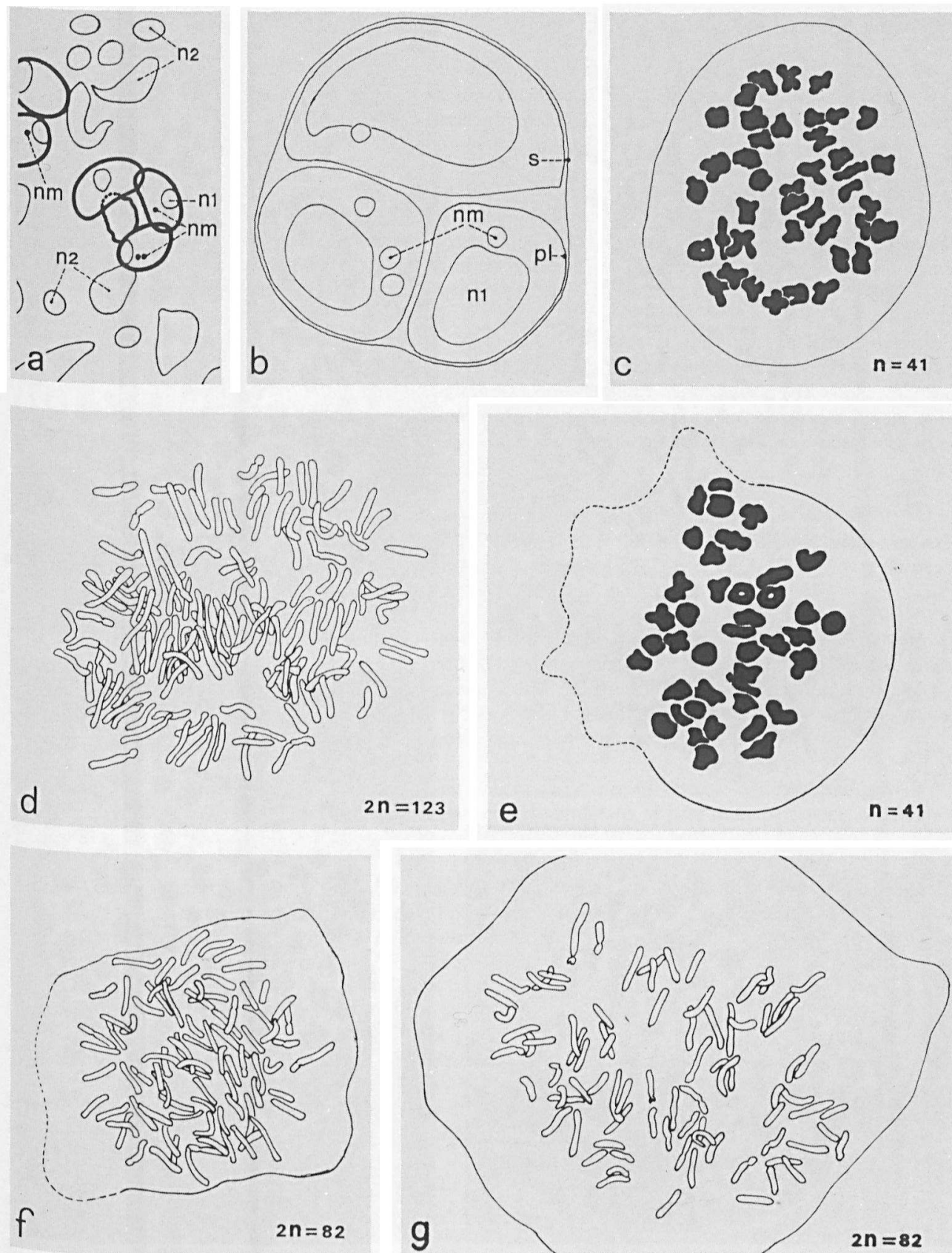

$2 n=123$
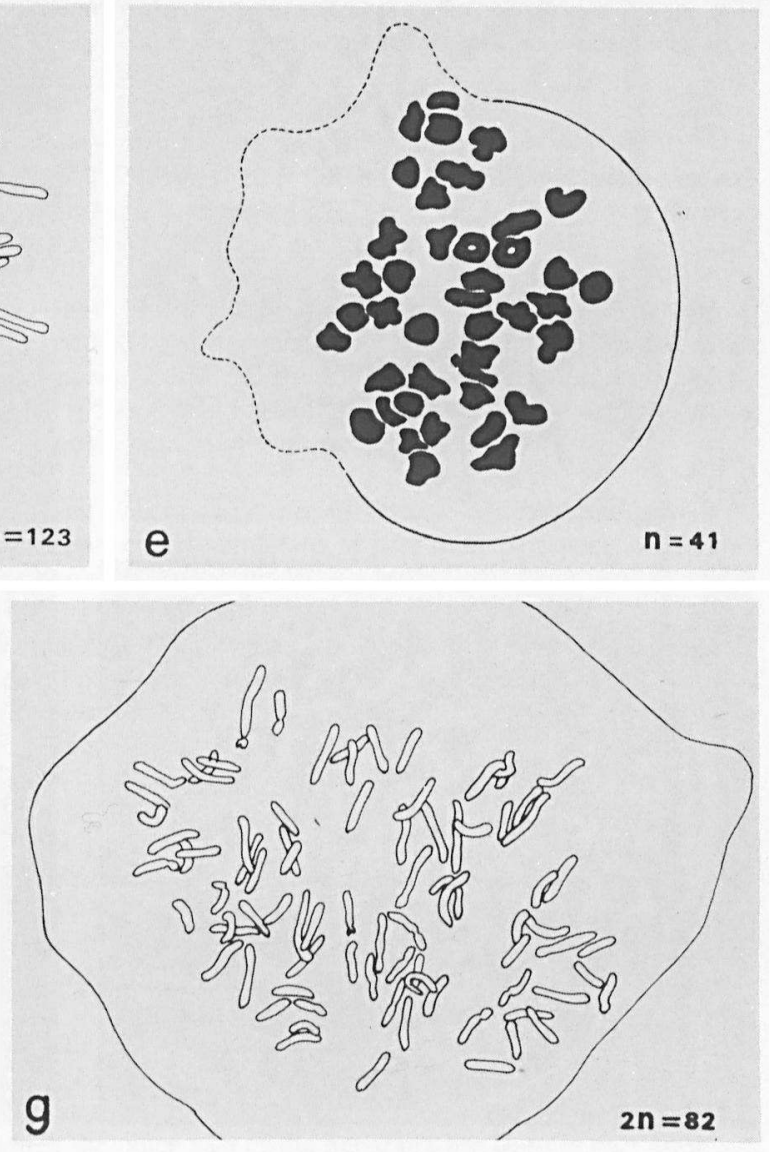

Fig. 31 


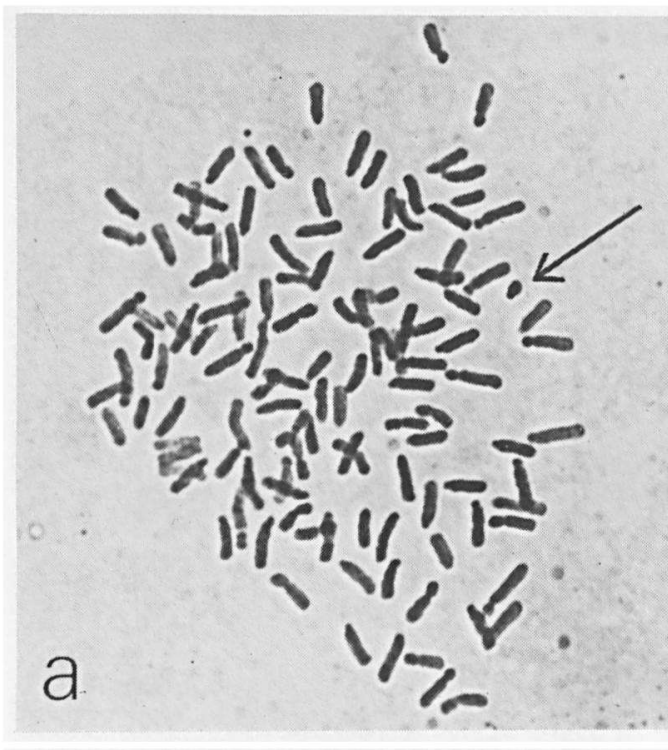

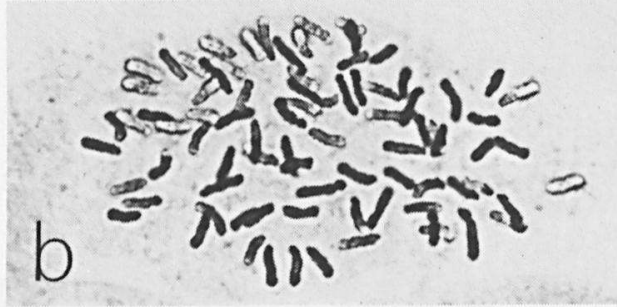

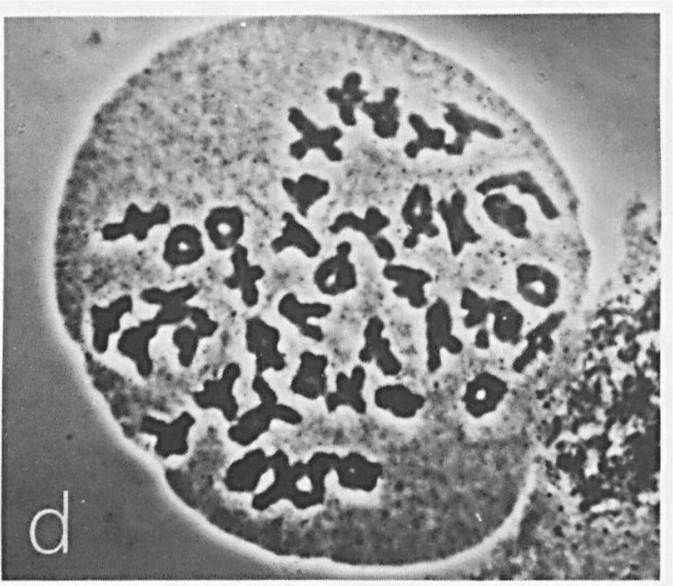

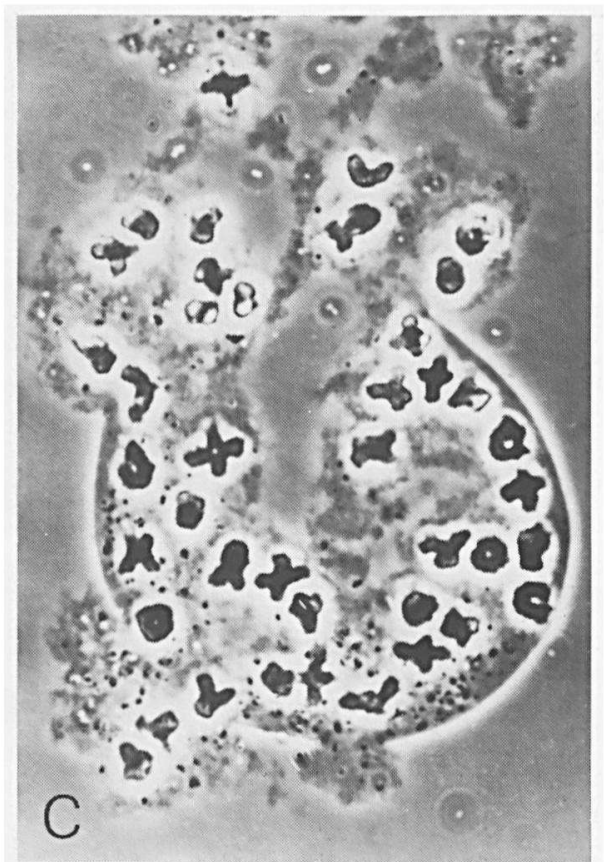

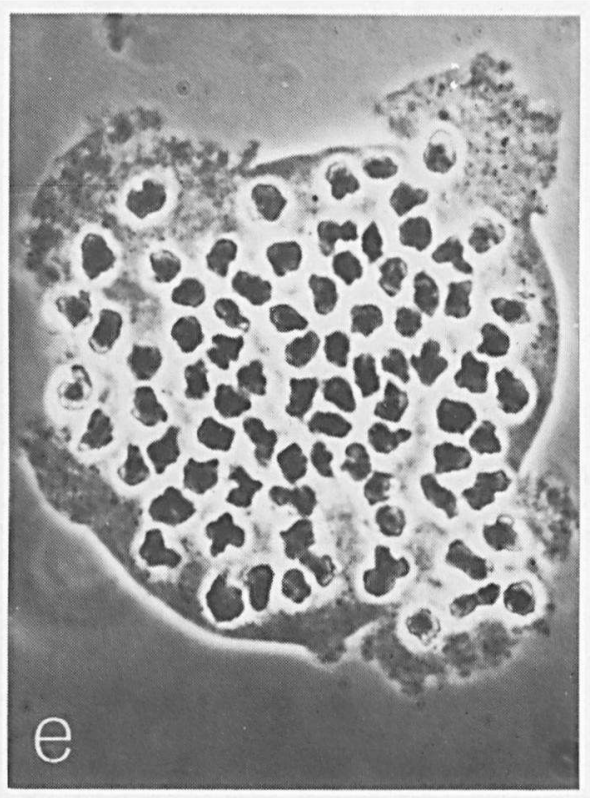

Plate 12 


\section{EXPLANATION OF PLATES}

Plate 1. a-c. Venation patterns and sporangial insertion of central parts of fertile pinnae, $\times 2$. a. Bolbitis auriculata; b. B. serratifolia; c. B. acrostichoides (a. Kew Distr. no. 1175, L; b. Balansa 2852, L; c. Young 547, L):-d-f. Venation patterns of central parts of sterile pinnae, $\times 2$. d. B. nicotianifolia; e. B. major; f. B. bernoullii (d. Maxon 9316, US; e. Levinge s.n., P; f. Greeves \& Ray 490, U).

Plate 2. Cross-sections showing anatomy, $\times 12 .-$ a. Bolbitis sinensis, rhachis - b. B. angustipinna, rhachis $-c$. B. sinuata, rhizome and petiole near rhizome apex (a. Hennipman 3229, L; b. H. 3536, L; c. H. 4052, L). ae = aerenchym, al $=$ anterior leaf trace, $d=$ dorsal bundle, $g=$ ground tissue, $1=$ lateral ridge, $l t=$ leaf trace, $\mathrm{m}=$ median ridge, $\mathrm{pi}=$ pinna trace, $\mathrm{pl}=$ posterior leaf trace, $\mathrm{r}=$ root, $\mathrm{rt}=$ root trace, $\mathrm{s}=$ sclerenchyma sheath, $\mathbf{s c}=$ sclerenchyma strands of ground tissue, $\mathrm{sl}=\mathrm{scale}, \mathrm{v}=$ ventral bundle.

Plate 3. a, b. Cross-sections of laminar bulbils, $\times 24$. a. Bolbitis repanda; b. B. sinuata (a. Hennipman 4023, L; b. H. 4052, L). 1 = lamina, $\mathrm{lt}=$ leaf trace, $\mathrm{rt}=$ root trace.-c-f. Fertile pinnae. c, d. Bolbitis angustipinna. c. cross-section of pinna, $\times 16$; $d$. ibid. of sporangia, $\times 125 .-\mathrm{e}$. Bolbitis appendiculata var. vivipara, crosssection of fertile pinna, $\times 30$. - f. Bolbitis crispatula, projection photograph of part of cleared pinna, $\times 14$ (c, d. Hennipman 3536, L; e. H. 3488, L; f. H. 3632, L). c = costa, gl = glandular hair, $\mathrm{r}=$ receptacle, $\mathrm{sp}=$ sporangium.

Plate 4. Spores. -a, b. Bolbitis appendiculata ssp. appendiculata. a. $\times 1000$; b. detail of a, $\times 2000 .-\mathrm{c}, \mathrm{d}$. B. appendiculata ssp. vivipara var. vivipara. c. $\times 500$; d. detail of c, $\times 1000 .-\mathrm{e}$, f. B. appendiculata ssp. vivipara var. neglecta. e. $\times 1000$; f. detail of e, $\times 4000$ (a, b. Hennipman 3527; c, d. H. 3119; e, f. Van Borssum Waalkes $603, \mathrm{~L}$ ).

Plate 5. Cross-sections of mature sporangia.-a-d. Bolbitis appendiculata ssp. appendiculata. a. part of sporangium, $\times 500$; $b$. detail of a, showing the residual layer connected with crests of the perispore, $\times 1000$; c. detail of (interconnecting) crests of different spores, $\times 2000$; d. ibid., $\times 4000 .-\mathrm{e}, \mathrm{f}$. Bolbitis angustipinna . e. arrangement of spores within the sporangium, $\times 500$; f. detail of e, near annulus, $\times 1000$ (a-d. Hennipman 3527 ; e, f. H. 3536). ip = inner perispore, op = outer perispore, $\mathrm{rl}=$ residual layer.

Plate 6. Spores of Bolbitis sinensis. a, b. spore with a normally shaped, cristate-undulate perispore. a. $\times$ 2000 ; b. $\times 4000$. c. spore with an abnormal, in part cristate-undulate, in part spiny perispore, $\times 2000$; . detail of a spiny perispore (with perforations), also showing a residual body (arrow), $\times 8000$ (Rock 2913, US).

Plate 7. Cross-section of a mature sporangium of Bolbitis sinensis. a. sporangium, $\times 500 ;$ b. detail of $\mathrm{a}, \times$ 1000 ; c. cross-section of spore, $\times 1000$; d detail of the perispore, $\times 4000$; e. detail of the arrangement of the perispore of adjacent spores, $\times 4000$ (Hennipman 3229 , L). ex $=$ exospore, ip $=$ inner perispore, op $=$ outer perispore, $\mathrm{rl}=$ residual layer, $\mathrm{s}=\mathrm{scar}$ of spore.

Plate 8. Spores.-a, b. Bolbitis bipinnatifida. a. $\times 1000$; b. detail of a, $\times 2000 .-c$, d. Bolbitis quoyana. c. $\times 1000$; d. $\times 2000$. - e, f. Bolbitis nicotianifolia. e. general view, $\times 1000$; f. detail of e showing also a residual body (arrow), $\times 2000$ (a, b. Jeffrey 759, L; c, d. Millar \& Holttum NGF 15887, K; e. f. Herminier s.n., L).

Plate 9. Spores.-a-c. Bolbitis angustipinna. a. $\times 1000$; b, c. $\times 2000 .-$ d, e. Bolbitis semicordata $\mathrm{d} . \times 2000$; e. $\times 5000$ (a-c. Hennipman 3229, L; d, e. Van Hardeveld \& Van der Werff $323 A, \mathrm{~L}$ ).

Plate 10. a, b, d-f. Aberrant spores. a, b. Bolbitis appendiculata ssp. appendiculata. a. $\times 1000 ; \mathrm{b} . \times 5000$. $\mathrm{d}-\mathrm{f}$. clustered spores (the total output of one sporangium) of triploid $B$. repanda. d, e. $\times 500$; $f$. detail of the 'perispore' of the cluster, $\times 2000$ (a, b. Anonymus in herb. Hooker \& Thomson, BM; d-f. Hennipman $4028, \mathrm{~L}) .-\mathrm{c}$. Bolbitis heudelotii, spore of which the outer part of the perispore has been in part removed, $\times 1000$ (Hepper 1826, K). 
Plate 11. a, b. Spore tetrads. a. Bolbitis heteroclita 'flagellifera', contents of young sporangium (tetrad already without S.M.C.-membrane), $\times 250$; b. B. repanda 'München' (triploid), spore tetrad with S.M.C.-membrane, $\times 1250$ (a. Hennipman 4027, L; b. H. 4022, L.).-c-g. Chromosome portraits, $\times 1000$. c. Bolbitis heteroclita 'malaccensis'; d. B. sinuata 'gigas'; e. B. heteroclita 'cuspidata $2 n$ '; f. B. rhizophylla (Price 351, L); g. B. heudelotii. See also table 1 and fig. 31 .

Fig. 31. Explanatory diagrams of plate $11 . \mathrm{n}_{1}=$ nucleus of spore, $\mathrm{n}_{2}=$ nucleus of tapetum, $\mathrm{nm}=$ nuclear material originating from lagging chromosomes, $\mathrm{pl}=$ plasmalemma of spore, $\mathrm{s}=$ S.M.C.-membrane.

Plate 12. Chromosome portraits, $x$ 1000. a. Bolbitis sinuata 'typical', $2 \mathrm{n}=122+$ fragment (arrow); $\mathrm{b}$. B. virens f. virens 'Hennipman', $2 \mathrm{n}=82$; c. B. sinuata 'sinuata', $\mathrm{n}=41$; d. B. sinensis 'Nooteboom', $\mathrm{n}=41$; e. B. repanda 'Holttum', $\mathrm{n}=82$. See also table 1 . 
Taxonomic Part 


\section{NOTES ON THE PRESENTATION OF DATA}

The presentation of the taxa is alphabetical within the alphabetically arranged series and within the Species incertae sedis. The synonymy of the taxa is listed in full. Basionyms and the homotypic synonyms of each basionym are listed chronologically. Relevant literature is included. The names of the authors have been abbreviated as much as possible according to the Kew Index of Author Abbreviations. Nearly all the type collections have been studied; those for which I searched in vain are designated by 'n.v.' (non vidi). Herbaria are abbreviated according to the Index Herbariorum. Lectotypes have generally been indicated only for taxa accepted in this paper. The additions 'fert.' ( = fertile) and 'ster.' (= sterile) to type collections are given to indicate that the type material consists only of fertile and sterile material respectively. These additions are also added when but little material of a certain taxon was available.

The material studied represents almost exclusively adult plants. It is often incomplete. Only part of the collections consists of plants with a rhizome. When leaves are available only, they often lack part of the petiole or the apex of the lamina. Incomplete leaves have been included in the description when parts of it provided relevant information. Fertile leaves not rarely have immature sporangia and spores. Mature fertile leaves often do not show mature spores for the obvious reason that when fertile leaves are collected with mature sporangia and spores, the sporangia open during the drying process, and the mature spores generally disappear together with the drying paper. Socalled intermediate leaves, dwarfs, and aberrant specimens are generally not considered in the descriptions. They are discussed in the notes when necessary. A survey of the identity of all the numbered collections studied is given in the Index of collections; the numbers following the collection numbers refer to the numbers assigned to the taxa.

Descriptions are prepared from adult plants. The term segment is used for a portion of the lamina that has an axis (R. M. Tryon Jr, Taxon 9, 1960, 104-109).

The shape of the lamina and the pinnae is indicated by the index (length/width ratio) and the place where the segment is widest. The number of pinnae given refers to the total number of pinnae to a leaf. Caudate apices of pinnae are not included in the measures given for the length of the pinnae. The width of the fertile pinnae and laminas (in case the leaf is entire) is sometimes difficult to ascertain from herbarium material as the margin of the pinnae is not rarely rolled up.

Primordia of bulbils can be traced when the sterile leaves are examined in transmitted light. If present, they appear as small knobs terminally, or subterminally either in the axil of a primary and a secondary vein (in pinnate leaves) or in the axil of a secondary and a tertiary vein (in simple leaves).

Venation pattern. The costa of the pinna and the simple lamina are regarded to represent the primary vein. The pinnately arranged lateral veins arising from these primary veins are designated as the secondary veins. Tertiary veins are the veins between adjacent secondary veins. The venation pattern as found between two adjacent secondary veins is usually described in general terms only. Both the descriptions and the illustrations of the venation patterns are as a rule taken from central parts of either central 
pinnae (in pinnate leaves), or central lobes (in pinnatipartite leaves), or the lamina (in entire leaves). The venation pattern is illustrated for all species. The drawings ( $4 / 5$ natural size) were taken from projection photographs showing the venation pattern four times magnified, and were afterwards reduced. Elements of the description that are similar in the sterile and the fertile leaves are not repeated in the description of the fertile leaves. Some of the terms relating to the fern leaf and used in this paper are schematically set forth in fig. 32 .

The information on the spores as given in the separate heading is generally taken from collections randomly selected from the available appropriate material.

Chromosome numbers are taken from the list supplied in chapter 7; this list also includes references. S.M.C. stands for Spore Mother Cell.

The distribution is given following political boundaries. Large countries have been subdivided. In order to supply also an indication of the frequency of a certain taxon throughout its geographical range, both the countries (or its administrative units) and the number of collections studied from each of them, is indicated. If there are more than 25 collections available from a certain area, they have been generally denoted as 'many collections'.

Distribution maps have been compiled for most of the species from the collections studied. As a precise indication of the locality especially of the older collections has often been neglected, I was unable to spot all the localities, in spite of the fact that different atlases, indices, as well as miscellaneous information regarding localities and itineraries were consulted.

Data regarding the habitat conditions and the altitude of the taxa are compiled from the usually scanty information on the labels and summarized under separate headings. Some species were studied in the field.

Vernacular names are only rarely recorded on the labels. They have not been taken into consideration.

Notes are added to most of the species. They contain amongst other things remarks on the taxonomic position and the relationship of the respective species, and should therefore be regarded as an important element.

Illustrations of specimens in outline were generally prepared from dried material and on translucent paper, natural size. These drawings were reduced afterwards. Fertile parts are given in solid black.

Keys. The keys to the species are based on characters of the sterile leaves, and sometimes on those of the spores. The fertile leaves generally do not provide important additional characters. Identification will be possible for most of the material using a good hand lens and preferably also a sourse of transmitted light to study the venation pattern. For a limited part of the material examination of the spores may be necessary. The keys also include hybrids and doubtful species in case these are known from more than one locality. Several species are inconsistent in the characters employed; they appear on more than one place in the key. Keys to infraspecific taxa are given under the species.

It seems more practical to provide keys for each of the continents and including also 


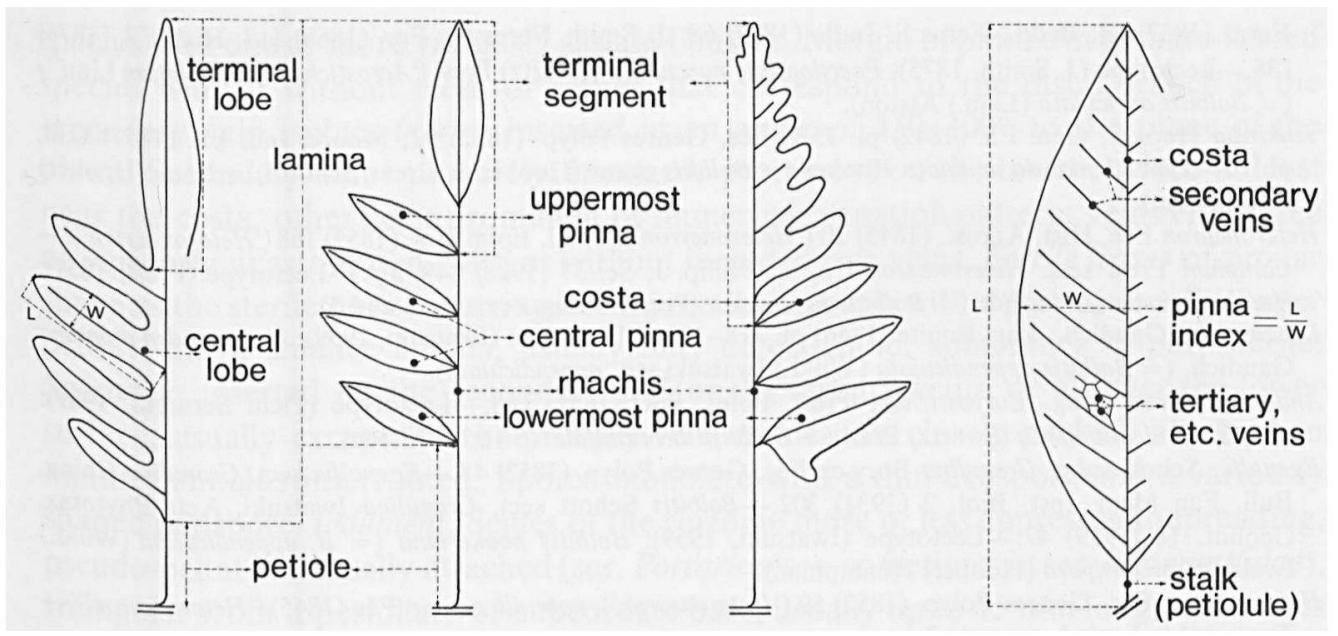

Fig. 32. Schematic illustration explaining the terms used in the Taxonomic Part.

doubtful species and hybrids, than to provide a key to all the recognized species only. The relationships between species of different regions is-where necessary-elucidated in the notes to the species.

Hybrids, dubious species and excluded species are separately listed.

\section{BOLBITIS}

Bolbitis Schott, Gen. Fil. (1835) adtab. (13) (excellent); C. Chr., Ind. Fil., Suppl. 3(1934) 102; Tardieu-Blot \& C. Chr., Not. Syst. 7 (1938) 99; Backer \& Posthumus, Varenfl. Java (1939) 80; Tardieu-Blot \& C. Chr. in Lecomte, Fl. Gen. I.-C. 7 (1941) 431; Copel., Gen. Fil. (1947) 115; Holttum, Ferns Malaya (1954) 461; Iwatsuki, Acta Phytotax. Geobot. 18 (1959) 44; Copel., Fern Fl. Philip. (1960) 254; Nayar \& Kaur, Bull. Nat. Bot. Gard. Lucknow 88 (1964) 1; Pichi Sermolli, Webbia 23(1969) 383; DeVol \& Kuo in FI. Taiwan 1 (1975) 347-Cyrtogonium J. Smith, in Hook., J. Bot (1841) 154, nom. illeg. (art. 63). - Leptochilus Kaulf. sect. Bolbitis C. Chr., Bot. Tidsskr. 26 (1904) 283-Leptochilus subg. Bolbitis C. Chr., Ind. Fil. (1906) Xxvi.-Lectotype (C. Chr., 1906): Bolbitis serratifolia (Kaulf.) Schott.

Egenolfia Schott, Gen. Fil. (1835 or 1836) ad tab. (16) (excellent); Fée, Genres Polyp. (1852) 48, 358; J. Smith, Hist. Fil. (1875) 131; Copel., Univ. Cal. Publ. Bot. $16(1928) 64$ (= Or. Gen. Polyp.); C. Chr., Contr. U.S. Nat. Herb. 26 (1931) 290; Ching, Bull. Fan Mem. Inst. Biol. 2 (1931) 297; Backer \& Postumus, Nat. Tijd. N. I. 93 (1933) 152; Tardieu-Blot \& C. Chr., Not. Syst. 7 (1938) 98; Backer \& Posthumus, Varenfl. Java (1939) 84; Tardieu-Blot \& C. Chr. in Lecomte, Fl. Gén. I.-C. 7 (1941) 423; Copel., Gen. Fil. (1947) 116; Holttum, Ferns Malaya (1954) 459; Copel., Fern Fl. Philip. (1960) 265; Nayar \& Kaur, Bull. Nat. Bot. Gard. Lucknow 100 (1964) 1; DeVol \& Kuo in Fl. Taiwan 1 (1957) 350.-Polybotrya Humb. \& Bonpl. ex Willd. subg. Egenolfia Fée, Hist. Acrost. (1845) 14.-Polybotrya subg. Ectoneura Fée, Hist. Acrost. (1845) 75 ('Ectonevra'), nom. illeg., see note 4.-Acrostichum L. subg. Egenolfia Hooker, Spec. Fil. 5 (1864) 251; Baker in Hooker \& Baker, Syn. Fil. (1868) 415.-Polybotrya Humb. \& Bonpl. ex Willd. sect. Egenolfia Diels in E. \& P., Nat. Pfi. Fam. 1 (1900) 195, fig. 102; v.A.v.R., Handb. Mal. Ferns (1908) 722.Type: Egenolfia hamiltoniana Schott (= Bolbitis appendiculata (Willd.) Iwatsuki ssp. appendiculata).

Campium Presl, Tent. Pterid. (1836) 238, pl. X: 22, 23; Epim. Bot. (1851) 169; Copel., Philip. J. Sc. 37 (1928) 341, p.p.; Backer \& Posthumus, Nat. Tijd. N. I. 93 (1933) 157, p.p.-Lectotype (C. Chr., 1906): Campium virens (Hooker \& Grev:) Presl (= Bolbitis virens (Hooker \& Grev.) Schott var. virens, see note 5).

roecilopteris [Eschweiler, Linnaea 2 (1827) 117, nom. nud. ('Poikilopteris')] Presl, Tent. Pterid. (1836) 241, pl. X: 18, 19, pl. XI: 1, 2; Epim. Bot. (1851) 171; Moore, Ind. Fil. (1857) xx, pl. 7; J. Smith, Cat. Cult. 
Ferns (1857) 23; Bedd., Ferns S. India (1864) 68; J. Smith, Ferns Br. For. (1866) 117; Hist. Fil. (1875) 136.-Lectotype (J. Smith, 1875): Poecilopteris punctulata (Swartz) Presl ('Acrostichum punctulatum Linn.') (= Bolbitis auriculata (Lam.) Alston).

Jenkinsia Hooker, Gen. Fil. (1842) pl. 75B; Fée, Genres Polyp. (1852) 92; Moore, Ind. Fil. (1857) xxiii, pl. 10-Type: Jenkinsia undulata Hooker (= Bolbitis virens (Hooker \& Grev.) Schott f. undulata (Hooker) Hennipman).

Heteroneuron Fée, Hist. Acrost. (1845) 20 ('Heteronevron'); Presl, Epim. Bot. (1851) 168 ('Heteroneurum').Campium Presl sect. Heteroneuron Copel., Philip. J. Sc. 37 (1928) 349, p.p.-Lectotype (Presl, 1851): Heteroneuron argutum Fée (= Bolbitis heteroclita (Presl) Ching, see note 2 and 3).

Lacaussadea Gaudich., Voy. Bonite (1846) pl. 118-120._Lectotype (Iwatsuki, 1959): Lacaussadea montana Gaudich. ( = Bolbitis appendiculata (Willd.) Iwatsuki ssp. appendiculata).

Anapausia Presl subg. Euryostichum Presl, Epim. Bot. (1851) 188.-Lectotype (Pichi Sermolli, 1969): Anapausia nicotianifolia (Swartz) Presl ( = Bolbitis nicotianifolia (Swartz) Alston).

Egenolfia Schott subg. Granulina Bory ex Fée, Genres Polyp. (1852) 48.-Egenolfia sect. Granulina Ching, Bull. Fan Mem. Inst. Biol. 2 (1931) 302.-Bolbitis Schott sect. Granulina Iwatsuki, Acta Phytotax. Geobot. 18 (1959) 47.-Lectotype (Iwatsuki, 1959): Bolbitis hookeriana (= B. appendiculata (Willd.) Iwatsuki ssp. vivipara (Hooker) Hennipman).

Heteroneuron Fée, Genres Polyp. (1852) 59 ('Heteronevron'), nom. illeg., non Fée (1845) ('Heteronevron').Type: not indicated, see note 2.

Edanyoa Copel., Philip. J. Sc. 81 (1952) 22, pl. 17.-Type: Edanyoa difformis Copel. (= Bolbitis heteroclita (Presl) Ching).

Bolbitis Schott sect. Lysidictyon Iwatsuki, Acta Phytotax. Geobot. 18(1959) 47.-Type: Bolbitis laxireticulata Iwatsuki (= Bolbitis spec. dub.).

Small to medium-sized, terrestrial, low-epiphytic, or usually low-climbing ferns. Rhizome short- to long-creeping, unbranched or with accessory branches, slender or stout, almost terete to dorsiventrally flattened, dorsally and/or laterally with 2-6 rows of leaves and in the apical part with variously shaped aerophores occurring in a line with those on the petiole (distinct in living rhizomes only), roots usually ventral, sometimes also lateral, either in two longitudinal rows or irregularly arranged; anatomy: ground tissue parenchymatous, when living light green, longitudinal sclerenchyma strands, if present, scattered in the ground tissue, \pm cylindrical, with a sheet of sclerenchyma of varying thickness situated just below the epidermis and interrupted by aerophores, vascularization dictyostelic, markedly dorsiventral, with a broad gutter-shaped ventral bundle giving off root and leaf traces, and 1-5 narrower to almost cylindrical dorsal ones giving off leaf traces. Leaves spaced to aggregate, dimorphic, simple, pinnate, or rarely bipinnate. Sterile leaves: petioles usually shortdecurrent on the rhizome, sometimes either almost perpendicular to the rhizome or (in epiphytes) long-decurrent on the rhizome, near the base dark brown or blackish, otherwise light brown or stramineous, near the base adaxially flattened or convex, abaxially terete, near the apex adaxially usually \pm flat with one or three median, longitudinal ridges and on either side 1 (2) lateral ones, sometimes adaxially round or convex, aerophores linear, pale, present laterally on either side and especially conspicuous in the basal part of young leaves; anatomy: ground tissue parenchymatous, in the basal region with or without sclerenchyma strands similar to those in the rhizome, with a firm sheet of sclerenchyma situated just below the epidermis and interrupted by aerophores, with a more or less U-shaped arrangement of vascular bundles of which the two anterior ones are largest; lamina green, above somewhat shining, texture mostly more or less herbaceous, sometimes coriaceous or pergamentaceous, in most 
species with one or more variously situated bulbils. Margin of pinnae in pinnate-leaved species with or without teeth or spines that correspond to the distal branch of the secondary vein, spines in vivo inserted at an angle of $150-100^{\circ}$ to the plane of the pinna. Secondary veins pinnately arranged, prominent on the lower surface at least near the costa, other veins prominent or immersed; venation pattern: veins either free or variously anastomosed, with or without included free veins. Fertile leaves of similar shape as the sterile ones occurring in conjunction, though with a proportionally longer petiole and a smaller lamina, usually fully acrostichoid, sometimes in part sterile. Sporangia inserted on the secondary and/or the tertiary veins, or all over the lower surface, usually except for the primary vein, numerous, closely packed, black when mature, annulus interrupted. Spores monolete with a thin exospore and a variously shaped perispore. Indument. Scales of the rhizome more or less appressed to spreading, pseudo-peltate or basally attached (ser. Portoricenses, sometimes in ser. Bipinnatifidae), triangular from a perfoliate or subcordate base, usually up to $15 \mathrm{~mm}$ long, sometimes up to $25(-40) \mathrm{mm}$ long, membranaceous to firmly herbaceous, light brown to blackish, opaque to almost clathrate, of the same colour throughout or with a paler marginal strip, margin almost entire, in one species (B. bernoullii) conspicuously denticulate, when mature sparsely set with slender, uniseriate, usually few-celled, thin-walled glandular hairs. Scales of the petiole in part \pm similar to those on the rhizome, usually on the basal part only, rarely on petiole and rhachis throughout, in part appressed, of smaller size and \pm orbicular. Scales of the lamina scattered on the lower face, especially on the rhachis at the junction with the pinnae, \pm similar to the appressed type of scales of the petiole. Leaves usually glabrescent. The crozier on either side densely covered with 2 - or 3(-5)-celled uniseriate hairs, the terminal and sometimes also the subterminal cell of which are glandular.

Chromos omes. $\mathrm{n}=41,82 ; 2 \mathrm{n}=($ c.) $82,(c)$.123 , and 164 .

$\mathrm{Ga}$ a t oph y te. Cordate or elongate.

Distribution. Pantropic. See fig. 26.

$\mathrm{H}$ a b i t a t. Mostly creeping on rocks, sometimes creeping in soil or epiphytic, in moist places-often nearby and even in streams-in monsoon and evergreen forest. Altitude: $0-1500(-2000) \mathrm{m}$.

Notes. 1. There is disagreement about the sequence of the plates in Schott's Genera Filicum, due to the fact that the plates have not been numbered. The 20 plates and descriptions were issued between 1834-1836 as 4 fascicles containing five plates each (Stafleu, Taxonomic Literature, 1967). At the British Museum I found Fée's copy of this book in which the plates are bound alphabetically. The number of the fascicles and the plates are indicated on the cover of that book in Fée's characteristic handwriting, except for the plates of Nephrodium and Taenitis. The copy at the Rijksherbarium shows Nephrodium to represent the fifth plate of fascicle 2 (plate 10 ), which makes the plate of Taenitis the fifth plate of fascicle 4 (plate 20). In conclusion, the following sequence is proposed: Fascicle 1 (1834): Anaxetum (1), Aspidium (2), Nephrolepis (3), Sphaeropteris (4), Trichopteris (5)-Fascicle 2 (1834): Olfersia (6), Polybotrya (7), Cystopteris (8), Polystichum (9), Nephrodium (10)-Fascicle 3 (1835): Lonchitis (11), Meniscium (12), 
Bolbitis (13), Hemionitis (14), Marattia (15)—Fascicle 4 (1835 or 1836): Egenolfia (16), Cochlidium (17), Monogramma (18), Aglaomorpha (19), Taenitis (20).

2. The genus Heteroneuron Fée (1845) included 16 species. It was typified by Presl (1851) who excluded all but one of Fée's species (H. argutum) and added a second. The remaining 15 species which Fée attributed to Heteroneuron were transferred by Presl to the genera Campium and Poecilopteris at the same time. Presl's spelling 'Heteroneurum' should be regarded as an orthographic variant. The effort of Pichi Sermolli (1969) to recognize a genus Heteroneurum Presl beside a genus Heteroneuron Fée, is contrary to the Code. The same holds for the selection of another type for Heteroneuron Fée, as was done by Iwatsuki $(1959 ;$ H. diversifolium $)$ and Pichi Sermolli( $1969 ; H$. scalpturatum). Heteroneuron Fée (1852) is a different genus, as Fée excluded H. argutum.

3. Heteronevron, the original spelling also adopted by Pichi Sermolli (1969), has according to the Code (art. 73) to be replaced by Heteroneuron.

4. When dealing with the genera of acrostichoid ferns, Fée (1845: 14) divided the genus Polybotrya into two subgenera, '1. Egenolfia, Schott; Gen. Filic.' and '2. Eupolybotrya'. In the part where the species are described (op. cit.: 72 and 75, resp.) he divided it into the subgenera 'I. Eupolybotrya' and 'II. Ectonevra' '(Egenolfia, Schott)'. Therefore, the subgenera Egenolfia and Ectoneura are homotypic synonyms.

5. Iwatsuki (1959) overlooked Christensen's publication (1906) and selected Campium punctulatum Presl ( = Bolbitis spec. dub.) as the type of Campium.

6. Synonyms have been listed only when their type species are part of this genus. Other genera to which species of the present genus have been referred include Acrostichum, Aspidium, Asplenium, Chrysodium, Diplazium, Gymnogramma, Gymnopteris, Hypoderris, Leptochilus, Lomariopsis, Meniscium, Nephrodium, Neurocallis, Notholaena, Polybotrya, Polystichum, and Stenosemia.

7. Etymology. As fully explained by Pichi Sermolli (Webbia 12, 1969, 386) the genus was probably so named on account of a bulbil on its leaves.

\section{KEYS TO SPECIES AND HYBRIDS}

H: Hybrids (p. 284 onwards). D: Species dubiae (p. 302 onwards)

\section{KEY TO THE SPECIES AND HYBRIDS OF AFRICA AND MADAGASCAR}

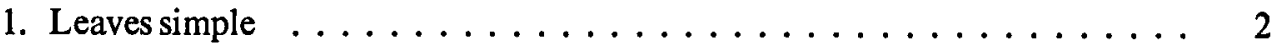

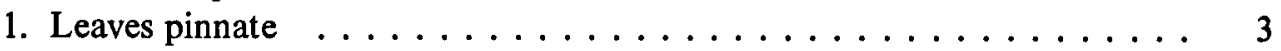

2. Lamina with a subterminal bulbil. Venation pattern intricate, part of the areoles with re-and/or excurrent included free veins ....... 20. B. gaboonensis

2. Lamina without a bulbil. Veins forming a regular network of angulate areoles without included free veins $\ldots \ldots \ldots \ldots \ldots \ldots \ldots$ 3. B. fluviatilis 
3. Terminal segment with a bulbil in the basal part; veins forming an intricate network which is locally irregular, part of the areoles with usually few ex- or recurrent included free veins $\ldots \ldots \ldots \ldots \ldots \ldots \ldots$ 36. B. gemmifera

3. Terminal segment without bulbils or with a subterminal bulbil $\ldots \ldots \ldots 4$

4. Venation pattern with ex- and recurrent included free veins ....... 5

4. Venation pattern without or with only excurrent included free veins . . . 7

5. Bulbils absent. Usually in streams . . . . . . . . . 27. B. heudelotii

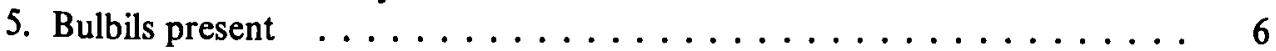

6. Upper half of lamina almost entire, not prolonged. Scales up to $5 \mathrm{~mm}$ long. Congo

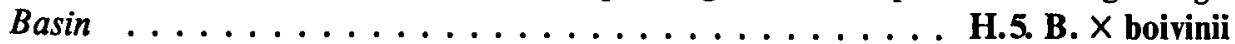

6. Upper half of lamina deltoid, regularly lobed, tapering towards the acute or flagelloid apex. Scales up to $12 \mathrm{~mm}$ long. Madagascar . . . . . 37. B. humblotii

7. Terminal segment triangular, as long as to longer than the remaining part of the

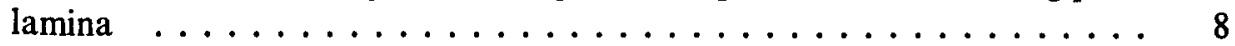

7. Terminal segment usually either conform to the pinnae or flagelloid, sometimes narrowly triangular and much shorter than the remaining part of the lamina 9

8. Margin of pinnae \pm entire. Terminal segment usually asymmetrical, consisting of few (2-5) lobes, not flagelloid. Veins regularly reticulate, without included free veins

2. B. auriculata

8. Margin of pinnae lobed $3 / 7-5 / 7$ towards the costa; a tooth present in the sinuses. Terminal segment symmetrical, composed of many lobes, sometimes flagelloid. Veins regularly reticulate, at least the costal arch with few short excurrent included free veins

4. B. bipinnatifida

9. Terminal segment flagelloid. Perispore cristate. Madagascar.

D. 5. B. longiflagellata

9. Terminal segment conform to the pinnae or somewhat prolonged. Perispore un-

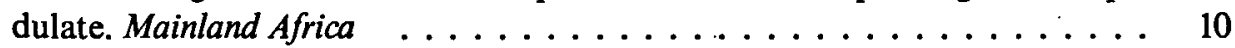

10. Pinnae 8-20 $\mathrm{mm}$ wide. Venation pattern without or with only one or a few excurrent included free veins. Terminal segment not prolonged. Fertile pinnae fully acrostichoid, the sporangia inserted all over the lower surface 9. B. salicina

10. Pinnae $15-45 \mathrm{~mm}$ wide. Venation pattern usually with several excurrent included free veins. Terminal segment usually somewhat prolonged. Fertile pinnae usually hemionitoid, sometimes acrostichoid, the sporangia inserted exclusively or mainly on the veins

5. B. acrostichoides

\section{KEY TO THE SPECIES OF AMERICA}

1. Scales of the rhizome $10-20 \mathrm{~mm}$ long or longer. Tertiary veins prominent throughout. Leaves in 2-6 rows. High-climbing plants 
1. Scales of the rhizome up to $10(-15) \mathrm{mm}$ long. Tertiary veins prominent near the costa or near the secondary veins only. Leaves in $2(-5)$ rows. Terrestrial, sometimes on the bases of trees ................... 3

2. Margin of the scales entire. Veins forming a regular network of \pm isodiametric or elongate areolae

28. B. lindigii

2. Margin of the scales denticulate. Veins forming an irregular network, part of the areoles with mostly excurrent included free veins ...... 35. B. bernoullii

3. Leaves either simple, or pinnate with the terminal segment usually conform to the pinnae, rarely narrowly triangular and much shorter than the remaining

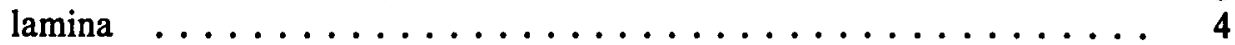

3. Leaves pinnate with the terminal segment triangular and longer than the remaining

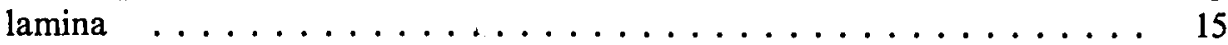

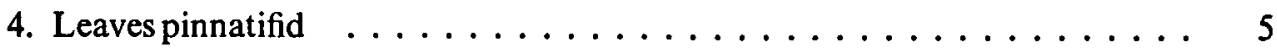

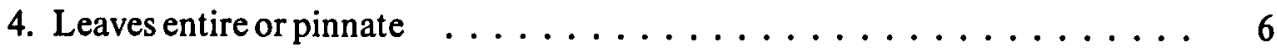

5. Leaves with a subterminal bulbil. The majority of the areoles along the leaf margin with usually one recurrent included free vein. Mexico . . . . . 29. B. hastata

5. Leaves without a bulbil. The majority of the areoles along the margin without included free veins. South America . . . . . . . . . . 42. B. semipinnatifida

6. Leaves entire and with a subterminal bulbil, or pinnate . . . . . . . 7

6. Leaves entire, without a subterminal bulbil (uncommon forms of generally pinnate species) . . . . . . . . . . . . . . . . . . . 13

7. Venation pattern without recurrent included free veins; excurrent free veins present or not. Perispore undulate $\ldots \ldots \ldots \ldots \ldots \ldots \ldots$

7. Venation pattern with both re- and excurrent included free veins. Perispore cristate

8. Rhachis with a narrow wing. Lamina with 4-8 pinnae, without bulbils.

12. B. serrata

8. Rhachis not winged. Lamina with $9-30$ or more pinnae and (a primordium of) a subterminal bulbil . . . . . . . . . . 13. B. serratifolia

9. Bulbils absent $\ldots \ldots \ldots \ldots \ldots \ldots \ldots \ldots \ldots \ldots \ldots \ldots$

9. Bulbils present, either axillary or subterminal . . . . . . . . . 11

10. Scales of the rhizome soft, rusty brown, bullate. Texture of leaves pergamentaceous; terminal segment if present \pm rhomboid; secondary veins prominent nearly to the

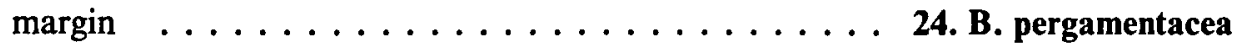

10. Scales of the rhizome firm, dark brown or blackish, flat, appressed. Texture of leaves thin-herbaceous; terminal segment if present biconvex; secondary veins slightly prominent and only to some distance from the margin.

22. B. nicotianifolia

11. Bulbil subterminal $\ldots \ldots \ldots \ldots \ldots \ldots \ldots \ldots$ 30. B. pandurifolia

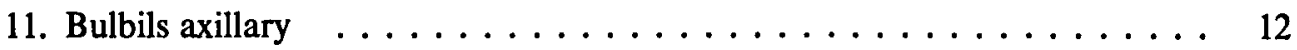

12. Leaves ternate; pinnae (sub) opposite; base of terminal segment (almost) symmetrical. The Andes

23. B. oligarchica 
12. Leaves with 3-9 usually alternate pinnae; base of terminal segment (strongly)

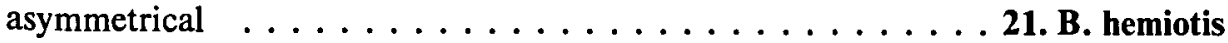

13. Scales of the rhizome bullate, rusty brown. Secondary veins prominent nearly to the margin $\ldots \ldots \ldots \ldots \ldots \ldots$ 24. B. pergamentacea

13. Scales of the rhizome flat, blackish. Secondary veins towards the margin either prominent or inconspicuous . . . . . . . . . . . . . . . 14

14. Base of the lamina truncate to subcordate, sometimes asymmetrical. Trinidad, Venezuela . . . . . . . . . . . . . . . . . . 21. B. hemiotis

14. Base of the lamina \pm acute, symmetrical . . . . . 22. B. nicotianifolia

15. Venation pattern without included free veins or with only an incidental short, simple, included free vein . . . . . . . . . . . . . . . . 16

15. Venation pattern with many recurrent (and excurrent) included free veins some of which are once forked . . . . . . . . . . . . . . . . 17

16. Leaves tufted. Margin of pinnae with teeth in the sinuses. Leaves green when dried. Mexico

44. B. umbrosa

16. Leaves spaced. Margin of pinnae without teeth in the sinuses (if present). Leaves usually dark green or blackish when dried $\ldots \ldots \ldots \ldots \ldots$ 1. B. aliena

17. Bulbils present. Terminal segment regularly triangular, composed of many lobes, apex often flagelloid. Scales of the rhizome up to $10 \mathrm{~mm}$ long, basally attached. Venation pattern: see fig. 68: e-j . . . . . . . . 31. B. portoricensis

17. Bulbils absent. Terminal segment either asymmetrical or symmetrical, composed of but few (up to 6) lobes, apex acute. Scales of the rhizome up to $4 \mathrm{~mm}$ long, pseudopeltate. Venation pattern: see fig. 79: $\mathrm{g}$, h

42. B. semipinnatifida

\section{KEY TO THE SPECIES AND HYBRIDS OF ASIA AND THE PACIFIC}

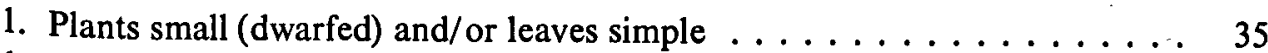

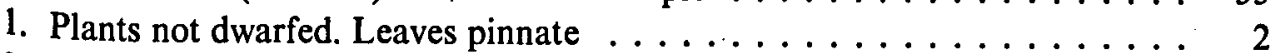

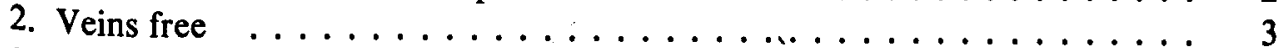

2. Veins anastomosing $\ldots \ldots \ldots \ldots \ldots \ldots \ldots \ldots \ldots$

3. Bulbil \pm terminal on the lamina. Spines on the margin of the pinnae \pm flattened at their bases. Base of pinnae symmetrical. Margin entire or shallowly lobed. Taiwan, Philippines $\ldots \ldots \ldots \ldots \ldots \ldots \ldots \ldots \ldots \ldots$ 18. B. rhizophylla

3. Bulbil subterminal on the lamina. Spines on the margin not flattened at their bases. Base of pinnae symmetrical or asymmetrical. Margin entire or lobed . . . 4

4. Rhizome long-creeping. Petiole with $>10$ vascular bundles $\ldots$ 17. B. major

4. Rhizome short-creeping. Petiole with $<10$ vascular bundles $\ldots \ldots \ldots 5$

5. Perispore smooth. Base of pinnae symmetrical, margin lobed $1 / 3-2 / 3(-3 / 4)$ towards the costa . . . . . . . . . . . . . . . . 6

5. Perispore reticulate. Base of pinnae either symmetrical or asymmetrical; if symmetrical the margin of the pinna \pm entire or lobed to $1 / 3(-1 / 2)$ towards the costa.

16. B. appendiculata 
6. Perispore cristate-undulate. Petiole and rhachis glabrescent

19. B. sinensis

6. Perispore cristate. Petiole and rhachis scaly throughout . . D. 7. B. tonkinensis

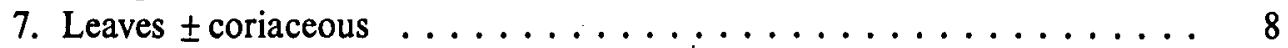

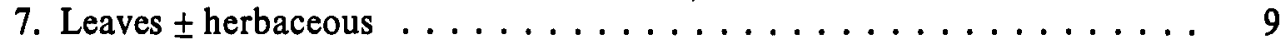

8. Terminal segment conform to the pinnae, though usually larger . 26. B. sinuata

8. Terminal segment triangular . . . . . . . . . . 33. B. rivularis

9. Central pinnae asymmetrical. Rhachis narrowly winged, at least in the upper part. Venation pattern regular, without or with but one short excurrent included free vein. Pinnae $25-100$ by $7-25 \mathrm{~mm} \ldots \ldots \ldots \ldots$

9. Central pinnae \pm symmetrical. Rhachis not winged and lamina with a \pm regular venation pattern, or rhachis with a narrow wing in the upper part and lamina with a very irregular venation pattern. Venation pattern and size of the pinnae

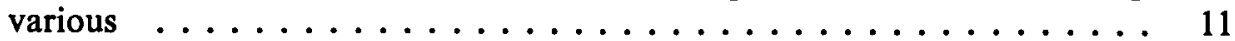

10. Terminal segment conform to the pinnae. S. India . . . . 11. B. semicordata

10. Terminal segment triangular. S. Japan . . . . . . . D. 4. B. laxireticulata

11. Venation pattern without excurrent included free veins, or with both ex- and re-

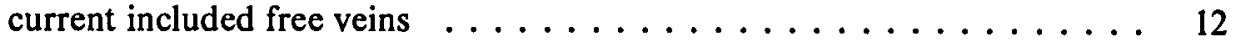

11. Venation pattern with excurrent included free veins $\ldots \ldots \ldots \ldots$

12. Terminal segment \pm conform to the pinnae $\ldots \ldots \ldots \ldots \ldots \ldots \ldots$

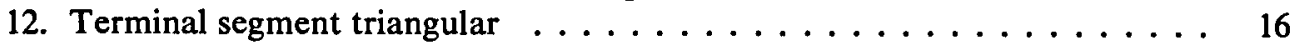

13. Veins forming a costal areole, otherwise free ... . . 8. B. crispatula

13. Veins more intricately anastomosing $\ldots \ldots \ldots \ldots \ldots \ldots$

14. Dried leaves purplish. Pinnae 4-24 to a leaf . . . . . . 10. B. scalpturata

14. Dried leaves greenish. Pinnae $2-50$ to a leaf $\ldots \ldots \ldots \ldots \ldots 15$

15. Pinnae $2-10(-15)$ to a leaf $\ldots \ldots \ldots \ldots \ldots \ldots$ 25. B. heteroclita

15. Pinnae $20-50$ to a leaf $\ldots \ldots \ldots \ldots \ldots \ldots \ldots$ 6. B. angustipinna

16. Bulbil \pm terminal. Small plants $\ldots \ldots \ldots \ldots \ldots \ldots$ 39. B. novoguineensis

16. Bulbil subterminal or absent $\ldots \ldots \ldots \ldots \ldots \ldots \ldots \ldots \ldots$

17. Leaves purplish when dried. Perispore undulate . . . . 10. B. scalpturata

17. Leaves greenish when dried. Perispore cristate or undulate . . . . . 18

18. Terminal segment narrowly triangular, much shorter than the remaining lamina. Perispore undulate $\ldots \ldots \ldots \ldots \ldots \ldots$ 6. B. angustipinna

18. Terminal segment triangular. Perispore cristate or cristate-undulate . . 19

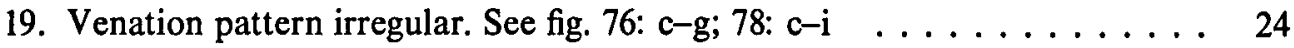

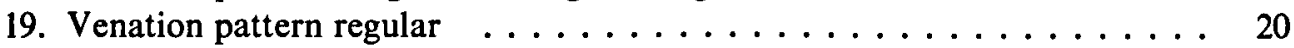

20. Base of pinnae narrow-acute or -cuneate. Pinnae $75-120$ by $11-18 \mathrm{~mm}$. Philippines $\ldots \ldots \ldots \ldots \ldots \ldots \ldots \ldots \ldots$ H. 12. B. $\times$ sinuosa

20. Base of pinnae wider. Pinnae larger or not $\ldots \ldots \ldots \ldots 21$

21. Terminal segment shorter than the remaining part of the lamina ..... 22

21. Terminal segment longer than to about as long as the remaining part of the

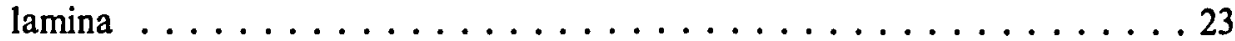

22. Venation pattern symmetrical. Margin usually with distinct spines. E. Malesia to the Pacific, Bonin Is.

32. B. quoyana 
22. Venation pattern asymmetrical (see fig. 86: f). Margin with inconspicuous teeth in the sinuses. Tonkin, S. China

D. 2. B. christensenii

23. Veins forming a costal areole, without or with but few smaller distal areoles. Queensland

34. B. taylorii

23. Veins forming a costal areole and many smaller distal areoles. Pacific.

D. 6. B. sagenioides

24. Venation pattern as in fig. 76: c-g. E. Malesia . . . . . . 41. B. repanda

24. Venation pattern as in fig. 78: c-i. Pacific . . . . . . 38. B. lonchophora

25. Terminal segment conform to the pinnae or somewhat prolonged (or flagelloid)

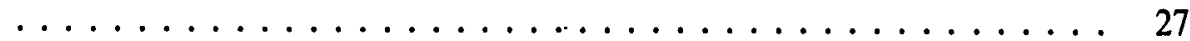

25. Terminal segment triangular, not flagelloid . . . . . . . . . 26

26. Pinnae 4-28 to a leaf. Margin of pinnae usually lobed, with spines or teeth. Venation pattern as in fig. 81: b-d. Perispore cristate or cristate-undulate.

43. B. subcordata

26. Pinnae $20-50$ to a leaf. Margin of pinnae usually \pm entire, usually without spines or teeth. Venation pattern as in fig. 40: $d-f$. Perispore undulate.

6. B. angustipinna

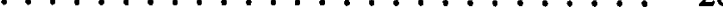

27. Leaves greenish when dried $\ldots \ldots \ldots \ldots \ldots \ldots$

28. Pinnae $20-40 \mathrm{~mm}$ wide. Venation pattern as in fig. 43: c, d . . 10. B. scalpturata

28. Pinnae $40-90 \mathrm{~mm}$ wide. Venation pattern as in fig. $42: \mathrm{d}-\mathrm{f}$. . . 7. B. costata

29. Pinnae up to $15 \mathrm{~mm}$ wide. Without bulbil(s). $S$. India . . . . 40. B. presliana

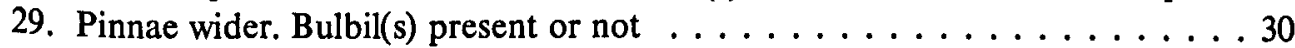

30. Venation pattern \pm regular $\ldots \ldots \ldots \ldots \ldots \ldots \ldots \ldots \ldots \ldots \ldots \ldots$

30. Venation pattern very irregular. S. India $\ldots \ldots \ldots \ldots \ldots . \ldots . \ldots 34$

31. Venation pattern asymmetrical; included free veins few. Pinnae $20-50$ to a leaf.

6. B. angustipinna

31. Venation pattern \pm symmetrical; included free veins several to many. Pinnae 3-22 to a leaf . . . . . . . . . . . . . . . . . . 32

32. Venation pattern as in fig. 86: b-d. Perispore cristate or spiny. Tonkin, S. China.

D. 1. B. cadieri

32. Venation pattern as in fig. 46: $b, c, f ; 48: d, e, m \ldots \ldots \ldots$

33. Venation pattern as in fig. 46: b, c, f. S. India and Ceylon .. 14. B. subcrenata

33. Venation pattern as in fig. 48: $d, e, m \ldots \ldots \ldots$ 15. B. virens

34. Terminal segment narrowly triangular, rhachis not winged: Pinna index usually $5-12$, pinnae generally more than $10 \mathrm{~cm}$ long. Venation patterns as illustrated in fig. 84: $b-g \quad \ldots \ldots \ldots \ldots \ldots \ldots \ldots \ldots$. . . 8. B. $\times$ lancea

34. Terminal segment usually conform to the pinnae, rhachis narrowly winged. Pinnae index usually 3-6, pinnae generally shorter than $15 \mathrm{~cm}$. Venation patterns as illustrated in fig. 84: $\mathrm{j}-\mathrm{m} \ldots \ldots \ldots \ldots \ldots$. . . 10. B. $\times$ prolifera

35. Bulbil \pm terminal. Pinnae $>16-30$. New Guinea $\ldots \ldots$ 39. B. novoguineensis

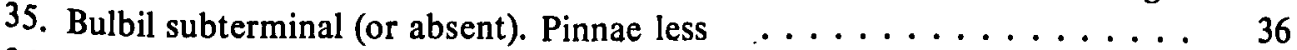

36. Veins anastomosing, without free veins or with few ex- and recurrent free veins. 
Leaves entire $\ldots \ldots \ldots \ldots \ldots \ldots \ldots \ldots \ldots \ldots \ldots$

36. Veins anastomosing, usually with many ex- and recurrent free veins. Leaves entire

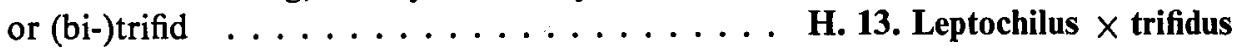

37. Leaves herbaceous . . . . . . . . . . . . . . . . . 39

37. Leaves coriaceous . . . . . . . . . . . . . . . . 38

38. Areoles (except the costal areole) all of about the same shape, decreasing in size towards the margin . . . . . . . . . . . 33. B. rivularis

38. Areoles of differing size and shape, not decreasing in size towards the margin.

26. B. sinuata

39. Leaves pinnate. Terminal segment triangular, venation as in fig. 22: $d$.

32. B. quoyana

39. Leaves entire or, if pinnate, with a venation as in fig. 20: a

25. B. heteroclita

\section{DESCRIPTIONS}

\section{Series Alienae Hennipman, ser. nov.}

Rhizoma longe repens gracile, sine fasciculis sclerenchymatorum. Folia dispersa biseriata. Lamina simplex, vel pinnata segmento terminali triangulari, bulbillis axillaribus raris. Pinnae $0-11$, marginibus sine spinis vel dentibus. Ordinatio venularum: venulae regulariter reticulatae, areolis angulatis isodiametris vel elongatis. Sporae perisporio laevi cristato.

Typus: Bolbitis aliena (Swartz) Alston.

Terrestrial. Rhizome usually long-creeping, up to $20(-30) \mathrm{cm}$ long, slender, with 2 rows of leaves, without sclerenchyma strands. Scales of the rhizome up to $15 \mathrm{~mm}$ long. Leaves spaced, up to $40 \mathrm{~mm}$ apart. Sterile leaves simple or pinnate; petiole with 5-7 vascular bundles; lamina sometimes with usually \pm spherical, sometimes elongate, axillary bulbils; pinnae $0-11$, the margin without teeth or spines; terminal segment triangular; venation pattern: veins regularly reticulate, areoles angulate, \pm isodiametric or somewhat elongate, decreasing in size towards the margin. Spores with a smooth cristate perispore.

Chromosomes. $\mathrm{n}=($ c.) 82 (B. aliena, $B$. auriculata).

D is tribution. Tropical America, tropical Africa, Madagascar, and the Mascarenes. See fig. 27.

1. Bolbitis aliena (Swartz) Alston-Fig. 33.

B. aliena (Swartz) Alston, Kew Bull. (1932) 310; Vareschi in Lasser, Fl. Venezuela 1 (1969) 375, p.p. -Acrostichum alienum Swartz, Schrader J. Bot. 1800, 2 (1801) 13; Syn. Fil. (1806) 13; Willd., Spec. Pl. 5 (1810) 119, p.p.; Poiret in Lam., Enc., Suppl. 1 (1810) 123; Sprengel, Syst. Veg. 4 (1827) 37, p.p.; Hooker, Spec. Fil. 5 (1864) 272, p.p.; Baker in Martius, Fl. Brasil. 1, 2 (1870) 589, p.p., pl. 51; fig. iii; Jenman, Bull. Bot. Dept. Jamaica (Kingston) n.s. 5 (1898) 153, p.p.-Gymnopteris aliena Presl, Tent. Pterid. (1836) 244; Hooker \& Bauer, Gen. Fil. (1842) pl. 85, quoad nomen solum; Fée, Hist, Acrost. (1845) 84, ibid.-Anapausia aliena Presl, Epim. Bot. (1851) 187, p.p._-Chrysodium alienum Mettenius, Fil. Lips. (1856) 21, pl. 10: fig. 5, quoad nomen solum.-Leptochilus alienus C.Chr., Bot. Tidsskr. 26 (1904) 285, p.p.; Ark. Bot. 9 (1910) 35, 


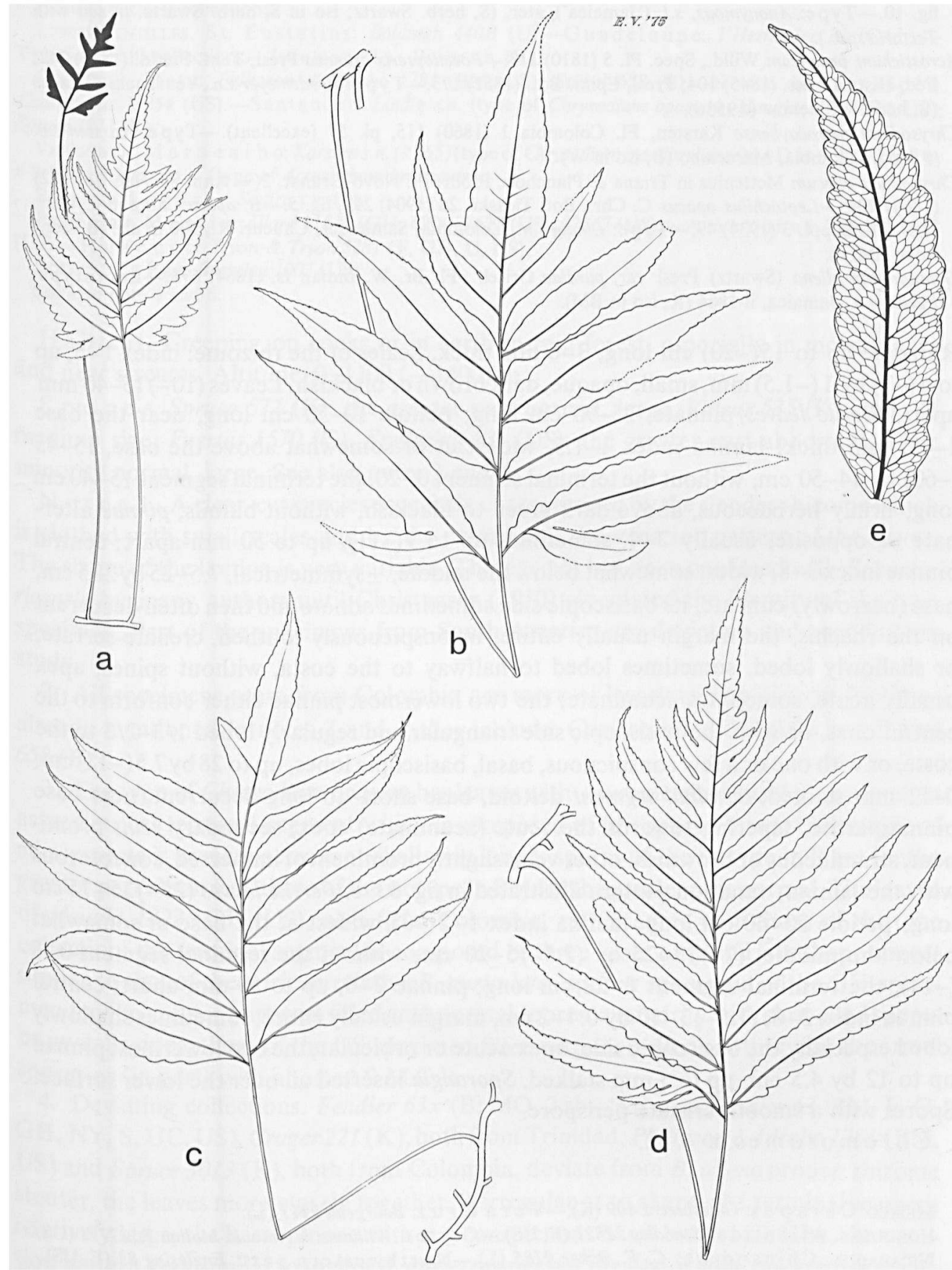

Fig. 33. Bolbitis aliena.-a-d. sterile leaves, $\times 1 / 5$; e. venation pattern of sterile pinna, $\times 4 / 5$ (a. Sintenis 5166, B; b. Spruce 577, K; c. Lindig s.n., P, a possible isotype of Chrysodium opacum; d. Valeur 535, K; e. Proctor \& Mullings 21999, U). 
fig. 10.-Type: Anonymous, s.l. ('Jamaica'), ster. (S, herb. Swartz; iso in S, herb. Swartz, mixed with Tectaria spec.).

Acrostichum brunneum Willd., Spec. Pl. 5 (1810) 113.-Poecilopteris brunnea Presl, Tent. Pterid. (1836) 242;

Fée, Hist. Acrost. (1845) 104: Presl, Epim. Bot. (1851) 173.-T y p e: Bredemeyer s.n., Venezuela, Caracas (B, herb. Willdenow (19536).

Chrysodium maracaybense Karsten, Fl. Colombia 1 (1860) 115, pl. 57 (excellent).-Type: Karsten s.n. (? 165), Colombia, Maracaibo (B; iso in W).

Chrysodium opacum Mettenius in Triana \& Planchon, Prodr. Fl. Novo-Granat. 2(= Ann. Sc. Nat. Bot. V.2) (1864) 204.-Leptochilus opacus C. Chr., Bot. Tidsskr. 26 (1904) 292, fig. 5. - B. opaca Ching in C. Chr., Ind. Fil., Suppl. 3 (1934) 49.-T ype: Lindig s.n., Colombia, Santander, Chucuri R., $900 \mathrm{~m}$ alt. (B, ster.; iso in BM, P).

Gymnopteris aliena (Swartz) Presl var. purdiae Griseb., Fl. Br. W. Indian Is. (1864) 674._Lectotype: Purdie s.n., Jamaica, ii-1864 (K; iso in BM).

Rhizome up to $15(-20) \mathrm{cm}$ long, 3-6 mm thick. Scales of the rhizome: index 1-5, up to $4(-7)$ by $1(-1.5) \mathrm{mm}$, small, opaque, dark brown or blackish. Leaves (10-) $15-40 \mathrm{~mm}$ apart. Sterile leaves pinnate, $25-90 \mathrm{~cm}$ long; petiole $10-50 \mathrm{~cm}$ long, near the base 1-4.5 mm thick; lamina index 1-1.5, widest at or somewhat above the base, 15-45 $(-60)$ by $14-50 \mathrm{~cm}$, without the terminal segment $0-20$, the terminal segment $15-40 \mathrm{~cm}$ long, firmly herbaceous, above dark green to blackish, without bulbils; pinnae alternate or opposite, usually $2-4$, sometimes up to $9(-11)$, up to $50 \mathrm{~mm}$ apart; central pinnae index 3-8, widest somewhat below the middle, \pm symmetrical, $7.5-25$ by $2-5 \mathrm{~cm}$, base (narrowly) cuneate, its basiscopic side sometimes adnate and then often decurrent on the rhachis, the margin usually entire, inconspicuously toothed, crenate-serrate, or shallowly lobed, sometimes lobed to halfway to the costa, without spines, apex usually acute, sometimes acuminate; the two lowermost pinnae either conform to the central ones, or with the basiscopic side triangular and regularly lobed $1 / 3-2 / 3$ to the costa, or with one or a few conspicuous, basal, basiscopic lobes, up to 28 by $7.5(-11) \mathrm{cm}$, 0-12 mm stalked; terminal segment deltoid, base short- to long-decurrent, near base pinnatipartite, tapering towards the acute (acuminate) apex; secondary veins prominent, stramineous or brownish, other veins slightly prominent or immersed, concolorous with the lamina; venation pattern illustrated in fig. 33: e. Fertile leaves (25-) $35-75 \mathrm{~cm}$ long; petiole $20-60 \mathrm{~cm}$ long; lamina index $1-2(-3)$, widest at the base or somewhat below the middle, (7-) $13-25$ by $(2.5-) 5-20 \mathrm{~cm}$, without the terminal segment $0-8$ $(-14)$, the terminal segment $6-22 \mathrm{~cm}$ long; pinnae $2-6$, up to $50 \mathrm{~mm}$ apart; central pinnae index $2-8,(1.5-) 3-10$ by $0.4-2 \mathrm{~cm}$, margin usually entire, sometimes shallowly lobed especially the basiscopic side, apex acute or orbicular; the two lowermost pinnae up to 12 by $4.5 \mathrm{~cm}$, up to $5 \mathrm{~mm}$ stalked. Sporangia inserted all over the lower surface. Spores with a smooth cristate perispore.

Chromosomes. $\mathrm{n}=82$.

Mexico. C h i a p a s: Ghiesbrecht 409 (K). - V e r a C r u z: Bourgeau 2147 (L).

Honduras. Atla ntida: Standley 52821 (F, US). - Cort es: Williams \& Antonia Molina R. 17771 (US). NiCaragua. Chin andega: C. F. Baker 2185 (L).-Northwestern part: Englesing 83 (F, US). Cuba. 11 collections.

JAMAICA. 8 collections.

Dominican Republic. 10 collections. 
Puerto Rico. 9 collections.

Lesser Antilles. St. Eustatius: Boldingh $440 B$ (U). -Guadeloupe: l'Herminier 21 (CAL).-

Trinidad: 10 collections.-Le ew a rd I s.: Holme s.n. (K).

Colombia. Meta: Philipson \& Idrobo 1781 (BM, US); Stübel 658 (B).-Norte de Santander: Cuatrecasas 13254 (US).-Sant ander: Lindig s.n. (type of Chrysodium opacum); Haught 1322 (GH, US); Spruce 3013 (K).

VeNEZUELA. M a r a c a i b o: Karsten s.n. (? 165)(type of Chrysodium maracaybense).-D is $t$ r i t o F e d er a l: Bredemeyer s.n. (type of Acrostichum brunneum).

ECuAdor. Los Ri o s: Sodiro s.n. (B).

Peru. S a n M a r t i n: Allard 21558 (GH, US), 21672 (US), 22367 (US).-A m a z o n as: Wurdack 2033 (US). - H u a n u c o: Tryon \& Tryon 5281 (F, GH, U, US).

Bolivia. La P az: Buchtien 1002 (US).

See also note 4 and 6.

$\mathrm{H}$ abitat. Creeping on rocks or in earth in rain forest, especially in moist places and near streams. Altitude: $0-1100(-1400) \mathrm{m}$.

S p o r e s. Spruce 577 (K): the greater part normal, large. Valeur 535 (K): ibid., of medium size. Fuertes 1570 (G), Englesing 83 (US): the greater part abnormal, a fair minority normal, large. See also notes 3 and 4.

$\mathrm{N}$ o t e s. 1. A clear-cut species especially characterized by the slender rhizome which is clothed with small scales and by the colour and the venation pattern of the leaves. The shape of the lamina is very variable. This species has been confused with B. portoricensis by many authors until Christensen (1910) elucidated the identity of the type specimen. Part of the specimens from South America are deviating and need further study.

2. All specimens seem from Colombia are more or less deviating. Except for those already mentioned in notes 3 and 4, they include: Cuatrecasas 13254 (US) and Stübel 658 (B).

3. The type of Chrysodium opacum has leaves with \pm symmetrical basal pinnae and an asymmetrical terminal segment which is composed of few spaced lobes; the margin of all segments is crenate-serrate or shallowly lobed; spores of the isotype in $P$ are for the greater part abnormal, those of the isotype in BM for the greater part normal. The leaves of Haught 1322 (GH, US), also from Colombia, rather agree with those of the type collection; the terminal segment is composed of three lobes only and is in shape almost similar to that of the African relative B. auriculata; spores: sporangia of the GH-specimen for the greater part part filled with normal spores, a minority with shrivelled spores or juvenile ones; those of the US-specimen filled with shrivelled or otherwise abnormal spores or (in part) with aborted S.M.C.'s.

4. Deviating collections. Fendler 63x (B; MO, 2 sh., '63'; not Fendler 63, BM, F, G, GH, NY, S, UC, US), Cruger 221 (K), both from Trinidad, Philipson \& Idrobo 1781 (BM, US) and Spruce $3013(\mathrm{~K})$, both from Colombia, deviate from B. aliena proper: rhizome stouter, the leaves more closely together, \pm irregular as to shape, the terminal segment relatively large, the basal pinnae with a \pm conspicuous basal basiscopic lobe, the venation pattern very similar to that of $B$. aliena proper but the size of adjacent areolae sometimes very different, some areolae with an unbranched, ex- or recurrent, included free vein. Spores: Philipson \& Idrobo 1781 (BM): the majority of the sporangia filled with 
normal spores, a minority with aborted spores; ibid.(US): nearly all sporangia filled with aborted S.M.C.'s and/or shrivelled spores, an incidental normal spore also present in the preparation; Fendler $63 x$ (MO, a separately mounted fertile leaf): sporangia filled with clusters of aborted spores or with aborted S.M.C.'s.

The outline of these plants is strongly reminiscent of that described for B. semipinnatifida. However, the venation pattern is different, showing far fewer free included veins than is generally found in B. semipinnatifida. These collections need further study; they are not plotted on the distribution map.

5. Christensen (1904) excluded Leptochilus alienus ('essentially different') from Leptochilus sect. Bolbitis. In this section he included Leptochilus opacus $(=$ B. aliena $)$, the venation pattern of which he judged 'very irregular' and to resemble that of Leptochilus contaminoides ( $=B$. serratifolia).

6. The species is absent from eastern Nicaragua towards western Colombia. Lack of exploration seems not to be the obvious reason to explain this disjunction as much field work has been performed at least in Costa Rica. A.F. Tryon (Contr. Gray Herb. 200, $1970,104,110)$ reported an almost similar disjunction for Eriosorus hirtus and $E$. hispidulus, which she thought more widely distributed in the past.

7. I suppose that a species like $B$. aliena has given rise to $B$. semipinnatifida through hybridization with a species of ser. Euryostichae. See also under $B$. semipinnatifida.

\section{Bolbitis auriculata (Lam.) Alston-Fig. 34, 35.}

B. auriculata (Lam.) Alston, J. Bot. 72, Suppl. 2 (1934) 3; C.Chr., Ind. Fil., Suppl. 3 (1934) 47, 197; Alston, J. Bot. 77 (1939) 284; in Exell, Cat. Vasc. Pl. S. Tomé (1944) 68; Tardieu-Blot, Mém. I.F.A.N. 28(1953) 112, pl. 18: fig. 6-8; Alston in Hutch. \& Dalziel, Fl. West Trop. Afr. ed. 2, Suppl. (1959) 68; Tardieu-Blot in Humbert, Fl. Madag. 2 (1960) 60, fig. 16: 3-4, p.p.; Harley, Handb. Liberian Ferns (1963) 42, fig.; Tardieu-Blot in Aubrév., Fl. Gabon 8 (1964) 187; in Aubrév., Fl. Cameroun 3 (1964) 318.-Acrostichum punctatum L. f., Spec. Pl., Suppl. (1781) 444, nom. illeg., non L.; Carruth., Cat. Afr. Pl. Welwitsch 2, 2(1901) 277.-A. auriculatum Lam., Enc. 1 (1783) 36; Poiret in Lam., Enc., Suppl. 1 (1810) 122('auritum'). - A. punctulatum Swartz, Schrader J. Bot. 1800, 2 (1801) 12; Syn. Fil. (1806) 13; Willd., Spec. Pl. 5(1810) 118; Hooker, Spec. Fil. 5 (1864) 258; Baker in Hooker \& Baker, Syn. Fil. (1868) 419. - Poecilopteris punctulata Presl, Tent. Pterid. (1836) 242; Epim. Bot. (1851) 173; J. Smith, Ferns Br. For. (1866) 117.-Cyrtogonium punctulatum J. Smith, Hook. J. Bot.4(1841) 154. - Heteroneuron punctulatum Fée, Hist. Acrost. (1845) 91, pl. 54 ('Heteronevron'). - Chrysodium punctulatum Mettenius, Fil. Lips. (1856) 21; Ettingsh., Denkschr. K. Ak. Wiss. M.-N. Kl. Wien 22 (1854) 58, pl. 7: fig. 8, pl. 8: fig. 2('punctata').-C. punctatum Mettenius ex Kuhn, Fil. Afric. (1868) 51.-Gymnopteris punctata Christ, Farnkr. Erde (1897) 50.-Leptochilus punctatus C. Chr., Bot. Tidsskr. 26 (1904) 292._L. auriculatus C. Chr., Ind. Fil. (1906) 384; Henriques, Bol. Soc. Brot. 27 (1917) 178.-Gymnopteris auriculata Engler, Veg. Erde 9, 2 (1908) 16, fig. 11.-Campium auriculatum Copel., Philip. J. Sc. 37 (1928) 398, fig. 49; C. Chr., Dansk Bot. Ark. 7 (1932) 70.-T y p e: Sonnerat per Thouin s.n., Mascarenes, Réunion (Isle de Bourbon) (iso in C. herb. Vahl, fert., S-PA, herb. Swartz, fert.). Acrostichum mascarenense Sprengel, Syst. Veg. 4 (1827) 37.-T y p e: Sieber s.n. (?24). (n.v.); see note 3.

Leptochilus auriculatus (Lam.) C. Chr. var. undulati-crenatus Hieron., Bot. Jahrb.46(1911)345; Bonap., Notes Ptérid. 1 (1915) 51; ibid. 10 (1920) 85.-B. auriculata (Lam.) Alston var. undulato-crenata Kunkel, Ber. Schweiz. Bot. Ges. 73 (1963) 187, comb. invalid. (art. 33). -L e ct o t y pe: Zenker 1598, Cameroun, EpossiNtonga near'Bipindi, $500 \mathrm{~m}$ alt. (B, 2 sh.).

B. fluviatilis (Hooker) C. Chr. var. crenatis Kunkel, Ber. Schweiz. Bot. Ges. 73 (1963) 188.-T y p e: Kunkel F-200, Liberia, Western Prov., Gola Forest, 300-400 m alt. (private herb. Dr. Kunkel).

B. fuviatilis auct. non (Hooker) C. Chr.: Harley, Handb. Liberian Ferns (1963) 43, fig.; Kunkel, Ber. Schweiz Bot. Ges. 73 (1963) 187, pp. 


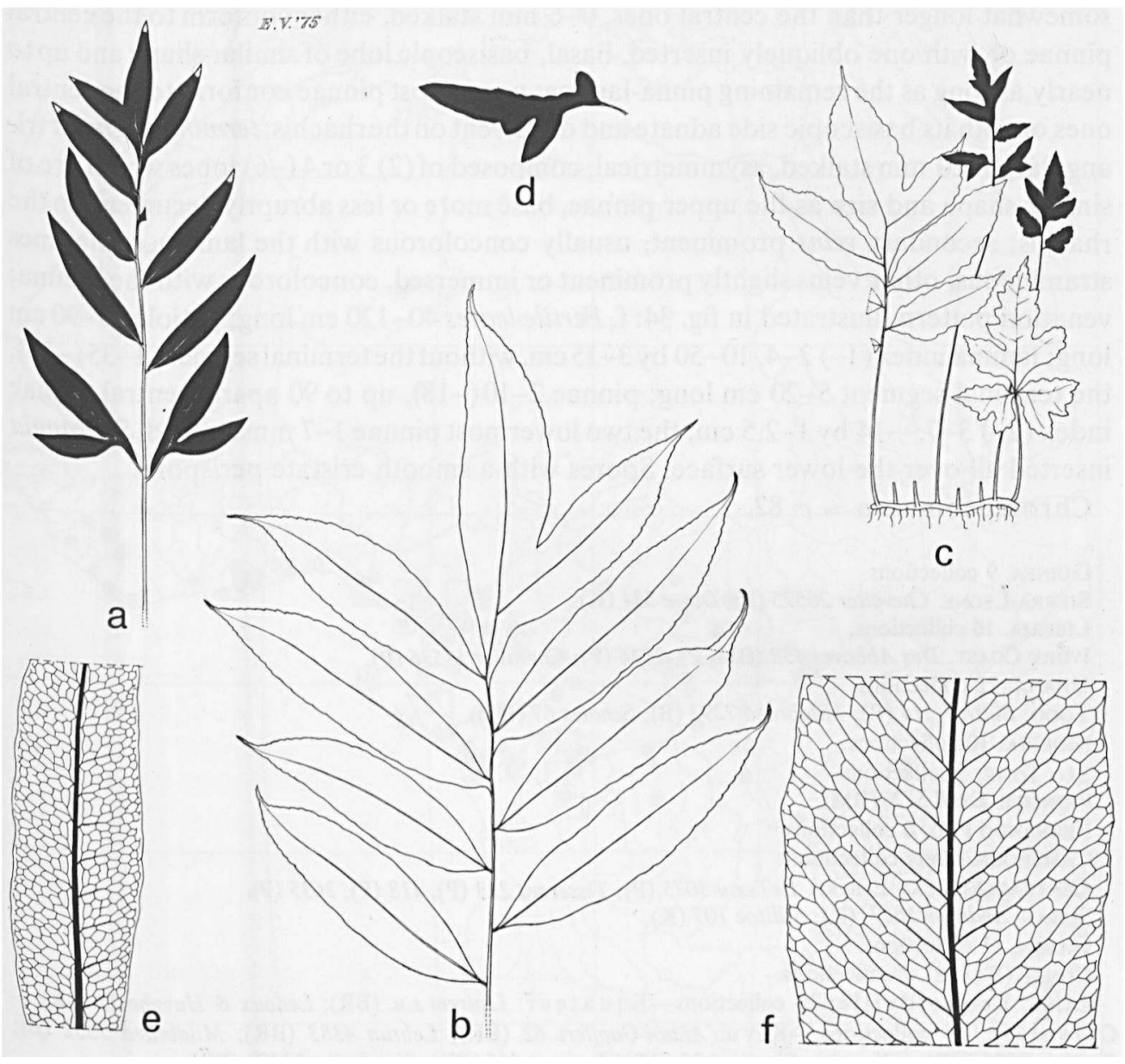

Fig. 34. Bolbitis auriculata.-a-c. sterile and fertile leaves, $\times 1 / 5$; d. basal pinna of fertile leaf, $\times 1 / 5$; e. venation pattern of fertile pinna, $\times 4 / 5$; f. ibid. of sterile pinna (a. Sonnerat per Thouin s.n., S-PA, holotype of B. auriculata; b. Hildebrandt 1803, L; c. Zenker 2751, B; d. Barter 1170, K; e. Kew Distr. no. 1175 , L; f. Boivin 2847, L).

Rhizome up to a few dm long, 3-7 mm thick. Scales of the rhizome: index 2-5, up to 7 by $0.5-1(-1.5) \mathrm{mm}$, opaque, brown or blackish. Leaves $15-40 \mathrm{~mm}$ apart. Sterile leaves pinnate, $30-115 \mathrm{~cm}$ long; petiole $15-65 \mathrm{~cm}$ long, near the base $2-4(-6) \mathrm{mm}$ thick; lamina index $1-2$, widest about or below the middle, $15-55(-70)$ by $15-40(-50) \mathrm{cm}$, without the terminal segment $0-35$, the terminal segment $15-40 \mathrm{~cm}$ long, usually herbaceous, sometimes papyraceous, olivaceous, rarely with a spherical or elongate axillary bulbil; pinnae (2-) 4-10, up to $60 \mathrm{~mm}$ apart; central pinnae index 2-6, widest about or below the middle, symmetrical, $8.5-30$ by $2.5-7.5 \mathrm{~cm}$, base cuneate, margin usually entire, sometimes finely serrate-crenate or unevenly and shallowly lobed, without spines or distinct teeth, apex acute or acuminate; the two lowermost pinnae somewhat shorter to 
somewhat longer than the central ones, 0-6 mm stalked, either conform to the central pinnae or with one obliquely inserted, basal, basiscopic lobe of similar shape and up to nearly as long as the remaining pinna-lamina; uppermost pinnae conform to the central ones or with its basiscopic side adnate and decurrent on the rhachis; terminal segment triangular, 0-20 mm stalked, asymmetrical, composed of $(2) 3$ or $4(-6)$ lobes which are of similar shape and size as the upper pinnae, base more or less abruptly decurrent on the rhachis; secondary veins prominent, usually concolorous with the lamina, sometimes stramineous, other veins slightly prominent or immersed, concolorous with the lamina; venation pattern illustrated in fig. 34 : f. Fertile leaves $40-120 \mathrm{~cm}$ long; petiole $35-90 \mathrm{~cm}$ long; lamina index (1-) $2-4,10-50$ by $3-15 \mathrm{~cm}$, without the terminal segment $1-35(-50)$, the terminal segment 5-20 cm long; pinnae 2-10(-18), up to 90 apart; central pinnae index (2-) 3-7,-14 by $1-2.5 \mathrm{~cm}$; the two lowermost pinnae 1-7 $\mathrm{mm}$ stalked. Sporangia inserted all over the lower surface. Spores with a smooth cristate perispore.

Chromosomes. $\mathrm{n}=c .82$.

GUINEA. 9 collections.

Sierra Leone. Chevalier 20575 (P); Dawe 534 (K).

LIBERIA. 16 collections.

IvORY COAST. Des Abbayes 438 (BM, P), 2146 (P); Chevalier 17536 (P).

GHaNA. 22 collections.

Togo. Büttner 514 (B); Mildbraed 7292 (B); Scholes 67 (BM).

Nigeria. 10 collections.

SÁo TOME. 5 collections.

PRINCIPE, Exell 572 (BM).

Fernando Poo. 6 collections.

Cameroun. many collections.

Central African Empire. LeTestu 3075 (P); Tisserant 223 (P), 418 (P), 2635 (P).

SUdAN. Andrews 1627 (K); Sillitoe $107(\mathrm{~K})$.

GABON. 11 collections.

Congo(Brazz). 7 collections.

ZAIRE. Le op old ville: 29 collections-Equateur: Laurent s.n. (BR); Ledoux \& Huyghe 23 (BR). Orientale: 14 collections.-Kivu: Antun-Gupffert 62 (BR); Lebrun 4383 (BR); Mildbraed 2332 (B); Troupin 2995 (BR). -K a s a i: Flamigni 35 (BR); Laurent 446 (BR); Vanderijst 22409 (BR).

ANGOla. Cabind a: Gossweiler 6591 (BM).-Cuanza Nort e: Welwitsch 153 (BM, K).

Uganda. We stern: Kassner $3132 b$ (P); Sangster $S 87$ p.p. (BM), 192 (BM), 292 (BM). - B u ganda: Longfield 13 (BM); Maitland 667 (K); Thomas 1390 (K).-Mu l a n ge: Dümmer 4471 (K).

TANGanYIKa. Tanga: 7 collections. - Southern Highland: Schweinfurth 3102 (B).-Eastern: Schlieben 2875 (B, BR). 'Ruwenzori Exp.': Scott Elliot 7096 (BM, K).

Comores. G ra nd e Com or e: Humblot s.n. (P). -A n jou a n (= Johanna Is.): Hildebrandt 1803 (B, K, L, P).-Mo helli: Benson 135A (BM).-M a y ot t e: Boivin 2847 (B, BM, L, P), Marie 151 (P), 188 (G).

MadaGasCar. 7 collections.

MASCAREnes. Réu nion: 6 collections.-Ma uritius: 8 collections.

$\mathrm{H}$ a bit at. On rocks or in earth in humid or swampy places (frequently in and near streams) in primary and secondary forest. Altitude: $0-1200 \mathrm{~m}$.

S p or e s. Félix 41 (S-PA), Harley F 111 (K), F 180 (GH), De Jussieu s.n. (S-PA): the majority normal; the sporangia of De Jussieu s.n. and Harley F 111 filled with over 50 spores of varying size. Peter 18921 (C): in part (the majority ?) normal. Baldwin 6709 (GH), Harley F $76 A(\mathrm{GH})$, Sonnerat per Thouin s.n. (type of Acrostichum punctulatum): the greater part abnormal. 


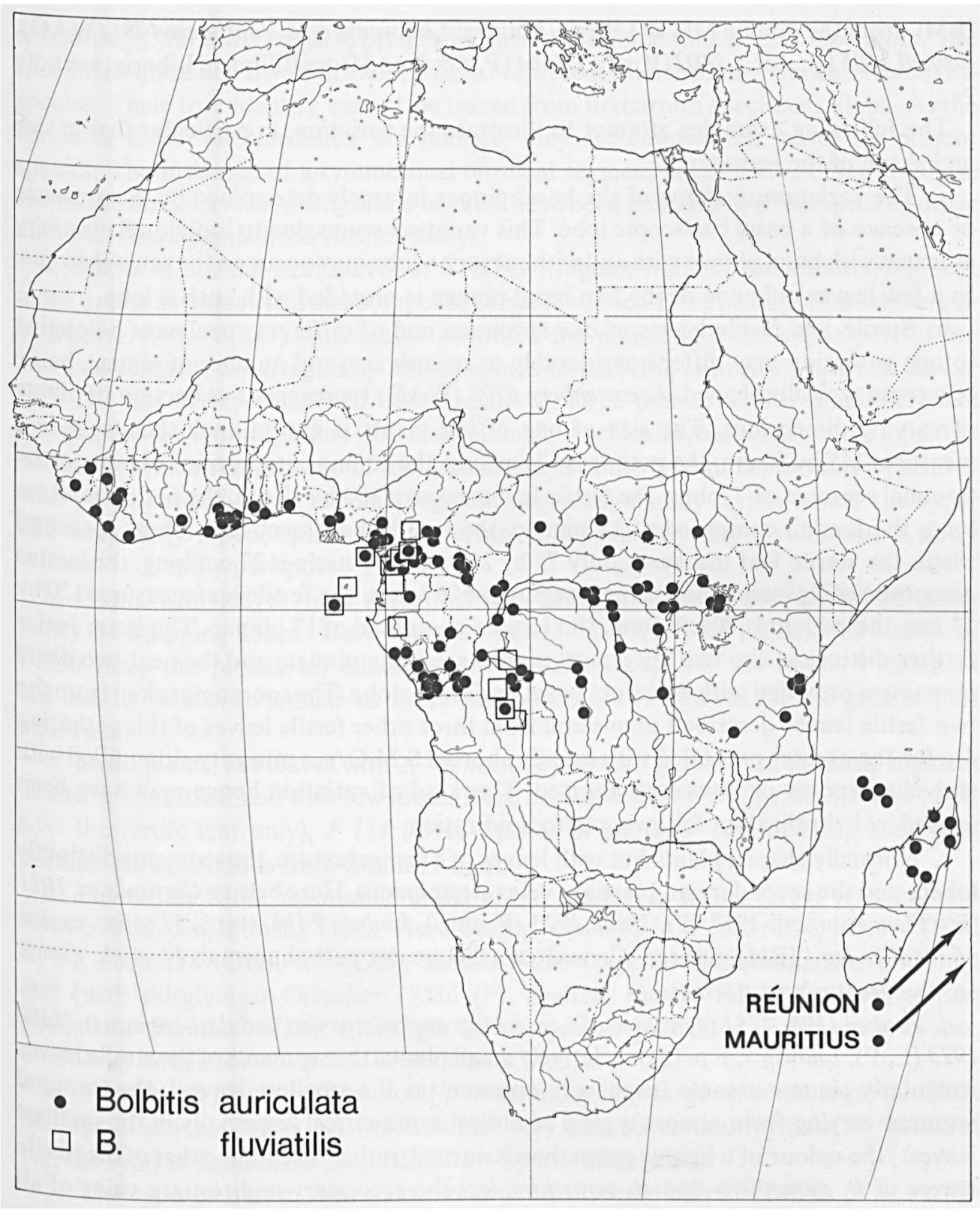

Fig. 35. Distribution of Bolbitis auriculata and B. fluviatilis.

Notes. 1. Although normally shaped specimens of the present species are obviously common throughout its geographical range, a considerable number of more or less atypical collections was found as well. Part of these collections have been gathered from but few localities. Such local outbursts of different forms are for instance known from the Puso Puso Ravine in Ghana: Adams 331 (BM; M, ster.), 415 (BM, K, P, all ster), 1892 
(BM), from the Nimba Mts. in Liberia/Guinea: Leeuwenberg \& Voorhoeve 4781 (WAG), Schnell 1105 (P, ster.), 1402 (P, ster.), 1464 (P, ster.), and from Bilimu in Liberia: see note 2c.

The following examples attempt to illustrate the variation, in particular that in size and shape of the leaves.

a. The variation in shape of the basal pinnae is largely determined by the presence or absence of a basal basiscopic lobe. This variation seems due to individual plasticity as leaves with basal pinnae with and without such a lobe may occur on the same rhizome. In a few leaves only one of the two basal pinnae is provided with such a lobe.

b. Sterile and fertile leaves of one specimen and of different specimens belonging to one gathering may differ considerably as regards size and number of pinnae, being otherwise typically shaped. Leeuwenberg 6797 (WAG) represents a collection of plants of varying dimensions. The size of one of the plants is exceptional: the sterile leaf measures 145 by $50 \mathrm{~cm}$, the petiole is $67 \mathrm{~cm}$ long, the lamina composed of 14 pinnae, the terminal segment of 7 lobes; the fertile leaf measures $160 \mathrm{by} 19 \mathrm{~cm}$, the petiole is $90 \mathrm{~cm}$ long, the lamina composed of 18 pinnae, the terminal segment of 6 lobes. A second plant: the sterile leaf measures only 75 by $26 \mathrm{~cm}$, the petiole is $27 \mathrm{~cm}$ long, the lamina composed of 7 pinnae, the terminal segment of 6 lobes; the fertile leaf measures 120 by $13 \mathrm{~cm}$, the petiole is $80 \mathrm{~cm}$ long, the lamina composed of 17 pinnae. The latter leaf is further distinct as the two lowermost pinnae are again pinnate and the next two distal pinnae are provided with a distinct basal basiscopic lobe. The sporangia taken from the two fertile leaves described above and from three other fertile leaves of this gathering are for the greater part filled with usually aborted S.M.C.'s, a minority either filled with shrivelled spores or obviously aborted. This kind of variation hence may have been caused by hybridization following autoploidization.

c. Normally shaped plants but with leaves of a firmer texture, the segments distinctly lobed, and the secondary and tertiary veins stramineous. Here belong Cervoni s.n., 1911 (P), Fleury s.n., vii-1917 (P), Hallé 1923 (P, ster.), Ludwig 7 (M, ster.), Fl. Afric. exsicc. 66 (Moller leg.) (BM). Spores: Cervoni s.n.: the greater part abnormal. Fl. Afric. exsicc. 66: the greater part normal.

d. Zenker 1598, 2751 (syntypes of Leptochilus auriculatus var. undulati-crenatus), Halle 1923 (L, P), Ludwig 7, p.p. (M): relatively small plants, the segments of the sterile leaves irregularly sinuate-crenate (especially apparent in the smallest leaves), the terminal segment varying from almost typical to almost symmetrical (especially in the smallest leaves); the colour of a lighter green than is normal, rather resembling that of the sterile leaves of $B$. gemmifera and $B$. acrostichoides; the secondary and tertiary veins of all except Zenker 2751 stramineous. Spores: Zenker 2751 (B): in part normal. The question arises if these collections result from hybridization between the present species and B. acrostichoides.

2. Plants with leaves intermediate between those of $B$. auriculata and B. fluviatilis. Generally, $B$. auriculata and $B$. fuviatilis are easily distinguished because of differences in leaf architecture, in the prominence of the secondary veins, and also more or less 
in texture. B. fluviatilis seems to prefer a more humid habitat than does B. auriculata. The specimens discussed below are supposed to represent intermediates between these two species. Their true identity cannot be traced from herbarium specimens alone. As the leaves of these 'intermediates' are pinnate, they are enumerated under the present species. The presence of a symmetrical terminal segment in the leaves of part of these seems the obvious reason why some of them have been included in B. fuviatilis by other authors. These plants need further study.

a. Plants of normal size; leaves of a rather irregular outline; the terminal segment more or less asymmetrical and composed of but few lobes. Here belongs Leeuwenberg \& Voorhoeve 4781 (WAG) with leaves of which the pinnae and the upper part of the terminal segment are irregularly lobed; spores in part (the minority?) normal. Barker $1170(\mathrm{~K})$ has leaves composed of six relatively large pinnae with a sinuate-crenate margin, the lowermost pinnae with several small, £orbicular, basal basiscopic lobes the outline of which reminiscent of the lamina shape of the type of Leptochilus grossedentatus ( $=$ B. fluviatilis), the terminal segment large, only slightly asymmetrical, the upper half irregularly lobed; spores: all abnormal, the normally shaped ones with a finely cristate or \pm spiny perispore.

b. Plants with leaves with a symmetrical terminal segment provided with many partly overlapping lobes. Here belongs Adams 1892 (= 3851; BM), the leaves of which have the pinnae all adnate to the rhachis except for the lowest two which are sessile, otherwise similar to those mentioned above. Spores for the greater part abnormal.

c. Small plants, the leaves with 2(-4) normally shaped pinnae, the terminal segment almost symmetrical and with few lobes. Here belong Harley $F 76(\mathrm{~K}$, ster.), $F 76 A(\mathrm{GH}$, p.p.: the fertile leaf only), $F 114(\mathrm{GH})$. Spores of Harley $F 114$ for the greater part normal. All collections from Bilimu, Liberia.

d. Plants like those mentioned under $\mathrm{c}$. but the leaves with a larger terminal segment provided with many lobes. Here belong Adams 331 (= 2635; BM, M), 415 (BM, $\mathrm{K}, \mathrm{P})$, Cook $138(\mathrm{GH}), 167(\mathrm{GH})$, Kunkel F-200 (typè of B. fluviatilis var. crenatis), and (with misgivings) Chevalier 17536 (P). Spores: Kunkel F-200: the greater part abnormal; Chevalier 17536: sporangia for the greater part filled with normal spores, a minority with aborted spores or aborted S.M.C.'s.

e. Small-sized plants with two odd pinnae and a very large terminal segment. Here belongs Linder s.n., $1926(\mathrm{~K})$ which comes very near to certain forms of B. fluviatilis, e.g. the type of Leptochilus grossedentatus. Spores abnormal.

3. The type of Acrostichum mascarenense. In the original publication of $A$. mascarenense a type specimen was not indicated. Sprengel referred to a plant named ' $A$. punctulatum Sieb.', a MS name. The type is presumably Sieber (Syn. Fil. no.) 24 from Mauritius (B, BR, G, K, L, MO, P, UC, W) or Sieber s.n. from the same place which may both belong to the same collection.

4. It is supposed that a $B$. auriculata-like fern may be one of the parents of $B$. gemmifera, B. longiflagellata (see: spec. dub.), Leptochilus gemmifer var. latipinnata (see: $B$. acrostichoides), and hybridized with $B$. heudelotii (see: hybrids). 
3. Bolbitis fluviatilis (Hooker) Ching-Fig. 35, 36.

B. fluviatilis (Hooker) Ching in C. Chr., Ind. Fil., Suppl. 3 (1934) 48; Alston, J. Bot. 77 (1939) 284; in Exell, Cat. Vasc. Pl. S. Tomé (1944) 69; Tardieu-Blot, Mém. I.F.A.N. 28 (1953) 110, pl. 18: fig. 4 \& 5; Adams \& Alston, Bull. Br. Mus. Nat. Hist. 1 (1955) 172; Alston, Bull. I.F.A.N. ser. A, 21 (1959) 447; in Hutch. \& Dalziel, Fl. West Trop. Afr. ed. 2, Suppl. (1959) 68; Kunkel, Ber. Schweiz. Bot. Ges. 73(1963) 187, p.p.; Tardieu-Blot in Aubrév., Fl. Gabon 8 (1964) 186, pl. 33: fig. 1-3; in Aubrév., Fl. Cameroun 3 (1964) 316.-Acrostichum fluviatile Hooker, Spec. Fil. 5 (1864) 274; Baker in Hooker \& Baker, Syn. Fil. (1868) 422.-Chrysodium fuviatile Kuhn, Fil. Afric. (1868) 51.-Poecilopteris fuviatilis J. Smith, Hist. Fil. (1875) 137.-Leptochilus fuviatilis C. Chr., Ind. Fil. (1906) 385.-Gymnopteris fuviatilis Engler, Veg. Erde 9, 2 (1908) 16.-Campium fuviatile Copel., Philip. J. Sc. 37 (1928) 399, fig. 50, pl. 32.-Lect o t y pe: Mann 442, Fernando Poo (K, 2 sh.; iso in GH, Kew Distr. no. 1181).

Acrostichum phanerodictyon Baker in Henriq., Bol. Soc. Brot. 4 (1886) 156, pl. 4: fig. C; Copel., Philip. J. Sc. 37 (1928) 401.-Leptochilus phanerodictyus C. Chr., Ind. Fil. (1906) 387.-Gymnopteris phanerodictya Engler, Veg. Erde 9, 2 (1908) 16. - B. phanerodictya Ching in C. Chr., Ind. Fil., Suppl. 3(1934) 49.-T у р e: Fl. Afric. exsicc. 68 (Moller, not Quintas leg.), Sao Tomé, $200 \mathrm{~m}$ alt. (K; iso in B, BM, BR, C, G, K, MO, P). See note 7 .

Leptochilus grossedentatus Bonap., Notes Ptérid. 14 (1923) 217.-B. grossedentata C. Chr., Ind. Fil., Suppl. 3 (1934) 48. - T y p e: LeTestu 1570, Gabon, Mayombe Bayaka, Djengila, Ravine of Dougouatzi (P).

B. fluviatilis (Hooker) Ching var. crenatis auct. non Kunkel: Kunkel, Ber. Schweiz. Bot. Ges. 73 (1963) 188.

Rhizome up to $20 \mathrm{~cm}$ long, up to $7 \mathrm{~mm}$ thick. Scales of the rhizome: index 1-6, up to 15 by $2.5 \mathrm{~mm}$, large, opaque, castaneous. Leaves up to $15(-25) \mathrm{mm}$ apart. Sterile leaves either entire or pinnatipartite, $15-85 \mathrm{~cm}$ long. Entire leaves: petiole $4-20 \mathrm{~cm}$ long, near the base 1-3.5(-5) mm thick; lamina index 3-7, widest about the middle, $10-60$ by $2-12 \mathrm{~cm}$, (thin-)herbaceous, usually olivaceous or blackish, without bulbils, base short- to long-decurrent, margin usually entire, sometimes in the basal half shallowly and irregularly lobed, without teeth or spines, apex acute to acuminate; costa prominent on either side, other veins immersed or the secondary veins near the costa slightly prominent; venation pattern: see fig. $36: \mathrm{h}$, i. Pinnatipartite leaves: petiole $4-35(-45) \mathrm{cm}$ long; lamina index $1-4$, widest below the middle, $20-50$ by $5-25(-35)$ $\mathrm{cm}$; lobes 2-8, usually opposite, those of one side usually spaced, sometimes overlapping, straight or the upper half somewhat falcate, those of one leaf rather varying in size, up to $20(-24)$ by $6.5(-8) \mathrm{cm}$, margin usually entire, sometimes shallowly and irregularly lobed, apex usually acute or acuminate, sometimes orbicular; the lamina at the sinuses above the two lowermost lobes $1-3.5 \mathrm{~cm}$ wide; terminal segment relatively long, $2.5-10 \mathrm{~cm}$ wide, usually entire, sometimes pinnatifid near the base; otherwise similar to the entire leaves. - Fertile leaves $15-90 \mathrm{~cm}$ long. Entire leaves: petiole $10-30 \mathrm{~cm}$ long, near the base $1-4 \mathrm{~mm}$ thick; lamina index 5-10(-18), usually widest about (to below) the middle, $4-40$ by $1-3 \mathrm{~cm}$. Pinnatipartite leaves: petiole $10-55 \mathrm{~cm}$ long; lamina index $2-8,9-40$ by $1-10(-20) \mathrm{cm}$; lobes $2-8$, up to $9(-13)$ by $2(-3) \mathrm{cm}$, the lamina at the sinuses above the two lowermost lobes $0.5-1.5 \mathrm{~cm}$ wide; terminal segment $1.5-3.5 \mathrm{~cm}$ wide. Sporangia inserted usually all over the lower surface; sometimes a narrow strip along the costa in the basal half and along the secondary veins of pinnatipartite leaves devoid of sporangia. Spores usually with a smooth cristate perispore. 

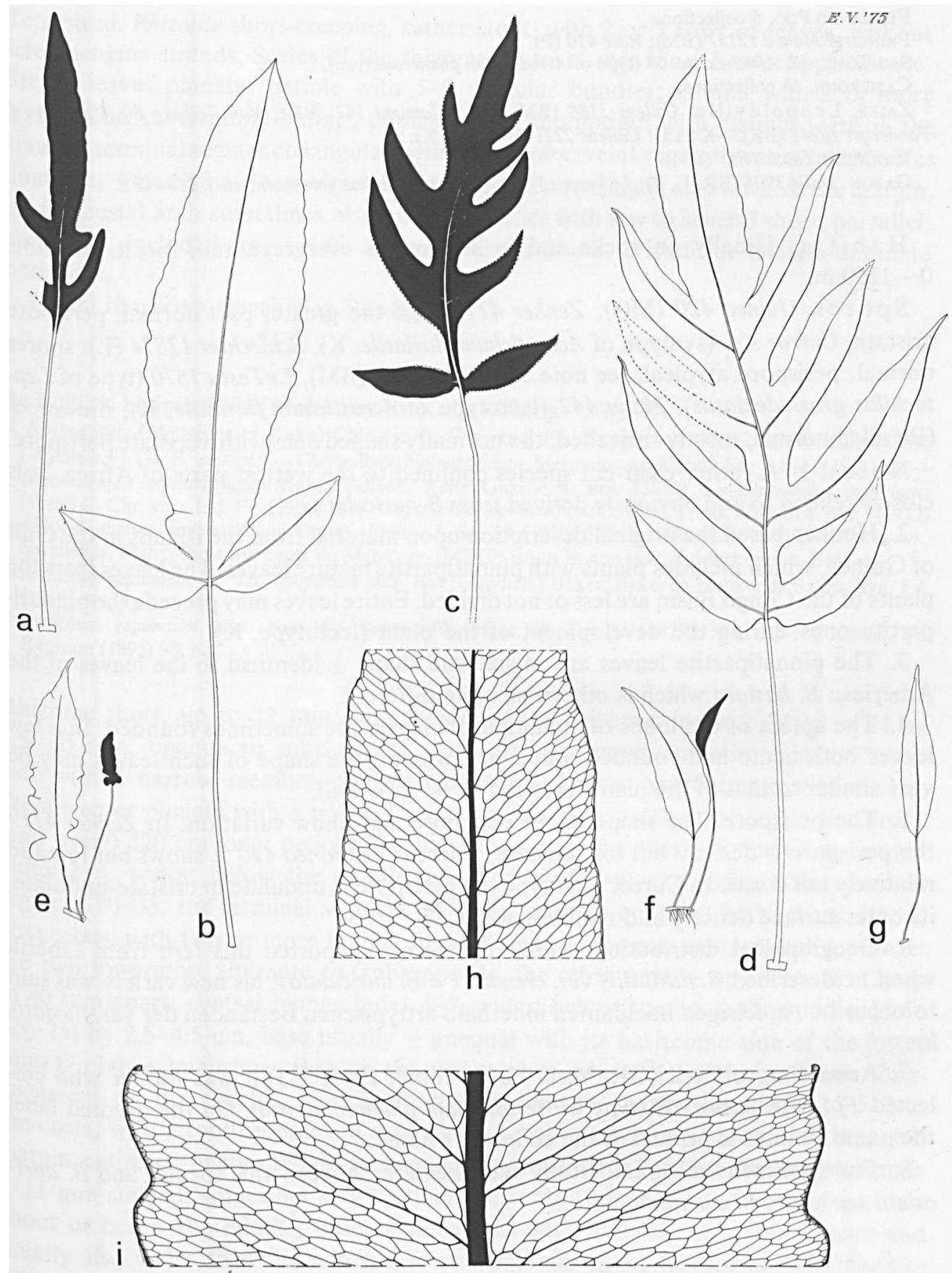

Fig. 36. Bolbitis fuviatilis. $-\mathrm{a}-\mathrm{g}$. sterile and fertile leaves, $\times 1 / 5 ; \mathrm{h}$, i. venation pattern of sterile laminas, $\times 4 / 5$ (a. Zenker 4119, G; b, i. Raynal 10705, P; c, d. Curror s.n., K, paratype of B. fuviatilis; e. Linder s.n., 1926, K; f, g. Fl. Afric. exsicc. 68, K, holotype of Acrostichum phanerodictyon; h. Schlechter 12874, L). 
Fernando Poo. 5 collections.

PrINCIPE. Monod 12137 (BM); Rose 410 (P).

SAO TOME. Fl. Afric. exsicc. 68 (type of Acrostichum phanerodictyon).

Cameroun. 14 collections.

ZaIRE. L e opoldvill e: Callens 3188 (BM, BR); Flamigni 141 (BR); Van Telborg 80 (P).-Kivu:

Vanderijst 16241 (BR).--K a s ai: Callens 2221 (BM, BR, K).

Rio MuNI. Guinea 855 (BM).

GABON. Halle 3906 (BR, L, P); LeTestu 1570 (type of Leptochilus grossedentatus), 2184 (P).

$\mathrm{Habitat.} \mathrm{Usually} \mathrm{on} \mathrm{rocks} \mathrm{and} \mathrm{in} \mathrm{streams} \mathrm{in} \mathrm{evergreen} \mathrm{rain} \mathrm{forest.} \mathrm{Altitude:}$ $0-1250 \mathrm{~m}$.

S pores. Guinea 420 (MO), Zenker $4119(\mathrm{G})$ : the greater part normal, perispore cristate. Curror s.n. (syntype of Acrostichum fluviatile, K), Schlechter 12874 (L): spores normal, perispore atypical, see note 5. Callens 3188 (BM), LeTestu 1570 (type of Leptochilus grossedentatus), Mann 442 (lectotype of Acrostichum fluviatile, K), Nickles 92 (P): all abnormal, mostly shrivelled, the normally shaped ones with a cristate perispore.

Notes. 1. A rather clear-cut species confined to the wettest parts of Africa. It is closely related to and obviously derived from $B$. auriculata.

2. Hooker based the original description upon material from the islands in the Gulf of Guinea, which includes plants with pinnatipartite mature leaves. The leaves from the plants of the Congo Basin are less or not divided. Entire leaves may precede the pinnatipartite ones during the development of the plant (lectotype, K).

3. The pinnatipartite leaves are in size and shape \pm identical to the leaves of the American $B$. hastata which is otherwise quite different.

4. The apices of the lobes of pinnatipartite leaves are sometimes rounded. In a few leaves both acute and rounded apices are present; the shape of such leaves may be very similar to that of the plants referred to $B . \times$ boivinii.

5. The perispore. The shape of the perispore may show variation. In Zenker 4119 the perispore is densely but low-cristate, whereas in Guinea 420 it shows but few and relatively tall crests. In Curror's syntype the perispore is undulate or cristate-undulate, its outer surface densely and minutely warty.

6. Geographical distribution. Indirectly, Kunkel reported this fern from Liberia when he described $B$. fluviatilis var. crenatis $(=B$. auriculata $)$; his new variety was said to occur '.. . in felsigen Bachläufen innerhalb arttypischen Beständen der var. fluviatilis ....

7. According to Exell (Cat. Vasc. PI. S. Tomé, 1944, 383) it was Moller who collected Fl. Afric. exsicc. 68 (type of Acrostichum phanerodictyon). On the original label the name Quintas is printed as the collector's name.

8. For specimens morphologically intermediate between this species and B. auriculata see under $B$. auriculata note 2 .

\section{Series Bipinnatifidae Hennipman, ser. nov.}

Seriei Quoyanae similis, differt marginibus pinnarum dentibus in sinubus munitis, ordinatione venularum venulis liberis paucis ad pluribus brevibus excurrentibus, positis in arcu costali, interdum etiam in arcu distali.

Ty pus: Bolbitis bipinnatifida (Mettenius ex Kuhn) Ching. 
Terrestrial. Rhizome short-creeping, rather stout, with 2 or 3 rows of leaves, without sclerenchyma strands. Scales of the rhizome up to $11 \mathrm{~mm}$ long. Leaves approximate. Sterile leaves pinnate; petiole with 5-9 vascular bundles; lamina with 2 or more \pm spherical subterminal bulbils, pinnae 16-22, the margin with distinct teeth in the sinuses; terminal segment triangular; venation pattern: veins regularly reticulate, areoles angulate, \pm isodiametric or somewhat elongate, decreasing in size towards the margin, on the costal arch sometimes also on distal arches with few to several short, parrallel, excurrent, included free veins. Spores with a smooth, cristate or cristate-undulate perispore.

Distribution. Seychelles. See fig. 30.

4. Bolbitis bipinnatifida (Mettenius ex Kuhn) Ching-Fig. 37.

B. bipinnatifida (Mettenius ex Kuhn) Ching in C. Chr., Ind. Fil., Suppl. 3 (1934) 47, non Iwatsuki (1959) ('bipinnata') $(=$ B. sinensis). - Chrysodium bipinnatifidum Mettenius ex Kuhn, Fil. Afric. (1868) 50.Heteroneuron bipinnatifidum Kuhn, Forsch. Reise Gazelle 4, Bot. (1889) 6.-Leptochilus cuspidatus (Presl) C. Chr. var., Ind. Fil. (1906) 181._Leptochilus bipinnatifidus C. Chr., Trans. Linn. Soc. Bot. 7(1912) 414.-Campium bipinnatifidum Copel., Philip. J. Sc. 37 (1928) 377, fig. 30.-L ect o t y pe: Boivin s.n., Seychelles, Mahé, 'bois des hauts de Mahé', iv-1848 ( $\mathrm{P}$; iso in B, one ster. and fert. pinna, BM).

Acrostichum repandum Bl. var. quoyanum auct. non (Gaudich.) Baker. Baker in Hooker \& Baker, Syn. Fil. (1868) 419, p.p.

Acrostichum repandum auct. non Bl.: Baker, Fl. Maur. \& Seych. (1877) 514, p.p.; Cordem., Fl. Réunion (1895) 98, p.p.

Rhizome short, up to $12 \mathrm{~mm}$ thick. Scales of the rhizome: index 2-7, up to 11 by $1.5(-2) \mathrm{mm}$, opaque to subclathrate, dark greyish brown, sometimes in the lower half with a narrow membranous marginal strip. Leaves approximate, glabrescent. Sterile leaves pinnate with a triangular terminal segment, rather varying in size, up to (and over?) $120 \mathrm{~cm}$ long; petiole $20-30 \mathrm{~cm}$ long, near the base 3-6 mm thick; lamina index $2-4$, widest below the middle, up to 95 by $20-40 \mathrm{~cm}$, without the terminal segment 20-35, the terminal segment 30-65 (or more?) cm long, herbaceous, (light) olivaceous, with two or more bulbils situated several $\mathrm{cm}$ apart subterminally; pinnae: the two lowermost alternate to (sub)opposite, the other ones \pm opposite, 16-22, up to $40 \mathrm{~mm}$ apart; central pinnae index 4-7, widest below (to about) the middle, 11.5$22(-25)$ by $2.5-4.5 \mathrm{~cm}$, base usually \pm unequal with its basiscopic side of the lower central pinnae angustate, that of the upper ones adnate to and sometimes longdecurrent on the rhachis, its acroscopic side cuneate, margin lobed $3 / 7$ to $5 / 7$ to the costa, with a tooth present in each sinus, lobes overlapping to somewhat spaced, margin entire or finely crenate-serrate, apex \pm rounded; the two lowermost pinnae 3-11 mm stalked, somewhat shorter than the central pinnae, $3.5-8 \mathrm{~cm}$ wide, widest about or below the middle, base asymmetrical with its basiscopic side shorter and usually also wider than the acroscopic one, margin of the basiscopic side lobed to $9 / 10$ to the costa, lobes usually entire or crenate-serrate, sometimes lobed to $2 / 5$ towards the costule; terminal segment relatively large, base short- to long-decurrent, margin near the base deeply pinnatipartite, tapering towards the apex, apex acute (in small leaves) or flagelloid, flagellum near the base with lobes which are spaced, \pm tri- 

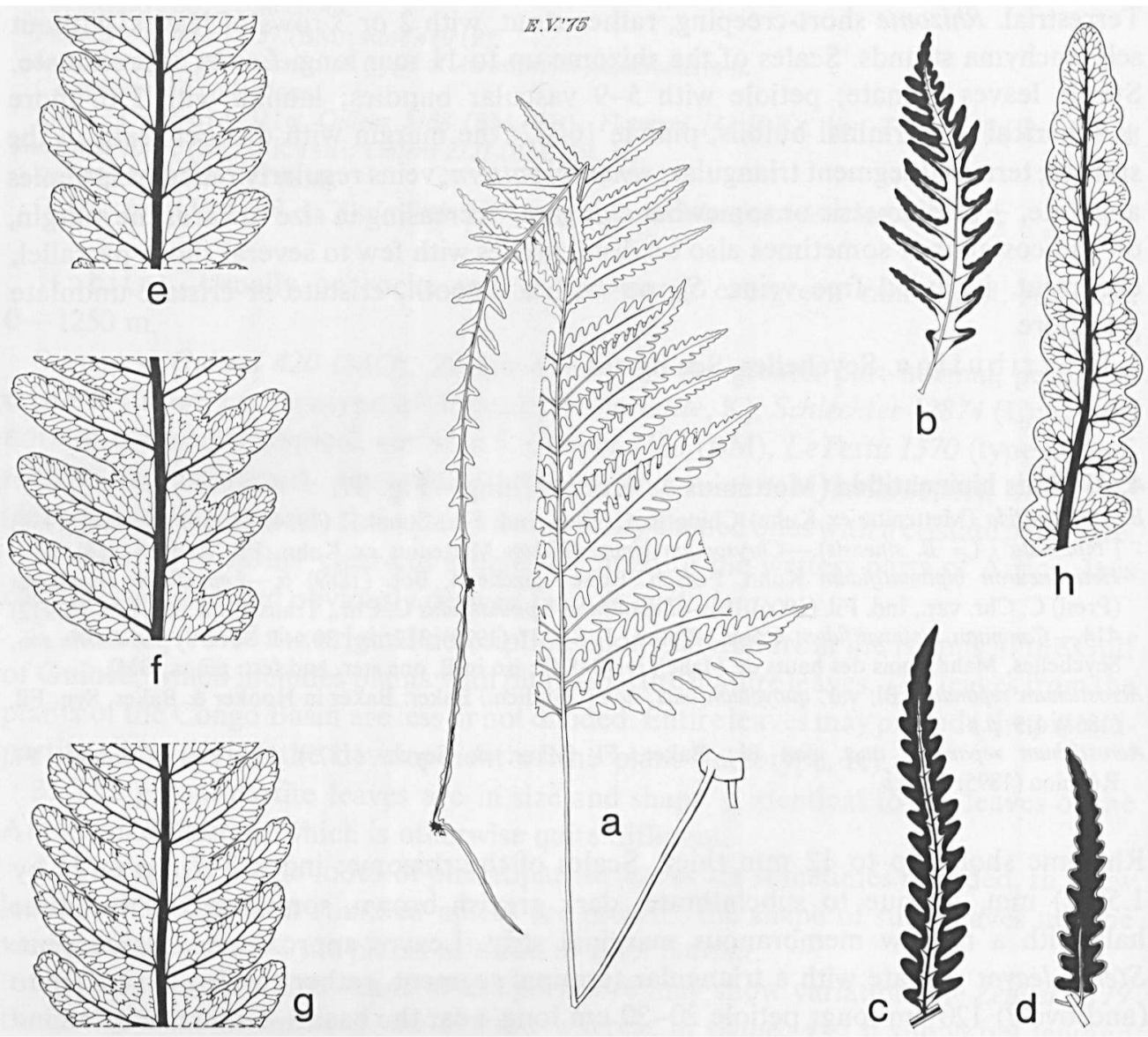

Fig. 37. Bolbitis bipinnatifida. -a. sterile leaf, $\times 1 / 5$; b. basal pinna of fertile leaf, $\times 2 / 5$; c, d. central pinnae of fertile leaf, $\times 2 / 5$; e-g. venation pattern of sterile pinnae, $\times 4 / 5$; h. ibid. of fertile pinna, $\times 4 / 5$ (a, $h$. Jeffrey 759, L; b. Horne 166, K; c. Jeffrey 759, K; d. Horne s.n., K; e. Brauer 12, B; f, g. Horne s.n., B).

angular, unequal with the margin of the basiscopic side long-decurrent on the rhachis, flagellum \pm sinuate towards the apex; costa and secondary veins stramineous, prominent on either side, other veins immersed or almost so, concolorous with the lamina; venation pattern: see fig. $37 \mathrm{e}-\mathrm{g}$. Fertile leaves. Only two complete and fully acrostichoid fertile leaves available: Jeffrey 759 (L): leaf $73 \mathrm{~cm}$ long, petiole $35 \mathrm{~cm}$ long, near the base $c .5 \mathrm{~mm}$ thick; ibid. (K): leaf $108 \mathrm{~cm}$ long, petiole $74 \mathrm{~cm}$ long, near the base c. $5 \mathrm{~mm}$ thick. Lamina index $2-3$, widest below the middle, $30-38$ by $10-20 \mathrm{~cm}$, without the terminal segment $18-28$, the terminal segment 6-20 cm long; pinnae 14-22, up to $50 \mathrm{~mm}$ apart; central pinnae index 5-8, widest about the middle, 7-9.5 by 1-2 $\mathrm{cm}$, margin lobed $1 / 5$ to $4 / 5$ towards the costa, a tooth in the sinus not always distinct; the two lowermost pinnae 5-8 $\mathrm{mm}$ stalked, sometimes slightly longer than the 
central ones, up to $3.5 \mathrm{~cm}$ wide. Sporangia inserted all over the lower surface or on part of the lamina (see note 3 ).

SeYchelles. Mah é: Boivin s.n. (lectotype); Bouton s.n. (K); Brauer 10, 11, 12 (all in B); Gardiner s.n., 1908 (K); Horne 166 (B, BM, K); Kew 1174, ex coll. Rawson W. Rawson 890 (L); Meriau s.n., 1903 (P); Rawson W. Rawson 1059 (BM); Perville 74 (P); slopes of Morne Blane Jeffrey 584 (K); Niole: Jeffrey 759 (K, L, P); Cascade Estate: Thomasset 113 (K).-Silh ou et te I.: Gardiner s.n., $1908(\mathrm{~K})$. - S in e lo c.: Horne s.n. (B, BM); Kew 1174 (B, GH, P, W); Ward s.n. (P).

H abitat. 'one of the commonest ferns in damp woods' (Horne 166); 'on rocks and boulders' (Jeffrey 759). Altitude: $200 \mathrm{~m}$ (Jeffrey 759).

Sp or es. Horne s.n. (B), Jeffrey 759 (L): by far the greatest part normal.

Notes. 1. A very distinct species which relationship is not obvious. Because of its venation pattern it seems related to $B$. quoyana (eastern Malesia to the Pacific) and to the American $B$. umbrosa. On the other hand it shares several features with the American B. portoricensis (ser. Portoricenses) but not with B. quoyana (ser. Quoyanae): scales (perfoliate in B. quoyana), the projection in the sinus (a spine in B. quoyana), and the shape of the terminal segment (not flagelloid and with only one bulbil in B. quoyana). Relationship to $B$. portoricensis seems at first sight not so likely as its venation pattern is quite different from that of the present species. The shape of the leaf and the venation patterns of the juvenile leaves of the present species and B. portoricensis (and also of $B$. quoyana) are, however, similar. A juvenile leaf of a cultivated specimen of B. bipinnatifida at Kew even showed incidental short recurrent veins (absent in mature leaves). Therefore, these two species may be related after all.

2. If the basal pinnae are not conform to the central ones, they are widest about or below the middle and not at the base as in all other species where the basal pinnae differ from the central ones.

3. Only few complete sterile and fertile leaves of this plant were available for study. Among the fertile leaves there were quite a few that were only in part acrostichoid, having a \pm conspicuous band along the main veins devoid of sporangia.

4. The locality 'Ile de Bourbon' printed on the labels of Boivin's material at $P$ has been scratched out and was replaced by 'Iles Seychelles' in Boivin's handwriting. It is therefore very likely that the same kind of printed labels with Boivin's collections in B and $\mathrm{L}$ should be corrected in the same way.

5. The epithet 'bipinnata' should not be regarded a new name in the genus Bolbitis as Iwatsuki (1959: 49) erroneously referred to 'Egenolfia bipinnata J. Sm.' (= Egenolfia bipinnatifida J. Smith $=$ B. sinensis).

\section{Series Bolbitianae Hennipman, ser. nov.}

Bolbitis Schott, Gen. Fil. (1835) pl. s.n. (13), typo incl., pro genere.

Terrestrial or low-epiphytic. Rhizome short-creeping, \pm stout, with $2-4$ rows of leaves, with or without sclerenchyma strands. Scales of the rhizome up to $10 \mathrm{~mm}$ long. Leaves close together. Sterile leaves pinnate; petiole with 5-20 vascular bundles; lamina with a (primordium of a) \pm spherical, subterminal (or terminal) bulbil; pinnae 3-50, the margin sometimes with inconspicuous spines in the sinuses; terminal segment usually con- 

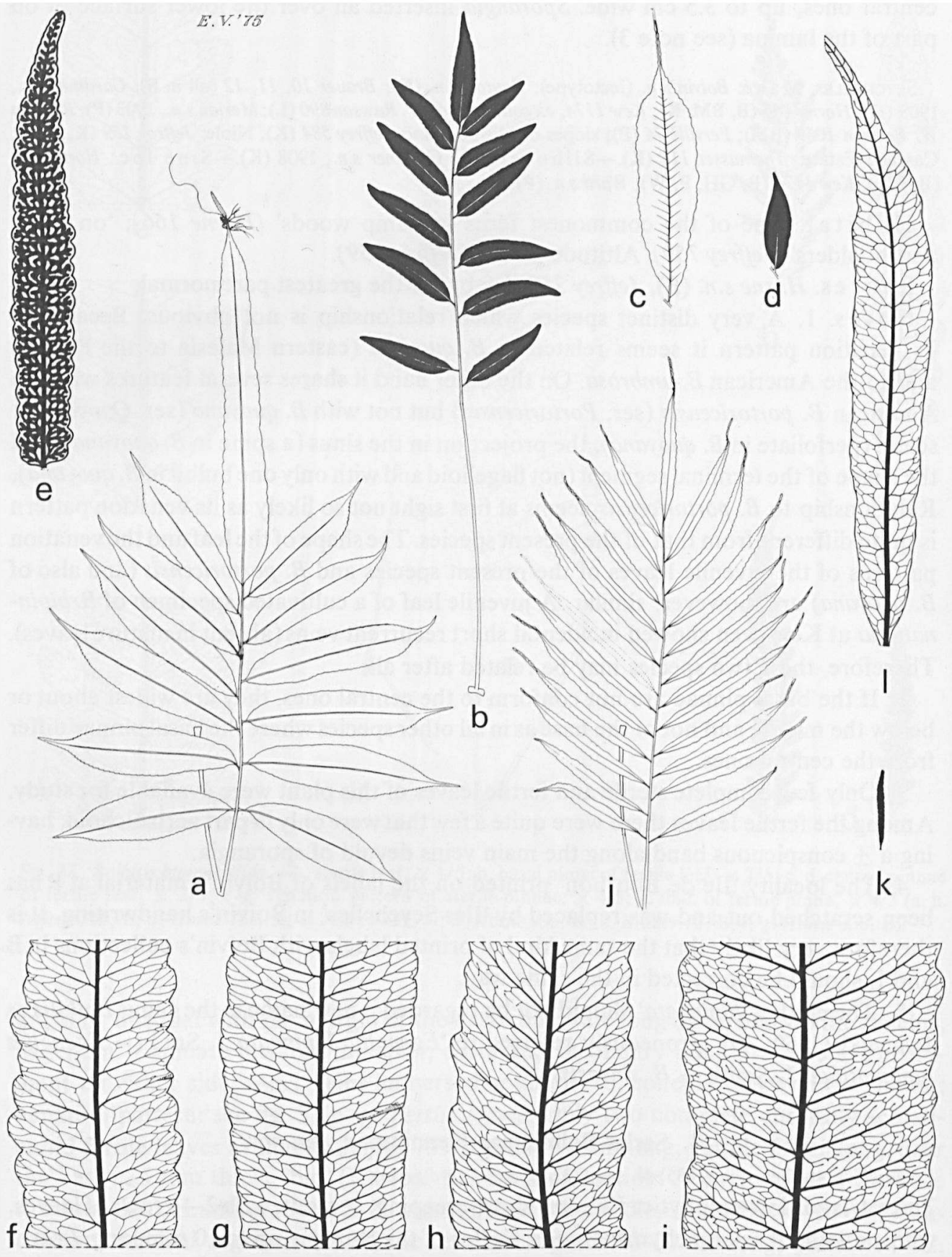

Fig. 38. a-i. Bolbitis acrostichoides. a, b. sterile and fertile leaf resp., $\times 1 / 5$; c, d. sterile and fertile pinna resp., $\times 1 / 5$; e. receptacles of fertile pinna, $\times 4 / 5 ; \mathrm{f}-\mathrm{i}$. venation pattern of sterile pinnae, $\times 4 / 5(\mathrm{a}, \mathrm{b} . T$. S. Jones 321, BM; c, d. Staudt 463, W; e, f. Young 547, L; g. W. de Wilde c.s. 4323, WAG; h. LeTestu 3076, S-PA; i. Félix 4la, S-PA). -j-1. Bolbitis salicina. j. sterile leaf, $\times 1 / 5 ; \mathbf{k}$. fertile pinna, $\times 1 / 5 ; 1$. venation pattern of sterile pinna, $\times 4 / 5$ (j, k. Halle 4009, P; 1. Kew Distr. no. 1182, Barter leg., GH). 
form to the pinnae, sometimes narrowly triangular; secondary veins prominent on either side, stramineous or concolorous with the lamina, tertiary and other veins slightly prominent or immersed; venation pattern: veins variously anastomosing, always with a costal areole, with or without excurrent included free veins. Spores with a smooth undulate (in B. semicordata: \pm verrucate) perispore which is usually situated close to the exospore in one species $(B$. costata) widely spaced from the exospore.

Ch rom o so mes. $\mathrm{n}=41$ (B. acrostichoides, $B$. crispatula, B. semicordata, B. virens); $2 \mathrm{n}=$ (c.) 82 (B. subcrenata, B. virens).

Distribution. Pantropical. See fig. 28.

Note. A very homogeneous series, the only pantropical one. The two American and the two African species are intimately related to each other. In Asia seven distinct species occur which are primarily characterized by venation pattern and leaf architecture. B. angustipinna, the most widespread Asian representative, is most closely related to the species from America and Africa.

\section{Bolbitis acrostichoides (Swartz) Ching - Fig. 38: a-i; 39.}

B. acrostichoides (Swartz) Ching in C. Chr., Ind. Fil., Suppl. 3 (1934) 47; Alston, J. Bot. 77 (1939) 286,p,p.; in Exell, Cat. Vasc. Pl. S. Tomé(1944) 68, p.p.; in Hutch. \& Dalziel, Fl. West Trop. Afr. ed. 2, Suppl. (1959) 68, p.p.; Tardieu-Blot, Mém. I. F. A. N. 28 (1953) 115, p.p., pl. 19: fig. 4; Harley, Handb. Liberian Ferns (1963) 38, fig.; Tardieu-Blot in Aubrév., Fl. Gabon 8(1964) 190, p.p.; in Aubrév., Fl. Cameroun 3(1964) 320, p.p., pl. 51: fig. 4. - Hemionitis acrostichoides Swartz, Schrader J. Bot. 1800, 2(1801) 17; Syn. Fil. (1806) 21; Willd., Spec. Pl. 5 (1810) 130; Poiret in Lam., Enc., Suppl. 3 (1813) 38; J. E. Smith in Rees, Cyclop. 17 (1811) s. pag. - Gymnogramma? acrostichoides Desvaux, Mag. Ges. Naturf. Freunde Berlin (Berl. Mag.) 5 (1811) 304; J. Bot. Soc. Ann. (not: Desv. J. Bot.) 3 (1814) 25; Kaulf., Enum. Fil. (1824) 80; Sprengel, Syst. Veg. 4 (1827) 40-Polybotrya acrostichoides Mettenius ex Kuhn, Fil. Afric. (1868) 52.-Acrostichum afzelii Carruth., Cat. Afr. Pl. Welwitsch 2, 2(1901) 77. -Leptochilus acrostichoides C. Chr., Ind. Fil. (1906) 384. - Gymnopteris acrostichoides Engler, Veg. Erde 9, 2(1908) 16. - Campium acrostichoides Copel., Philip. J. Sc. 37 (1928) 394, fig. 44, pl. 30. - Lect ot y pe: Afzelius s.n., p.p., Sierra Leone(S-PA). See note 2.

Leptochilus acrostichoides (Swartz) C. Chr. var. cuneatus Bonap., Notes Ptérid. 7 (1918) 208; ibid. 15 (1924) 19, quoad nomen solum; Copel., Philip. J. Sc. 37 (1928) 398, quoad nomen solum. -S y n t y p es:MugnierSerand I $C^{\prime \prime}, 2 C^{\prime \prime}, 3 C^{\prime \prime}$, Guinea, Iles de Los (Conakry), 40-50 m alt. (P).

Leptochilus diversibasis Bonap., Notes Ptérid. 14 (1924) 216.-B. diversibasis Ching in C. Chr., Ind. Fil., Suppl. 3 (1934) 48. -T y pe: LeTestu 1589, Gabon, Mayombe Bayaka, Midouanga (P, 3 sh.).

Acrostichum virens et syn. homot. auct. non Hooker \& Grev.: auct. div. p.p.

Leptochilus gemmifer Hieron. var. latipinnatus auct. non Hieron.: Hieron., Bot. Jahrb. 46 (1911) 346, p.p. Campium gemmiferum auct. non (Hieron.) Copel.: Copel., Philip. J. Sc. 37(1928) 397,p.p.

Rhizome up to $12 \mathrm{~cm}$ long, up to $10 \mathrm{~mm}$ thick, with 2 rows of leaves. Scales of the rhizome: index $3-7$, up to 8 by $1-1.5(-2) \mathrm{mm}$, opaque to subclathrate, blackish. Sterile leaves pinnate, $30-110 \mathrm{~cm}$ long; petiole $10-40(-70) \mathrm{cm}$ long, near the base $1.5-5 \mathrm{~mm}$ thick, with 7-11 vascular bundles; lamina index 1-3, widest about or below the middle, 20-80 by $10-35 \mathrm{~cm}$, without the terminal segment $6-50$, the terminal segment $7-45 \mathrm{~cm}$ long, herbaceous, usually light green to olivaceous, sometimes with a purple tinge, with a subterminal bulbil; rhachis not winged; pinnae alternate or opposite, 7-27 or rarely more, up to $65 \mathrm{~mm}$ apart; central pinnae index 3-8, widest about (to below) the middle, mostly the central part with parallel margins, $7.5-24$ by $1.5-4.5 \mathrm{~cm}$, base usually \pm symmetrical, sometimes its basiscopic side shorter and/or narrower, angustate to cuneate, margin \pm 


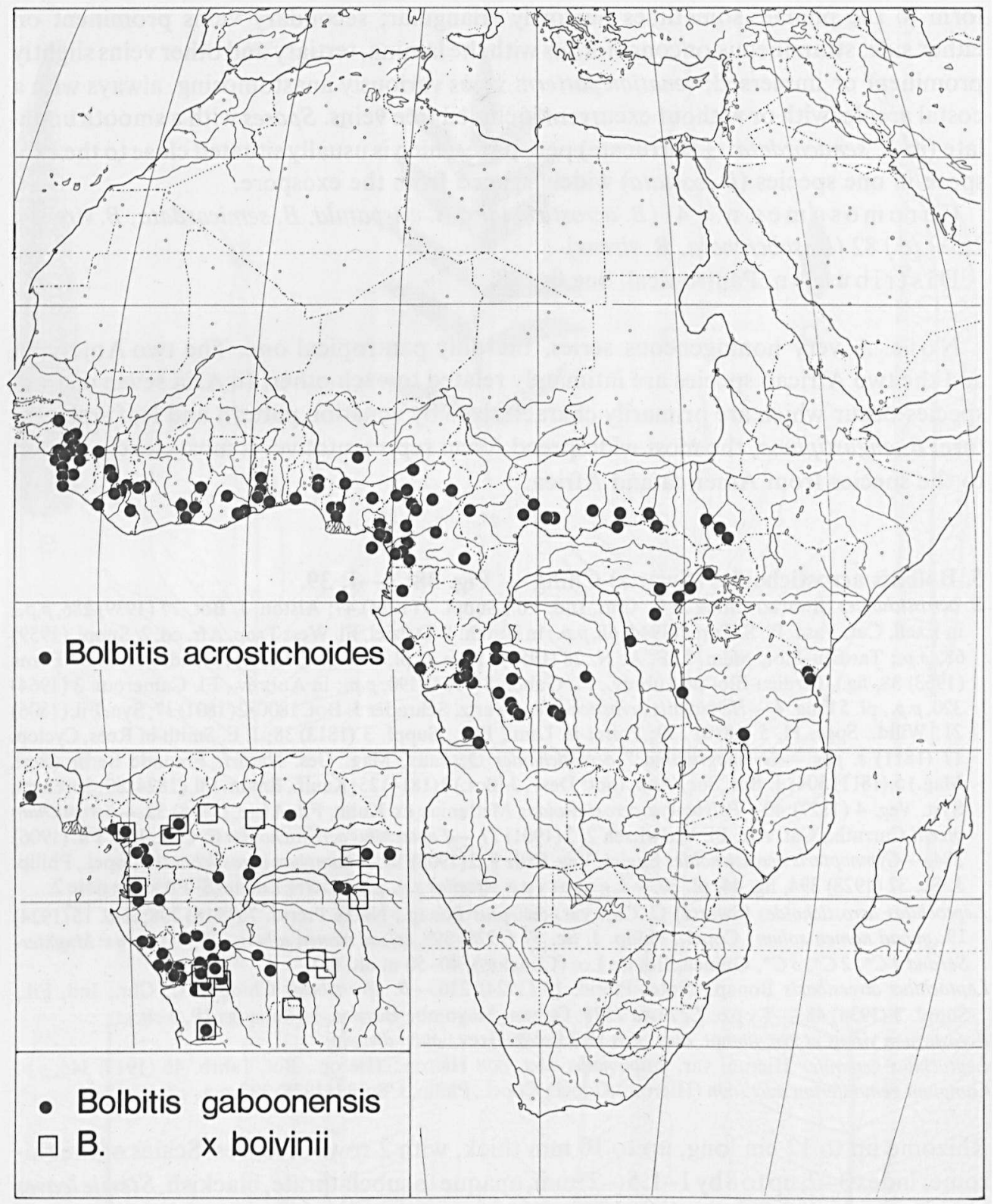

Fig. 39. Distribution of Bolbitis acrostichoides, B. gaboonensis, and B. $\times$ boivinii.

entire or finely (doubly-) serrate, without spines, apex acute to long-acuminate; the two lowermost pinnae \pm conform to the central ones, $1-10 \mathrm{~mm}$ stalked; terminal segment usually joined to the rhachis, sometimes \pm subarticulate, usually conform to the central pinnae but (much) prolonged, sometimes narrowly triangular; venation pattern: veins forming a costal areole and few to many smaller distal areoles, with few to many excur- 
rent included free veins; see also fig. 38: $\mathrm{f}-\mathrm{i}$. Fertile leaves $35-140 \mathrm{~cm}$ long; petiole $18-70$ $\mathrm{cm}$ long; lamina index $1-6,10-75$ by $5-30 \mathrm{~cm}$, without the terminal segment $7-60$, the terminal segment 5-30 cm long; pinnae 7-27, up to $80 \mathrm{~mm}$ apart; central pinnae index 4-15, 3-17.5 by $0.5-1.8 \mathrm{~cm}$; the lowermost pinnae stalked 1-20 mm. Sporangia inserted usually \pm on the veins, arranged gymnogrammoid, rarely inserted all over the lower surface, arranged acrostichoid.

Chromosomes. $n=41$.

Senegal. Heudelot 702 (B, K, P).

GUINEA. 42 collections.

SIERRA LEONE. 22 collections.

LIBERIA. 14 collections.

IVORY COAST. 13 collections.

GHANA. 11 collections.

ToGo. Busse 3534 (B); Büttner 57 (B); Mildbraed 7364 (B); Mischlich s.n. (P).

Nigeria. 14 collections.

SAO TOME. Moller $63(\mathrm{~K})$; Welwitsch $63 b(\mathrm{BM})$.

FERnando Poo. Henderson s.n. (K).

Cameroun. 26 collections.

Central African Empire. 13 collections.

SUDAN. 10 collections.

GABON. Halle 2300 (P); LeTestu 1589 (type of Leptochilus diversibasis).

Congo(Brazz.). Cervoni s.n. (P); Charvet 79 (P); Descoing 11319 (P).

ZAIRE. Le o pold vill e: 18 collections. - O r i e n t a l e: Germain 4410 (BR, P). -K i vu: Antun-Gupffert 11

(BR); Bequaert 6593 (BR, P), Mildbraed 2215 (B), 2330 (B).

Angola. Lunda: Gossweiler 14069 (K, P); Young 547 (L). - C u a n a Norte: Welwitsch 134 (K), 153 (K), 154 (BM).

UGanda. Wester n: Sangster 190 (BM), 293 (BM); Thomas 1527 (K).

TAnZania. T a ga: Peter 18129 (BM, BR, K, P); Verdcourt 90 (BM, K). -W estern: Jefford et al. 2720 (K); Newbould \& R. M. Harley 4447 (K). -S o u thern Highla nds: Schweinfurth 3034 (B, G, K, $\mathrm{P}, \mathrm{W})$.

$\mathrm{H}$ a bi t a t. Usually creeping on rocks, sometimes in soil in (seasonally) humid places in primary and secondary forest. Altitude: $0-1000(-1500) \mathrm{m}$. Locally common.

Spores. Hepper $1476(\mathrm{~K})$, LeTestu 2533 (GH), Nielsen 1667 (C), Schweinfurth 2235 (W): the greater part normal. Afzelius s.n. (lectotype of Hemionitis acrostichoides). W. de Wilde c.s. 4323 (WAG): in part normal. Jones $348(\mathrm{GH})$ : all shrivelled. See also notes 4 and 5.

N o tes. 1. A variable species, especially regarding the venation pattern and the size of the leaves. It is intimately related to, or possibly even conspecific with, the American $B$. serratifolia. $B$. salicina, a rather critical species, is considered by me a derivative of the present species. See also $B$. serratifolia.

2. The material upon which the original description was based is a mixture of a fertile and a sterile leaf of $B$. acrostichoides and a fertile leaf belonging to $B$. gemmifera.

3. There is strong evidence that a fern like $B$. acrostichoides is one of the parents of B. gemmifera from which it is easily distinguished because of differences in the venation pattern, in the place of insertion of the bulbil, and in the shape of the perispore.

4. Some collections have relatively wide acrostichoid fertile pinnae rather resembling the central pinnae of $B$. auriculata. Their sporangia are inserted mainly on the veins or all 
over the lower surface. Here belong Annet 298(P), Ludwigs7,p.p.(M), Staudt 463(B, GH, $\mathrm{K}$, W; syntype of Leptochilus gemmifer var. latipinnatus), Zenker 4120 (G, K, L, MO, SPA, W), all from Cameroun, and Hallé 2300 (P) from Gabon. Spores: Ludwigs 7, Staudt 463, Zenker 4120 (G): all abnormal. Possibly hybrids with $B$. auriculata.

5. Plants morphologically intermediate between the present species and $B$. salicina include Callens 2233 (K), Harley F 56 (BM, GH, K), and Kunkel F-30 (WAG). These specimens have leaves with relatively narrow pinnae, a venation pattern with but very few included free veins and a terminal segment which is not prolonged but conform to the central pinnae; spores of all these collections are abnormal. Here may also belong LeTestu 1589 (type of Leptochilus diversibasis) of which the large and relatively narrow sterile leaves are composed of a high number of pinnae (over 50 ) and a relatively short terminal segment; the venation pattern shows but occasionally an included free vein; sporangia inserted all over the lower surface of the fertile leaf; spores obviously for the greater part normal. Mainly because of the width of the pinnae this rather dubious specimen is included here.

\section{Bolbitis angustipinna (Hayata) Ito - Fig. 40: a-f.}

B. angustipinna (Hayata) Ito, J. Jap. Bot. 14 (1938) 443.-Leptochilus angustipinnus Hayata, Ic. Pl. Formos. 5 (1915) 297, fig. 119.-Campium angustipinnum Copel., Philip. J. Sc. 37 (1928) 381, fig. 33; C. Chr., Contr. U.S. Nat. Herb. 26 (1931) 292.-L ec tot y p e: (Iwatsuki, Acta Phytotax. Geobot. 18, 1959, 53): Owatari s.n., Taiwan, near Hokkokei, 20-i-1898 (TI, n.v.; L, phot.).

Leptochilus cuspidatus (Presl) C. Chr. var. crenatus Rosenst., Hedwigia 56 (1915) 348.-T y p e: Faurie 281 (= Rosenstock, Fil. Formos. exsicc. 198), Taiwan, Takao, Bankinsing, $800 \mathrm{~m}$ alt. (B; iso in BM, L, M, MICH, fragm., PNH, S-PA, UC).

[Acrostichum contaminans Wall., Cat. (1829) no. 22, nom. nud.-Poecilopteris contaminans Moore, Ind. Fil. (1857) 8, nom. nud.]-Acrostichum crispatulum Clarke var. contaminans Clarke, Trans. Linn. Soc. Bot. 1 (1880) 580, pl. 84: fig. 2A, 2C.-B. contaminans Ching in C. Chr., Ind. Fil., Suppl. 3 (1934) 47; Iwatsuki, Acta Phytotax. Geobot. 18 (1959) 53, fig. 9; DeVol \& Kuo in Fl. Taiwan 1(1975) 348. - T y p e: Wallich 22, Nepal, between Helounda and Bhimpedy, 1820 (K, herb. Wallich; iso in K, p.p.). See note 4.

Acrostichum semicordatum auct. non Baker. Baker, Syn. Fil. (1868) 422, p.p.

Gymnopteris contaminans auct. non Bedd.: Bedd., Handb. Ferns Br. India (1883) 435, p.p.; ibid., Suppl. (1892) 105; Hayata, Bot. Mag. Tokyo 23 (1906) 26, p.p.

B. scalpturata auct. non (Fée) C. Chr.: Ito, J. Jap. Bot. 14(1938) 439, fig.; Nayar \& Kaur, Bull. Nat. Bot. Gard: Lucknow 88 (1964) 55.

Rhizome up to $10(-20) \mathrm{cm}$ long, $10-20 \mathrm{~mm}$ thick, with 2-4 rows of leaves. Scales of the rhizome: index 3-8, 3-6(-8) by $0.5-1.5(-2.5) \mathrm{mm}$, subclathrate, dark brown or blackish throughout. Sterile leaves pinnate, $55-150 \mathrm{~cm}$ long; petiole $20-60 \mathrm{~cm}$ long, near the base $2.5-7 \mathrm{~mm}$ thick, with 7-16 vascular bundles; lamina index $1-3$, widest somewhat below the middle, $30-90$ by $20-45 \mathrm{~cm}$, without the terminal segment $25-80$, the terminal segment 8-25 cm long, (firm) herbaceous, usually light to dark green, sometimes with a purple tinge, with a subterminal bulbil on terminal segment (and pinnae); rhachis not winged; pinnae alternate or opposite, $20-50,5-45(-90) \mathrm{mm}$ apart; central pinnae index $5-12$, usually widest about (to below) the middle, the central part usually with parallel margins, $11-30$ by $2-3(-5) \mathrm{cm}$, base \pm symmetrical, acute, broadly attenuate or truncate, margin usually slightly serrate-crenate, sometimes lobed to $1 / 3$ to the costa and with a short or inconspicuous spine in each sinus, apex acute or acuminate; the twolowermost pinnae \pm conform to the central ones, $0-10 \mathrm{~mm}$ stalked; the pinnae in the upper 

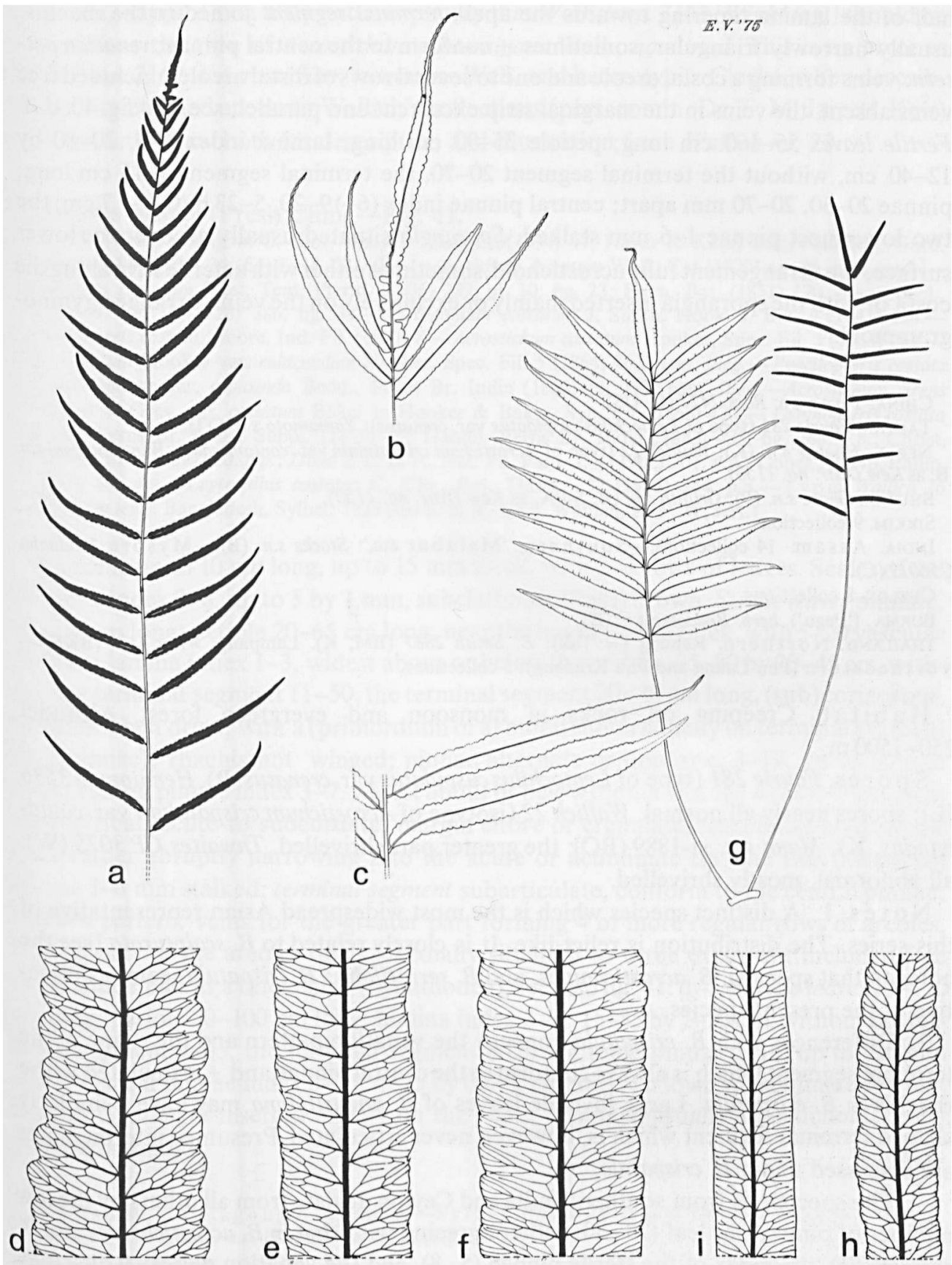

Fig. 40. a-f. Bolbitis angustipinna. a. fertile leaf, $\times 1 / 5$; b. terminal part of sterile leaf, $\times 1 / 5$; c. central pinnae, $\times 1 / 5 ; d-f$. venation pattern of sterile pinnae, $\times 4 / 5$ (a, d. Hennipman $3536, L ; b, c, e . H .3637$, L; f. Tagawa et al. T 380, L).-g-i. Bolbitis crispatula. $-\mathrm{g}$, h. var. crispatula. g. sterile and fertile leaf, $\times$ $1 / 5$; h. venation pattern of sterile pinna, $\times 4 / 5$. - i. var. copelandii, venation pattern of sterile pinna, $\times$ 4/5 (g. Mann s.n., ix-1889, M; h. Austen s.n., US 418607; i. Van Beusekom 3204, L). 
half of the lamina tapering towards the apex; terminal segment joined to the rhachis, usually narrowly triangular, sometimes \pm conform to the central pinnae; venation pattern: veins forming a costal areole and one to several rows of distal areoles, included free veins absent, the veins in the marginal strip excurrent and parallel; see also fig. 40: $d-f$. Fertile leaves $55-160 \mathrm{~cm}$ long; petiole $35-90 \mathrm{~cm}$ long; lamina index $1-4,20-80$ by $12-40 \mathrm{~cm}$, without the terminal segment $20-70$, the terminal segment $3-15 \mathrm{~cm}$ long; pinnae $20-50,20-70 \mathrm{~mm}$ apart; central pinnae index (6-) $9-20,5-23$ by $0.4-1.7 \mathrm{~cm}$; the two lowermost pinnae 1-6 mm stalked. Sporangia situated usually all over the lower surface, the arrangement fully acrostichoid, sometimes either with a sterile strip along the costa or with the sporangia inserted mainly or exclusively on the veins, arranged gymnogrammoid.

China. Yunna n: Rock 2427 (C, US).

TAIWAN. Faurie 281 (type of Leptochilus cuspidatus var. crenatus); Yamamoto s.n. (TI).

NEPAL. Hooker s.n. (K); Wallich 22 (type of Acrostichum crispatulum var. contaminans); Winterbottom s.n. (B, as Kew Distr. no. 1150).

Bhutan. Ghose s.n. (P); Griffith s.n. (B; S-PA, as Kew Distr. no. 1150).

SIKKIM. 9 collections.

INDIA. A s s a m: 14 collections.-Southern. 'Malabar etc.': Stocks s.n. (B).-Mysore: Saldanha C56012(C).

CEYLON. 8 collections.

BURMa. ('Pegu'), herb. Beddome s.n. (K).

ThaILAND. Northern. Raheng (= Tak): E. Smith 2837 (BM, K). Lampang: Winit 1126 (BKF).Northe astern (Phu Luang and Phu Kradeng): 5 collections.

$\mathrm{Habitat}$. Creeping on rocks in monsoon and evergreen forest. Altitude: $250-1500 \mathrm{~m}$.

S p o r e s. Faurie 281 (type of Leptochilus cuspidatus var. crenatus, B), Hennipman 3536 (L): spores nearly all normal. Wallich 22 (isotype of Acrostichum crispatulum var. contaminans, K), Mann s.n. xi-1889 (BO): the greater part shrivelled. Thwaites CP 3075 (W): all abnormal, mostly shrivelled.

Notes. 1. A distinct species which is the most widespread Asian representative of this series. The distribution is relict-like. It is closely related to $B$. scalpturata (see the notes to that species), $B$. acrostichoides, and $B$. serratifolia: $B$. crispatula seems a derivative of the present species.

2. Differences with $B$. crispatula concern the venation pattern and the shape of the terminal segment which is always conform to the central pinnae and \pm articulated to the rhachis in $B$. crispatula. Large juvenile leaves of $B$. angustipinna may have a similarly shaped terminal segment which is, however, never articulated. Presumed intermediates are discussed under $B$. crispatula.

3. The specimens from southern India and Ceylon deviate from all others by the low number of pinnae per leaf (20-30; rather agreeing with that in $B$. acrostichoides and $B$. serratifolia), the index of the sterile pinnae $(5-8)$, and the venation pattern which may show irregularities. Hybridization seems also involved. See also the note to $B$. subcrenata var. subcrenata.

4. Typification of Acrostichum crispatulum var. contaminans. Beddome (Handb. Ferns 
Br. India, Suppl. 1892: 105) stated explicitly that Wallich no. 2168 (Wight leg.) should be regarded as the type of Acrostichum contaminans Wall., nom. nud. This is an error as Wallich no. 2168 is Acrostichum terminans Wall. and heterotypic. Clarke, when describing the variety, had access to Wallich's collections (pers. comm. Dr. F. M. Jarrett, Kew) and correctly drew both his description and illustration from Wallich no. 22.

\section{Bolbitis costata (Presl) Ching-Fig. 41.}

B. costata (Presl) Ching in C. Chr., Ind. Fil., Suppl. 3(1934) 47; Nayar \& Kaur, Bull. Nat. Bot. Gard. Lucknow 88 (1964) 34; fig. 8, 16, 27, 60.-[Acrostichum costatum Wall., Cat (1829) no. 26, nom. nud.]Campium costatum Presl, Tent. Pterid. (1836) 239, pl. 10: fig. 23; Epim. Bot. (1851) 170, p.p.; Copel., Philip. J. Sc. 37 (1928) 386, fig. 37.-Cyrtogonium costatum J. Smith, Hook. J. Bot. 4 (1841) 154.Poecilopteris costata Moore. Ind. Fil. (1857) 8. -Acrostichum costatum Hooker, Spec. Fil. 5 (1864) 262.A. costatum Hooker var. rubicundum Hooker, Spec. Fil. 5 (1864) 262, nom. illeg. - Poecilopteris costata (Presl) Moore var. rubicunda Bedd., Ferns Br. India (1865) pl. 113, nom. illeg.-Acrostichum virens Hooker \& Grev. var. costatum Baker in Hooker \& Baker, Syn. Fil. (1868) 420.- Gymnopteris costata Bedd., Ferns Br. India, Suppl. (1876) 27; Handb. Ferns Br. India (1883) 438, fig. 266, p.p.; Christ, Farnkr. Erde (1897) 50, p.p.; Diels in E. \& P., Nat. Pfl. Fam. 1, 4 (1902) 201, p.p.; Copel., Polyp. Philip. (1905) 44, p.p.-Leptochilus costatus C. Chr., Bot. Tidsskr. 32 (1916) 344, p.p.-T y pe: Wallich 26 (De Sylva leg.), Bangladesh, Sylhet, 1823 (iso in B, K, herb. Wallich, K, P, W, fert.).

Rhizome up to $10 \mathrm{~cm}$ long, up to $15 \mathrm{~mm}$ thick, with 2-4 rows of leaves. Scales of the rhizome: index 3-8, up to 5 by $1 \mathrm{~mm}$, subclathrate, (light) brown. Sterile leaves pinnate, $55-140 \mathrm{~cm}$ long; petiole $20-65 \mathrm{~cm}$ long, near the base 2-7 mm thick, with 5-20 vascular bundles; lamina index $1-3$, widest about or below the middle, $35-80$ by $20-45 \mathrm{~cm}$, without the terminal segment $11-50$, the terminal segment $20-40 \mathrm{~cm}$ long, (sub) coriaceous, purplish when dried, with a (primordium of a) bulbil subterminally on terminal segment (and pinnae); rhachis not winged; pinnae alternate or opposite, 3-19, up to $80 \mathrm{~mm}$ apart, central pinnae index $3-7$, widest about or below the middle, $16-43$ by $4-9 \mathrm{~cm}$, base symmetrical, acute to subcordate, margin entire or crenulate, sometimes crisped, the apex rather abruptly narrowing into the acute or acuminate tip; the two lowermost pinnae 1-6 mm stalked; terminal segment subarticulate, conform to the central pinnae; venation pattern: veins for the greater part forming 4 or more regular rows of areoles, with in part of the areoles one ( \pm medially situated) to three excurrent included free veins which end in a thickening (hydathode?), see also fig. 41: d-f. Fertile leaves 40-170 $\mathrm{cm}$ long; petiole $20-100 \mathrm{~cm}$ long; lamina index $3-7,15-85$ by $7-30 \mathrm{~cm}$, without the terminal segment $9-65$, the terminal segment $8-20 \mathrm{~cm}$ long; pinnae 5-18, up to $130 \mathrm{~mm}$ apart; central pinnae index 6-15, 4-25 by $0.6-2 \mathrm{~cm}$, the two lowermost pinnae $1-6 \mathrm{~mm}$ stalked. Sporangia inserted mainly on the veins, arranged usually acrostichoid, rarely gymnogrammoid.

Bangladesh. Chit tag on g. 6 collections.-S y lhet: Wallich 26 (type of Campium costatum).

Nepal. E a st: Hooker s.n. (K), Clarke 31730 (K).

SIKKIM. 5 collections.

INDIA. A s a m: 18 collections.

Burma. B h a mo D ist. near Shwebo: Lace 5210 (K).-Moulmein: Parish s.n. (B).

Thalland. Nort hern. Lampang. Winit (Wanadorn) 58 (BKF, SING), $927=$ BKF 1368 (BKF, K). Doi Inthanon: Murata et al. T 15546 (L). 


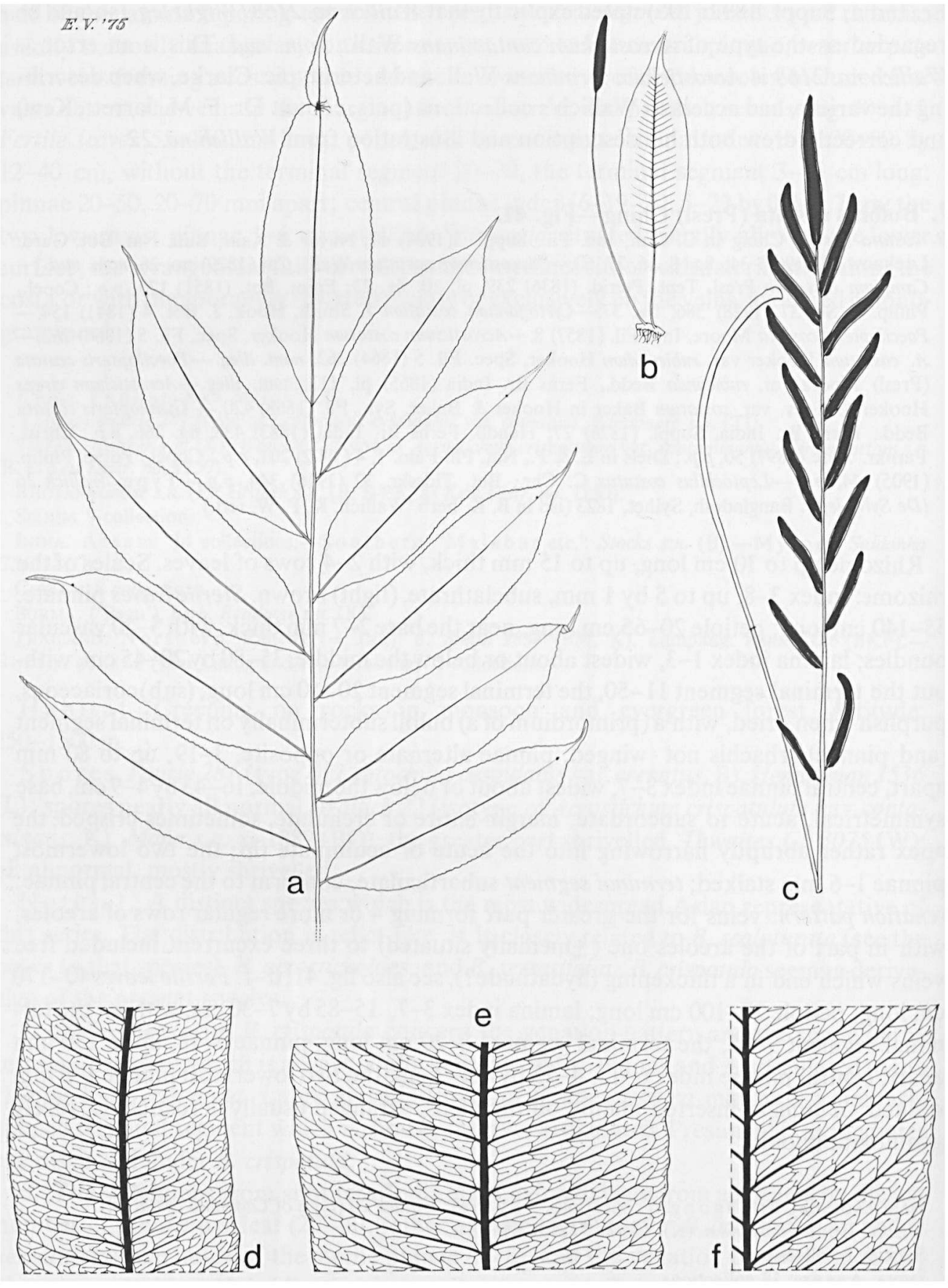

Fig. 41. Bolbitis costata. -a-c. sterile and fertile leaves, $\times 1 / 5 ; \mathrm{d}-\mathrm{f}$. venation pattern of sterile pinnae, X 4/5 (a, e. Anonymus s.n., W; b. Mann s.n., xii-1886, M; c. Mann s.n., ii-1886, S-PA; d. Hooker \& Johnson '110', GH; f. Anonymus s.n., Assam, B). 
$\mathrm{H}$ a bi t a t. Only few data available; obviously growing in forest. Altitude: 0-1200 m. According to Nayar \& Kaur (1964) specimens of this species grow closely together forming dense colonies in forests near streams; the rhizome partially covered with gravelly soil.

S p o r es. Mann s.n., Ukiam, ii-1889 (M), Nambur Forest, i-1888 (S-PA), Naga Hills, iii-1883 (L, 908.206-45), Wallich 26 (isotype of Acrostichum costatum, B), Winit 58 (SING): all or the greater part normal.

Notes. 1. A clear-cut species which is mainly characterized by the purplish colour of the dried leaves, the number of pinnae per leaf, and the shape and venation pattern of the pinnae.

2. The present species was validly published by Presl (1836), who mentioned the number of vascular bundles (12-18) in the petiole of this species in the description of his new genus Campium, and also provided a drawing of the venation pattern of part of a fertile pinna. Later (1851) he included in B. costata all the elements which Fée (1845) listed under Heteroneuron scalpturatum (see B. scalpturata, note 6). Hooker(1864) agreed with Presl (1851) but added Meniscium deltigerum $(=B$. virens var. deltigera $)$ and Jenkinsia undulata $(=B$. virens var. virens) as varieties of Acrostichum costatum. Baker (1868) united Acrostichum costatum sensu Hooker (1864) with A. virens sensu Hooker (1864). Clarke's concept (1880) is correct; he excluded from $A$. costatum the ferns described as Meniscium deltigerum and Jenkinsia undulata. Beddome (1883) largely agreed with Hooker (1864) but characterized Meniscium deltigerum and Jenkinsia undulata as 'only abnormal forms, such as occur more or less in nearly all the other species of Gymnopteris and cannot be recorded as varieties'; he strongly criticized the concepts of both Baker and Clarke. Christensen's concepts $(1906,1916)$ are discussed under $B$. scalpturata, note 6. Copeland (1928) based himself on Clarke.

3. Variation in size of the fertile leaves. Occasionally small fertile leaves are formed (e.g. Clarke 19653, K). One collection (Mann s.n., Besisto, Khasi Hills, xii-1886, M) comprises a simple fertile leaf (spores all abnormal) occurring in conjunction with a simple sterile leaf; the shape of the simple leaf agrees with the terminal segment of leaves of typical plants.

4. Winit 58 (BKF, 2 sh.) consists of one sterile and two fertile leaves (all separately mounted). The venation pattern of the sterile leaf looks intermediate between that of the present species and $B$. virens; the colour of the leaf is greenish. The colour of the two fertile leaves is typically purplish; the spores of one of the leaves are all shrivelled, those of the second leaf typical. Winit 59-928 (BKF), collected from the same place, represents $B$. virens; hybridization between the latter and the present species may underly the deviations shown in the material of Winit 58.

5. Spores. The perispore is situated at considerable distance from the exospore.

8. Bolbitis crispatula (Copel.) Ching-Fig. 40: g-i; 42.

B. crispatula (Copel.) Ching in C. Chr., Ind. Fil., Suppl. 3 (1934) 47; Nayar \& Kaur, Bull. Nat. Bot. Gard. Lucknow 88 (1964) 36, fig. 17, 33, 61, 65, 74, 86.-Acrostichum crispatulum [Wall., Cat.(1829) no. 24, nom. nud.] Clarke, Trans. Linn. Soc. Bot. 1 (1880) 580, pl. 84: fig. 2B, 2D, excl. var., nom. illeg., non 
Fée (1852) (= Elaphoglossum spec.).-[Cyrtogonium crispatulum J. Smith, Hook. J. Bot. 4 (1841) 154, nom. nud.]-Campium crispatulum [Presl, Epim. Bot. (1851) 171, nom. nud.] Copel., Philip. J. Sc. 37 (1928) 382, fig. 34, pl. 26. - [Poecilopteris crispatula J. Smith, Cat. Cult. Ferns (1857) 23, nom. nud.; Moore, Ind. Fil. (1857) 8, nom. nud.; J. Smith, Ferns Br. For.(1866) 117, nom. nud.] -Acrostichum virens Hooker \& Grev. var. crispatulum Hooker, Spec. Fil. 5 (1864) 261 ('var.?'); Baker in Hooker \& Baker, Syn. Fil. (1868)420.Gymnopteris contaminans (Bedd.) Bedd. var. crispatula Bedd., Handb, Ferns Br. India, Suppl. (1892) 105. -Leptochilus virens (Hooker \& Grev.) C. Chr. var. crispatulus C. Chr., Bot. Tidsskr. 32(1916) 344. - T y p e: Wallich 24 p.p. (Blinkworth leg.), Bangladesh, Kumaon (Kamoun?) (K, herb. Wallich; iso in $\mathrm{K}, \mathrm{MICH}$, fragm.).

B. copelandii Cing ex Tardieu-Blot \& C. Chr., Not. Syst. 7(1938) 101; Tardieu-Blot \& C. Chr. in Lecomte, Fl. Gén. I.-C. 7 (1941) 434, fig. 50: 1, 2.-T y pe: H.M. Smith 302, Cambodia, Angkor (BM; iso in MICH, US).

[B. copelandii Ching f. viridis Tardieu-Blot \& C. Chr., Not. Syst. 7 (1938) 102, nomen-T y p e: Pierre s.n., Cochinchina, Deon Ba, vii-1866 (iso in K).]

Gymnopteris contaminans auct. non (Bedd.) Bedd. (nom. illeg.): Bedd., Ferns Br. India, Suppl. (1876) 27, p.p.; Handb. Ferns Br. India (1883) 435, p.p.

Rhizome up to $10(-15) \mathrm{cm}$ long, up to $10 \mathrm{~mm}$ thick, with 2-4 rows of leaves. Scales of the rhizome: index 4-15, up to 8 by $1 \mathrm{~mm}$, subclathrate, blackish. Sterile leaves pinnate, $25-90 \mathrm{~cm}$ long; petiole $10-40(-50) \mathrm{cm}$ long, near the base $1.5-3 \mathrm{~mm}$ thick, with 5-8 vascular bundles; lamina index 1-3, widest about or below the middle, 15-50 by $9-25 \mathrm{~cm}$, without the terminal segment $10-35$, the terminal segment $4-15 \mathrm{~cm}$ long, firm-herbaceous, olivaceous, purplish or purplish-brown (light to dark green when living), usually without, sometimes with a \pm terminal bulbil; rhachis not winged; pinnae alternate or opposite, 10-30 (-60), 10-40 mm apart; central pinnae index 4-11, widest about or below the middle, $5-17.5$ by $0.9-2 \mathrm{~cm}$, base symmetrical, narrowly to broadly cuneate, margin \pm entire or finely serrate or serrate-crenate, \pm crisped, without spines, apex acute or acuminate; the two lowermost pinnae 0-4 mm stalked, base symmetrical or asymmetrical with the basiscopic side shorter, otherwise conform to the central pinnae; terminal segment subarticulate, usually conform to the central pinnae, sometimes inconspicuous(see note); venation pattern: veins forming a costal areole, otherwise free, excurrent, see also fig. 40: h, i. Fertile leaves $25-90 \mathrm{~cm}$ long; petiole $15-50(-65) \mathrm{cm}$ long, lamina index $1-3,9-40$ by $3-20 \mathrm{~cm}$, without the terminal segment $7-35$, the terminal segment 2-9 $\mathrm{cm}$ long; pinnae $10-30(-45)$, up to $50 \mathrm{~mm}$ apart, index $4-35,2-12$ by $0.2-1.2 \mathrm{~cm}$; the two lowermost pinnae $0-6 \mathrm{~mm}$ stalked. Sporangia either inserted all over the lower surface, arranged acrostichoid, or mainly inserted on the veins and along the margin, arranged \pm pteridioid.

Chromosomes. $\mathrm{n}=41$ (var. copelandii).

D is t ribution. Assam, Thailand, Indo-China. See fig. 42.

Habitat. Creeping on stones usually in riverbeds in monsoon forest. Altitude 0-1200 m.

Note. A distinct species which is closely related to B. angustipinna. For differences see under that species. For specimens with part of their features intermediate between the present species and $B$. angustipinna see var. copelandii, note 1 . The leaves of $B$. crispatula usually turn purplish upon drying, like those of $B$. scalpturata and $B$. costata. The material is easily divided into two allopatric varieties. 
1a. Fertile pinnae acrostichoid; index (7-) 10-35 . . . . . . a. var. crispatula

b. Fertile pinnae \pm pteridoid; index $4-10(-20) \quad \ldots \ldots \ldots$. b. var. copelandii

a. var. crispatula-Fig. 40: g, h; 42.

B. crispatula (Copel.) Ching.

Scales of the rhizome: index 4-10, up to 5 by $1 \mathrm{~mm}$, upper half acute. Sterile leaf $30-90$ $\mathrm{cm}$ long; petiole $10-40(-50) \mathrm{cm}$ long; lamina $20-50$ by $10-25 \mathrm{~cm}$, without the terminal segment 15-35, the terminal segment 5-15 cm long, without bulbils; pinnae 10-26; central pinnae index (4-)7-11, usually widest about, sometimes below the middle. Fertile leaves $35-90 \mathrm{~cm}$ long; petiole $20-50(-65) \mathrm{cm}$ long; lamina $10-40$ by $4-20 \mathrm{~cm}$, without the terminal segment 7-35, the terminal segment 2-9 cm long; pinnae 10-25; central pinnae index (7-) $10-35,2-12$ by $0.2-0.5 \mathrm{~cm}$. Sporangia inserted all over the lower surface.

Bangladesh. Sylhet: Carke 42452 (K); Wallich 24 p.p. (Gomez leg.) (K, herb. Wallich).-Not t $\mathrm{r}$ a c ed: Kumaon: Wallich 24 p.p. (Blinkworth leg.; type of Acrostichum crispatulum).

INDIA. A s s a m. Garo Hills, Khasya Hills, Jaintia Hills: 15 collections.

$\mathrm{H}$ abit a t. See the species.

S pores. Austen s.n. (MICH), Nayar et al. $L W G 63414$ (LWG): the greater part normal. Hooker \& Thomson '107' (BM), Mann s.n., ix-1889 (L): all abnormal, mostly shrivelled.

b. var. copelandii (Ching) Hennipman, stat. nov.-Fig, 40 : i; 42.

B. copelandii Ching ex Tardieu-Blot \& C.Chr., Not. Syst. 7(1938) 101.

Scales of the rhizome: index $4-15$, up to 8 by $1 \mathrm{~mm}$, upper half acute or hair-like. Sterile leaf $25-60(-85) \mathrm{cm}$ long; petiole $10-35 \mathrm{~cm}$ long; lamina $15-45$ by $9-25 \mathrm{~cm}$, without the terminal segment $11-35$, the terminal segment $4-10 \mathrm{~cm}$ long, sometimes aborted or absent, a bulbil if present situated at the base of the terminal segment or pseudo-terminal; pinnae 15-30 (-60); central pinnae index 4-8, widest below or about the middle. Fertile leaves 25-60 (-90) cm long; petiole 15-35 $(-50) \mathrm{cm}$ long; lamina 9-25 $(-40)$ by $3-11 \mathrm{~cm}$, without the terminal segment 7-22 $(-35)$, the terminal segment $2-6 \mathrm{~cm}$ long; pinnae $12-30(-45)$; central pinnae index $4-10,2-8$ by $0.2-1.2 \mathrm{~cm}$. Sporangiainserted on the veins and along the margin only.

Thailand. Northern. Lampang. Winit 1026 (BKF, K, SING).-Northe as tern. Phu Pan: Hansen et al. 11300 (C). Phu Luang. Hennipman 3632 (BKF, KYO, L); Tagawa et al. T 1910 (L, US).Eastern. Nangrawng Waterfall: Kerr 8218 (K); Smitinand \& Floto 6093 (= BKF 24438) (BKF).Sou the ast ern. Koh Kut (island in the Gulf of Thailand): Van Beusekom 3204 (L).

CAmbodia. Angkor: Lecomte \& Finet 1796 (P), 1813 (P); H. M. Smith 298 (C, GH, US), 302 (type of B. copelandii). Pnom-chom: Poilane 28825 (P). 


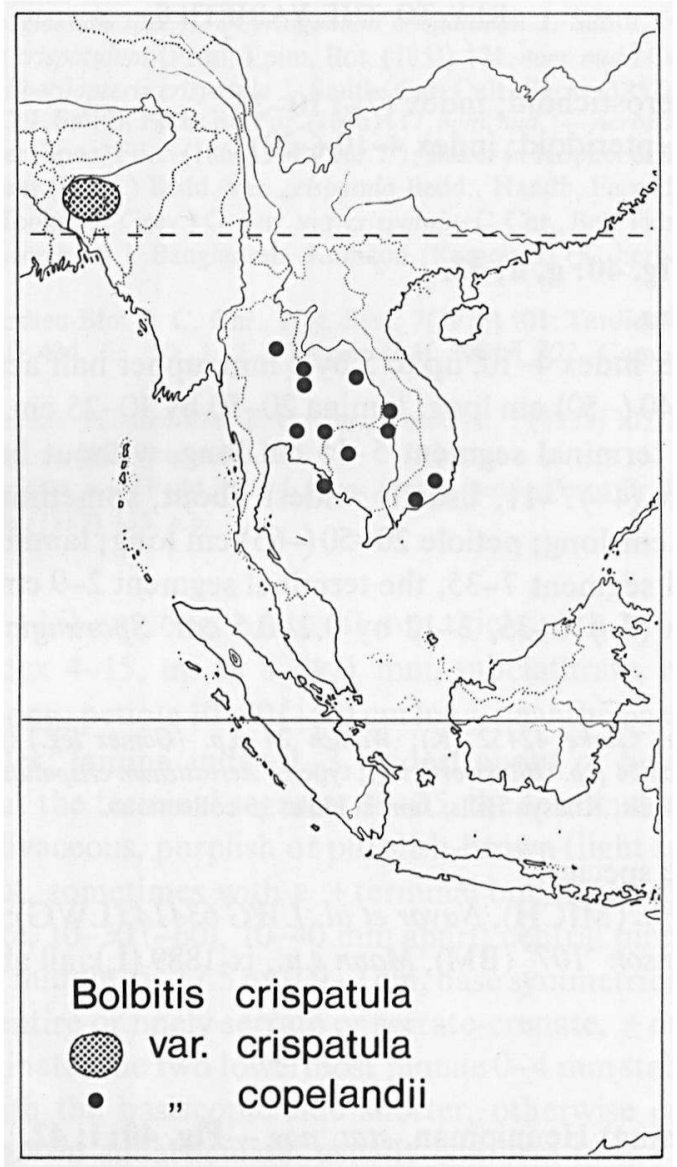

Fig. 42. Distribution of Bolbitis crispatula.

LAOs. Khong: Thorel 2480 (P). Bassac: Thorel s.n. (P). Oudang: Thorel s.n. (P).

VIEtnam. Cochinchina: Chevalier 29851 (P); Matthew s.n. (K); Pierre 1300 (BM, BO, GH, K, P), 1300 bis (P), s.n., vii-1866 (K, P); Poilane 2564 (P).-S. A n n a m: Squires 845 (B, BO, GH, K, M, MO, SING, S-PA, W).

H a bit a t. See the species.

Sp o res. Van Beusekom 3204 (L, sh. no. 8), Hennipman 3632 (L), Matthew s.n. (K), H.M. Smith 302 (type of B. copelandii, US), Squires 845 (BO): the greater part normal. Tagawa et al. T 1910 (L): in part (the majority?) abnormal.

Notes. 1. This variety is heterogeneous. It includes the following elements.

a. The collections from Thailand except those from Koh Kut. Part of the sterile leaves occurring in conjunction with the fertile ones are large and consist of a great number of pinnae per leaf (the figures given between brackets in the description refer to these specimens). The shape, size, and venation pattern of these pinnae are \pm interme- 
diate between those of typical plants and those of $B$. angustipinna. The shape of the pinnae of the fertile leaves is uniform throughout the material attributed to this variety. These collections from Thailand are further of great interest for two reasons. Firstly, they all have a conspicuous bulbil situated at the articulation of the terminal segment of both the sterile and the fertile leaf. Secondly, the shape of the terminal segment varies; usually the terminal segment is either conform to the central pinnae, or inconspicuous, or absent, rarely (in a few fertile leaves only) narrowly triangular. In leaves where the terminal segment is absent or inconspicuous, the localization of the bulbil is terminal or almost so. Such a terminal localization of the bulbil is characteristic for $B$. rhizophylla and $B$. novoguineensis. For a correct interpretation of these facts it seems of interest to know that the smaller-sized sterile leaves occurring on the same rhizome as the deviating ones agree in all details (including size!) with the mature sterile leaves of the nondeviating collections of this species.

b. Lecomte \& Finet 1796 and 1813, Thorel s.n., from Oudang, and Pierre 1300 (p.p.) rather agree with the collections mentioned above, but the obviously mature specimens lack a bulbil.

2. One out of eight fertile plants of Van Beusekom 3204. (Thailand, Koh Kut) has fertile leaves agreeing as to shape of the pinnae with those of the typical variety. The sporangia are situated on the veins (all over the lower surface in the typical variety).

3. Cultivated material. One out of three living plants sent by Van Beusekom from Koh Kut, Thailand, and belonging to the gathering Van Beusekom 3204, shows abnormally shaped sterile and fertile leaves in cultivation. These sterile leaves are bipinnate in the lower half of the lamina, the two lowermost pinnae again pinnate with a terminal segment conform to the central pinnules, up to 18 by $10 \mathrm{~cm}$; pinnules up to 14 , up to 55 by $11 \mathrm{~mm}$; the terminal segment of the leaf is sometimes in the lower half either lobed to halfway towards the costa, or shows some odd free pinnules at the base of the segment. The fertile leaves are usually \pm normal, sometimes the lowest pinnae with some odd pinnules at the acroscopic side of the base (Hennipman 4087, L).

\section{Bolbitis salicina (Hooker) Ching-Fig. 38: $\mathbf{j}-\mathbf{l}$.}

B. salicina (Hooker) Ching in C. Chr., Ind. Fil., Suppl. 3 (1934) 50; Alston, J. Bot. 77 (1939) 286; TardieuBlot, Mém. I.F.A.N. 28 (1953) 116, pl. 19: fig. 5; Alston in Hutch. \& Dalziel, Fl. West Trop. Afr. ed. 2 , Suppl. (1959) 69; Harley, Handb. Liberian Ferns (1963) 40, fig.; Tardieu-Blot in Aubrév., Fl. Gabon 8 (1964) 189.-Acrostichum salicinum Hooker, Spec. Fil. 5 (1864) 265; Baker in Hooker \& Baker, Syn. Fil. (1868) 422.-Chrysodium salicinum Kuhn, Fil. Afric. (1868) 52.-Gymnopteris salicina J. Smith, Hist. Fil. (1875) 138.-Leptochilus salicinus C. Chr., Ind. Fil. (1906) 387.-Campium salicinum Copel., Philip. J. Sc. 37 (1928) 395, fig. 46.-Lect ot y pe (Tardieu-Blot, op. cit., 1964, 'holotype'): Barter s.n. (Niger Expedition), Sierra Leone (K).

Rhizome up to $10 \mathrm{~cm}$ long, up to $7 \mathrm{~mm}$ thick, with 2(3) rows of leaves. Scales of the rhizome: index 2-6, up to 3.5 by $1(-1.5) \mathrm{mm}$, opaque to subclathrate, blackish, sometimes with a paler marginal strip. Sterile leaves pinnate, $30-60 \mathrm{~cm}$ long; petiole $5-35 \mathrm{~cm}$ long, near the base $1.5-3 \mathrm{~mm}$ thick, with $3-7$ vascular bundles; lamina index $1-3$, widest about or below the middle, $15-45$ by $8-20 \mathrm{~cm}$, without the terminal segment $10-35$, the 
terminal segment 5-15 cm long, firm-herbaceous, light to dark green, usually with a distinct bulbil subterminally on the terminal segment (and pinnae); rhachis not winged; pinnae alternate or opposite, 14-23, up to $45(-60) \mathrm{mm}$ apart; central pinnae index 5-15, widest about (to below) the middle, $6.5-16$ by $0.8-2 \mathrm{~cm}$, base symmetrical, usually narrowly cuneate, margin \pm entire or finely crenate-serrate, without spines, apex acute to long-acuminate; the two lowermost pinnae up to $10 \mathrm{~mm}$ stalked, otherwise similar to the central pinnae; terminal segment joined to the rhachis or subarticulate, usually conform to the pinnae, rarely narrowly triangular; venation pattern: veins forming a regular network of a costal areole and usually one transverse row of smaller distal ones, sometimes the costal arch with a single excurrent included free vein, see also fig. 38: 1 . Fertile leaves $40-90 \mathrm{~cm}$ long; petiole $24-55 \mathrm{~cm}$ long; lamina index 2-6, 15-35 by 3$10 \mathrm{~cm}$, without the terminal segment $10-30$, the terminal segment $4-6 \mathrm{~cm}$ long; pinnae $12-25$, up to $60 \mathrm{~mm}$ apart, central pinnae index $7-13,2.5-9$ by $0.2-0.7 \mathrm{~cm}$; the two lowermost pinnae up to $10 \mathrm{~mm}$ stalked. Sporangia usually inserted all over the lower surface, arranged acrostichoid, sometimes on and near the veins only.

GUINEA. 13 collections.

SiERRA LEONE. 5 collections.

LiBERIA. 10 collections.

Ivory Coast. Chaper s.n. (P); Chevalier 17545 (L, P); Portères s.n. (P).

Grana. Adams 1978 (=3877) (BM, K, P).

NigerIA. Baldwin Jr. 13763 (BM); Jones \& Onochie FHI 18931 (BM); Talbot s.n. (BM).

FERNANDo Poo. Henderson s.n. (K); Mann 133? (not 1339; syntype of Acrostichum salicinum, K). Portères s.n. (P).

Cameroun. De Wit 7030 (WAG).

GABON. Halle 3919 (P), 4009 (BR).

CONGO (BRAZZ.). 5 collections.

ZAIRE L eopoldvill e: 6 collections.

$\mathrm{H}$ a bit a t. Usually on rocks, either in streams or in streambeds, in forest; obviously seasonally submerged. Altitude: $0-1200 \mathrm{~m}$.

S p or es. Cook $163(\mathrm{GH})$, Cremers $306(\mathrm{P})$, Harley F 77(K), Leeuwenberg \& Voorhoeve 4735 (WAG): all or the greater part normal. See also the notes.

Notes. 1. A medium-sized species from wet habitats, obviously derived from a fern like $B$. acrostichoides. Most of the collections attributed to this have a combination of characters that justifies keeping $B$. salicina and $B$. acrostichoides separate. I have strong doubts, however, whether this classification is correct, as it remains uncertain to which degree the morphological characteristics of these two species are determined by habitat conditions. Specimens \pm intermediate are discussed under $B$. acrostichoides, note 5; see also note 2 below.

2. The identification of some small-sized fertile plants is problematical. Their sterile leaves resemble juvenile leaves of $B$. acrostichoides. Here I think belong Mann's syntype (a collection consisting of 14 sterile leaves and one fertile leaf), Henderson s.n. (K), and Portères s.n. (P), all with abnormal spores, all from Fernando Poo.

3. Occasionally the terminal segment of the lamina is narrowly triangular. Both types of terminal segment may occur on the same rhizome (e.g. Wellens 327 , BR). 
4. Copeland regarded the present species (as Campium salicinum) and Campium feeianum ( $=\boldsymbol{B}$. presliana) as more closely related to each other than to any other known species.

10. Bolbitis scalpturata (Fée) Ching-Fig. 43: a-d.

B. scalpturata (Fée) Ching in C. Chr., Ind. Fil., Suppl. 3 (1934) 50; Ito, J. Jap. Bot. 14 (1938) 439, fig., quoad nomen solum; Backer \& Posthumus, Varenfl. Java (1939) 82, p.p.; Posthumus, Ann. Bot. Gard. Btzg vol. hors série (1944) 62; Iwatsuki, Acta Phytotax. Geobot. 18 (1959) 59, see note 5; Nayar \& Kaur, Bull. Nat. Bot. Gard. Lucknow 88 (1964) 55, quoad nomen solum. - Heteroneuron scalpturatum Fée, Hist. Acrost. (1845) 95, pl. 56, p.p. ('Heteronevron').-Acrostichum scalpturatum Kunze, Bot. Zeit. (1848) 103, p.p., non Lindman (1903) $(=$ B. serratifolia), nec (Fée) Presl $(1851)(=$ Acrostichum aureum). - Chrysodium scalpturatum Mettenius, Fil. Lips. (1856) 21, p.p., non Fée (1845) (= Acrostichum aureum), nec Ettingsh. $(1864)(=B$ B. $\times$ lancea). - Leptochilus scalpturatus C. Chr., Ind. Fil. (1906) 387, p.p.; v.A.v.R., Handb. Malayan Ferns (1908) 743, p.p.; C. Chr., Bot. Tidsskr. 32 (1916) 344.-Campium scalpturatum Copel., Philip. J. Sc. 37 (1928) 383, fig. 35; Backer \& Posthumus, Nat. Tijdschr. Ned. Ind. 93 (1933)162.Le ct ot y pe: Gaudichaud s.n., Philippines, Manila, xi-1836 (P; iso in B, 2 sh., BM, P, 2 sh.). See note 6. Leptochilus reimersii Rensch, Hedwigia 74 (1934) 249, pl. 7: fig. 2.-T y pe: Rensch 636, Lesser Sunda Is., Sumbawa, Batu Dulang (B).

Gymnopteris costata (Presl) Bedd. var. undulata auct. non (Hooker) Christ: Christ, Bot. Tidsskr. 24 (1901) 109.

Leptochilus scalpturatus (Fée) C. Chr. var. undulatus auct. non (Hooker) C. Chr.: C. Chr., Bot. Tidsskr. 32 (1916) 344. See note 3.

Rhizome up to $10 \mathrm{~cm}$ long, up to $10 \mathrm{~mm}$ thick, with 2 rows of leaves. Scales of the rhizome: index 1-10(-15), up to 7 by $1.5 \mathrm{~mm}$, subclathrate, blackish. Sterile leaves pinnate, 25-90 cm long; petiole 7-40 $\mathrm{cm}$ long, near the base $1.5-4 \mathrm{~mm}$ thick, with 5-9 vascular bundles; lamina index $1-3$, widest below (to about) the middle, $20-55$ by $7-$ $30 \mathrm{~cm}$, without the terminal segment 4-40, the terminal segment 9-20 cm long, herbaceous to subcoriaceous, purplish or purplish-brown, with a bulbil subterminally on the terminal segment; rhachis not winged; pinnae alternate or opposite, 4-24, 15-50 mm apart; central pinnae index 3-7, widest about or below the middle, $5.5-17.5$ by $1.5-4 \mathrm{~cm}$, base \pm symmetrical, narrowly to broadly cuneate, margin \pm entire or finely serratecrenate, without spines, apex acute to (long-)acuminate; the two lowermost pinnae 1$7 \mathrm{~mm}$ stalked; terminal segment usually subarticulate, sometimes joined to the rhachis, usually conform to the central pinnae, sometimes narrowly triangular and/or somewhat prolonged; venation pattern: veins forming a network of a costal areole and one to few transverse rows of smaller distal ones, part of the areoles with few to several, mostly excurrent included free veins; see also fig. 43: c, d. Fertile leaves $30-70 \mathrm{~cm}$ long; petiole $15-50 \mathrm{~cm}$ long; lamina index $1-4,10-40(-45)$ by $3-15 \mathrm{~cm}$, without the terminal segment 4-35, the terminal segment 3-10 cm long; pinnae 4-26, up to $60 \mathrm{~mm}$ apart; central pinnae index $3-8,3-9$ by $0.6-1.5 \mathrm{~cm}$, the two lowermost pinnae $0-6 \mathrm{~mm}$ stalked. Sporangia inserted mostly on and near the veins, either all over the lower surface, arranged acrostichoid, or along the margin only, arranged pteridoid.

Burma. Moulmein: Parish s.n. (BM, K).

ThaILAND. Nort h ern: Molesworth Allen 2164 (BM, SING, S-PA, US).-N o r t h e a s t e r $\mathrm{n}$ : Hennipman 3688 (L). - So u th e a s ter n: Kerr 9751 (P); Schmidt 891 (C).-P en i n u lar: Seidenfaden 2123 (C).

Vietnam. Cochin chin a: Pierre s.n., 1886 (P). 


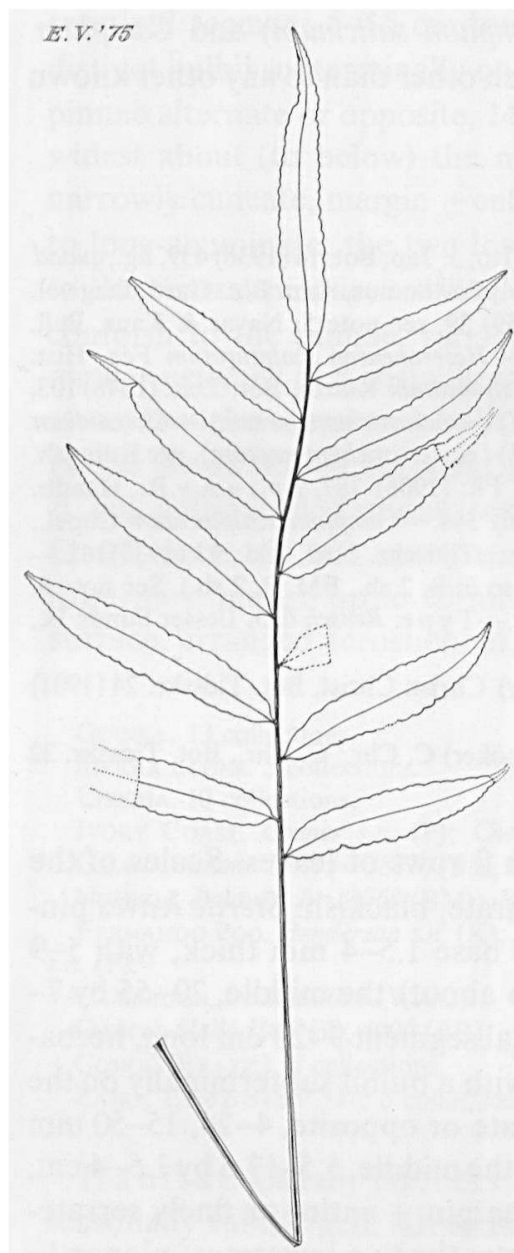

a

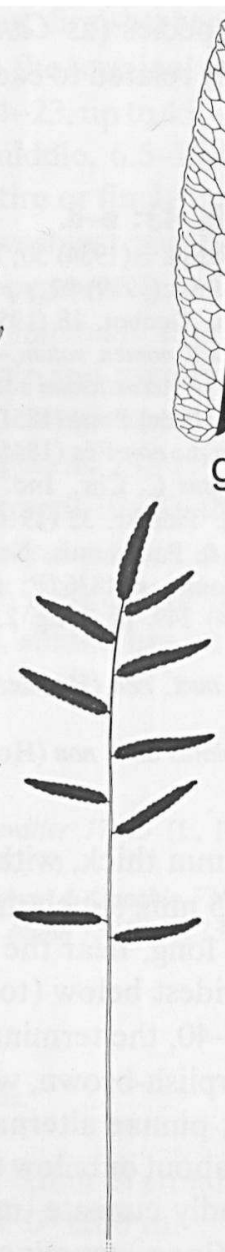

b

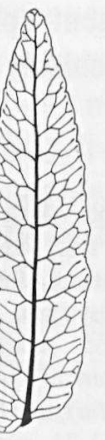

g
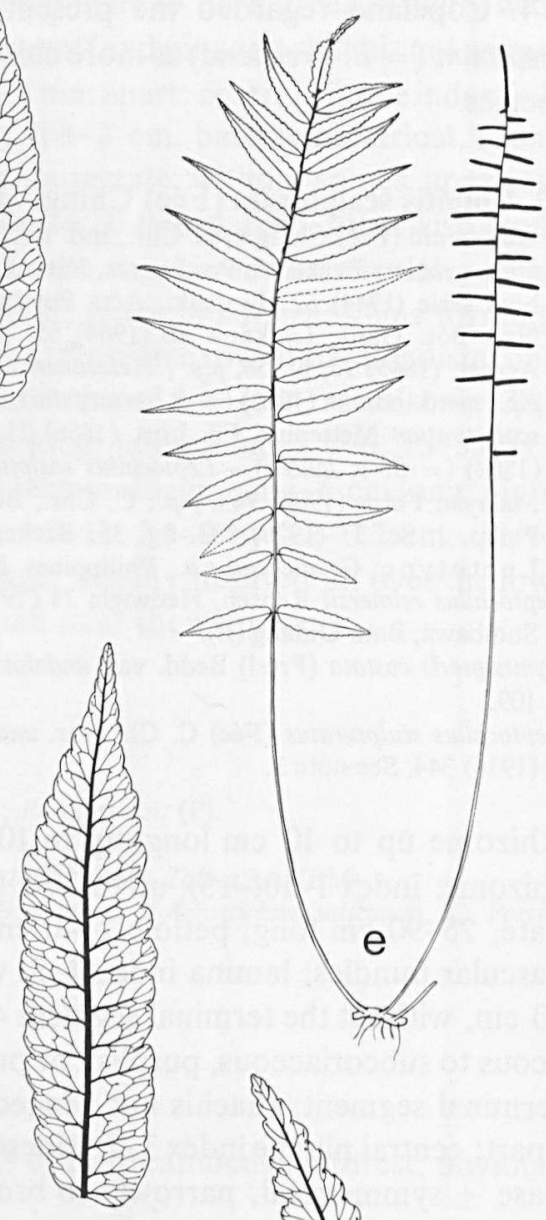

h
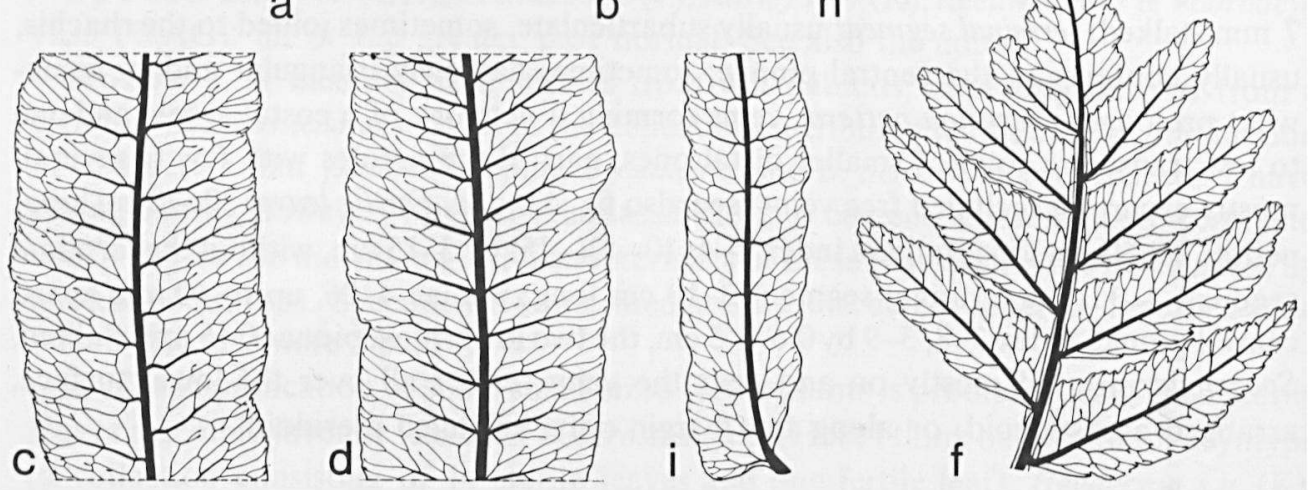

Fig. 43. a-d. Bolbitis scalpturata. a, b. fertile and sterile leaf resp., $\times 1 / 5$; , d. venation pattern of sterile pinnae, $\times$ 4/5 (a, b. Gaudichaud s.n., P, lectotype of B. scalpturata; c. Mousset 562, L; d. Posthumus 1126, BO). - $-\mathrm{j}$. Bolbitis semicordata. e. sterile and fertile leaf, $\times 1 / 5$; f. terminal part of sterile leaf, $\times 4 / 5$; g-i. Venation pattern of sterile pinnae, $\times$ 4/5 (e. Mclvor 4. 's.n.', BM, paratype of B. semicordata; $f$. Stocks \& Law s.n., C; g. Perrottet 1473, L; h. Wight 63, B; i. Anonymus s.n., iii-1838, BRI). 
Sumatra. La mpong Dist.: Posthumus 1103 (BO), 1126 (BO).

JAVA. East: 7 collections, presumably all from Mt. Tengger.

Lesser SuNDa Is. B ali: Posthumus 3708 (BO).-S u m b aw a: Rensch 636 (type of Leptochilus reimersii).

Flor es: Schmutz 3677 A (L).

PhiLippines. Pa la wa n: Merrill 7267 (MICH, P).-L u z o n: 6 collections.

Celebes. Sout hw es t: Sarasin 1193 (P).-Sal aj ar gro u p: Docters van Leeuwen 1621 (BO, U).

$\mathrm{H}$ a bit a t. On rocks in (exclusively monsoon?) forest; twice recorded from limestone (Thailand, Celebes). Altitude: $0-1200 \mathrm{~m}$.

S p o r e s. Allen 2164 (BM), Hennipman 3688 (L), Kerr 9751 (P), Posthumus 1103 (BO): nearly all normal. Allen 2164 (S-PA), Copeland 249 (MICH), Seidenfaden 2123 (C), Williams 30 (US), 234 (UC), Zollinger 2162 (G, P): the greater part abnormal. Gaudichaud s.n. (lectotype of Heteroneuron scalpturatum) (BM, P, 2 sh.): spores (nearly?) all abnormal, mostly shrivelled, with many small, smooth, brown, accessorial bodies inside each sporangium.

Notes. 1. A somewhat critical species which needs further study when more material is at hand. The venation pattern of the pinnae shows considerable variation and is usually locally \pm irregular; it may come near to that of $B$. angustipinna or $B$. virens. As in $B$. costata and $B$. crispatula the colour of the dried leaves is purplish. The scales of the rhizome are similar to those found in part of $B$. crispatula var. copelandii.

2. As regards the arrangement of the sporangia there occur two types of plants, one acrostichoid, the other \pm pteridoid. There is no distinct correlation between the arrangement of the sporangia and the geographical distribution. For instance, the specimens from Koh Chang (Thailand) and Palawan are \pm pteridoid whereas those from Luzon and Celebes are acrostichoid; both types of plants occur on Java.

3. The combination Gymnopteris costata var. undulata (Hooker) Christ was made to accommodate Schmidt 891. Christ referred to Notholaena undulata Wall. For full synonymy see B. virens. C. Christensen (1916) identified the same collection as follows: 'L. [eptochilus] scalpturatus (Fée) C. Chr. Ind. [1906] 387 (excl. syn. omnibus, Heteronevron scalpturatum Fée exceptum), var. undulatus var. nov. Jymnopteris costata var. undulata Christ, l.c. 109,-[Schmidt $]$ (no. 891).' He thus excluded Notholaena undulata as a synonym of Leptochilus scalpturatus, at the same time recognizing it as a variety of that species; obviously an error.

4. Allen 2146 and Hennipman 3688 from northern Thailand agree with B. virens as regards the number of pinnae and more or less so as regards venation pattern.

5. Iwatsuki listed $B$. scalpturata as a doubtful species but obviously only had in mind to cite a certain collection from Formosa as a doubtful specimen as he reported at the same time not to have examined any specimen of $B$. scalpturata proper.

6. The lectotypification of Heteroneuron scalpturatum Fée. The relevant entry in Fée's original description runs as follows:

Cyrtogonium costatum? J. Smith, in Journ. bot. Hook., IV, 1841 [nom. nud.].

Acrostichum costatum, Wall., herb., n. ${ }^{\circ} 26$ [nom. nud.].-Campium, Presl, Tentam. pterid., p. 239?

Habitat in India occidentali [orientali], Nepaul, Sylhet (Wallich); Manilla (Gaudichaud, Voy. de la Bonite, 1836). - V.S. in herb. Bory et Mus. Par., ex Gaudichaud.

Exsiccata: Hügel, in herb. imper. Vindob., $\mathrm{n}^{\text {os }} 2165,2161,4140,4974$. 
The protologue consists of the following elements:

A. The material. Firstly, the specimens annotated by Fée (in his characteristic elegant handwriting) as 'Heteronevron scalpturatum':

a. Hügel 2161, 2165, 4140, and 4974; all sterile (all in W). Referred to B. $\times$ lancea.

b. Wallich 26 , a fertile leaf (W). The sheet bears also Wallich's original label and identification. It represents an isotype of Acrostichum costatum (=B. costata).

c. Gaudichaud s.n., a sterile plant composed of three leaves (P). It is part of the lectotype of $B$. scalpturata.

Secondly, the material not annotated by Fée but also or possibly also studied by him.

a. Wallich $26(\mathrm{P})$.

b. Gaudichaud s.n. (P, 2 sh.). At least one of these sheets, comprising both sterile and fertile material, was studied by Fée as will be elucidated below.

B. The synonyms. The addition, by Fée, of queries to the names of Wallich, J. Smith, and Presl has been variously interpreted. Presl (1851) thought that the queries indicated that Fée doubted if J. Smith and he himself had studied original material of Wallich 26: 'Species a me et a J. Smith enumerata ad specimina Wallichiana autographa constituta fuit, inde signa interrogationis a clar. Fée illata plane inutilia censunda sunt.' Copeland, however, concluded that the queries were added because:'... Fée was questioning the identity of Campium costatum and his own species. What he described and knew positively as Heteronevron scalpturatum, was Gaudichaud's plant from the Philippines, which must accordingly be regarded the type.' Christensen(1906) listed Campium costatum as a synonym of Leptochilus scalpturatus. Later (1916), after studying the material of both taxa at $P$, he ranked them as different species.

$C$. The illustration. The material Fée studied being known, it should be easy, accordingly, to indicate the material from which the lithograph was drawn. Christensen (1916), who studied original material at Paris, stated: 'The form illustrated by Fée is according to authentical specimens examined by me, collected near Manila by Gaudichaud; .... Christensen is incorrect as neither the material of Gaudichaud at Paris, nor that of other collections studied by Fée at Paris or Vienna comprise leaves matching those of the lithograph satisfactorily.

The sterile leaf. The drawing of the sterile leaf remained a mystery until I understood that the beautifully depicted sterile leaf is a composition of elements belonging to three different sterile leaves present on three different sheets in two different herbaria:

a. The petiole and the terminal segment are drawn from Hügel 4140 .

b. The number of pinnae is taken from Gaudichaud s.n., the specimen annotated by Fée as: 'Heteronevron scalpturatum,-Espèce à faire figurer'.

c. The size and the venation pattern of the greater part of the pinnae are taken from a sterile leaf of Gaudichaud s.n., a sheet not annotated by Fée. The length of the lamina without the terminal segment of the latter leaf is $25 \mathrm{~cm}$ whereas it is $19 \mathrm{~cm}$ in the drawing.

The fertile leaf. It cannot be indicated with certainty from which specimen the illustration of the fertile leaf was drawn; the fertile leaves of B. costata and B. scalpturata 
may be much alike. The drawing shows a leaf with an incomplete petiole, the lamina $37 \mathrm{~cm}$ long (the terminal segment $9 \mathrm{~cm}$ long) and composed of 11 pinnae. These data neither correspond with Gaudichaud s.n. (P) nor with Wallich $26(\mathrm{~W})$. The spores illustrated look normal; the outer surface shows low crests which are lacking in both $B$. costata and $B$. scalpturata. Fée described the spores as irregular.

The rhizome. The rhizome pictured presumably has been taken from a plant of Gaudichaud as a rhizome is lacking in all other collections cited by Fée.

D. The description. Fée (1845) used to give relevant details of the plants, such as sizes of leaves, pinnae, etc. in the entry on 'Dimensions'. In this entry the length of the sterile leaf is given as $50-60 \mathrm{~cm}$. I found the length of the sterile leaves of Gaudichaud's plants to vary from $50-74 \mathrm{~cm}$, whereas that of Hügel's collections is much greater. The length of the petiole of the sterile leaves is given by Fée as $c .15 \mathrm{~cm}$. I found it to range between 17 and $32 \mathrm{~cm}$ in the leaves of Gaudichaud's collections, and to be $38 \mathrm{~cm}$ in Hügel 4140 and in Fée's drawing. The length of the fertile leaf is given as $92 \mathrm{~cm}$. The length of the fertile leaves of Gaudichaud's collections is $c$. 70 $\mathrm{cm}$. The length can only have been taken from Wallich 26 as all of Hügel's collections are sterile. It is further remarked that the measures given for the distance between two adjacent pinnae ('entre-noeuds') of the fertile and the sterile leaf have been interchanged.

Discussion. As regards the significance of the queries Fée added to the names of Wallich, J. Smith, and Presl, I share Copeland's conclusion but not his argumentation. Fée's had a very good knowledge not only of the material of Gaudichaud but also of that collected by Hügel and of Wallich 26 (type of Acrostichum costatum). In Fée's description elements taken from each of these different collections can be indicated. As Acrostichum costatum is not included with certainty in Fée's eventual concept of Heteronevron scalpturatum, the name Heteroneuron scalpturatum is not superfluous.

The discrepancies between the data provided in the description if compared with those of the drawing, have never been noticed before, presumably also since the quality of the text of Fée's Histoire des Acrostichées is much praised; the illustrations are generally regarded masterpieces, the aesthetic and scientific value of which are unquestioned. Amongst those misled in recent years is Pichi Sermolli (1969) who chose Heteroneuron scalpturatum as the type of Heteroneuron (the choice is against the Code, see the note to the genus) after careful examination of all species concerned and having taken into consideration '... the descriptions, the specimens studied and the illustrations given by Fée...

11. Bolbitis semicordata (Baker) Ching-Fig. 43 :e-j.

B. semicordata (Baker) Ching in C. Chr., Ind. Fil., Suppl. 3(1934) 50; Nayar \& Kaur, Bull. Nat. Bot. Gard. Lucknow 88 (1964) 57, fig. 22, 36, 68, 71, 90.-[Acrostichum virens Hooker \& Grev. var. minus, fuscatum Hooker, Sec. Cent. Ferns (1861) pl. 88, nom. inval. (art. 24 hence art. 32)]-Acrostichum semicordatum Baker in Hooker \& Baker, Syn. Fil. (April 1868) 422, p.p.-Poecilopteris semicordata Bedd., Ferns Br. India (1868) pl. 270 ( = Hooker, op. cit., pl. 88). - Gymnopteris semicordata J. Smith, Hist. Fil. (1875) 138. Campium semicordatum Copel., Philip. J. Sc. 37 (1928) 378, fig. 31, pl. 23, 24.-L e ct o t y p (Copel., op. cit., 'cotype'): Law s.n., India, Concan (K; iso in B, C, GH, K, P, S-PA, W). 
B. semicordata (Baker) Ching var. incisa Nayar \& Chandra, Amer. Fern J. 54 (1964) 14, fig. 10-15; Nayar \& Kaur, Bull. Nat. Bot. Gard. Lucknow 88 (1964) 61, fig. 37, 38, 91.-T y p e: Chandra et al. LWG 95145, India, Mysore, Castle Rock, Gudkewadi, 400 m alt., 9-xii-1962 (LWG; iso in LWG, 2 sh. ' 10-xii-1965').

Gymnopteris contaminans auct. non Bedd.: Bedd., Ferns Br. India, Suppl. (1876) 27, p.p.; Handb. Ferns Br. India (1883) 435, p.p., fig. 264; ibid., Suppl. (1892) 105, p.p.

Rhizome up to $10 \mathrm{~cm}$ long. $3-5(-8) \mathrm{mm}$ thick, with 2 rows of leaves. Scales of the rhizome: index 4-8, up to 6 by $1 \mathrm{~mm}$, opaque to subclathrate, blackish. Sterile leaves pinnate, $15-70 \mathrm{~cm}$ long; petiole 5-40 $\mathrm{cm}$ long, near the base $1.5-2.5 \mathrm{~mm}$ thick, with 3-7 vascular bundles; lamina index 1-3, widest about or below the middle, 10-30 by 5-13 $\mathrm{cm}$, without the terminal segment 8-23, the terminal segment 3-16 cm long, (firm-) herbaceous, light green to olivaceous, without or with 1(2) subterminal bulbil(s); rhachis narrowly winged; pinnae usually \pm opposite, $12-29$, up to $30 \mathrm{~mm}$ apart; central pinnae index (2-)3-6, widest somewhat below the middle, $2.5-10$ by $0.7-2.5 \mathrm{~cm}$, base \pm equally long-sided, oblique, its acroscopic side subcordate to broadly cuneate, its basiscopic side \pm narrowly cuneate, margin usually \pm entire or finely crenate-serrate, sometimes shallowly lobed, sometimes with teeth, apex acute or acuminate; the two lowermost pinnae deflexed, 0-3 mm stalked; terminal segment usually subarticulate and conform to the pinnae, sometimes joined to the rhachis and narrowly triangular; venation pattern: veins forming a costal areole, without or with few to several smaller distal areoles, usually with a short, excurrent, included, free vein on the distal part of the costal arch, see also fig. 43: g-i. Fertile leaves $20-60 \mathrm{~cm}$ long; petiole $10-45 \mathrm{~cm}$ long; lamina index 2-6, 7-30 by $2-13 \mathrm{~cm}$, without the terminal segment $4-25$, the terminal segment $1.5-7 \mathrm{~cm}$ long; pinnae $10-26$, up to $30 \mathrm{~mm}$ apart, usually perpendicularly spreading; central pinnae index $5-30,0.8-6.5$ by $0.15-0.4 \mathrm{~cm}$, the acroscopic side of the base sometimes auricled; the two lowermost pinnae 0-2 mm stalked. Sporangia inserted all over the lower surface or (in narrow pinnae) mainly on the costa, arranged acrostichoid.

Chromosomes. $n=41$.

INDIA. Western Ghats. Mysore, Madras, and Kerala: 21 collections.

H a bit at. Creeping (exclusively?) on rocks (often near streams) in forest. Presumably common. Altitude (but few records available): 400-1800 m.

S p o r e s. Barnes 545 (MICH), Chandra et al. LWG 95145 (type of B. semicordata var. incisa), Van Hardeveld \& Van der Werff $323 A$ (L), 323B (L), Law s.n. (lectot.dupl. of Acrostichum semicordatum. K): nearly all normal, perispore verrucate. Hooker \& Thomson s.n. (BM), Meebold 10905 (B), Law s.n. (isolectotype of Acrostichum semicordatum; 'Stocks, Law etc.', C) : nearly all normal, large, perispore thick, usually \pm spherical, sometimes with a few inconspicuous folds or verrucae.

Notes. 1. The retention of certain juvenile features has presumably played a decisive role in the origin of $B$. semicordata. See the general part.

2. Although a considerable part of the material fits the description, still a significant number of specimens is more or less divergent. Hybridization with the other members of this series, occurring in southern India, seems common. See also $B . \times$ lancea, $B . \times$ prolifera, and note 3. 
3. From the study of herbarium material of the Bolbitis species growing in southern India, I got the impression that these may grow intermingled, that hybridization between them is common, and that vegetative propagation is markedly developed in all. This impression was confirmed by the following collections may by Van Hardeveld \& Van der Werff, two students from Utrecht University. Their magnificent Bolbitis collections are all from one locality in Anaimalai Hills, State of Madras.

a. Van Hardeveld \& Van der Werff $323 B$ (L, 7 sh.) represents \pm typical B. semicordata. The shape of the terminal segment shows variation; it is either entire or near the base deeply lobed, or the base shows many odd pinnae. Both types of leaf are of comparable size and may occur on the same rhizome. Spores: the greater part normal, perispore verrucate. Referred to $B$. semicordata.

b. Ibidem $323 A$ (L, $4 \mathrm{sh}$.). Leaves almost similar to those mentioned above but somewhat larger and with the terminal segment always deeply lobed and (much) prolonged, with a wing along the rhachis of the upper half of the lamina only, the base of the central pinnae more or less oblique, the venation pattern rather irregular and rather resembling that pictured by Beddome for Poecilopteris repanda and Gymnopteris contaminans. Spores for the greater part normal, perispore verrucate. Referred to B. semicordata with misgivings.

c. Ibidem 323 (L, 4 sh.). Plants rather agreeing with those of $323 \mathrm{~A}$ but larger, the rhachis of the sterile leaves not winged, the venation pattern very irregular. Spores in part (the majority?) abnormal. As regards the outline of the leaves these plants agree with Acrostichum terminans (Wallich 2168). Referred to $B . \times$ prolifera.

4. One of the two isotypes of var. incisa studied has pinnae with a \pm entire margin. According to Nayar \& Kaur both varieties grow intermingled.

5. The basionym was validly published during 1868 by either Baker or Beddome. As Beddome referred to 'Poecilopteris semicordata (Baker)' I presume that Baker's publication was the earliest.

6. Posthumus (Ann. Bot. Gard. Btzg, vol. hors série 1944, 62) reported the present species from Sumba (Lesser Sunda Is.); I could not trace the reference collections.

\section{Bolbitis serrata (Kuhn) Ching-Fig. 44.}

B. serrata (Kuhn) Ching in C. Chr., Ind. Fil., Suppl. 3 (1934) 50.-Acrostichum serratum Baker in Hooker \& Baker, Syn. Fil. (1868) 524, nom. illeg., non Lam. \& Poiret (1796) (= Grammitidacea?).-Chrysodium serratum Kuhn, Linnaea 36 (1869) 63.-Leptochilus serratus C. Chr., Bot. Tidsskr. 26 (1904) 289, fig. 2.Ty p e: Spruce 4123, Peru, San Martin, near Tarapoto, 1855-56 (K; iso in BM, GH, NY, P, US). Acrostichum serratifolium auct. non Kaulf.: Hooker, Spec. Fil. 5 (1864) 253, p.p.

Rhizome up to $10(-15) \mathrm{cm}$ long, 2-5 mm thick, with 2 rows of leaves. Scales of the rhizome: index 2-4, up to 2 by $1 \mathrm{~mm}$, subclathrate, dark brown or blackish. Sterile leaves pinnate, $15-40 \mathrm{~cm}$ long; petiole 8-20 cm long, near the base 1-2 mm thick, with 3-5 vascular bundles; lamina index $1-3$, widest about or below the middle, $8-20$ by $3-12 \mathrm{~cm}$, without the terminal segment $1.5-10$, the terminal segment $6-12 \mathrm{~cm}$ long, herbaceous, light to dark green, without bulbils; rhachis narrowly winged; pinnae usually alternate, $4-8$, up to $25 \mathrm{~mm}$ apart; central pinnae index $3-6$, straight, widest about the middle, 


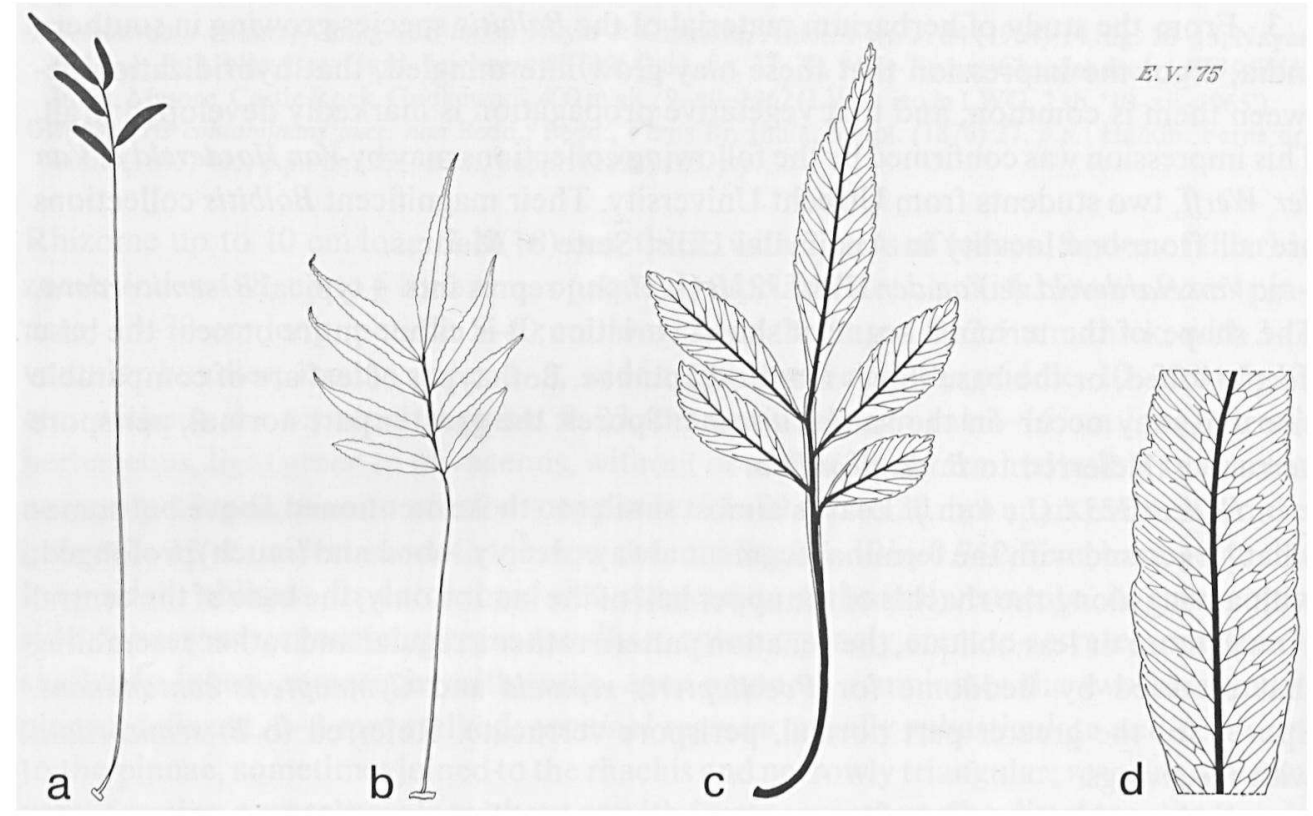

Fig. 44. Bolbitis serrata.-a, b. fertile and sterile leaf resp., $\times 1 / 5$; c. juvenile leaf, $\times 4 / 5$ d. venation pattern of sterile pinna, $\times 4 / 5$ (a. Spruce 4123, K; b, d. ibid., P; c. Wurdack 2110, U).

$3.5-12$ by $0.8-2.5 \mathrm{~cm}$, base symmetrical, narrowly cuneate or attenuate, margin entire or crenate-serrate (especially in the upper half), without spines, apex acute or acuminate; the two lowermost pinnae 0-1.5 mm stalked, otherwise conform to the central pinnae; terminal segment conform to the central pinnae, usually subarticulate, sometimes joined to the rhachis; venation pattern: veins forming a costal areole, smaller distal areoles present or not, included free veins absent, see also fig. 44:d. Fertile leaves $22-50 \mathrm{~cm}$ long; petiole $15-35(-40) \mathrm{cm}$ long; lamina index $1-3,7-12$ by $3-6 \mathrm{~cm}$, without the terminal segment 3-7, the terminal segment 3-6 cm long; pinnae 4-8, $10-25 \mathrm{~mm}$ apart; central pinnae index $3-8,1.5-5.5$ by $0.4-1 \mathrm{~cm}$; the two lowermost pinnae $0.5-7 \mathrm{~mm}$ stalked. Sporangia mainly inserted on and near the veins, arranged acrostichoid.

Colombia. Met a: Pinto \& Bischler 223 (G).

ECUADOR. A n tis a na: Grubb et al. 1710A (NY, US).-S a ntiago-Z a mo ra: Van der Werff 671 (L, U). Peru. A maz on as: Wurdack 2110 (G, NY, S, U, UC, US).-L or e to: Killip \& Smith 28495 (F, NY).San Martin: Spruce 4123 (type of Chrysodium serratum).-Sine loc.: Godet s.n. (B).

$\mathrm{H} \mathrm{a} \mathrm{bit} \mathrm{a} \mathrm{t.} \mathrm{Creeping} \mathrm{on} \mathrm{rocks} \mathrm{or} \mathrm{in} \mathrm{soil} \mathrm{(in} \mathrm{streambeds)} \mathrm{in} \mathrm{rain} \mathrm{forest.} \mathrm{Altitude:} 250$ $700 \mathrm{~m}$. Locally common (Wurdack 2110 ).

Spores. Wurdack 2110 (US): all normal, the perispore usually with few folds. Wurdack 2110 (UC): the greater part normal, the perispore usually with many folds. 
Killip \& Smith 28495 (NY): in part (the majority?) abnormal, the perispore situated close to the exospore and provided with low rather sharp crests. Spruce 4123 (GH, K): in part normal, the perispore with or without crest-like folds. See also note 2.

Notes. 1. A rather critical species closely related to and obviously derived from a fern like $B$. serratifolia. The differences between the present species and $B$. serratifolia are presumably caused by retention. A narrow wing along the rhachis is also found in $B$. semicordata which is otherwise different.

2. The collections from Colombia and Peru consist of relatively stout plants. The leaves are composed of 3-7 large pinnae, the venation pattern is intricate and resembles that found in part of B. serratifolia. Spores: Pinto \& Bischler $223(\mathrm{G})$ : the greater part nor$\mathrm{mal}$, the perispore rather densely folded; Grubb et al.1710A (US): sporangia filled with aborted spores and/or with aborted S. M. C.'s.

3. According to Baker, Acrostichum serratum should be near Acrostichum raddianum ( = Lomagramma guianensis). Christensen thought Leptochilus serratus closely related to Leptochilus guianensis (= Lomagramma guianensis). In my opinion the juvenile leaves of Lomagramma guianensis and the mature leaves of $B$. serrata are good examples of parallel evolution.

13. Bolbitis serratifolia (Mertens ex Kaulf.) Schott-Fig. 45.

B. serratifolia (Mertens ex Kaulf.) Schott, Gen. Fil. (1835) ad tab. (13).-Acrostichum serratifolium Mertens ex Kaulf.. Enum. Fil. (1824) 66; Hooker, Spec. Fil. 5 (1864) 263, p.p.; Baker in Hooker \& Baker, Syn. Fil. (1868) 423, p.p.; in Martius, Fl. Brasil. 1, 2 (1870) 590, pl. 38: fig. 18-22; Lindman, Ark. Bot. 1 (1903) 252, fig. 8; Hedwigia 43 (1904) 311. - Cyrtogonium serratifolium J. Smith, Hook. J. Bot. 4 (1841) 154.-Heteroneuron serratifolium Fée, Hist. Acrost. (1845) 94, pl. 55: fig. iiiB ('Heteronevron'); Fournier, Mex. Pl. I, 1 Crypt. (1872) 69, quoad nomen solum.-Chrysodium serratifolium Ettingsh., Denkschr. K. Ak. Wiss. M.-N. Kl. Wien 22 (1864) 57.-Gymnopteris serratifolia Diels in E. \& P., Nat. Pfl. Fam. I, 4 (1899) 201.-Leptochilus serratifolius C. Chr., Bot. Tidsskr. 26 (1904) 289, fig. 3.-T y p e: Mertens s.n., Brazil, Rio de Janeiro (n.v.; B?, LE?).

Acrostichum fraxinifolium Presl in Presl \& Presl, Deliciae Pragensis 1 (1822) 161, non R. Brown (1810) (= Acrostichum spec.).-Poecilopteris fraxinifolia Presl, Tent. Pterid. (1836) 242, pl. 11: fig. 1, 2; Hooker, Gen. Fil. (1842) pl. 81B; Presl, Epim. Bot. (1851) 173._Chrysodium fraxinifolium Mettenius, Fil. Lips. (1856) 21, non Fée (1845) (= Acrostichum speciosum Willd.).-Polybotrya fraxinifolia Mettenius, Fil. Lechl. 2(1859) 8.-T y p e: Pohl s.n., Brazil, Guanabara, Rio de Janeiro, Corcovado Mts. (PR).

Acrostichum triste Vellozo in Arrabida, Fl. Fluminensis 11 (1836) pl. 90, s. descr.; Arch. Mus. Nac. Rio de Jan. 5 (1881) 453.-T y p e: Vellozo s.n., Brazil, Guanabara, Rio de Janeiro, Mt. Nhamduaray, fert. (n.v.; R?).

Poecilopteris crenata Presl, Epim. Bot. (1851) 174; Underwood, Bull. Torr. Bot. Cl. 33 (1907) 603, fig. 15, 16.-Leptochilus crenatus C. Chr., Ind. Fil., Suppl. 1 (1913) 48.-B. crenata C. Chr., Ind. Fil. Suppl. 3 (1934) 47.-T y p e: Mikan s.n., Brazil, Guanabara, near Rio de Janeiro (n.v.; PR?).

Heteroneuron serratifolium (Kaulf.) Fée var. undulatum Fée, Crypt. Vasc. Brésil 1 (1869) 18. - S y n t y p e s (all from Brazil, Guanabara, Rio de Janeiro): Martius s.n. (n.v.; herb. Fée?), Glaziou 953 (BR), 2422 (BR, P).

Gymnopteris contaminoides Christ, Ann. Cons. Jard. Bot. Genève 3 (1899) 32.-Acrostichum contaminoides Lindman, Hedwigia 43 (1904) 311.-Leptochilus contaminoides C. Chr., Bot. Tidsskr. 26 (1904) 294, fig. 6a-c.-L e ct ot y pe (C. Chr., op. cit.): Balansa 2852, Paraguay, near Paraguari, mountain ridge near Tchololo (B, BM, G, L, 3 sh., P).

Acrostichum pervium Lindman, Ark. Bot. 1 (1903) 254, pl. 8: fig. 7.-T y p e: Mosén 75, Brazil, Guanabara, Corcovado Mts. near Rio de Janeiro (S-PA; iso in GH, P, S, U, US). 
Acrostichum curupirae Lindman, Hedwigia 43 (1904) 311.-Leptochilus curupirae C. Chr., Bot. Tidsskr. 26 (1904) 296, fig. 7.-B. curupirae Ching in C. Chr., Ind. Fil., Suppl. 3 (1934) 48.-T y p e: Exp. I. Regnell A 3061 Lindman leg.), Brazil, Mato Grosso, Mato do Curupira, ster. (S-PA; iso in BM, S, US). Leptochilus serratifolius (Kaulf.) C. Chr. f. caudatus C. Chr., Bot. Tidsskr. 26 (1904) 290.-S y n t y pes (both from Brazil, s. loc.): Langsdorff s.n. (B), Mendoza 1368 (B).

Leptochilus mexicanus Christ, Bull. Herb. Boiss. II, 7 (1907) 414.-B. mexicana C. Chr., Ind. Fil., Suppl. 3 (1934) 49.-T y pe: Münch 19, Mexico, Chiapas, San Christobal (iso in US; no specimens traced by me at $P$, nor by Dr. Farron, in litt., at BAS).

Leptochilus contaminoides (Christ) C. Chr. var. lobulatus Christ, Fedde Rep. 6 (1909) 351.-T y p e: Hassler 10377a, Paraguay, Sierra de Amambay (n.v.; Hassler 10377, Rojas leg., at B, K, L, P = B. serratifolia). Leptochilus serratifolius (Kaulf.) C. Chr. var. prolifer Rosenst., Fedde Rep. 20 (1924) 93.-Type: Von Luetzelburg 7180, Brazil, Espirito Santo, Rio Doce (M).

B. maguirei Maxon \& Morton, Bull. Torr. Bot. Cl. 75 (1948) 71.-T y p e: Maguire 24744, Surinam, Table Mt., near Augustus Falls (US; iso in NY, U).

Lomaria bredemeijeriana auct. non Klotzsch:Klotzsch, Linnaea 20 (1847) 346.

Acrostichum scalpturatum auct. non (Fée) Kunze: Lindman, Ark. Bot. 1 (1903) 253, pl. 8: fig. 9.

Rhizome up to $10 \mathrm{~cm}$ long, up to $15 \mathrm{~mm}$ thick, with 2 rows of leaves. Scales of the rhizome: index $2-4$, up to 6 by $2 \mathrm{~mm}$, subclathrate, dark brown or blackish. Sterile leaves pinnate, (35-) 50->135 cm long; petiole $18-50 \mathrm{~cm}$ long, near the base $2-5 \mathrm{~mm}$ thick, with 5-13 vascular bundles; lamina index $1-3$, usually widest below, sometimes about the middle, (20-) $25-90$ by $10-40 \mathrm{~cm}$, without the terminal segment $23-65 \mathrm{~cm}$, the terminal segment $10-30 \mathrm{~cm}$ long, herbaceous, usually light to dark green, sometimes with a purple tinge or purplish, with a (primordium of a) bulbil subterminally on the terminal segment (and the pinnae); rhachis not winged; pinnae alternate or opposite, 9-30 $(-42)$, up to $60 \mathrm{~mm}$ apart; central pinnae index 3-10(-14), widest usually about (to below) the middle, $7.5-28$ by $2-4 \mathrm{~cm}$, base usually equally long sided, either somewhat asymmetrical with its basiscopic side orbicular and sometimes narrower than the broadly attenuate or truncate acroscopic side, or symmetrical, (narrowly) cuneate to subcordate, margin usually entire, sometimes crenate-serrate or shallowly lobed, with or without indistinct teeth, sometimes somewhat crisped, apex acute to(long-) acuminate; terminal segment usually joined to the rhachis, sometimes subarticulate, usually \pm conform to the central pinnae, sometimes either prolonged or with some odd pinnae at the base; venation pattern: veins forming a costal areole and few to many smaller distal areoles, without or with few to many, excurrent, included, free veins; see also fig. 45: d-k. Fertile leaves $50-100(-140) \mathrm{cm}$ long; petiole $30-60(-80) \mathrm{cm}$ long; lamina index $2-6,17-65$ by $4-15 \mathrm{~cm}$, without the terminal segment $13-60$, the terminal segment 2-10 cm long; pinnae 12$30(-45)$, up to $70 \mathrm{~mm}$ apart; central pinnae index $2-9(-12), 2-11$ by $0.6-1.5 \mathrm{~cm}$; the lowermost pinnae $0-11 \mathrm{~mm}$ stalked. Sporangia mainly situated on and near the veins, less so on the tissue between the veins, arranged acrostichoid.

Mexico. Chi ap as: Ghiesbrecht $409(\mathrm{GH})$; Münch 19 (type of Leptochilus mexicanus).

Costa Rica. S a n J os.é: Skutch 3084 (US).

Panama. Pittier 5336 (US), 5608 (US); Williams 881 (NY, US).

Colombia. M a g d a l en a: Bennett 18 (US); Grant 10899 (US); H. H. Smith 2522 (BM, F, G, GH, K, MICH, MO, NY, P, S, UC, US, W).-Cu nd in a m a r ca: Grant 10463 (US); Stübel 673 (B), 690 (B), 695 (B).M e t a: Alston 7654 (BM); Idrobo 478 (US); Philipson \& Idrobo s.n. (BM)._Si n e lo c.: Joad s.n. (K); Moritz 129 (BM, fragm.). 

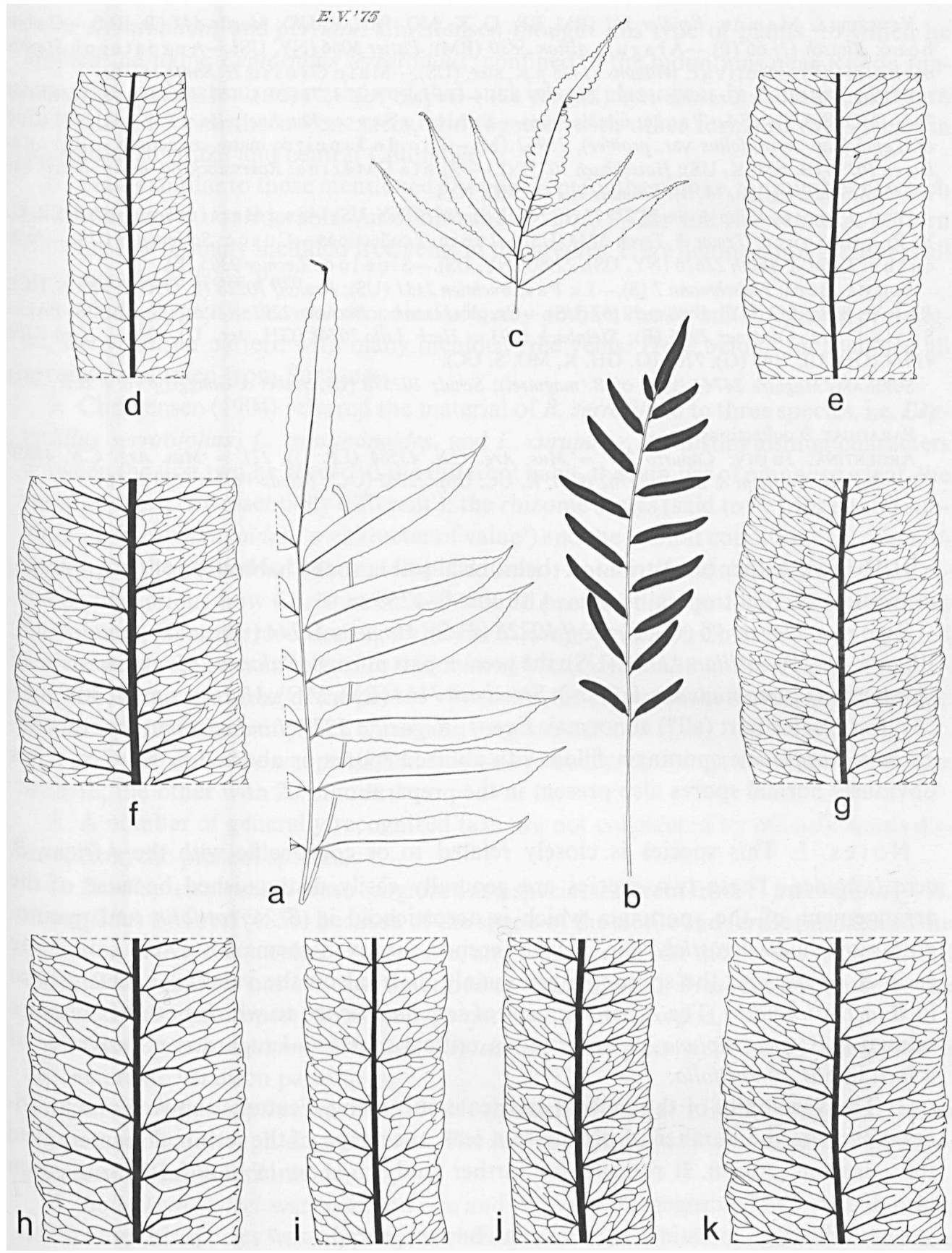

Fig. 45. Bolbitis serratifolia.-a, b. sterile and fertile leaf resp., $\times 1 / 5$; c. terminal part of sterile leaf, $\times$ $1 / 5$; d-k. venation pattern of sterile pinnae, $\times 4 / 5$ (a, b. Wagner 76, P; c. Skutch 3084, K; d. Anonymus s.n., L; e. Pohl \& Schott s.n., L; f. L. B. Smith 1214, US; g. Krukoff 10228, UC; h. Spruce 4736, P; i. Eyerdam \& Beetle 22762, US; j. ibid., UC; k. Schunke A 215, US). 
Venezuela. Merid a: Fendler 311 (BM, BR, G, K, MO, fert., P, US); Moritz 447 (B, BM).-Car ab o bo: Klotsch (?) 66 (P).-A ragua: Alston 5830 (BM); Pittier 6066 (NY, US).-A n zo at egu i: Steyermark 61144 (F).-B oli var. Williams 11438 p.p., ster. (US).-M a to G ross o: H. Smith 16(P).

BrazIL. Para: De Granville 1219 (Z), 1499 (Z).-Goias: Pohl 1871 (BR).-Min as Gerais: Macedo 2437 (MO, S, US).-Sã o Pa ulo: 6 collections.-Es pirit o S a n to: Von Luetzelburg 929 (M), 7180 (type of Leptochilus serratifolius var. prolifer), 18962 (M).-R io de J a ne ir o: many collections.-Parana: Dusén 10136 (B, BM, K, US); Hatschbach 10755 (L). -S a n t a Ca t a r i na: Rosenstock Fil. austro-brazil. exsicc. 5 (Schmalz leg.)(B, M, S); Schmalz 101 (F, MO, NY).

Peru. Lor e to: Killip \& Smith 27797 (F, NY, US), 28919 (NY, US).-S a n Martin: Spruce4726(BR, G, K, P).-H u a nu co: Tryon \& Tryon 5234 (US).-J u n in: 7 collections.-Cu z co: Scolnik 877 (US).-Ayacu ch o: Killip \& Smith 22626 (NY, US), 22995 (NY, US).-Si n e lo c.: Lechler 2305 (B).

Bolivia. B e n i: Fleischmann 7 (S)._L a P a z: Buchtien 2131 (US); Krukoff 10228 (F, G, GH, MO, S, UC); Rusby \& White 552 (NY); Williams 1187 (US)._Co c h a b a m ba: Buchtien 2221 (S-PA. US), 2223 (S-PA).S a n t a Cru z: Cárdenas 2996 (F); Steinbach $1093=$ Herb. Lillo 20768 (GH, ster., U), $3003=$ Herb. Lillo 41314 (GH, U), 7143 (G), 7163 (G, GH, K, MO, S, UC).

SURINAM. Maguire 24744 (type of B. maguirei); Schulz 10257a(U); Stahel \& Gonggrijp $76=$ B.W. 2933 (U).

Paraguay. 9 collections.

ARgentina. Jujuy: Capurro $217=$ Mus. Arg. C.N. 43584 (LP, U), $271=$ Mus. Arg. C.N. 43580 (U),-S al t a: Eyerdam \& Beetle 22762 (GH, K, UC, US), 22765 (UC); Schulz-Varela 5179 (LP).

$\mathrm{H} \mathrm{a} \mathrm{b} \mathrm{it} \mathrm{a} \mathrm{t.} \mathrm{Either} \mathrm{terrestrial} \mathrm{(on} \mathrm{rocks,} \mathrm{or} \mathrm{in} \mathrm{soil} \mathrm{in} \mathrm{rocky} \mathrm{habitats)} \mathrm{or} \mathrm{low-epiphytic,}$ in tropical and subtropical forests. Altitude: 0-1250 m.

S p o re s. Fiebrig $96(\mathrm{G})$, Krukoff 10228 (F, S), Maguire 24744 (type of B. maguirei, U), Wagner s.n. (P), Williams 1187 (US): the greater part normal. Balansa 2852 (lectotoype of Gymnopteris contaminoides, L, 3 sh.), Steinbach 7163 (BM, MO), Martius s.n. (L, 908.20655): the greater part (all?) abnormal. Exp. 1. Regnell A 2337 (Lindman leg.; type of Acrostichum curupirae): sporangia filled with aborted spores or aborted S. M. C.'s, a few obviously normal spores also present in the preparation.

Notes. 1. This species is closely related to or conspecific with the African $B$. acrostichoides. These two species are generally easily distinguished because of the arrangement of the sporangia which is acrostichoid in $B$. serratifolia and gymnogrammoid in $B$. acrostichoides. This difference, however, seems not a very sound one as in $B$. serratifolia the sporangia are mainly inserted on the veins ( \pm exclusively so in $B$. acrostichoides). The Asian element of ser. Bolbitianae to which both of them are related is $B$. angustipinna. $B$. serrata - a critical species-I regard as a derivative of a fern like $B$. serratifolia.

2. The variability of the present species is surprising. Features showing much variation include the number of pinnae to a leaf, the shape of the terminal segment, and the venation pattern. It remains for further study to show in how far hybridization also adds to the heterogeneity.

The following, \pm distinct elements can be recognized.

a. Plants small; sterile leaves 50-90 cm long, pinnae 10-20, usually 14 to a leaf, terminal segment conform to the central pinnae, venation pattern fully anastomosed and without or with but few included excurrent free veins ("more accidentally than normally', Christensen, 1904). These plants agree with the original descriptions of $A$ crost $i$ - 
chum serratifolium and pervium. Christensen thought this type of plants, to which he applied the name Leptochilus serratifolius, confined to the mountains near Rio de Janeiro. But explorations since showed that such plants also occur in Panama, northern Colombia, and northern Venezuela, and together with other forms of this species in southeastern Brazil and central Colombia.

b. Plants similar to those mentioned above except for the pinnae, the number of which to a leaf shows considerable variation ranging from 9-28, and the venation pattern showing few to many included free veins in the areoles. Here belong amongst others all specimens seen from Peru.

c. Plants large; the leaves composed of many pinnae, the terminal segment prolonged, the venation pattern with many included free veins. Here belong for instance all specimens studied from Paraguay.

3. Christensen (1904) referred the material of $B$. serratifolia to three species, i.e. Leptochilus serratifolius, $L$. contaminoides, and $L$. curupirae. As distinguishing characters between the first two he recorded the different habit, the number of pinnae to a leaf, the venation pattern ('essentially different'), the rhizome scales (said to be fringed and subulate in $L$. contaminoides, 'a character of value') and the habitat conditions ( $L$. contaminoides: 'on the ground in the dense tropical forest'; $L$. serratifolius: 'open ground'). It remains mysterious how Christensen was able to provide data on habitat conditions as such data are scantily recorded on the labels. L. curupirae, which Christensen recognized with some doubt, was supposed to differ from L. contaminoides in the shape of the lamIna, the margin and base of the pinnae, the scales, the colour of the leaves, and the shape of the terminal segment which should be long-stipitate.

4. Acrostichum triste was published only on a plate representing two fertile leaves, one with 18 , the other with 20 pinnae.

5 . A number of generally recognized taxa are not considered by me sufficiently distinct from the present species.

a. Poecilopteris crenata was recognized as a species different from $P$. fraxinifolia $(=B$. serratifolia) by Presl (1851) because of the shape of the sterile and fertile pinnae and the presence of ovate-lanceolate, acuminate, and ciliate scales on the petiole, rhachis, and costa. The venation pattern was described as anastomosed; Presl did not describe the included free veins reported for the type material by Underwood.

b. Gymnopteris contaminoides and Leptochilus mexicanus were based mainly on a characteristic venation pattern.

c. Acrostichum curupirae was described from sterile material. Lindman reported this as a common fern though always sterile. The leaves are provided with distinct bulbils, in part also with small plants.

d. Bolbitis maguirei was based on size and shape of the leaves. According to Maxon $\&$ Morton this species was supposed to differ from $B$. serratifolia by its uncinate-serrate rather than crenate-lobate pinnae, and from $B$. crenat $a(=B$. serratifolia $)$ by its venation pattern which should not show included free veins. Included free veins are, however, rather common in the large sterile leaf of the holotype, far less so in a small leaf of the isotype in NY. 
14. Bolbitis subcrenata (Hooker \& Grev.) Ching-Fig. 46, 47.

B. subcrenata (Hooker \& Grev.) Ching in C. Chr., Ind. Fil., Suppl. 3 (1934) 50; Backer \& Posthumus, Varenfl. Java (1939) 80, see note 4; Nayar \& Kaur, Bull. Nat. Bot. Gard. Lucknow 88 (1964) 63, fig. $2-6,11,14,23,32,41,43,46,50-52,59,72,73,76-80,83-85,92,93$.-Acrostichum subcrenatum Hooker \& Grev., Ic. Fil. (1829) pl. 110 (excellent); Clarke, Trans. Linn. Soc. Bot. 1 (1880) 581, p.p.Campium subcrenatum Presl, Tent. Pterid. (1836) 39; Hooker, Gen. Fil. (1842) pl. 80; Copel., Philip. J. Sc. 37 (1928) 391, fig. 42; Backer \& Posthumus, Nat. Tijdschr. Ned. Ind. 93 (1933) 158, excl. specim.Cyrtogonium subcrenatum J. Smith, Hook. J. Bot. 4 (1841) 154.-Heteroneuron subcrenatum Fée, Hist. Acrost. (1845) 93, quoad nomen solum ('Heteronevron'). - Poecilopteris subcrenata Moore, Ind. Fil. (1857) xx._Gymnopteris subcrenata Bedd., Handb. Ferns Br. India (1883) 437, fig. 265.—Leptochilus subcrenatus

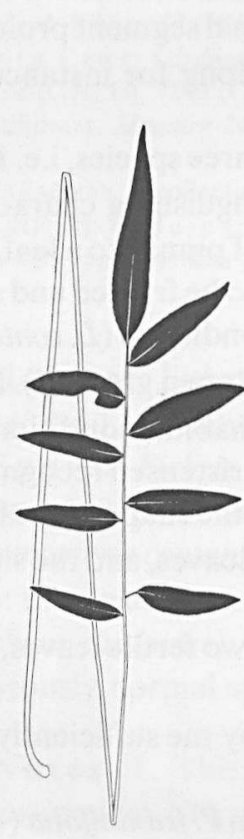

a
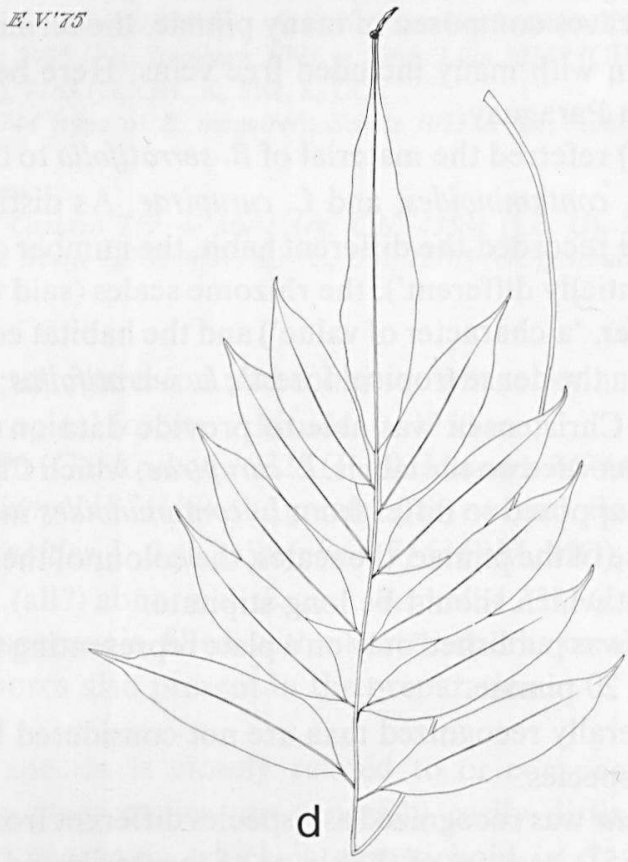

C

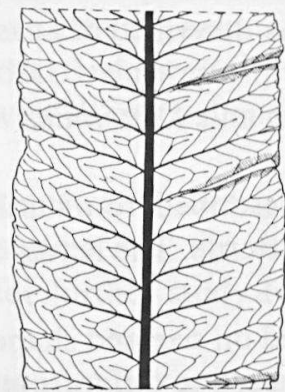

b
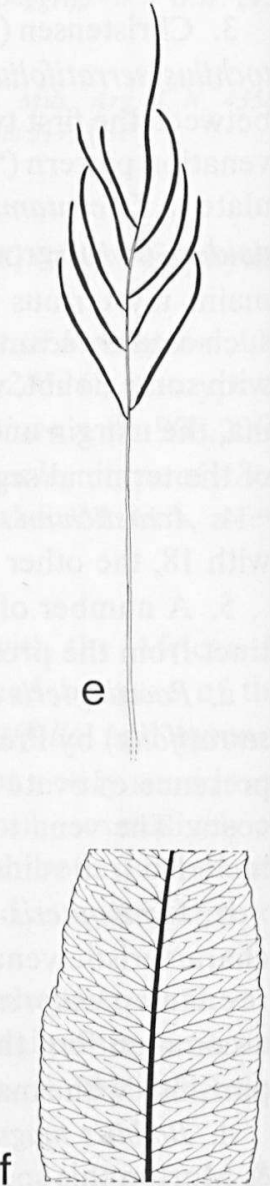

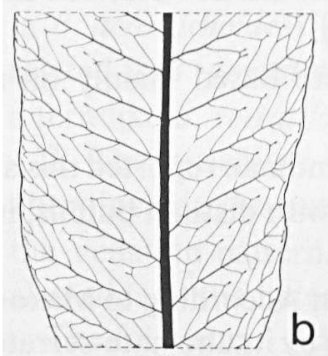

Fig. 46. Bolbitis subcrenata.-a-c. var. subcrenata. a. fertile leaf, $\times 1 / 5, b$, c. venation pattern of sterile pinnae, $\times 4 / 5 .-d-f$. var. prolifera. d, e. sterile and fertile leaf resp., $\times 1 / 5 ; \mathbf{f}$. venation pattern of sterile pinna, $\times 4 / 5$ (a. Thwaites CP 1313, P; b. Wall '60/107', BO; c. Thwaites CP 1313, B (sh. 1); d, e. Anonymus s.n.. iii-1838, BRI; f. Wight, Pen. Ind. Or. $3175, \mathrm{~L}$ ). 
C. Chr., Ind. Fil. (1906) 388.-T y p e: Emerson s.n., eastern Ceylon, near Saffragan (n.v.; not at K, GL). Acrostichum proliferum Hooker, Ic. Pl. 7 (1844) pl.681, 682, nom. illeg., non Bl. $(1828)(=B$. repanda).-Campium proliferum Presl, Epim. Bot. (1851) 170.-Polybotrya prolifera Mettenius, Fil. Hort. Lips (1856) 24 , pl. 2: fig. 11, nom. illeg., non Bory (1833) $(=B . \times$ prolifera).-Poecilopteris hookeriana Moore, Ind. Fil. (1857) xx; Thwaites, Enum. Pl. Zeyl. (1864) 380.-T y p e: Falconer s.n., southern India, Bombay (K). [Acrostichum terminans Wall., Cat. (1830) no. 2168, nom. nud., quoad nomen solum]-Poecilopteris terminans [Moore, Ind. Fil. (1857) 15, nom. nud., quoad nomen solum] Bedd., Ferns S. India (1864) 68, pl. 203. - L e ctot y pe: Beddome's plate drawn from a specimen from southern India.

Campium bradfordii Copel., Philip. J. Sc. 37 (1928) 390, fig. 40, pl. 28.-B. bradfordii Ching in C. Chr., Ind. Fil., Suppl. 3 (1934) 47.-T y p e: Bradford s.n. (1954?), s. loc. ('Ceylon')(UC, 267415).

Acrostichum virens auct. non Hooker \& Grev.: auct. p.p.

Rhizome up to $10 \mathrm{~cm}$ long, up to $12 \mathrm{~mm}$ thick, with 2(3) rows of leaves. Scales of the rhizome: index $2-8$, up to 7 by $1.5 \mathrm{~mm}$, opaque to subclathrate, brownish or blackish. Sterile leaves $40-125 \mathrm{~cm}$ long, pinnate; petiole $10-55 \mathrm{~cm}$ long, near the base 2-4 mm thick, with 5-13 vascular bundles; lamina index $1-3(-5)$, widest below (to about) the middle, $20-75$ by $8-30 \mathrm{~cm}$, without the terminal segment $3-30$, the terminal segment $15-50 \mathrm{~cm}$ long (possibly longer), \pm subcoriaceous, light to dark green, with a (primordium of a) bulbil subterminally on the terminal segment (and pinnae); rhachis usually not winged, sometimes inconspicuously winged in the upper half of the lamina; pinnae alternate or opposite, $3-19$, up to $45 \mathrm{~mm}$ apart, central pinnae index $2-6$, widest usually about, sometimes below the middle, $6-21$ by $2.5-4(-5.5) \mathrm{cm}$, base almost symmetrical, usually narrowly cuneate or attenuate, margin \pm entire. or finely serrate or (bi) crenateserrate especially in the upper half, not crisped, without teeth or spines, apex acute to (long-)acuminate; the two lowermost pinnae 1-8 mm stalked; terminal segment joined to the rhachis or subarticulate, either conform to the pinnae, or prolonged, or flagelloid; venation pattern: the corresponding tertiary veins of two adjacent secondary veins fusing in pairs, the costal arch with usually one, sometimes 2 or 3 excurrent included free veins, or with a small, medially situated, distal areole, the distal arches usually with one medially situated excurrent free vein ( \pm meniscioid), see also fig. 46 : b, c, f. Fertile leaves $30-120 \mathrm{~cm}$ long; petiole $18-90 \mathrm{~cm}$ long; lamina index $1-4,7-45$ by $4-20 \mathrm{~cm}$, without the terminal segment 3-30, the terminal segment 4-20 cm long; pinnae 3-17, up to $60 \mathrm{~mm}$ apart; central pinnae index $4-50,2.5-16$ by $0.2-1.4 \mathrm{~cm}$; the two lowermost pinnae $1-8(-13) \mathrm{mm}$ stalked. Sporangia inserted mainly on and near the veins, arranged acrostichoid.

Chromosomes. $2 \mathrm{n}=c .82$ (var. subcrenata).

Distribution. Southern India and Ceylon. See fig. 47.

$\mathrm{H}$ abitat. See the varieties.

Notes. 1. A clear-cut species easily recognized because of its venation pattern reminiscent of that of the meniscioid ferns. The species is closely related to $B$. virens.

2. Hooker, in his Species Filicum, merged both Acrostichum subcrenatum and $A$. proliferum into $A$. virens which in Hooker's construction is a receptacle of different elements. See $B$. virens, note 2 . Beddome had eventually (1883) a good concept of this species. Copeland (1928) included northern India in the 'sure geographical range' of Campium subcrenatum and described a pauci-pinnate form from Ceylon as Campium bradfordii. 
3. Backer \& Posthumus (1939) reported this fern from West Java, near Tjikoja and on Mt. Salak. Posthumus based himself on a sterile specimen which he found mixed with Zollinger $2162(=B$. scalpturata), but which he thought to be part of Zollinger $884(=B$. repanda $; 884 x=B$. heteroclita). The herbarium material at Bogor is labelled 'Zollinger $2162,4856 \mathrm{H}$. Bog.' and has the locality 'Modjopahit' (East Java, south of Surabaya). This specimen shows a venation pattern characteristic for the typical variety of the present species. Therefore, and also as the sheet bears a record number of the garden, I suppose that the material was cultivated at Bogor, and originated from Ceylon.

4. Beddome, who validly published Poecilopteris terminans, erroneously referred to Acrostichum terminans Wall. The latter was based on Wallich 2168 which represents $B$. $\times$ prolifera.

\section{KEY TO THE VARIETIES}

1a. Fertile pinnae index 3-10; terminal segment of sterile leaves usually conform to the central pinnae but (much) prolonged, sometimes conform or subflagelloid. Ceylon . . . . . . . . . . . . . . . . . . var. subcrenata

b. Fertile pinnae index $>10$; terminal segment of sterile leaves usually flagelloid, sometimes (mostly in small-sized leaves) conform to the central pinnae. Southern India

b. var. prolifera

a. var. subcrenata-Fig. 46: a-c.

B. subcrenata (Hooker \& Grev.) Ching.-Campium bradfordii Copel.

Sterile leaves: lamina $20-50 \mathrm{~cm}$ long, without the terminal segment 3-23, the terminal segment $15-33 \mathrm{~cm}$ long; venation pattern: if two veins arise distally on the costal arch, these may fuse into a small areole with a short excurrent vein distally. Fertile leaves: lamina $7-45 \mathrm{~cm}$ long, without the terminal segment 3-30, the terminal segment $4-13 \mathrm{~cm}$ long; central pinnae index $4-10,2.5-8$ by $0.6-1.4 \mathrm{~cm}$.

CEYlon. Ballard 1525 (K); Bradford s.n. (type of Campium bradfordii); Ferguson 210 (US), s.n. (GH); Leschenault s.n. (P); Skinner s.n. (K); Thwaites CP 1313 (B, BM, BO, GH, K, L, P, W); Wall 10562 (BO), '60/107' (GH).

$\mathrm{H}$ a bit at. No information on the labels available. According to Manton \& Sledge (op cit.) this taxon should occur 'in the wetter forests of the central and southern provinces among dense undergrowth.'

S por es. Ballard $1525(\mathrm{~K})$ : all normal. See also the note.

Note. Some of the collections show some irregularities in the venation vattern. $\mathrm{Hy}$ bridization, presumably with $B$. angustipinna, seems to occur. Examples: Thwaites $C P$ 1313 (B, sh. 1); spores: all shrivelled, ibid. (B, sh. 2) the greater part abnormal, in part normally shaped. 


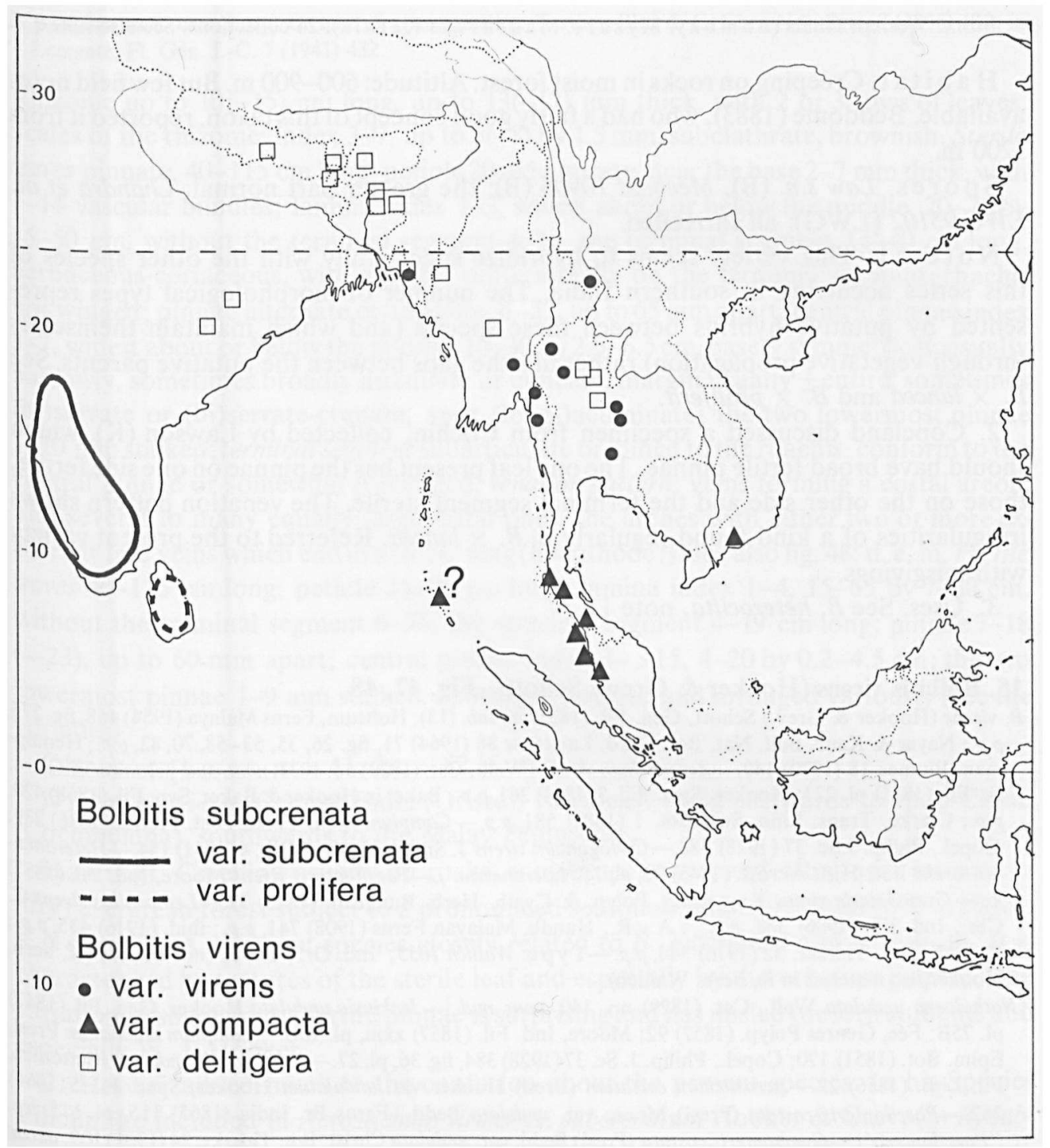

Fig. 47. Distribution of Bolbitis subcrenata and B. virens.

b. var. prolifera (Hooker) Hennipman, stat. nov. - Fig. 46: d-f.

Basionym: Acrostichum proliferum Hooker, Ic. Pl. 7 (1844) pl. 681, 682.-Poecilopteris terminans Bedd.

Sterile leaves: lamina $30-75 \mathrm{~cm}$ long, without the terminal segment $7-30$, the terminal segment $23-50 \mathrm{~cm}$ long, possibly (much) longer; venation pattern: if two or three veins arise distally on the costal arch, these run free and excurrent. Fertile leaves: lamina $12-35 \mathrm{~cm}$ long, without the terminal segment 5-20, the terminal segment $6-20$ $\mathrm{cm}$ long; pinnae index $10-50,4-16$ by $0.2-0.6 \mathrm{~cm}$. 
INDIA. Western Ghats (Bombay, My sore, Madras and Kerala): 24 collections. See also note 2.

$\mathrm{H}$ a b i t a t. Creeping on rocks in moist forest. Altitude: $600-900 \mathrm{~m}$. But few field notes available. Beddome (1883), who had a fairly good concept of this taxon, reported it from $1200 \mathrm{~m}$.

Spores. Law s.n. (B), Meebold 10906 (B): the greater part normal. Chandra et al. LWG 95102 (LWG): all shrivelled.

Notes. 1. This variety seems to hybridize successfully with the other species of this series occurring in southern India. The number of morphological types represented by putative hybrids between these species (and which maintain themselves through vegetative propagation) rather fills the gaps between the putative parents. See $B . \times$ lancea and $B . \times$ prolifera.

2. Copeland discussed a specimen from Cochin, collected by Lawson (K), which should have broad fertile pinnae. The one leaf present has the pinnae on one side fertile, those on the other side and the terminal segment sterile. The venation pattern shows irregularities of a kind found regularly in $B . \times$ lancea. Referred to the present variety with misgivings.

3. Uses. See B. heteroclita, note 11 .

15. Bolbitis virens (Hooker \& Grev.) Schott--Fig. 47, 48.

B. virens (Hooker \& Grev.) Schott, Gen. Fil. (1835) ad tab. (13); Holttum, Ferns Malaya (1954) 468, fig. 275, p.p.; Nayar \& Kaur, Bull. Nat. Bot. Gard. Lucknow 88 (1964) 71, fg. 26, 35, 53-58, 70, 82, p.p.; Hennipman, Blumea 18 (1970) 149.-Acrostichum virens [Wall., Cat. (1829) no. 1033, nom. nud.] Hooker \& Grev., Ic. Fil. (1831) pl. 221; Hooker, Spec. Fil. 5 (1864) 261, p.p.; Baker in Hooker \& Baker, Syn. Fil. (1868) 420, p.p.; Clarke, Trans. Linn. Soc. Bot. 1 (1880) 581, p.p. - Campium virens Presl, Tent. Pterid. (1836) 239; Copel., Philip. J.Sc. 37 (1928) 388.-Cyrtogonium virens J. Smith, Hook. J. Bot. 4(1841) 154.-Heteroneuron virens Fée, Hist. Acrost. (1845) 93, p.p. ('Heteronevron')._-Poecilopteris virens Moore, Ind. Fil. (1857) xx.-Gymnopteris virens Keyserling, Polyp. \& Cyath. Herb. Bungeani (1873) 33.-Leptochilus virens C. Chr., Ind. Fil. (1906) 388, p.p.; v.A.v.R., Handb. Malayan Ferns (1908) 741, p.p.; ibid. (1916) 435, p.p.; C. Chr., Bot. Tidsskr. 32 (1916) 344, p.p.-T y p e: Wallich 1033, 'Ind. Or.', Tovag (not traced) (K, herb. Hooker; no material in $\mathrm{K}$, herb. Wallich).

[Notholaena undulata Wall., Cat. (1829) no. 140, nom. nud.]-Jenkinsia undulata Hooker, Gen. Fil. (1842) pl. 75B; Fée, Genres Polyp. (1852) 92; Moore, Ind. Fil. (1857) xxiii, pl. 10B.-Campium undulatum Presl, Epim. Bot. (1851) 170; Copel., Philip. J. Sc. 37 (1928) 384, fig. 36, pl. 27.-Lomariopsis undulata Mettenius, Fil. Lips. (1856) 22.-Acrostichum costatum (Presl) Hooker var. undulatum Hooker, Spec. Fil. 5(1864) 262.-Poecilopteris costata (Presl) Moore var. undulata Bedd., Ferns Br. India (1865) 115, pl. 115, p.p. ('Poecilopteris').-Gymnopteris costata (Presl) Bedd. var. undulata Christ, Bot. Tidsskr. 24 (1901) 109, quoad nomen solum. - Leptochilus scalpturatus (Fée) C. Chr. var. undulatus C. Chr., Bot. Tidsskr. 32 (1916) 344, quoad nomen solum; Bonap., Notes Ptérid. 7 (1918) 103.-B. undulata Ching in C. Chr., Ind. Fil., Suppl. 3 (1934) 50.-T y p e: Wallich 140, Burma, Martaban (north of Moulmein) (K, the fertile leaf only; iso in BM). Poecilopteris costata (Presl) Moore var. deltigera Bedd., Ferns Br. India (1865) 114, pl. 114 ('Paecilopteris'). Meniscium deltigerum [Wall., Cat. (1829) no. 59, nom. nud.] Clarke, Trans. Linn. Soc. Bot. 1(1880) 572.Campium deltigerum Copel., Philip. J. Sc. 37 (1928) 387, fig. 38.-B. deltigera C. Chr., Ind. Fil., Suppl. 3(1934) 48; Tagawa \& Iwatsuki, Southeast Asian Studies 5(1967) 91.-B. virens (Hook. \& Grev.) Schott var. deltigera Hennipman, Blumea 18 (1970) 149.-T y pe: Wallich 59, Nepal, between Katmandu and Bhimpedy, above Mt. Chesapang ( $K$, herb. Wallich; iso in $K$ ).

B. virens (Hooker \& Grev.) Schott var. compacta Hennipman, Blumea 18(1970) 149.-T y pe: Tagawa et al. T. 6802, Peninsular Thailand, Nakhon Sri Thammarat, Trang, Khao Chong, $600-1100 \mathrm{~m}$ alt. (L; iso in KYO).

Gymnopteris contaminans auct. non Bedd.: Bedd., Ferns Br. India, Suppl. (1876) 27, p.p.; Handb. Ferns Br. India, Suppl. (1892) 105, p.p.

Leptochilus scalpturatus auct. non (Fée) C. Chr.: C. Chr., Ind. Fil. (1906) 387, p.p. 
B. prolifera auct. non (Bory) C. Chr. \& Tardieu-Blot: Tardieu-Blot \& C. Chr., Not. Syst. 7 (1938) 102; in Lecomte, Fl. Gén. I.-C. 7 (1941) 432.

Rhizome up to $10(-15) \mathrm{cm}$ long, up to $13(-17) \mathrm{mm}$ thick, with 2 or 3 rows of leaves. Scales of the rhizome: index 3-7, up to $5(-8)$ by $1.5 \mathrm{~mm}$, subclathrate, brownish. Sterile leaves pinnate, $40-115 \mathrm{~cm}$ long; petiole $20-65 \mathrm{~cm}$ long, near the base $2-7 \mathrm{~mm}$ thick, with $7-14$ vascular bundles; lamina index $1-3$, widest about or below the middle, $20-75$ by $15-50 \mathrm{~cm}$, without the terminal segment 4-50, the terminal segment $14-40 \mathrm{~cm}$ long, herbaceous-coriaceous, with a bulbil subterminally on the terminal segment; rhachis not winged; pinnae alternate or opposite, $6-22$, up to $65 \mathrm{~mm}$ apart; central pinnae index $3-7$, widest about or below the middle, $10-30$ by $2.5-6.5 \mathrm{~cm}$, base \pm symmetrical, usually narrowly, sometimes broadly attenuate or cuneate, margin usually \pm entire, sometimes (bi)serrate or (bi)serrate-crenate, apex (long-)acuminate; the two lowermost pinnae 1-10 mm stalked; terminal segment subarticulate or joined to the rhachis, conform to the central pinnae or somewhat prolonged; venation pattern: veins forming a costal areole and several to many equally large distal ones, the arches with either two or more excurrent free veins which end in a thickening(hydathode?); see also fig. 48: d, e, m. Fertile leaves $40-125 \mathrm{~cm}$ long; petiole $25-70 \mathrm{~cm}$ long; lamina index $1-4,15-65$ by $7-30 \mathrm{~cm}$, without the terminal segment 6-55, the terminal segment 4-19 cm long; pinnae 7-18 $(-23)$, up to $60 \mathrm{~mm}$ apart; central pinnae index $3->15,4-20$ by $0.2-4.5 \mathrm{~cm}$; the two lowermost pinnae 1-9 mm stalked. Sporangia inserted and arranged variously (see the varieties).

Chromosomes. $\mathrm{n}=41 ; 2 \mathrm{n}=82$ (f. virens).

Distribution. Central India (Orissa), Northeast India eastwards to Indo-China (Cochinchina), southwards to the Malay Peninsula.

$\mathrm{Hab}$ it a t. Creeping usually on rocks, sometimes in soil and often near streams in (dry) evergreen forest subject to a pronounced seasonal climate. Altitude: $200-1100 \mathrm{~m}$.

Notes. 1. A clear-cut species closely related to $B$. subcrenata and B. costata. It is characterized by features of the sterile leaf and especially by the venation pattern. The variation in shape of the fertile pinnae is surprising and has misled the fern students and especially W. J. Hooker.

2. Hooker added much to the confusion about the present species. In his Species Filicum he included in Acrostichum virens: $A$. subcrenatum Hooker \& Grev. $(=B$. subcrenata), $A$. proliferum Hooker $(=B$. subcrenata), A. terminans Wall. nom. nud. $(=B$. prolifera), $A$. contaminans Wall. ( $=B$. angustipinna), and $A$. crispatulum Wall., nom. nud. ( = B. crispatula). He cited material from tropical Africa belonging to $B$. acrostichoides (the basionym Hemionitis acrostichoides and its homotypic synonyms are erroneously not included in the Species Filicum), and material collected by G. Gardner, said to originate from Ceylon but actually coming from Brazil, representing Cyclodium meniscioides (syn.: Campium molle Copel.; see excluded species). Hooker's choice of the name Acrostichum virens (date of publication: 1831) over $A$. subcrenatum (date of publication: 1829) is against the present Code. In the Species Filicum Meniscium deltigerum Wall., nom. nud. ( $=B$. virens var. deltigera) and Jenkinsia undulata Hooker $(=B$. virens f. undulata) were given varietal status under $A$ crostichum costatum $(=B$. costata). 

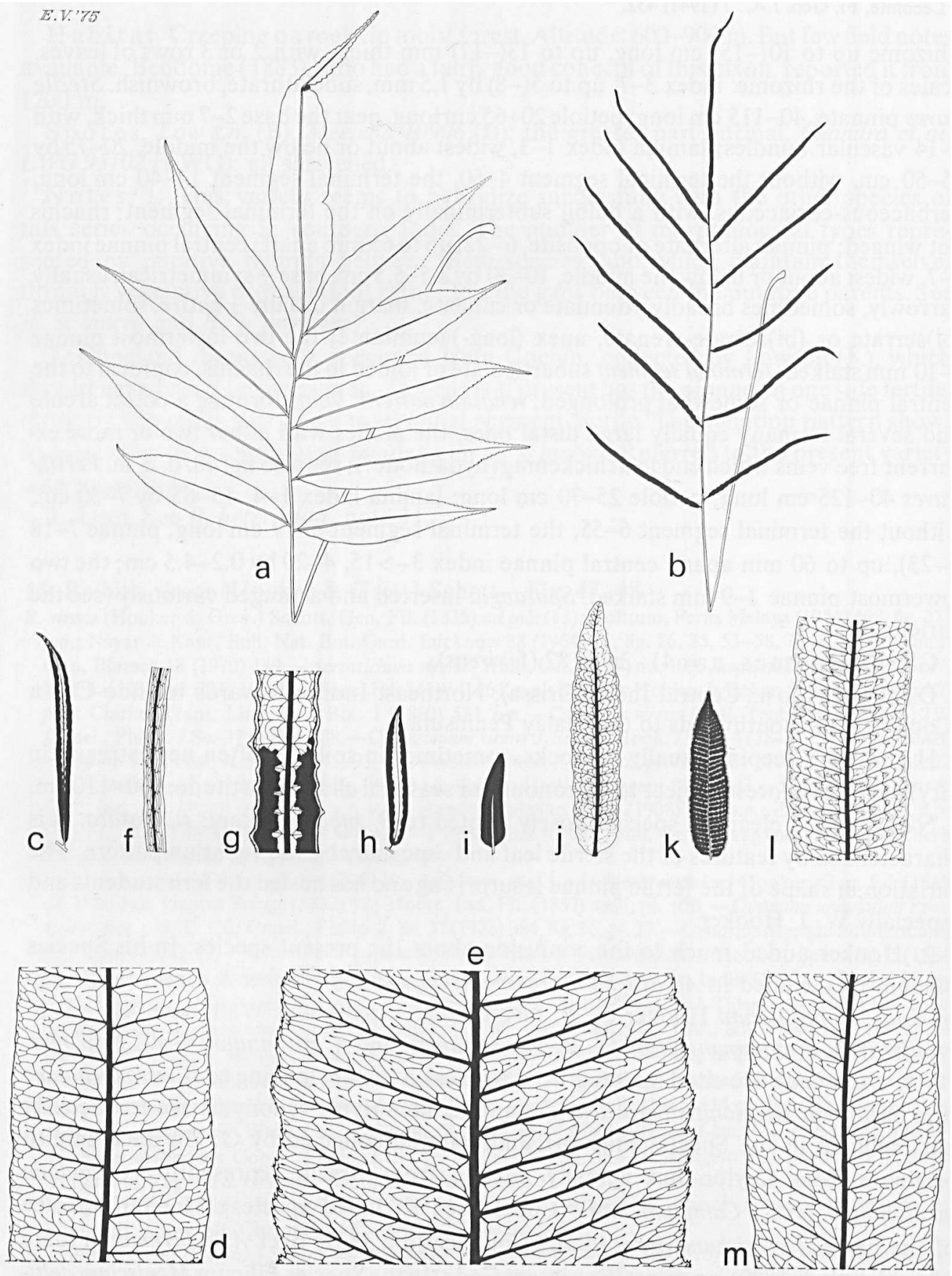


\section{KEY TO INFRASPECIFIC TAXA}

la. Fertile pinnae either \pm gymnogrammoid or with irregular patches of sporangia on the veins between the secondary veins and near the margin.

c. var. deltigera

b. Fertile pinnae either fully acrostichoid or with sporangia along the margin

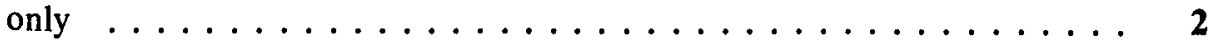

2a. Sporangia along the margin only ............. var. virens $\mathrm{f}$. undulata

b. Sporangia all over the lower surface $\ldots \ldots \ldots \ldots \ldots$

3a. Central pinnae index $>15 \ldots \ldots \ldots \ldots$... var. virens $f$. virens

b. Central pinnae index $3-8 \ldots \ldots \ldots$. . . . . . . . . compacta

a. var. virens-Fig. $47 ; 48: a-h$.

B. virens (Hooker \& Grev.) Schott.-Jenkinsia undulata Hooker.

Fertile leaves: central pinnae index $4->15,5.5-20$ by $0.2-1.5 \mathrm{~cm}$. Sporan̈gia inserted either all over the lower surface, or on the veins near the margin only; arranged either acrostichoid or \pm pteridoid.

Distribution. See the formae.

$\mathrm{H}$ abit at. See the species.

\section{a.1. f. virens-Fig. 48: a-g. \\ B. virens (Hooker \& Grev.) Schott.}

Fertile leaves: central pinnae index $>15,8-20$ by $0.2-0.9 \mathrm{~cm}$. Sporangia arranged acrostichoid.

China. Y u n n a n: Wang 78807 (A).

BANGladesh (Chittagong). Clarke 19840 (K).

Burma. Sou th e rn. Beddome s.n. (K); Dickason 6711 (BM, GH, L, MICH, US).

Thailand. N or $t \mathrm{~h}$ e $\mathrm{r}$ n: 20 collections, mainly from Doi Chieng Dao and Doi Suthep.-N or $\mathrm{t}$ h e a s $\mathrm{t}-$ e $\mathrm{r}$ n: 6 collections, from Phu Luang and Phu Kradeng.-S o u t h w e s t e r n. Khao Yai: Van Beusekom $164(\mathrm{~L})$.

H a bit at. See the species.

S pores. Hennipman 3136 (L), 3159 (L): in part (the majority?) normal.

Fig. 48. Bolbitis virens. -a-h. var. virens.-a-g. f. virens. a, b. sterile and fertile leaf resp., $\times 1 / 5$; c. aberrant fertile pinna, $\times 1 / 5$; d, e. venation pattern of sterile pinnae, $\times 4 / 5 ; f, g$. venation pattern of fertile pinnae, $\times 4 / 5$. - h. f. undulata. fertile pinna, $\times 1 / 5 .-i$, j. var. compacta. i. fertile pinna, $\times 1 / 5$; j. venation pattern of fertile pinna, $\times 4 / 5 .-\mathrm{k}-\mathrm{m}$. var. deltigera. $\mathbf{k}$. fertile pinna, $\times 1 / 5 ; 1$. venation pattern of fertile pinna, $\times 4 / 5$; m. venation pattern of sterile pinna, $\times 4 / 5$ ( $a$, f. Hennipman $3159, \mathrm{~L}$;

b. Van Beusekom 164, L; c, g. Hennipman 3136, L; d. Hansen \& Smitinand 11836, C; e. Tagawa et al. T 932, L; h. Dickason 7014, US; i. Tagawa et al. T 6802, L; j. Henderson SF 29084, BO; k. Mann s.n., xii1888, US; 1, m. Dickason 7766, US). 
$\mathrm{N}$ o t e. One sheet of Hennipman $3136(\mathrm{~L})$ has a fertile leaf with pinnae of intermediate shape: the basal and apical part acrostichoid, the central part \pm pteridoid.

a.2. f. undulata (Hooker) Hennipman, stat. nov.-Fig. 48: h.

Jenkinsia undulata Hooker.-For references see the species.

Fertile leaves: central pinnae index $4-8,5.5-11$ by $1-1.5 \mathrm{~cm}$; sporangia arranged \pm pteridoid.

Burma. Te nas s e ri m. Moulmein: 5 collections. Thaton Dist.: Dickason 7014 (BM, GH, US).

THAILAND. N or th er n: Winit 1034 (SING).

$\mathrm{H}$ abitat. See the species.

S p or es. Lobbs.n.(K): the greater part normal. Winit 1034 (SING): abnormal, mostly shrivelled.

N o t e. Wallich $141(\mathrm{~K})$ is presumably mislabelled; it has the identification 'Taenitis blechnoides' and the locality Penang in Wallich's handwriting.

b. var.compacta Hennipman-Fig. 47; 48: i, j.

B. virens (Hooker \& Grev.) Schott var. compacta Hennipman.

Fertile leaves: central pinnae index $3-8,4-11.5$ by $0.8-2 \mathrm{~cm}$; sporangia inserted all over the lower surface, arranged acrostichoid.

VIEtnam, Cochin chin a: Chevalier 2544 (Poilane leg.) (P); Matthew 34 (K).

THAiland. Pen in sular: Haniff \& Nur SF 3830 (SING); Hansen \& Smitinand $11836(\mathrm{C}) ;$ Murton $10(\mathrm{~K})$; Seidenfaden 2123 (BM, C); Tagawa \& Yamada T 214 (BKF); Tagawa et al. T6802 (type of B. virens var. compacta).

Malay Peninsula. K ed a h: Ridley 15707 (BM, K, SING).-S el a ng or. Holttum s.n. (BO, K); Matthew s.n. (K); Ridley 1682 (SING). - L a n g k a wi I.: Henderson SF 29084 (BO, K); Robinson 6358 (K). - Penang: Curtis 628 (MICH, fragm., SING, US).

INDIA. Ni c o b a I I s. (probably): Helfer s.n. (B).

Habitat. See the species.

S p o r es. Curtis 628 (SING), Tagawa et al. T 6802 (holotype of B. virens var. compacta): the greater part normal. Chevalier 2544 (P): the greater part abnormal, mostly normally shaped. Henderson SF 29084 (BO): abnormal, mostly shrivelled.

c. var. deltigera (Bedd.) Hennipman-Fig. 47; 48: k-m.

Poecilopteris costata (Presl) Moore var. deltigera Bedd.

Fertile leaves: central pinnae index $3-5,5-17$ by $1.5-4.5 \mathrm{~cm}$; sporangia inserted on the veins and near the margin only, arranged non-acrostichoid.

Bangladesh. Chittagong: Clarke 19841 (K).

NEPAL. Wallich 59 (type of Meniscium deltigerum). 
BHUTAN. Griffith s.n. (B, BM, K, S-PA).

SIKKIM. 7 collections.

INDIA. D a rje eling: Clarke 9090 (K), 36582 (G, K, P);Gamble s.n. (P); Warburg 988 (B).-A s s a m: 5 collections. - O ris s a: Mooney 675 (K); Sanyal 2639 (K).

Burma. Chin Hills: Dickason 7766 (BM, GH, MICH, US).-S o u the er n B u r m a: Dickason 7029 (BM, $\mathrm{GH}, \mathrm{MICH})$.

Thailand. Northern: Tagawa \& Fukuoka T 2110 (L, US); Winit 59 (SING), 1034 (BKF).

H a bit a t. See the species.

Spores. Mann s.n. (L, 908.206-47), Winit 59 (SING): the greater part normal. Tagawa \& Fukuoka T2110 (L): in part (the majority?) normal.

\section{Series Egenolfianae Hennipman, ser. nov.}

Egenolfia Schott, Gen. Fil. (1835 or 1836) pl. s.n. (16), typo incl., pro genere.

Terrestrial or low-epiphytic. Rhizome usually short-creeping and stout, in one species (B. major) long-creeping and slender, with 2 or 3 (4) rows of leaves, sclerenchyma strands usually present. Scales of the rhizome up to $7.5 \mathrm{~mm}$ long. Leaves usually close together, in one species (B. major) spaced. Sterile leaves pinnate (to bipinnate); petiole with usually $5-7$, in one species (B. major) with $12-18$ vascular bundles; lamina with one \pm spherical, usually subterminal, in one species (B. rhizophylla) \pm terminal bulbil; pinnae 14-90, the margin with spines corresponding to the most distal acroscopic tertiary vein of each secondary vein; terminal segment triangular; venation: secondary veins (slightly) prominent on either side, tertiary veins slightly prominent or immersed; venation pattern: veins free. Fertile leaves pinnate (to tripinnate). Spores with either a smooth, cristate or cristate-undulate perispore, or with a reticulate \pm cristate perispore.

Ch ro mo s o me s: $\mathrm{n}=41,2 \mathrm{n}=82$ (B. appendiculata, B. rhizophylla, B. sinensis).

Distribution. Southern India and Ceylon to western Malesia, northwards to southern Japan. See fig. 30.

Note. In B. rhizophylla the spines on the margin are often flattened at the base and tooth-like.

16. Bolbitis appendiculata (Willd.) Iwatsuki-Fig. 49-51.

B. appendiculata (Willd.) Iwatsuki, Acta Phytotax. Geobot. 18 (1959) 48; Tagawa \& Iwatsuki, Southeast Asian Studies 5 (1967) 90; Hennipman., Blumea 18 (1970) 147.-Acrostichum appendiculatum Willd., Spec. Pl. 5 (1810) 114; Hooker, Exot. Fl. 2 (1825) 108, pl. 108; Spec. Fil. 5 (1864) 251; p.p.; Thwaites, Enum. Pl. Zeyl. (1864) 380; Baker in Hooker \& Baker, Syn. Fil. (1868) 415; Clarke, Trans. Linn. Soc. Bot. 1 (1880) 577 , p.p.; Henry, Trans. As. Soc. Jap. 24, Suppl. (1896) 116.-Gymnogramma auriculata Kaulf., Enum. Fil. (1824) 79 , non Bl. (1828) (= Thelypteris spec.).-Polybotrya appendiculata J. Smith, Hook. J. Bot. 4(1841) 150; Bedd., Ferns S. India (1864) 66, pl. 194; Ferns Br. India (1865) 111, pl. 111, p.p.; Handb. Ferns Br. India (1883) 424, excl. var. p.p.; Kuhn, Ann. Mus. Bot. Lugd.-Bat. 4(1869) 294; Christ, Farnkr. Erde (1897) 43, p.p.; v.A.v.R., Handb. Mal. Ferns (1908) 724, p.p.; C. Chr., Bot. Tidsskr. 32 (1916) 343._Lacaussadea appendiculata Gaudich., Voy. Bonite Bot. (1852) pl. 119.-Egenolfia gaudichaudiana Fée, Genres Polyp (1852) 358, nom. illeg. - Egenolfia appendiculata J. Smith, Ferns Br. For. (1866) 111, fig.; Ching, Bull. Fan Mem. Inst. Biol. 2 (1931) 308; Nakai, Bot. Mag. Tokyo 47 (1933) 169, p.p.; Backer \& Posthumus, Nat. Tijdschr. Ned. Ind. 93 (1933) 153; Tardieu-Blot \& C. Chr., Not. Sys. 7 (1938) 98; Backer \& Posthumus, 


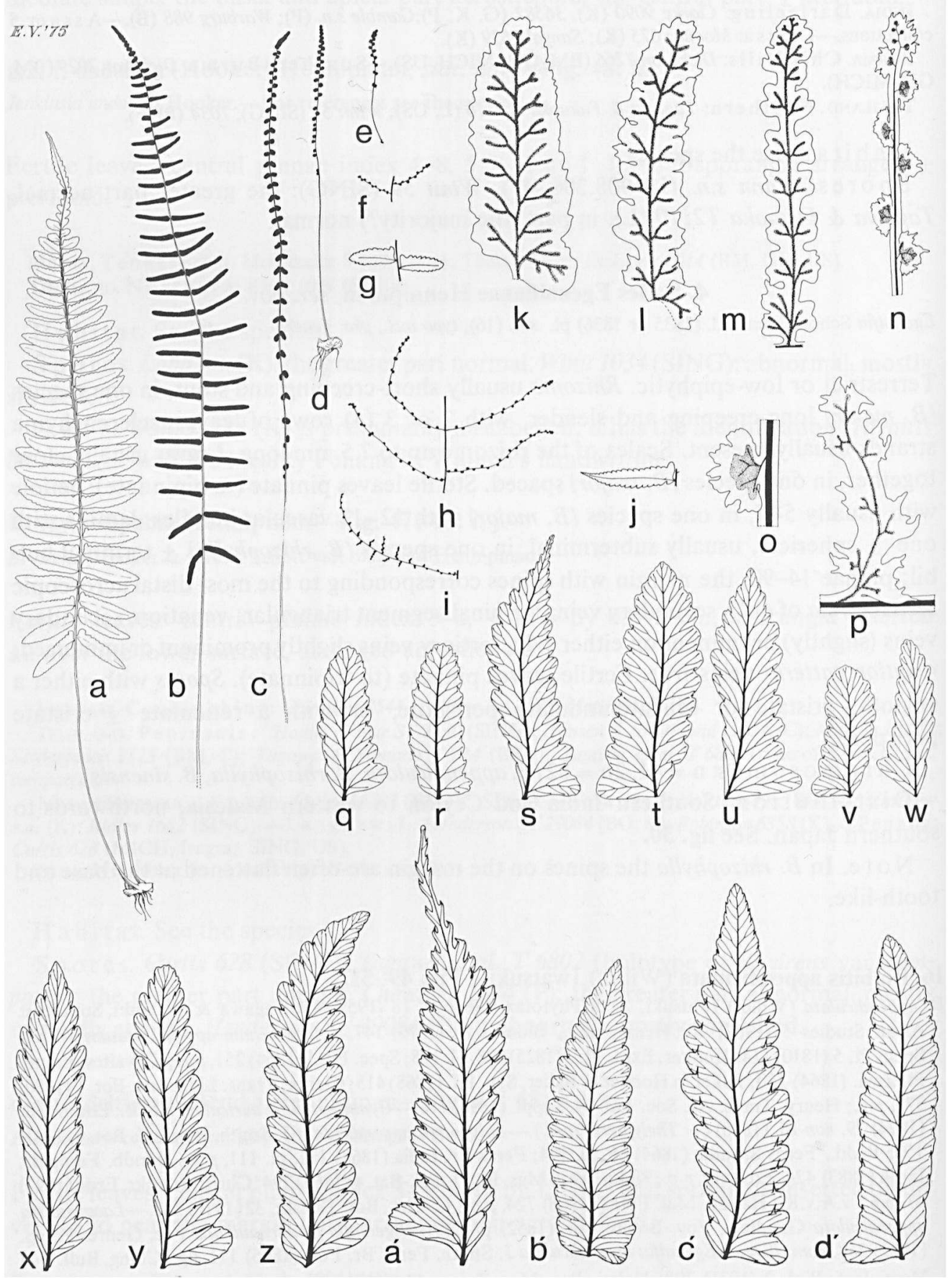


Varenfl. Java (1939) 84, fig. 14; Tardieu-Blot \& C. Chr. in Lecomte, Fl. Gén. I.-C. 7 (1941) 427; Holttum, Ferns Malaya (1954) 459, fig. 270; Copel., Fern FI. Philip. (1960) 266; Nayar \& Kaur, Bull. Nat. Bot. Gard. Lucknow 100 (1964) 17, fig. 9, 25; DeVol \& Kuo in Fl. Taiwan 1 (1975) 350, pl. 123.-T y p e: Klein 912, 'Ind. Or.', iii-1803 (B, herb. Willdenow 19551).

Polybotrya vivipara Hamilton ex Hooker, Exot. Fl. 2 (1825) 107, pl. 107; Sprengel, Syst. Veg. 4 (1827) 33.-Egenolfia vivipara C. Chr., Ind. Fil., Suppl. 3 (1934) 102; Tardieu-Blot \& C. Chr. in Lecomte, Fl. Gén. I.-C. 7 (1941) 423; Nayar \& Kaur, Bull. Nat. Bot. Gard. Lucknow 100(1964) 35, fig. 1, 2, 6, 8, 11, 12 , 17, 32.-Egenolfia appendiculata (Willd.) J. Smith var. vivipara Deb, Taxon 14 (1965) 110, p.p. - B. hookeriana Iwatsuki, Acta Phytotax. Geobot, 18 (1959) 49.-B. appendiculata (Willd.) Iwatsuki ssp. vivipara Hennipman, Blumea 18 (1970) 147.-T y p e: Wallich 29 p.p. (Hamilton leg), India, Assam, Goalpara, 1808 ( $\mathrm{K}$, herb. Hooker; iso in $\mathrm{K}$, herb. Wallich).

Acrostichum viviparum Hamilton ex Sprengel, Syst. Veg. 4 (1827) 36, nom. illeg., non L.f. (1781) (= Asplenium spec.), nec Cav. (1802) (= Onychium?).-T y pe: Wallich 28 p.p. (Hamilton leg.), India, Assam, Kamrup, Nabovi, 1801 (K, herb. Hooker, n.v.; iso in K, herb. Wallich?, see note 2).

Polybotrya marginata BI., Enum. Pl. Jav. (1828) 100, nom. superfl., see note 3; Fl. Java Filices (1829) 18, pl. 3; Presl, Tent. Pterid. (1836) 231, quoad nomen solum; Fée, Hist. Acrost. (1845) 75; Hance, J. Linn. Soc. Bot. 13 (1873) 138; Hayata, Ic. PI. Formos. 5 (1915) 306.-Polybotrya appendiculata (Willd.) J. Smith var. marginata C. Chr., Bot. Tidsskr. 32 (1916) 343.-T y p e: Blume s.n., West Java, G. Burangrang (L, 908. 316-449).

Acrostichum asplenifolium Bory in Bélanger, Voy. Ind. Or. Bot. 2 (1833) 21, pl. 3.-Polybotrya asplenifolia Presl, Tent. Pterid. (1836) 231; Fée, Hist. Acrost. (1845) 77; Ettingsh., Denkschr. K. Ak. Wiss. M.-N. Kl. Wien 22 (1864) 65, fig 6, pl. 1: fig. 4, 12, 13; Bedd., Ferns S. India (1964) 66, pl. 195.-Polybotrya appendiculata (Willd.) J. Smith var. asplenifolia Bedd., Handb. Ferns Br. India (1883) 424, fig. 255. - Egenolfia asplenifolia Fee, Genres Polyp. (1852) 358; Ching, Bull. Fan Mem. Inst. Biol. 2 (1931) 309; Tardieu-Blot \& C. Chr., Not. Syst. 7 (1938) 98; in Lecomte, Fl. Gén. I.-C. 7 (1941) 427; Nayar \& Kaur, Bull. Nat. Bot. Gard. Lucknow 100 (1964) 19, fig. 3-5, 14, 18, 29.-B asplenifolia Iwatsuki, Acta Phytotax. Geobot. 18 (1959) 49.-T y p e: Bélanger s.n., Southern India, Madura, Dendigal, 1831 (P, herb. Bory 26.149 or 26.549$)$.

Polybotrya nodiflora Bory in Bélanger, Voy. Ind. Or. Bot. 2 (1833) 17; Fée, Hist. Acrost. (1845) 77, pl. 38: fig. 2; Ching, Bull. Fan Mem. Inst. Biol. 2 (1931) 304.-Egenolfia nodiflora Fé, Genres Polyp. (1852) 48. - T y p e: Bélanger s.n., Burma, Rangoon, 1831 (P, herb. Bory 26.27 or 26.47; iso in L, '451').

Egenolfia hamiltoniana Schott, Gen. Fil. (1835 or 1836) pl. (16), non Fée (1852) (= B. appendiculata var. vivipara).-Egenolfia schottii Fée, Genres Polyp. (1852) 48, nom. illeg.-T y p i fi c a t i o n: see note 4.

Acrostichum alatum Roxb. ex Griff., Calc. J. Nat. Hist. 4(1844) 480, non Fée(1845) (= Elaphoglossum spec.). -T y pe: Roxburgh s.n., 'Malay Islands' (BR; BM, phot.).

Acrostichum seetacoonense Roxb. ex Griff., Calc. J. Nat. Hist. 4 (1844) 480.-Ty pe: Roxburgh s.n., Bangladesh, Chittagong, Seetacoond (BR; BM, phot.).

[Acrostichum hamiltonianum Wall., Cat (1829) no. 28, nom. nud.]-Polybotrya hamiltoniana [Presl, Tent. Pterid. (1836) 236, nom. nud.] Fé, Hist. Acrost. (1845) 78, nom. superf.-Egenolfia hamiltoniana Fée, Genres Polyp. (1852) 48, non Schott (1835 or 1836) (= B. appendiculata ssp. appendiculata).Acrostichum appendiculatum Willd. var. hamiltonianum Baker in Hooker \& Baker, Syn. Fil. (1868) 415;

Fig. 49. Bolbitis appendiculata ssp. appendiculata. $-\mathrm{a}-\mathrm{e}$. sterile and fertile leaves, $\times 1 / 5 ; \mathrm{f}-\mathrm{j}$. (parts of leaves with) sterile and fertile pinnae, $\times 1 / 5 ; \mathbf{k}-\mathbf{n}$. venation patterns and receptacles (dotted) of fertile pinnae, $\times 3 ; 0$, p. ibid. of fertile pinnules, $\times 6$; q-d'. shape and venation pattern of sterile pinnae, $x$ 4/5 (a, k, l, s-w. Hennipman 3527, L; b-d. H. 3721, L; e. Hansen \& Smitinand 12205, C; f, g. Helfer s.n., holotype of Polybotrya helferiana, B, leaf $1 ;$ h, i. ibid., leaf $2 ; \mathrm{j}$, p. Rock 759 , US, isotype of Egenolfia helferiana var. incisa; m. Winit 53b, SING; n, o. Kew Distr. no. 1154, B; q. Edaño BS 76512, UC; r. Loher s.n., UC, 229550; x Thwaites CP 1314, SING; y. Bory s.n., P, holotype of Acrostichum asplenifolium; z. Anonymus s.n., Ceylon, GH; $\mathrm{a}^{\prime}$. Holttum SF 19307, SING; b'. Norris s.n., B; c'. Holttum s.n., SING 20982; d'. Molesworth Allen 1538, SING). 
Clarke, Trans. Linn. Soc. Bot. 1 (1880) 578.-Polybotrya appendiculata (Willd.) J. Smith var. hamiltoniana Bedd., Handb. Ferns Br. India (1883) 426, fig. 256, p.p.; v.A.v.R., Handb. Mal. Ferns (1908) 724, quoad nomen solum; ibid. (1916) 431, ibid.-T y p e: Wallich 29 p.p. (De Sylva leg.), Bangladesh, Sylhet (iso in B, BM, G, ster., K, US).

Polybotrya helferiana Kunze, Farrnkr. Erde 2 (1849) 35, pl. 94 (excellent); Mettenius, Fil. Hort. Lips. (1856) 24, pl. 2: fig. 7.-Egenolfia appendiculata (Willd.) J. Smith var. helferiana Christ, Bot. Tidsskr. 24 (1901) 109, quoad nomen solum; Bull. Herb. Boiss. II, 4 (1904) 610, ibid.-Polybotrya appendiculata (Willd.) J. Smith var. helferiana Bonap., Notes Pterid. 7 (1918) 102.-Egenolfia helferiana C. Chr., Contr. U.S. Nat. Herb. 26 (1931) 292; Ching, Bull. Fan Mem. Inst. Biol. 2 (1931) 303, p.p.; Nayar \& Kaur, Bull. Nat. Bot. Gard. Lucknow 100 (1964) 28, p.p. - B. helferiana Iwatsuki, Acta Phytotax. Geobot. 18 (1959) 49. -T y pe: Helfer s.n., Burma, Tenasserim (B; iso in B, BR, K, M, MICH, S-PA, UC). See also note 3.

Lacaussadea montana Gaudich., Voy. Bonite Bot. (1852) pl. 118.-Egenolfia montana Fée, Genres Polyp. (1852) 358. - Egenolfia asplenifolia (Bory) Fée var. montana Tardieu-Blot \& C. Chr., Not. Syst. 7 (1938) 99.-Egenolfia appendiculata (Willd.) J. Smith var. montana Tardieu-Blot \& C. Chr. in Lecomte, Fl. Gén. I.-C. 7 (1941) 427.-T y p e: Gaudichaud 24, Vietnam, Annam, Tourane (P).

Polybotrya appendiculata (Willd.) J. Smith 'var.' Bedd., Ferns Br. India (1865) 111, pl. 111: the 3 left-hand top figs.-Polybotrya appendiculata (Willd.) J. Smith var. subintegra Bedd., Handb. Ferns Br. India (1883) 427.-Egenolfia appendiculata (Willd.) J. Smith var. subintegra C. Chr., Contr. U.S. Nat. Herb. 26 (1931) 291.-T y p e: Parish s.n., Burma, Moulmein (K, ster.).

Egenolfia helferiana (Kunze) C. Chr. var. incisa Ching, Bull. Fan Mem. Inst. Biol. 2 (1931) 304.-T y p e: Rock 759, Lower Burma, Oktada beyond Paung, Martaban Hills, Kalama Range (C; iso in US).

Egenolfia asplenifolia (Bory) Fée var. moniliformis Tardieu-Blot \& C. Chr., Not. Syst. 7 (1938) 99.Egenolfia appendiculata (Willd.) J. Smith var. moniliformis Tardieu-Blot \& C. Chr. in Lecomte, Fl. Gén. I.-C. 7 (1941) 427.-Sy nt y pes: Harmand 96, Vietnam, Cochinchina, P. Condore (P); Poilane 18179, Vietnam, Annam, near Kontum, $1000 \mathrm{~m}$ alt. (P); Poilane 21668, ibid., near Bien Hoa (P); Thorel s.n., Laos, Bassac, 1866-68 (BM, P).

Egenolfia keralensis Nayar \& Kaur, Bull. Nat. Bot. Gard. Lucknow 94 (1964) 4, fig. 1, 2B; ibid. 100 (1964) 30, fig. 15, 28.-T y p e: Chandra LWG 77752, Southeast India, Kerala (LWG, n.v.).

B. appendiculata (Willd.) Iwatsuki var. neglecta Hennipman, Blumea 18 (1970) 147.-T y pe: Van Borssum Waalkes 603, Java, P. Panaitan, G. Panjaweran near Legon Kadam, $70 \mathrm{~m}$ alt. (L; iso in BO).

Egenolfia appendiculata $\times$ helferiana.-Holttum, Dansk Bot. Ark. 23 (1965) 237.

See for misidentifications under the infraspecific taxa.

Rhizome with 2 or 3 rows of leaves. Scales of the rhizome: index $2-10$, up to $7(-15)$ by $1(-2) \mathrm{mm}$, opaque or subclathrate, usually blackish. Sterile leaves pinnate, 15-100 $\mathrm{cm}$ long; petiole 3-25 $\mathrm{cm}$ long, near the base 1-4 mm thick; lamina index 2-6(-8), usually widest somewhat below the middle, $10-80$ by $2.5-20 \mathrm{~cm}$, without the terminal segment $10-77$, the terminal segment $1-10 \mathrm{~cm}$ long, herbaceous, usually olivaceous; rhachis with or without a narrow wing; pinnae alternate to opposite, (20-)25-60(-85), up to $30 \mathrm{~mm}$ apart; central pinnae index $2-5(-7)$, widest about or below the middle, $1.5-10(-15)$ by $0.5-2.5 \mathrm{~cm}$, base symmetrical to strongly oblique, margin \pm entire, finely serrate, or lobed to $1 / 3(-1 / 2)$ towards the costa, apex acute or acuminate; the two lowermost pinnae (strongly) deflexed, $0-2(-5) \mathrm{mm}$ stalked, conform to the central pinnae; terminal segment triangular, apex sometimes somewhat prolonged; venation pattern: secondary veins on either side with 2 or 3 tertiary veins, see also fig. 49: q-d'; 50: $\mathrm{c}-\mathrm{g}, \mathrm{k}-\mathrm{m}$. Fertile leaves pinnate to tripinnate, $15-90 \mathrm{~cm}$ long; petiole $10-45 \mathrm{~cm}$ long; lamina index $1-20(-40), 3-55$ by $0.7-12 \mathrm{~cm}$, without the terminal segment $3-52$, the terminal segment $0.5-5 \mathrm{~cm}$ long; pinnae $15-65(-90)$, up to $60 \mathrm{~mm}$ apart, variously shaped, $0.2-11$ by $0.15-1.7 \mathrm{~cm}$; the two lowermost pinnae $0-6 \mathrm{~mm}$ stalked. Sporangia inserted all over the lower surface or \pm restricted to the veins. Spores with a reticulate cristate perispore. 
Chromosomes. $\mathrm{n}=41$ (ssp. appendiculata, ssp. vivipara var. vivipara); $2 \mathrm{n}=82$ (ssp. appendiculata).

Distribution. Southern India and Ceylon; northeast India eastwards to southern Japan and Luzon, southwards to Java.

$\mathrm{H}$ a bit a t. Creeping on rocks or in soil in deciduous and evergreen forest. Altitude: $0-1500 \mathrm{~m}$.

$S$ pores. See the infraspecific categories.

Notes. 1. Typification of Polybotrya vivipara. Wallich 29 p.p. (Hamilton leg.) with Hamilton's MS name in Hooker's handwriting, which agrees with Hooker's plate (Exot. Fl. 2, 1825, pl. 107) is the holotype. This sheet lacks an authentic label of Hamilton, which was obviously retained by Wallich together with a duplicate specimen $(\mathrm{K}$, herb. Wallich) not studied by Hooker. Wallich 29 p.p. (De Sylva leg.) is the type of Polybotrya hamiltoniana.

2. Typification of Acrostichum viviparum Hamilton ex Sprengel. Sprengel listed A. viviparum Hamilton, which he referred to 'A. appendiculatum Hook. [Exot. Fl. 2, 1825, pl. 108] excl. syn. W [illdenow, 1810]'. Hooker (1825) referred A. appendiculatum to 'A. appendiculatum? Willd. Sp. Pl. v. 5, p. 114.' and to 'A. viviparum Hamilton's MSS'. As Sprengel supplied a description, $A$. viviparum Hamilton ex Sprengel is a new species, typified by Wallich 28 p.p. (Hamilton leg.) from which Hooker's plate was drawn; the name is a later homonym and therefore illegitimate. I failed to trace (overlooked?) the type specimen of this at $\mathrm{K}$, herb. Hooker. Wallich 28 p.p. (Hamilton leg.), sheet $\mathrm{C}$ in $\mathrm{K}$, herb. Wallich, and with the locality 'Nibari' (sphalm.? = Nabovi) possibly represents an isotype. Wallich 28 p.p. (De Sylva leg.) is B. appendiculata ssp. appendiculata proper, whereas Wallich 28 p.p. (Anonymus leg.) agrees with the type of Polybotrya helferiana (see spp. appendiculata, note 2B).

3. Polybotrya marginata $\mathrm{Bl}$. is a superfluous name as in the 'Addenda et Emendanda' of the original publication Blume included in it references to Acrostichum viviparum Sprengel (nom. illeg.) and A. appendiculatum Willd. ('Hooker') which is legitimate.

4. The typification of Egenolfia hamiltoniana Schott. The relevant entry in Schott's Original publication of the genus Egenolfia (1835 or 1836) runs as follows:

S p e ci es E. hamiltoniana (Acrost. viviparum Hamilton, Polybotrya marginata Blume).'

The three elements involved in the construction of $E$. hamiltoniana are: (a) the material, the plate, and the description of Schott, (b) Acrostichum viviparum Hamilton ex Sprengel, nom. illeg., and (c) Polybotrya marginata Bl., nom. illeg.

E. hamiltoniana should be regarded a new species; it is not a nom.nov. as it is based on two heterotypic synonyms.

The material from which Schott's plate was drawn was not included in the loan I received from Vienna. If lectotypification becomes necessary I recommend to select Acrostichum viviparum Sprengel as lectotype, as Hamilton's material is well-described and illustrated by Hooker (1825).

5. Fée (1825) retained the name Egenolfia hamiltoniana (Fée) Fée (bas.: Polybotrya hamiltoniana [(Wallich) Presl ex] Fée, 1845) and renamed E. hamiltoniana Schott into $E$. schottii. This procedure is against the present Code. 
6. Ching (1931) recognized Egenolfia nodifiora (Bory) Fée (bas.: Polybotrya nodiflora Bory)in which he included Polybotrya hamiltoniana [(Wallich) Presl ex] Fé (1845), Polybotrya vivipara Hooker (1925) ('Acrostichum'), and P. appendiculata var. subintegra Beddome (1883). The correct name for this taxon, however, in the genus Egenolfia is $E$. vivipara (Hooker) C. Chr., and in the genus Bolbitis is $B$. hookeriana Iwatsuki, as there is already a B. vivipara (bas.: Campium viviparum $\mathrm{Kjellberg}=B$. quoyana).

7. Koorders 119 (BO, ster.) from Karimondjawa Is. and Palmer \& Bryant 1268 (US, ster.) from West Java, Palabuan Ratu, could not be identified infraspecifically.

8. The present species is amongst others characterized by the shape of the perispore which is unique in the genus. See chapter 6.

\section{KEY TO INFRASPECIFIC TAXA}

1a. Fertile pinnae with a lamina. Sporangia situated on the lower surface of the leaf. a. ssp. appendiculata

b. Fertile pinnae without a lamina or with a narrow strip of lamina present along the costa only. Sporangia facing to all directions . . . . . . . 2

2a. Fertile pinnae without a lamina, moniliform. Base of sterile pinnae symmetrical. Mainland Asia . . . . . . . . . . . b. b.1. ssp. vivipara var. vivipara

b. Fertile pinnae with a narrow strip of lamina along the costa, \pm moniliform. Base of sterile pinnae variously shaped. Andaman and Nicobar Is., W. Java.

b.2. ssp. vivipara var. neglecta

\section{a. ssp. appendiculata-Fig. 49.}

Acrostichum appendiculatum Willd.-A. viviparum Hamilton ex Sprengel.-Polybotrya marginata Bl. -Acrostichum asplenifolium Bory.-Egenolfia hamiltoniana Schott.-Acrostichum alatum Roxb. ex Griff.-Polybotrya helferiana Kunze.-Lacaussadea montana Gaudich.-Egenolfia helferiana (Kunze) C. Chr. var. incisa Ching.-E. asplenifolia (Bory) Fée var. moniliformis Tardieu-Blot \& C. Chr.E. keralensis Nayar \& Kaur. $-E$. appendiculata $\times$ helferiana.

Polybotrya appendiculata (Willd.) J. Smith var. helferiana auct. non (Kunze) Christ: Christ, Bot. Tidsskr. 24 (1901) 109; Bull. Herb. Boiss. II, 4 (1904) 610.

Rhizome 3-9 mm thick, with usually 3 rows of leaves, usually with many sclerenchyma strands. Sterile leaves $15-60(-80) \mathrm{cm}$ long; petiole 3-25 cm long, with a narrow wing at least in the upper part either with spreading scales mainly present at the petiole base, or glabrescent; lamina $10-45(-55)$ by $2.5-13 \mathrm{~cm}$, without the terminal segment $10-40$ $(-50)$, the terminal segment $1-7 \mathrm{~cm}$ long; rhachis with a narrow wing; pinnae $25-60(-85)$;

Fig. 50. Bolbitis appendiculata ssp. vivipara. -a-h. var. vivipara. a, b. sterile and fertile leaf resp., $\times 1 / 5$; $c-g$. shape and venation pattern of sterile pinnae, $\times 4 / 5$; the asterisk indicates anastomosing veins; $h$. venation pattern and receptacle (dotted) of basal part of fertile pinna, $\times 3 .-\mathrm{i}-\mathrm{n}$. var. neglecta. $\mathrm{i}, \mathrm{j}$. fertile and sterile leaf resp., $\times 1 / 5 ; \mathrm{k}-\mathrm{m}$. shape and venation pattern of sterile pinnae, $\times 4 / 5$; n. venation pattern and receptacle (dotted) of fertile pinna, $\times 3$ (a, b. Hennipman 3119, L; c-g. H. 3488, L; h. Rock 823, US; i, j, l, m. Van Borssum Waalkes 603, L, holotype of B. appendiculata var. neglecta; k. Backer s.n., BO; n. Korthals s.n., Java, L). 


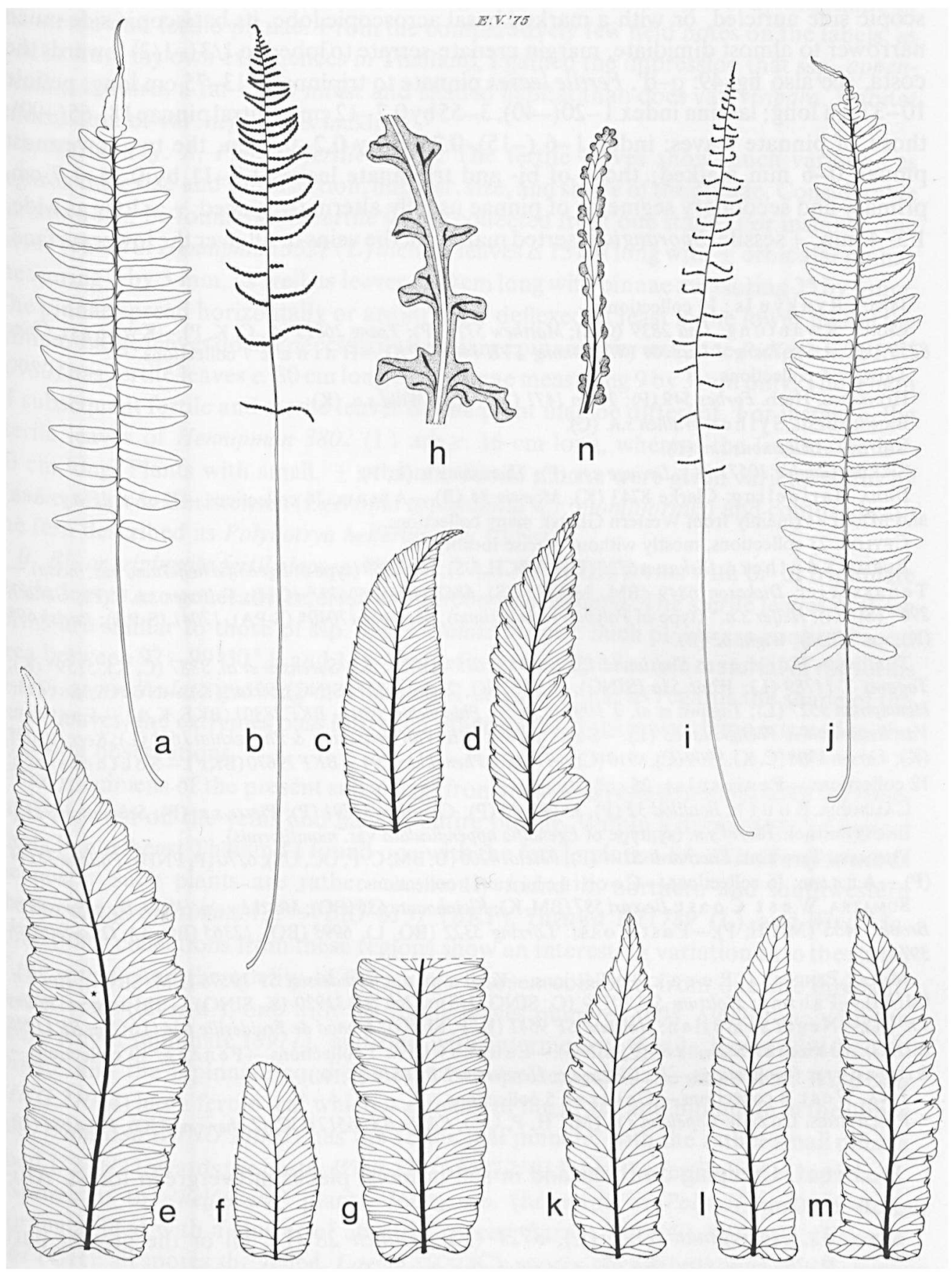


central pinnae index $2-5,1.5-7.5$ by $0.5-1.7(-2) \mathrm{cm}$, base oblique either with its acroscopic side auricled, or with a marked basal acroscopic lobe, its basiscopic side much narrower to almost dimidiate, margin crenate-serrate to lobed to $1 / 3(-1 / 2)$ towards the costa, see also fig. 49: q-d'. Fertile leaves pinnate to tripinnate, $13-75 \mathrm{~cm}$ long; petiole $10-37 \mathrm{~cm}$ long; lamina index $1-20(-40), 3-55$ by $0.7-12 \mathrm{~cm}$; central pinnae $18-65(-90)$; those of pinnate leaves: index $1-6(-15), 0.25-4$ by $0.2-0.7 \mathrm{~cm}$, the two lowermost pinnae $0-6 \mathrm{~mm}$ stalked; those of bi- and tripinnate leaves $1.2-11$ by $0.15-1.7 \mathrm{~cm}$, primary and secondary segments of pinnae usually alternate, spaced, \pm as long as wide, 1.5-4 mm, \pm sessile. Sporangia inserted mainly on the veins or all over the lower surface.

JAPAN. R y u k yu I s.: 10 collections.

CHINA. Kwa nt ung: Lau 2839 (GH); Matthew 5113 (P); Tsang 26823 (A, C, K, P).-K wang si: Ching 8238 (UC, US); Tsang LU 23204 (W); Whong SYU 140 (S-PA).-H a i n a n: 7 collections.

TAIWAN. 6 collections.

HonKong. Herb. Forbes 549 (P); Taam 1471 (UC, US); Willd s.n. (K).

BaNGLADESH. S y lhe t: Wallich s.n. (C).

BhUTAN. Anonymous s.n. (P).

SIKKIM. Gamble 10571 (K); Levinge s.n. (P); Thomson s.n. (L, P).

InDia Darje eling: Clarke 8743 (K), Moewis 34 (B).-A s s a m: 16 collections.-Ker ala, Mys ore, and $\mathrm{Madr}$ a (mainly from Western Ghats): many collections.

CEYLON. 23 collections, mostly without precise locality.

BURMA. S o u t h e r n: Dickason 6789 (GH, MICH, US); Rock 759 (type of Egenolfia helferiana var, incisa).Tenasserim: Dickason 6849 (BM, MICH, US), 6867 (BM), 6867*1) (GH); Falconer s.n.* (P); Gallatly 296* (SING); Helfer s.n.* (type of Polybotrya helferiana); Meebold 17040* (S-PA), 17041 (S-PA); Parish 69* (K), s.n.* (BM); Wight $62(\mathrm{~B})$.

THAILAND. Nor the rn: Smitinand $132=$ BKF 6157 (BKF, K); S申rensen et al. $3887(\mathrm{C}, \mathrm{K}), 5159^{*}(\mathrm{~K})$; Tagawa T 11789 (L); Winit 53a (SING), 53b (SING, 2 sh.), $53 b^{*}$ (SING), $922 a^{*}(\mathrm{~K})$.- N or the astern: Hennipman 3527 (L); Tagawa et al. T 1094 (L, US); Phloenchit $2030=$ BKF 2830I (BKF, K, L). - E a st e r n: Van Beusekom \& Phengkhlai 83 (L).-S o u t h w est ern: Van Beusekom \& Phengkhlai 163*(L); Kerr 10502* (K); Larsen 8908 (C, K), 8909 (C), 8910 (C) $8911^{*}$ (C); Phengkhlai $294=B K F 29670$ (BKF).-S o u th a st e r n: 12 collections. - Peninsular: 25 collections.

Cambodia. S o u t h: Bouillod 32 (P), 33 (P), 46 (P); Chevalier 31791 (P); Pierre s.n. (P): Schmidt 349 (C). LAOS (Bassac). Thorel s.n. (syntype of Egenolfia appendiculata var. moniliformis).

VIETNAM. To n kin: Eberhardt 5194 (P); Pételot 4205 (B, BM, C, P, UC, US), 6270 (P, PNH); Poilane 25144 (P).-A n n a m: 16 collections. - C o ch in ch in a: 11 collections.

Sumatra. W e s t C o as t: Beccari $587(\mathrm{BM}, \mathrm{K})$; Kleinhoonte 635(BO); Matthew s.n. $\{$ K).-Be n c o ole n: Brooks 145S (MICH, P).-East Coast: Lörzing 5322 (BO, L), 6993 (BO), 12365 (BO, L); Otto-Surbeck 397 (L).

Malay Peninsula. P e r a k: 8 collections.-K e 1 a n t a n: Henderson SF 19578 (BM, SING); Nur s.n. (SING).-Pah a ng: Holttum SF 20799 (C, SING, US); Kiah SF 31920 (K, SING).-S elang or: Ridley s.n. (K). - Negr i S e m b il a n: Holttum SF 9541 (K, P, SING); Monod de Froideville 654 (L); Nur SF 11848 (SING). - J o hore: Ridley s.n. (P, SING).-La ng k awi Is.: 7 collections.-Pen a ng: 10 collections.Sing a por e: 6 collections.-P. T i o $\mathrm{m}$ a n: Henderson SF 18614 (SING).

JAva. West: 8 collections. -Central: 5 collections.

PhILIPPINES. Lu z o n: Copeland s.n. (MICH, P, UC); Edaño BS 76512 (UC); Loher s.n. (UC); Price 884 (L).

$\mathrm{H}$ a bitat. Creeping on rocks and in soil in moist places in evergreen forest. Altitude: $0-1500 \mathrm{~m}$.

Spores. Hennipman 3527 (L), 3721 (L), Wallich 28 (L): all or the greater part normal. Walker s.n. (P): the greater part abnormal. See also the notes.

${ }^{1)}$ The collections with an asterisk agree with Polybotrya helferiana; see note 2B. 
Notes. 1. A distinct taxon easily distinguished from var. vivipara by the shape of the sterile and fertile pinnae. From the comparatively few field notes on the labels, as well as from my own experiences in Thailand, I gained the impression that ssp. appendiculata requires a far more moist and shaded habitat than does var. vivipara. Habitat information of var. neglecta is lacking.

2. Variability. A. Pinnate fertile leaves. The fertile leaves show much variation as regards their size and the insertion, number, size, and shape of the pinnae. Considerable variation can be found in the fertile leaves collected from one stand. For instance, the fertile leaves of Hennipman 3527 (L) include leaves $c .15 \mathrm{~cm}$ long with \pm orbicular pinnae measuring 3 by $3 \mathrm{~mm}$, as well as leaves $c$. $70 \mathrm{~cm}$ long with pinnae measuring 35 by $4 \mathrm{~mm}$. The pinnae spread horizontally or are strongly deflexed at least in the lower half of the lamina. Large leaves do not necessarily bear large pinnae. For instance Ridley s.n. (SING 20980 ) has fertile leaves $c .80 \mathrm{~cm}$ long with pinnae measuring 9 by $3 \mathrm{~mm}$ only. The length of subsequent fertile and sterile leaves of one plant may be different. For instance, the sterile leaves of Hennipman 3802 (L) are c. $16 \mathrm{~cm}$ long, whereas the fertile one is $c$. $40 \mathrm{~cm}$ long. Plants with small, \pm orbicular fertile pinnae were given varietal status by Tardieu-Blot \& Christensen (Egenolfia asplenifolia var. moniliformis) and confused with the fern described as Polybotrya helferiana by Christ.

$B$. Bi- or tripinnate fertile leaves. Fig. $49: \mathrm{f}, \mathrm{h}, \mathrm{i}, \mathrm{n}-\mathrm{p}$. The forms with bi- or tripinnate fertile leaves are generally referred to Polybotrya helferiana. The sterile leaves of these forms are similar to those of ssp. appendiculata proper. Such plants are confined to an area between $97^{\circ}-99^{\circ} 30^{\prime} \mathrm{E}$ and $14^{\circ}-19^{\circ} \mathrm{N}$. From a study of ample material of the forms of ssp. appendiculata growing in Thailand, I conclude that as regards the shape of the fertile leaves, the extremes pass into each other smoothly as is also demonstrated by the following observations.

The specimens of the present subspecies from mainland Asia generally have the sporangia inserted on the veins and have \pm entire pinnae. Within a dense stand of typical plants in northeast Thailand I found one with the fertile pinnae lobed halfway towards the costa. Such plants are rather commonly found in northern and southwestern Thailand, e.g. Smitinand $132=$ BKF 6157 (BKF), Tagawa T11789 (L), and Winit $53 a$. Some other collections from these regions show an interesting variation as to the shape of the pinnae and the quality of the spores. Larsen collected from one site three different forms (all in $\mathrm{C}$ ), one with the fertile leaves pinnate $(8908,8909)$, one with the fertile leaves bipinnate $(8911)$, and one with intermediate fertile leaves $(8910)$. The pinnate and the bipinnate condition may be represented in the same leaf: Winit $53 b$ (SING 21014) has a fertile leaf which is pinnate in the lower and bipinnate in the upper half; Winit $53 b$ (SING 21002) has the fertile leaf pinnate, with the rather small pinnae lobed 1/2-1/3 towards the costa; Winit $53 b(S I N G 21013)$ has the fertile leaves bipinnate. Spores of the 'helferiana' plants. Helfer s.n. (holotype of Polybotrya helferiana): sporangia filled with a cluster of abortive spores. Larsen 8910 (C), 8911 (C), Dickason 6867 (GH): all spores shrivelled. Larsen 8909 (C): spores normally shaped but contents abnormal. Van Beusekom \& Phengkhlai 163A,163B,163C (all from the same site, all in L): $163 A$ : nearly all sporangia with $>50$ normal spores, a few sporangia with abortive 
spores; $163 \mathrm{~B}$ : ibid., but with proportionally more sporangia with abnormal spores; $163 C$ : all sporangia filled with usually abortive S.M.C.'s some in part also with aborted spores.

One of the sterile leaves of Larsen 8911 (C) has a few of its central pinnae atypically shaped; these pinnae are lobed to halfway towards the costa, lack spines in the sinuses, and have a glaucous tinge (absent from all other pinnae).

The 'helferiana' plants discussed above might be the result of divergent evolution. The observation that plants with lobed fertile pinnae (and normal spores) are found occasionally within a stand of typical specimens is in support of this. However, the information from the spores of the 'helferiana' forms and the incidental occurrence of abnormally shaped pinnae might be indications that these plants are of hybrid origin. As var. vivipara is the nearest related taxon which, moreover, has moniliform fertile pinnae, it seems possible that in case 'helferiana' has a hybrid origin, its parents are ssp. appendiculata and var. vivipara.

C. The sterile pinnae. The mcst distinct form is that described as Acrostichumasplenifolium. The base of the sterile pinnae is very oblique, the margin deeply lobed with conspicuous spines in the sinuses. Such plants occur in southern India and Ceylon. The majority of the specimens from southern India belongs to 'asplenifolium'; it is rare in Ceylon, where it fully integrates with more typical forms. A specimen of 'asplenifolium' kept in cultivation at Singapore for many years retained its shape (Holttum, 1954). Part of the specimens from Vietnam have \pm similarly shaped sterile pinnae. Spores (of specimens from southern India): Chandra $L W G 77703$ (LWG): all abnormal. Van Hardeveld \& Van der Werff 322 (L), Nayar LWG 45552 (LWG): the majority abnormal, a minority normal with a densely cristate perispore. A minority of the plants from southern India is much different from 'asplenifolium' as to the size and shape of the pinnae. For instance Anonymus s.n. (in herb. Hooker \& Thomson; BM, C, P, W) from Mysore has large sterile pinnae (up to 90 by $22 \mathrm{~mm}$ ) the base of which varies from slightly asymmetrical to normal; the fertile pinnae also are large (up to 30 by $7 \mathrm{~mm}$ ), the spores are either all shrivelled (the specimen in $\mathrm{P}$ ) or \pm spherical, proportionally large $(40-45 \mu \mathrm{m})$, the perispore with few and low crests. The specimen with the latter spores presumably represents an (auto)ploid; its spores are discussed in chapter 6 , and illustrated in plate $10: a, b$.

3. Geographical distribution of the 'helferiana' form. Ching (1931) erroneously located Helfer's type in Assam. Nayar \& Kaur also reported this fern (as: Egenolfia helferiana) from Assam. The reference sheet cited, Nayar LWG 77005 (LWG) is sterile, however! These authors also reported this fern from the Andamans; the reference sheet cited belongs to var. neglecta.

4. The type of Egenolfia helferiana var. incisa was said to have sterile pinnae which are deeply lobed and of a different texture. Actually, the sterile pinnae are typical, but the fertile leaves are \pm atypical being tripinnate.

5. The specimens from the Philippines are somewhat aberrant as regards the sterile pinnae which have acute or serrate lobes ( \pm orbicular in ssp. appendiculata proper) and which are reminiscent of those of B. rhizophylla. Spores: Copeland s.n. (MICH), Loher 


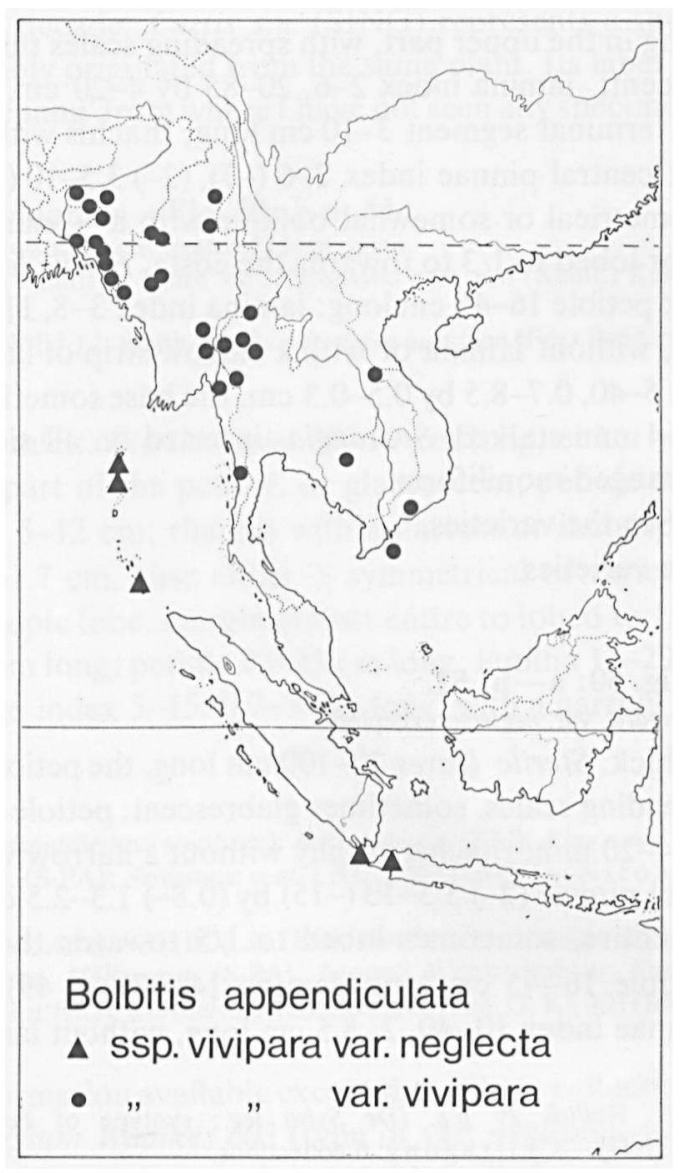

Fig. 51. Distribution of Bolbitis appendiculata ssp. vivipara.

s.n. (UC), Price 884 (L): normal, the perispore rather erosed. Edaño BS 76512 (UC): normal.

6. Pételot 4083 (Colani leg.)(B. MICH, P, US) from a limestone hill in Pha Vang, Laos, is abnormal as to the shape of the sterile pinnae which have a \pm symmetrical narrow base, the rather terminal position of the bulbil, and the purplish tinge present on part of the leaves. Spores: all abnormal. Possibly a hybrid with $B$. crispatula var. copelandii.

b. ssp. vivipara (Hooker) Hennipman-Fig. 50,51.

Polybotrya vivipara Hamilton ex Hooker. $-P$. nodiffora Bory.-Acrostichum seetacoonense Roxb. ex Griff. - Polybotrya hamiltoniana Fée.-P. appendiculata (Willd.) J. Smith var. subintegra Bedd.-B. appendiculata (Will.) Iwatsuki var. neglecta Hennipman.

Rhizome 2.5-12 mm thick, with 2 or 3 rows of leaves, without or with but few sclerenchyma strands. Sterile leaves $20-100 \mathrm{~cm}$ long; petiole $5-25 \mathrm{~cm}$ long, the margins acute 
or with a narrow wing in the upper part, with spreading scales throughout or mainly at the base, or glabrescent; lamina index $2-6,20-85$ by $4-20 \mathrm{~cm}$, without the terminal segment $12-77$, the terminal segment $3-10 \mathrm{~cm}$ long; rhachis with or without a narrow wing; pinnae 20-60; central pinnae index 2-6 (-7), (2-) 3.5-10 (-15) by (0.8-) 1.1-2.5 $\mathrm{cm}$, base either symmetrical or somewhat oblique with a \pm marked basal acroscopic lobe, margin entire or lobed to $1 / 3$ to towards the costa. Fertile leaves pinnate or bipinnate, $30-90 \mathrm{~cm}$ long; petiole $16-45 \mathrm{~cm}$ long; lamina index $3-8,11-37(->49)$ by $0.7-10$ $\mathrm{cm}$; pinnae 15-> 55, without lamina or with a narrow strip of lamina along the costa; central pinnae index $5-40,0.7-8.5$ by $0.5-0.3 \mathrm{~cm}$, the base sometimes wider; the lowermost two pinnae 0-4 mm stalked. Sporangia inserted on all sides of the costa (and costules), usually arranged moniliform.

Distribution: See the varieties.

$\mathrm{H}$ a bitat. See the varieties.

\section{b.1. var. vivipara-Fig. 50: a-h; 51.}

For synonyms see ssp. vivipara, var. neglecta excepted.

Rhizome 5-12 mm thick. Sterile leaves $20-100 \mathrm{~cm}$ long, the petiole and rhachis usually throughout with spreading scales, sometimes glabrescent; petiole $5-25 \mathrm{~cm}$ long; lamina index $2-5,20-85$ by $4-20 \mathrm{~mm}$; rhachis usually without a narrow wing in the basal part; pinnae 33-60; central pinnae (2-) 3.5-10 (-15) by $(0.8-) 1.3-2.5 \mathrm{~cm}$, base \pm symmetrical, margin usually entire, sometimes lobed to $1 / 5$ towards the costa. Fertile leaves $40-90 \mathrm{~cm}$ long; petiole $16-45 \mathrm{~cm}$ long; lamina $14-37(->49)$ by $4-10 \mathrm{~cm}$; pinnae $35->55$; central pinnae index $10-40,2-8.5 \mathrm{~cm}$ long, without lamina.

Bangladesh. Sylhet: Wallich 29 p.p. (De Sylva leg.; syntype of Polybotrya hamiltoniana).Tip pera: Biswas 5083 (PNH).-Chit tagong: 6 collections.

INDIA. A s a m: 12 collections.

Burma. A rakan: McKee 6109 (BM); NSW P8261 (US).-Northe ast ern: Belcher 610 (PNH, US); Lace 4508 (K).-Cent ral: Rock 823 (US).-S o u thern: Dickason 7120 (BM, GH, MICH, US); Lace 5023 (K). - T en as s erim: 8 collections ( 7 from Moulmein).

Thailand. Nor the $\mathrm{r}$ : 12 collections.

Cambodia. (Angkor). Smith 306 (C, GH, MICH, UC, US).

LAOS. Harmand s.n. (BM, P); Poilane 11388 (K, P).

VIeTNaM. Coch in ch in a: Pierre 1269 (P); Poilane 2541 (Chevalier leg.)(P).-Co n So n Is.: De Lanessan s.n. (P).

Habitat. Creeping on rocks or in soil in dry evergreen (or deciduous?) forest. Several times reported from limestone. Altitude: $0-900(-1200) \mathrm{m}$.

S p o re s. Hennipman 3488 (L), Smith 306 (UC): nearly all normal. Rock 823 (US): the greater part abnormal, mostly shrivelled.

Not es. Manton \& Sledge (1954) counted the chromosome number of a plant cultivated at the Botanic Garden at Singapore. They stated that the plant is native in southern Thailand from whence it was said to have been introduced to Penang by Curtis. From Penang it apparently was passed on to Singapore. I have not seen any specimen of this taxon from southern Thailand, a region at least locally thoroughly explored for 
ferns during the last decade. Curtis s.n. (SING) represents a specimen cultivated at Penang, and presumably originated from the same plant. Its label bears the annotation 'Wild on the hills in Penang' from where I have not seen any specimen either.

b.2. var. neglecta Hennipman-Fig. $50: \mathbf{i}-\mathbf{n} ; \mathbf{5 1}$.

$B$. appendiculata (Willd.) Iwatsuki var, neglecta Hennipman.

Polybotrya appendiculata (Willd.) J. Smith var. rhizophylla auct. non (Kaulf.) Kuhn: Kuhn, Ann. Mus. Bot. Lugd.-Bat. 4 (1869) 225.

Polybotrya appendiculata (Willd.) J. Smith var. hamiltoniana auct. non (Fée) Bedd.: v.A.v.R., Handb. Malayan Ferns (1916) 431.

Rhizome $2.5-7 \mathrm{~mm}$ thick. Sterile leaves $30-50 \mathrm{~cm}$ long, either with spreading scales mostly on the basal part of the petiole, or glabrescent; petiole $6-15 \mathrm{~cm}$ long; lamina index $3-6,20-45$ by $5-12 \mathrm{~cm}$; rhachis with a narrow or inconspicuous wing; pinnae $20-54,3.5-8$ by $1.1-1.7 \mathrm{~cm}$, base either \pm symmetrical or variously oblique with a \pm marked basal acroscopic lobe, margin almost entire to lobed to $1 / 3$ towards the costa. Fertile leaves $30-70 \mathrm{~cm}$ long; petiole $22-35 \mathrm{~cm}$ long; lamina $11-29$ by $0.7-5 \mathrm{~cm}$; pinnae 15-45; central pinnae index $5-15,0.7-3 \mathrm{~cm}$ long, with a narrow strip of lamina along the costa.

INDIA. A nd a ma n Is. (middle and southern): Buchanan s.n. (BM); King's coll. s.n. (B, P, U, W); Kurz s.n. (P); E.H. Man (Mau?) s.n. (S-PA); Srivastava et al. LWG 79581 (LWG). - N i c o b a r I s.: Galathea Exp. 2858 (C), 2900 (C).

JAVA. We st. P. Panaitan: Amdjah 31 (BO), 32 (BO); Van Borssum Waalkes 603 (type of var. neglecta). West Priangan, Palabuhan Ratu: Möller s.n. (S-PA). Around Wijnkoopsbaai: Kuntze 5017 (P).-Sine loc.: Anonymus 385 (B); Backer s.n. (BO); Korthals s.n. (L); Lobb 200 (BM, G, K), 203 (BM).

$\mathrm{H}$ a bit a t. No information available except that of type collection: 'on stone', at $70 \mathrm{~m}$. $\mathrm{Sp}$ ores. Van Borssum Waalkes 603 (type of var. neglecta): nearly all normal. King s.n. (W), Lobb 200 (BM): all abnormal, mostly shrivelled.

Note. The moniliform shape of the fertile pinnae is sometimes obscured when the pinnae bear mature sporangia. In such cases they are easily confused with the fertile pinnae of ssp. appendiculata if mounted with the abaxial side up.

17. Bolbitis major (Bedd.) Hennipman, stat. nov. -Fig. 52: a-c.

Polybotrya appendiculata (Willd.) J. Smith var. major Bedd., Handb. Ferns Br. India (1883) 424.-T y p e: Atkinson s.n., Sikkim, $1200 \mathrm{~m}$ alt. (K; iso in BM).

Rhizome up to c. $20 \mathrm{~cm}$ long, up to $7 \mathrm{~mm}$ thick, with 2 rows of leaves. Scales of the rhizome: index $2-5$, up to 2.5 by $1 \mathrm{~mm}$, opaque, brownish to blackish. Sterile leaves pinnate, the two complete ones 110 and $160 \mathrm{~cm}$ long; petioles $45-65 \mathrm{~cm}$ long, near the base 4-7 $\mathrm{mm}$ thick with more than 10 vascular bundles; lamina index $2-4$, widest somewhat below the middle, $60-95$ by $24-30 \mathrm{~cm}$, without the terminal segment $54-80$, the terminal segment 6-14 cm long, herbaceous, dark brown or blackish, the rhachis densely covered with small, appressed, dark brown, \pm permanent scales, not winged; pinnae alternate to opposite, $52->64$, up to $70 \mathrm{~mm}$ apart; central pinnae index 5-9, widest about the 


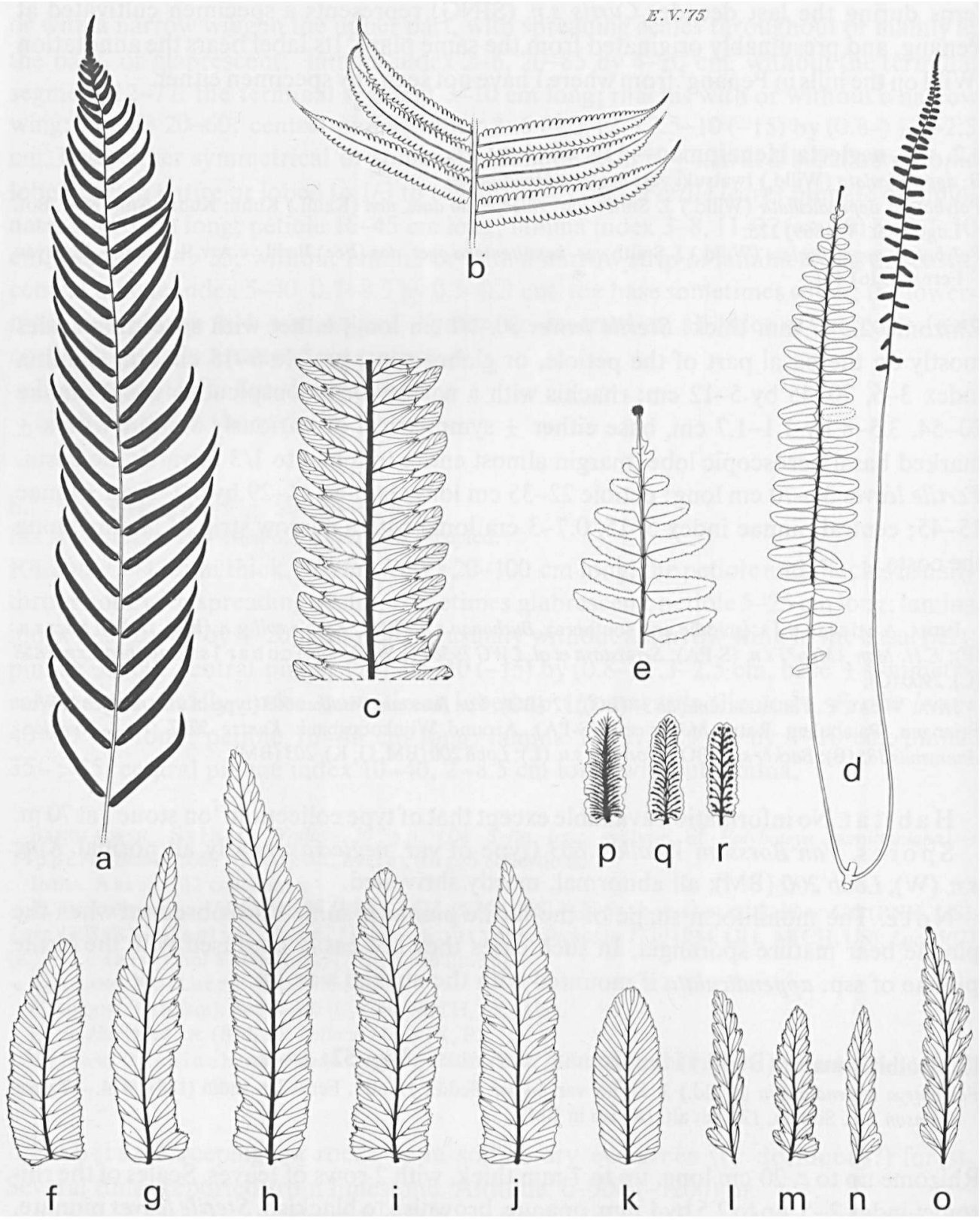

Fig. 52. a-c. Bolbitis major. a. fertile leaf, $\times 1 / 5$; b. central part of sterile leaf, $\times 1 / 5$; c. venation pattern of sterile pinna, $\times 4 / 5$ (a, b. Atkinson s.n., K, holotype of B. major; c. Levinge s.n., P).-d-r. Bolbitis rhizophylla. d. sterile and fertile leaf, $\times 1 / 5$; e. terminal part of sterile leaf with bulbil, $\times 2 / 5 ; \mathrm{f}-0$. shape and venation pattern of sterile pinnae, $\times 4 / 5$; p-r. ibid., fertile pinnae, $\times 4 / 5$ (d. Hallier 4218, L; e. Fénix BS 12984, L; f. Loher 1262, US; g. Elmer 15899, UC; h. Elmer 16793, GH; i. Sinclair \& Edaño 9476, SING; j. LeRoy Topping 655, US; k. Copeland 1983, MICH; l-n. Ramos \& Edaño BS 46986, B, isotype of Egenolfia fuviatilis; o. ibid., UC; p. Ramos BS 77179, MICH; q. Copeland s.n., MICH, herb. Copel. 4343; r. Elmer 15899, BO). 
middle, $13.5-20$ by $2-3 \mathrm{~cm}$, base symmetrical, broadly cuneate, attenuate or truncate, margin lobed $2 / 5-3 / 5$ towards the costa, with a conspicuous spine in the sinuses, lobes somewhat falcate, c. 4-5 mm wide near the base, apex oblique, truncate or orbicular, the two lowermost pinnae \pm sessile, otherwise conform to the central pinnae; terminal segment relatively small, triangular, apex acute; venation pattern: secondary veins on either side with $>6$ tertiary veins, see fig. 52: c. Fertile leaves pinnate, the two complete ones 137 and $165 \mathrm{~cm}$ long; petiole 67 and $91 \mathrm{~cm}$ long resp.; lamina index $c .5,70$ by $c$. $18 \mathrm{~cm}$ and 74 by c. $15 \mathrm{~cm}$ resp.; pinnae 54 and 60 resp., up to $55 \mathrm{~mm}$ apart; central pinnae index $13-17$, widest somewhat below the middle, $7.5-10$ by $0.5-0.6 \mathrm{~cm}$, the margin \pm entire. Sporangia inserted all over the lower surface. Spores with a smooth cristate perispore.

SIKkIM. Atkinson s.n. (type); Gamble 8556 (K); Levinge s.n., 26-ix-1883 (P, W, ster.).

INDIA. Assam: Mann s.n., i-1891 (K, UC).

BANGLADESH. Chitt a g o ng: Carke 19621 (BM, K). See also note 3.

H abit a t. No information available.

S pores. Atkinson s.n. (BM, K; type): all normal. Gamble 8556 (K), Levinge s.n. (P): all abnormal.

Notes. 1. An apparently rare fern characterized by the long-creeping rhizome with widely spaced big leaves, the large number of vascular bundles in the petiole, and the leaf architecture. The leaves have a dark colour as in those of $B$. tonkinensis. Its position within this series seems isolated.

2. Typification. Beddome did not mention a (type) collection. At the time Beddome described var. major, however, only Atkinson's material of this taxon was in his herbarium, which is accordingly the type.

3. Duthie 10654 (K, P, UC, all ster.) from Madhya Pradesh (Central India) possibly belongs here.

18. Bolbitis rhizophylla (Kaulf.) Hennipman-Fig. 52: d-r; 53.

B. rhizophylla (Kaulf.) Hennipman, Blumea 18 (1970) 148.-Gymnogramma rhizophylla Kaulf., Enum. Fil. (1824) 78 ('rhizophyllum')._Polybotrya rhizophylla Presl, Tent. Pterid. (1836) 231; Fée, Hist. Acrost. (1845) 77; Mettenius, Fil. Hort. Lips. (1856) 24; Ettingsh., Denkschr. K. Ak. Wiss. M.-N. Kl. Wien 22(1864) pl. 7: fig. 9. - Lacaussadea rhizophylla Gaudich., Voy. Bonite Bot. (1852) pl. 120, s. descr.-Egenolfia rhizophylla Fee, Genres Polyp. (1852) 48; Copel., Fern Fl. Philip. (1960) 266; DeVol \& Kuo in Fl. Taiwan 1 (1975) 352.-Polybotrya appendiculata (Willd.) J. Smith var. rhizophylla Kuhn, Ann. Mus. Bot. Lugd.-Bat. 4 (1869) 295, quoad nomen solum. - T y p e: Chamisso s.n., Philippines, Manila (n.v., LE?; iso in B).

Polybotrya intermedia [J. Smith, Hook. J. Bot. 3(1841) 72, nom. nud.] Fée, Hist. Acrost. (1845) 76, pl. 40: fig. 1; Ettingsh., Denkschr. K. Ak. Wiss. M.-N. KI. Wien 22 (1864) 63, pl. 6: fig. 7.-Egenolfia intermedia Fée, Genres Polyp. (1852) 48; Ching, Bull. Fan Mem. Inst. Biol. 2(1931) 308; Copel., Fern Fl. Philip. (1960) 266. - B. intermedia Iwatsuki, Acta Phytotax. Geobot. 18 (1959) 49.-T y p e: Cuming 269, Philippines, Luzon, Ilocos (G; iso in BM, GH, K, P, UC, US, W).

Polybotrya neglecta Fée, Hist. Acrost. (1845) 76, pl. 39: fig. ii.-T y p e: Cuming 47, Philippines, Luzon, Laguna (P, herb. Bory). See note 2.

Polybotrya serrulata [J. Smith, Hook. J. Bot. 3 (1841) 401, nom. nud.] Fé, Hist. Acrost. (1845) 76, pl. 39: fig. ii*; Mettenius, Fil. Hort. Lips. (1856) 24; Ettingsh., Denkschr. K. Ak. Wiss. M.-N. KI. Wien 22(1864) 64, pl. 1: fig. 3.-Egenolfia serrulata Fée, Genres Polyp. (1852) 358; Ching, Bull. Fan Mem. Inst. Biol. 2(1931) 
307; Nakai, Bot. Mag. Tokyo 47 (1933) 170; Tagawa, Acta Phytotax. Geobot. 16(1956) 175.-B. serrulata Iwatsuki, Acta Phytotax. Geobot. 18(1959) 49.-S y n t y pes: Cuming 47 p.p., Philippines, Luzon, Laguna (BM, G, GH, K, L, P, US, W); Hügel 4636, Philippines, Luzon, Manila (W).

Polybotrya exaltata Brackenr. in Wilkes, U.S. Expl. Exp. 16(1854) 78.-T y p e: Brackenridge s.n., Philippines, Luzon, Mt. Makiling (US, 62635).

Polybotrya duplicato-serrata Hayata, Ic. Pl. Formos. (1915) 305, fig. 123 A.T y p e: Nakahara s.n., Taiwan, Akocho, Tanashu, i-1905 (TI, n.v.; L. phot.).

Egenolfia fluviatilis Copel., Philip. J. Sc. 38 (1929) 152, pl. 5; Fern Fl. Philip. (1960) 267.-B. copelandii Iwatsuki, Acta Phytotax. Geobot. 18 (1959) 49, nom. illeg., non Ching ex Tardieu-Blot \& C. Chr. (1938) (=B. crispatula var. copelandii). - T y p e: Ramos \& Edaño BS 46986, Philippines, Luzon, Isabela, San Mariano, Mt. Yalobo region, $700 \mathrm{~m}$ alt. (iso in B, UC, US).

Rhizome with 2 or 3 rows of leaves. Scales of the rhizome: index $2-6$, up to $3(-4)$ by $1 \mathrm{~mm}$, subclathrate, brownish or blackish, with or without a paler marginal strip. Sterile leaves pinnate, $20-90 \mathrm{~cm}$ long; petiole 8-25 cm long, near the base $1.5-3 \mathrm{~mm}$ thick; lamina index 3-9, widest usually below, sometimes about the middle, $18-80$ by $4.5-15$ $\mathrm{cm}$, without the terminal segment $15-75$, the terminal segment $1-6 \mathrm{~cm}$ long, herbaceous, green to blackish, the petiole and lower side of the basal part of the rhachis (apart from the appressed scales) usually with small, blackish, spreading, \pm permanent scales; rhachis with a narrow wing except for the lowest part; pinnae alternate to opposite, 35-90, up to $35 \mathrm{~mm}$ apart; central pinnae index $2-6$, widest usually below, sometimes about the middle, $2.5-8$ by $0.8-2 \mathrm{~cm}$, base usually symmetrical, (narrowly) cuneate to subcordate, margin finely serrate, biserrate, or serrately lobed to $1 / 3$ towards the costa, the spines often tooth-like, apex orbicular or acuminate; the two lowermost pinnae deflexed or not, conform to the central pinnae, $0-2 \mathrm{~mm}$ stalked; terminal segment narrowly triangular, apex acute; venation pattern: secondary veins on either side with 1-3 tertiary veins, see also fig. 52: $\mathrm{f}-\mathrm{o}$. Fertile leaves $20-80 \mathrm{~cm}$ long; petiole $10-45 \mathrm{~cm}$ long; lamina index $5-20,7-45$ by $0.8-4.5(-7) \mathrm{cm}$, without the terminal segment $6-43$, the terminal segment $0.5-2.5 \mathrm{~cm}$ long; pinnae $22-65$, up to $55 \mathrm{~mm}$ apart; central pinnae index $1-6$, $0.4-3(-4)$ by $0.3-0.7 \mathrm{~cm}$. Sporangia inserted on and near the veins. Spores with a smooth cristate-undulate perispore.

Chromosomes. n. $=41$.

Talwan. Bankinsing: Faurie 279 (BM, P); Henry 81 (K, P), 81 (B, US); Rosenstock, Fil. Formos. exsicc. 197 (Faurie leg.) (B, GH, MICH, S-PA, UC). -T a k a o: Ogata s.n. (BM). - T a n a s h u: Nakahara s.n. (type of Polybotrya duplicato-serrata). - Nor the r $n$ Formos a': Maries s.n. (K).

PhilipPINes. B a laba c Is.: Mangubat 388 (B, BO, GH, P, US).-Palawan: Merrill 1236 (B, BO, BRI, CAL, GH, L, P, SING), 9243 (GH, SING, UC, US)._-M in d o r o: Ramos BS 39793 (BO, P, US); Sulit \& Conklin PNH 16693 (A, K, MICH, PNH). - L u z o n: 74 collections (from all over the island but especially from the central and northern provinces).

$\mathrm{H}$ abitat. Usually creeping on rocks in moist forests and often near riverbanks. Altitude: $0-1000 \mathrm{~m}$.

Spores. Copeland s.n. (MICH), Cuming 269 (type of Polybotrya intermedia; US, W), Merrill 1236 (GH), Ramos BS 77186 (MICH), Rosenstock, Fil. Formos. exsicc. 197 (UC): all, or the greater part normal, those of Merrill 1236large. Brackenridge s.n. (type of Polybotrya exaltata), Elmer 15899 (GH), LeRoy Topping 655 (GH), Ramos \& Edaño BS 46986 (type of Egenolfia fuviatilis, US): in part normal, in part abnormal. Chamisso 


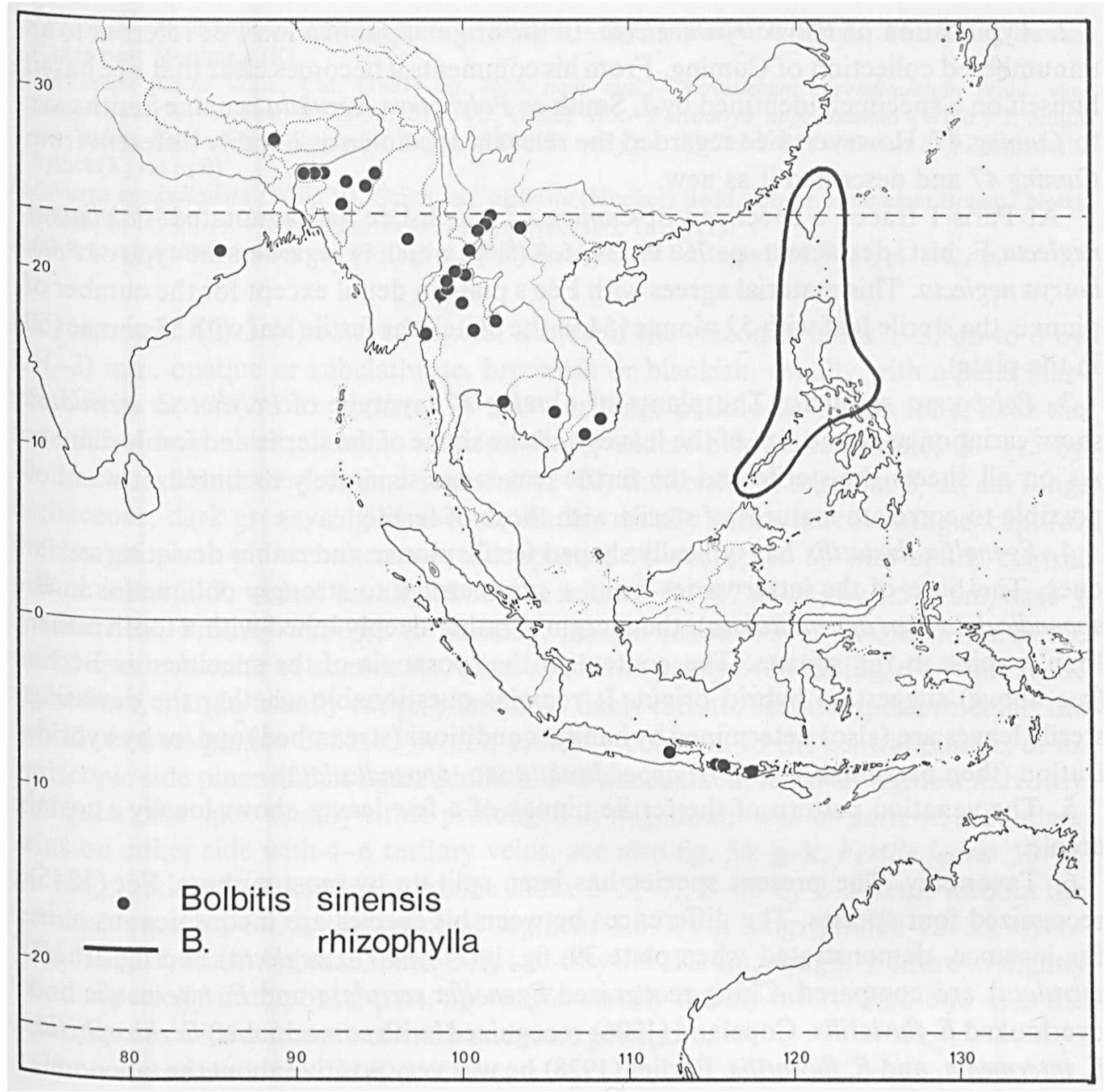

Fig. 53. Distribution of Bolbitis rhizophylla and B. sinensis.

s.n. (type of Gymnogramma rhizophylla), Cuming 47 (syntype of Polybotrya serrulata, $\mathrm{BM}$, both the normally shaped and the irregularly shaped fertile leaf; $P$, herb. Bory), Curran 8982 (US), Elmer 16793 (L), Usteri s.n. (MICH): the greater part abnormal. Ramos \& Edaño BS 46986 (type of Egenolfia fluviatilis, B): sporangia usually filled with aborted spores and/or aborted S.M.C.'s, a few sporangia with mostly normal rather large spores.

Notes. 1. A distinct species easily characterized by the shape of the sterile and fertile pinnae, the terminal bulbil, and the shape of the perispore. The shape of the sterile pinnae and the indument rather resemble those of $B$. appendiculata var. vivipara. The perispore is similar to that of $B$. sinensis. 
2. Typification of Polybotrya neglecta. In the original publication Fée referred to an unnumbered collection of Cuming. From his comments it becomes clear that Fée based himself on a specimen identified by J. Smith as Polybotrya serrulata, a name Smith gave to Cuming 47. However, Fée regarded the relevant specimen as notably different from Cuming 47 and described it as new.

At Paris I traced a specimen of Cuming 47 which Fée had annotated 'Polybotrya neglecta F. hist. des acrost. p. 76. Pl. 39, f. 2 ('1')', which is regarded the type of Polybotrya neglecta. This material agrees with Fée's plate in detail except for the number of pinnae: the sterile leaf with 52 pinnae ( 54 in the plate), the fertile leaf with 53 pinnae ( 50 in the plate).

3. Polybotrya serrulata. The plants of Cuming 47 (syntype of Polybotrya serrulata) show variation as to the size of the leaves and the shape of the sterile and fertile pinnae. As on all sheets the sterile and the fertile leaves are separately mounted, it was not possible to correlate features of sterile with those of fertile leaves.

4. Egenolfia fluviatilis has typically shaped fertile pinnae and rather deviating sterile ones. The base of the latter varies from \pm symmetrical to strongly oblique (as in $B$. appendiculata ssp. appendiculata), the margin is rather deeply lobed with a tooth rather than a spine in the sinuses. The content of the sporangia of the specimen in Berlin (see above) suggests a hybrid origin. It remains questionable whether the deviating sterile leaves are (also) determined by habitat conditions (streambed) and/or by hybridization (then presumably with $B$. appendiculata ssp. appendiculata).

5. The venation pattern of the fertile pinnae of a few leaves shows locally a costal areole.

6. Taxonomy. The present species has been split up by most authors. Fée (1845) recognized four species. The differences between his species are inconspicuous as is, for instance, demonstrated when plate 39 , fig. ii (Polybotrya neglecta) and fig. $\mathrm{ii}^{*}(P$. serrulata) are compared. Ching recognized Egenolfia serrulata and $E$. intermedia and overlooked E. fluviatilis. Copeland (1906) recognized (with some doubts) E. rhizophylla, E. intermedia, and E. fluviatilis. Earlier (1928) he was very positive about the taxonomic value of less salient characters which would have been 'refused recognition as of specific value by those blinded by the common characters of the genus.'

\section{Bolbitis sinensis (Baker) Iwatsuki-Fig. 53, 54.}

B. sinensis (Baker) Iwatsuki, Acta Phytotax. Geobot. 18 (1959) 49.-Acrostichum sinense Baker, Kew Bull. (1906) 14.-Egenolfia sinensis Maxon, Proc. Biol. Soc. Wash. 36 (1923) 173; Ching, Bull. Fan Mem. Inst. Biol. 2 (1931) 305; Tardieu-Blot \& C. Chr. in Lecomte, Fl. Gén. I.-C. 7 (1941) 424, fig. 48, Nayar \& Kaur, Bull. Nat. Bot. Gard. Lucknow 100 (1964) 32.-Campium sinense C. Chr., Contr. U.S. Nat. Herb. 26 (1931) 292, excl. specim. p.p.-T y p e: Henry 12494, China, Yunnan, forest of Szemao ( $\mathrm{K}$; iso in $\mathrm{B}, \mathrm{BM}, \mathrm{US})$.

Acrostichum appendiculatum Willd. var. costulatum Hooker, Spec. Fil. 5(1864) 252('costulata')._Polybotrya appendiculata (Willd.) J. Smith var. costulata Bedd., Ferns Br. India (1865) 110, pl. 110; Handb. Ferns Br. India (1883) 426, fig. 257; Christ, Farnkr. Erde (1897) 44, fig. 102, the upper two drawings of sterile parts only.-Egenolfia bipinnatifida J. Smith, Hist. Fil. (1875) 132; Backer \& Posthumus, Varenfl. Java (1939) 85; Nayar \& Kaur, Bull. Nat. Bot. Gard. Lucknow 100 (1964) 24, fig. 30.-B. bipinnatifida Iwatsuki, Acta Phytotax. Geobot. 18 (1959) 49 ('bipinnata', sphalm.), nom. illeg, non (Kuhn) Ching in C. Chr., 1934.-B. sinensis (Baker) Iwatsuki var. costulata Tagawa \& Iwatsuki, Acta Phytotax. Geobot. 
22 (1967) 102; Southeast Asian Studies 5 (1967) 91.-T y pe: Parish 60, Burma, Tenasserim, Dawna Range near Moulmein (K).

[Acrostichum ludens Wall., Cat. (1831) no. 2685, nom. nud.]_Acrostichum appendiculatum Willd. var. ludens Baker in Hooker \& Baker, Syn. Fil. (1868) 415.-Polybotrya appendiculata (Willd.) J. Smith var. ludens Bedd., Ferns Br. India, Suppl. (1876) 26.-T y p e: Wallich 2685 (= Gomez 87), Bangladesh, Sylhet $(\mathrm{K}$; iso in $\mathrm{B})$.

Polybotrya appendiculata (Willd.) J. Smith var. costulata (Hooker) Bedd. subvar. petiolulata Bonap., Notes Ptérid. 14 (1923) 33.-T y pe: Parish 69, Burma, Nwalabo, Tavoy (P).

Polybotrya appendiculata auct. non (Willd.) J. Smith: Bedd., Ferns Br. India (1865) 111, pl. 111, p.p.

Rhizome with (2) 3 (4) rows of leaves. Scales of the rhizome: index 1-5, up to 6 by $1.5(-2) \mathrm{mm}$, opaque or subclathrate, brownish or blackish, usually with a paler marginal strip. Sterile leaves pinnate, $35-145 \mathrm{~cm}$ long; petiole $10-35 \mathrm{~cm}$ long, near the base $1.5-4 \mathrm{~mm}$ thick; lamina index $1-4(-5)$, widest below the middle, $20-115$ by $10-35 \mathrm{~cm}$, without the terminal segment $12-80$, the terminal segment $8-35 \mathrm{~cm}$ long, herbaceous, dark green, \pm glabrescent; rhachis usually with a narrow wing except for the lowest part; pinnae alternate to opposite, $14-40$, up to $65 \mathrm{~mm}$ apart; central pinnae index 3-7, widest usually about the middle, $5.5-17.5(-21)$ by $1.5-5 \mathrm{~cm}$, base \pm symmetrical, broadly cuneate to auricled, margin lobed $1 / 3-2 / 3(-3 / 4)$ towards the costa, apex orbicular or acuminate, lobes spaced to partly overlapping, c. 6-8 $\mathrm{mm}$ wide at the base, margin usually entire, sometimes finely crenate-serrate, apex orbicular; the two lowermost pinnae deflexed or not, either \pm conform to the central pinnae, or its basiscopic side pinnatifid or again pinnate, 1-8 $\mathrm{mm}$ stalked; terminal segment narrowly triangular, the apex usually either prolonged or flagelloid; venation pattern: secondary veins on either side with 4-6 tertiary veins, see also fig. 54: $\mathrm{g}-\mathrm{k}$. Fertile leaves 18-85 cm long; petiole $8-50 \mathrm{~cm}$ long; lamina index $2-5(-7), 8-40$ by $2-13 \mathrm{~cm}$, without the terminal segment 5-35, the terminal segment $1.5-7 \mathrm{~cm}$ long; pinnae $12-35$, up to $70 \mathrm{~mm}$ apart; central pinnae index $2-8,1.3-6$ by $0.4-1.2 \mathrm{~cm}$, margin \pm entire to slightly lobed especially in the basal part. Sporangia usually mainly inserted on and near the veins. Spores with a smooth cristate-undulate perispore.

Chromosomes. $n=41 ; 2 n=82$.

CHINA. S. Yun nan: 6 collections.

BANGLADESH. Syl he t: Wallich 2685 (type of Acrostichum appendiculatum var. ludens).

INDIA. Assam: 25 collections (13 collections from the Khasya Hills).-Orissa: Mooney 1468 (K), $4097(\mathrm{~K})$.

Burma. Central: Dickason 6621 (BM, GH), 6657 (BM); Lace $4900(\mathrm{~K})$.-Southern Shan States:

Dickason $9140(\mathrm{GH})$. - T en a s s e ri m: Parish 60 (type of Acrostichum appendiculatum var. costulata).

THAiland. Northern: many collections. - Northeastern: Hennipman 3624 (L); Tagawa et al. T

1100 (L, US); Shimizu et al. $T 11381$ (L). -Southeastern: Iwatsuki \& Fukuoka T7169(L).

CAmbodia. Poilane 28761 (P).

VIETnam. Coch in chin a: Matthew s.n. (K), -A n n a m. South: Poilane 23888 (P), 24408 (BM, P).

JAVA. Ea s t: Mousset 873 (MICH).

Lesser SUNDA IsLands. B ali: Holstioogd 809 (L), 873 (L); Posthumus 3704 A (BO, ster.).-Su mbawa: Rensch 630 (B, ster.).

H a bitat. Usually terrestrial, creeping either in soil or on rocks, sometimes lowepiphytic (up to $1 \mathrm{~m}$ ), in (hill) evergreen (monsoon) forest. I found it in several places 

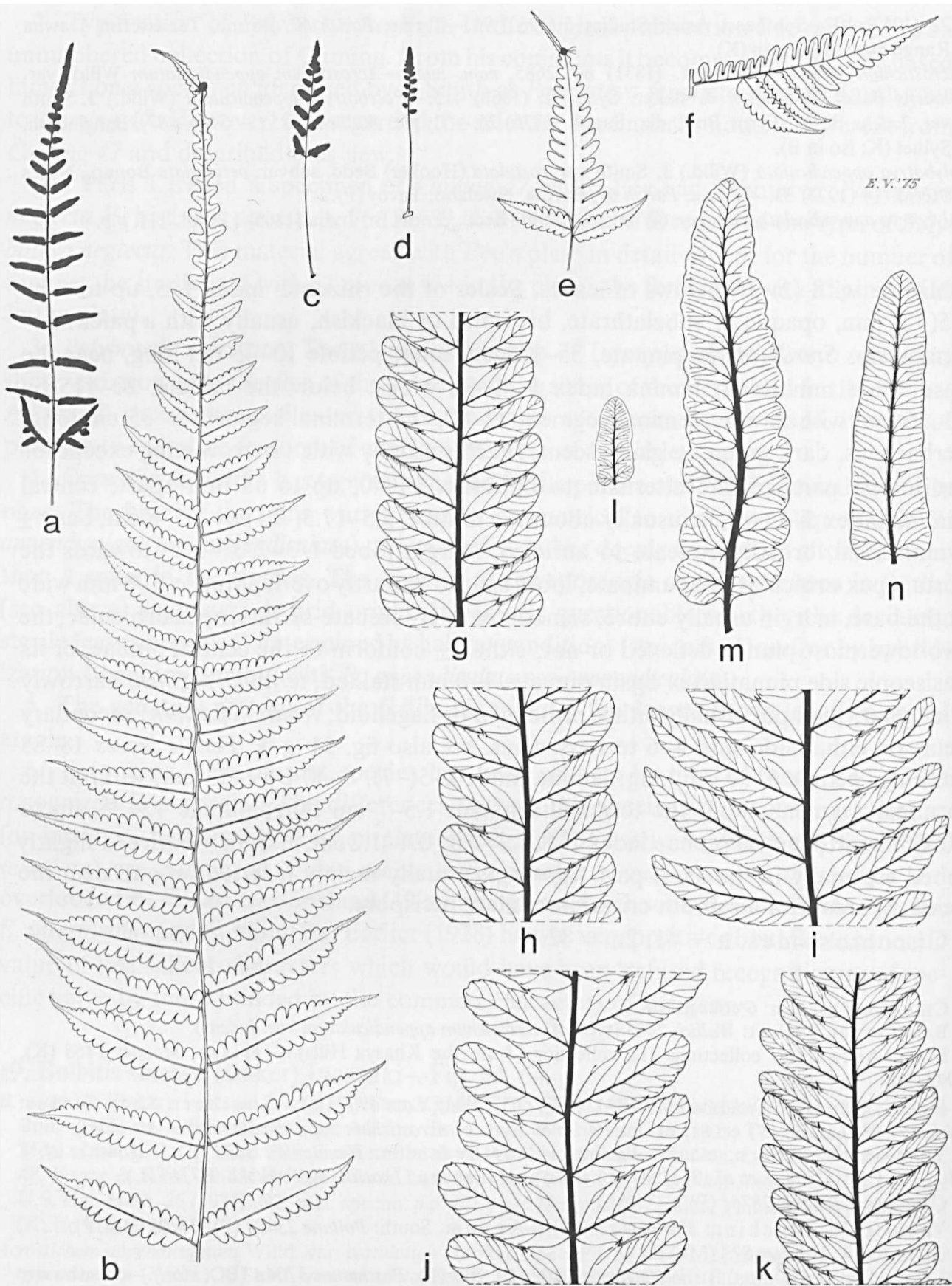
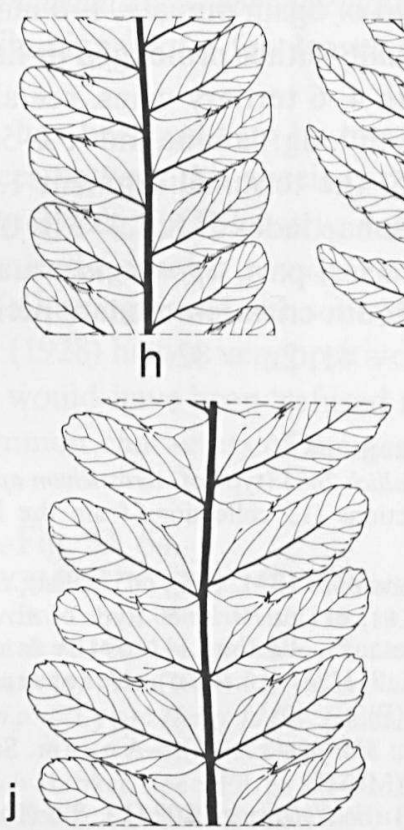

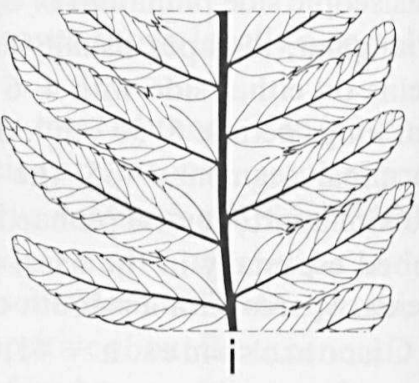

Fig. 54. Bolbitis sinensis. $-a-d$. sterile and fertile leaves, $\times 1 / 5$; e. terminal part of sterile leaf, $\times 1 / 5$; $\mathrm{f}$. lowermost pinna of sterile leaf, $\times 1 / 5 ; \mathrm{g}-\mathrm{k}$. venation pattern of sterile pinnae, $\times 4 / 5 ; 1-n$. ibid. of fertile pinnae (a. Parish $60, \mathrm{~K}$, holotype of Acrostichum appendiculatum var. costulatum; b, e, f, j, l-n. Hennipman 3229, L; c. H. 3389, L; d. Beddome s.n., K; g. Hennipman 3229A, L; h, i. Van Beusekom 1328, L; k. Rock 2913, US). 
in northern Thailand to be locally the dominant ground fern forming stands of many specimens densely growing together. Altitude: $0-1900 \mathrm{~m}$.

S p ores. Van Beusekom 1328 (L), Hennipman 3155 (L), 3229 (L), Henry 12494 (type of Acrostichum sinense, US), Holstvoogd 809 (L), Rock 2657 (C, US): the greater part normal. Cavalerie 4230 (L): in part normal. Griffith s.n. (BM), Parish 60 (type of Acrostichum appendiculatum var. costulatum): the greater part (all?) abnormal.

Notes. 1. A clear-cut species primarily characterized by the combination of free veins, symmetrical pinnae, and an undulate-cristate perispore. It may be related to $B$. appendiculata and is certainly so to B. tonkinensis (= spec. dub., see also note 2 ). The relationship to $B$. major, which has \pm similarly shaped sterile and fertile pinnae, is obscure.

The species is confined to regions in Asia with a monsoon climate in both the northern and the southern hemisphere.

Most authors recognized two species within this complex which are primarily based on differences in shape of the two lowermost pinnae (see note 4).

2. Variability.

a. The collections of Rock. Rock made several collections in southern Yunnan which were critically studied by Christensen. The latter author noticed the difference in venation pattern of the sheets he received: $\operatorname{Rock} 2657(\mathrm{C} ;=B$. sinensis $)$ with free veins; Rock 2913 (BM; = B. sinensis s.l., see below) with a venation pattern sometimes showing a costal areole; and Rock $2636(=B$. angustipinna $\times$ sinensis $)$ with the veins rather irregularly anastomosing into a pattern that Christensen thought not to be materially different from that of Campium angustipinnum $(=B$. angustipinna). Christensen commented: 'It is without question that the specimens mentioned belong to the same species, ... and as mentioned before, it is very probable that Egenolfia and Campium ( = Bolbitis) should be united.' Iwatsuki (1959) largely based himself on Christensen when he formalized this view.

Except for the venation pattern, Rock 2913 (BM) is almost typical. The venation pattern of the pinnae not only deviates by the occurrence of costal areoles (which are occasionally also present in Rock 2657, US) but also as the most distal tertiary vein generally springs directly from the costa and not from the secondary vein. The small fertile leaf is heavily attacked by a fungus. Still, nearly all sporangia are mature and not infected. The majority of these is filled with shrivelled spores, a minority with either aborted S.M.C.'s or with obviously normal spores.

Rock 2913 (US) differs somewhat from the specimen in BM as regards details of colour, texture, and shape of the sterile leaf. The shape of the fertile leaf is typical. Spores: nearly all sporangia filled with 64 normal, or largely normal spores having an undulate-cristate or a spiny perispore. The spores of this specimen are described in detail in chapter 6.

b. Two plants which originated from northern Thailand were cultivated side by side in the Botanic Garden, Leiden. One has been collected by Nooteboom on Doi Suthep, the other by myself (Hennipman 3229) on Doi Chieng Dao. Both are diploid. During two seasons with excellent growth these two plants behaved differently. The plant of Nooteboom formed small, rather erect sterile and fertile leaves, whereas mine formed 
much larger and nearly exciusively sterile, paler leaves which touched the ground, the bulbils developing into small plants.

c. Plants more or less intermediate between $B$. sinensis and $B$. appendiculata ssp. appendiculata are known from Tonkin and Burma. They agree fairly well with $B$. tonkinensis (see Spec. dub.) but are smaller and the leaves lack residual scales. These plants include Cadière 1146 (P) and Herb. Ec. Sup. Agric. \& Sylvic. Hanoi 4078 (Colani leg.) (BM, MICH, P, UC), both from Tonkin, and Parish 69 (type of Polybotrya appendiculata subvar. petiolulata) and 71 from Burma, Tenasserim. Beddome's figures (1865, pl. 111, the two upper right figures) were prepared from these Burmese collections.

3. A considerable part of the specimens has fertile leaves with a fungal infection. The fungus forms a variously thick whitish mycelium around all parts of the leaf. Hyphae are found between the sporangia and inside part of mostly juvenile sporangia. Occasionally sporangia of mature size were found with a number of normal spores embedded in a loose reticulum of hyphae (Hennipman 3229, L).

4. The present species was first recognized by Hooker (1864)-who lumped all the egenolfioid ferns into Acrostichum appendiculatum-as var. costulata. He characterized this variety by the central pinnae which are symmetrical and pinnatifid, and the two lowermost pinnae which are bipinnatifid. As Hooker already remarked, the shape of the two lowermost pinnae shows considerable variation; they may also be \pm conform to the central pinnae. Lowermost pinnae representing both extremes are found on different leaves from the same plant. The intermediate condition is frequent (for instance the type of Acrostichum sinense).

Tagawa \& Iwatsuki (1967), in contrast with Iwatsuki (1959), agreed with the view that differences in the shape of the two lowermost pinnae have no taxonomic value. Still, they recognized a var. costulata of $B$. sinensis representing plants 'which are usually larger than those of the type variety'. However, the shape of the basal pinnae is not correlated with the size of the leaves: the type of var. costulata represents a small plant.

The differences given by Nayar \& Kaur between Egenolfia sinensis and E. bipinnatifida are either trifling or not represented in the reference sheets of both studied by me ( $L W G 83037$ and $L W G$ 77006; both in LWG).

5. The present species was reported from East Java by Backer \& Posthumus and by De Joncheere (Amer. Fern J. 43, 1953, 45). The material upon which Backer \& Posthumus based themselves was not traced, that of De Joncheere was lost during World War II (Van Steenis-Kruseman, Fl. Mal. I, 1, 1950, 265).

6. See also B. bipinnatifida, note 6 .

\section{Series Euryostichae Hennipman, ser. nov.}

Anapausia Presl subgen. Euryostichum Presl, Epim. Bot. (1951) 188, typo incl, pro subgenere.

Terrestrial or on tree bases. Rhizome short-creeping, slender or not, with 2, in one species $(B$. nicotianifolia) with $2-5$, rows of leaves, with sclerenchyma strands. Scales 
of the rhizome usually \pm appressed, in one species (B. pergamentacea) almost spreading, up to $10 \mathrm{~mm}$ long, usually flat, in one species $(B$. pergamentacea $) \pm$ bullate. Leaves approximate or up to $40 \mathrm{~mm}$ apart. Sterile leaves usually pinnate, sometimes simple; petiole with 5-20 vascular bundles; lamina: bulbil(s) if present either elongate and subterminal on the terminal segment or \pm spherical and axillary, appressed scales
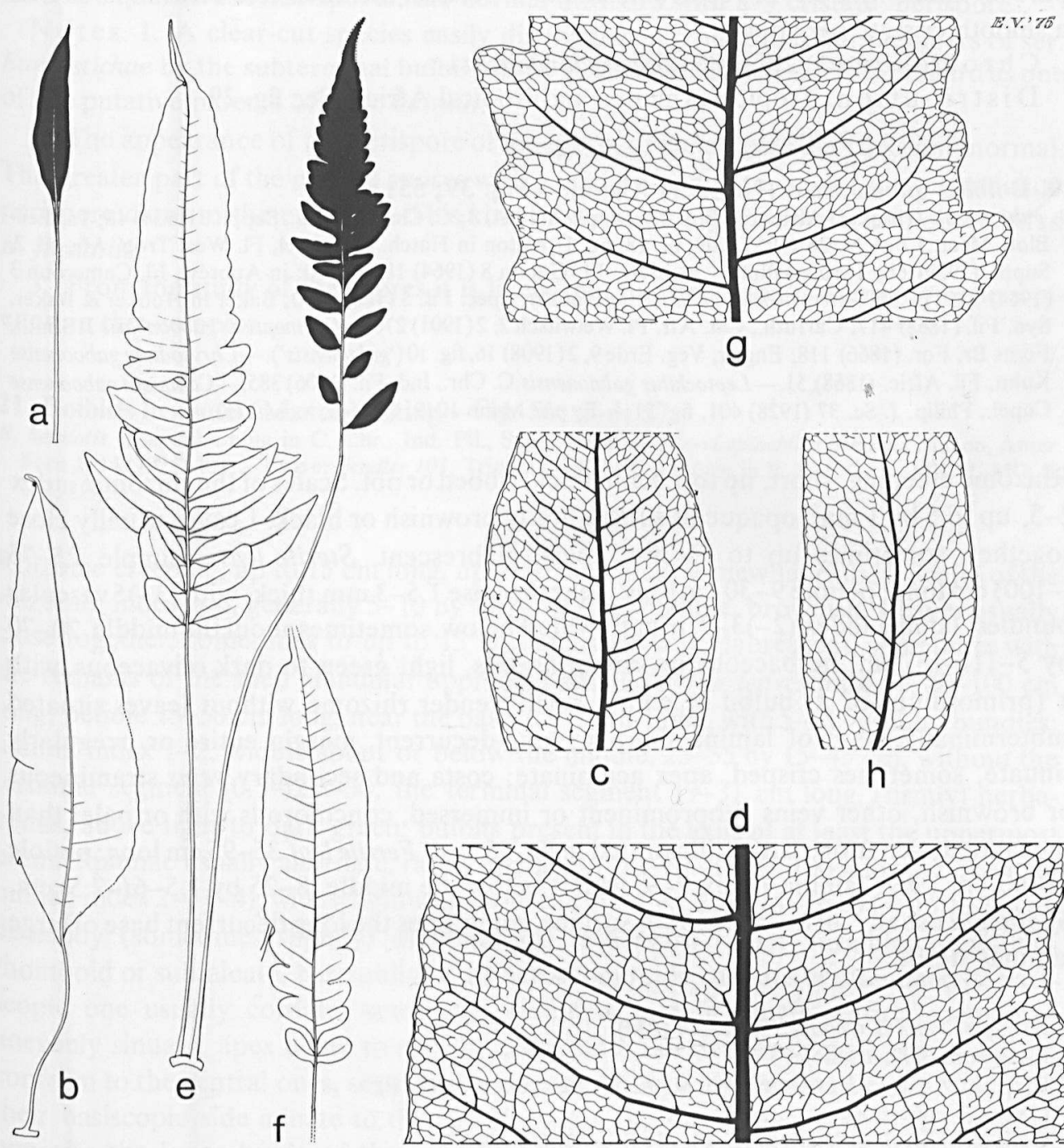

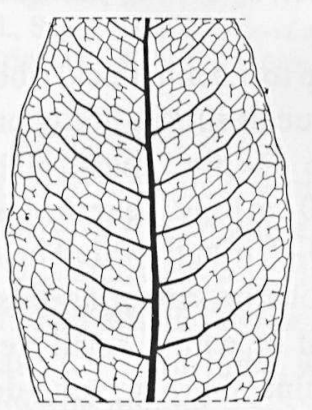

C

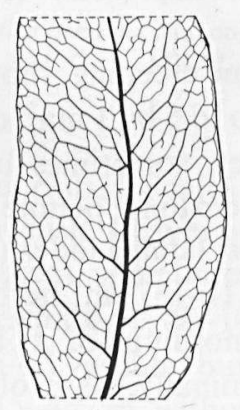

$\mathrm{h}$

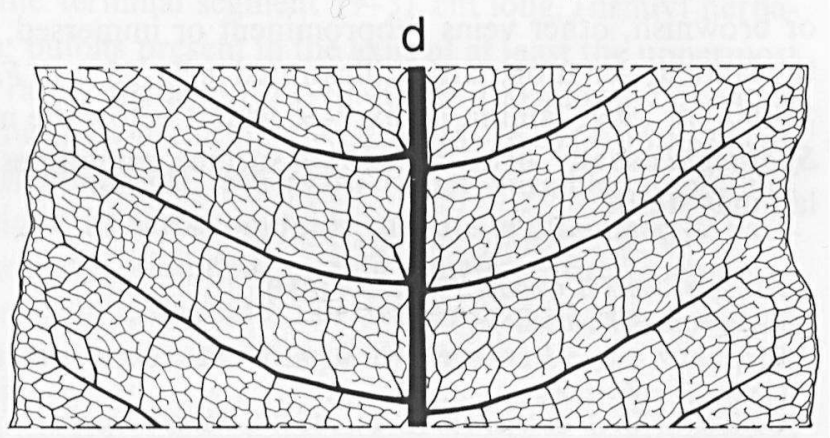

Fig. 55. a-d. Bolbitis gaboonensis, a, b. sterile and fertile leaf resp., $\times 1 / 5$; c, d. venation pattern of sterile laminas, X 4/5 (a, b. Mann 1049, K, holotype of B. gaboonensis; c, d. Zenker 2456, L).-e-h. Bolbitis $\times$ boivinit. e, f. sterile and fertile leaves, $\times 1 / 5$; g. venation pattern of sterile lamina, $\times 4 / 5 ; \mathrm{h}$. ibid. of sterile pinna (e. Welwitsch $156, \mathrm{~K}$, lectotype of $B$. X boivinii; f. Anonymus s.n., 17-viii-1946, P; g. Letouzey 2925bis, L; h. Welwitsch 156, BM, see e). 
on the rhachis deeply cut, the ultimate divisions ending in a glandular cell; rhachis not winged; pinnae $2-8$, the margin without teeth or spines; terminal segment \pm conform to the pinnae though somewhat, in one species (B. oligarchica) much larger; venation: secondary veins prominent on either side, tertiary veins prominent or immersed; venation pattern: secondary veins connected by \pm curved transverse tertiary veins, the areoles including a network of smaller areoles varying in size and shape in part of which with ex- or recurrent, simple or forked, included free veins. Spores with a smooth cristate perispore.

Chromosomes. $2 \mathrm{n}=82$ (B. pergamentacea).

Distribution. Tropical America and tropical Africa. See fig. 29.

20. Bolbitis gaboonensis (Hooker) Alston.-Fig. 39; 55: a-d.

B. gaboonensis (Hooker) Alston, J. Bot. 72, Suppl. 2 (1934) 3; C. Chr., Ind. Fil., Suppl. 3 (1934) 48; TardieuBlot, Mém. I.F.A.N. 28 (1953) 109, pl. 18: fig. 1; Alston in Hutch. \& Dalziel, Fl. West Trop. Afr. ed. 2, Suppl. (1959) 68; Tardieu-Blot in Aubrév., Fl. Gabon 8 (1964) 184, pl. 30; in Aubrév., Fl. Cameroun 3 (1964) 315, pl. 50.-Acrostichum gaboonense Hooker, Spec. Fil. 5 (1864) 270; Baker in Hooker \& Baker, Syn. Fil. (1868) 417; Carruth., Cat. Afr. Pl. Welwitsch 2, 2(1901) 276.-Gymnopteris gaboonensis J. Smith, Ferns Br. For. (1866) 118; Engler, Veg. Erde 9, 2 (1908) 16, fig. 10 ('gabunensis'). -Chrysodium gaboonense Kuhn, Fil. Afric. (1868) 51. - Leptochilus gaboonensis C. Chr., Ind. Fil. (1906) 385. - Campium gaboonense Copel., Philip. J. Sc. 37 (1928) 401, fig. 51.-T y p e: Mann 1049, Guinea, 'coast' (K).

Rhizome creeping, short, up to $4.5 \mathrm{~mm}$ thick, ribbed or not. Scales of the rhizome: index $2-5$, up to 5 by $1 \mathrm{~mm}$, opaque to subclathrate, brownish or black. Leaves usually close together, sometimes up to $15 \mathrm{~mm}$ apart, glabrescent. Sterile leaves simple, 25-75 $(-100) \mathrm{cm}$ long; petiole $9-30 \mathrm{~cm}$ long, near the base $1.5-3 \mathrm{~mm}$ thick, with (3-)5 vascular bundles; lamina index (2-)3-7, usually widest below, sometimes about the middle, 20-70 by $5-11(-13) \mathrm{cm}$, herbaceous or subcoriaceous, light green to dark olivaceous, with a (primordium of a) bulbil or with a small slender rhizome without leaves situated subterminally, base of lamina short- to long-decurrent, margin entire or irregularly sinuate, sometimes crisped, apex acuminate; costa and secondary veins stramineous or brownish, other veins subprominent or immersed, concolorous with or paler than the lamina; venation pattern illustrated in fig. $55: \mathrm{c}$, d. Fertile leaf $35-95 \mathrm{~cm}$ long; petiole $15-60 \mathrm{~cm}$ long; lamina index $2-9$, widest below the middle, $8-25$ by $1.5-6(-7.5) \mathrm{cm}$. Sporangia usually all over the lower surface, sometimes the long decurrent base of large laminas sterile.

NigerIA. Jones \& Onochie FHI 17514 (BM).

CAMeroun. 10 collections.

Central African Republic. Cervoni s.n. (P); Tisserant 2716 (L, P).

GABON. 5 collections.

Congo (BrazZ). Charvet 160 (L, P), 184 (P); Thollon 719 (P).

ZaIRE. Le o pold vill e: 29 collections.-Equat e u r: Laurent s.n., 22-xii-1903 (BR); Néré 2226(P).Orient a le: 5 collections. -K as a i: Laurent s.n., xi-1895 (BR).

ANGOLA. Ca bind a: Gossweiler 7647 (BM, P). - Cu a n z a Nort e: Welwitsch 155 (BM, K).

$\mathrm{H}$ abitat. Moist places in rain forest; reported from streambanks and from rocks in rivulets. Altitude: $0-500 \mathrm{~m}$. 
Spores. Luja 216 (BR): the majority normal with a cristate perispore. Vanderijst 8019 (BR): likewise but the appearance of the perispore different, see note 2. Thollon 719 (P): in part normal. Callens 2819 (BM), Welwitsch 155 (BM, K): the majority of the sporangia filled with aborted spores, a minority of the sporangia with aborted S.M.C.'s: some normal spores also present in the preparations. Mann 1049 (type of Acrostichum gaboonense), Zenker 3806 (BM, K, L): sporangia filled with either (aborted and 'normal') S.M.C.'s and/or aborted spores, the normal S.M.C.'s with a \pm cristate 'perispore'.

Notes. 1. A clear-cut species easily distinguished from the other members of ser. Euryostichae by the subterminal bulbil on the sterile leaf. B. gaboonensis I regard as one of the putative parents of $B . \times$ boivinii.

2. The appearance of the perispore of the spores of Vanderijst 8019 (BR) is abnormal. The greater part of the normal spores with a cristate-undulate, a few with an undulate perispore close to the exospore. This kind of variation in perispore is also observed in B. fluviatilis.

3. From the study of the spores it is likely that hybridization is a common phenomenon in this complex.

21. Bolbitis hemiotis (Maxon) Ching-Fig. 56: a-d; 57.

B. hemiotis (Maxon) Ching in C. Chr., Ind. Fil., Suppl. 3 (1934) 48.-Leptochilus hemiotis Maxon, Amer. Fern J. 14 (1925) 101.- Ty p e: Fendler 101, Trinidad, 1877-80 (US; iso in B, BM, G, K, MICH, MO, S, UC, US).

Rhizome creeping, up to $15 \mathrm{~cm}$ long, up to $10 \mathrm{~mm}$ thick, somewhat ribbed. Scales of the rhizome: index $2-6$, generally $5-10$ by $1-3(-4) \mathrm{mm}$, opaque, brownish. Leaves usually close together, sometimes to up to $15 \mathrm{~mm}$ apart, usually glabrescent, sometimes with the remains of the shed glandular appressed scales. Sterile leaves pinnate, $40-100 \mathrm{~cm}$ long; petiole $15-50 \mathrm{~cm}$ long, near the base $2.5-7 \mathrm{~mm}$ thick, with 5-11 vascular bundles; lamina index $1-2$, widest about or below the middle, $25-55$ by $15-45 \mathrm{~cm}$, without the terminal segment (0.5-)2.5-30, the terminal segment 19-31 cm long, (firmly) herbaceous, above light to dark green, bulbils present in the axils of at least the uppermost pinnae; pinnae usually alternate, rarely opposite, (2) 3-6(-8), 40-100 mm apart; central pinnae index $2-3(-4)$, widest below or about the middle, (8.5-) $15-26$ by (4-) $5.5-12 \mathrm{~cm}$, distinctly (sometimes slightly) asymmetrical, otherwise rather variable, sometimes rhomboid or subfalcate, base oblique, its acroscopic side cuneate or attenuate, its basiscopic one usually cordate, sometimes rounded, margin usually entire, sometimes unevenly sinuate, apex acute to shortly acuminate; the two lowermost pinnae almost conform to the central ones, sessile or up to $3 \mathrm{~mm}$ stalked, uppermost pinnae often with their basiscopic side adnate to the rhachis, the uppermost pinna sometimes inserted opposite the longest side of the usually unequally based terminal segment; terminal segment $7.5-13.5 \mathrm{~cm}$ wide, sometimes almost symmetrical and either cuneate or decurrent; rhachis, costae, and secondary veins stramineous or brownish, other veins immersed or almost so, concolorous with the lamina; venation pattern illustrated in fig. 56: d. Fertile leaf $40-115 \mathrm{~cm}$ long; petiole 30-65 cm long; lamina index 1-3(-4), widest about or below the middle, (10-)15-35(-50) by 5-17 cm, without the terminal segment 

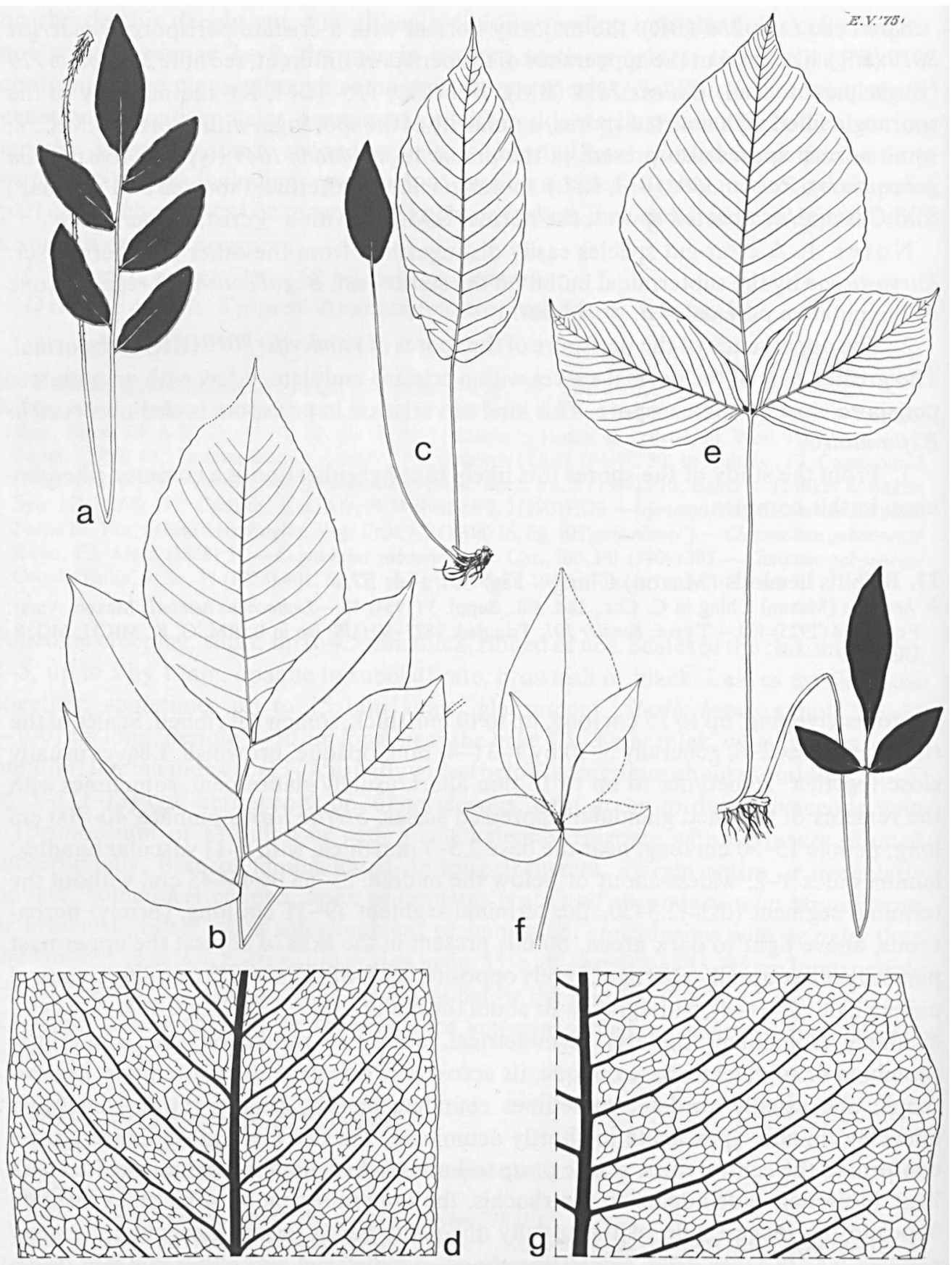

Fig. 56. a-d. Bolbitis hemiotis. a-c. sterile and fertile leaves, $\times 1 / 5$; $d$. venation pattern of sterile pinna, $X$ 4/5 (a. Broadway s.n., BM; b. Fendler 101, BM, isotype of B. hemiotis; c. Aldridge s.n., BM; d. Jermy 2642 , BM). -e-g. Bolbitis oligarchica. e. sterile and fertile leaf, $\times 1 / 5$; f. sterile leaf with developing bud, $X$ 1/5; g. venation pattern of sterile pinna, $\times 4 / 5$ (e. Rojas 98 , US; f. Skutch 2972 , US; g. Idrobo \& Schultes 1113 , US). 
1.5-22(-37), the terminal segment 7-14 cm long; pinnae (2)3-9, 15-100 mm apart; central pinnae index $2-4,4-12.5$ by $1.5-5 \mathrm{~cm}$, apex round; terminal segment $2.5-5.5 \mathrm{~cm}$ wide. Sporangia all over the lower surface except for a narrow marginal strip.

TrINIDAD. Northern part: 16 collections.

VeneZUELA. Su c r e: Steyermark \& Agostini 91193 (US); Steyermark \& Rabe 96119 (U, ster.)._A r a gua: Oberwinkler 14678 (M, ster.).

See also note 4.

$\mathrm{H} \mathrm{a} \mathrm{b} \mathrm{i} \mathrm{t} \mathrm{a} \mathrm{t.} \mathrm{On} \mathrm{the} \mathrm{ground} \mathrm{or} \mathrm{on} \mathrm{rocks} \mathrm{in} \mathrm{wet} \mathrm{forest} \mathrm{gullies.} \mathrm{Altitude:} 200-900 \mathrm{~m}$. S por e s. Jermy 2638 (BM), 2640 (BM), 2906 (BM): the majority normal, a minority shrivelled. Fendler 101 (isotype, GH): \pm equal parts normal and abnormal. Fendler 101 (isotype, BM), Hombersley $61 b$ (US): the majority abnormal. Fendler 101 (holotype): all abnormal, the greater part shrivelled, a few normally shaped but empty; a few sporangia with aborted S.M.C.'s.

Not e s. 1. Closely related to the other species of the series, to B. oligarchica in particular. The latter differs amongst others in leaf architecture, in texture, and in the shape of the base of the pinnae.

2. A few plants were found with leaves composed of only two alternately placed pinnae and a markedly asymmetrical terminal segment. Fendler 101 (MO) contains also a plant consisting of four small simple sterile leaves with a \pm symmetrical base. One simple fertile leaf was found, see fig. 56: $\mathrm{c}$.

3. In the greatest part of the material the rhachis is more or less flexuous and often finely spotted by the darker scars of the scales.

4. I have my doubts about the following locality records: Jamaica(Fielding s.n., BRI), and Ecuador (Hinds s.n., 'brought on board', US); these are excluded from the distribution map.

22. Bolbitis nicotianifolia (Swartz) Alston-Fig. 58: a-e.

B. nicotianifolia (Swartz) Alston, Kew Bull. (1932) 310; C. Chr., Ind. Fil., Suppl. 3(1934) 49, 198; Vareschi in Lasser, Fl. Venezuela 1 (1969) 373, p.p. -Acrostichum nicotianifolium Swartz, Syn. Fil. (1806) 13, 199 ('nicotianaefolium'); Poiret in Lam., Enc., Suppl. 1 (1810) 122; Willd., Spec. P1. 5 (1810) 118; Hooker, Garden Ferns (1862) pl. 26 (excellent); Spec. Fil. 5 (1864) 275, p.p.; Baker in Hooker \& Baker, Syn. Fil. (1868) 419, p.p.; in Martius, Fl. Bras. 1, 2 (1870) 589, pl. 39: fig. 9, p.p.-Gymnopteris nicotianifolia Presl, Tent. Pterid. (1836) 244; Fée, Hist. Acrost. (1845) 86, quoad nomen solum; J. Smith, Ferns Br. For. (1866) 118, p.p.; Christ, Farnkr. Erde (1897) 51, p.p. - Anapausia nicotianifolia Presl, Epim. Bot. (1851) 189.Chrysodium nicotianifolium Mettenius, Fil. Lips. (1856) 22, quoad nomen solum.-Leptochilus nicotianifolius C. Chr., Bot. Tidsskr. 26 (1904) 285; Maxon, Sc. Surv. Porto Rico \& Virgin Is. 6 (= Pterid. Porto Rico \& Virgin Is.) (1926) 459.-T y pe: Ventenat s.n., Lesser Antilles, Virgin Is., St. Thomas (S-PA). See note 5. Acrostichum acuminatum Willd., Spec. PI. 5(1810, January) 116, p.p., non Jussieu ex Poiret in Lam. (1810, September).-Gymnopteris acuminata Presl, Tent. Pterid. (1836) 244; Fée, Hist. Acrost. (1845) 85, p.p., pl. 46: fig. ii.-Anapausia acuminata Presl, Epim. Bot. (1851) 188.-Chrysodium acuminatum Mettenius, Fil. Lips. (1856) 22. - Photinopteris acuminata Morton, Contr. U.S. Nat. Herb. 38 (1967) 31.-L e c tot y p e (Presl, 1851): Plumier, Tract. Fil. Amer. (1705) 100, pl. 115, 'Lingua cervina, scandens, Citrei foliis, major' drawn from Plumier s.n., Lesser Antilles, eastern Martinique, St. Maria (P-JU). See note 6.

Gymnopteris acuminata Presl var. heterophylla Fée. Hist. Acrost.(1845)86. - T y p e:Anonymous (Herminier?), Lesser Antilles, Guadeloupe (P, 3 sh., 'Acrostichum fallax Bory', MS).

Acrostichum nicotianifolium Swartz var. saxicolum Jenman, Bull. Bot. Dept. Jamaica (Kingston) n.s. 5(1898) 153.-T y p e: Jenman s.n., Greater Antilles, Jamaica (K). 


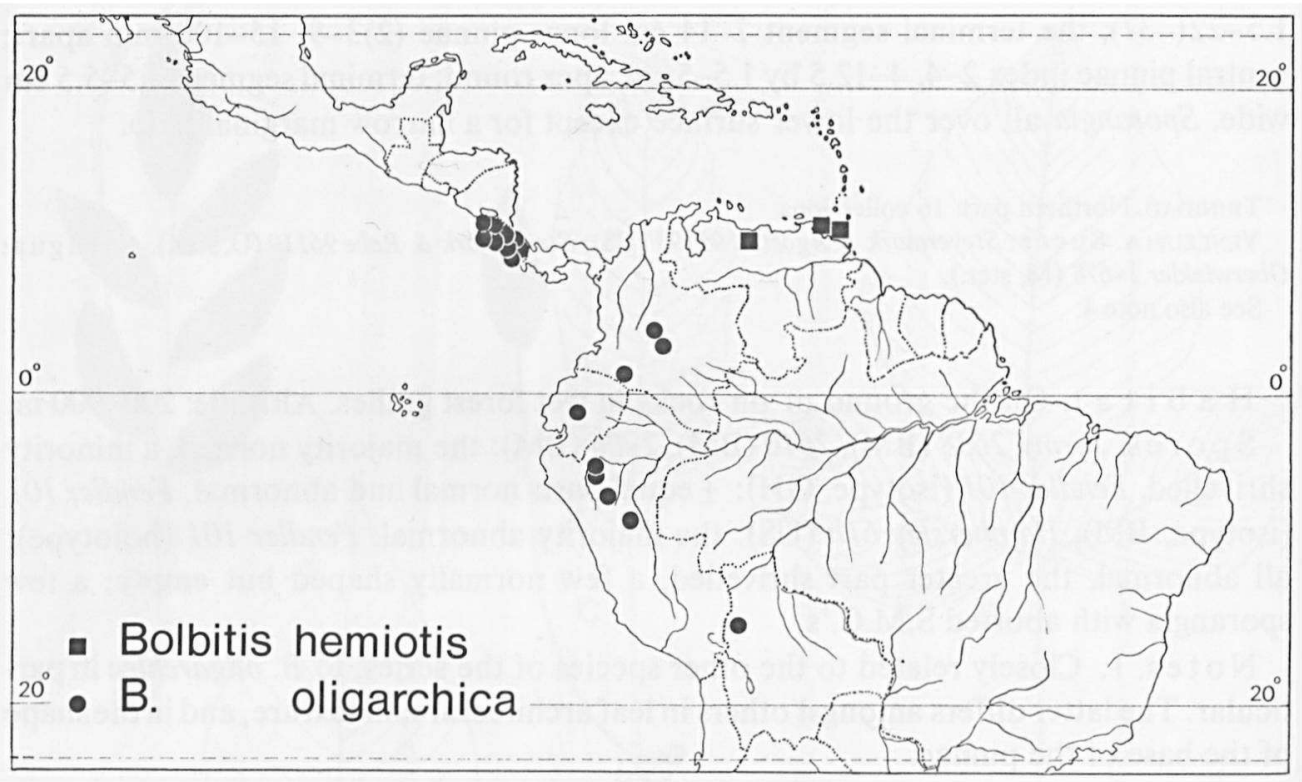

Fig. 57. Distribution of Bolbitis hemiotis and B. oligarchica.

Leptochilus nicotianifolius (Swartz) C. Chr. var. simplex Rosenst., Fedde Rep. 22 (1926) 23.-T y p e: Brade ('Brade \& Brade') 552, Costa Rica, Llanuros de San Carlos, $200 \mathrm{~m}$ alt. (S-PA).

Leptochilus killipii Maxon, Amer. Fern J. 21 (1931) 138.-B. killipii Lellinger, Fern Gaz. 11 (1975) 107.T y p e: Killip 2778, Panama, 4 miles above Juan Diaz (US; iso in B, P, S-PA).

B. bradeorum auct. non (Rosenst.) Ching. Ching in C. Chr., Ind. Fil., Suppl. 3 (1934) 47, p.p.

Rhizome creeping or climbing, up to $15 \mathrm{~cm}$ long, up to $15 \mathrm{~mm}$ thick, slightly ribbed. Scales of the rhizome: index 4-9, 5-10 by $0.5-1.5 \mathrm{~mm}$, opaque to subclathrate, dark brown or blackish. Leaves rather close together to up to $45 \mathrm{~mm}$ apart, glabrescent. Sterile leaves usually pinnate, rarely simple. Pinnate leaves $40-125 \mathrm{~cm}$ long; petiole $14-$ $55 \mathrm{~cm}$ long, near the base $2.5-6 \mathrm{~mm}$ thick, with $7-18$ vascular bundles; lamina index $1-2(-3)$, usually widest about, sometimes below the middle, $20-65(->70)$ by $15-40(-60)$ $\mathrm{cm}$, without the terminal segment $0.5-25(-40)$, the terminal segment $20->45 \mathrm{~cm}$ long, thin-herbaceous, light to dark green, without bulbils; pinnae usually alternate, 2-10, 0-80 mm apart; central pinnae index $2-5$, usually widest about or below, rarely above the middle, (5.5-) $13-37$ by (2-)3.5-9.5 cm, usually with a proportionally large central part with parallel margins, sometimes biconvex, the upper half slightly falcate or not, the basal half usually somewhat asymmetrical, base variously truncate, margin entire or slightly sinuate, apex attenuate or acuminate; the two lowermost pinnae usually \pm conform to the central ones, sometimes with a more or less marked basal basiscopic lobe, sessile or up to $7 \mathrm{~mm}$ stalked, the uppermost pinnae usually sessile, sometimes the basiscopic side of the base shortly adnate to the rhachis; terminal segment $5-14 \mathrm{~cm}$ wide; 
costa and secondary veins stramineous, tertiary veins slightly prominent, stramineous or concolorous, other veins immersed or almost so, usually concolorous with the lamina, sometimes stramineous, the secondary veins spreading and ending some distance from the margin; venation pattern illustrated in fig. 58: d. Simple leaves $29-44 \mathrm{~cm}$ long; petiole $7-16 \mathrm{~cm}$ long; lamina index $3-4,21-30$ by $6-9 \mathrm{~cm}$, otherwise similar to the terminal segment of pinnate leaves. - Fertile leaves. Pinnate leaves 30-110 cm long; petiole $20-65 \mathrm{~cm}$ long; lamina index $1-3$, widest somewhat below or about the middle, $12-50$ by $7-25 \mathrm{~cm}$, without the terminal segment $2-30$, the terminal segment $8-20 \mathrm{~cm}$ long; pinnae 2-10, $20-85 \mathrm{~mm}$ apart; central pinnae index $2-6,4-14.5$ by $0.8-3 \mathrm{~cm}$, base truncate to subcordate, the apex acute or orbicular; the two lowermost pinnae sessile or up to $7(-14) \mathrm{mm}$ stalked, the two uppermost pinnae sessile to shortly adnate to the rhachis; terminal segment $1.5-4.5 \mathrm{~cm}$ wide. Simple leaves $32-40 \mathrm{~cm}$ long; petiole $20-28 \mathrm{~cm}$ long; lamina index $4-7,10-18$ by $2-4.5 \mathrm{~cm}$, otherwise similar to the terminal segment of pinnate leaves. Sporangia all over the lower surface, except for a usually indistinct marginal strip.

Guatemala. Standley 24202 (GH, US), 49365 (US); Von Türckheim II 1077 (BR), 8829 (US).

Honduras. Ray 2139 (GH); Shank \& Antonio Molina R. 4873 (GH); Standley 52943 (F, US), 54164 (US).

Costa Rica. 10 collections.

JAMAICA. 8 collections.

DOMINICAN REPUBLIC. 10 collections.

Puerto Rico. 10 collections.

VIRGIN Is. Ventenat s.n. (type of Acrostichum nicotianifolium).

GuADELOUPE. 11 collections.

MARTINIQUe. Bélanger s.n. (P); Duss 1699 (B, US); Hahn 10 (BM, P).

British GuYana. Appun 882 (K); Jenman 2142 (K, U); Mell \& Mell 207 (US); Schomburgk s.n. (K).

SURINAM. Kappler 8 (L); Schulz 8922 (GH), 10252 (US).

French GuYana. LePrieur 62 (G, P); Mélinon 364 (P); (ex herb.?) Rudge s.n. (BM).

Panama. 12 collections.

Colombia. 10 collections.

ECUADOR. Sodiro s.n., 1883 (K, UC), s.n., 1891 (P); Verleysen s.n. (P).

Peru. 7 collections.

See also note 8 .

$\mathrm{H}$ a bit a t. Terrestrial or on tree bases; in rain forest. Altitude: up to $600 \mathrm{~m}$.

Sp ores. Haught 1432 (US), Herminier s.n. (L, 908.328-291), Killip \& Garcia 33530 (US): the greater part normal. Cuatrecasas \& Llano 24199 (US), Schunke 372 (US): the greater part abnormal. Haught 2037 (GH): sporangia filled either with aborted often clustered S. M. C.'s, or with shrivelled spores. See also notes 4, 5, and 7.

Notes. 1. A variable species which is in particular characterized by the combination of features from rhizome scales and leaves (architecture and texture). It is very close to $B$. oligarchia which has, to mention a salient character, ternate leaves with axillary bulbils, and to $B$. pergamentacea with which it was long confused by many authors.

2. Generally the specimens from Central America and western tropical South America have more prominent and more stramineous veins which are, moreover, arranged in a more coarsely reticulated venation pattern, if compared with those from the West Indies and Guyana.

3. The terrestrial specimens usually have their leaves in two rows. Large epiphytes 
(only known from Colombia and Peru) may have their leaves arranged in 4 or 5 rows. The rhizome anatomy of these epiphytes is similar to that of $B$. lindigii and $B$. bernoullii, but sclerenchyma strands are included in the ground tissue.

4. Specimens with simple sterile and fertile leaves. Simple sterile leaves precede pinnate ones during development. The following specimens have both simple fertile and sterile leaves:

Spruce 4636 (syntype of Acrostichum oligarchicum). Spores nearly all shrivelled or otherwise abnormal, a few obviously normal.The sterile laminas agree in detail with the sterile pinnae of Spruce $28(\mathrm{P})$, which represents a large plant of which the greater part of the spores is abnormal.,

Killip 2778 (type of Leptochilus killipii). Spores: the US specimen: nearly all normal, a considerable number of the sporangia young; the S-PA specimen: likewise but young sporangia lacking. Except for the leaf architecture the plants agree in detail with those of Killip 2753 (GH, US) which was collected from a similar habitat ('muddy banks of a small stream') near the site where Killip 2778 was gathered. The GH sheet of Killip 2753 comprises a plant with pinnate leaves as well as one with simple sterile leaves. The latter matches those of Killip 2778 in detail.

Brade 552 (type of Leptochilus nicotianifolius var. simplex). Spores all shrivelled or otherwise abnormal.

5. The holotype of Acrostichum nicotianifolium. Ventenat's collection represents an intermediate leaf composed of two fertile pinnae to one side, two sterile pinnae to the other, and a fertile terminal segment. The few scales present on the petiole base are proportionally wide, opaque, and light brown. They resemble those present on the rhizome of Von Türckheim 2822 (L), regarded as a deviating specimen and presumably a hybrid between the present species and $B$. pergamentacea (see note 7). The shape, texture, and venation pattern of the sterile pinnae are typical. The fertile pinnae have a narrow sterile marginal strip. The strip is inconspicuous in the present species and usually \pm conspicuous in $B$. pergamentacea. A considerable number of the relatively few sporangia are juvenile, the mature ones present are for the greater part filled with mostly aborted S.M.C.'s and less so with abnormal spores. B. pergamentacea has not been recorded from St. Thomas.

6. Morton lectotypified Acrostichum acuminatum by the other syntype: Anonymus s.n., Asia ('Malaspina, Peru'), ster. (B, herb. Willdenow 19539) which represents the common Malesian fern Photinopteris speciosa (Bl.) Presl. Consequently, he proposed the new combination Photinopteris acuminata (Willd.) Morton. His efforts were against the Code (Hennipman, Blumea 22, 1974, 51).

7. Deviating specimens. A considerable number of more or less atypical specimens were met. From their shape and the data of the spores it is concluded that hybridization is commonly encountered in this species.

a. Specimens with but two alternate pinnae, a rather firm texture and a rather coarse venation pattern. They are reminiscent of $B$. oligarchica. Here belong: Allen 2132 from Panama (MO), a small specimen, the fertile leaf showing abnormal spores only; Wendland 787, from Costa Rica (W), a large specimen, the fertile leaf with sporangia mostly filled with a cluster of abnormal spores, some obviously normal spores also present. 


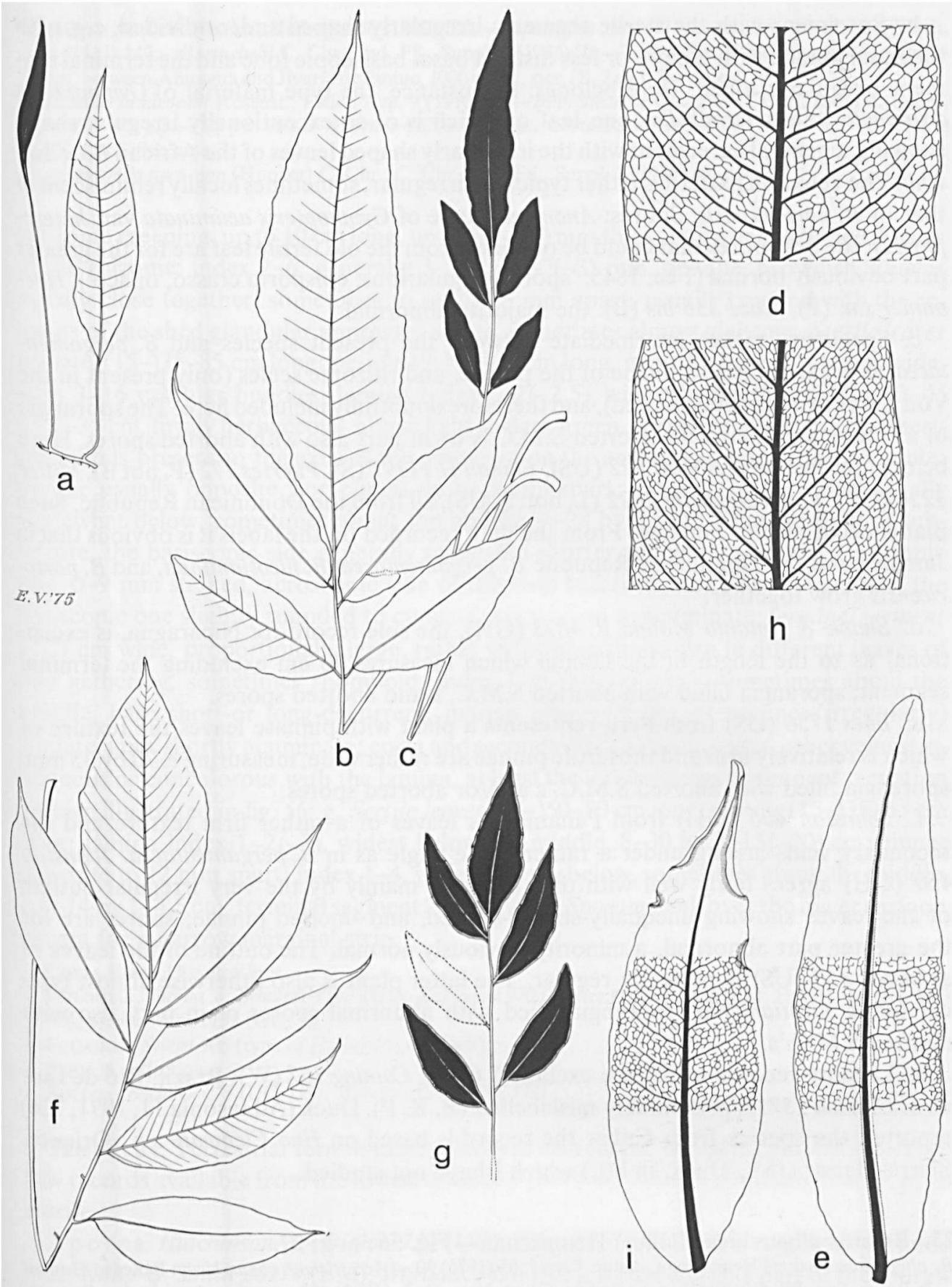

Fig. 58. a-e. Bolbitis nicotianifolia. a-c. sterile and fertile leaves, $\times 1 / 5$; d. venation pattern of sterile pinna, $\times 4 / 5$; e. ibid. of fertile pinna (a. Killip 2778, P, isotype of Leptochilus killipii; b, c. Herminier s.n., L 908.289-052; d. Maxon 9316, US; e. Anonymus (Wright?) 690, L).-f-i. Bolbitis pergamentacea. f, g. fertile and sterile leaf resp., $\times 1 / 5 ; h$. venation pattern of sterile pinna, $\times 4 / 5 ; i$. ibid. of fertile pinna (f, i. Wright 788, L; g. Eggers 2755, L; h. Linden 2117, L). 
b. Specimens with the sterile segments irregularly shaped and/or divided, e.g. with the basal pinnae with a more or less distinct basal basiscopic lobe and the terminal segment irregularly lobed. Here belongs, for instance, the type material of Gymnopteris acuminata var. heterophvlla. one leaf of which is of an exceptionally irregular shape showing a general agreement with the irregularly shaped leaves of the African $B . \times$ boivinii. The venation pattern is either typical or irregular, sometimes locally reminiscent of that of B. portoricensis. Spores: Anonymus (type of Gymnopteris acuminata var. heterophylla): the few spores that could be obtained from the old fertile leaf are for the greater part obviously normal (Fée, 1845: 'sporis irregularibus, episporio crasso, opaco'). Herminier s.n. (P), Mazé 336 bis (B): the majority abnormal.

c. Specimens rather intermediate between the present species and B. pergamentacea as regards texture, shape of the pinnae, and rhizome scales (only present in the Von Türckheim collection cited), and therefore doubtfully included here. The sporangia of all these are filled with aborted S.M.C.'s or in part also with aborted spores. Here belong, for instance, Abbott 822 (US), Ekman $H 11197$ (S), Fuertes 772 (P, not B), Miller 1226 (US), Von Türckheim 2822 (L, not: S, US), all from the Dominican Republic. Such plants occur also in Jamaica. From the data recorded on the labels it is obvious that in Jamaica and the Dominican Republic B. pergamentacea, B. nicotianifolia, and B. portoricensis grow together.

d. Shank \& Antonio Molino R. $4873(\mathrm{GH})$, the sole record for Nicaragua, is exceptional as to the length of the lamina which measures $40 \mathrm{~cm}$ excluding the terminal segment; sporangia filled with aborted S.M.C.'s and aborted spores.

e. Bües 1756 (US) from Peru represents a plant with pinnate leaves the texture of which is relatively firm and the fertile pinnae are rather wide, measuring $c .70$ by $35 \mathrm{~mm}$; sporangia filled with aborted S.M.C.'s and/or aborted spores.

f. Johnston $406(\mathrm{GH})$ from Panama has leaves of a rather firm texture and the secondary veins arising under a rather acute angle as in B. pergamentacea. Johnston $450(\mathrm{GH})$ agrees fairly well with this, differing mainly by the very irregular outline of the leaves showing unequally-sized, -shaped, and -incised pinnae; spores are for the greater part abnormal, a minority obviously normal. The outline of the leaves of Johnston 450 (US) is perfectly regular. The latter plant is also otherwise almost typical for B. nicotianifolia; sporangia filled with abnormal spores or in part also with aborted S.M.C.'s.

8. Locality records which are excluded: Chile, Cuming 157 (W); Brazil, Rio de Janeiro, Glaziou 15715, presumably mislabelled (B, K, P). Duek (Adansonia 11, 1971, 726) reported the species from Cuba; the record is based on Hno Clemente s.n., Oriente, Sierra Maestra (SV, Duek, in litt.) which I have not studied.

23. Bolbitis oligarchica (Baker) Hennipman-Fig. 56: e-g; 57.

B. oligarchica (Baker) Hennipman, Amer. Fern J. 65 (1975) 30. - Acrostichum oligarchicum Baker in Hooker \& Baker, Syn. Fil. (1868) 418, p.p.; Sodiro, Crypt. Vasc. Quit. (1893) 493, quoad nomen solum. - Gymnopteris oligarchica J. Smith, Hist. Fil. (1875) 138.-Chrysodium oligarchicum Salomon, Nomencl. Gefässkr. (1883) 136.-Leptochilus oligarchicus C. Chr., Bot. Tidsskr. 26 (1904) 285.-L e c to t y p e (Maxon, Amer. Fern J. 21, 1931, 139): Spruce 4737, Peru, San Martin, Mt. Guayrapurima near Tarapoto (K). See note 1. 
Hypoderris stuebelii Hieron., Hedwigia 46 (1907) 323.-Leptochilus stuebelii Maxon, Proc. Biol. Soc. Wash. 46 (1933) 142. - B. stuebelii C. Chr., Ind. Fil., Suppl. 3 (1934) 50.-T y p e: Stübel 906, Ecuador, Tunguragua, between Abitagua and Jivaria de Pintuc, $1000 \mathrm{~m}$ alt., ster. (B, 2 sh.). See note 4.

Leptochilus bradeorum Rosenst., Fedde Rep. 9 (1910) 70.-B. bradeorum Ching in C. Chr., Ind. Fil., Suppl. 3(1934) 47, p.p.-T y pe: Brade \& Brade 367('s.n.'), p.p., Costa Rica, La Palma, $1400 \mathrm{~m}$ alt. (UC; iso in NY, ster.). See note 3.

B. pandurifolia auct. non (Hooker) C. Chr.: C. Chr., Ind. Fil., Suppl. 3 (1934) 49, p.p.

Rhizome creeping, up to $10 \mathrm{~cm}$ long, up to $10(-15) \mathrm{mm}$ thick, more or less ribbed. Scales of the rhizome: index $2-6$, generally $3-10$ by $1-2(-3) \mathrm{mm}$, opaque, brownish. Leaves usually close together, sometimes to up to $15 \mathrm{~mm}$ apart, usually covered with the remains of the shed glandular appressed scales, sometimes almost glabrous. Sterile leaves ternate, (15-)35-85 cm long; petiole (5-) 10-45 cm long, near the base $1.5-6 \mathrm{~mm}$ wide, with 3-16 vascular bundles; lamina index $1-2(-2.5)$, widest below the middle, 10-45 by $7-35 \mathrm{~cm}$, firmly herbaceous, above light to dark green, (primordia of) almost spherical bulbils present in the axils of the pinnae or on the acroscopic side of the petiolule; pinnae usually opposite, sometimes up to $5 \mathrm{~mm}$ apart; index $1-3(-4)$, widest usually somewhat below, sometimes about the middle, $4-28$ by $2.5-13.5 \mathrm{~cm}$, straight or subfalcate, the basiscopic side generally somewhat shorter and wider than the acroscopic one, $0-9 \mathrm{~mm}$ stalked, acroscopic side of the base (narrowly to) broadly cuneate, the basiscopic one slightly rounded to cuneate, apex acute to acuminate; terminal segment $7-20 \mathrm{~cm}$ wide, proportionally large, rather variable in shape also in different leaves of one gathering, sometimes rhomboid, index $1-3$, widest below, sometimes about the middle, base short-or long-decurrent, margin almost entire, sometimes (irregularly) sinuate, apex shortly acuminate; costa and secondary veins stramineous, other veins stramineous or concolorous with the lamina, at least the tertiary veins prominent; venation pattern illustrated in fig. 56 : g. Fertile leaves (25-) $50-80 \mathrm{~cm}$ long; petiole (15-) $35-65 \mathrm{~cm}$ long; lamina index $1-2(-3)$, widest below the middle, $8-30$ by $3-15(-20) \mathrm{cm}$; pinnae opposite to $12 \mathrm{~mm}$ apart; index $1-4$, widest usually below, sometimes about the middle, $2.5-14$ by $1-4.5 \mathrm{~cm}$; terminal segment $2-9 \mathrm{~cm}$ wide. Sporangia all over the lower surface, except for a narrow marginal strip.

Costa Rica. 13 collections.

Colombia. Idrobo \& Schultes 1113 (US); Kalbreyer 970 (B, ster.); Klug 1873 (BM, F, GH, MICH, MO, NY, S); Plowman 2132 (GH, L).

ECUADOR. Stübel 906 (type of Hypoderris stuebelii).

PERU. 8 collections.

Bolivia. Buchtien 273 (UC, US); Williams 1293 (GH, US).

$\mathrm{H} \mathrm{a} \mathrm{b} \mathrm{i} \mathrm{t} \mathrm{a} \mathrm{t.} \mathrm{Terrestrial} \mathrm{fern} \mathrm{in} \mathrm{moist} \mathrm{places} \mathrm{in} \mathrm{rain} \mathrm{forest.} \mathrm{Altitude:} 300-1600 \mathrm{~m}$. Only few records available from the lowest altitudes. Obviously locally common, though often sterile.

S pores. Hutchinson \& Wright 3712 (UC), Klug 1873 (MICH): the greater part normal. Killip \& Smith 26174 (BM), Wurdack 1896(NY): a considerable part of the sporangia aborted; a minority of the spores normal, the others, though usually normally shaped, mostly small and empty, sometimes shrivelled.

Notes. 1. Maxon (1931) first noted that the material upon which Baker based his 
'inadequate and misleading' description of Acrostichum oligarchicum is a mixture of two elements. Maxon referred the other syntype, Spruce 4636 from the same place, to Leptochilus killipii $(=B$. nicotianifolia). Ching, during 1931, annotated the material of the present species in Kew and Paris as Bolbitis oligarchica (Baker) Ching. Christensen (1934) who as a rule based himself on Ching as far as the new combinations in Bolbitis were concerned, obviously ignored Ching as regards the nomenclature of this species and referred Baker's species to $B$. pandurifolia.

2. Apart from B. oligarchica (see note 1) Ching (in C. Chr., 1934) also recognized $B$. bradeorum to which he referred Leptochilus killipii $(=B$. nicotianifolia $)$ as a synonym.

3. Apart from the type collection of Leptochilus bradeorum I found two other collections from Costa Rica annotated by Rosenstock as 'Leptochilus Bradeorum Ros.n.sp.' which are, however, not cited with the original description: Brade \& Brade 367, p.p. (B, S-PA, US), gathered from the type locality on a different date (22-vi-1910) and also representing this species, and Brade 552, Llanuros de San Carlos, $200 \mathrm{~m}$ alt., v-1910 (S-PA), type of Rosenstock's Leptochilus nicotianifolius var. simplex $(=$ B. nicotianifolia !!

4. The typification of Hypoderris stuebelii. Hieronymus reported this fern from two localities. He based himself solely on one collection composed of two sterile leaves. On the label of this sheet Stiibel mentioned the second locality where he had seen (not collected!) this fern.

5. The petiole of this species is rather fleshy and has proportionally slender vascular bundles.

6. Juvenile leaves. A few, old, decaying leaves have in the axils of the two pinnae a short rhizome bearing one small simple leaf with an inconspicuous bulbil situated at the lamina base (fig. 56:f). The venation pattern of the small leaf is somewhat simpler than that of the large ones.

7. The species is closest to $B$. hemiotis. For differences see there. It occurs in part sympatric with $B$. nicotianifolia, but is apparently confined to higher altitudes.

\section{Bolbitis pergamentacea (Maxon) Ching-Fig. 58: $f-i ; 59$.}

B. pergamentacea (Maxon) Ching in C. Chr., Ind. Fil., Suppl. 3 (1934) 49; Vareschi in Lasser, Fl. Venezuela 1 (1969) 372.-Leptochilus pergamentaceus Maxon, J. Wash. Acad. Sc. 14 (1924) 144; Sc. Surv. Porto Rico \& Virgin Is. 6 (= Pterid. Porto Rico \& Virgin Is.) (1926) 460.-T y p e: Underwood 1426, Jamaica, Green River Valley, $750 \mathrm{~m}$ alt. (US, 2 sh.).

Leptochilus turrialbae Rosenst., Fedde Rep. 22 (1925) 22.-B. turrialbae Ching in C. Chr., Ind. Fil., Suppl. 3 (1934) 50.-T y p e: Brade \& Brade 370, Costa Rica, Turrialba, $650 \mathrm{~m}$ alt. (S-PA).

Acrostichum nicotianifolium auct., non Swartz c. syn. homot.: auct. p.p., see B. nicotianifolia.

Rhizome creeping, up to $15 \mathrm{~cm}$ long, up to $10 \mathrm{~mm}$ thick, slightly ribbed. Scales of the rhizome: index $1-2$, up to $6(-15)$ by $4(-10) \mathrm{mm}$, the central and upper part (sub) clathrate, the basal part opaque, light or rusty brown. Leaves rather close together to $35 \mathrm{~mm}$ apart, glabrescent. Sterile leaves pinnate, (25-)50-140 cm long; petioles 


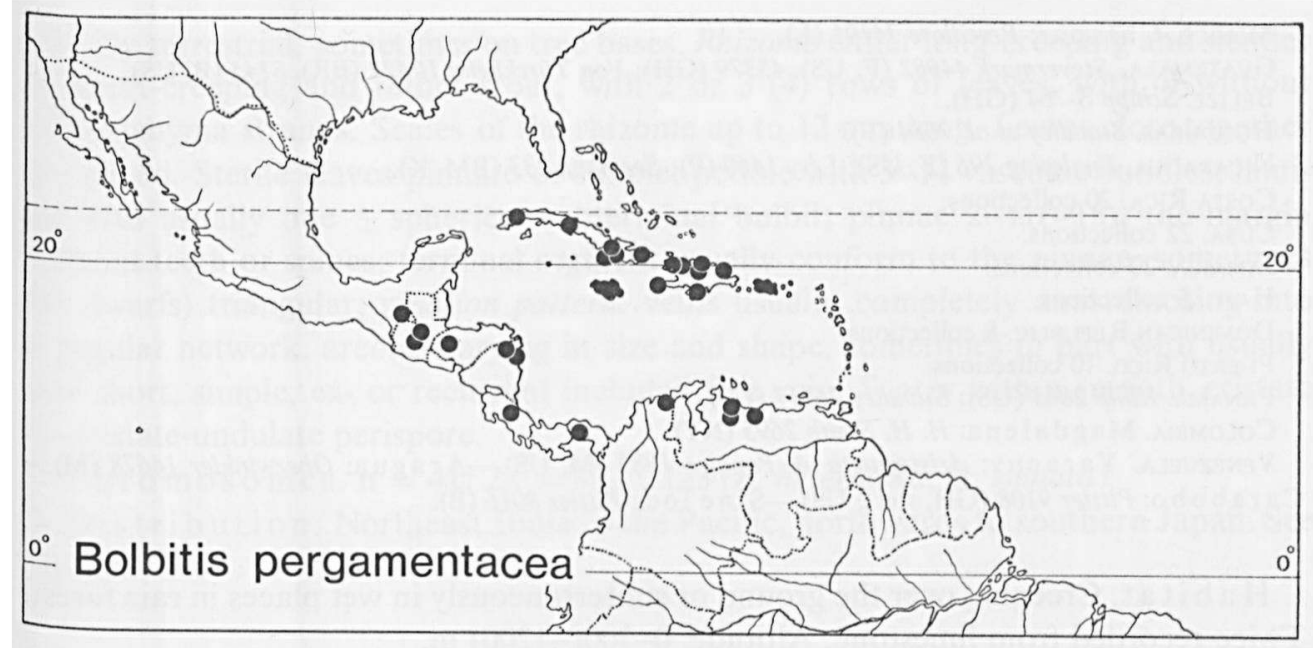

Fig. 59. Distribution of Bolbitis pergamentacea.

(10-)20-80 cm long, near the base 2-10 mm thick, firm, with 9-20 vascular bundles; lamina index 1-2, widest usually below, sometimes about the middle, (15-)25-60 by (9-)20-50 cm, without the terminal segment 0-20, the terminal segment $15-45 \mathrm{~cm}$ long, pergamentaceous, light (to dark) green, usually with a glaucous tinge, without bulbils; pinnae usually alternate, 2-6, 0-60 mm apart; central pinnae index (1-)2-4 $(-5)$, widest usually about, sometimes below or above the middle, ( $8-) 13-32.5$ by (2.5-) $4-15 \mathrm{~cm}$, usually somewhat asymmetrical with the basiscopic side larger than the acroscopic one, and the basiscopic margin convex, the acroscopic one convex or sigmoid, base usually oblique with its acroscopic side narrowly cuneate, its basiscopic one (broadly) cuneate to rounded, sometimes symmetrical, cuneate, margin usually entire, sometimes especially towards the apex (irregularly) sinuate, apex acuminate; the two lowermost pinnae 0-6 mm stalked, conform to the central pinnae; uppermost pinna usually with the basiscopic side shortly adnate to the rhachis, rarely sessile; terminal segment usually about rhomboid, rarely biconvex, 60-200 mm wide; costa and secondary veins stramineous, other veins immersed or almost so, concolorous with the lamina, secondary veins arising under an acute angle, conspicuous nearly to the margin; venation pattern illustrated in fig. $58:$ h. Fertile leaves (25-) $50-135 \mathrm{~cm}$ long; lamina index 1-4, usually widest somewhat below, sometimes about the middle, $12-45$ by $5-25 \mathrm{~cm}$, without the terminal segment $0-30$, the terminal segment $9-20 \mathrm{~cm}$ long; pinnae 2-8, 0-100 mm apart; central pinnae index 3-6, (3.5-)6-15.5(-19) by $1-5.5 \mathrm{~cm}$, apex acute or rounded; the two lowermost pinnae $1-12(-30) \mathrm{mm}$ stalked; terminal segment $20-60 \mathrm{~mm}$ wide. Sporangia all over the lower surface except for a generally conspicuous sterile marginal strip.

Chromosomes. $2 n=82$. 
Mexico. Chi a a s: Breedlove 34191 (L).

GuATEMAla. Steyermark 44982 (F, US), 45379 (GH); Von Türckheim II 422 (BR), 8145 (B, US).

BELIZE. Schipp S-764 (GH).

Honduras. Standley et al. 7034 (F).

Nicaragua. Englesing 296 (F, US); Lévy 1460 (P); Seemann 233 (BM, K).

Costa Rica. 20 collections.

CUBA. 22 collections.

JAMAICA. 20 collections.

HAITI. 5 collections.

Dominican Republic. 8 collections.

Puerto Rico. 10 collections.

Panama. Killip 2863 (US); Standley 26217 (US).

Colombia. Magdalen a: H. H. Smith 2695 (NY).

Venezuela. Yara cuy: Aristeguieta \& Pannier 1885 (M, US).-A ragua: Oberwinkler 14678 (M).Ca ra bob o: Pittier 9108 (GH, ster.; US).-Si n e loc.: Pittier 8016 (B).

$\mathrm{H}$ a bit a t. Creeping over the ground or subterraneously in wet places in rain forest. Twice recorded from limestone. Altitude: $0-700(-1200) \mathrm{m}$.

S p o r e s. Eggers 2755 (L), Webster $4158(\mathrm{MICH})$ : nearly all normal. Underwood 1426 (holotype of Leptochilus pergamentaceus): a considerable part (the majority?) shrivelled or otherwise abnormal. Anonymus s.n. (P, the specimen illustrated by Fée, 1845, 'Linden 2117'), Aristeguieta \& Pannier 1885 (US), Hioram s.n. (GH), Pittier 9108 (GH), Valeur 340 (SING): the majority of the spores shrivelled or otherwise abnormal. Brade \& Brade 370 (type of Leptochilus turrialbae): the mature sporangia present all filled with (a cluster of) abnormal mostly shrivelled spores.

Notes. 1. A clear-cut species which, however, has been combined with B. nicotianifolia by most authors. Fée (1845) already recognized these two species; incorrectly he used the names Gymnopteris acuminata and G. nicotianifolia. Both Fée and Maxon (1924) listed a number of differences between the two species. But the best distinguishing characters are features of the rhizome scales and the texture of the sterile leaf, not mentioned by them. $B$. nicotianifolia grows (exclusively?) on rocks and trees, whereas $B$. pergamentacea grows on or in soil.

2. A few specimens were found with all but one of the features of $B$. pergamentacea. Blauner 303 (BM) has the outline of the segments of the sterile leaf intermediate between the present species and $B$. nicotianifolia; spores: the greater part shrivelled. Von Türckeim 2648 (W) has one of the two lowermost pinnae with a distinct basal basiscopic lobe, the other with a free pinnule; spores: sporangia filled with aborted S.M.C.'s and/or aborted spores. The outline of the sterile leaves of the latter is reminiscent of that described for B. nicotianifolia in note 7 .

\section{Series Heteroclitae Hennipman, ser. nov.}

Lamina simplex vel pinnata pinna terminali, bulbillo subterminali, marginibus pinnarum integris, sine spinis vel dentibus. Ordinatio venularum: venulae plerumque regulariter reticulatae, areolis statura ac forma diversis; interdum venulis inclusis liberis. Sporae perispora laevi cristata vel cristato-undulata.

Ty pus: Bolbitis heteroclita (Presl) Ching. 
Usually terrestrial, sometimes on tree bases. Rhizome either long-creeping and slender or short-creeping and rather stout, with 2 or 3 (4) rows of leaves, with or without sclerenchyma strands. Scales of the rhizome up to $12 \mathrm{~mm}$ long. Leaves close together to spaced. Sterile leaves pinnate or simple; petiole with 3-14 vascular bundles; lamina with usually one \pm spherical subterminal bulbil; pinnae $2-11(-15)$, the margin without teeth or spines; terminal segment usually conform to the pinnae, sometimes (in dwarfs) triangular; venation pattern: veins usually completely anastomosing into a regular network, areoles varying in size and shape, sometimes in part with usually one short, simple, ex- or recurrent included free vein. Spores with a smooth, cristate or cristate-undulate perispore.

Chromosomes. $\mathrm{n}=41 ; 2 \mathrm{n}=82, c .123$ (B. heteroclita, B. sinuata).

Distribution. Northeast India to the Pacific, northwards to southern Japan. See fig. 27.

\section{Bolbitis heteroclita (Presl) Ching-Fig. 60.}

B. heteroclita (Presl) Ching in C. Chr., Ind. Fil., Suppl. 3 (1934) 48; Tardieu-Blot \& C. Chr., Not. Syst. 7 (1938) 102; Backer \& Posthumus, Varenfl. Java (1939) 83, fig. 13; Tardieu-Blot \& C. Chr. in Lecomte, Fl. Gén. I.-C. 7 (1941) 435; Holttum, Ferns Malaya (1954) 462, fig. 271; Iwatsuki, Acta Phytotax. Geobot. 18 (1959) 57, fig. 12; Copel., Fern Fl. Philip. (1960) 257; Ching, Fl. Hain. (1964) 165, fig. 75; DeVol \& Kuo in Fl. Taiwan 1 (1975) 348, pl. 122.-Acrostichum heteroclitum Presl, Rel. Haenk. (1825) 15, pl. 2: fig. 2; Epim. Bot. (1851) 172.-Heteroneuron heteroclitum Fée, Hist. Acrost. (1845) 92 ('Heteronevron'). - Chrysodium heteroclitum Kuhn, Ann. Mus. Bot. Lugd.-Bat. 4 (1869) 294 ('heterocliton')._Leptochilus heteroclitus C. Chr., Ind. Fil. (1906) 385; Christ, Bull. Herb. Boiss. II, 6 (1906) 1005; Philip. J. Sc. 2 (1907) Bot. 159; v.A.v.R., Handb. Malayan Ferns (1908) 739._Campium heteroclitum Copel., Philip. J. Sc. 37 (1928) 359, fig. 13; Backer \& Posthumus, Nat. Tijdschr. Ned. Ind. 93 (1933) 163.Ty p e: Haenke s.n., Phillipines, Luzon, Sorsogon (PRC; iso in B, 2 sh., BR, W, 2 sh.; all ster.).

Nephrodium cuspidatum Presl, Rel. Haenk. (1825) 31 ('Nephrodium?').-Polystichum cuspidatum Presl, Tent. Pterid. (1836) 82.-Heteroneuron cuspidatum Presl, Epim. Bot. (1851) 169.-Chrysodium cuspidatum Kuhn, Ann. Mus. Bot. Lugd.-Bat. 4 (1869) 293, p.p. - Leptochilus cuspidatus C. Chr., Ind. Fil. (1906) 384, quoad nomen solum.-Campium cuspidatum Copel., Philip. J. Sc. 37 (1928) 365, fig. 19.-B. cuspidata Ching in C. Chr., Ind. Fil., Suppl. 3 (1934) 48; Copel., Fern Fl. Philip. (1960) 259.-T y pe: Haenke s.n., Philippines, Luzon, Sorsogon, ster. (PRC; iso in W, '77').

Acrostichum flagelliferum Wall. ex Hooker \& Grev., Ic. Fil. (1827) pl. 23, p.p.; Bl., Enum. Pl. Jav. (1828) 104; Fl. Java Fil. (1829) 37, pl. 13, p.p.; Kunze, Bot. Zeit. (1848) 102; Hooker, Spec. Fil. 5 (1864) 258, p.p.; Baker in Hooker \& Baker, Syn. Fil. (1868) 418, p.p.; Clarke, Trans. Linn. Soc. Bot. 1 (1880) 579, p.p.-B. flagellifera Schott, Gen. Fil (1835) ad tab. (13).-Cyrtogonium flagelliferum J. Smith, Hook. J. Bot. 4 (1841) 154.-Chrysodium flagelliferum Mettenius, Fil. Lips. (1856) 21, pl. 20: fig. 11; Ettingsh., Denkschr. K. Ak. Wiss. M.-N. KI. Wien 22 (1864) 58, pl. 8: fig. 9-Poecilopteris flagellifera J. Smith, Cat. Cult. Ferns (1857) 23; Bedd., Ferns Br. India (1865) pl. 112, p.p.; J. Smith, Ferns Br. For. (1866) 117.Gymnopteris flagellifera Bedd., Ferns Br. India, Suppl. (1876) 27; Handb. Ferns Br. India (1883) 433; Christ, Farnkr. Erde (1897) 49; Copel., Polyp. Philip. (1905) 42, p.p. - T y p e: Wallich s.n., a specimen of unknown origin cultivated at the Botanic Garden, Calcutta (K. herb. Hooker).

Acrostichum proliferum Bl., Enum. PI. Jav. (1828) 104, non Hooker $(1844)(=$ B. subcrenata).-T y p e: Blume s.n., Java, Mt. Parang (n.v.).

Leptocnilus linnaeanus Fée, Hist. Acrost. (1845) 87, pl. 47: fig. 2, excl. syn.; v.A.v.R., Handb. Malayan Ferns (1908) 735; ibid., Suppl. (1917) 60.-Dendroglossa linnaeana Fée, Gen. Polyp. (1852) 81.-Acrostichum linnaeanum Hooker, Sec. Cent. Ferns (1861) pl. 26, p.p.; Spec. Fil. 5 (1864) 278; Baker in Hooker \& Baker, Syn. Fil. (1868) 417; Christ, Bull. Herb. Boiss. 6 (1898) 206.-Chrysodium linnaeanum Kuhn, Ann. Mus. Bot. Lugd.-Bat. 4 (1869) 293, p.p.-Chrysodium linnaeanum Kuhn var. sinuatum Kuhn, Ann. Mus. Bot. Lugd.-Bat. 4 (1869) 293 ('sinuata'), nom. illeg. -Gymnopteris linnaeana Christ, J. de Bot. 19 (1905) 125; Copel., Polyp. Philip. (1905) 41.-Leptochilus heteroclitus (Presi) C. Chr. 
var. linnaeanus Christ, Philip. J. Sc. 2 (1907) Bot. 160.-Campium linnaeanum Copel., Philip. J. Sc. 37 (1928) 343, quoad nomen solum; Backer \& Posthumus, Nat. Tijdschr. Ned. Ind. 93(1933) 159, excl. syn. - B. linnaeana C. Chr., Ind. Fil., Suppl. 3 (1934) 198; Backer \& Posthumus, Varenfl. Java (1939) 80. -T y pe: Zollinger 1441, West Java, Mt. Salak, Tjikoja (P; iso in BM, G).

Cyrtogonium acuminatum Brackenr. in Wilkes, U.S. Expl. Exp. 16 (1854) 86.-T y p e: Brackenridge s.n., Philippines, Luzon, near Los Baños (US, 51087).

Poecilopteris stenophylla Kurz ex Teijsmann \& Binnendijk, Nat. Tijdschr. N. I. 27 (1864) 15; Copel., Philip. J. Sc. 37 (1928) 393, pro spec. dub.-B. stenophylla Ching in C. Chr. Ind. Fil., Suppl. 3 (1934) 50.-T y pe: Kurz s.n., West Java, Mt. Salak, 900-1200 m alt. (L, 908.289-062; iso in BM). See note 3.

Chrysodium heteroclitum (Presl) Kuhn var. subcrenatum Kuhn, Ann. Mus. Bot. Lugd.-Bat. 4 (1869) 294, p.p. ('heterocliton', 'subcrenata').-T y p e: Zollinger $884 x$, West Java, Mt. Salak, Tjikoja (P; iso in $\left.B, K,{ }^{\prime} 884^{\prime}\right)$. See note 4.

Acrostichum modestum Baker, J. Linn. Soc. 22 (1886) 231; C. Chr. in Copel., Philip. J. Sc. 37 (1928) 411.-Leptochilus modestus C. Chr., Ind. Fil. (1906) 386.-Campium modestum Copel., Brittonia 1 (1931) 76, fig. 1.-B. modesta Ching in C. Chr., Ind. Fil., Suppl. 3 (1934) 49.-T y pe: C. Hose 159, Borneo, Sarawak, Kako, Kriau (K; iso in BM, SING).

Gymnopteris inconstans Copel. in Perkins, Fragm. Fl. Philip. (1905) 177; Polyp. Philip. (1905) 43.Leptochilus inconstans Christ, Bull. Herb. Boiss. II, 6 (1906) 1005.-Leptochilus heteroclitus (Presl) C. Chr. var. inconstans Christ, Philip. J. Sc. 2 (1907) Bot. 160.-B. inconstans Ching in C. Chr., Ind. Fil., Suppl. 3 (1934) 48.-Sy nt y pesı Copeland 251, Philippines, Luzon, Bataán, Mt. Mariveles, along Lamao R. (B, BM, P); Merill 3128 , ibid. (B, K, P, US).

Leptochilus heteroclitus (Presl) C. Chr. var. eurybasis Christ, Philip. J. Sc. 2 (1907) Bot. 159. -Campium heteroclitum (Presl) Copel. var. eurybasis Copel., Philip. J. Sc. 37 (1928) 361.-T y p e: Clemens 552, Philippines, Mindanao, Lake Lanao, Camp Keithley, $660 \mathrm{~m}$ alt. (P; iso in MICH).

Leptochilus heteroclitus (Presl) C. Chr. var. foxworthyi Christ, Philip. J. Sc. 2 (1907) Bot. 160.-T y p e: Foxworthy BS 68, Philippines, Luzon, Prov. Rizal, Bosoboso (P; not: MICH which is the type of Campium foxworthyi $=$ B. heteroclita).

Leptochilus sumatranus v.A.v.R., Bull. Jard. Bot. Btzg II, 23 (1916) 15, pl. 2: fig. la-c; Handb. Malayan Ferns, Suppl. 1 (1917) 436; ibid., Corrections (1917) 60.-T y p e: Brooks 260.S, South Sumatra, Bencoolen, Lebong Tandai (BO; iso in BM, P).

Campium nigrum Copel., Philip. J. Sc. 37 (1928) 361, fig. 14, pl. 10.-B. nigra Ching in C. Chr., Ind. Fil., Suppl. 3 (1934) 49.-T y p e: Karigone 69, Caroline Is., Ponape, ster., 1925 (UC, 267415).

Campium pseudoscalpturatum Copel., Philip. J. Sc. 37 (1928) 363, fig. 16, pl. 11.-B. pseudoscalpturata Ching in C. Chr., Ind. Fil., Suppl. 3 (1934) 49; Copel., Fern Fl. Philip. (1960) 261.-T y pe: Ramos BS 14816, Philippines, Mindanao, Camiguin de Mindanao (MICH).

Campium foxworthyi Copel., Philip. J. Sc. 37 (1928) 364, fig. 17, pl. 12.-B. foxworthyi Ching in C. Chr., Ind. Fil., Suppl. 3 (1934) 48; Copel., Fern Fl. Philip. (1960) 258.-T y p e: Foxworthy BS 68, Philippines, Luzon, Prov. Rizal, Bosoboso (MICH; not: $\mathrm{P}$ which is the type of Leptochilus heteroclitus var. foxworthyi).

Campium tenuissimum Copel., Philip. J. Sc. 37 (1928) 364, fig. 18, pl. 13.-B. tenuissima Copel., Fern Fl. Philip. (1960) 259. - T y pe: Copeland s.n., Philippines, Luzon, Laguna Prov., Los Baños, 17-iii-1906 (MICH, herb. Copeland 290).

Leptochilus simplicifolius Holttum, Gard. Bull. S. S. 4 (1929) 409,-B. simplicifolia Ching in C. Chr., Ind. Fil., Suppl. 3 (1934) 50; Holttum, Ferns Malaya (1954) 464, fig. 272.-T y p e: Henderson SF 17977, Malay Peninsula, Pahang, Cameron Highlands, Robinson's Falls, c. $1350 \mathrm{~m}$ alt. (K; iso in SING).

Campium membranaceum Copel., Philip. J. Sc. 40 (1929) 307, pl. 7.-B. membranacea C. Chr., Ind. Fil., Suppl. 3 (1934) 198; Fern Fl. Philip. (1960) 261.-T y pe: Copeland s.n., Philippines, Mindanao, Mt. Matutum, v-1917 (MICH, herb. Copeland 11538; iso in UC, 352513).

B. koidzumii Tagawa, Acta Phytotax. Geobot. 6 (1937) 93; Ito, Fil. Liuk. (1938) 644.-T y pe: Koidzumi s.n., Japan, Ryukyu Is., Iriomote, vii-1923 (KYO, n.v., L, phot.; iso in KYO, 2 sh., n.v., L, phot., US).

B. annamensis Tardieu-Blot \& C. Chr., Not. Syst. 7 (1938) 100; in Lecomte, Fl. Gén. I.-C. 7 (1941) 436, fig. 50: 3 \& 4.-T y pe: Cadière 149, Vietnam, Annam, Thanh Tan, 100-200 m alt. (BM; iso in P, 3 sh.). B. edanyoi Copel., Philip. J. Sc. 81 (1952) 22, pl. 15; Fern Fl. Philip. (1960) 258.-T y pe: Edaño PNH8811, Philippines, Mindanao, Davao Prov., Baguan R., $1525 \mathrm{~m}$ alt. (MICH; iso in PNH).

Edanyoa difformis Copel., Philip. J. Sc. 81 (1952) 22, pl. 17; Fern Fl. Philip. (1960) 265.-T y p e: Edaño PNH 5213, Philippines; Negros I., Oriental, Lake Balinsasayo (MICH; iso in PNH, 2 sh.). 
[Gymnopteris trilobata J. Smith, Hook. J. Bot. (1841) 403, nom. nud.]

Cyrtogonium diversifolium auct. non (B1.) J. Smith: J. Smith, Hook. J. Bot. 3 (1841) 403.

Heteroneuron diversifolium auct. non (Bl.) Fée: Fée, Hist. Acrost. (1845) 91, p.p. ('Heteronevron').

Dendroglossa lanceolata auct. non (L.) Presl: Presl, Epim. Bot. (1851) 149, p.p.

Leptochilus diversifolius auct. non (B1.) C. Chr.: Christ, Philip. J. Sc. 2 (1907) Bot. 160.

Leptochilus cuspidatus auct. non (Presl) C. Chr.: auct. japon.p.p. See: Tagawa, Acta Phytotax. Geobot. 6(1937) 93

Gymnopteris repanda auct. non (Bl.) C. Chr.: auct. japon. p.p. See: Tagawa, Acta Phytotax. Geobot. 6(1937) 93.

B. interlineata auct. non (Copel.) Ching. Ito, J. Jap. Bot. 14 (1938) 439, fig.

B. diversifolia auct. non (Bl.) Schott: Nayar \& Kaur, Bull. Nat. Bot. Gard. Lucknow 88 (1964) 42, fig. 12, $19,29,62$.

B. subsimplex auct. non (Fée) Ching. Nayar \& Kaur, Bull. Nat. Bot. Gard. Lucknow 88(1964) 68, fig. 24, 64, 69.

Rhizome usually short-, sometimes long-creeping, up to $c .20 \mathrm{~cm}$ long, $2-7(-10) \mathrm{mm}$ thick, with sclerenchyma strands. Scales of the rhizome: index 3-6, 3-9 by $0.5-1.5$ $(-2) \mathrm{mm}$, usually subclathrate, sometimes opaque, brownish or blackish, usually with a paler marginal strip. Leaves usually \pm spaced, up to $50 \mathrm{~mm}$ apart, sometimes rather close together. Sterile leaves simple or pinnate, $15-100 \mathrm{~cm}$ long. Pinnate leaves $25-100$ $\mathrm{cm}$ long; petiole 3-35(-55) cm long, near the base 1.5-4(-7) $\mathrm{mm}$ thick; lamina index of non-flagelloid leaves $1-5$, that of flagelloid leaves sometimes more, widest below the middle, $10->75$ by $5-30 \mathrm{~cm}$, without the terminal segment $0-25$, the terminal segment to $>75 \mathrm{~cm}$ long, herbaceous, usually greenish, sometimes purplish; rhachis not winged; pinnae alternate to opposite, $2-10(-15)$, up to $85 \mathrm{~mm}$ apart; central pinnae index $2-5(-7)$, widest usually about the middle, $1-26$ by $0.7-6.5 \mathrm{~cm}$, base attenuate to truncate, margin usually \pm entire, sometimes sinuate or sinuate-serrate or finely repand without teeth or spines, apex acute to caudate; the twolowermost pinnae usually \pm conform to the central ones, rarely with a \pm marked basal basiscopic lobe, 0-6 mm stalked; terminal segment usually \pm conform to the pinnae though generally somewhat (to much) larger, the apex acute to flagelloid, sometimes the terminal segment flagelloid; secondary veins usually stramineous, sometimes blackish, usually slightly prominent nearly to the margin; venation pattern: see fig. $60: \mathrm{t}-\mathrm{z}$. Simple leaves $15-45 \mathrm{~cm}$ long; petiole $3-18 \mathrm{~cm}$ long, near the base $0.5-3 \mathrm{~mm}$ thick; lamina index $3-7(-17)$, widest usually about, sometimes below the middle, $10-30$ by (1-)2-7 cm, otherwise similar to the terminal segment of pinnate leaves. - Fertile leaves $14-75 \mathrm{~cm}$ long. Pinnate leaves 30 $75 \mathrm{~cm}$ long; petiole $10-52 \mathrm{~cm}$ long; lamina index $1-4,8-32$ by $2-25 \mathrm{~cm}$, without the terminal segment $0-20$, the terminal segment $6-18(-30) \mathrm{cm}$ long; pinnae $2-10$, up to $60 \mathrm{~mm}$ apart; central pinnae index $2-7,1-13$ by $0.5-2.5(-4) \mathrm{cm}$; the two lowermost pinnae 0-6 mm stalked. Simple leaves $14-40(->45) \mathrm{cm}$ long; petiole $10-30(->35) \mathrm{cm}$ long; lamina index 3-10, 4-13 by $0.5-2 \mathrm{~cm}$. Sporangia inserted all over the lower surface, arranged acrostichoid. Spores with a smooth, cristate or cristate-undulate perispore.

Chrom os omes. $n=41$; at meiosis with 41 uni- and 41 bivalents; $2 n=82, c .123$.

JAPAN. S. R y u ky u I s. Iriomote: 6 collections. Ishigaki Shima: Fosberg 37801 (L, US); Nozu s.n. (TI).

China. Kwei chow: Cavalerie s.n. (BM, S-PA).-S. Y unnan: 7 collections.-K wangtung: Mat- 


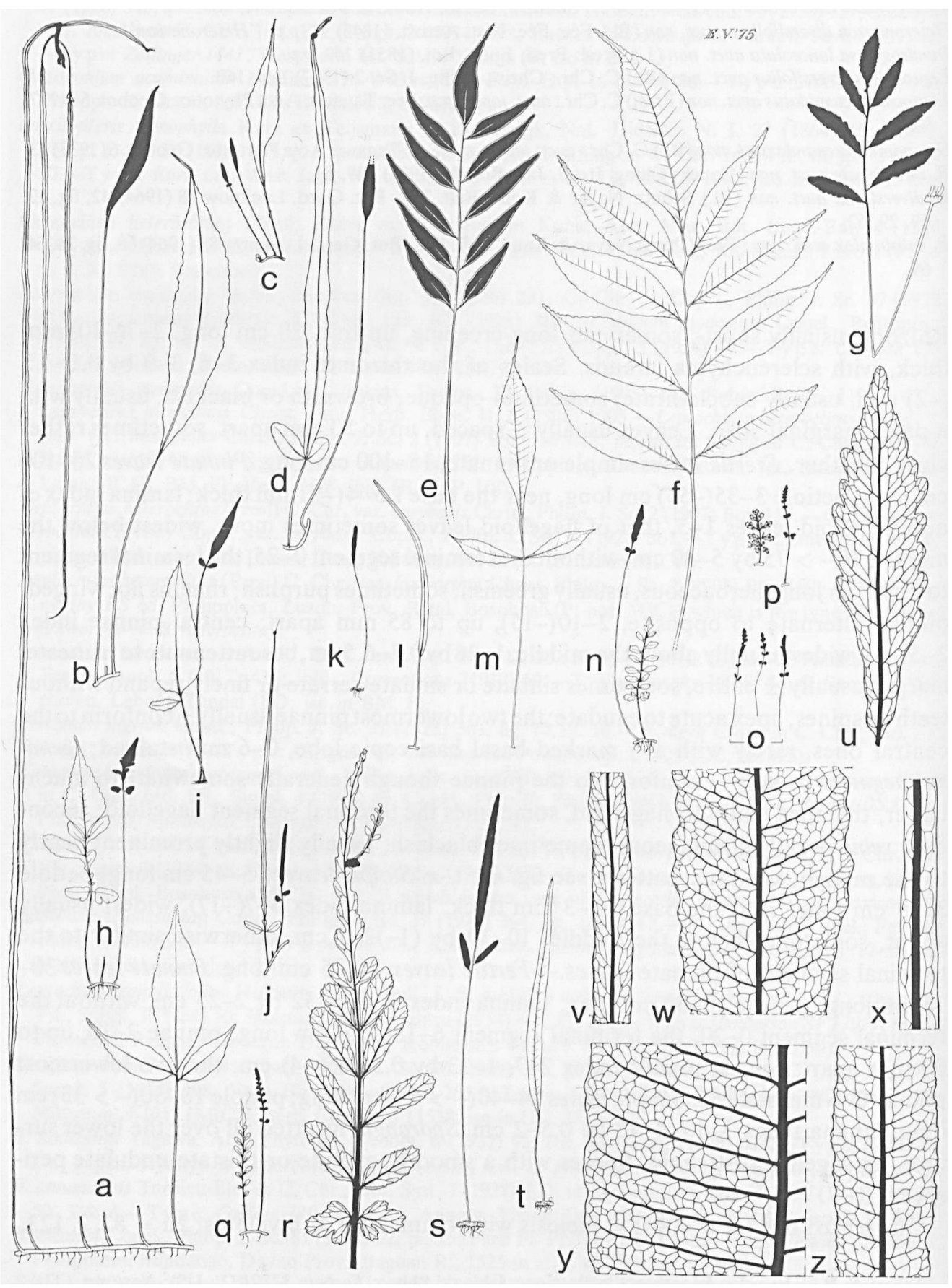


thew s.n. (P); Tsoong 2842 (UC).-Kwangsi: Ko 56007 (A),-Hainan: Hancock 10 (K, US); Tsang 382 (= LU 17131) (B, BO, GH, K, UC, US).

TAIWAN. Tato, Gukutsu, Kwarenko: Matsuda s.n. (UC). Mt. Koto: Miyake s.n. (TI).

BANGLADESI. 9 collections.

NePAL. E a t: Hara et al. 6305227 (US); Hooker s.n. (K).

SIKKIM. 5 collections.

INDIA. A s s a m: 14 collections.

Burma. Nort heast: Kaulback 250 (BM); Kingdon Ward 21615 (BM).-S o ut hern: Meebold 17039

(S-PA).-Ten ass e ri m: Falconer s.n. (L, M); Gallatly 64 (K); Parish s.n. (P).

Malay Peninsula. Kedah: Namen SF 38015 (A, BO, BRI, PNH, SING).-Pe rak: 7 collections.Kel a ntan: Haniff \& Nur SF 10354 (BM, BO, K, SING); Yapp 210 (B, K).-Treng g a u: Holttum s.n. (SING).-Pahang: 13 collections.-Selangor: 5 collections.-J o h ore: Holttum SF 17700 (SING); Ridley 12510 (SING).-Lang kawi Is.: Curtis s.n. (SING)._P. Penang: Anonymus s.n. (SING).Sing a por e. Bt. Panjang (= Panjam): 5 collections.-P. Tiom a n: Henderson SF 18590 (BM, SING).

Thailand. Northern: 6 collections. - Southeastern: 7 collections. - Southwestern: Van Beusekom 293 (L); Larsen 9588 (C).-Peninsula: Hennipman 3800 (L), 3835 (L); Tagawa et Yamada T 211 (BKF, L); Tagawa et al. T 5289 (L).

VIETNAM. To $\mathrm{k}$ in: 9 collections. - A n n a m: 12 collections.-Coch in ch in a: Pierre 5723 (P); Poilane 2482 (P, UC), 19467 (MICH, P).

Sumatra. A tje h: Asdat 54 (BO, K, L), 55 (BO, L).-T a panuli: Rahmat si Boeea 5374 (L), 5577 (L); Surbeck 660 (L).-West Coast: Alston 13756 (BM), 14266 (BM).-East Coast: 5 collections.Bencoolen (3 localities): Brooks 95.S (P), 99.S (BM, MICH, P), 226.S (BM, BO), 260.S (type of Leptochilus sumatranus), 7924 (BM); Hancock $56(\mathrm{~K})$; Van Steenis 3632 (BO).-La m pong Dist ri ct s. Mt. Radjabasa: Posthumus 1106 (BO), 1112 (BO), 1128 (BO).-P. Si m a l u r: Achmad 447 (BO, L, SING, U).-P. Engg a n o: Lütjeharms 3944 (BO, GH, L), 4040 (BO, GH, K, L, P).

JAva. West: 15 collections.-C entral: Docters von Leeuwen-Reijnvaan 929 (BO, L), 2007 (BO, L); Kostermans \& Van Woerden 18I (BO).-East: 7 collections.-Christmas I.: 6 collections.

Borneo. Sa rawak: 10 collections.-S a bah: Allen 1631 (S-PA, SING).-Indonesian Borneo. West: Teuscher s.n. (BO, L); Teijsmann s.n. (BO). East: Kostermans 9518 (BO, L). Southeast: Hub. Winkler 2225 (B). Northeast: (3 localities): Amdjah 443 (BO, L), 444 (BO), 562 (BO), 600 (BO), 670 (BO).An a mbas I s.: Van Steenis 953 (BO).

Philippines. Palawan: 7 collections. - Busuanga: Marche s.n. (P).-Mindoro: 6 collections.B a t a nes I s.: Ramos BS 80160 (MICH, SING, UC), BS 80351 (BR, MICH, UC).-B a b u y a n I s.: Fénix BS 3912 (US). - L u z o n: many collections. -R o m bl on: Bartsch 307 (US), 313 (US). -S a ma r: Wagner \& Grether 4229 (MICH, UC, US).-N egros: Copeland s.n. (B, MICH, US); Edaño PNH 21374 (PNH); Elmer 9705 (BM, BO, K, L, MICH, MO, P, US, W), 10041 (G, MICH, P).-P a n ay: Paniza PNH 9243 (PNH).-Sulu I s. Jolo: Williams 3114 (UC, US). Tawitari: Kondo \& Edaño PNH 38631 (K, PNH), PNH 38696 (K, L, PNH); Ramos \& Edaño PNH 44024 (B, BO, P, SING, UC, US, W).-Minda n a o: 15 collections.

Celebes. North: 5 collections.-W est Central: Posthumus 2323 (BO), 2331 (BO).-Southwest: Bünnemeijer 10914 (BO). - S o u th e as t: Kjellberg 408 (BO, S-PA).

Moluccas. Sul a I s. Mangole: Bloembergen 4800 (BO, K, L, PNH, SING).

New Guinea. W est. Babo: Aët 93 (BO, L); Sorong: Djamhari 562 (BO); Vink (BW) 17580 (L). Bernhard Camp: Brass 13242 (GH, MICH, UC), 13902 (GH).-Nor the ast: 5 collections. $-P$ a pua: 6 collections.-M i s ö̈l: Teijsmann s.n. (BO, P).

Fig. 60. Bolbitis heteroclita. $-\mathrm{a}-\mathrm{s}$. sterile and fertile leaves, $\times 1 / 5 ; \mathrm{t}-2$. venation pattern of sterile pinnae, $x$ 4/5 (a. Price 303, L; b. Holttum SF 31352, SING; c. Foxworthy BS 68 , MICH, holotype of Campium foxworthyi; d. Edaño PNH 8811, MICH, holotype of B. edanyoi; e, f, m. Jacobs 7950, L; g, u. Price 452, L; h. Copeland 279, US; i. Holttum SF 21625, SING; j. Copeland s.n., MICH, holotype of Campium tenuissimum; k, v. Richards 2233, US; 1. Hose s.n., SING, isotype of Acrostichum modestum; n. Ramos \& Edaño BS 28843, P; o. Merrill 3128, L, 3 fertile leaves, syntype of Gymnopteris inconstans; p. Price 2518A, L, sterile and fertile leaf; q, r. Price 35I, L; s, t. Backer 21291, BO; w. Tagawa \& Iwatsuki 4690, L; x. Fox PNH 5060, MICH; y. Hennipman 3836, L; z. Holttum SF 24699, C). 
Solomon Is. B o u ga invill e: Wakefield 1156 (BM); Waterhouse 411 B (K), - N ew G e o r gi a. Kolombangara: Braithwaite R.S.S. 4356 (L), R.S.S. 4442 (L); Whitmore \& Grubb BSIP 2120(US).—Mala it a: Templeton Crocker Exp. s.n. (BISH).

Caroline Is. Po n a pe: 9 collections.-K us a i e: Takamatsu 531 (BISH, BO, K, MICH).

H a bitat. Creeping on rocks, in soil or on bases of trees in moist places in rain forest (often near streams), sometimes rheophytic. Altitude: 0-1500(-1750) $\mathrm{m}$.

S pores. Bamler 97 (UC), Copeland, Pterid. Philip. exsicc. 279 (GH), Elmer 18068 (L), Hennipman 3836 (L), Merrill 3128 (US, syntype of Gymnopteris inconstans), Price 452 (L), Richards 2233 (US): all or the greater part normal. Braithwaite R.S.S. 4442 (L), Brooks 260.S (BO, type of Leptochilus sumatranus): about equal parts normal and abnormal. Henderson SF 17977 (SING, type of Leptochilus simplicifolius): the majority abnormal. Clemens 22001 (BO), Copeland s.n. (holotype of Campium membranaceum): the greater part abnormal. Elmer 17554 (C), Jacobs 7950 (L), Tagawa \& Iwatsuki 4732 (US): all abnormal. Anonymus 33 (BISH), Backer 21291 (BO), Ledermann 13176 (B), 13279 (B), 13765 (B), Takamatsu 953 (BISH): sporangia filled with aborted S.M.C.'s and/or aborted spores. See also the entries on variation.

Not es. 1. This species is closely related to $B$. sinuat $a$ from which it differs especially in the texture of the sterile leaves. Possible intermediates are discussed under B. sinuata, note 1 .

Most of the material is easily recognizable. However, a considerable part represents (much) deviating facultative rheophytes and dwarfs. The latter material is not included in the description; it is discussed below. The present species takes part in the parentage of several hybrids (see there).

2. Acrostichum proliferum and $A$. repandum were described by Blume (1828) at the same time. A. proliferum was based on sterile material collected by Blume on Mt. Parang in Java. I have not found a proper type collection (obviously there has been a mixing of specimens of this species and of $B$. repanda when Blume's herbarium was remounted during 1908 and 1909) but the description is almost similar to that given by Blume for $A$. flagelliferum. Both should have ternate leaves which are typical for $B$. heteroclita and never occur in $B$. repanda to which Blume (1829) referred $A$. proliferum.

3. It remains uncertain whether Teijsmann \& Binnendijk described Poecilopteris stenophylla from wild or from cultivated material.

4. Zollinger 884, the type of Chrysodium heteroclitum var. subcrenatum Kuhn, was also cited by Kuhn under Chrysodium cuspidatum in the same publication.

5. The geographical distribution. B. heteroclita is absent from the lowland plains and the proportionally dry areas in Burma, Thailand, Laos, Cambodia, and the Lesser Sunda Is.

The following collections have not been included in the distribution map:

a. W. Smith s.n. (M) from Anaimallai Hills, southern India; Carruthers s.n., Ceylon (P, 'Kaulfuss Tauschverein'). From these areas the present species was unkown from the wild to Beddome, who knew the species of southern India particularly well, and to Blatter \& d'Almeida (Ferns of Bombay, 1922). B. heteroclita was not rarely found cultivated in southern India. 
b. Dietrich s.n., southern Queensland, Brisbane R. (B). Possibly a mislabelled collection. This record awaits confirmation.

c. Buchanan 118, Fiji (B). The outline of the leaves is reminiscent of the type of Poecilopteris stenophylla. Presumably mislabelled. Buchanan eventually was Superintendent of the Botanic Garden at Calcutta; he made two or three voyages to eastern Malesia (not the Pacific) from which no herbarium material is known (Van Steenis-Kruseman, Fl. Mal. I, 1, 1950, 214).

6. Iwatsuki discussed the occurrence of B. heteroclita in Taiwan as reported in some papers by Japanese authors. According to him all these records are unreliable.

7. Ching (in C. Chr., 1934) regarded Campium tenusissimum and B. inconstans as synonymous, but kept $B$. cuspidata separate. Copeland (1960)argued about this view: 'but as I know these variable, "inconstant" little ferns in the field, B. tenusissima is distinguishable, but $B$. inconstans is $B$. cuspidata.'

8. Apart from $B$. heteroclita two names listed by Nayar \& Kaur (1964) apply to the present species, i.e. $B$. diversifolia and $B$. subsimplex in their sense. The discriminating characters between these three species as given in their key did not hold in the reference material of $\boldsymbol{B}$. heteroclita and $\boldsymbol{B}$. subsimplex I received on loan. For $\boldsymbol{B}$. diversifolia no reference material is cited; the description gave sufficient information.

9. Nayar \& Kaur (1966) amply described the venation pattern of the fertile leaf of $B$. subsimplex in their sense, which should be diplodesmic (a term not mentioned by them). The only reference material cited is a badly preserved specimen in which I was unable to trace such a pattern. See also the general chapters.

10. As measured from cross-sections (paraffine method) of cultivated material, the thickness of the leaves of triploid $B$. heteroclita (origin: the botanic garden, Groningen) varies from $85-110 \mu \mathrm{m}$, that of triploid $B$. sinuata (origin: Johnson s.n., Singapore) varies from $285-315 \mu \mathrm{m}$.

11. Uses. Hooker \& Greville received the type of Acrostichum flagelliferum with Dr. Wallich's MS name and reference to Rheede's Pannamara-maravara (Hort. Malab. 12, 1703, 39, pl. 19). As already communicated by Hooker \& Greville, Rheede ascribed great medicinal value to this plant. However, Rheede's plant cannot be properly identified; see Species dubiae no. 11.

12. Plants with leaves of a texture supposedly intermediate between those of the present species and of $B$. sinuata are discussed under $B$. sinuata (note 2). Copeland 1543 (B, $\mathrm{MICH}, \mathrm{P}, \mathrm{US}$ ), by Christ referred to Leptochilus diversifolius, has slightly thicker leaves than normal.

Variation. The morphological variation is outstanding. The following types are included:

A. Specimens with usually pinnate leaves of which the terminal segment is either conform to the pinnae or conform-flagelloid. The venation pattern shows maximum development or nearly so. These ferns include the type specimens of Acrostichum heteroclitum. A. Alagelliferum, Cyrtogonium acuminatum, Chrysodium heteroclitum var. subcrenatum, Campium membranaceum, C. nigrum, C. pseudoscalpturatum, and Bolbitis koidzumii. Campium nigrum was described from a sterile leaf from Ponape. Copeland preferred to keep this collection separate from Campium heteroclitum: 'the combination of frond 
color and opacity and of the striking scaliness of the dark stout stipe marks it off clearly enough'. I found the type material to have a somewhat thicker leaf than is usually found. The colour of the leaf does not deviate, but the light brown scales which lack a paler marginal strip and which seem more persistent than those usually found, give it a rather distinct appearance. The sporangia of several collections from Ponape studied invariably contained either aborted S.M.C.'s or aborted S.M.C.'s and/or aborted spores. Possibly all the morphologically strikingly similar plants from Ponape are part of the same clone.

The epiphytic specimens of northeastern India are stout plants. The sterile pinnae are typical as to size. The venation pattern of part of the material shows irregularly arranged excurrent free veins in a considerable part of the areoles. The fertile pinnae are large, measuring $60-130$ by $17-35 \mathrm{~mm}$. Spores: Mann s.n., vi-1889 (UC): nearly all normal. Ibid. (US): a majority normal, the perispore rather densely cristate. Jerdon s.n. (MICH): the (apparent) majority normal, rather large (the longest axis $c .50-55 \mu \mathrm{m}$ ), the perispore densely cristate. Mann s.n., xi-1885 (S-PA), vii-1888 (SING), vi-1889 (L): all or the greater part of the sporangia with abnormal spores or abnormal S.M.C.'s. The triploid specimens of the present species which are in cultivation at the botanic gardens of Berlin, Groningen, Utrecht, and Leiden and which presumably originate all from the same gathering, are very similar to these Indian plants.

The type material of Campium membranaceum deviates as to the leaf architecture only. The basal pinnae of the sterile leaves are either entire, or provided with a small, basal, basiscopic lobe, or have a basal basiscopic pinnule. One of the fertile leaves shows three pinnae inserted at the same place of the rhachis. Spores of the holotype largely abnormal.

B. Specimens with mostly simple leaves, sometimes with leaves that have two small pinnae. The venation patterm is variously developed. The shape of the lamina or of the terminal segment (in case the leaf is pinnate) is similar to the (upper part of the) terminal segment as found in the specimens mentioned under $\mathrm{A}$. The specimens with narrow leaves (leaf index 10-20(-40)) are generally rheophytic. Here belong the type specimens of Bolbitis annamensis from Indo-China, B. edanyoi, Campium foxworthyi, C. tenuissimum, all from the Philippines, Leptochilus linnaeanus from Java, Acrostichum modestum from Borneo, Leptochilus simplifolius from the Malay Peninsula and L. sumatranus from Sumatra.

Acrostichum modestum, with very narrow and small leaves, was regarded by Christensen (quoted by Copeland, 1928) 'a simple fronded Tectaria, in habit resembling Diplazium lanceum'.

Leptochilus simplicifolius, amply discussed by Holttum (1954), was regarded by him as the mountain form of $B$. heteroclita. The differences between these two species as given by Holttum appeared to be quantitative only.

B. annamensis was supposed by Tardieu-Blot \& C. Christensen to be closely related to Campium hydrophyllum $(=B$. sinuata). The type rather agrees with that of Leptochilus linnaeanus, also in venation pattern, which these authors incorrectly regarded as being totally different. 
Plants intermediate between A and B include the type of Poecilopteris stenophylla (from Java). The outline of this plant is reminiscent of Leptochilus malaccensis which is regarded as parallel variation of $B$. sinuata.

C. Small plants with pinnate leaves, with a well-developed triangular terminal segment. The venation pattern of the two lowest pinnae without or with but few areoles. Here belong the type of Nephrodium ? cuspidatum, part of the type of Gymnopteris inconstans, and part of Cuming 161 (the type of Heteroneuron argutum excluded).

Plants intermediate between B and C include Copeland 33 (GH), Elmer 17554(L), Le Roy Topping 386 (US), 444 (GH, US), Merrill3128(B, syntype of Gymnopterisinconstans), all from the Philippines. The outline of these plants not rarely shows irregularities.

D. Small plants with bipinnate leaves and a well-developed terminal segment; veins free or anastomosing. Here belongs the type material of Edanyoa difformis.

Edanyoa difformis has bipinnate sterile leaves up to $4 \mathrm{~cm}$ long; veins are free. The plants of the type collection recall the genera Microstaphyla and Peltapteris (derivatives of Elaphoglossum). In the type locality of $E$. difformis (Negros I.) Mr. Price collected ample material fully matching the type (Price $2518 \mathrm{~A}, \mathrm{~L}$ ), as well as slightly different plants (Price $2518 \mathrm{~B}, \mathrm{~L}$ ) that also show leaves of a different shape on a single rhizome. These leaves include ternate leaves of a shape as found in juvenile leaves of $B$. heteroclita proper though much smaller, as well as leaves the terminal segment of which is shaped exactly like sterile segments as found in Heteroneuron argutum $(=B . \times$ arguta); all leaves have free veins. Spores: Edaño PNH 5213 (isotype of Edanyoa difformis, PNH), Price 2518 A (CAHUP, L), 2518B (CAHUP, L): sporangia filled with aborted S.M.C.'s and/or aborted spores.

Sterile leaves like those of the type of Edanyoa difformis were also met in several other collections. The leaves on the bulbils of the sterile collections of Edaño PNH 21374 (MICH), also from Negros I., are small, and either similar to the leaves of the type of $E$. difformis or less complex. The leaves that bear the small plants are up to $20 \mathrm{~cm}$ long; the lamina consists of two small basal pinnae and a proportionally long, flagelloid terminal segment, and is similar to that of the larger juvenile leaves as found in well-developed specimens; veins are anastomosed. All kind of intermediate leaves are present in this collection as well. The variation in leaf shape as found in this and other collections justifies the inclusion of $E$. difformis in the present species.

The types of the fertile dwarfs included here in $B$. heteroclita have sterile leaves matching the juvenile leaves formed on the bulbils of (more) well-developed plants. The ample herbarium material of these dwarfs cannot be satisfactorily classified using herbarium methods only.

Cy tol ogy. I have attempted to add further to the understanding of the present complex by cultivating a number of specimens and including some dwarfs of conspicuously different habit. In cultivation these plants show considerable common variation as to the shape of their juvenile leaves in particular; the fertile adults are slightly, though conspicuously dissimilar.

The chromosome numbers of well-developed plants (showing the complex venation pattern) are $2 n=82$ and $2 n=123$. The triploid is so similar to the diploid that the latter 
may have arisen through autoploidization. Jerdon's herbarium collection from northeastern India, mentioned above because of its large spores, possibly represents an autotetraploid. The 'linnaeana' dwarf collected by Prof. Holttum at Pahang, Malaya, is an autotriploid'. 'Cuspidata' dwarfs from the Philippines have $2 \mathrm{n}=c$. 82 , and $2 \mathrm{n}=c$. 123. The diploid and the triploid are so similar that they were originally collected and cultivated under a single number. I only came across the mixture when preparing a count from a meiosis after the material had become fertile. A somewhat larger specimen, also from the Philippines, shows in the wild and in cultivation an astonishing variation of the juvenile leaves; it is a diploid. (Auto)-tetraploids have not (yet) been recorded from the Philippines. They are likely to occur there.

All diploid dwarfs have a \pm irregular meiosis when in cultivation, which is reflected by the abortive spores found in the wild gatherings. The degree of chromosome paring at metaphase 1 of the meiosis varies from sporangium to sporangium. Some karyotypes showed mainly (exclusively?) bivalents.

D is cussion. Because of the shape of the leaves, both the triploid and the diploid dwarfs are likely to be the result of the same process. The abnormal spores formed by the diploids are at first sight strong evidence that these diploids originate from a cross between different species. However, these diploids showed at metaphase 1 a varying number of bivalents, and not univalents only (as seems the rule). They did not originate by simple retention from well-developed diploids as the spores are abnormal. I further fail to recognize substantial differences between the leaves of these diploids and the juvenile leaves as found in well-developed specimens. The question then arises in how far this kind of heterogeneity can be caused by autoploidization as well. The reversal of the polyploid to the diploid condition is not otherwise known from collections occurring in the wild. However, Manton (1950) found, as a result of repeated large-scale sowings of spores of an artificially produced autotriploid of Osmunda regalis L., that most of the 24 , sexually produced eventual sporophytes had \pm diploid somatic cells. Of the few plants that became fertile she made exact counts: two plants were exact diploids, one was a 'diploid plus 1', and two were 'diploids plus 2'. Her illustration of the chromosomes of the 'diploid plus 1' at meiosis shows, apart from one univalent, bivalents only. She did not further report on the meiosis and spores resulting from the 'exact' diploids. These \pm diploid plants of $O$ smunda regalis showed quite a diversity as to the shape of the leaves. Some plants were perfectly normal, the others were smaller and showed either irregularities in the leaves or remained permanently in the juvenile condition.

This or a comparable process as found by Manton in artificial Osmunda regalis autoploids might be a possible explanation for the existence of the diploid dwarfs with aborted spores in $B$. heteroclita. The chance that a viable spore is produced by an autotriploid is exceedingly small. But when formed in Bolbitis heteroclita the resulting sporophyte has means for maintaining itself in the wild through vegetative propagation (bulbils and/or accessorial branching). The same holds for the triploids, whether

'Manton, in a letter to Prof. Holttum, 1958; published with kind permission of Dr. Manton. 


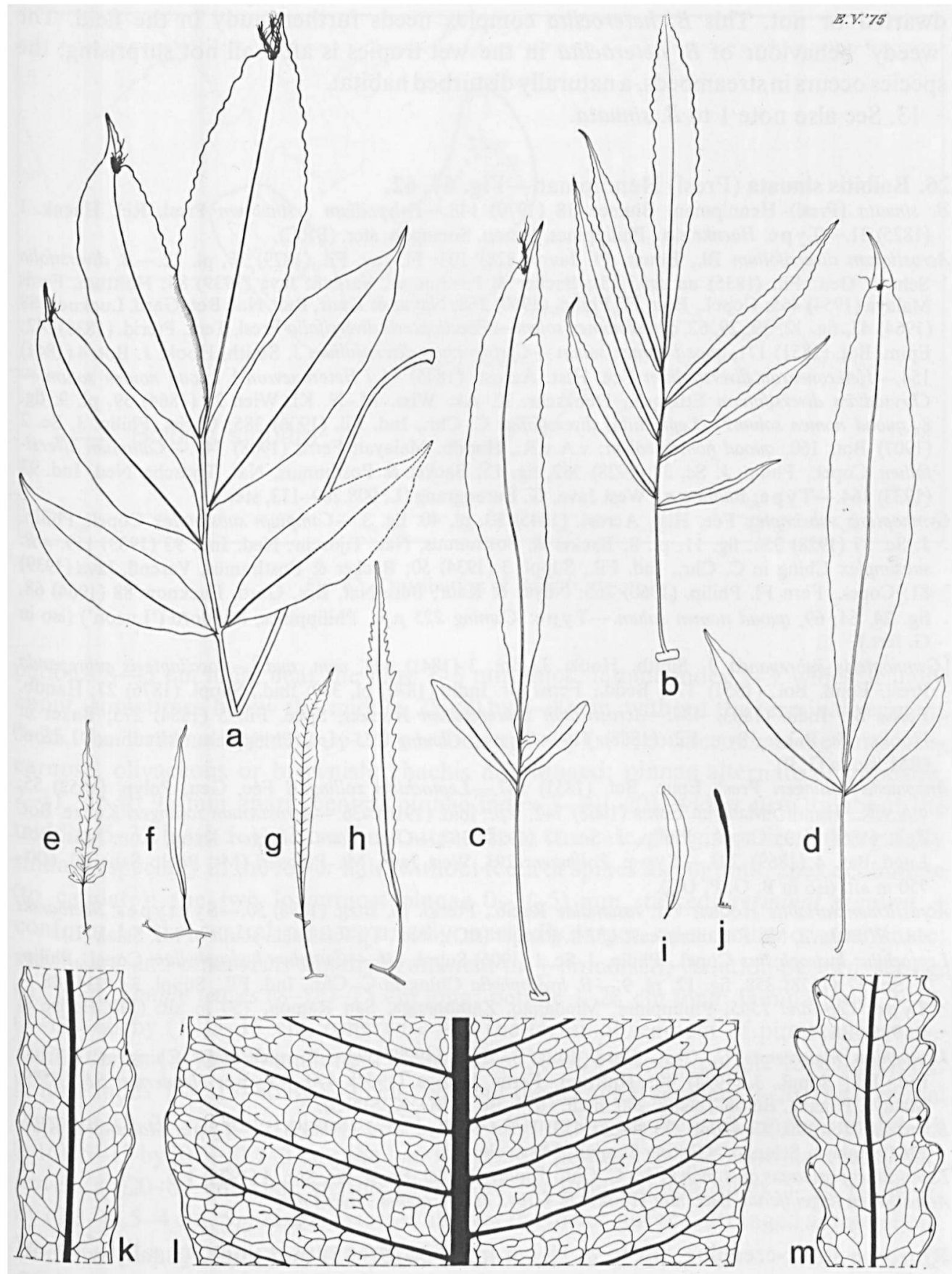

Fig. 61. Bolbitis sinuata.-a, b, o-j. sterile and fertile leaves, $\times 1 / 5 ; \mathrm{c}$, d. sterile leaves, $\times 1 / 7 ; \mathrm{k}-\mathrm{m}$. venation pattern of sterile leaves, $\times$ 4/5 (a. Van Beusekom 550, L; b, k. Price 291, L; c, d. Price 604, L, triploid; e. Hennipman 4100, L, diploid; f. Aloasid \& Edaño PNH 4748, MICH; g. Hennipman 4117, L, diploid; h. Edaño BS 79653, MICH; i, j. Copeland 1565, MICH, holotype of Campium hydrophyllum; 1 . Johnson s.n., L 965.110-982, L, triploid; m. Hennipman 4064, L, diploid). 
dwarfed or not. This $B$. heteroclita complex needs further study in the field. The 'weedy' behaviour of $B$. heteroclita in the wet tropics is after all not surprising; the species occurs in streambeds, a naturally disturbed habitat.

13. See also note 1 to $B$. sinuata.

26. Bolbitis sinuata (Presl) Hennipman-Fig. 61, 62.

B. sinuata (Presl) Hennipman, Blumea 18 (1970) 148.-Polypodium ? sinuatum Presl, Rel. Haenk. 1 (1825) 21.-T y p e: Haenke s.n., Philippines, Luzon, Sorsogon, ster. (PRC).

Acrostichum diversifolium Bl., Enum. Pl. Jav. (1828) 103; Fl. Jav. Fil. (1829) 36, pl. 12.-B. diversifolia Schott, Gen. Fil. (1835) ad tab. (13); Backer \& Posthumus, Varenfl. Java (1939) 84; Holttum, Ferns Malaya (1954) 465; Copel., Fern Fl. Philip. (1960) 258; Nayar \& Kaur, Bull. Nat. Bot. Gard. Lucknow 88 (1964) 42, fig. 12, 19, 29, 62, quoad nomen solem.-Poecilopteris diversifolia Presl, Tent. Pterid. (1836) 242; Epim. Bot. (1851) 171, quoad nomen solum.-Cyrtogonium diversifolium J. Smith, Hook. J. Bot. 4(1841) 154.-Heteroneuron diversifolium Fée, Hist. Acrost. (1845) 91 ('Heteronevron'), quoad nomen solum.Chrysodium diversifolium Ettingsh., Denkschr. K. Ak. Wiss. M.-N. Kl. Wien 22 (1864) 59, pl. 9: fig. 8, quoad nomen solum?-Leptochilus diversifolius C. Chr., Ind. Fil. (1906) 385; Christ, Philip. J. Sc. 2 (1907) Bot. 160, quoad nomen solem; v.A.v.R., Handb. Malayan Ferns (1908) 740._Campium diversifolium Copel., Philip. J. Sc. 37 (1928) 362, fig. 15; Backer \& Posthumus, Nat. Tijdschr. Ned. Ind. 93 (1933) 164.-T y p e: Blume s.n., West Java, G. Burangrang (L, 908.289-113, ster.).

Gymnopteris subsimplex Fée, Hist. Acrost. (1845) 83, pl. 40: fig. 3.-Campium subsimplex Copel., Philin. J. Sc. 37 (1928) 356, fig. 11, pl. 8; Backer \& Posthumus, Nat. Tijdschr. Ned. Ind. 93 (1933) 159.-B. subsimplex Ching in C. Chr., Ind. Fil., Suppl. 3 (1934) 50; Backer \& Posthumus, Varenfl. Java (1939) 81; Copel., Fern Fl. Philip. (1960) 265; Nayar \& Kaur, Bull. Nat. Bot. Gard. Lucknow 88 (1964) 68, fig. 24, 64, 69, quoad nomen solum. - T y p e: Cuming 225 p.p., Philippines, Mindoro ('Luzon') (iso in $\mathrm{G}$, fert.).

[Gymnopteris subrepanda J. Smith, Hook. J. Bot. 3 (1841) 403, nom. nud.]-Poecilopteris subrepanda Presl, Epim. Bot. (1851) 171; Bedd., Ferns Br. India (1870) pl. 339; ibid., Suppl. (1876) 27; Handb. Ferns Br. India (1883) 434.-Acrostichum subrepandum Hooker, Spec. Fil. 5 (1864) 275; Baker in Hooker \& Baker, Syn. Fil. (1868) 419.-Ty pe: Cuming 225 p.p., Philippines, Mindoro, 'Luzon' (BM; iso in $G, P$ ).

Anapausia zollingeri Presl, Epim. Bot. (1851) 187.-Leptochilus zollingeri Fée, Gen. Polyp. (1852) 55; v.A.v.R., Handb. Malayan Ferns (1908) 742, p.p.; ibid. (1916) 436.-Acrostichum zollingeri Kunze, Bot. Zeit. (1864) 419; Copel., Philip. J. Sc. 37 (1928) 357.-Chrysodium zollingeri Kuhn, Ann. Mus. Bot. Lugd.-Bat. 4 (1869) 293.-T y pe: Zollinger 1293, West Java, Mt. Pulosari (Mt. Poelo Saharie), 600$750 \mathrm{~m}$ alt. (iso in $\mathrm{B}, \mathrm{G}, \mathrm{P}, \mathrm{UC}$ ).

Acrostichum variabile Hooker var. rasamalae Racib., Pterid. Fl. Btzg. (1898) 50.-S y n t y pes: Raciborski s.n., West Java, Mt. Rasamala east of Mt. Pantjar (BO); ibid., Tji Rasamala south of Mt. Salak (L).

Leptochilus hydrophyllus Copel., Philip. J. Sc. 1 (1906) Suppl. 146.-Campium hydrophyllum Copel., Philip. J. Sc. 37 (1928) 358, fig. 12, pl. 9.-B. hydrophylla Ching in C. Chr., Ind. Fil., Suppl. 3 (1934) 48.Ty pe: Copeland 1565, Philippines, Mindanao, Zamboanga, San Ramon, $150 \mathrm{~m}$ alt. (MICH; iso in B, P, S-PA, US).

Leptochilus malaccensis C. Chr., Gard. Bull. S. S. 4 (1929) 394.-B. malaccensis Ching in C. Chr., Ind. Fil., Suppl. 3 (1934) 49; Holttum, Ferns Malaya (1954) 470.-T y pe: Henderson SF 18589, Malaya, Pahang, Buloh Telang, $240 \mathrm{~m}$ alt. (BM; iso in BO, K, SING).

B. nitens Holttum, Kew Bull. 13 (1958) 453; Ferns Malaya ed. 2 (1966) 635.-T y pe: Molesworth Allen 3384, Malaya, Selangor, Ginting Simpah, $240 \mathrm{~m}$ alt. (K).

Leptochilus preslianus auct. non (Fée) C. Chr.: Ridley, J. Mal. Br. R. As. Soc. 4 (1926) 115.

Acrostichum flagelliferum auct. non Hooker \& Grev., et syn. homot.: auct., p.p.

Rhizome short-creeping, up to $c .15 \mathrm{~cm}$ long, 3-10(-15) $\mathrm{mm}$ thick, usually without, sometimes with few sclerenchyma strands. Scales of the rhizome: index 3-10, up to 12 by $1.5 \mathrm{~mm}, \pm$ opaque, blackish. Leaves rather close together to $20(-30) \mathrm{mm}$ apart. Sterile leaves simple or pinnate, (8-)20-100 cm long. Pinnate leaves $35-100 \mathrm{~cm}$ long; 


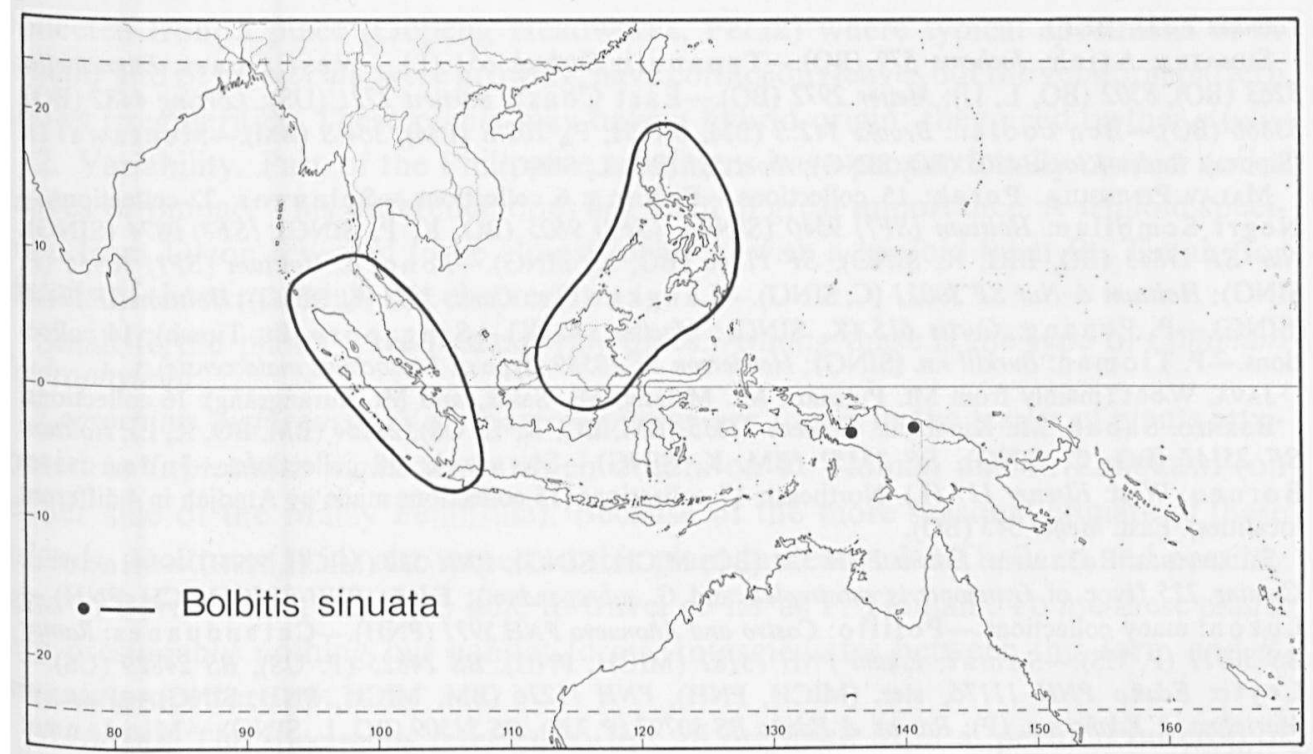

Fig. 62. Distribution of Bolbitis sinuata.

petioles $8-45 \mathrm{~cm}$ long, near the base $1-5 \mathrm{~mm}$ thick; lamina index $1-3$, widest usually about, sometimes below the middle, $25-60$ by $8-40 \mathrm{~cm}$, without the terminal segment $0-25$, the terminal segment $15-50 \mathrm{~cm}$ long, usually (sub)coriaceous, sometimes subcarnose, olivaceous or brownish; rhachis not winged; pinnae alternate or opposite, $2-11$, up to $95 \mathrm{~mm}$ apart; central pinnae index $3-10(-20)$, widest usually about the middle, $5-35$ by $(0.5-) 1-7 \mathrm{~cm}$, base attenuate to truncate, margin entire or irregularly sinuate especially in the lower half, without teeth or spines throughout, apex acuminate (to caudate); the two lowermost pinnae $0-3(-5) \mathrm{mm}$ stalked; terminal segment \pm conform to the central pinnae, usually markedly larger, apex acute or acuminate; secondary and other veins slightly prominent or \pm immersed; venation pattern: see fig. 61: $\mathrm{k}-\mathrm{m}$. Simple leaves (8-)20-75 cm long; petiole 1-28 cm long; lamina index 3-10, (7-)20-60 by (1-)3-15 cm, otherwise like the terminal segment of pinnate leaves.Fertile leaves (8-)20-90 cm long. Pinnate leaves $30-90 \mathrm{~cm}$ long; petiole $10-65 \mathrm{~cm}$ long; lamina index $1-4,15-40$ by $8-25 \mathrm{~cm}$, without the terminal segment $0-20$, the terminal segment 11-28 cm long; pinnae 2-10, up to $60 \mathrm{~mm}$ apart; central pinnae index 4-10 $(-16), 5-17$ by $(0.5-) 0.7-3 \mathrm{~cm}$; the two lowermost pinnae $1.5-3(-6) \mathrm{mm}$ stalked. Simple leaves (8-)20-60 cm long; petiole (4-) $10-40 \mathrm{~cm}$ long; lamina index 5-18, (4-)8-35 by (0.3-) $0.5-4 \mathrm{~cm}$. Sporangia inserted all over the lower surface, arranged acrostichoid. Spores with a smooth cristate perispore.

Chromosomes. $n=41 ; 2 n=122+$ fragm.

INDIA. Ni c o bar I s.: Galathea Exp. 2691 (C), 2859 (BM, C), s.n. (US).

THailand. Peninsular: Van Beusekom 550 (L); E. Smith 541 (K), 2019 (K, SING); Tagawa \& 
Yamada T 213 (BKF).

Sumatra. A tjeh: Jochems 579 (BO).-Tapanuli: Surbeck 651 (L).-West Coast: Bünnemeijer 3263 (BO), 8502 (BO, L, U); Meijer 2972 (BO).-East Coast: Bartlett 7721 (US); Lörzing 4482 (BO), $5386 b$ (BO).-Ben coolen: Brooks 142.S (BM, MICH, P), 202.S (BM), 364.S (BM).-M en tawai Is. (Sipora): Boden-Kloss 14805 (BO, SING); Iboet 531 (BO, L, SING).

Malay Peninsula. Perak: 15 collections. - Pahang: 6 collections.-Selangor: 22 collections.Negri Sembilan: Holttum (SF?) 9540 (SING), (SF?) 9605 (BO, K, P, SING), (SF?) 9874 (SING); Nur SF 11845 (BO, BRI, K, SING), SF 11846 (BO, K, SING).-J o hore: Holttum (SF?) 10956 (P, SING); Holttum \& Nur SF 20021 (C, SING). - La n g k a wi I s.: Curtis 3371 (K, SING); Holttum SF 17440 (SING).-P. Penang: Curtis 615 (K, SING); Mactier s.n. (K).-S ing a pore (Bt. Timah): 14 collections.-P. Ti o m a n: Burkill s.n. (SING); Henderson SF 18589 (type of Leptochilus malaccensis).

JAvA. West (mainly from Mt. Pulosari, Mt. Malang, Mt. Salak, and Mt. Burangrang): 16 collections.

Borneo. S a b a h. Mt. Kinabalu: Clemens 27635 (BM, BO, K, L, US), 28234 (BM, BO, K, L); Holttum SF 25141 (BO, C, SING), SF 25352 (BM, K, SING).-Sarawak: 18 collections.-Indonesian B orn e o. West: Elsener 127 (L). Northeast: 17 collections (15 collections made by Amdjah in 4 different localities). East: Meijer 545 (BO).

PhilupPINes. Pa a a a n: Edano PNH 524 (BO, MICH, SING), PNH 532 (MICH, PNH).-Mindor o: Cuming 225 (type of Gymnopteris subsimplex and G. subrepandum); Edaño PNH 3876 (MICH, PNH).Luzon: many collections.-Polillo: Castro and Añonuevo PNH 5971 (PNH). - Ca t a nd u a n es: Ramos BS 30311 (P, US).-S a mar: Edaño PNH 15182 (MICH, PNH), BS 24825 (P, US), BS 24829 (US).L ey te: Edaño PNH 11176, ster. (MICH, PNH), PNH 11226 (BM, MICH, PNH, SING),-P a n ay: Martelino \& Edanto s.n. (P); Ramos \& Edaño BS 30797 (P, US), BS 31309 (BO, L, SING)._Min da na o: 8 collections.

New GuineA. West. Albatros bivouac. Docters van Leeuwen 9135 (BO, K, L). Sine loc.: Beccari s.n. (K). -Nor t he as t. Torricelli Mts.: Schlechter 14442 (B).

Habitat. Terrestrial and low epiphytic in evergreen forest. Altitude: 0-1200 $(-1800) \mathrm{m}$.

S pores. Allen 3405 (S-PA), Van Beusekom 550 (L), Copeland s.n. (MICH. herb. Copeland 10212), Elsener H 127 (L), Henderson SF 18589 (type of Leptochilus malaccensis), Kiah s.n. (SING): the greater part normal. Elmer 7629 (P): about equal parts normal and abnormal. Copeland 1565 (MICH, type of Leptochilus hydrophyllus), Cuming 225 (BM, type of Gymnopteris subrepanda), Elmer 9041 (MICH), Holttum SF 25352 (SING), Zollinger 1293 (P, type of Acrostichum zollingeri): the greater part abnormal. Backer 14467 (BO), Brooks 364.S (BM), Curtis 3371 (SING, paratype of Leptochilus malaccensis), Johnson s.n. (L, a triploid), Nur s.n. (SING), Ramos \& Edaño BS 36971 (L), Williams 205 (NY): only abnormal spores and aborted S.M.C.'s present.

Not es. 1. A rather heterogeneous species readily recognized through the combination of texture-markedly different from that of $B$. heteroclita, its closest relativeleaf architecture, and venation pattern. A texture similar to that of the present species is also found in $B$. rivularis which belongs to a different series. $B$. sinuata is confined to the wettest parts with evergreen forest in Malesia. $B . \times$ singaporensis is a hybrid between the present species and $B$. appendiculata spp. appendiculata. $B$. sinuata is also included in the parentage of Leptochilus $\times$ trifidus. Hybrids with $B$. heteroclita are likely to be formed. The texture of the leaves, which is the best differentiating character, is difficult to evaluate from herbarium material. Plants with a seemingly less coriaceous texture than normal include Zollinger 1293 (type of Leptochilus zollingeri, Java) and Elmer 7629 (BO, K, L, M, P) from the Philippines, which is also otherwise very similar to Zollinger 1293. Molesworth Allen 4365 (S-PA), 4556 (S-PA), 4731 (S-PA), all 
collected from a place (Gopeng Headworks, Perak) where typical specimens of $\boldsymbol{B}$. sinuata and $B$. heteroclita were growing, have coriaceous leaves but the venation pattern shows irregularities. These plants may have a hybrid origin; they need further study.

2. Variability. Part of the Philippine specimens have proportionally narrow entire leaves or pinnae. This difference does not seem of great importance. A triploid specimen from Luzon revealed to be almost identical with a triploid from Bt. Timah (for details of these materials see chapter 7).

Small fertile plants were found a few times. Among these is the type of Campium hydrophyllum.

Leptochilus malaccensis. Very narrow pinnae are found in the leaves of plants attributed to Leptochilus malaccensis which occurs on P. Tioman and P. Langkawi (on either side of the Malay Peninsula). Because of the more seasonal climate of these islands, Holttum (1954) also expected this plant in Peninsular Thailand and possibly also in Borneo 'as it has been able to travel so far as P. Tioman'. To me these plants are presumably nothing but habitat forms. Intermediates between this form and $B$. sinuata proper are not rare.

$B$. nitens. The specimens referred to this all originate from a 'peculiar outcrop of white quartzite (?), of $c .100 \times 60$ feet which apparently becomes very dry' and 'which may well simulate a more seasonal climate than is experienced by the ferns in the normal rainforest a few yards away' (Holttum, 1958). The only feature by which the material from this area deviates distinctly is that of the scales on the petiole, which seem permanent or almost so. In how far such a condition is not the result of the different habitat conditions remains to be demonstrated. Mrs. Allen (in Holttum, 1958) was of opinion that B. nitens also occurred in a similar habitat in Sarawak. This is incorrect; the material she had in mind (e.g. Lörzing 12405 p.p., L) represents Leptochilus $\times$ trifidus.

7. Series Heudelotianae Hennipman, ser. nov.

Terrestres, crescunt in locis temporaliter inundatis vel in fluminibus permanentibus. Lamina pinnata segmento terminali triangulari, sine bulbillis, marginibus edentatis vel espinosis. Ordinatio venularum is series Portoricensium similis. Sporae perispora laevi undulata.

Ty pus: Bolbitis heudelotii (Bory ex Fée) Alston.

Terrestrial in seasonally flooded habitats or in permanent streams. Rhizome \pm longcreeping, slender to rather stout, with 2 rows of leaves, without sclerenchyma strands. Scales of the rhizome up to $10 \mathrm{~mm}$ long. Leaves \pm spaced. Sterile leaves pinnate; petiole with 5-11 vascular bundles; lamina without bulbils; pinnae 8-21(-26), the margin without teeth or spines; terminal segment triangular; venation pattern: veins forming an intricate network, areoles varying in size and shape, the majority with one or a few ex- or recurrent, simple or forked, included free veins. Spores with a smooth undulate perispore.

Chromosomes. $2 \mathrm{n}=82$.

Distribution. Tropical Africa. See fig. 30, 64 . 
27. Bolbitis heudelotii (Fée) Alston-Fig. 63 : a-i; 64.

B. heudelotii (Bory ex Fée) Alston, J. Bot. 72, Suppl. 2 (1934) 3; C. Chr., Ind. Fil., Suppl. 3 (1934) 48, 198, Alston, J. Bot. 77 (1939) 285; in Hutch. \& Dalziel, Fl. West Trop. Afr. ed. 2, Suppl. (1959) 68; Harley, Handb. Liberian Ferns (1963) 41, fig.; Tardieu-Blot in Aubrév., Fl. Gabon 8 (1964) 188; in Aubrév., Fl. Cameroun 3 (1964) 319, pl. 51: fig. 1 \& 2; Arnoult, Bull. Assoc. Franç. Aquariophiles n.s. 19 (1964) 4; Pichi Sermolli, Webbia 23 (1969) 388, fig. 1-3; Benl, Heine \& Michel, Pisciculture Franç. 20 (1969) iv-xi, pl. 1-2, phot.; Schelpe, Contr. Bolus Herb. 1 (1969) 41.-Gymnopteris heudelotii Bory ex Fée, Hist. Acrost. (1845) 84, pl. 45; Christ, Farnkr. Erde (1897) 51, fig. 1-3; Engler, Veg. Erde 9, 2 (1908) 6.-Anapausia heudelotii Presl, Epim. Bot. (1851) 187.-Acrostichum heudelotii Hooker, Spec. Fil. 5 (1864) 264; Baker in Hooker \& Baker, Syn. Fil. (1868) 224.-Acrostichum heudelotii Hooker var. major Hooker, Spec. Fil. 5 (1864) 264, nom. illeg.-Chrysodium heudelotii Kuhn, Fil. Afric. (1868) 51.Leptochilus heudelotti C. Chr., Ind. Fil. (1906) 385.-Campium heudelotii Copel., Philip. J. Sc. 37 (1928) 396.-T y pe: Heudelot 805 ('s.n.'), Guinea, Fouta-Djallon ('Senégambie, Foula-Dhiallon') (P; iso in $B$, some sterile and fertile pinnae, $K, L$ ).

Acrostichum heudelotii (Fée) Hooker var. minor Hooker, Spec. Fil. 5 (1864) 264.-S y n t y p es: Barter s.n., Sierra Leone (K); Kirk s.n., Malawi, Manganja country, $90 \mathrm{~m}$ alt. (K).

Campium angustifolium Copel., Philip. J. Sc. 37 (1928) 396, fig. 47, pl. 31.-B. heudelotii var. angustifolia Tardieu-Blot, Not. Syst. 14 (1953) 349.-Ty p e: Mildbraed 9404, Cameroun, Buar, c. $1000 \mathrm{~m}$ alt. (K, 2 sh.).

B. felixii Tardieu-Blot, Not. Syst. 13 (1948) 169.-T y pe: Félix 933, Guinea, vicinity of Macenta (P).

Rhizome up to $20 \mathrm{~cm}$ long, up to $10 \mathrm{~mm}$ thick. Scales of the rhizome: index 2-5, up to 10 by $2 \mathrm{~mm}$, opaque, light to dark brown. Sterile leaves $30-85 \mathrm{~cm}$ long; petiole 8-40 cm long, near the base 2-6 mm thick; lamina index 1-4, widest about or below the middle, $25-50$ by $12-25 \mathrm{~cm}$, without the terminal segment $6-35$, the terminal segment 10-22 cm long, herbaceous, green to blackish; rhachis not winged; pinnae alternate or opposite, 8-21(-26), 15-30 mm apart; central pinnae index 5-14, widest about or below the middle, $5.5-17$ by $1-3 \mathrm{~cm}$, base \pm symmetrical, cuneate, margin usually \pm entire, sometimes serrate, without teeth or spines, apex acute, acuminate, or orbicular; the two lowermost pinae $2-8 \mathrm{~mm}$ stalked, usually \pm conform to the central pinnae, sometimes asymmetrical with the basiscopic side wider and lobed to $3 / 5$ towards the costa; the uppermost pinnae usually adnate at the basiscopic side of the base; terminal segment triangular, deeply lobed near the base, tapering towards the acute or acuminate apex; secondary and other veins slightly prominent or \pm immersed; venation pattern: see fig. 63: e, f. Fertile leaves $25-100 \mathrm{~cm}$ long; petiole $25-55 \mathrm{~cm}$ long; lamina index $2-5,15-45$ by $9-17 \mathrm{~cm}$, without the terminal segment $10-35$, the terminal segment 5-13 cm long; pinnae 11-28, 10-30 mm apart; central pinnae index $8-15(-20)$, widest below the middle, $4-14.5$ by $0.4-1.3 \mathrm{~cm}$; the two lowermost pinnae 3-8 mm stalked. Sporangia inserted all over the lower surface. Spores large, with a smooth undulate perispore usually situated at considerable distance from the exospore.

Chromosomes. $2 n=82$.

Fig. 63. a-i. Bolbitis heudelotii. a, b. sterile and fertile leaf resp. of a terrestrial plant, $\times 1 / 5$; c. lowermost pinna of a fertile leaf, $\times 1 / 5$; d. sterile leaf of an aquatic plant, $\times 1 / 5 ; \mathrm{e}, \mathrm{f}$. shape and venation pattern of pinnae of an aquatic plant, $\times 4 / 5$; g. venation pattern of a fertile pinna, $\times 4 / 5 ; \mathrm{h}$, i. ibid. of sterile pinnae (a. Stolz 1003, M; b. Hepper 1826, K; c. Cook 66, GH; d, f. Linder 1154, GH; e. Dusén s.n., 1892, P: o. Peter 10301. S-PA: h. Stolz 1003. S-PA: i. ibid.. L. - $\mathrm{i}-\mathrm{m}$. Bolbitis lindigii. j. juvenile leaf, $\times 1 / 5$; k. sterile leaf, $\times 1 / 5 ; 1, \mathrm{~m}$. venation pattern of sterile pinnae, $\times 4 / 5$ (j. Lewy 1851, $\mathrm{P}$, isotype of Chrysodium pellucens; k. Alston 7836, BM; 1. Gascoyne-Cecil 88, L; m. Lancaster 588, US). 


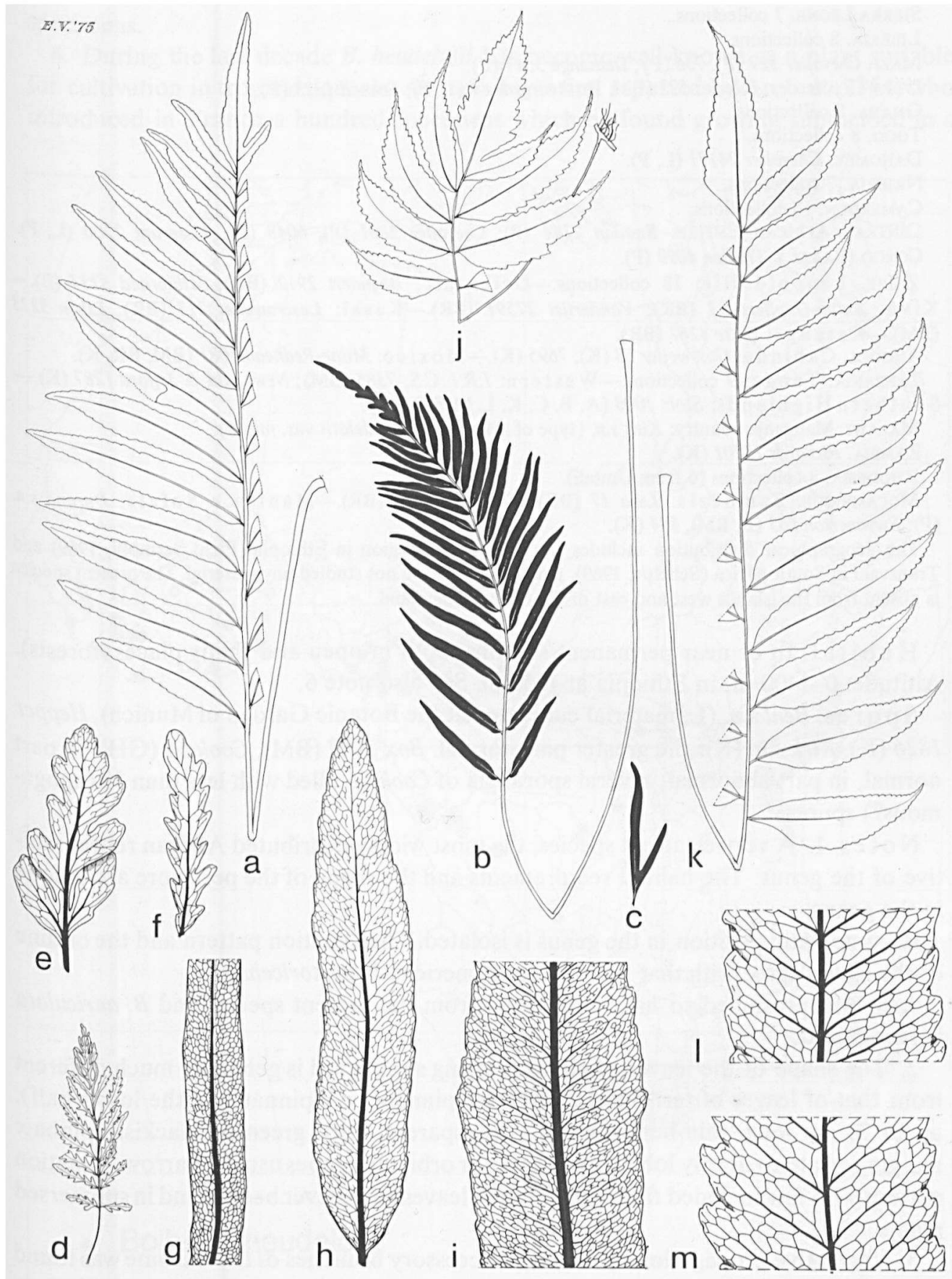


GUINEA. 15 collections.

SIERRA LEONE. 7 collections.

LIBERIA. 8 collections.

MaLI. Chevalier 529 (P), 756 (P); Demange 3547 (P).

Ivory Coast. des Abbayes 593 (BM, P); Anonymus 4 (L, P); Schnell 1323 (P).

Ghana. 8 collections.

Togo. 8 collections.

DAHOMEY. Chevalier 24171 (L, P).

Nigeria. 7 collections.

Cameroun. 18 collections.

Central African Empire. Baudon 2244 (P); Chevalier 5801 (P), 6048 (P); Tisserant 2200 (L, P).

Congo (BrazZ.). Thollon 4099 (P).

ZaIRE. Leopoldville: 18 collections.-Orientale: Bequaert 2912 (BR); Mildbraed 3134 (B).-

Kivu: Antun-Gupffert 33 (BR); Vanderijst 17591 (BR).-Kasai: Lescrauwaet 213 (BR); Liben 3223 (BM).-Ka t a ng a: Witte 4267 (BR).

Angola. C a b in da: Gossweiler 58 (K), 7695 (K).-Moxi co: Milne-Redhead 4081 (BM, BR, K).

TANZANIA. T ang a: 9 collections.-W estern: I.R.L.C.S. 7385 (BM); Newbould \& Jefford 1287 (K).Sout hern Highl ands: Stolz 1003 (A, B, C, K, L, M, S-PA, U).

MALAWI. Manganja country: Kirk s.n. (type of Acrostichum heudelotii var. minor).

ZAMBIA. Richards 13201 (K).

RHODESIA. 8 collections (6 from Umtali).

Mocambique, Zambezia: Luja 17 (BR), 421 (BR), 456 (BR).-Manica e S ofala: Durand s.n. (P); Swynnerton 833 (B, BM), 834 (K).

The geographical distribution includes the Lake Tana region in Ethiopia (Pichi Sermolli, 1969) and Transvaal in South Africa (Schelpe, 1969), from where I have not studied any material. The present species is absent from the islands west and east of the tropical mainland.

$\mathrm{H}$ abit at. In or near permanent streams, both in open and shady places (forests). Altitude: $0-1400 \mathrm{~m}$; in Ethiopia at $1850 \mathrm{~m}$. See also note 6.

Sp or es. Benl s.n. (L, material cultivated at the Botanic Garden of Munich), Hepper 1826 (K), Kirk s.n. (K): the greater part normal. Box 2083 (BM), Cook 66 (GH): in part normal, in part abnormal; several sporangia of Cook 66 filled with less than 32 (apogamous?) spores.

Notes. 1. A very clear-cut species, the most widely distributed African representative of the genus. The habitat requirements and the shape of the perispore are unique in the genus.

Its systematic position in the genus is isolated. The venation nattern and the nutline of the leaves agree with that found in the American B. portoricensis.

A hybrid presumed to have originated from the present species and $B$. auriculata is recognized.

2. The shape of the leaves of plants growing submersed is generally much different from that of leaves of terrestrials. They are pinnate or bipinnate (in the lower half), up to $30 \mathrm{~cm}$ long, thin-herbaceous, \pm transparent, dark green to blackish; pinnae: margin serrate to deeply lobed, apex acute or orbicular; lobes usually narrow. Venation pattern with few included free veins. Fertile leaves have never been found in submersed plants.

3. Vegetative propagation by means of accessory branches of the rhizome was found in all plants in cultivation whether growing submersed (when growth is slow) or terrestrial (when growth may be rapid). The accessory branches formed in association with the formation of the leaf (see fig. 2: b) are well-developed on the oldest part of 
the rhizome only; this is the reason why they are generally not present in herbarium specimens.

4. During the last decade $B$. heudelotii has become well-known as a plant suitable for cultivation in tropical aquaria. As such it was first reported by Arnoult (1964) who introduced in France a hundred specimens which he found growing submersed in a

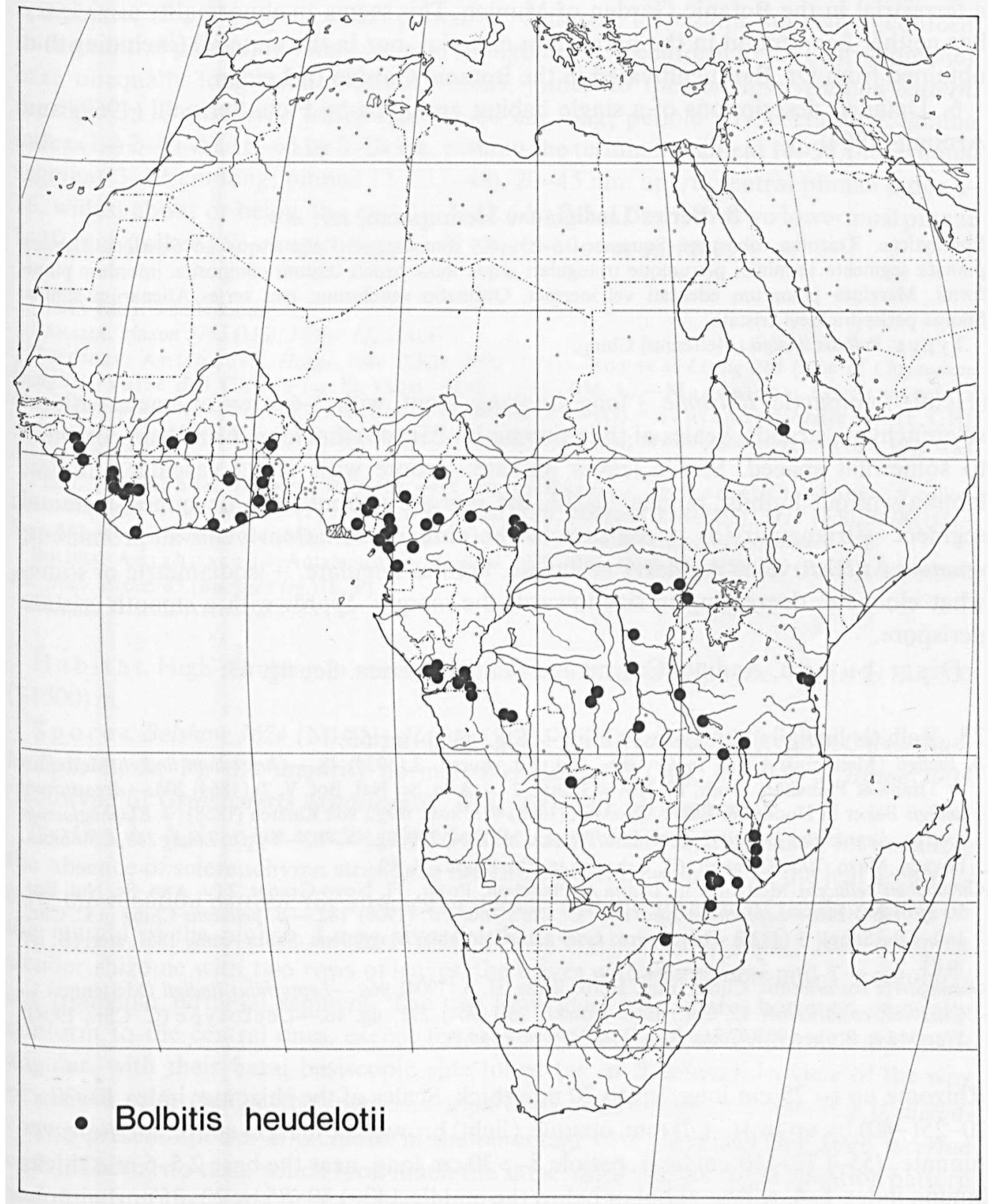

Fig. 64. Distribution of ser. Heudelotianae (monotypic). 
river in Upper Volta. He treated these like aquatics and all but one perished. The remaining plant was multiplied by means of vegetative propagation (accessory branches of the rhizome). Later, the species was introduced from other localities as well. Benl et al. (1969) cultivated the material as a terrestrial and were the first to obtain fertile leaves in cultivation.

5. Benl et al. (1969) reported a case of vivipary on the leaves of a plant grown as a terrestrial in the Botanic Garden of Munich. This seems an abnormality as vivipary has neither been found in the herbarium material, nor in the material (including that obtained from Dr. Benl) cultivated in the Botanic Garden of Leiden.

6. Detailed descriptions of a single habitat are given by Pichi Sermolli (1969) and Arnoult (1964).

\section{Series Lindigianae Hennipman, ser. nov.}

Epiphyticae. Rhizoma robustum. Squamae $15-25(-40) \mathrm{mm}$ longae. Folia usque ad 6-seriata. Lamina pinnata segmento terminali plerumque triangulari atque lobis paucis tantum composita, interdum pinniformi. Margines pinnarum edentati vel inermes. Ordinatio venularum: eius series Alienarum similis. Sporae perispora laevi cristata.

Ty p u s: Bolbitis lindigii (Mettenius) Ching.

(Hemi-?) epiphytic. Rhizome \pm long-creeping, stout, with 2-6 rows of leaves without sclerenchyma strands. Scales of the rhizome 10-25(-40) $\mathrm{mm}$ long. Leaves approximate to somewhat spaced. Sterile leaves pinnate; petiole with 10-22 vascular bundles; lamina without bulbils; pinnae $12-37$, the margin without teeth or spines; terminal segment \pm triangular (or \pm conform to the pinnae); venation: veins all prominent; venation pattern: veins regularly reticulate, areoles angulate, \pm isodiametric or somewhat elongate, decreasing in size towards the margin. Spores with a smooth cristate perispore.

Distribution. Andine. Central and South America. See fig. 65.

28. Bolbitis lindigii (Mettenius) Ching-Fig. 63: $\mathbf{j}-\mathrm{m} ; 65$.

B. lindigii (Mettenius) Ching in C. Chr., Ind. Fil., Suppl. 3 (1934) 48.-Chrysodium lindigii Mettenius in Triana \& Planchon, Prodr. Fl. Novo-Granat. 2 (= Ann. Sc. Nat. Bot. V, 2) (1864) 204.-Acrostichum lindigii Baker in Hooker \& Baker, Syn. Fil. (1868) 423, nom. illeg., non Karsten $(1858)(=$ Elaphoglossum spec.).-Leptochilus lindigii C. Chr., Bot. Tidsskr. 26 (1904) 290, fig. 4a, 4b. - T y p e:Lindig 258, Colombia, Boyaca, Muzo, 700-900 m alt. (B, 2 sh.; iso in BM, fragm., K, P).

Chrysodium pellucens Mettenius in Triana \& Planchon, Prodr. Fl. Novo-Granat. 2 (= Ann. Sc. Nat. Bot. V, 2) (1864) 205. - Leptochilus spec. dub. C. Chr., Ind. Fil. (1906) 182. - B. pellucens Ching in C. Chr., Ind. Fil., Suppl. 3 (1934) 49.-T y pe: Lewy s.n., Colombia, Magdalena, Santa Anna, 1851, ster. (B; iso in $\mathrm{P})$.

Gymnopteris costaricensis Christ, Bull. Herb. Boiss. II, 4 (1904) 964.-Leptochilus lindigii (Mettenius) C. Chr. var. costaricensis C. Chr., Bot. Tidsskr. 26 (1904) 291, fig. 4b.-L e c to ty p e (C. Chr., 1904): Werckle \& Brune 1903, Costa Rica, s. loc. (P; iso in BM).

Rhizome up to $25 \mathrm{~cm}$ long, up to $20 \mathrm{~mm}$ thick. Scales of the rhizome: index 10-30,c. $10-25(-40)$ by up to $1(-1.7) \mathrm{mm}$, opaque, (light) brown, the margin entire. Sterile leaves pinnate, (55-) 70-110 cm long; petiole $8->30 \mathrm{~cm}$ long, near the base $2.5-6 \mathrm{~mm}$ thick; lamina index $1-4$, widest about or below the middle, ( $40-) 50-85$ by $20-35 \mathrm{~cm}$, without 
the terminal segment (20-)35-73, the terminal segment 10-21 cm long, firmly herbaceous, light to dark green; rhachis not winged; pinnae usually alternate, $12-37,25-60 \mathrm{~mm}$ apart; pseudo-articulate, straight to sigmoid; central pinnae index 3-8, widest about or below the middle, $12-26$ by $2.5-5 \mathrm{~cm}$, base usually \pm symmetrical, (narrowly)cuneate (to subcordate), sometimes \pm oblique, margin usually finely crenate-serrate especially towards the apex, or crenate-serrately lobed to $1 / 5$ towards the costa, apex (long-) acuminate; the two lowermost pinnae \pm deflexed, $1.5-12 \mathrm{~mm}$ stalked, \pm conform to the central pinnae; terminal segment triangular (or \pm conform to the central pinnae), base unequally long-sided, without bulbils, joined to the rhachis; venation pattern: see fig. 63: 1, m. Fertile leaves (25-)35-80 cm long; petiole 10-30 cm long; lamina index (1-) $2-4(-5), 16-65$ by $8-20 \mathrm{~cm}$, without the terminal segment $14-58$, the terminal segment 3-12 cm long; pinnae 12-33 (-48), 20-45 mm apart; central pinnae index 416 , widest about or below the middle, $5-12.5$ by $0.5-1.5 \mathrm{~cm}$; the two lowermost pinnae 1-10 mm stalked. Sporangia inserted all over the lower surface.

Costa Rica. 8 collections.

Panama. Maxon 5745 (US); Pittier 4225 (US).

Colombia. Antioquia: Hodge 7044 (GH), 7072 (GH).-B oyaca: Lindig 258 (type of Chrysodium lindigii).-Valle del Cauca (= El Valle): Alston 7836 (BM, L).-Magdalena: Lewy s.n. (type of Chrysodium pellucens).-Norte de S an tander: Bischler 2609 (G). - S an tand e r: Hodge 6512 (F, GH). ECUAdOR. Grubb et al. 1651 (US); Jvosio(?)-Morecalli 45/8 (K); Mexia 7260 (GH, MICH, UC, US); Stübel 929 (B).

Peru. A mazonas: Tessmann 4001 (B), 4415 (B).-H u a nu co: Stork s.n. (UC).-Junin: 9 collections.-L or et o: Schunke 333 (F, NY, S, US), 14076 (US); Williams 3240 (US), 3416 (US), 3418 (US).Sa n Mart in: Belshaw 3624 (MICH, UC).-S in e loc.: Matthew 1107 (K); Sandeman 3446 (K).

BoliviA. A n ta hu a ca (= Antahuacana): Buchtien 2220 (BM, G, S-PA, US), s.n. (P, US); Rosenstock, Fil. Boliv. exsicc. 85 (Buchtien leg.)(L, P).

BRAZIL. South. Acre: Ule 9138 (B).

$\mathrm{H}$ a bit a t. High-climbing in rain forest, also reported as epiphytic. Altitude: $0-1200$ $(-1500) \mathrm{m}$.

S p o r es. Belshaw 3624 (MICH), Hodge 6512 (F, GH), Lindig 258 (type of Chrysodium lindigii, B, K, P): the majority normal. Wendland 507 (W), Werckle \& Brune 1903 (lectotype of Gymnopteris costaricensis, BM), 1904 (P): all abnormal.

Notes. 1. A clear-cut species related to $B$. aliena with which it shares, for instance, the absence of sclerenchyma strands in the rhizome, the essentials of the leaf architecture, the venation pattern, the absence of bulbils, and the absence of teeth or spines on the margin of the pinnae. Large juvenile plants are terrestrial, with a long-creeping slender rhizome with two rows of leaves, the leaves with few pinnae and a \pm regularly cut triangular terminal segment. The two lowermost pinnae are, however, generally conform to the central ones, except for those of Wendland $507(\mathrm{~W})$ which are \pm triangular, with their basal basiscopic side lobed (as in $B$. aliena). In view of the way of growth of $B$. lindigii, the species is apparently a derivative of a fern like $B$. aliena.

The present species is included in the parentage of $B$. bernoulli (see Spec. incertae sedis). These two taxa, which look much the same, differ mainly in the venation pattern and details of the scales of the rhizome. 


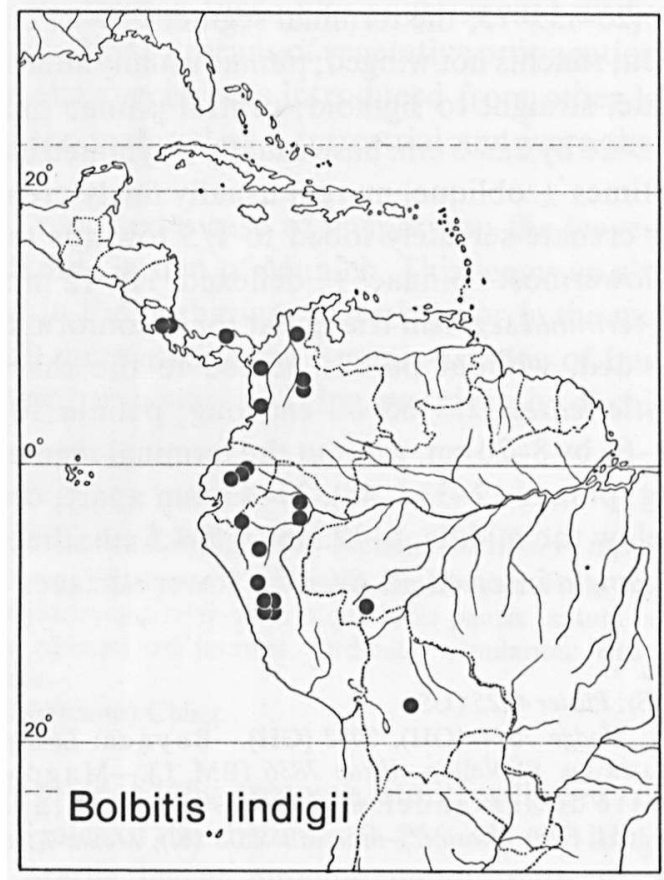

Fig. 65. Distribution of ser. Lindigianae (monotypic).

2. Chrysodium pellucens was not understood by Christensen (op. cit. 1904, 1906). The type material consists of small sterile plants, the leaves of which are composed of only six pinnae. The margin of the pinnae is lobed to $1 / 4$ towards the costa. They represent juvenile leaves.

\section{Series Portoricenses Hennipman, ser. nov.}

Squamae basi affixae. Lamina simplex, vel pinnata segmento terminali pinnatiformi vel triangulari, bulbillis unico vel plerisque subterminalibus. Ordinatio venularum: venulae in reticulationem intricatam anastomosentes, areolis in parte venulis liberis plerumque recurrentibus.

Ty pus: Bolbitis portoricensis (Sprengel) Hennipman.

(Exclusively?) terrestrial. Rhizome \pm short-creeping, \pm stout or rather slender, with 2 rows of leaves, with or without sclerenchyma strands. Scales of the rhizome up to $10 \mathrm{~mm}$ long. Leaves approximate to somewhat spaced. Sterile leaves pinnate or simple; petiole with 3-11 vascular bundles; lamina with one or more \pm spherical subterminal bulbil(s); pinnae up to $14(-18)$, the margin sometimes with teeth in the sinuses; terminal segment either triangular or conform to the pinnae; secondary veins slightly prominent at the base, others veins immersed; venation pattern: veins forming an intricate network, areoles varying in size and shape, part of which with one or more, (mostly) 
recurrent, simple or forked, included free veins. Spores with a smooth, cristate or cristate-undulate perispore.

Distribution. (Sub)tropical America. See fig. 30, 67.

\section{Bolbitis hastata (Fournier) Hennipman-Fig 66: a-c; 67.}

B. hastata (Fournier) Hennipman, Amer. Fern J. 65 (1975) 30.-Acrostichum hastatum Liebmann, Kong. Dansk. Vid. Selsk. Skrift. V, 1 (1849) 172, nom. illeg., non Thunb. (1784) (= Pyrrosia spec.).-Gymnopteris hastata Fournier, Mex. Pl. 1 (1872) 70.-Leptochilus liebmannii Maxon, Proc. Biol. Soc. Wash. 43(1930) 86, nom. illeg. (art. 72). - B. liebmannii C. Chr., Ind. Fil., Suppl. 3 (1934) 49.-T y pe: Liebmann s.n. (in part distributed as Liebmann Pl. Mexic. 2440), Mexico, Vera Cruz, Arroyo de Isapa, Baranca de Jovo, near St. Maria Tlapacoyo (= Tlapacoyan), 450-600 m alt. (C; iso in B, K, US).

Acrostichum alienum auct. non Swartz c. syn. homot.: auct. p.p.

Rhizome rather slender, c. $5 \mathrm{~mm}$ thick (Barrington 403, GH). Scales of the rhizome: index 3-4, up to 4 by $1 \mathrm{~mm}$, opaque, light brown. Leaves somewhat spaced. Sterile leaves pinnatipartite, the three complete ones available 63,84 , and $95 \mathrm{~cm}$ long, resp. (Liebmann: 45-65); petiole 23, 40, and $41 \mathrm{~cm}$ long, resp., near the base $c .3 \mathrm{~mm}$ thick, with 5-7 vascular bundles; lamina index 1-2, widest somewhat below the middle, $28-54$ by $17-35 \mathrm{~cm}$, herbaceous, olivaceous, sometimes with a glaucous tinge, with one subterminal bulbil on terminal (and lateral) segment(s); lateral segments usually \pm opposite, 4-8, spaced; the two lowermost segments: index 3-5, widest about the middle, $13-22$ by $3-5 \mathrm{~cm}$, margin \pm entire, with an inconspicuous \pm translucent membrane, apex acute or acuminate, the sinus between the segments orbicular or truncate, without teeth or spines; the lamina at the sinuses above the two lowermost lobes 8$30 \mathrm{~mm}$ wide; terminal segment conform to the lateral ones but larger; venationpattern: secondary veins of the segments somewhat unequally dichotomous quite near the costa, see also fig. 66: c. Fertile leaves: only incomplete leaves available; lamina index c. 2, 15-20 by $6-11 \mathrm{~cm}$; segments $4-7$, the two lowermost segments: index 3-4, 4.5-7 by c. $1.5 \mathrm{~cm}$; the lamina above the two lowermost segments $0.7-1.6 \mathrm{~cm}$ wide. Sporangia inserted all over the lower surface. Spores with a smooth cristate or cristate-undulate perispore.

Mexico. Chi ap as: Ghiesbrecht 370 ('Chiapas, etc.'; BM, GH, K).-O ax a ca. Dist. Choapan: Hallberg 1597 (L, NY).-Vera Cruz: Liebmann s.n. (type of Acrostichum hastatum); Barrington 403 (GH).

Dr. J. T. Mickel (in litt.) kindly communicated three other localities in the State of Oaxaca: Dist. Villa Alta: Hallberg 1616 (NY); Dist. Tuxtepec: Mickel 5903 (NY); Dist. Ixtlan: Mickel 6420 (NY).

$\mathrm{Habit}$ at. Terrestrial in moist ravines in the subtropical forest region; on wet rock ledges or in sand. Altitude: $100-850 \mathrm{~m}$.

Spores. Ghiesbrecht 370 (BM, GH, K): the greater part normal, perispore cristate. Liebmann s.n. (type of Acrostichum hastatum, US): ibid., perispore cristateundulate.

Notes. 1. Liebmann regarded the present species as one of the rarest Mexican ferns. It is closely related to $B$. portoricensis which differs in the shape of the leaves and the rhizome, as well as in the relative position of the leaves on the rhizome. I 

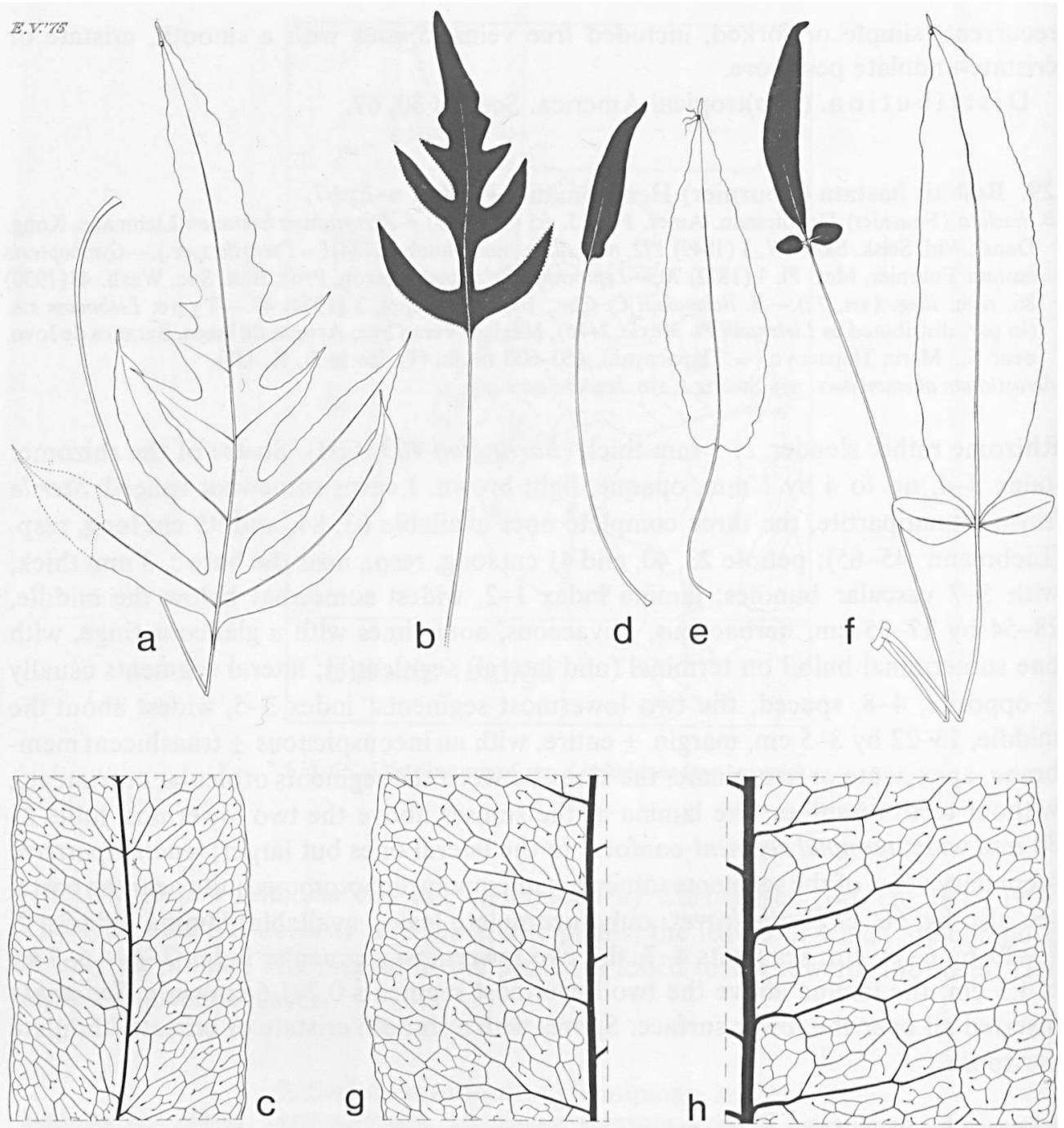

Fig. 66. a-c. Bolbitis hastata. a, b. sterile and fertile leaf resp., $\times 1 / 5$; c. venation pattern of lobe of sterile leaf, $\times 4 / 5$ (a, b. Ghiesbrecht 370, K; c. Liebmann s.n., K, isotype of B. hastata).-d-h. Bolbitis pandurifolia. $\mathrm{d}-\mathrm{f}$. sterile and fertile leaves, $\times 1 / 5$; g. venation pattern of central part of terminal segment, $\times 4 / 5$; h. ibid. of lamina (d, e. Spruce 4741, BR, isotype of B. pandurifolia; f. Spruce 5684, K; g. ibid., P; h. Spruce 4741 , BM, see d).

regard the present species a derivative of a fern like $B$. portoricensis. The derived shape of the leaves agrees with that of Phymathodes scolopendria (as was already noticed by Liebmann) and also with that found in the African B. fluviatilis which is otherwise quite different.

2. The altitude records on the labels of the type specimens show variation; it is given as $450 \mathrm{~m}$ (iso in.US) and as $600 \mathrm{~m}$ (iso in $\mathrm{K}$ ). 
30. Bolbitis pandurifolia (Hooker) C. Chr.-Fig. 66: d-h; 67.

B. pandurifolia (Hooker) C. Chr., Ind. Fil., Suppl. 3 (1934) 49, p.p. (excl. syn. Leptochilus oligarchicus; 'oligogarchicus').-Gymnopteris pandurifolia Hooker, Sec. Cent. Ferns (1861) pl. 87 (excellent).Acrostichum pandurifolium Hooker, Spec. Fil. 5 (1864) 271; Baker in Hooker \& Baker, Syn. Fil. (1868) 422; Sodiro, Crypt. Vasc. Quit. (1893) 497.-Leptochilus pandurifolius C. Chr., Bot. Tidsskr. 26 (1904) 286.-Ty p e: Spruce 4741, Peru, San Martin, Mt. Guayrapurima near Tarapoto (K; iso in BM, BR, P). Acrostichum oligarchicum auct. non Baker: Sodiro, Crypt. Vasc. Quit. (1895) 493.

Rhizome \pm stout, $c, 4 \mathrm{~mm}$ thick, without sclerenchyma strands (Killip \& Smith 26039, US). Scales of the rhizome: index 3-8, up to 7 by $1.5 \mathrm{~mm}$, opaque, brown. Leaves \pm approximate. Sterile leaves simple or ternate with proportionally small pinnae, $20-60 \mathrm{~cm}$ long. Ternate leaves $45-60 \mathrm{~cm}$ long; petiole $16-27 \mathrm{~cm}$ long, near the base 3-4 mm thick, with $3(-5$ ?) vascular bundles; lamina index $2-5$, widest usually below (to about) the middle, $26-36$ by $6-12 \mathrm{~cm}$, firm-herbaceous to subcarnose, greenish, with one subterminal bulbil; rhachis not winged; pinnae \pm opposite, index 1-3, widest about or below the middle, $2-10$ by $1.8-4 \mathrm{~cm}, 0-2 \mathrm{~mm}$ stalked, base \pm symmetrical to slightly oblique with the basiscopic side larger, cuneate, margin usually entire, sometimes slightly repand or sinuate especially in the basal part, without teeth or spines, apex acute to acuminate; terminal segment not articulated to the rhachis, $6-9 \mathrm{~cm}$ wide, base (short-) decurrent, otherwise like the pinnae; venation pattern: areolae relatively large, free included veins relatively long, see also fig. 66 : g. Simple leaves $20-50 \mathrm{~cm}$ long; petiole 3-17 cm long; lamina index 2-5, widest somewhat below (to about) the middle, part of the leaves also broadened near the base but never conspicuously panduriform, $15-35$ by $4.5-10 \mathrm{~cm}$, base (narrowly to) broadly cuneate to auriculate, otherwise like the terminal segment of pinnate leaves.-Fertile leaves $20-56 \mathrm{~cm}$ long. Ternate leaves $(2$ leaves of Spruce 5684) 48 and $56 \mathrm{~cm}$ long; petiole 32 and $42 \mathrm{~cm}$ long, resp.; lamina index c. $4, c .4 \mathrm{~cm}$ wide; pinnae shortly stalked, resp. c. 2 by 1 , and 1.5 and 2.5 by $c .0 .8 \mathrm{~cm}$; terminal segment $c .2 \mathrm{~cm}$ wide, base narrowly cuneate. Simple leaves $20-45 \mathrm{~cm}$ long; petiole $13-30 \mathrm{~cm}$ long; lamina index $4-8,7-17$ by $1.2-2.7 \mathrm{~cm}$, base usually gradually decurrent, sometimes subcordate and long-decurrent. Sporangia inserted all over the lower surface. Spores with a smooth cristate perispore.

Ecuador. Chi m b or azo: Spruce 5684 (BM, K, NY, P).-S in e lo c.: Sodiro s.n., viii-1891 (B, P, UC). Peru. A mazonas: Wurdack 1897 (NY).-San Martin: Spruce 4741 (type of Gymnopteris panduri: folia)._J u nin: Killip \& Smith 26039 (NY, US).

H a bit a t. Rain forest. Terrestrial (Wurdack 1897) or (low?) epiphytic(Killip \& Smith 26039). Altitude: 350-500 m (Wurdack 1897); 1100 m (Killip \& Smith 26039).

Sp or es. Spruce 4741 (type of Gymnopteris pandurifolia, BM, K), 5684 (K), Wurdack 1897 (NY): the greater part normal. Killip \& Smith 26039 (NY): sporangia mostly young, the mature ones with aborted spores or aborted S.M.C.'s.

Notes. 1. A somewhat critical species of which the details of the venation pattern and the shape of the bulbil suggest relationship to $B$. portoricensis and $B$. hastata rather than to a representative of ser. Euryostichae with a similar shape of the leaves. The 


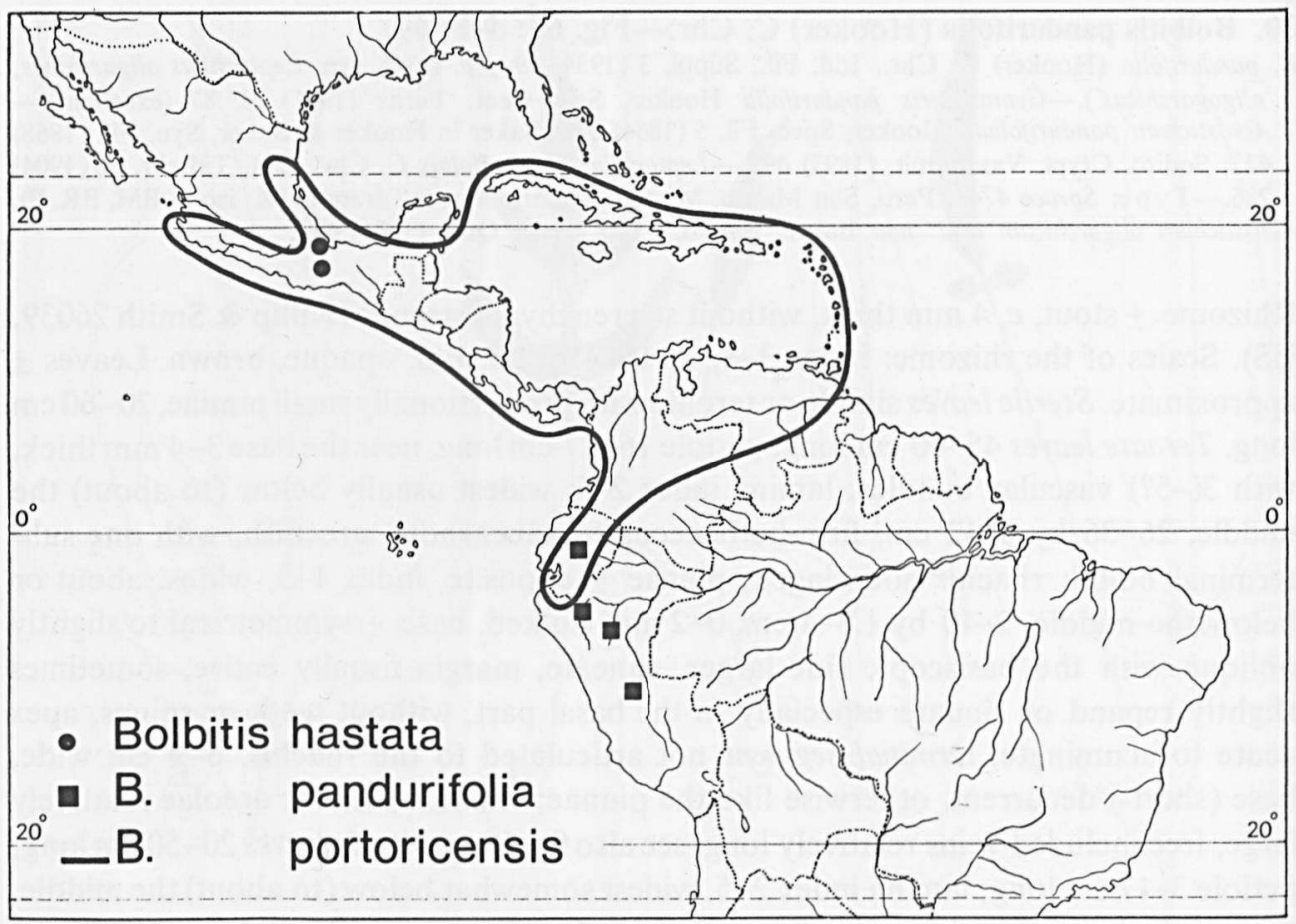

Fig. 67. Distribution of ser. Portoricenses: Bolbitis hastata, B. pandurifolia, and B. portoricensis.

configuration of the vascular strands in the one rhizome sectioned is also unlike that found in ser. Euryostichae. The appressed scales on the petiole could not be studied in sufficient detail.

2. Variation in the shape of the leaves. The collections from Ecuador have ternate leaves with a long and firm petiole, whereas those from Peru have simple leaves with a relatively shorter and carnose petiole.

31. Bolbitis portoricensis (Sprengel) Hennipman-Fig. 67, 68.

B. portoricensis (Sprengel) Hennipman, Amer. Fern J. 65 (1975) 30.-Acrostichum portoricense Sprengel, Nova Acta Caes. Leop. Carol. 10 (1821) 226.-Gymnopteris portoricensis Fée, Hist. Acrost. (1845) 85.Anapausia portoricensis Presl, Epim. Bot. (1851) 187.-T y pe: Bertero s.n., West Indies, Puerto Rico (G; iso in P, herb. Bory, fert., TO, fert.).

Acrostichum cladorrhizans Sprengel, Nova Acta Caes. Leop. Carol. 10 (1821) 225; Syst. Veg. 4 (1827) 37.Leptochilus cladorrhizans Maxon, Sc. Surv. Porto Rico \& Virgin Is. 6 (= Pterid. Porto Rico \& Virgin Is.) (1926) 460.-B. cladorrhizans Ching in C. Chr., Ind. Fil., Suppl. 3 (1934) 47; Vareschi in Lasser, Fl. Venezuela 1 (1969) 374, pl. 70.-T y p e: Bertero s.n., West Indies, Puerto Rico (G; iso in P, herb. Bory, fert., TO, fert.).

Acrostichum irregulare Liebmann, Kong. Dansk. Vid. Selsk. Skrift. V, 1 (1849) 173._Gymnopteris irregularis Fournier, Mex. Pl. 1 (1872) 70.-T y pe: Liebmann s.n., Mexico, Vera Cruz, Baranca de St. Maria Tlatetlá, $450-540 \mathrm{~m}$ alt. (iso in B). 
Poecilopteris lobulosa Presl, Epim. Bot. (1851) 174; C. Chr., Bot. Tidsskr. 26 (1904) 297; Ind. Fil. (1906) 503.-T y pe: Friedrichsthal s.n., Guatemala, 1841, fert. (W).

Acrostichum alienum Swartz var. flagellum Jenman, Bull. Bot. Dept. Jamaica (Kingston) n.s. $5(=11)(1898)$ 154.-Leptochilus alienus (Swartz) C. Chr. var. flagellus Bonap., Notes Ptérid. 1 (1915) 164.-T y pe: Jenman s.n., Jamaica (n.v.).

Acrostichum alienum Swartz var. semipinnatifidum auct. non (Fée) Baker: Christ, Bull. Soc. Bot. Belg. 35 (1896) 246; Jenman, Bull. Bot. Dept. Jamaica (Kingston) n.s. 5 (1898) 154.

Acrostichum alienum auct. non Swartz c. syn. homot.: auct. p.p.

[Filix latifolia laciniata, et ad lacinias molliter aculeata, Plumier, Tract. Fil. Amer. (1705) 9, pl. 9.-L e g.: Plumier s.n., Santo Domingo (P, herb. Vaillant).]

[Filix latifolia, in pinnulas obtusas, et leviter crenatas divisa, Plumier, Tract. Fil. Amer. (1705) 10, pl. 10.L eg.: Plumier s.n., Martinique (P, herb. Surian, n.v.).]

Rhizome stout, up to $15(-20) \mathrm{mm}$ thick, with sclerenchyma strands. Scales of the rhizome: index 3-8, up to 10 by $2 \mathrm{~mm}$, usually opaque, sometimes subclathrate, reddish brown. Leaves approximate. Sterile leaves pinnate, $20->200 \mathrm{~cm}$ long; petiole 4-60 cm long, near the base $1.5-7 \mathrm{~mm}$ thick, with 5-11 vascular bundles; lamina of non-flagelloid leaves: index 1-3, widest usually somewhat below the middle, sometimes at the base, $15-80$ by $7-50 \mathrm{~cm}$, without the terminal segment $0-35(-45)$, the terminal segment $15-55 \mathrm{~cm}$ long, herbaceous, usually light or bright green, usually without conspicuous bulbils; rhachis not winged; lamina of flagelloid leaves up to $c .200 \mathrm{~cm}$ long, without the terminal segment $6-50$, the terminal segment up to $150 \mathrm{~cm}$ long, possibly even longer, the flagellum with 3-5 bulbils situated $10-30 \mathrm{~cm}$ apart; pinnae usually \pm opposite, 2-14(-18), up to $110 \mathrm{~mm}$ apart; central pinnae index (2-)3-8, widest about or below the middle, $3.5-30(-35)$ by $1.3-7 \mathrm{~cm}$, base \pm symmetrical, variously shaped, margin either entire or (bi)serrate-crenate or lobed to $2 / 3$ towards the costa, with or without \pm distinct teeth (in the sinuses), apex acute or acuminate, lobes or segments of pinnae obliquely inserted, straight or subfalcate, the margin entire to (bi)serrate-crenate; the two lowermost pinnae $0-10 \mathrm{~mm}$ stalked, usually asymmetrical with the acroscopic side conform to that of the other pinnae, the basiscopic side triangular to about as long as wide, deeply pinnatifid near the base, segments of about similar shape as those of proximal pinnae; terminal segment triangular, near the base deeply pinnatifid, gradually less incised towards the apex, base narrowly cuneate, apex acute, acuminate, or (long-) flagelloid, base of segments usually abruptly long-decurrent; venation pattern: rather variable as to the size of the areoles, see also fig. 68: e-j. Fertile leaves with an acute or somewhat prolonged apex, 12-135 cm long; petiole 7-85 cm long; lamina index 1-4, $5-50$ by $2.5-25 \mathrm{~cm}$, without the terminal segment $0-30$, the terminal segment $5-30 \mathrm{~cm}$ long; pinnae 2-12, 15-85 mm apart; central pinnae index 3-15, widest about the middle, $0.9-17$ by $0.3-3.5 \mathrm{~cm}$; the two lowermost pinnae $0-8 \mathrm{~mm}$ stalked. Sporangia inserted usually all over the lower surface, sometimes along the main veins with a narrow sterile strip. Spores with a smooth cristate or cristate-undulate perispore.

Mexico. Central and southern states: many collections.

BELIZE. 6 collections.

Guatemala. Many collections.

HoNDURAs. 13 collections. 
Salvador. 6 collections.

Nicaragua. 14 collections.

Costa RicA. Many collections.

CUBA. 13 collections.

JAMAICA. Hart 63 (US); Jenman 26 (K); Watt 118 (P, U, US).

DOMinican RepUbLic. 8 collections.

HaITI. 5 collections.

Puerto Rico. 9 collections.

Lesser Antilles. (St. Eustatius, St. Kitts, Montserrat, Guadeloupe, Dominica, Martinique, St. Lucia, St. Vincent, Trinidad, and Tobago). 60 collections.

Panama. Many collections.

Colombia. Antioquia: Haught 4629 (F, US).-Guajira: Haught 4293 (S, US).-Magdalena: Bennett 17 (F, US); Purdie s.n. (BM, K); H. H. Smith 1054 (B, BM, BR, F, G, GH, K, L, MO, NY, P, U, UC, US).-Meta: Philipson et al. 2055 (BM).-Norte de S antander: Bischler 2418 (G).

Venezuela. Aragua: 5 collections.-A nzoategui: Steyermark 61142 (F, US).-Bolivar: $L$. Williams 11438 (F, US).-Carabobo: Alston 6161 (BM); Linden 1573 (BR).-Merida: Fendler 336 (BR, G, GH, K, MO); Vogl s.n. (M).-Y a r a cu y: Vareschi \& Pannier 2619 (US).

ECUADOR. Seemann $368(\mathrm{~K})$; Sodiro s.n. (P); Verleysen s.n. (P).

$\mathrm{H}$ a bi t a t. Usually creeping on rocks (several times reported from sandstone), sometimes in soil, in usually wet places in forests. Altitude: $0-1250$ (in Guatemala -1800 ) $\mathrm{m}$.

Spores. Herminier s.n. (L), Proctor 19262 (U), Schipp 526 (K): the greater part normal, the perispore densely and low cristate. Friedrichsthal s.n. (type of Poecilopteris lobulosa, W): ibid., the perispore with few inconspicuous crests, sometimes locally with minute spines. Liebmann s.n. (type of Acrostichum irregulare, B): ibid., the perispore cristate-undulate. Bertero s.n. (type of Acrostichum portoricense, G), ibid. (type of $A$. cladorrhizans, P), Sintenis 6261 (L), H. M. Smith 1054 (L): abnormal, the normally shaped ones with a cristate perispore.

Notes. 1. A clear-cut species which is exceedingly variable as regards the shape of both the leaves and the pinnae. B. portoricensis is easily recognized especially by the combination of leaf-shape and venation pattern. It is closely related to $B$. hastata. The relationship to $B$. pandurifolia is less apparent. The present species has been confused with $B$. aliena by others. It is presumably included in the parentage of $B$. semipinnatifida.

2. The type specimens of Acrostichum portoricense and $A$. cladorrhizans, though with leaves of a rather different outline, easily fit into the species as delimited here. Fée (1845) who first united these species under the name $A$. portoricense, used characters of the sporangia (size, shape, and number of indurated cells) to discriminate between these two and Gymnopteris aliena sensu Fée $(=B$. portoricensis). However, these features show considerable variation in a number of collections studied (the material studied by Fée included).

3. Poecilopteris lobulosa. Presl (1851) based himself on fertile material collected by Friedrichsthal and referred to a collection which Fée had earlier annotated as Heteronevron .... As Presl did not annotate this specimen, the original material of this could be traced only thanks to Fée's annotation. The type matches completely the piece of fertile leaf present on Friedrichsthal 1044 (W, 3 sh.) that Presl annotated as Anapausia aliena. 

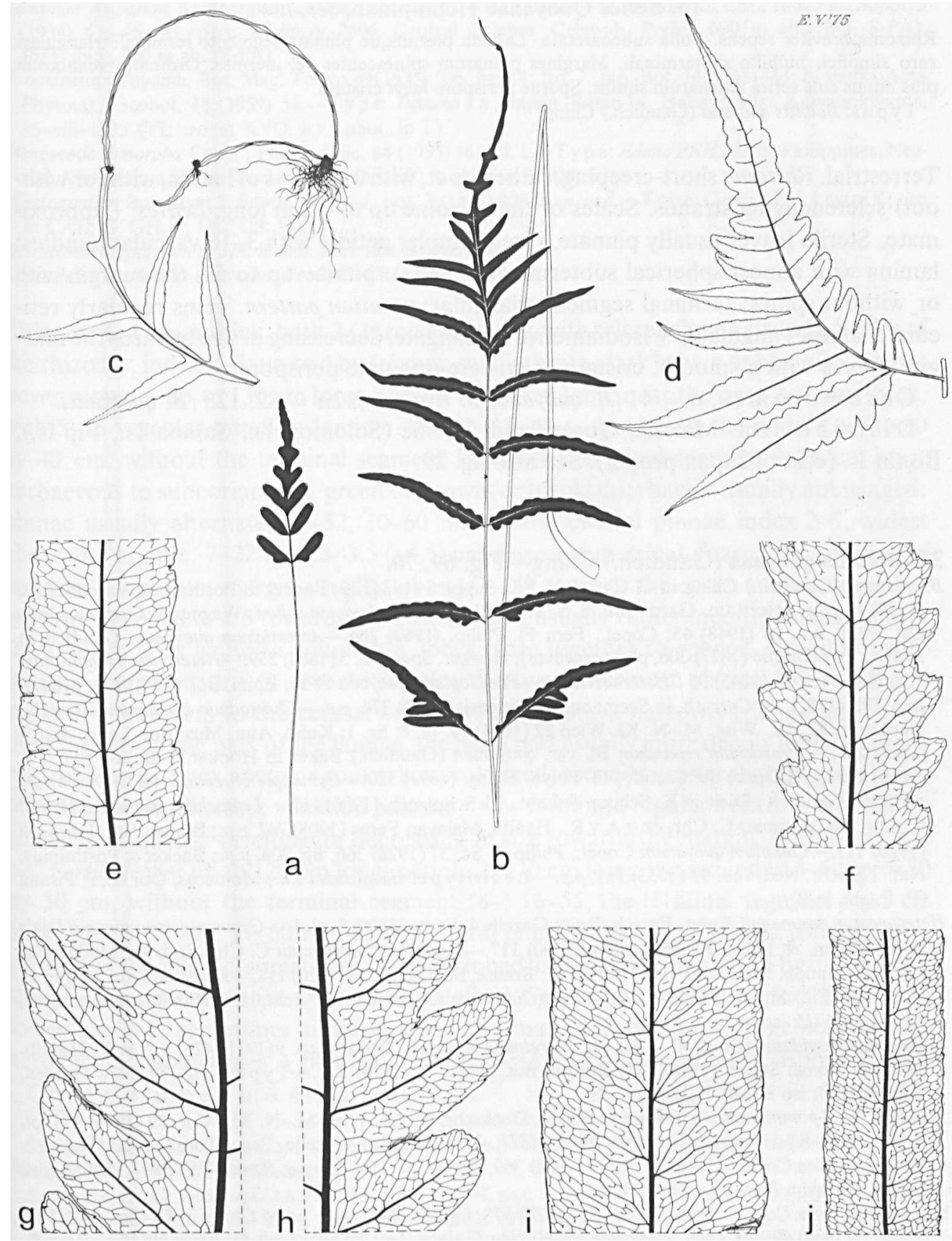

Fig. 68. Bolbitis portoricensis. -a, b. fertile leaves, $\times 1 / 5$; c. terminal part of sterile leaf, $\times 1 / 5$; d. basal pinna of a sterile leaf, $\times 1 / 5 ; \mathrm{e}-\mathrm{j}$, venation pattern of sterile pinnae, $\times 4 / 5$ (a. Skutch $2577, \mathrm{~K} ; \mathrm{b}$. Dodge \& Allen 17329, US; c. H. H. Smith 1054, K; d. Kupper 739, M; e. Walker T 10495, BM; f. Von Türckheim 2728, P; g. Herminier s.n., L; h. Alston 5826, BM; i. Alfaro 16535, P; j. C. F. Baker 2185, P). 


\section{Series Quoyanae Hennipman, ser. nov.}

Rhizoma breviter repens. Folia subcoarctata. Lamina plerumque pinnata segmento terminali triangulari, raro simplici, bulbillo subterminali. Margines pinnarum spinescentes vel inermes. Ordinatio venularum: plus minus eius series Alienarum similis. Sporae perispora laevi cristata.

Ty pus: Bolbitis quoyana (Gaudich.) Ching.

Terrestrial. Rhizome short-creeping, rather stout, with 2(3) rows of leaves, with (or without) sclerenchyma strands. Scales of the rhizome up to $4 \mathrm{~mm}$ long. Leaves \pm approximate. Sterile leaves usually pinnate, rarely simple; petiole with 3-16 vascular bundles; lamina with almost spherical subterminal bulbil(s); pinnae up to 52, the margin with or without spines; terminal segment triangular; venation pattern: veins regularly reticulate, areoles angulate, \pm isodiametric or elongate, decreasing in size towards the margin. Spores with a smooth, cristate or cristate-undulate perispore.

Chromosomes. $\mathrm{n}=41$ (B. quoyana, B. rivularis), $2 \mathrm{n}=82,123$ (B. quoyana).

Distribution. Malesia, Queensland, Pacific (Solomon Is., Samoa Is., Fiji Is.), Bonin Is. (east of Japan proper). See also fig. 29.

\section{Bolbitis quoyana (Gaudich.) Ching-Fig. 69, 70.}

B. quoyana (Gaudich.) Ching in C. Chr., Ind. Fil., Suppl. 3 (1934) 49; Backer \& Posthumus, Varenfl. Java (1939) 81, p.p.; Holttum, Garc. Bull. S. S. 11 (1947) 271, quoad nomen solum: Wagner \& Grether, Univ. Cal. Publ. Bot. 23 (1948) 45; Copel., Fern Fl. Philip. (1960) 264.-Acrostichum quoyanum Gaudich. in Freyc., Voy. Uranie (1827) 306, pl. 3 (excellent); Hooker, Spec. Fil. 5(1864) 259.- Heteroneuron quoyanum Fte, Hist. Acrost. (1845) 96 ('Heteronevron'). - Poecilopteris quoyana Presl, Epim. Bot. (1851) 173; Moore, Ind. Fil. (1857) 13; Carruth. in Seemann, Fl. Vitiensis (1873) 374, p.p. - Chrysodium quoyanum Ettingsh., Denkschr. K. Ak. Wiss. M.-N. Kl. Wien 22 (1864) 59, pl. 8: fig. 1; Kuhn, Ann. Mus. Bot. Lugd.-Bat. 4 (1869) 293.-Acrostichum repandum Bl. var. quoyanum (Gaudich.) Baker in Hooker \& Baker, Syn. Fil. (1868) 419, p.p.; Drake del Castillo, Fl. Polyn. Frans. (1893) 320._Gymnopteris repanda (B1.) Christ var. quoyana (Gaudich.) Diels in K. Schum. \& Laut., Fl. Schutzgeb. (1900) 117. - Leptochilus cuspidatus (Presl) C. Chr. var. quoyanus C. Chr. ex v.A.v.R., Handb. Malayan Ferns (1908) 742, p.p.; Brause, Bot. Jahrb. 56 (1920) 117.-Campium quoyanum Copel., Philip. J. Sc. 37 (1928) 366, fig. 20a, p.p.; Backer \& Posthumus, Nat. Tijdschr. Ned. Ind. 93 (1933) 161, p.p. - L e ct o t y pe: Gaudichaud s.n., Moluccas, Obi Is., P. Pisang (P; fragm. in B).

Heteroneuron naumannii Kuhn, Forsch. Reise Gazelle 4, Farne (1889) 5, pl. 1.-Gymnopteris naumannii Diels in K. Schum. \& Laut., Fl. Schutzgeb. (1900) 117.-Leptochilus naumannii C. Chr., Ind. Fil. (1906) 386; v.A.v.R., Handb. Malayan Ferns (1908) 742; Brause, Bot. Jahrb. 56 (1920) 118.-B. naumannii Ching in C. Chr., Ind. Fil., Suppl. 3 (1934) 49.-T y pe: Naumann s.n., Bismarck Arch., New Hanover, south coast, 23-vii-1875 (B; iso in B).

Leptochilus cuspidatus (Presl) C. Chr. var. marginalis Rosenst., Fedde Rep. 9(1911) 426; v.A.v.R., Handb. Malayan Ferns, Suppl. (1916) 435; Brause, Bot. Jahrb. 56(1920) 117.-T y pe: King 144, New Guinea, Ambasi (BO; iso in $\mathrm{MICH}, 3 \mathrm{sh}$.).

Aspidium novo-pommeranicum Brause ex Rech., Denkschr. K. Ak. Wiss. M.-N. Kl. Wien 89 (1914) 471, pl. 3: fig. 8b.-Syntypes: Rechinger 3836, 4371, New Britain, Gazelle Pen., Toma, ster. (W, n.v.). Campium validum Copel., Philip. J. Sc. 37 (1928) 369, fig. 22, pl. 15.-T y pe: Ramos BS 13807, Philippines, Luzon, Cagayan Prov. (n.v.; MICH?).

Campium parvum Copel., Philip. J. Sc. 37 (1928) 375, fig. 28, pl. 21.-B. parva Ching in C. Chr., Ind. Fil., Suppl. 3 (1934) 49.-T y pe: Schlechter 16163, New Guinea, Terr. of New Guinea, $300 \mathrm{~m}$ alt. (UC; iso in B, L, P).

Campium enorme Copel., Philip. J. Sc. 40(1929) 307, pl. 8. - B. enormis C. Chr., Ind. Fil., Suppl. 3(1934) 197; Copel., Fern Fl. Philip. (1960) 264.-Ty pe: Copeland s.n., Philippines, Mindanao, Mt. Matatum, 1-v-1917 (MICH, herb. Copeland 11539, 11540; iso in UC, 353983). 
Campium viviparum Kjellberg in C. Chr., Bot. Jahrb. 66 (1933) 50.-B. vivipara C. Chr., Ind. Fil., Suppl. 3 (1934) 51.-Syntypes: Kjellberg 2506, Central Celebes, Celewab, Preho, $600 \mathrm{~m}$ alt. (BO, S-PA), 3635 , ibid., $500 \mathrm{~m}$ alt. (S-PA).

B. boninensis Tuyama, Bot. Mag. Tokyo 49(1935) 506, fig. 14; Ito, J. Jap. Bot. 14(1938) 443; Iwatsuki, Acta Phytotax. Geobot. 18 (1959) 54.-T y p e: Tuyama s.n., Japan, Bonin Is., Hahajima Is., Kuwanokiyama, 15-viii-1933 (TI; iso in KYO, n.v., phot. in L).

Stenosemia dimorpha Copel., Philip. J. Sc. 84 (1955) 161, pl. 1.-T y p e: Edaño PNH 21388, Philippines, Negros, Kinabkaban River, Canlaon Volcano, $650 \mathrm{~m}$ alt. (iso in BM, L).

[Cyrtogonium laciniatum J. Smith, Hook. J. Bot. 3 (1841) 403, nom. nud.-Ty p e: Cuming 294, Leyte (K; iso in CAL, G, GH, P, UC, US, W).]

Acrostichum repandum et syn. homot. auct. non B1.: auct. p.p.

Leptochilus cuspidatus auct. non (Presl) C. Chr.: auct. p.p.

Rhizome 6-13 mm thick, with 2 (3) rows of leaves, with sclerenchyma strands. Scales of the rhizome: index 2-7, up to 4 by $1.5 \mathrm{~mm}$, subclathrate, dark brown or blackish. Sterile leaves pinnate, up to $130 \mathrm{~cm}$ long; petiole $10-50 \mathrm{~cm}$ long, near the base $2-9 \mathrm{~mm}$ thick, with 5-16 vascular bundles; lamina index $1-3(-4)$, widest below the middle, up to 80 by $40 \mathrm{~cm}$, without the terminal segment $12-70$, the terminal segment 9-26 cm long, herbaceous to subcoriaceous, green or brown (or blackish); rhachis usually not winged; pinnae usually alternate, $13-52,10-60 \mathrm{~mm}$ apart; central pinnae index 2-7, widest about the middle, $7-22$ by $1.3-3.5(-4.5) \mathrm{cm}$, base symmetrical, (narrowly to) broadly cuneate to cordate, margin usually lobes $1 / 3-2 / 3$ towards the costa, sometimes either \pm entire or lobed to $4 / 5$ towards the costa, with a usually rather inconspicuous spine in the sinuses, apex acute to acuminate, lobes close together to spaced, straight or subfalcate, margin entire or crenate-serrate; the two lowermost pinnae 1-9 (-13) mm stalked, \pm conform to the central ones; terminal segment narrowly triangular, shorter than the remaining part of the lamina, the basal part usually more deeply lobed than the central pinnae, apex acute or somewhat prolonged; secondary veins slightly prominent, other veins \pm immersed; venation pattern: veins forming a regular network, areoles angulate isodiametric or elongate decreasing in size towards the margin, see also fig. 69: l-s. Fertile leaves up to $120 \mathrm{~cm}$ long; petiole $25-70 \mathrm{~cm}$ long; lamina index 3-6, up to 70 by $30 \mathrm{~cm}$, without the terminal segment (8-) $16-55$, the terminal segment $4-15 \mathrm{~cm}$ long; pinnae 11-42, 17-60 mm apart; central pinnae index 5-15, widest usually about (to below) the middle, sometimes as deeply lobed as the sterile ones, $3-15$ by $0.4-1.7 \mathrm{~cm}$; the two lowermost pinnae up to $13 \mathrm{~mm}$ stalked. Sporangia usually inserted all over the lower surface, sometimes situated along the margin only, the arrangement usually acrostichoid, sometimes \pm pteridoid. Spores with a smooth cristate perispore.

Chromosomes. $n=41 ; 2 n=82,123$.

JAPAN. B on in Is.: Tuyama s.n. (type of B. boninensis); Warburg s.n. (B). Additional collections cited by Iwatsuki (op. cit.) under B. boninensis.

JAVA. Cent r al: Raciborski s.n. (L, 938.297-481).-E as t: Franck 37 (C, US); Rosenstock Fil. Jav. Or. exsicc. 107 (Mousset leg.) (P).-S in e lo c.: Hildebrand s.n. (P); Zippel s.n. (B).

Phillipines. Lu z o n: 7 collections. -S a m a r: Edaño BS 24868 (US); Ramos BS 17556 (K, MICH, US).Le y te: Cuming 294 (type of Cyrtogonium laciniatum); Edario PNH 11238 (MICH, PNH), PNH 11252 (MICH, PNH); Wenzel 432 (GH), 732 (MO).-N e g r os: Edaño PNH 21388 (type of Stenosemia dimorpha). -P a n a y: Ramos \& Edar̃o $B S 30847$ (K, P, US). - Min d a n a o: 6 collections.

Celebes. Central: Kjellberg 2506 (syntype of Campium viviparum; BO, S-PA), 3635 (ibid.; S-PA).- 


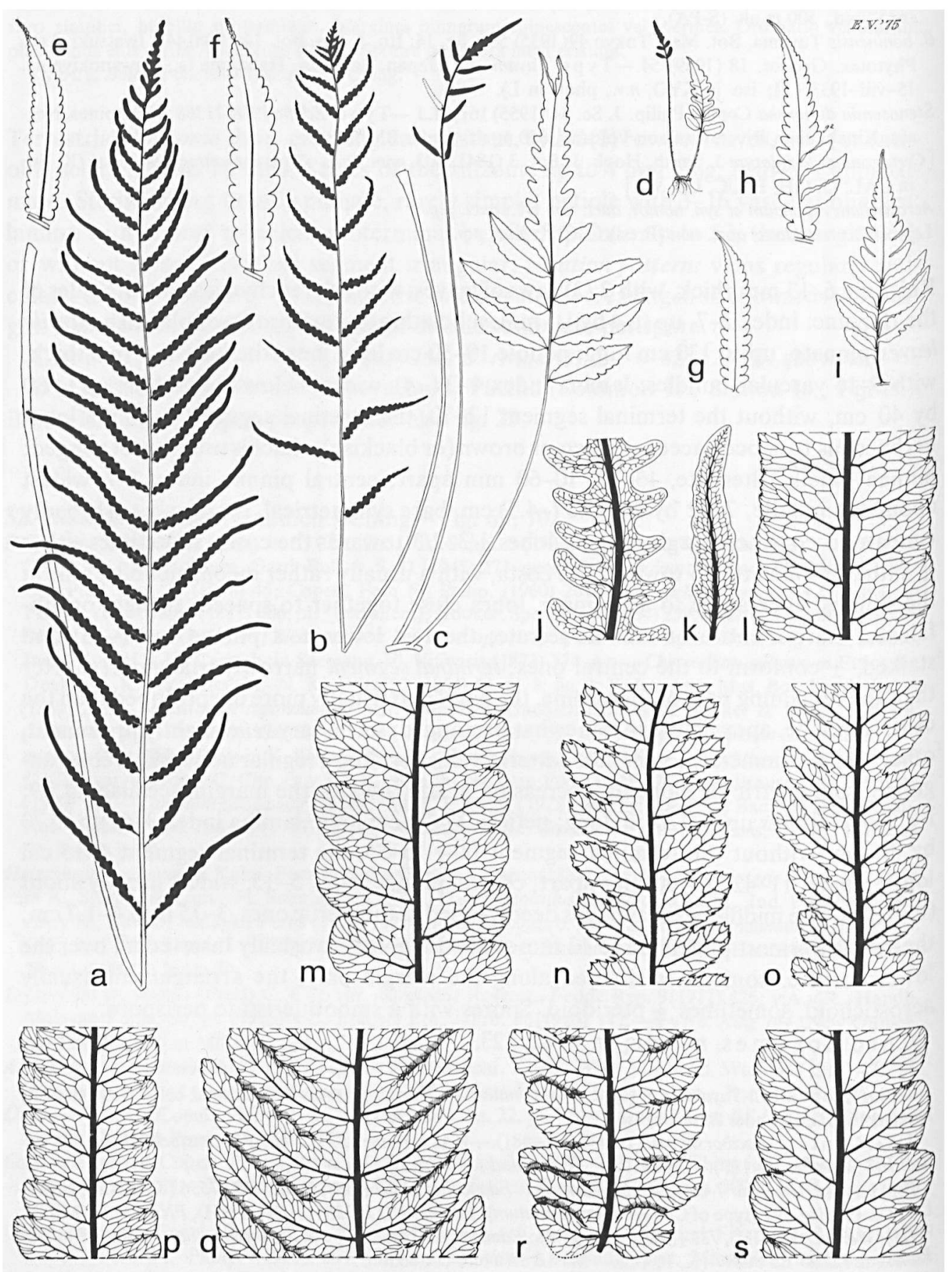


North Peninsula: Alston 15766 (BM); Posthumus 2280 (BO); Savinnière s.n. (P); Wisse 72 (BO, WAG). Moluccas. P. Morotai: Reimschlüssel 10 (US).-Halmaheira: Teijsmann s.n. (BO).-Ternat e: Alston 16656 (BM): Bequin 1012 (BO, L): Von Martens s.n. (B).-P. Pis a ng: Gaudichaud s.n. (lectotype of Acrostichum quoyanum).-A m b o n: Rant 388 (BO); Zippel 207 (B), s.n. (L).

NEw GuINEA. 70 collections from the mountainous regions all over the island.-D'Entrecastea ux I s.: Brass 25931 (GH, L, PNH), 27162 (K, L).-B i a k: Grether \& Wagner 4218 (UC).-A ru I s.: Buwalda 5078 (BO).

Bismarck Archipelago. New Britain: 10 collections. -New Ireland: Anonymus 94 (B); Lahaie 766 (P).-New Ha nover: Naumann s.n. (type of Heteroneuron naumannii).-Admiralty Is.: Grether 4583 (UC); Grether \& Wagner 4036 (UC). Moseley s.n., Challenger exp. (K); Wagner 3277 'US).

Solomon Is. Buka: Waterhouse 47 (K).-B ougainville: Wakefield 1037 (BM), 1161 (BM).Shortland I.: Guppy 99 (BM).-Santa Ysabel: Braithwaite R.S.S. 4527 (K).-Vella La Vella: Wall \& Nakisi BSIP 17502 (L).-Gu a d a l ca n al: Braithwaite R.S.S. 4130 (K, L); McKee 1612 (LAE).

SAMOA I s. U polu: Flohr s.n. (S-PA); Rechinger 626 (W), 783 (W).-S in e lo c.: Reinecke $5 b$ (G).

FIJI Is. Sin e l oc.: Horne s.n. (K).

Australia. Qu e ens 1 and: 6 collections.

$\mathrm{H}$ a bit a t. Creeping on rocks and in soil in rain forest, mostly near streams. Several times reported from limestone. Altitude: 0-1200(-1700) $\mathrm{m}$.

S p o r es. Brass 5621 (BM), Gaudichaud s.n. (type of Acrostichum quoyanum), Jermy 9604 (diploid; BM), Kostermans 2909 (L), Raciborski s.n. (L, 938.297-481), Schlechter 16163 (type of Campium parvum; B, L): all or the greater part normal. Copeland s.n. (type of Campium enorme; UC): part of the sporangia filled with normal spores, part with normal and abnormal spores, part with only abnormal spores. Brass 23616 (A), Grether 3601 (MICH), Rechinger 3650 (W): the majority (?) normal. Brass 12833 (GH), Mendoza \& Convocar PNH 8606 (MICH), Ramos BS 17556 (MICH): the greater part abnormal. Tuyama s.n. (type of B. boninensis; TI), Walker T 10053 (triploid; BM): sporangia filled with usually aborted S.M.C.'s.

Notes. 1. A distinct species which has been confused by most authors with Bolbitis repanda. It is closest to $B$. rivularis, its coriaceous counterpart (and also showing a tendency to dwarfing), to $B$. taylorii which differs especially in leaf architecture and venation pattern, and to $B$. sagenioides (see: Spec. dub.). B. bipinnatifida and B. subcordata are more distant relatives.

The present species is best recognized by the shape and venation of the pinnae or, when dwarfs are at hand, of the terminal segment.

The study of the spores has contributed considerably to the present classification as it allowed me to exclude an essential part of the morphological heterogeneity which I first had assembled and in which I was unable to draw lines. Amongst these excluded specimens are $B . \times$ foxii, $B$. $\times$ sinuosa, and the type of Heteroneuron argutum $(=B$. heteroclita s.l.).

Fig. 69. Bolbitis quoyana.-a-f. sterile and fertile leaves, $\times 1 / 5 ; \mathrm{g}$, h. central pinnae, $\times 1 / 5$; i. lowermost pinna, $\times 1 / 5 ; j$, k. venation pattern of fertile pinnae, $\times 4 / 5 ; 1-s$. ibid. of sterile pinnae (a, e, p. Walker T 10052, BM, triploid; b, f, m. Walker T 9604, BM, diploid; c. Walker T 9300, BM; d. Schlechter 16163, $\mathrm{L}$, isotype of Campium parvum; g-i. Braithwaite RSS 4527, leaf C, K; j. Ledermann 10487a, BO; k. Ramos BS 7522, B; 1. Pleyte 452, BO; n. Elmer 13468a, BO; o. Brass 13473, GH; q. Grether \& W. H. Wagner 4036, UC; r. Teijsmann s.n., BO; s. Walker T 9615, BM, triploid). 
2. Variability. The features varying most markedly are the size of the leaves and the shape of the pinnae. The two extremes are represented by the type specimens of Campium parvum (New Guinea) and by Braithwaite R.S.S. nos. 4130 and 4527 (Solomon Islands).

a. Campium parvum is in a fertile condition only known by the type collection (10 plants) upon which the following description is based. Rhizome up to $3 \mathrm{~mm}$ thick with 2 rows of leaves. Scales of the rhizome up to 2 by $0.8 \mathrm{~mm}$. Leaves rather spaced. Sterile leaves pinnate with a triangular terminal segment, up to $15 \mathrm{~cm}$ long; petiole up to 7 $\mathrm{cm}$ long, with 3 vascular bundles; lamina index $1-3$, widest below the middle, up to 8.5 by $5.5 \mathrm{~cm}$, with primordia of bulbils subterminally on the terminal (and lateral) segments; pinnae $2-5$, up to $7 \mathrm{~mm}$ apart; central pinnae index $1-3$, widest about the middle, up to 35 by $13 \mathrm{~mm}$, the margin slightly crenate-serrate; the two lowermost pinnae up to $2 \mathrm{~mm}$ stalked; terminal segment near the base deeply lobed, with distinct spines in the sinuses; venation pattern in the basal part of the terminal segment \pm similar to that found in the central pinnae of $B$. quoyana proper; the venation pattern of the pinnae simpler. Fertile leaves up to $19 \mathrm{~cm}$ long; petiole up to $15 \mathrm{~cm}$ long; lamina index $2-3$, up to 4 by $2 \mathrm{~cm}$; pinnae 4 . Sporangia inserted all over the lower surface.

In a sterile condition known by two other collections from New Guinea: King 269 (MICH) and Werner $41=$ Rosenstock Fil. Novog. exsicc. 11 (S-PA, p.p., UC, p.p.). The sterile leaves agree completely with leaves grown on bulbils situated on typical specimens of $B$. quoyana.

Fertile plants intermediate between Campium parvum and typical B. quoyana are represented bỹ Walker $T 7882$ (BM) and $T 9300$ (BM), both from New Guinea.

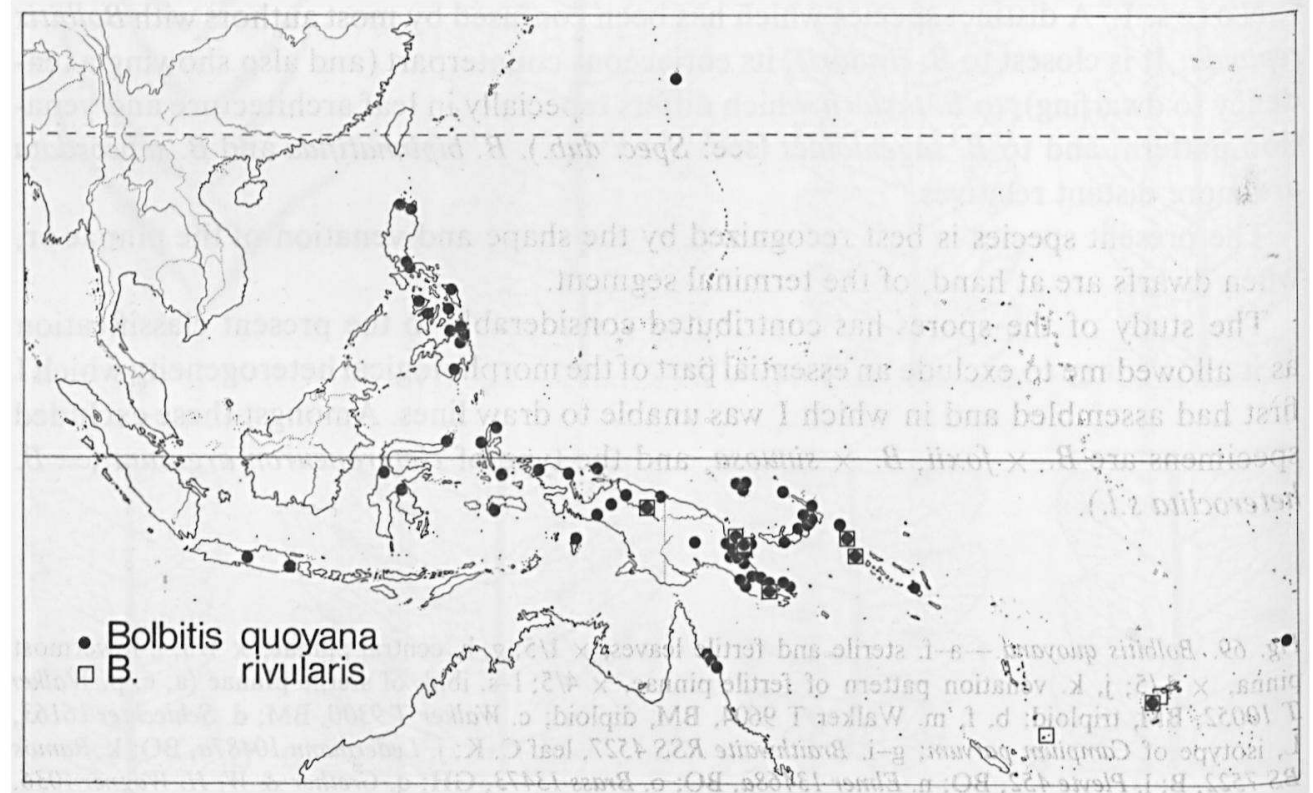

Fig. 70. Distribution of Bolbitis quoyana and B. rivularis. 
b. Braithwaite's collections from the Solomon Islands. Braithwaite R.S.S. 4130 (L). Sterile leaves pinnate, $155 \mathrm{~cm}$ long; petiole $80 \mathrm{~cm}$ long; lamina 75 by $45 \mathrm{~cm}$; pinnae 32 , normally shaped, the largest $c$. 24 by $5 \mathrm{~cm}$, lobes rather spaced. Spores: sporangia with aborted S.M.C.'s only.

Braithwaite R.S.S. 4527 of which I was kindly permitted by Dr. F. M. Jarrett, Kew, to study all the material prior to the distribution of duplicates; it was provisionally identified by Prof. Holttum as 'Bolbitis spec. nov.'. The plants revealed features similar to typical $B$. quoyana except for the shape of the leaves. The lowest part of the leaves is bipinnate; the pinnae are composed of up to 10 pinnules. The bipinnate condition gradually passes into the pinnate one in the central and upper part of the leaf. Spores in part normal, large (the longest axis up to $50 \mu \mathrm{m}$ long), in part shrivelled. The bipinnate condition is not consistent; it covers a variously large part of the three sterile leaves studied. The shape of the pinnae of typical specimens from the Admiralty Is. and Solomons shows considerable variation. The plants from these regions are in need of cytological investigation.

3. The differences between the diploids and triploids collected by Walker(vouchers in BM) mainly regard the number of pinnae to a leaf.This number is 17 in Walker T9603; 19-22 in $T$ 9604, $T 9605$ (all diploids); 26-30 in T 9616 (= Jermy 4945), 40 and 46 in $T 10052$ (both triploids). The fertile pinnae of the diploids are smaller than those of the triploids but otherwise not much different. As the differences between the diploid and triploid specimens are otherwise trifling, the triploids are likely to be the result of autoploidization. However, Walker (in litt.) informed me that the meiosis of the triploids showed the chromosomes nearly all to be present as univalents, only very few as bior trivalents.

4. Copeland's classification of the specimens from the Philippines and New Guinea is confusing. As regards the specimens from the Philippines, he included Cuming 294, the type of Cyrtogonium laciniatum, in Bolbitis quoyana. Cyrtogonium laciniatum deviates in the following characters: sterile leaves bear comparatively few (13-16) pinnae lobed $1 / 2-4 / 5$ towards the costa, the lobes are remote and \pm serrate. A specimen similar to the latter was described by Copeland (1951) as Stenosemia dimorpha! Campium validum, which he (1928) newly described, was regarded as synonymous with $B$. $\operatorname{sinuosa}(=B . \times$ sinuosa. later (1960). Copeland thought $B$. sinuosa related to $B$. presliana which is quite different. The type specimen of Campium validum (as far as could be studied from the plate) and that of Campium enorme are strikingly similar to that of Acrostichum quoyanum (which was not studied by Copeland).

5. The typification of Acrostichum quoyanum. In the original description Acrostichum quoyanum was reported from several localities. Only the collection from P. Pisang, from which the plate and the original description obviously were drawn, could be traced.

33. Bolbitis rivularis (Brackenr.) Ching-Fig. 70, 71.

B. rivularis (Brackenr.) Ching in C. Chr., Ind. Fil., Suppl. 3 (1934) 50; Backer \& Posthumus, Varenfl. Java (1939) 82 ('B. ? rivulare').-Cyrtogonium rivulare Brackenr. in Wilkes, U.S. Expl. Exp. 16(1854) 85, pl. 11 : 
fig. 2.-Neurocallis rivularis Moore, Ind. Fil. (1861) 276.-Acrostichum rivulare Hooker, Spec. Fil. 5 (1864) 263; Baker in Hooker \& Baker, Syn. Fil., 2nd ed. (1874) 524. - Poecilopteris rivularis Carruth. ex Seemann, Fl. Vitiensis (1873) 374.-Leptochilus rivularis C. Chr., Ind. Fil. (1906) 387.-Campium rivulare Copel., Philip. J. Sc. 37 (1928) 373, fig. 27, pl. 20; Backer \& Posthumus, Nat. Tijdschr. Ned. Ind. 93 (1933) 161/'C ?rivulare').-T y p e: Brackenridge s.n., Fiji Is., Ovalau (US, 51090).

Campium kajewskii Copel., Philip. J. Sc. $60(1936)$ 112, pl. 22.-T y p e: Kajewski 1763, Solomon Is., Bougainville, Kupei Gold Field, $1000 \mathrm{~m}$ alt. (iso in A, one of the two sheets with this number; BRI).

Rhizome up to $6 \mathrm{~mm}$ thick, with 2 rows of leaves, with sclerenchyma strands. Scales of the rhizome: index 1-3, up to 4 by $1.5(-2) \mathrm{mm}$, subclathrate, dark brown or blackish. Sterile leaves usually pinnate, rarely simple, $10-70 \mathrm{~cm}$ long. Pinnate leaves $10-70 \mathrm{~cm}$ long; petiole $3-35 \mathrm{~cm}$ long, near the base $2-5 \mathrm{~mm}$ thick, with 5-12 vascular bundles; lamina index 1-3 $(-5)$, widest usually below (to about) the middle, $7-40$ by $3-25 \mathrm{~cm}$, without the terminal segment $0-15$, the terminal segment $5-30 \mathrm{~cm}$ long, fleshy-coriaceous, olivaceous to brownish; rhachis not winged; pinnae alternate to opposite, 2-11, up to $50 \mathrm{~mm}$ apart; central pinnae index 1-5, widest about or below the middle, $1.5-16$ by $0.8-4.5 \mathrm{~cm}$, straight to subfalcate, base \pm symmetrical, cuneate to subcordate, margin \pm entire to lobed to $1 / 5(-2 / 5)$ towards the costa, without or with but an inconspicuous tooth or spine in the sinuses, apex acute or acuminate; the two lowermost pinnae usually opposite, 0-4 $\mathrm{mm}$ stalked; terminal segment triangular, as long as to longer than the remaining part of the lamina, apex acute or acuminate; secondary and other veins slightly prominent or \pm immersed; venation pattern: veins forming a regular network of angulate, isodiametric or elongate areoles which decrease in size towards the margin; see also fig. 71: i-1. Simple leaves $15-30 \mathrm{~cm}$ long; petiole $3-9 \mathrm{~cm}$ long, near the base $1-1.5 \mathrm{~mm}$ thick; lamina index 4-7, widest about or somewhat below the middle, $12-23$ by $2.5-4.5$ $\mathrm{cm}$, base cuneate to subcordate, margin \pm entire to lobed to $2 / 5$ towards the costa, apex acute or acuminate, otherwise similar to the terminal segment of pinnate leaves.Fertile leaves. Pinnate leaves $20-75 \mathrm{~cm}$ long; petiole $13-45 \mathrm{~cm}$ long; lamina index 1-4, $5-35$ by $2-20 \mathrm{~cm}$, without the terminal segment $0.5-23$, the terminal segment $2.5-15 \mathrm{~cm}$ long; pinnae 2-12, up to $80 \mathrm{~mm}$ apart; central pinnae index 1-6, widest about or below the middle, $1.2-9$ by $0.3-1.5 \mathrm{~cm}$, the two lowermost pinnae $1-10 \mathrm{~mm}$ stalked; terminal segment with an acute apex. Simple leaf (Graeffe 108, P) $33 \mathrm{~cm}$ long; petiole $22 \mathrm{~cm}$ long, near the base $c .1 \mathrm{~mm}$ thick; lamina 11 by $2 \mathrm{~cm}$, widest below the middle, base acute, margin slightly lobed in the lower half, otherwise \pm entire, apex acute. Sporangia inserted all over the lower surface. Spores with a smooth cristate perispore.

Chromosomes. $n=41$.

New GuineA. West. Berhard Camp: Brass 13833 (A), 13856 (A)._East. 15 collections.

Solomon Is. Bougainville: Kajewski 1763 (type of Campium kajewskii); Wakefield 1101 (BM).Shortland Is.: Guppy 98 (BM).

New Hebrides. A n eit y u m: Kajewski 893 (BISH, BO, K, US).

FIJI Is. OvalaU: Brackenridge s.n. (type of Cyrtogonium rivulare); Graeffe 108 (P).-Viti Le vu: Degener 14410 (A, MICH); Gillespie 2622 (BISH, K, MICH, UC). -S i n e lo c.: Horne 814 (K).

$\mathrm{Habitat}$. Terrestrial and on rocks in moist places in rain forest; several times reported to grow near or in streams. Altitude: 0-2000 m. 

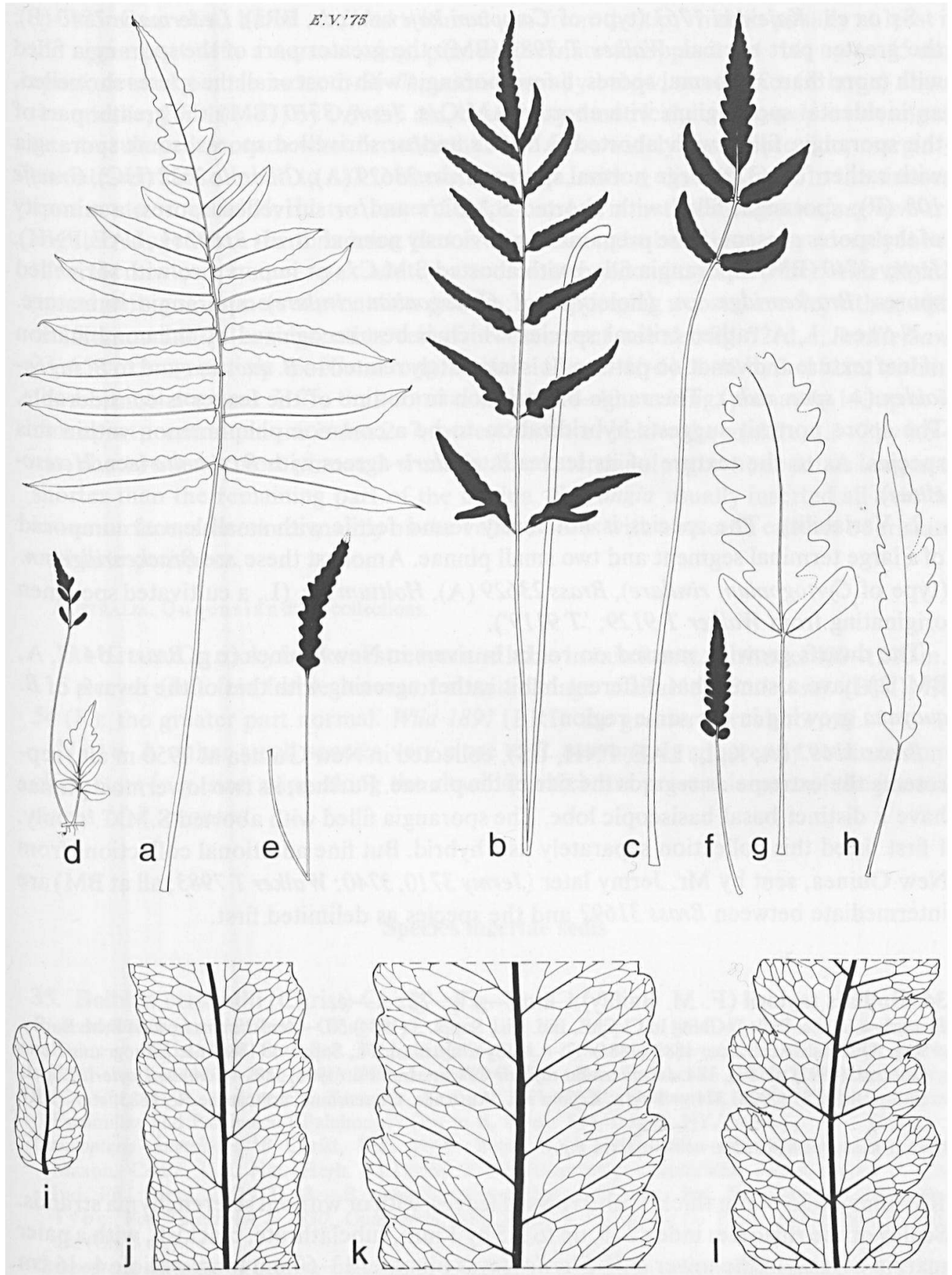

Fig. 7l. Bolbitis rivularis. - a-h. sterile and fertile leaves, $\times 1 / 5 ; \mathrm{i}-1$. venation pattern of sterile pinnae, $\times$ 4/5 (a. Gillespie 2622, MICH; b, k. Brass 31692, L; c. Jermy 3740, BM; d. Brass 23627, L; e. Graeffe 108, P; f-h. Walker T 9127, BM; i, j. Gillespie 2622, UC; 1. Kajewski 893, BO). 
Sp or es. Kajewski 1763 (type of Campium kajewskii; A, BRI), Ledermann 7840 (B): the greater part normal. Walker $T 7983$ (BM): the greater part of the sporangia filled with more than 32 normal spores, a few sporangia with most or all the spores shrivelled, an incidental sporangium with aborted S.M.C.'s. Jermy 3710 (BM): the greater part of the sporangia filled with aborted S.M.C.'s and/or shrivelled spores, some sporangia with rather few (32?) large normal spores. Brass 23629 (A), Gillespie 2622 (UC), Graeffe 108 (P): sporangia filled with aborted S.M.C.'s and/or shrivelled spores, a minority of the spores present in the preparations obviously normal. Brass 31692 (L, LAE, PNH), Jermy 3740 (BM): sporangia filled with aborted S.M.C.'s or in part also with shrivelled spores. Brackenridge s.n. (holotype of Cyrtogonium rivulare): sporangia immature.

Notes. 1. A rather critical species which is best recognized by the combination of leaf texture and venation pattern. It is intimately related to $B$. quoyana and to $B$. sagenioides $(=$ spec. dub.). The range of variation in outline of the leaves is considerable. The spore portrait suggests hybridization to be a common phenomenon within this species. As to the texture of its leaves $B$. rivularis agrees with $B$. sinuata (ser. Heteroclitae).

2. Variability. The species is not rarely found fertile with small leaves composed of a large terminal segment and two small pinnae. Amongst these are Brackenridge s.n. (type of Cyrtogonium rivulare), Brass 23629 (A), Holttum s.n. (L, a cultivated specimen originating from Walker $T$ 9129; 'T 9119').

The dwarfs growing massed on rocks in rivers in New Guinea (e.g. Brass 23417, A, $B M, L)$ have a somewhat different habit rather agreeing with that of the dwarfs of $B$. quoyana growing in the same region.

Brass 31692 (A, K, L, LAE, PNH, US), collected in New Guinea at $1950 \mathrm{~m}$ alt., represents the extreme as regards the size of the pinnae. Further, its two lowermost pinnae have a distinct basal basiscopic lobe. The sporangia filled with aborted S.M.C.'s only. I first listed this collection separately as a hybrid. But fine additional collections from New Guinea, sent by Mr. Jermy later (Jermy 3710, 3740; Walker T 7983; all at BM) are intermediate between Brass 31692 and the species as delimited first.

34. Bolbitis taylorii (F. M. Bailey) Ching-Fig. 72: a-e.

B. taylorii (F. M. Bailey) Ching in C. Chr., Ind. Fil., Suppl. 3 (1934) 50.-Acrostichum taylorii F.M. Bailey, Rep. Queensl. Accl. Soc. 1883 (1884) 11, n.v.; Syn. Queensl. Fl., Suppl. 1 (1886) 65; Lithograms Ferns Queensl. (1892) pl. 183, 184. - Leptochilus taylorii C. Chr., Ind. Fil.(1906) 388._Campium taylorii Copel., Philip. J. Sc. 37 (1928) 374.-T y pe: Kefford s.n., Australia, Queensland, Johnstone R., 1883, ster. (BRI, 50121).

Acrostichum repandum auct. non Bl.: auct. p.p.

Rhizome up to $6 \mathrm{~mm}$ thick, with 2 rows of leaves, with or without sclerenchyma strands. Scales of the rhizome: index $2-5$, up to 3.5 by $1 \mathrm{~mm}$, subclathrate, blackish, with a paler margin at least in the lower half. Sterile leaves pinnate, $13-60 \mathrm{~cm}$ long; petiole 4-16 cm long, near the base $0.5-2.5 \mathrm{~mm}$ thick, with 3-5 vascular bundles; lamina index $1.5-4$, widest usually below (to about) the middle, $8-50$ by $4-22 \mathrm{~cm}$, without the terminal segment $1-20$, the terminal segment $7-30 \mathrm{~cm}$ long, herbaceous, bright to dark green, the 
(primordium of a) bulbil situated within $2 \mathrm{~cm}$ from the tip; rhachis usually at least in the upper part with a narrow wing; pinnae alternate to opposite, 4-14, up to $45 \mathrm{~mm}$ apart; central pinnae index 2-5(-7), widest usually below, sometimes (if large) about the middle, $2.5-10(-17)$ by $0.9-2.5 \mathrm{~cm}$, base \pm asymmetrical, its acroscopic side angustate to subcordate, somewhat broader than the more widely basiscopic side, margin \pm crenate-serrate to lobed to $1 / 2$ towards the costa, with \pm distinct teeth in the sinuses, apex acute or rounded; the two lowermost pinnae 1-4 mm stalked; terminal segment triangular, longer than the remaining part of the lamina, the basal part more deeply lobed than the central pinnae, apex acute or acuminate; secondary and other veins slightly prominent or \pm immersed; venation pattern: veins forming a costal areole, with or without few small distal areoles, other veins free, excurrent, see also fig. 72: d, e. Fertile leaves $23-65 \mathrm{~cm}$ long; petiole $16-37 \mathrm{~cm}$ long; lamina index $2-4(-6), 7-40$ by $3-11 \mathrm{~cm}$, without the terminal segment 3-25, the terminal segment 4-13 cm long; pinnae 6-14, up to 45 $\mathrm{mm}$ apart; central pinnae index 2-7, widest about or below the middle, $1.3-7$ by $0.5-1$ $\mathrm{cm}$; the two lowermost pinnae 2-4 mm stalked; terminal segment about as long as to shorter than the remaining part of the lamina. Sporangia usually inserted all over the lower surface, sometimes mainly on the veins. Spores with a smooth cristate or cristateundulate perispore.

Australia. Qu eensland: 17 collections.

$\mathrm{H} \mathrm{a} \mathrm{bit} \mathrm{a} \mathrm{t.} \mathrm{On} \mathrm{rocky} \mathrm{banks} \mathrm{of} \mathrm{streams} \mathrm{and} \mathrm{lakes} \mathrm{in} \mathrm{rain} \mathrm{forest.} \mathrm{Altitude:} 400-1200 \mathrm{~m}$.

Spores (but few fertile leaves of a suitable age available). Bailey s.n. (BRI), Hill 54 (K): the greater part normal. Wild 1891 (BRI): the greater vart abnormal.

Note. A rather small species very close to $B$. quoyana. It apparently retained some characters (e.g. size, wing along the rhachis) which are present in part of the juvenile stages of $B$. quoyana.

\section{Species incertae sedis}

35. Bolbitis bernoullii (Christ) Ching-Fig. 72 : $\mathbf{f - k} ; 73$.

B. bernoullii (Kuhn ex Christ) Ching in C. Chr., Ind. Fil., Suppl. 3 (1934) 47.-Acrostichum bernoullii Kuhn ex Christ, Bull. Soc. Bot. Belg. 35 (1896) 244.-Gymnopteris bernoullii Diels in E. \& P., Nat. Pfl. Fam 1, 4 (1899) 201.-Leptochilus bernoullii C. Chr., Bot. Tidsskr. 26 (1904) 296.-L ect o ty pe (C. Chr., 1904, 'holotype'): Bernoulli \& Cario 382 ('282'), Guatemala, Costa Grande, between Escamillas and Palahueco ('Palahucco') (iso in B, holo?, BM, fragm., NY, fragm.).

Gymnopteris donnell-smithii Christ, Bull. Herb. Boiss. II, 6 (1906) 289.-Poecilopteris donnell-smithii Maxon, Contr. U.S. Nat. 'Herb. 13 (1909) 20 ('Poikilopteris').-Leptochilus donnell-smithii C. Chr., Ind. Fil., Suppl. 1 (1913) 48.-B. donnell-smithii Ching in C. Chr., Ind. Fil., Suppl. 3 (1934) 48.Type: Von Türckheim $N$ 8830, Guatemala, Alta Verapaz, Cubilquitz (= Finca de Cubilhuitz), $350 \mathrm{~m}$ alt. (US, 3 sh.).

Gymnopteris tuerckheimii Christ, Bull. Herb. Boiss. II, 6 (1906) 290.-Leptochilus tuerckheimii C. Chr., Ind. Fil,, Suppl. 1 (1913) 48.-Ty pe: Von Türckheim N 8831, Guatemala, Alta Verapaz, Cubilquitz (= Finca de Cubilhuitz), $350 \mathrm{~m}$ alt. (US, 2 sh.). Acrostichum serratifolium auct. non Kaulf.: Baker in Hooker \& Baker, Syn. Fil. (1868) 423,p.p:

Heteroneuron serratifolium auct. non (Kaulf.) Fournier: Fournier, Mexic. P1. 1, 1 Crypt. (1872) 69. 

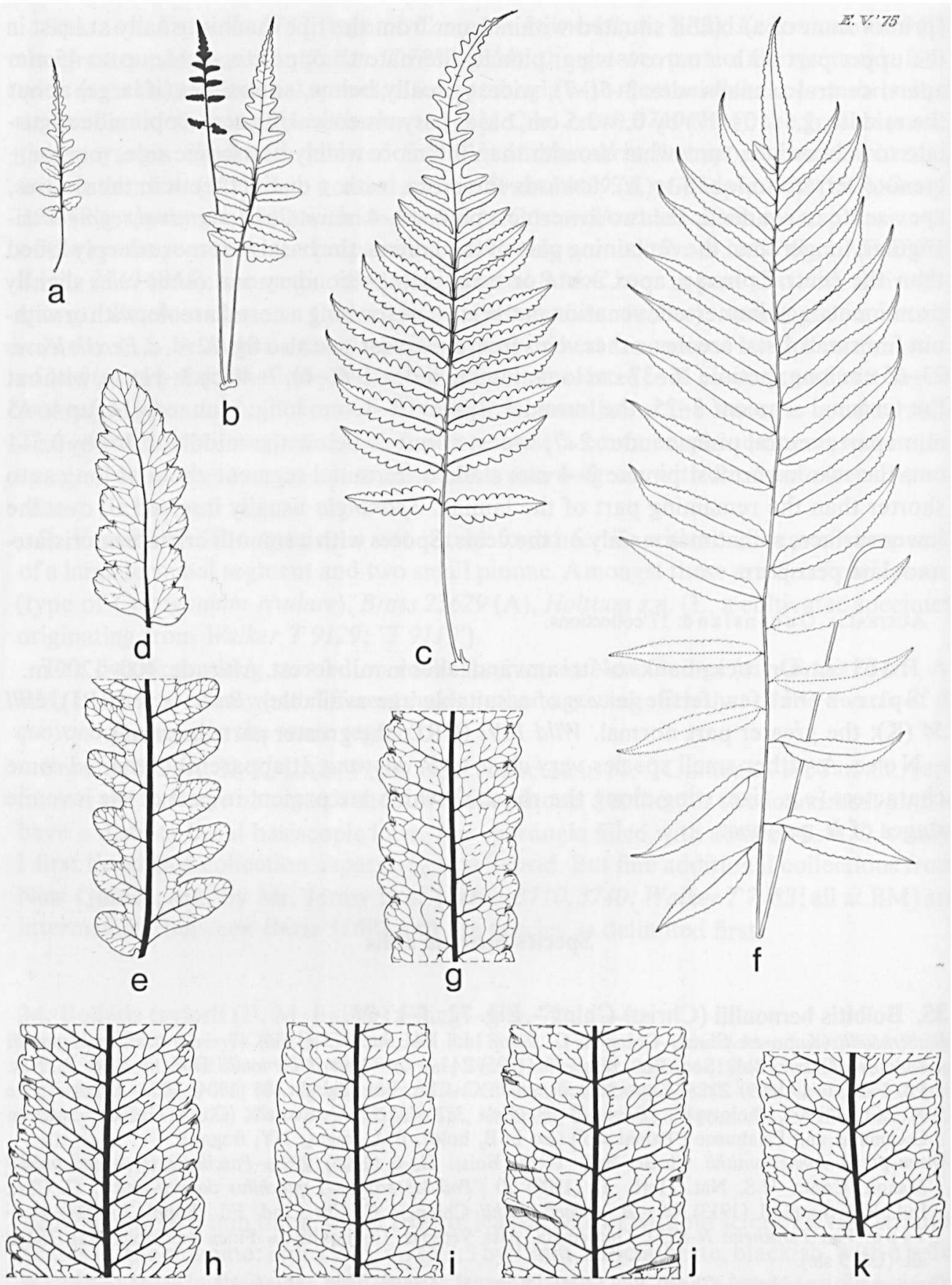

Fig. 72. a-e. Bolbitis taylorii. a-c. sterile and fertile leaves, $\times 1 / 5$; $d$, e. venation pattern of sterile pinnae, $\times 4 / 5$ (a. Kefford s.n., BRI 50121, holotype of B. taylorii; b. Messmer P 6854, BRI; c. Goy 436, BRI; d. Brass 20273, L; e. Messmer P 6932, NSW). -f-k. Bolbitis bernoullii. f. sterile leaf, $\times 1 / 5$; g-k. venation pattern of sterile pinnae, $\times$ 4/5 (f, g. Bourgeau 2008, L; h. Stübel 929a, B; i. Von Türckheim 4034, L; j. k. Steeves \& Ray 490, L). 


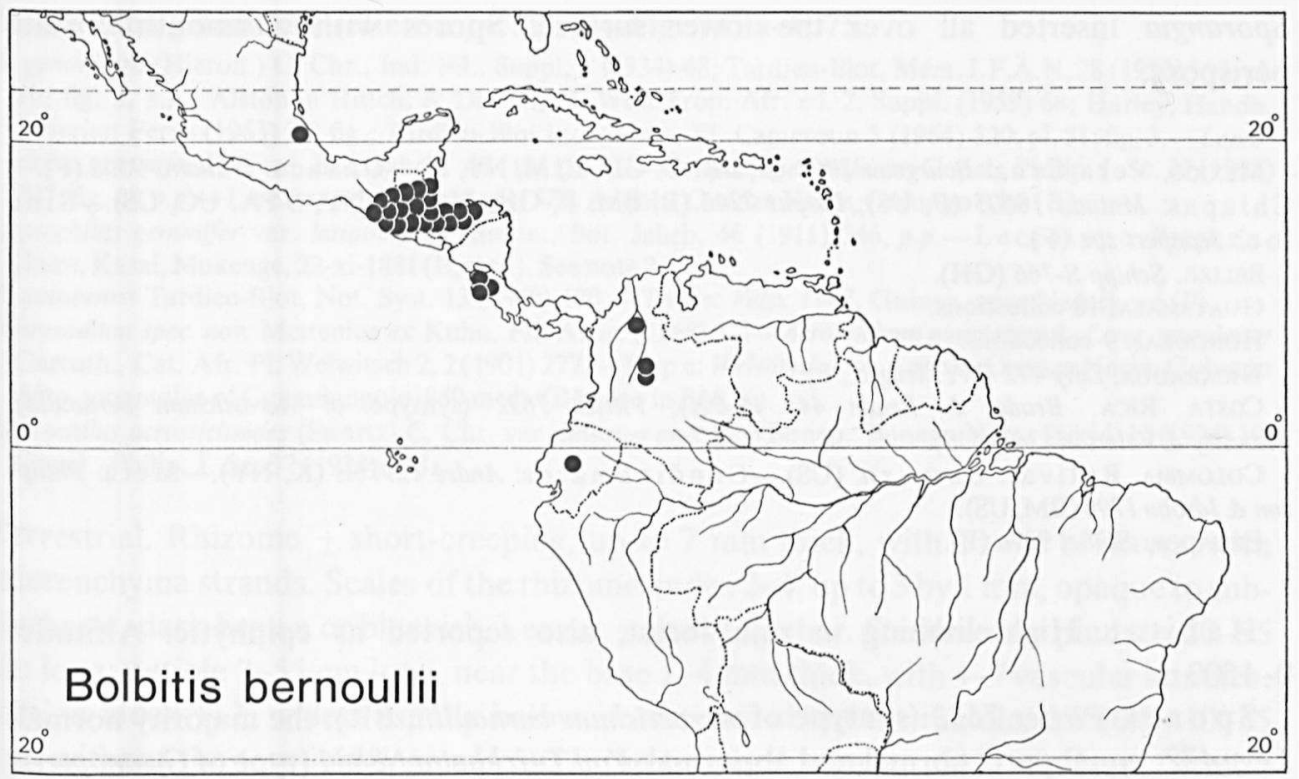

Fig. 73. Distribution of Bolbitis bernoullii.

Rhizome \pm long-creeping, without sclerenchyma strands, up to $20 \mathrm{~cm}$ long, up to $30 \mathrm{~mm}$ thick, with 2-6 rows of leaves. Scales of the rhizome: index (5-)20-30, c. 10-20 by $0.5-1.5(-3) \mathrm{mm}$, opaque, red-brown, the margin conspicuously denticulate. Leaves approximate to somewhat spaced. Sterile leaves pinnate, $60-135 \mathrm{~cm}$ long; petiole $14-40$ $\mathrm{cm}$ long, near the base $2.5-5 \mathrm{~mm}$ thick with 10-22 vascular bundles; lamina index (1-) $2-4$, widest usually somewhat below, sometimes about the middle, $35-110$ by $15-50 \mathrm{~cm}$, without the terminal segment 24-94, the terminal segment 7-16 cm long, firmly herbaceous, light to dark green, without bulbils; rhachis not winged; pinnae alternate or opposite, 16-48, 20-60 mm apart, pseudo-articulate, straight to sigmoid; central pinnae index 4-9, widest about or below the middle, $9-26$ by $2-4 \mathrm{~cm}$, base \pm symmetrical, narrowly to broadly cuneate, margin usually either entire or especially towards the apex crenate-serrate, sometimes lobed to 1/6 towards the costa, without spines or teeth, apex (long-) acuminate; the two lowermost pinnae usually \pm deflexed, \pm conform to the central pinnae, $1.5-12 \mathrm{~mm}$ stalked; terminal segment joined to the rhachis, usually triangular and composed of but few lobes, sometimes \pm conform to the central pinnae and with an unequally long-sided base; veins all prominent; venation pattern: veins forming areoles varying in shape and size, the tertiary veins with few to several mostly excurrent free veins ending in a thickening (hydathode?); see also fig. 72: g-k. Fertile leaves $35-90 \mathrm{~cm}$ long; petiole $9-23 \mathrm{~cm}$ long; lamina index $2-5,20-70$ by (5-) 9-23 cm, without the terminal segment 18-67, the terminal segment 2-9 cm long; pinnae 16-49, (10-) 25-65 mm apart; central pinnae index 5-15 (-20), widest about or below the middle, $3.5-15.5$ by $0.6-1.2(-1.7) \mathrm{cm}$; the two lowermost pinnae $2-10 \mathrm{~mm}$ stalked. 
Sporangia inserted all over the lower surface. Spores with a smooth cristate perispore.

Mexico. Vera Cruz: Bourgeau 2008 (B, BR, G, GH, L, M, NY, P).-O ax a ca: Williams 9508 (F).Chi a pas: Matuda 18605 (F, US); Purpus 7246 (B, BM, F, GH, M, MO, NY, S-PA, UC, US).-Sine lo c.: Jeanpert s.n. (F).

BeLIZE. Schipp S-766 (GH).

Guatemala. 18 collections.

HoNDURAS. 9 collections.

Nicaragua. Lévy 472 (NY, fragm., P).

Costa Rica. Brade \& Brade 443 (S-PA); Pittier 7632 (syntype of Acrostichum bernoullii); Standley \& Valerio 44743 (US).

Colombia. B olivar: Curran s.n. (US).-Cundina marca: André 1297 bis (K, NY).-Met a: Philipson \& Idrobo 1796 (BM, US).

ECUADOR. Stübel 929a (B).

Habitat. High-climbing in rain forest, also reported as epiphytic. Altitude: 0-1500 m.

Spores. Pittier 7632 (syntype of Acrostichum bernoullii, BR): the majority normal. Lévy 472: equal parts normal and abnormal. Von Türckheim N8831 (type of Gymnopteris tuerckheimii, US): a minority normal. Contreras 2260 (US), Von Türckheim N8830 (type of Gymnopteris donnell-smithii, US), Bernoulli \& Cario 382 (lectotype of Acrostichum bernoullii, B): (nearly) all abnormal.

Notes. 1. A clear-cut species which is of particular interest as part of its features suggests that it is closely related to $B$. lindigii, whereas other features such as the scales and the venation pattern show interesting differences with those of $B$. lindigii.

In contrast to the scales, the venation pattern shows much variation. It is superficially rather similar to that of $B$. serratifolia, and Christ regarded the venation pattern of Gymnopteris donnell-smithii and G. tuerckheimii as of the same 'type' as that of $G$. contaminoides $(=B$. serratifolia). The venation pattern of all specimens of the present species shows at least local irregularities. This $I$ regard as a strong indication for hybridity. As the venation pattern of quite a few specimens has at least locally areoles like those typical for $B$. lindigii, and as excurrent free veins are always frequent, the venation pattern of $B$. bernoullii seems intermediate between that described for $B$. lindigii and a pattern with exclusively or mainly free excurrent veins. Acrostichoids of the New World with the latter type of venation pattern and with a presumably similar growth pattern are found in the genera Polybotrya (Soromanes) and Lomariopsis. Terrestrial ferns that may be involved are B. serratifolia and Cyclodium meniscioides (Willd.) Presl. The precise identity of $B$. bernoullii seems a promising field for further study.

2. Gymnopteris donnell-smithii and G. tuerckheimii were regarded by Christ to be related to the following species: $G$. costaricensis $(=B$. lindigii), G. guianensis (= Lomagramma guianensis), and $G$. serratifolia $(=B$. serratifolia). Christ did not refer to Acrostichum bernoullii which he himself had described ten years earlier and in which both fit easily. 
36. Bolbitis gemmifera (Hieron.) C. Chr.-Fig. $74:$ a $-\mathbf{f} ; 75$.

B. gemmifera (Hieron.) C. Chr., Ind. Fil., Suppl. 3 (1934) 48; Tardieu-Blot, Mém. I.F.A.N. 28 (1953) 113, pl. 19; fig. 3, p.p.; Alston in Hutch, \& Dalziel, Fl. West Trop. Afr. ed. 2, Suppl. (1959) 68; Harley, Handb. Liberian Ferns (1963) 39, fig.; Tardieu-Blot in Aubrév., Fl. Cameroun 3 (1964) 320, pl. 51: fig. 3.-Leptochilus gemmifer Hieron., Bot. Jahrb. 46 (1911) 345.—Campium gemmiferum Copel., Philip. J. Sc. 37 (1928) 387, fig. 48,p.p. - L e ct o t y pe: Welwitsch $157 b$, Angola, Cuanza Norte, Golungo Alto(B).

Leptochilus gemmifer var. latipinnatus Hieron., Bot. Jahrb. 46 (1911) 346, p.p.-L e c to t y pe: Pogge s.n., Zaire, Kasai, Mukenge, 22-xi-1881 (B, ster.). See note 2.

B. guineensis Tardieu-Blot, Not. Syst. 13 (1948) 170.-T y p e: Félix 1147, Guinea, near Nzérékoré (P).

[Chrysodium spec. nov. Mettenius ex Kuhn, Fil. Afric. (1868) 52] - Acrostichum punctatum L.f. var. angolense Carruth., Cat. Afr. Pl. Welwitsch 2, 2 (1901) 277.-T y p e: Welwitsch 157, Angola, Cuanza Norte, Golungo Alto, mountains of Cungulungulo, $850 \mathrm{~m}$ alt. (BM; iso in BM, $\mathrm{K}$ ).

Leptochilus acrostichoides (Swartz) C. Chr. var cuneatus auct. non Bonap.: Bonap., Notes Ptérid. 15(1924) 19; Copel., Philip. J. Sc. 37 (1928) 398.

Terrestrial. Rhizome \pm short-creeping, up to $7 \mathrm{~mm}$ thick, with 2 rows of leaves, with sclerenchyma strands. Scales of the rhizome: index $2-4$, up to 3 by $1 \mathrm{~mm}$, opaque to subclathrate, dark brown or blackish. Leaves \pm close together. Sterile leaves pinnate, 20-95 cm long; petiole 7-55 cm long, near the base 1-4 mm thick, with 4-7 vascular bundles; lamina index 1-2, widest usually below, sometimes about the middle, 15-50 by 10-35 $\mathrm{cm}$, without the terminal segment 5-37, the terminal segment 6-17 cm long, (firmly) herbaceous, green, sometimes with a purple tinge, with a distinct, usually globose (elongate) bulbil inserted laterally on the basal part of the terminal segment; rhachis not winged; pinnae alternate or opposite, 6-25, 10-50 mm apart; central pinnae index (2-) 3-6, widest usually about, sometimes below the middle, 7-21 by $2-4.5(-6) \mathrm{cm}$, base usually \pm symmetrical, \pm cuneate, sometimes asymmetrical, usually its acroscopic side broader, rarely with one odd acroscopic pinnule, margin usually irregularly lobed with adjacent lobes often (much) differing in size and shape, sometimes \pm entire without teeth or spines, apex acute to shortly caudate; the two lowermost pinnae \pm conform to the central pinnae, 1-10 mm stalked; terminal segment joined to the rhachis, either \pm cors form to the central pinnae or with the base strongly asymmetrical with its longest side sometimes with a \pm distinct lobe; secondary and other veins slightly prominent or $\pm \mathrm{im}$ mersed; venation pattern: veins forming a locally irregular pattern of \pm isodiametric areoles varying in shape and size, few areoles with usually one, mostly simple, re- or excurrent included vein; see also fig. 74: e, f. Fertile leaves 30-85 cm long; petiole 20-60 cm long; lamina index $1-4,9-40$ by $5-15 \mathrm{~cm}$, without the terminal segment $6-36$, the terminal segment 1-8 cm long; pinnae 6-22, 15-70 mm apart; central pinnae index 3-6, widest about or below the middle, $2.5-9$ by $0.8-1.6 \mathrm{~cm}$; the two lowermost pinnae 1-12 mm stalked. Sporangia inserted all over the lower surface. Spores with a cristate perispore.

SENEGAL. Dakar: Anonymus 1556 (P).

GUINEA. 5 collections.

Sierra Leone, 9 collections.

LiBERIA. 6 collections.

Ivory COAST. Hallé 96 (P), 279 (P); Leeuwenberg 3834 (WAG); Rousseau s.n. (P).

GHaNA. 6 collections. 


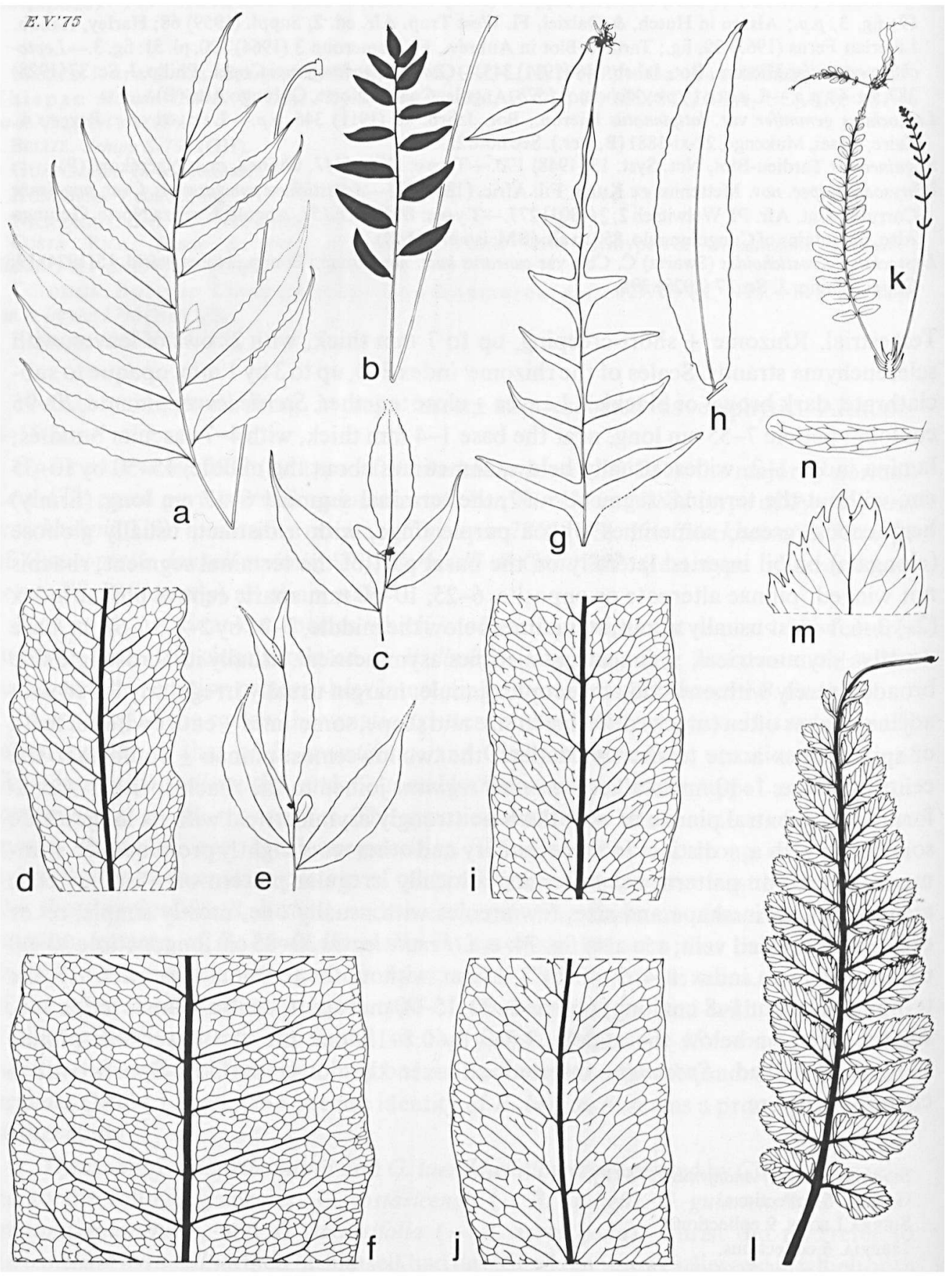


Cameroun. 8 collections.

Central African Republic. Anonymus 'C' (P); Leeuwenberg 7091 (U, WAG).

ZAIRE. Le o p old vill e: 22 collections. - Eq u a t e u r:De Joncheere BCO 23 (private herb. De Joncheere); Kassner 3409 (L, P).-Orientale: 14 collections.-Kivu: Antun-Gupffert 4 (BR); Gillet 2309 (BR).K a s a : 7 collections. -K a t a n ga: Mullenders 2248 (BR).

ANGola. Cabinda: Gossweiler 9037 (BM).-Cuanza Nort e: Welwitsch 157 (type of Acrostichum punctatum var. angolense), $157 b$ (lectotype of Leptochilus gemmifer).

Uganda. East e rn:Wood 788 (BM, K), - B u g a n da:Dümmer 698 (BM, MICH, fragm., P, fragm.); Longfield 16 (BM); Thomas 1344 (K).

TANGANYIKa. Tanga: 9 collections.

ZAMBIA (N. Rhodesia). Carson s.n. (K).

Habitat. In primary and secondary forest. Altitude: 0-1000 (in Tanganyika: $-1200) \mathrm{m}$.

S pores. Holst 2277 (K, paratype of Leptochilus gemmifer), Barter s.n. (GH): all normal, c. 60 spores in each sporangium. Engler 619 (B): a distinct minority abnormal. Jensen s.n., xii-1922 (C): all abnormal.

Notes. 1. A distinct species easily recognized by the localization of the bulbil, the shape of the terminal segment, and the venation pattern. The shape of the terminal segment seems intermediate between that found in B. auriculata and that of a fern with simple leaves or, if pinnate, with a terminal segment which is conform to the pinnae. The venation pattern seems intermediate between the pattern of $B$. auriculata and a pattern with part of its veins not anastomosing. The origin of the present species seems bifold. Apart from a fern like $B$. auriculata, a fern either like $B$. gaboonensis or like $B$. acrostichoides seems involved. The anatomy of the rhizome, the shape of the perispore, and the shape of the bulbil (which is sometimes elongate) point to a fern like B.gaboonensis rather than to one like $B$. acrostichoides.

2. From the description of Leptochilus gemmifer var. latipinnatus it becomes clear that Hieronymus based himself on the sterile leaves of Pogge s.n. only. The other two specimens cited (Pogge 1620 and Staudt 463 ) represent B. acrostichoides s.l.

\section{Bolbitis luumblotii (Baker) Ching-Fig. 74 : g-j.}

B. humblotii (Baker) Ching in C. Chr., Ind. Fil., Suppl. 3 (1934) 48; Tardieu-Blot in Humbert, Fl. Madag. Fam. V, 2 (1960) 58, fig. xvi: 1-2.-Acrostichum humblotii Baker, J. Bot. 22 (1884) 114. - Leptochilus humblotii C. Chr., Ind. Fil. (1906) 385.-Campium humblotii Copel., Philip. J. Sc. 37 (1928) 395, quoad nomen solum; C. Chr., Dansk Bot. Ark. 7(1932) 70, pl. 22: fig. 1-4.-T y p e: Humblot 300, Madagascar, ster. (K; iso in BM, fragm., $\mathrm{P}, 3$ sh., W).

Chrysodium boivinii auct. non Mettenius ex Kuhn: Kuhn, Fil. Afric. (1868) 51,p.p.

Fig. 74. a-f. Bolbitis gemmifera. a, b. sterile and fertile leaf resp., $\times 1 / 5$; c, d. terminal part of sterile leaves with bulbil, $\times 1 / 5$; e, f. venation pattern of sterile pinnae, $\times 4 / 5$ (a. Peter 21872, C; b. De Joncheere BCO 23, herb. De Joncheere; c. Harley F I20, GH; d. Callens s.n., 27-xi-1947, GH; e. Peter 16901, S-PA; f. Pogge s.n., B, paratype of B. gemmifera). $-\mathrm{g}-\mathrm{j}$. Bolbitis humblotii. g. terminal part of sterile leaf, $x$ $1 / 5 ; \mathrm{h}$. sterile pinna, $\times 1 / 5 ; i$, j. venation pattern of sterile pinnae, $\times 4 / 5(g, h$. Humblot $300, P$, isotype of B. humblotii; i. Last s.n., 1890, BM; j. Humblot 300, W, see g).-k-n. Bolbitis novoguineensis. k. sterile and fertile leaf, $\times 1 / 5 ; 1$. sterile leaf, $\times 4 / 5 ; \mathrm{m}$. terminal part of sterile pinna, $\times 3 ; \mathrm{n}$. venation pattern of fertile pinna, $\times 3(\mathrm{k}, \mathrm{m}, \mathrm{n}$. Brass 28008 , L, holotype of B. novoguineensis; 1. Brass $27916, \mathrm{~L})$. 


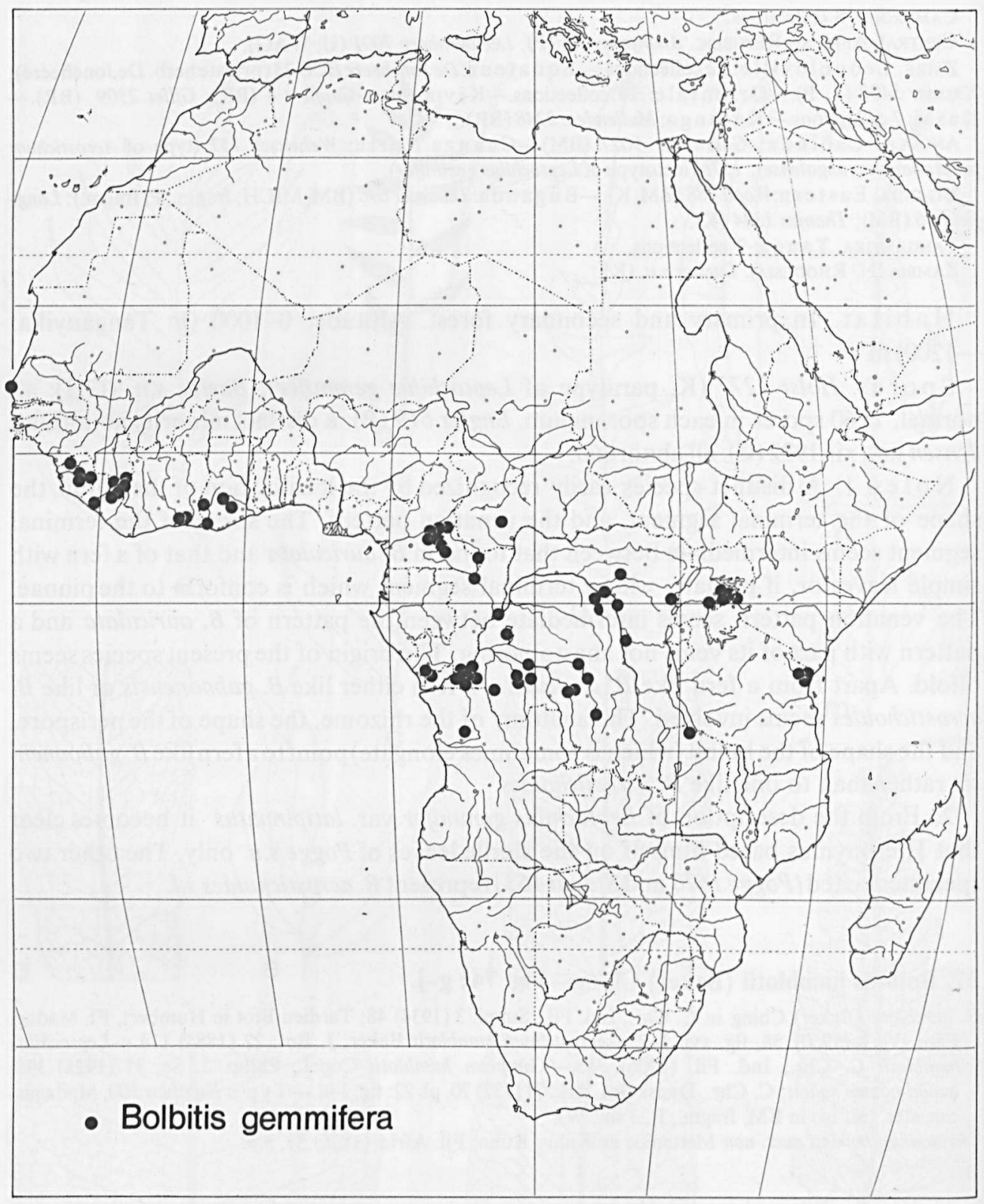

Fig. 75. Distribution of Bolbitis gemmifera.

Terrestrial. Rhizome insufficiently known, that of Cours 3410 (P) short-creeping, $10 \mathrm{~mm}$ thick, with 2 rows of leaves, with sclerenchyma strands. Scales of the rhizome:index 3-4, large, up to 12 by $3 \mathrm{~mm}$, subclathrate, brownish or blackish. Leaves \pm close together, the material available largely incomplete. Sterile leaves pinnate, the only complete one 
$90 \mathrm{~cm}$ long; petiole $c .40$ and $50 \mathrm{~cm}$ long, near the base 3-5 mm thick, with 5-7 vascular bundles; lamina index $1-4$, widest about or below the middle, $30-85$ by $20-40 \mathrm{~cm}$, without the terminal segment 6-32, the terminal segment $25-60 \mathrm{~cm}$ long, firm-herbaceous (rather fleshy when living?), dark olivaceous to blackish, with a spherical bulbil just below the apex; rhachis not winged; pinnae alternate or opposite, 6-14, up to $20-45 \mathrm{~mm}$ apart; central pinnae index 3-7, widest about the middle, $11-26.5$ by $2.5-5.5 \mathrm{~cm}$, base \pm asymmetrical, its basiscopic side more or less adnate, decurrent, the acroscopic side acute to subcordate, margin \pm entire, without teeth or spines, apex acute to acuminate; the two lowermost pinnae (slightly) asymmetrical, the basiscopic side somewhat shorter and narrower than the acroscopic side, 0-2 $\mathrm{mm}$ stalked; terminal segment triangular, deeply pinnatifid near base, large, apex acute to flagelloid; venation: secondary veins usually not well discernable; venation pattern: veins forming an irregular pattern of areoles decreasing in size towards the margin, in part of the areoles, and especially in those about halfway between margin and costa with one or few simple or branched, exor recurrent free included veins, see also fig. 74: i, j. Fertile leaves 87 and $100 \mathrm{~cm}$ long; petiole 56 and $59 \mathrm{~cm}$ long resp.; lamina index $2-4,30-41$ byc. $13 \mathrm{~cm}$, without the terminal segment 12-23, the terminal segment $11-18 \mathrm{~cm}$ long; pinnae 8-12, up to $60 \mathrm{~mm}$ apart; central pinnae index $5-7$, widest about the middle, $6.5-9$ by $1.1-1.5 \mathrm{~cm}$; the two lowermost pinnae 0-1 mm stalked. Sporangia inserted all over the lower surface except for a narrow strip along the primary veins of the segments. Spores with a smooth cristate perispore.

Madagascar. S a m bi i a n o. Nossi-bé: Boivin s.n, 1846/1848 (W, fert.), iii-1851 (P). Massif of Manongarivo: Perrier de la Bâthie 7489 (P); Last s.n., iv/vi-1890 ('Be kilus Mts.')(K).-E a s t. St Marie Is., Tafondro: Boivin 1581/2 (P). Ambatosoratra: Cours 3410 (P).-Central. Northern part, source of Sambirano R. (= Tsaratanana Mt.): Last s.n., xii-1890 (K). Lokoho Valley: Humbert \& Cours 22971 (P). Central part, Tananarive: Warpur (Warbur?) s.n., 19-vii-1900 (K). South of Moramanga: Decary 18306 (P).-Si n e lo c.: Humblot 300 (type of Acrostichum humblotii).

$\mathrm{H}$ a bit at. Terrestrial in forest. Altitude: $75-1000 \mathrm{~m}$.

S p or s. Last s.n., iv/vi-1890 (BM): the majority normal. Last s.n., xii-1890 (K): the greater part abnormal, mostly normally shaped and with a perispore with rather thick, minutely spiny crests. Boivin s.n., iii-1851 (W): sporangia with mostly aborted S.M.C.'s or aborted spores.

Notes. The relationship of this obviously locally common and very distinct fern is not at all clear. It seems not related to species of the series Bolbitianae, Egenolfianae, Euryostichae, Heteroclitae, or Lindigianae.

$B$. humblotii may possibly have arisen through hybridization, and if this assumption is correct, the venation pattern of $B$. humblotii points to a fern like $B$. auriculata as one of the parents.

The present species seems to have taken part in the origin of $B$. longiflagellata $(=$ spec. $d u b$.$) .$

2. In spite of the firm texture of the leaves, vegetative propagation by means of bulbils seems common. 
3. It is doubtful whether the locality Mayotte (Comores Is.) as given on part of Boivin's collection (W, ster.) is reliable, as the species has not been collected on that island since. The label of a fertile leaf of this (W) gives Nossi-be as the locality. Boivin visited both islands; his labels cannot always be trusted (C. Chr., 1932, viii).

\section{Bolbitis lonchophora (Fée) C. Chr.-Fig. 76: a-g; 77.}

B. lonchophora (Fée) C. Chr., Ind. Fil., Suppl. 3 (1934) 49; Bull. Bish. Mus. 177 (1943) 103, p.p.; Yuncker, Bull. Bish. Mus. 220 (1959) 41; Brownlie, Fl. Nouv.-Caléd. 3 (1969) 259, pl. 34: fig. 5, 6.-Acrostichum lonchophorum Kunze, Farrnkr. 1 (1840) 5, pl. 2, nom. illeg.-Heteroneuron lonchophorum Fée, Hist. Acrost. (1845) 94 ('Heteronevron'; nom. leg., art. 72).-Poecilopteris repanda (Bl.) Presl var. lonchophora Presl, Epim. Bot. (1851) 172, p.p.-Acrostichum repandum Bl. var. lonchophorum Powell, J. Bot. 6 (1868) 341, quoad nomen solum?-Campium lonchophorum Copel., Bull. Bish. Mus. 93 (1932) 14, 75, p.p.-T y p e: Cuming 1416, Austral Is. (= Society Is. s.l.), Otaroha (= Rurutu) (iso in BM, K).

Cyrtogonium palustre Brackenr. in Wilkes, U.S. Expl. Exp. 16 (1854) 86, pl. 12.-Acrostichum repandum Bl. var. palustre Powell, J. Bot. 6 (1868) 341.-Campium palustre Copel., Philip. J. Sc. 37 (1928) 371; Wilder, Bull. Bish. Mus. 86 (1931) 9.-B. palustris Hennipman, Blumea 18 (1970) 148.-T y p e: Brackenridge s.n., Society Is., Tahiti (US, 51085).

Acrostichum repandum auct. non B1.: auct. p.p.

Leptochilus cuspidatus auct. non (Presl) C. Chr.: auct. p.p.

Terrestrial. Rhizome short-creeping, rather stout, up to $c .20 \mathrm{~cm}$ long, up to $10 \mathrm{~mm}$ thick, with (2) 3 rows of leaves, with few sclerenchyma strands. Scales of the rhizome: index $1-4$, up to 5 by $2 \mathrm{~mm}$, opaque to subclathrate, brown to blackish. Leaves \pm close together. Sterile leaves pinnate, 30-95 cm long; petiole 7-35 cm long, near the base 1-5 $\mathrm{mm}$ thick, with 5-9 vascular bundles; lamina index 1-4, widest usually below the middle, sometimes at the base, $23-65$ by $8-25 \mathrm{~cm}$, without the terminal segment $10-43$, the terminal segment 8-28 cm long, herbaceous-chartaceous, dark green with sometimes a purple tinge, with a spherical subterminal bulbil; rhachis not winged; pinnae alternate (or opposite), $12-25,10-45 \mathrm{~mm}$ apart; central pinnae index $3-6$, widest usually about, sometimes either below or above the middle, $6-20$ by $1.7-4.5 \mathrm{~cm}$, base $\pm \mathrm{sym}$ metrical, angustate or narrowly cuneate, margin usually lobed $2 / 5(-3 / 5)$ towards the costa, sometimes \pm entire especially in the upper part, with distinct teeth in the sinuses, apex acute to caudate, lobes of the margin narrow, c. $5 \mathrm{~mm}$ wide, entire or crenulate; the two lowermost pinnae $1-10 \mathrm{~mm}$ stalked, usually \pm asymmetrical with the acroscopic side longer and more narrowly angustate, the basiscopic side sometimes with a \pm distinct basal basiscopic lobe; the uppermost pinnae with the basiscopic side decurrent along the rhachis; terminal segment triangular, deeply lobed near the base, tapering towards the usually flagelloid, sometimes acute or prolonged apex; secondary and other veins \pm immersed, angle between costa and secondary vein narrow; venation pattern irregular, veins in part forming areoles varying in size and shape, in part (towards the margin) free, excurrent, the areoles sometimes with usually one ex- or recurrent, included free vein, see also fig. 76: c-g. Fertile leaves 35-65(-75) cm long; petiole 24$45 \mathrm{~cm}$ long; lamina index 2-6, 11-35 by $2-12 \mathrm{~cm}$, without the terminal segment $9-31$, the terminal segment 2-8 cm long; pinnae $12-23,10-50 \mathrm{~mm}$ apart; central pinnae index $3-6$, widest below the middle, often somewhat falcate, $2.5-8$ by $0.7-1.5 \mathrm{~cm}$; the two 


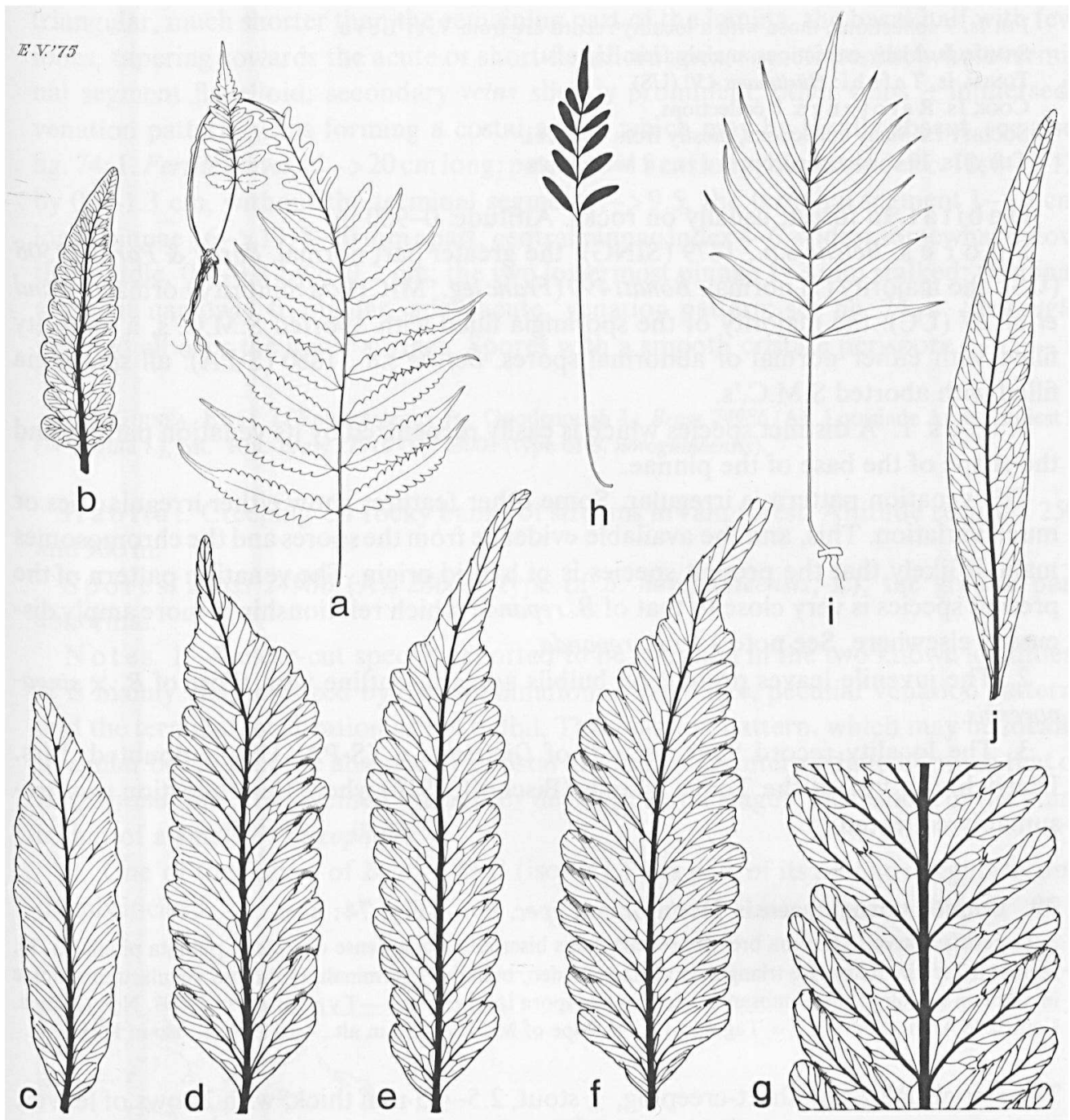

Fig. 76. a-g. Bolbitis lonchophora. a. sterile leaf, $\times 1 / 5$; b. venation pattern of a fertile pinna, $\times 4 / 5 ; \mathrm{c}-\mathrm{g}$. venation pattern of sterile pinnae, $\times 4 / 5$ (a. Safford 974, GH; b. Tilden 87, Leland et al. leg., UC; c. MacDaniels 1279, UC; d. St. John 16654, BO; e. Kew Distr. no. 1173, L; f. St. John 16768, UC; g. Degener I4351, GH). $-\mathrm{h}-\mathrm{j}$. Bolbitis presliana. $\mathrm{h}, \mathrm{i}$. fertile and sterile leaf resp., $\times 1 / 5 ; \mathrm{j}$. venation pattern of sterile pinna, $\times$ 4/5 (h. Hügel 3854, W, holotype of B. presliana; i. Beddome s.n., BM; j. Hooker \& Thomson s.n., B).

lowermost pinnae 1-12 mm stalked. Sporangia inserted all over the lower surface. Spores with a smooth cristate-undulate perispore.

Chromosomes. $\mathrm{n}=82$.

New Hebrides. B a n k s Is.: Palmer 9 (K), 10 (K).

New CALEDonia. 17 collections from all over the island. 
FuI Is. 9 collections; those with a locality record are from Viti Levu.

SAMOA Is. Many collections, mainly from Upolu.

TONGA Is. T a f a hi: Hürlimann 450 (US).

Cook Is. R a r o t on ga: 8 collections.

SOCIETY Is. Many collections, mostly from $\mathrm{T}$ a h it $\mathrm{i}$.

TUBAI Is. 10 collections from $R$ u r $u$ t $u$ and $R$ a $p$ a.

$\mathrm{H}$ a bi t a t. In forest; usually on rocks. Altitude: 0-900 m.

S p o r e s. Betche s.n., 1879 (SING): the greater part normal. Parks \& Parks 22508 (US): the majority (?) normal. Bonati 490 (Francleg., MICH): a minority normal. Leland et al. 87 (UC): the majority of the sporangia filled with aborted S.M.C.'s, a minority filled with either normal or abnormal spores. Betche s.n., 1880 (S-PA): all sporangia filled with aborted S.M.C.'s.

$\mathrm{N}$ o t e s. 1. A distinct species which is easily recognized by its venation pattern and the shape of the base of the pinnae.

The venation pattern is irregular. Some other features show either irregularities or much variation. This, and the available evidence from the spores and the chromosomes make it likely that the present species is of hvbrid origin. The venation nattern of the present species is very close to that of $B$. repanda which relationship is more amply discussed elsewhere. See note 1 to $B$. repanda.

2. The juvenile leaves present on bulbils agree in outline with those of $B . \times$ singaporensis.

3. The locality record 'Queensland' of Dietrich s.n. (S-PA, US) is doubted. Mrs. Dietrich also visited the Tonga Islands (Bischoff, 1916) where this collection was presumably collected.

\section{Bolbitis novoguineensis Hennipman, spec. nov.-Fig. 74: k-n.}

Terrestris, parva. Rhizoma breviter repens, foliis biseriatis. Folia dense coarctata, pinnata pinnis 20-30, segmento terminali anguste triangulari vel flagelloideo, bulbillo \pm terminali. Ordinatio venularum: venulae in aerolam costalem anastomosantes. Sporae perispora laevi cristata.-T y pu s: Brass 28078, New Guinea, Louisiade Arch., Sudest I. (= Tagula I.), west slope of Mt. Riu, $250 \mathrm{~m}$ alt., 4-ix-1956 (L; iso in K, L.).

Terrestrial. Rhizome short-creeping, \pm stout, $2.5-4.5 \mathrm{~mm}$ thick, with 2 rows of leaves, without sclerenchyma strands. Scales of the rhizome: index 1-4, up to $1.5(-2)$ by $0.5 \mathrm{~mm}$, subclathrate, reddish brown. Leaves \pm close together. Sterile leaves pinnate, $9-21 \mathrm{~cm}$ long; petiole 2-5 cm long, near the base $1-1.5 \mathrm{~mm}$ thick, with 3 vascular bundles; lamina index 3-5, widest somewhat below the middle, $7-17$ by $2-4 \mathrm{~cm}$, without the terminal segment 5-13, the terminal segment $1.5-4 \mathrm{~cm}$ long, firm-herbaceous (to subcarnose?), olivaceous, with a \pm terminal bulbil; rhachis with a narrow wing throughout or in the upper half only; pinnae alternate to opposite, $>16$ to $30,5-10 \mathrm{~mm}$ apart; central pinnae index $1-3$, widest about or below the middle, $1-2.4$ by $0.7-0.8 \mathrm{~cm}$, base usually symmetrical, cuneate, sometimes somewhat asymmetrical, its basiscopic side cuneate, the acroscopic side either broadly attenuate or with a basal acroscopic lobe, margin (bi)crenate-serrate with distinct spines in the sinuses, apex acute or rounded; the two lowermost pinnae 0.5-1.5 mm stalked, \pm conform to the central pinnae; terminal segment usually narrowly 
triangular, much shorter than the remaining part of the lamina, the basal half with few lobes, tapering towards the acute or short-flagelloid apex, sometimes the whole terminal segment flagelloid; secondary veins slightly prominent, other veins \pm immersed; venation pattern: veins forming a costal areole which may be locally absent, see also fig. 74: 1 . Fertile leaves $15->20 \mathrm{~cm}$ long; petiole $6-15 \mathrm{~cm}$ long; lamina index $c .10,8->12$ by $0.8-1.3 \mathrm{~cm}$, without the terminal segment $7->9.5$, the terminal segment $1->3 \mathrm{~cm}$ long; pinnae 16-> 19, 8-20 mm apart; central pinnae index 4-6, widest somewhat below the middle, $0.6-0.8$ by $c .0 .2 \mathrm{~cm}$; the two lowermost pinnae $1-3 \mathrm{~mm}$ stalked; terminal segment narrowly triangular, apex acute; venation pattern: see fig. 74: n. Sporangia inserted all over the lower surface. Spores with a smooth cristate perispore.

New GuINEA. E a st. D'Entrecasteaux Is., Goodenough I.: Brass 24986 (A); Louisiade Arch., Sudest I. (= Tagula I.), Mt. Riu: Brass 27916 (L), 28008 (type of B. novoguineensis).

$\mathrm{H} \mathrm{a} \mathrm{bit} \mathrm{a} \mathrm{t.} \mathrm{Creeping} \mathrm{on} \mathrm{rocky} \mathrm{banks} \mathrm{of} \mathrm{streams} \mathrm{in} \mathrm{rain} \mathrm{forest.} \mathrm{Altitude} \mathrm{records:} 250$ and $900 \mathrm{~m}$.

Spores. Brass 24986 (A), 28008 (type of B. novoguineensis, L): the greater part abnormal.

Notes. 1. A clear-cut species reported to be common in the two known localities. It is mainly characterized by the combination of small size, peculiar venation pattern, and the terminal localization of the bulbil. The venation pattern, which may be locally irregular because of the absence of a costal areole, seems intermediate between that of $B$. quoyana and a free-veined species (or developmental stage). The aspect of the plant is that of a small $B$. rhizophylla.

2. One of the leaves of Brass 28008 (iso in L) has part of its pinnae irregularly and deeply incised.

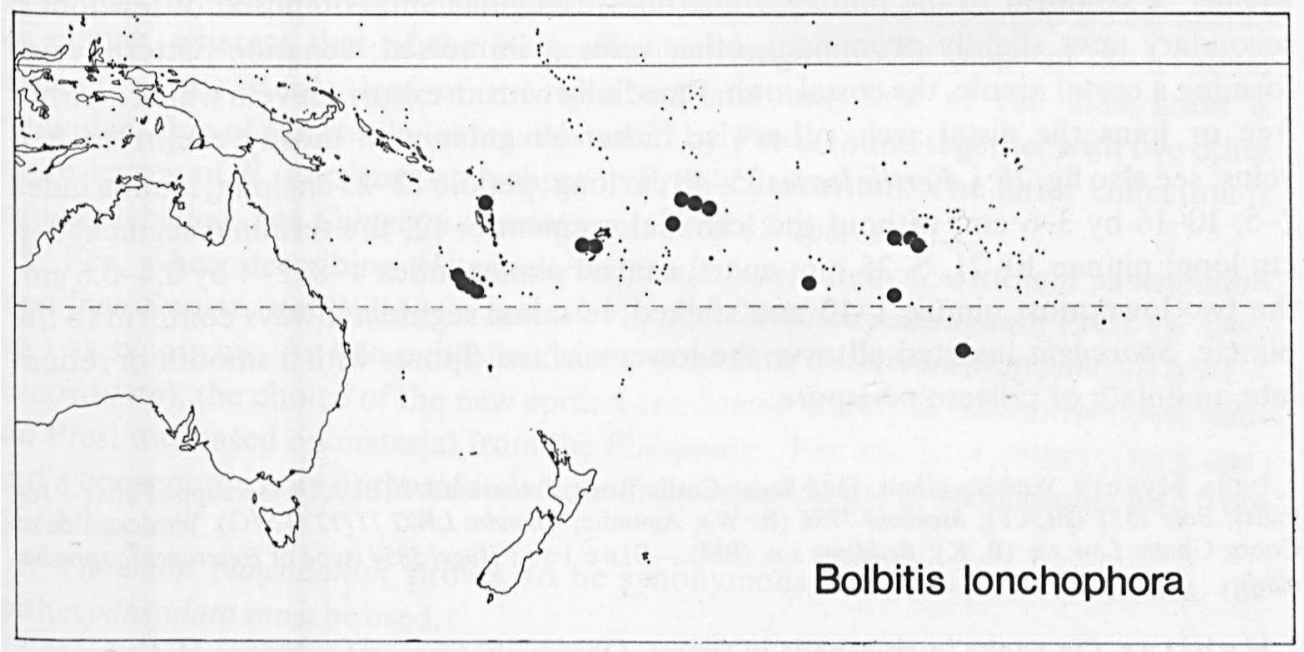

Fig. 77. Distribution of Bolbitis lonchophora. 
3. A small thickening presumably representing a primordium of a bulbil is found terminally on the costae.

40. Bolbitis presliana (Fée) Ching-Fig. 76: $\mathbf{h}-\mathbf{j}$.

B. presliana (Fée) Ching in C. Chr., Ind. Fil., Suppl. 3(1934) 49, p.p.; Copel., Fern Fl. Philip. (1960) 260, p.p.; Nayar \& Kaur, Bull. Bot. Gard. Lucknow 88 (1964) 53, fig. 21, 34, 67, 81, 89, p.p.-Heteroneuron preslianum Fée, Hist. Acrost. (1845) 92, pl. 39: fig. i, p.p. ('Heteronevron').-Poecilopteris presliana Moore, Ind. Fil. (1857) xx, p.p.; Bedd., Ferns Br. India (1868) pl. 269, c. descr., p.p.-Acrostichum preslianum Hooker, Spec. Fil. 5 (1864) 265, p.p., nom. illeg., non Fée (1845) (= Elaphoglossum spec.).-Gymnopteris presliana J. Smith, Hist. Fil. (1875) 138, p.p.; Bedd., Handb. Ferns Br. India (1883) 439, fig. 267,p.p.-Leptochilus preslianus C. Chr., Ind. Fil. (1906) 387, p.p.; v.A.v.R., Handb. Malayan Ferns (1908) 704, p.p.; Ridley, J. Mal. Br. R. As. Soc. 4 (1916) 115, quoad nomen solum; v.A.v.R., Handb. Malayan Ferns, Suppl. 1 (1917) 435, p.p. - Campium feeianum Copel., Philip. J. Sc. 37 (1928) 392, fig. 43, nom. illeg.-T y pe: Hügel 3854, 'Asia' (W; iso in PR, ster.). See note 2.

Terrestrial. Rhizome short-creeping, up to $10 \mathrm{~cm}$ long, 4-6 mm thick, with 2 rows of leaves, without sclerenchyma strands. Scales of the rhizome: index 2-5, up to 3(-4) by $1(-1.5) \mathrm{mm}$, opaque to subclathrate, dark reddish brown or blackish. Leaves \pm close together. Sterile leaves pinnate, 20-50 cm long; petiole 5-24 cm long, near the base 1-3 $\mathrm{mm}$ thick, with 5-7 vascular bundles; lamina index $1-3$, widest usually somewhat below (to about) the middle, $12-26$ by $5-15 \mathrm{~cm}$, without the terminal segment $7-18$, the terminal segment 4-12 cm long, \pm herbaceous, dark green to blackish, without bulbils; rhachis sometimes with a narrow wing in the upper half of the lamina; pinnae alternate or opposite, 8-22, up to $25 \mathrm{~mm}$ apart; central pinnae index 4-11, widest above to below the middle, tapering towards both ends, $4-10$ by $0.6-1.3 \mathrm{~cm}$, base angustate, margin \pm entire or slightly and irregularly sinuate, without teeth or spines, apex acute to acuminate; the two lowermost pinnae $3-10 \mathrm{~mm}$ stalked, usually conform to the central pinnae, rarely with a basal acroscopic lobe; terminal segment joined to the rhachis, usually \pm conform to the pinnae, sometimes triangular and composed of few lobes; secondary veins slightly prominent, other veins \pm immersed; venation pattern: veins forming a costal areole, the costal arch \pm medially with an excurrent vein which is either free or joins the distal arch, otherwise rather irregular with mostly excurrent free veins, see also fig. 76 : j. Fertile leaves $25-40 \mathrm{~cm}$ long; petiole $12-25 \mathrm{~cm}$ long; lamina index $2-5,10-16$ by $3-6 \mathrm{~cm}$, without the terminal segment $6-12$, the terminal segment $3-6$ $\mathrm{cm}$ long; pinnae 10-21, 5-25 mm apart; central pinnae index $4-8,2-4$ by $0.4-0.6 \mathrm{~cm}$; the two lowermost pinnae 1-10 mm stalked; terminal segment always conform to the pinnae. Sporangia inserted all over the lower surface. Spores with a smooth or reticulate, undulate or cristate perispore.

InDiA. Mys o r e. Western Ghats: Dnd Sagar-Castle Rock, d'Almeida 311 (BLAT); Gersoppa Falls (= Jog Falls), Bole 1531 (BLAT); Meebold 7734 (B, W); Agumbe, Chandra LWG 77712 (LWG). Western side of Coorg Ghats: Law s.n. (B, K); Beddome s.n. (BM).-Sin e lo c.: Hügel 3854 (type of Heteroneuron preslianum).

$\mathrm{H}$ a b it a t. On rocks in riverbeds in forest. Obviously (in part) submersed during the rainy season. Altitude records; $600 \mathrm{~m}$ (Meebold 7734); 'sea level'(Bedd., op. cit., 1883). 
S p o res. Hügel 3854 (W, holo): the greater part normal, perispore usually smooth, sometimes reticulate, usually with variously tall crests, sometimes undulate. Bole 1531: the greater part normal, perispore smooth cristate. Chandra LWG 77712: in part normal, the perispore usually smooth undulate with minute spines, sometimes smooth cristate (or the crests reticulate?). Beddome s.n. (BM, 2 sh.): the majority normal, the perispore reticulate and low cristate.

Notes. 1. A small and presumably rare fern which is easily recognized by the colour of the dried leaves, the shape of the pinnae, the absence of bulbils, and the venation pattern. As regards its relationships Hooker (1864) thought its affinities to be 'certainlv with Acrostichum Heudelotii' ( $=B$. heudelotii), with which it seems to share the preference for wet habitats only; Copeland, however, regarded the present species (as Campium feeianum) as very close to $B$. salicina, also a fern of very moist places.

$B$. presliana shows characters which suggest hybrid origin. Firstly, the perispore shows much variation and seems intermediate between that of $B$. appendiculata and ser. Bolbitianae. Secondly, the outline of the leaves shows irregularities. Odd pinnae occur in quite a number of the leaves, and a few leaves have one or more pinnae showing a basal acroscopic lobe. The venation pattern also shows irregularities and may resemble the pattern of $B$. subcrenata and a pattern with free veins (ser. Egenolfianae). The absence of a bulbil is noteworthy as a bulbil is present in species of the series to which I regard B. presliana as possibly related (ser. Egenolfianae and ser. Bolbitianae; both also in Mysore).

2. In the original publication Fée cited Hügel 3854 (W) from southern India as 'exsiccatum'. Fée's excellent illustration shows two sterile leaves and a fertile one attached to a single rhizome. The type specimen at Vienna shows a sterile leaf corresponding with the largest of those illustrated, and a separately mounted fertile leaf also corresponding with that of the illustration except for the shape of the terminal segment. The terminal segment of the fertile leaf of the type specimen is conform to the pinnae, whereas that of the illustration is triangular. The shape of the terminal segment of the sterile leaf of the type material and that of the illustration is triangular. One of the sterile leaves illustrated by Fée is found together with two other sterile leaves of $B$. presliana on a sheet in Presl's herbarium. The latter collection is wrongly labelled. See Campium punctulatum Presl (= spec. dub).

3. Fée, when describing Heteroneuron preslianum, cited Acrostichum punctulatum Presl (non Swartz, nec Willd.) (= spec. dub.), and Campium punctulatum Presl (= spec. $d u b$.) as synonyms. As Fée published at the same time a Heteroneuron punctulatum (= B. auriculata), the choice of the new epithet preslianum is correct. Acrostichum punctulatum Presl was based on material from the Philippines, Fée and later authors(for Copeland's concept see note 4) therefore erroneously reported the present species also from the Philippines.

If Campium punctulatum proves to be synonymous with the present species, the epithet punctulata must be used.

4. Copeland (1928) listed the present species as Campium feeianum. He copied Fée's original description verbatim and only added 'This is a rare fern of southern India'. The 
reason why Copeland did not use the epithet preslianum in the genus Campium is obscure. He possibly wanted to restrict that epithet to the Philippine element of Fée's species. In his Fern Flora of the Philippines (1960) he cited Campium feeianum as a synonym under $B$. presliana; the locality 'southern India' is given with a query.

5. Van Alderwerelt van Rosenburgh (1908) erroneously reported this fern from Madagascar, and later (1917) also from Singapore (following Ridley).

41. Bolbitis repanda (B1.) Schott-Fig. 78.

B. repanda (Bl.) Schott, Gen. Fil. (1835) ad tab. (13).-Acrostichum repandum Bl., Enum. Pl. Jav. (1828) 104; Fl. Jav. Fil. (1829) 39, pl. 14, 15, p.p.; Hooker, Spec. Fil. 5 (1864) 260, p.p.; Baker in Hooker \& Baker, Syn. Fil. (1868) 419, p.p. - Campium repandum Presl, Tent. Pterid. (1836) 239.-Cyrtogonium repandum J. Smith, Hook. J. Bot. 3 (1841) 403.-Heteroneuron repandum Fé, Hist. Acrost. (1845) 96, pl. 59 ('Heteronevron'). -Poecilopteris repanda Presl, Epim. Bot. (1851) 172; Bedd., Ferns S. India (1864) 68, pl. 202, quoad nomen solum. -Chrysodium repandum Mettenius, Novara Exp. Bot. 1 (1870) 202, p.p. -Gymnopteris repanda Christ, Farnkr. Erde (1897) 50, p.p.; Diels in E. \& P., Nat. Pfl. Fam. 1, 4 (1901) 201, p.p.; Copel., Publ. Bur. Gov. Lab. 28 (= Polyp. Philip.) (1905) 43, p.p.-T y p e: Reinwardt s.n., E. Java, Besoeki, Banjuwangi, xii-1821 (L, 908.288-262, ster.).

Leptochilus cuspidatus auct. non (Presl) C. Chr.: auct. div., p.p.

Campium quoyanum auct. non (Gaudich.) Copel.: Copel., Philip. J. Sc. 37 (1928) 366, p.p.; C. Chr. \& Holttum, Gard. Bull. S.S. 7 (1934) 262.

B. quoyana auct. non (Gaudich.) C. Chr.: Backer \& Posthumus, Varenfl. Java (1939) 82, p.p.

Usually terrestrial, sometimes low-epiphytic. Rhizome short-creeping, up to $10(-15)$ $\mathrm{cm}$ long, up to $9 \mathrm{~mm}$ thick, with 2 or 3 rows of leaves, with few sclerenchyma strands. Scales of the rhizome: index $2-5$, up to 4 by $1.5 \mathrm{~mm}$, subclathrate, brown to blackish, usually with a paler margin. Leaves \pm close together. Sterile leaves pinnate, 35-100 $(-120) \mathrm{cm}$ long; petiole $10-40 \mathrm{~cm}$ long, near the base $1-4 \mathrm{~mm}$ thick, with 5-7 vascular bundles; lamina index of non-flagelloid leaves $1-3$, of flagelloid leaves up to 7 , widest below the middle, $15-70(-100)$ by $6-30 \mathrm{~cm}$, without the terminal segment $7-40$, the terminal segment 8-60 $(-70) \mathrm{cm}$ long, herbaceous-pergamentaceous, green to blackish, with a (primordium of a) spherical subterminal bulbil; rhachis not winged; pinnae alternate or opposite, 8-20 (-24), 20-50 mm apart; central pinnae index (2-) 3-6(-8), widest about (to below) the middle, $4.5-22$ by $1.5-4.5 \mathrm{~cm}$, base \pm symmetrical, angustate to broadly cuneate, margin lobed $1 / 4-1 / 2$ towards the costa, usually with a distinct tooth in each sinus, apex acute to long-acuminate, lobes usually finely serrate-crenate, sometimes entire; the two lowermost pinnae $1-11 \mathrm{~mm}$ stalked, either \pm conform to the pinnae, or asymmetrical with its basiscopic side shorter, wider, and more deeply lobed than the acroscopic side; terminal segment triangular, deeply lobed near the base, tapering towards the acute to flagelloid apex; secondary veins slightly prominent, other veins \pm immersed; venation pattern irregular, veins forming areoles varying in shape and size, some of which with usually one, mostly excurrent included free vein, see also fig. 78: c-i. Fertile leaves $35-85 \mathrm{~cm}$ long; petiole $18-45 \mathrm{~cm}$ long; lamina ind ex 2-5 $(-7), 8-45$ by $2-10 \mathrm{~cm}$, without the terminal segment $5-35$, the terminal segment $3-11$ $\mathrm{cm}$ long; pinnae 9-20 (-23), 20-60 mm apart; central pinnae index 3-9, widest usually below, sometimes about the middle, $1.5-7$ by $0.4-1.5(-2) \mathrm{cm}$; the two lowermost 


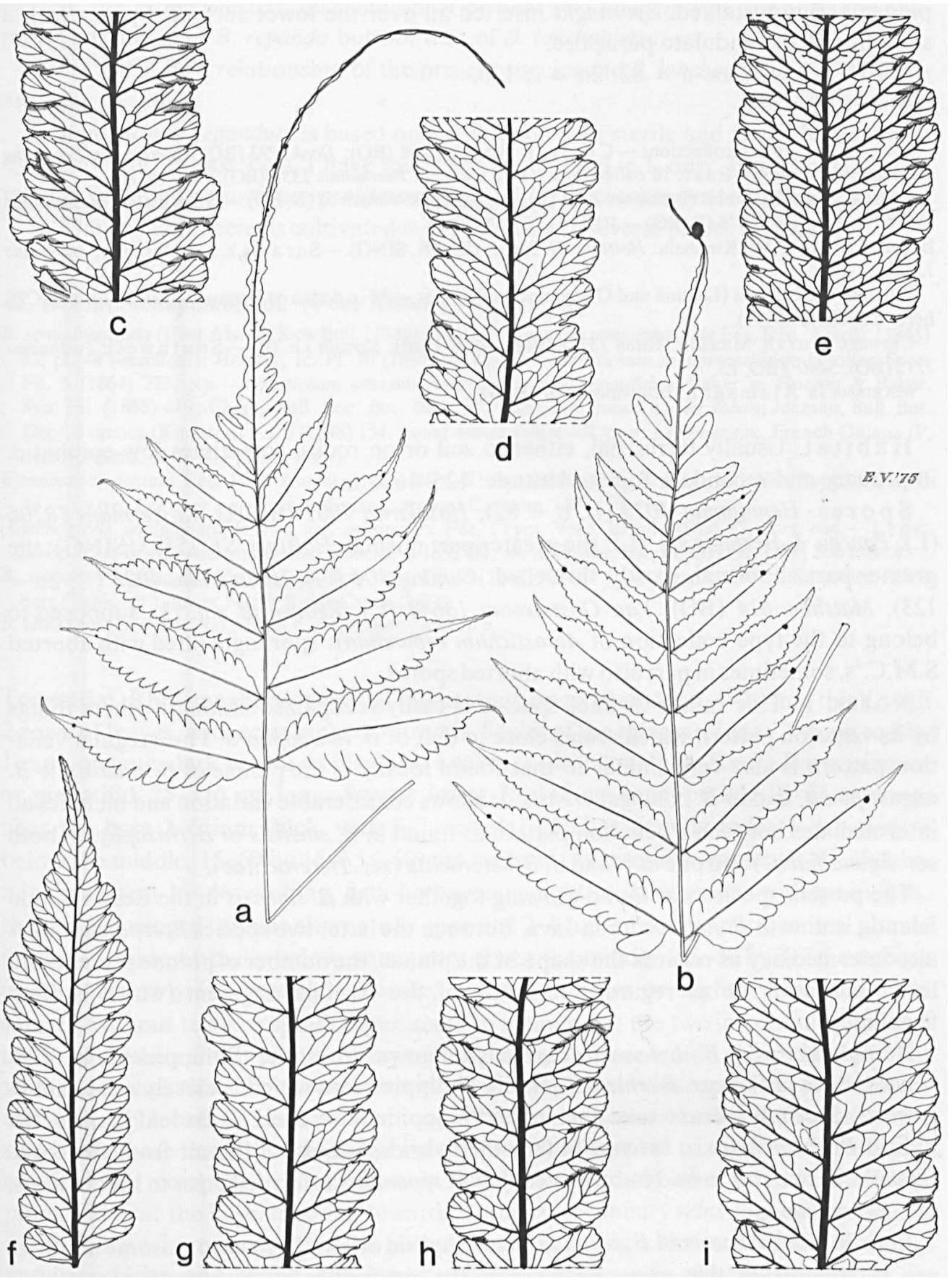

Fig. 78. Bolbitis repanda. - a, b. sterile leaf of a triploid and a tetraploid specimen resp., $\times 1 / 5$; c-i. venation pattern of sterile pinnae, $\times 4 / 5$ (a. Hennipman 4032, L, culta, triploid; b. $H$. 4026, L. culta, tetraploid; c. H. s.n., L, culta, tetraploid; d. H. s.n., L, culta, triploid; e. Van Ooststroom 13644, L; f. Korthals 205, L; g. Donk 54, BO; h. Holttum SF 25585, SING; i. Blume s.n., L). 
pinnae 1-8 mm stalked. Sporangia inserted all over the lower surface. Spores with a smooth cristate-undulate perispore.

Chromosomes. $\mathrm{n}=82 ; 2 \mathrm{n}=$ c. 120 .

JAVA. West: many collections.-Central: Beumée 1108 (BO); Donk P92 (BO); Docters van LeeuwenReijnvaan 1121 (BO).-E a st: 16 collections.-P. B a we a n: Posthumus 1331 (BO), 1369 (BO).

Lesser Sunda Is B ali: Posthumus 3704 (L).-L o m b ok: Rensch 52 (B, BO).-S u m b a w a: Kostermans $18206(\mathrm{~K}, \mathrm{~L})$; Rensch $576(\mathrm{~B}, \mathrm{BO}) .-\mathrm{F}$ lo r e s: 5 collections.

BorNeo. S a b a h. Mt. Kinabalu: Holttum SF 25585 (BM, K, SING),-S a r a w a k. Mt. Merinjak: Anonymus $133(\mathrm{P})$.

PhILIPPINEs. Luz on (Laguna and Quezon): 6 collections.-Mind a n a o. Davao: Copeland s.n. (MICH, herb. Copeland 10199).

Celebes. N or th. Manado: Alston 15774 (BM), 16231 (BM); Sarasin s.n. (P).-S o ut h w es t: Posthumus $2715(\mathrm{BO}), 3440$ (BO, L).

MARIANA Is. A la maga n: Hosokawa 7901 (BISH).

$\mathrm{H}$ a bit a t. Usually terrestrial, either in soil or on rocks, sometimes low-epiphytic, in primary and secondary forest. Altitude: $125-1650 \mathrm{~m}$.

S pores. Hennipman 4023 (L, $\mathrm{n}=82$ ), Hosokawa 7901 (BISH), Kostermans 18206 (L), Pancho \& Hernaez s.n. (L): the greater part normal. Holttum SF 25585(SING): the greater part abnormal, mostly shrivelled. Cuming 104 (G), Hennipman 4028 (L, 2n = 123), Matthew 614 (BO), Van Ooststroom 13644 (L), Reinwardt s.n. (L, supposed to belong to the type collection of Acrostichum repandum): sporangia filled with aborted S.M.C.'s, sometimes in part also with aborted spores.

Notes. 1. This rather distinct species is easily recognized amongst others things by its venation pattern which seems close to that of $B$. lonchophora. The irregular venation pattern is also very similar to that found locally in the pinnae of $B$. sinensis $\times B$. angustipinna, and in $B . \times$ singaporensis. It shows considerable variation and includes all intermediates between a venation pattern as found in $B$. sinensis or $B$. rhizophylla (both ser. Egenolfianae) and one as found in $B$. heteroclita (ser. Heteroclitae).

The present species was found growing together with $B$. sinensis in the Lesser Sunda Islands, and with $B$. heteroclita in Java. Between the latter two species $B$. repanda shows also intermediacy as regards the shape of the pinnae, the number of pinnae present on a leaf, and rather so as regards the shape of the terminal segment (which is often flagelloid).

$B$. repanda and $B$. heteroclita are also known from the Philippines where $B$. sinensis does not occur. $B$. rhizophylla, the Philippine fern which is closely related to $B$. sinensis, does not seem to take part in the Philippine B. repanda, as the leaf shape of the hybrid $B$. rhizophylla $\times$ heteroclita (see the hybrids) is quite different from that of $B$. repanda. It remains to be demonstrated that $B$. repanda was formed prior to speciation in ser. Egenolfianae.

Both $B$. lonchophora and $B$. repanda seem of hybrid origin. Their chromosome numbers are in support of this view. Because of the similarities in the venation pattern it seems likely that the parentage of $B$. repanda is in part the same as or closely related to that of $B$. lonchophora. This suggestion is only supported as far as $B$. heteroclita is con- 
cerned; the rather western distribution of present ser. Egenolfianae supports the proposed parentage of $B$. repanda but not that of $B$. lonchophora.

There might be a relationship of the present species and B. lonchophora to B. novoguineensis.

2. Acrostichum repandum is based on a collection with sterile and fertile leaves made by Reinwardt in East Java. I have found sterile material in L only. Blume (1929) erroneously regarded Acrostichum proliferum Bl. $(=B$. heteroclita $)$ as synonymous.

3. The present species is cultivated in greenhouses in several places in Europe.

42. Bolbitis semipinnatifida (Fée) Alston-Fig. 79, 80.

B. semipinnatifida (Fée) Alston, Kew Bull.(1932) 310.-Gymnopteris semipinnatifida Fée, Hist. Acrost. (1845) 83, pl. 44 (excellent); Hooker, Ic. Pl. 10 (1854) pl. 71, 72.-Acrostichum semipinnatifidum Hooker, Spec. Fil. 5 (1864) 273, p.p.-Acrostichum alienum Swartz var. semipinnatifidum Baker in Hooker \& Baker, Syn. Fil. (1868) 419; Christ, Bull. Soc. Bot. Belg. 35 (1896) 246, quoad nomen solum; Jenman, Bull. Bot. Dept. Jamaica (Kingston) n.s. 5 (1898) 154, quoad nomen solum.-T y p e: LePrieur s.n., French Guiana (P, herb. Richard; iso in K, P, herb. Bory).

Gymnopteris dentata Fée, Hist. Acrost. (1845) 85.-B. dentata Ching in C. Chr., Ind. Fil., Suppl. 3 (1934) 48.-T y p e: LePrieur s.n., French Guiana, around Cayenne, 1834 (P).

[Gymnopteris semipinnatifida Fée var. subsimplex Fée, Crypt. Vasc. Brésil (1869) 17, nom. nud.-T y pe: Spruce 2121, Brazil, Amazonas, Sao Gabriel, ii-1852 (K; iso in B, BM, BR, G, GH, K, US) ] See note 6.

B. scopulina Maxon \& Morton, Bull. Torr. Bot. Cl. 75 (1948) 72.-T y p e: Maguire 24814, Surinam, Tafelberg(Table Mt.), lower North Ridge Creek (NY).

B. aliena auct. non (Swartz) Alston c. syn. homot.: auct. p.p.

Terrestrial. Rhizome short-creeping, rather stout, up to $10 \mathrm{~cm}$ long, 4-10 mm thick, with 2 rows of leaves, with sclerenchyma strands. Scales of the rhizome: index 2-6, up to 4 by $1 \mathrm{~mm}$, opaque, dark brown or blackish. Leaves \pm close together. Sterile leaves pinnate or pinnatifid, $25-115 \mathrm{~cm}$ long. Pinnate leaves $25-115 \mathrm{~cm}$ long; petiole $10-65 \mathrm{~cm}$ long, near the base 2-6 mm thick, with 5-7 vascular bundles; lamina index 1-1.5, widest below the middle, $15-60$ by $15-35(-50) \mathrm{cm}$, without the terminal segment $0-17$, the terminal segment $15-46 \mathrm{~cm}$ long, firm-herbaceous, light to dark green, without bulbils; rhachis not winged; pinnae alternate to opposite, 2-8, up to $55 \mathrm{~mm}$ apart; central pinnae index 3-5, widest usually below, sometimes about the middle, $9.5-30$ by $3-7.5 \mathrm{~cm}$, base usually narrowly (to broadly) cuneate, margin \pm entire or slightly and irregularly sinuate, without teeth or spines, apex acute to acuminate; the two lowermost pinnae \pm conform to the central pinnae or asymmetrical, its basiscopic side shorter and (much) wider $(-20 \mathrm{~cm})$ than the acroscopic side, with one basal basiscopic lobe or regularly and \pm deeply lobed, $0-3 \mathrm{~mm}$ stalked; uppermost pinnae sometimes with the basiscopic side of the base decurrent along the rhachis; terminal segment triangular, either asymmetrical and composed of usually only few (generally $2-4$ ) lobes or \pm symmetrical, pinnatifid near the base, tapering towards the apex; secondary veins slightly prominent, other veins \pm immersed; venation pattern: veins forming a dense network of areoles varying in size and shape, the larger areoles with usually one mostly simple, exor recurrent vein, see also fig.79: g, h. Pinnatifid leaves 50-105 cm long; petiole $20-50 \mathrm{~cm}$ long; lamina index $1-3(-5)$, widest below the middle, $30-60$ by $8-35 \mathrm{~cm}$, 


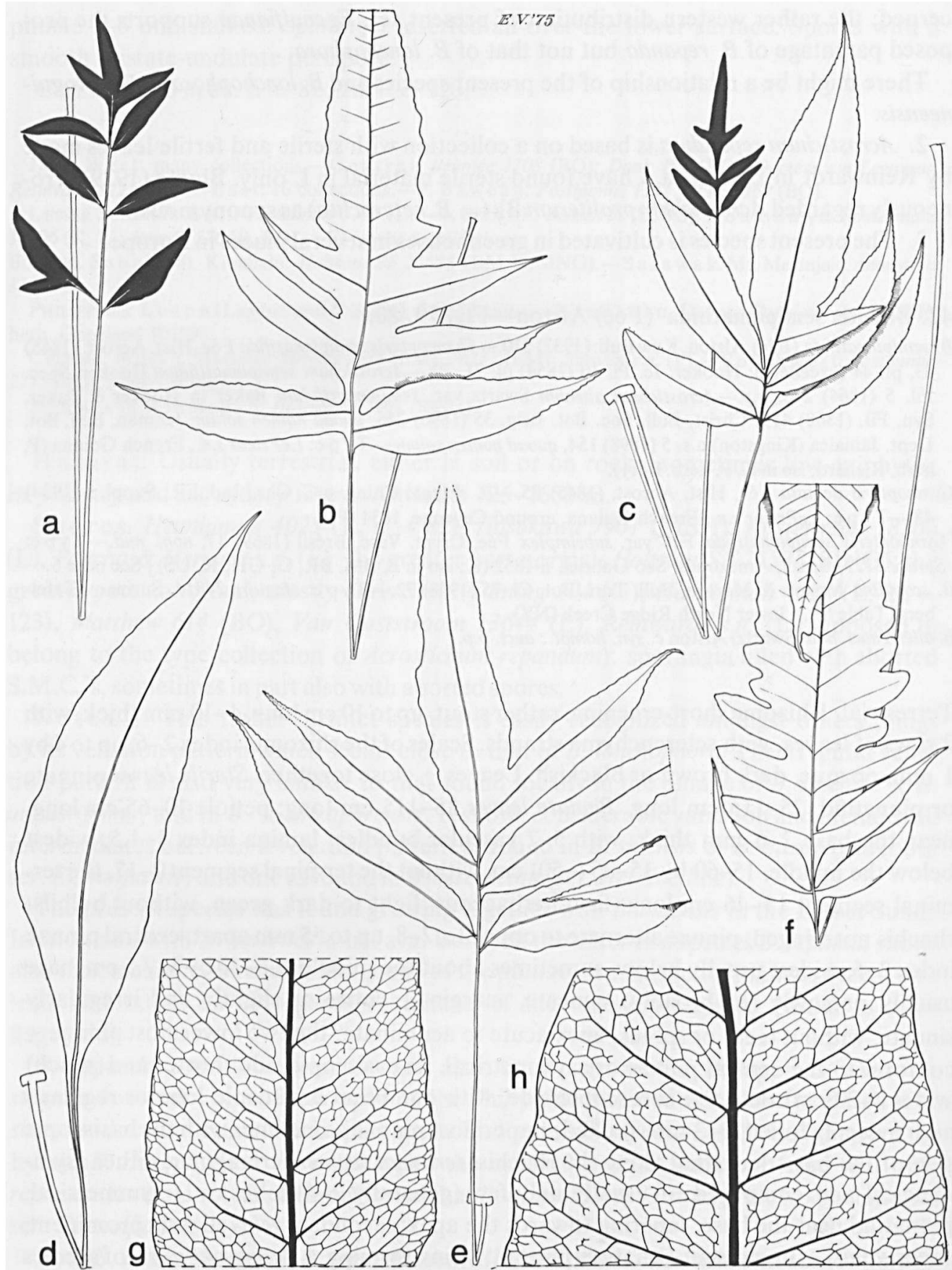

Fig. 79. Bolbitis semipinnatifida.-a-f. sterile and fertile leaves, $\times 1 / 5 ; \mathrm{g}, \mathrm{h}$. venation pattern of sterile pinnae, $\times$ 4/5 (a, e. Fanshawe 4822, BM; b. Daniëls \& Jonker 820, U; c. Maguire 24814, NY, holotype of B. scopulina; d. Croizat 793, U; f. Spruce 2212, K; g. Geijskes 1008, U; h. Lemos Froes 21474, NY). 
either regularly pinnatifid throughout or with 2 conspicuous basal lobes, margin usually \pm entire, sometimes irregularly and undeeply sinuate; otherwise similar to pinnate leaves. - Fertile leaves (25-) 35-110 cm long. Pinnate leaves (25-) 35-110 cm long; petiole (20-) 30-80 cm long; lamina index 1-3, (5-) 10-30 by $2-14 \mathrm{~cm}$, without the terminal segment 0-17, the terminal segment (5-) 8-18 cm long; pinnae 2-8, 0-70 mm apart; central pinnae index 3-7 $(-10), 3.5-10.5$ by $0.8-2 \mathrm{~cm}$, the two lowermost pinnae either \pm conform to the central pinnae and $0.8-2 \mathrm{~cm}$ wide, or asymmetrical and up to $5.5 \mathrm{~cm}$ wide. Pinnatifid leaves $55-90 \mathrm{~cm}$ long; petiole $40-70 \mathrm{~cm}$ long; lamina index 2-5, 14-25 by 3-9 $\mathrm{cm}$; the two lowermost lobes $20-70$ by $9-16 \mathrm{~mm}$; otherwise similar to pinnate leaves. Sporangia inserted all over the lower surface. Spores with a smooth, usually cristate, rarely cristate-undulate perispore.

\section{Lesser Antilles. Trinid a d: Prestoe s.n. (BM).}

French Guiana. 7 collections.

SuRINAM. 9 collections.

British GuIANA. 8 collections.

BRAZIL. A mazon as: Lemos Froes 21474 (NY); Von Luetzelburg 21294 (M); Spruce 2121 (type of Gymnopteris semipinnatifida var. subsimplex). - P a ra: Spruce 577 (B, K).

CoLOMBiA. V a u pes: Schultes \& Cabrera 12929 (US).

VenezUeLA. Boliva r: Wurdack \& Monachino 41313 (US).-A maz o n as: Ugueto R.: Croizat 793 (MO, U); Rusby \& Squires 380 (BM, F, G, GH, M, MO, NY, US).

$\mathrm{H}$ a bit a t. Rocky places along streams in rain forest. Altitude: 0-800 m. Jonker \& Wensink (Tijdschr. Kon. Ned. Aardr. Gen. II, 77, 1960, 145-161) reported the present species as the most common fern in 'high dryland forest' of the Emma Range in central Surinam.

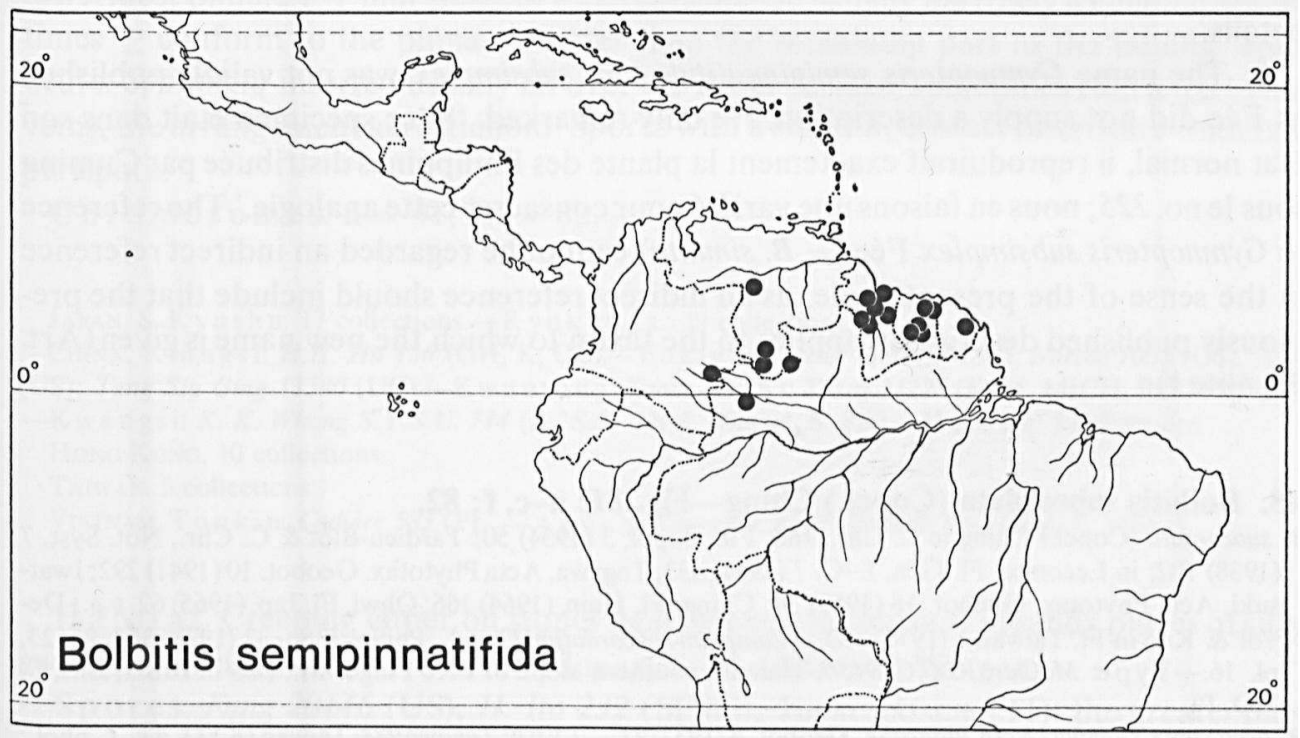

Fig. 80. Distribution of Bolbitis semipinnatifida. 
S p or es. Jenman s.n. (P), Rusby \& Squires 380 (MO, US), Maguire 24814 (type of $B$. scopulina NY), Wurdack \& Monachino 41313 (US): the greater part normal. Daniëls \& Jonker 820 (U), Fanshawe 4882 (BM): in part normal. Lemos Froés 21474 (NY). Von Luetzelburg 21294 (M), Spruce 2121 (type of Gymnopteris semipinnatifida var. subsimplex, BR, G, GH): all or the greater part abnormal.

Notes. 1. Apparently a good species which was not recognized by either Ching or Christensen. For a long time it was confused with $B$. portoricensis (as B. aliena auct. non (Swartz) Alston). Its relationships seem bifold. On one side it seems close to B. aliena with which it shares the outline of the leaves of a considerable part of the specimens, features of the rhizome, and the absence of a bulbil. On the other hand it seems also related to ser. Euryostichae because of features of the venation pattern and the outline of the leaves of another part of the material. It may well have arisen through hybridization.

2. The outline of the pinnate leaves is very variable. The extremes may be found in one collection, e.g. Rusby \& Squires 380 (US, 2 sh.).

3. Plants with pinnatifid sterile leaves are found outside the Guianas and west of $60^{\circ}$ W: Lemos Froés 21474, Von Leutzelburg 21294, Schultes \& Cabrera 12929, Spruce 2121, Wurdack \& Monachino 41314. The outline of these leaves rather agrees with that found in the African B. $\times$ boivinii which parentage includes a member of ser. Euryostichae. The plants with pinnatifid leaves were at first considered a different variety. Supposedly intermediately shaped leaves became available from plants collected in Surinam later, e.g. Daniëls \& Jonker 820 (IJ, U).

4. See also note 6 to $B$. aliena.

5. Gymnopteris semipinnatifida and G. dentata were described by Fée in the same part of his opus, both from collections made by LePrieur in French Guiana. The sterile leaves of the type specimens of both species are very similar in outline and identical in details.

6. The name Gymnopteris semipinnatifida var. subsimplex was not validly published as Fée did not supply a description. He only remarked: 'Si ce spécimen était dans son état normal, il reproduirait exactement la plante des Philippines distribuée par Cuming sous le no. 225; nous en faisons une variété pour consacrer cette analogie.' The reference to Gymnopteris subsimplex Fée $(=B$. sinuata) cannot be regarded an indirect reference in the sense of the present Code, as an indirect reference should include that the previously published description applies to the taxon to which the new name is given (Art. 32 , note 1 ).

\section{Bolbitis subcordata (Copel.) Ching-Fig. 81 : a-c, f; 82.}

B. subcordata (Copel.) Ching in C. Chr., Ind. Fil., Suppl. 3 (1934) 50; Tardieu-Blot \& C. Chr., Not. Syst. 7 (1938) 102; in Lecomte, Fl. Gén. 1.-C. 7 (1941) 433; Tagawa, Acta Phytotax. Geobot. 10(1941) 292; Iwatsuki, Acta Phytotax. Geobot. 18 (1959) 54; Ching, Fl. Hain. (1964) 166; Ohwi, Fl. Jap. (1965) 62, p.p.; DeVol \& Kuo in Fl. Taiwan 1 (1975) 350.-Campium subcordatum Copel., Philip. J. Sc. 37 (1928) 369, fig. 23, pl. 16.-T y pe: McClure C.C.C. 9436, Hainan, southern slope of Five Finger Mt. (iso in BISH, BM, C, $\mathrm{MO}, \mathrm{P})$.

B. formosana Tagawa, Acta Phytotax. Geobot. 6(1937)92.-T y p e: Tagawa984, Taiwan (KYO, n.v., L, phot.; iso in $\mathrm{TI}$, n.v., $\mathrm{L}$, phot.).

B. nakaii H. Ito, J. Jap. Bot. 14 (1938) 442. - L e ct ot y pe (Iwatsuki, 1959): H. Ito s.n., Japan, Ryukyu Is., 
Okinawa, Mt. Benokiyama (TI, n.v., L, phot.).

Gymnopteris repanda auct. non (BI.) Christ: auct. jap. p.p.

Acrostichum virens auct. non Hooker \& Grev. c. syn. homot.: auct. jap. p.p.

B. quoyana auct. non (Gaudich.) Ching: auct. jap. p.p.

Terrestrial. Rhizome short-creeping, \pm stout, $4-14 \mathrm{~mm}$ thick, with 2 or 3 rows of leaves, with sclerenchyma strands. Scales of the rhizome: index $1-4$, up to $4.5(-7)$ by $1(-1.5)$ $\mathrm{mm}$, subclathrate, brown or blackish. Leaves \pm close together. Sterile leaves pinnate, 20-115 cm long; petiole 6-52 cm long, near the base 1-5.5 mm thick, with 5-9 vascular bundles; lamina index $1-3$, widest below (to about) the middle, $13-70$ by $7-35 \mathrm{~cm}$, without the terminal segment $1.5-50$, the terminal segment $10-27 \mathrm{~cm}$ long, herbaceous, dark green or brownish; rhachis not winged; pinnae alternate or opposite, 4-28, 15-75 mm apart; central pinnae index $2-7$, widest below or about the middle, $3.5-22.5$ by $1.7-4 \mathrm{~cm}$, base \pm symmetrical, broadly cuneate to subcordate, margin usually lobed to $1 / 3(-1 / 2)$ towards the costa, sometimes entire, crenate-serrate, with \pm distinct spines (or teeth) in the sinuses, apex acute or acuminate; the two lowermost pinnae 0-8 $\mathrm{mm}$ stalked, \pm conform to the central pinnae; terminal segment narrowly triangular, longer to shorter than the remaining part of the lamina, near the base somewhat more deeply incised than the central pinnae, apex acute to short-flagelloid; secondary veins slightly prominent, other veins \pm immersed; venation pattern: veins forming a costal areole and several to many distal areoles, the veins towards the margin free, excurrent, areoles in part with usually one simple, excurrent free vein, see also fig. 81: b,c,f. Fertile leaves $23-125 \mathrm{~cm}$ long; petiole $15-70 \mathrm{~cm}$ long; lamina ind ex $2-10,16-60$ by $3-17 \mathrm{~cm}$, without the terminal segment 4-47, the terminal segment 4-13 cm long; pinnae 6-25, 20-80 mm apart; central pinnae index 3-10, widest about or below the middle, $2-8$ by $0.4-1.2 \mathrm{~cm}$; the two lowermost pinnae 1-7 mm stalked; terminal segment usually narrowly triangular sometimes \pm conform to the pinnae, shorter than the remaining part of the lamina, apex acute. Sporangia inserted usually all over the lower surface, sometimes restricted to the veins, the arrangement acrostichoid. Spores with a smooth, cristate or cristate-undulate perispore.

Chromosomes. $\mathrm{n}=41 ; 2 \mathrm{n}=82$.

JAPAN. S. K y u shu: 17 collections. - R yu k yu I s.: 19 collections.

CHINA. Kiang s i: H.H. Hu 156 (GH, K, UC).-F u ki e n: Metcalf 7380 (BM, C); Norton 1058 (US), 1059 (US); Tang Siu Ging 13580 (UC).-K want ung: Taam 221 (A); Tsang 21228 (K, M, MICH, P, S-PA, US).

-K wa ng si: K. K. Whong S.Y.S.U. 714 (B, 'S.S. Sin \& Whang', S-PA).-H a in a n: 5 collections.

HoNG Kong. 10 collections.

TaIWAN. 5 collections.

VIETnAm. To n ki n: Cadière 583 (P). -A n n a m: Chevalier 4167 (Poilane leg.) (BM, P, SING).

$\mathrm{H} \mathrm{a} \mathrm{bit} \mathrm{a} \mathrm{t.} \mathrm{Creeping} \mathrm{either} \mathrm{on} \mathrm{stones} \mathrm{near} \mathrm{streams} \mathrm{in} \mathrm{forest} \mathrm{or} \mathrm{in} \mathrm{sandy} \mathrm{places} \mathrm{of} \mathrm{silty}$ swamps. Altitude: from 0-600 $\mathrm{m}$ in Japan, $1100-1500 \mathrm{~m}$ in Vietnam.

S pores. Fung 20146 (US), H. Ito 232 (SING), Yamazaki s.n. (TI): the greater part normal. McClure C.C.C.9436 (type of Campium subcordatum, MO): the majority (?) normal. Tagawa 7704 (US): sporangia for a considerable part filled with rather large nor- 


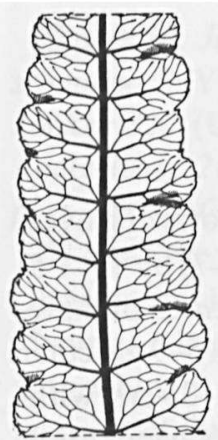

E. V' 75
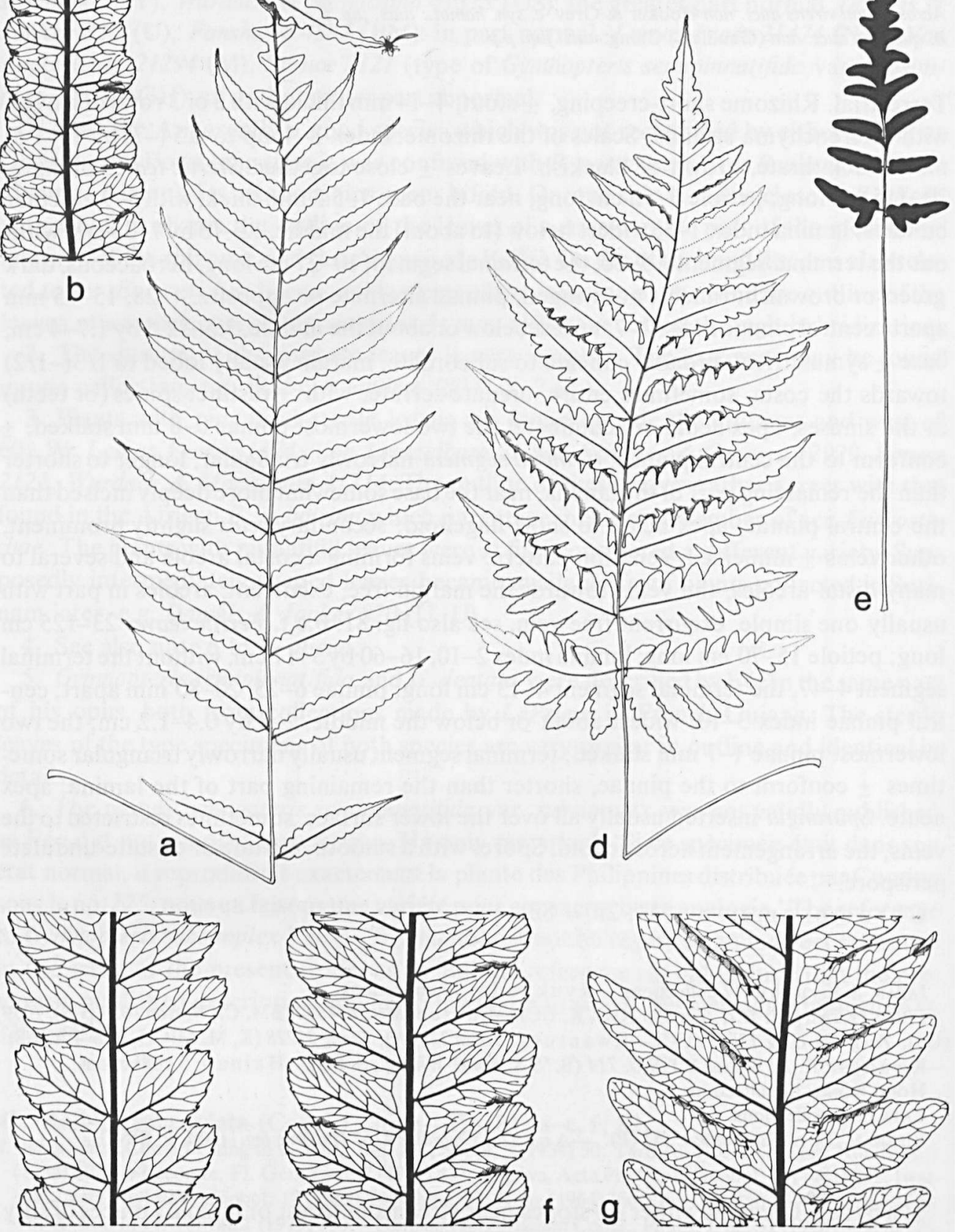

Fig. 81. a-c, f. Bolbitis subcordata. a. sterile leaf, $\times 1 / 5 ; b, c, f$. venation pattern of sterile pinnae, $\times 4 / 5$ (a. Tagawa 7704, L; b. Ohba 662254, L; c. Tagawa \& Iwatsuki 1496, L; f. Fosberg 37296, US).-d, e, g. Bolbitis umbrosa. $d$, e. sterile and fertile leaf resp., $\times 1 / 5 ; g$. venation pattern of sterile pinna, $\times 4 / 5$ (d, e. Purpus 4355, P; g. Liebmann s.n., L, paratype of $B$. umbrosa). 
mal as well as abnormal spores, some sporangia filled with aborted spores or aborted S.M.C.'s.

Notes. 1. A distinct species especially characterized by venation pattern and leaf architecture. The venation pattern looks intermediate between that of $B$. quoyana and one with mainly or exclusively free veins (that of $B$. angustipinna?). The pattern agrees with that sometimes found in $B$. scalpturata which is conspicuously different in several other features; there is also some resemblance to that of $B$. cadieri $(=s p e c$. dub. $)$. B. laxireticulata $(=$ spec. dub.) seems related on other grounds.

2. The largest plants are found in southern Japan. Specimens from more southern latitudes are often small with leaves composed of proportionally few (six or more) pinnae.

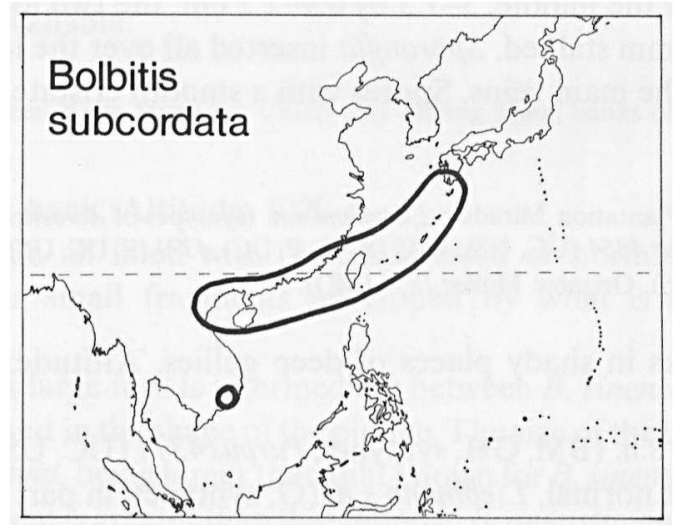

Fig. 82. Distribution of Bolbitis subcordata.

44. Bolbitis umbrosa (Liebmann) Ching-Fig. 81 : d, e, g.

B. umbrosa (Liebmann) Ching in C. Chr., Ind. Fil., Suppl. 3 (1934) 50.-Acrostichum umbrosum Liebmann, Kong. Dansk. Vid. Selsk. Skr. V, 1 (1849) 174. - Leptochilus umbrosus C. Chr., Ind. Fil. (1906) 388. - L e ctot y pe: Liebmann s.n. Mexico, Vera Cruz, near plantation Mirador, c. $60 \mathrm{~km}$ west of Paso de Ovejas, barranco de S. Francisco, 600-750 m alt., $x-1841$ (BM).

Terrestrial. Rhizome(only incomplete material available) short-creeping, $c .7 \mathrm{~mm}$ thick, with 2 rows of leaves, with few sclerenchyma strands. Scales of the rhizome: index 2-6, up to 5 by $2 \mathrm{~mm}$, subclathrate, brownish. Leaves \pm close together. Sterile leaves pinnate, $>50$ to $115 \mathrm{~cm}$ long; petiole $>19$ to $63 \mathrm{~cm}$ long, near to the base $2-5 \mathrm{~mm}$ thick, with 5-7 vascular bundles; lamina index 1-2(-3), widest somewhat below the middle, 32-72 by $20-35 \mathrm{~cm}$, without the terminal segment $0-16$, the terminal segment $29-56 \mathrm{~cm}$ long, thin-herbaceous, light green to olivaceous, without bulbils; rhachis not winged; pinnae alternate or opposite, 2-6, 0-65 mm apart; central pinnae index 2-4, widest about or below the middle, $12-21$ by $3.5-7 \mathrm{~cm}$, base usually asymmetrical, variously shaped, its basiscopic side narrower and usually decurrent along the rhachis, the acroscopic side free, margin lobed $2 / 5-4 / 5$ towards the costa, with a distinct tooth in the sinuses, apex 
acute to acuminate; lobes of the margin straight to falcate, \pm triangular or the basal part with parallel margins, margin crenate-serrate; the two lowermost pinnae asymmetrical, index 1-2(-4), widest somewhat below the middle or at the base, its basiscopic side broader, the margin lobed nearly to the costa, lobes up to 11 by $3 \mathrm{~cm}$, margin crenate-serrate or lobed to $2 / 5$ towards the costule; terminal segment proportionally long, deeply pinnatifid near the long-decurrent base, gradually narrowing towards the acute or acuminate apex; secondary and tertiary veins slightly prominent or immersed; venation pattern: veins forming a regular network of angulate areoles decreasing in size towards the margin, the areoles usually without, sometimes with a short, recurrent included free vein, see also fig. 81 : g. Fertile leaves $38-65 \mathrm{~cm}$ long; petiole $23-47 \mathrm{~cm}$ long; lamina index $1-3,14-32$ by $5-14 \mathrm{~cm}$, without the terminal segment $3-12$, the terminal segment 10-25 cm long; pinnae 4-8, 25-50 mm apart; central pinnae index (2-)3-5(-6), widest about or below the middle, $3-7.5$ by $0.9-2.2 \mathrm{~cm}$; the two lowermost pinnae 3.610.5 by $1-4 \mathrm{~cm}, 2-7 \mathrm{~mm}$ stalked. Sporangia inserted all over the lower surface leaving a sterile zone along the main veins. Spores with a smooth cristate or cristate-undulate perispore.

Mexico. Ver a Cruz. Plantation Mirador: Liebmann s.n. (syntypes of Acrostichum umbrosum); Ross 669 (BM, M). Zacuapam: Purpus 4354 (UC, US), 4355 (B, G, P, UC), 4371 (P, UC, US), 4471 (P), 11142 (F, UC), 12082 (MO, US), 16723 (US). Orizaba: Müller 1854 (MO).

H abitat. On rocks in shady places of deep gullies. Altitude: $450-1000 \mathrm{~m}$ (Liebmann, 1849).

S p o r e s. Liebmann s.n. (BM, GH, syntype), Purpus 4371 (UC, US), 11142 (US), 12082 (MO): the greater part normal. Liebmann s.n. (G, syntype): in part normal. Purpus 4355 (P): sporangia filled with (a cluster of) shrivelled spores.

N o tes. 1. A clear-cut species of which the relationships are not clear. It seems nearest to $B$. portoricensis. The venation pattern agrees with that of ser. Alienae. The outline of the leaves and the shape of the pinnae agree with those of $B$. bipinnatifida (endemic to the Seychelles). Unfortunately, the material at hand does not permit to study the features of the rhizome in sufficient detail.

2. Para(syn)types with a different combination of data (if at all recorded) and/or locality are in B, G, GH, K, L, NY, fragm., P, S-PA, ster., UC, US, fragm. 'Pl. Mexic. Liebmann 2689'.

\section{HYBRIDS}

H.1. Bolbitis angustipinna $\times$ sinensis-Fig. 83: a-c.

Campium sinense auct. non (Baker) C. Chr.: C. Chr., Contr. U.S. Nat. Herb. 26 (1931) 292, p.p.

Rhizome $9 \mathrm{~mm}$ thick, with 2 rows of leaves, with sclerenchyma strands. Leaves close together. Sterile leaves (one complete and one incomplete leaf available) pinnate, $165 \mathrm{~cm}$ long; petiole $58 \mathrm{~cm}$ long; laminas: (1) $>99$ by $35 \mathrm{~cm}$, without the terminal segment $61 . \mathrm{cm}$ long; (2) 107 by $40 \mathrm{~cm}$, without the terminal segment $55 \mathrm{~cm}$ long; 
herbaceous, olivaceous, with a spherical subterminal bulbil; rhachis without a narrow wing; pinnae subopposite, 22 (in both of the leaves), $70 \mathrm{~mm}$ apart; central pinnae index $5-8$, widest about or below the middle, $20-24$ by $3.5-4 \mathrm{~cm}$, base symmetrical, broadly attenuate, margin lobed to $1 / 3$ towards the costa, with a spine in each sinus, apex acute or acuminate; the two lowermost pinnae $2 \mathrm{~mm}$ stalked, \pm conform to the central ones; terminal segment narrowly triangular, pinnatifid near the base, otherwise flagelloid; venation pattern rather irregular: veins forming a costal areole and one or two smaller distal ones, excurrent free veins varying in length, see also fig. 83: b, c. Fertile leaf (but one incomplete leaf available) $>100 \mathrm{~cm}$ long; petiole $>51 \mathrm{~cm}$ long; lamina 49 by $15 \mathrm{~cm}$, without the terminal segment $39 \mathrm{~cm}$ long; pinnae 21, 75 $\mathrm{mm}$ apart; central pinnae index 7, the two largest ones 9.5 and 10 by $1.4 \mathrm{~cm}$; the two lowermost pinnae $6 \mathrm{~mm}$ stalked. Sporangia mainly inserted on the veins. Spores: normal spores not available.

China. Yunnan, between Keng Hung (= Cheli) and Muang Hing, banks of the Meh Nam Wäh: Rock 2636 (BM, C, ster., US).

$\mathrm{H}$ a bit a t. A river bank. Altitude: $1020 \mathrm{~m}$.

Spores. Sporangia all filled with (either aborted or normally shaped) S.M.C.'s, abnormal spores, or small fragments enveloped by what is supposed to be perisporial material.

$\mathrm{P}$ a re $\mathrm{nta} \mathrm{g}$ e. This large fern is intermediate between $B$. sinensis and $B$. angustipinna in venation pattern and in the shape of the pinnae. The size of the leaves agrees with that found in $B$. angustipinna, being larger than that known for $B$. sinensis. The relative length of the terminal segment is greater than that found in B. angustipinna and agrees with that sometimes found in $B$. sinensis. The number of pinnae to a leaf is more like that found in $B$. sinensis than in $B$. angustipinna.

Notes. 1. Ching, in his monograph of Egenolfia, did not cite this collection though he referred to Christensen (1931) who amply discussed the present fern in relation to the delimitation of the genus Bolbitis.

2. Rock's field-label reads: 'The long tip of this fern strikes root and produces new plants. Only one of the many seen had [a] separate fertile frond'.

H.2. Bolbitis appendiculata $\times$ sinensis-Fig. 83: d, e. Rhizome short-creeping, $8 \mathrm{~mm}$ thick, with 2 rows of leaves. Scales of the rhizome: index $3-6$, up to 4 by $1 \mathrm{~mm}$, dark brown, subclathrate. Leaves close together. Sterile leaf(only one complete leaf and two complete laminas present) pinnate with a triangular terminal segment, $104 \mathrm{~cm}$ long; petiole $47 \mathrm{~cm}$ long; laminas: (1) $57 \mathrm{by} 20 \mathrm{~cm}$, the terminal segment $5 \mathrm{~cm}$ long and with 38 pinnae, (2) 42 by $18 \mathrm{~cm}$, the terminal segment $10 \mathrm{~cm}$ long and with 26 pinnae, (3) 59 by $20 \mathrm{~cm}$, the terminal segment $6 \mathrm{~cm}$ long and with 38 pinnae, widest somewhat below the middle, herbaceous, light olivaceous, with a spherical subterminal bulbil; rhachis with a narrow wing in the upper part; pinnae alternate, up to $40 \mathrm{~mm}$ apart; central pinnae index 4-6, widest about the middle, the two largest ones 4-15 by 
$3 \mathrm{~cm}$, base \pm symmetrical, narrowly to broadly truncate, margin shallowly lobed, with a distinct spine in each sinus, apex acuminate; the two lowermost pinnae conform to the central ones, c. $2 \mathrm{~mm}$ stalked; terminal segment triangular; venation pattern: veins free, see also fig. 83: e. Fertile leaf: petiole $>40 \mathrm{~cm}$ long; lamina 34 by $9 \mathrm{~cm}$; pinnae 26 , the two largest ones 5 by 0.6 and 5.5 by $0.7 \mathrm{~cm}$. Sporangia inserted all over the lower surface. Spores: all abnormal.

Himalayas. Griffith s.n. (B, 2 sh., one also labelled as Kew Distr. no. 1155; BM, ster.).

Spores. All abnormal, mostly shrivelled, some with a normallv shaned reticulate cristate perispore, the meshes of the crests wider than in B. appendiculata.

$\mathrm{P}$ a r e $\mathrm{n}$ a ge. The size of the leaves and of the pinnae is that of $B$. sinensis. The base of the sterile pinnae agrees with that of $B$. sinensis and $B$. appendiculata ssp. vivipara, the margin of the pinna is slightly lobed (-1/5 towards the costa) rather like that of $B$. appendiculata ssp. appendiculata. The shape of the fertile pinnae resembles that of $B$. sinensis and $B$. appendiculata proper. The sporangia are inserted all over the lower surface as found in part of $B$. appendiculata proper. The shape of the perispore is intermediate between that found in the putative parents.

H. 3. Bolbitis appendiculata ssp. vivipara $x$ virens-Fig. 83:f-h.

Rhizome 90 by $9 \mathrm{~mm}$, with 2 rows of leaves. Scales of the rhizome up to 4.5 by $1 \mathrm{~mm}$, subclathrate, blackish. Leaves rather close together. Sterile leaves pinnate, 62 and $65 \mathrm{~cm}$ long; petioles 18 and $17 \mathrm{~cm}$ long resp.; laminas: (1) 44 by $23 \mathrm{~cm}$, the terminal segment $17 \mathrm{~cm}$ long, with 22 pinnae, (2) 48 by $27 \mathrm{~cm}$, the terminal segment $19 \mathrm{~cm}$ long, with 23 pinnae, firm-herbaceous, dark purplish brown, with a spherical subterminal bulbil; rhachis not winged; pinnae alternate, c. $20 \mathrm{~mm}$ apart; central pinnae index 4-6, widest about the middle, $12.5-14$ by $2.5-3.0 \mathrm{~cm}$, base subcordate to cuneate, margin irregularly and shallowly lobed, locally irregularly undulate, without spines or teeth, apex \pm acute; the two lowermost pinnae $1 \mathrm{~mm}$ stalked, \pm conform to the central ones; terminal segment triangular, deeply lobed near the base, apex acute or acuminate; venation pattern irregular: veins anastomosing into a costal areole (rarely absent) and several smaller distal ones varying in size and shape, see also fig. 83: h. Fertile leaf (but one available) $53 \mathrm{~cm}$ long; petiole $30 \mathrm{~cm}$ long; lamina 23 by $4.5 \mathrm{~cm}$, without the terminal segment $18 \mathrm{~cm}$ long; pinnae 16 , the two largest ones 3 and 3.2 by c. $0.15 \mathrm{~cm}$, margin obliquely dentate, between adjacent teeth with but little laminar tissue; terminal segment \pm conform to the

Fig. 83. a-c. Bolbitis angustipinna $\times$ sinensis. a. sterile pinna, $\times 2 / 5 ; b$, c. venation pattern of sterile pinnae, $\times$ 4/5 (a. Rock 2636, C; b, c. ibid., US). -d, e. Bolbitis appendiculata $\times$ sinensis. $d$. sterile pinna, $\times$ 1/5; e. venation pattern of sterile pinna, $x$ 4/5 (d, e. Kew Distr. no. 1155, Griffith leg., B). -f-h. Bolbitis appendiculata ssp. vivipara $\times$ virens. $f, g$. sterile and fertile leaf resp., $\times 1 / 5 ; \mathrm{h}$. venation pattern of sterile pinna, $\times$ 4/5 (f-h. Hayata s.n., 16-x-1921, TI).-i, j. Bolbitis $\times$ arguta, sterile and fertile leaf resp., $\times$ 1/5 (Cuming 161, P).-k. Bolbitis fluviatilis $\times$ heudelotii, venation pattern of sterile pinna (Callens 3183 , BM).- - - . Bolbitis heteroclita $\times$ rhizophylla, shape and venation pattern of sterile pinnae, $\times 4 / 5(1, \mathrm{~m}$. Copeland s.n., MICH, herb. Copel. 20358; n, o. Hennipman 4091, L, culta). 


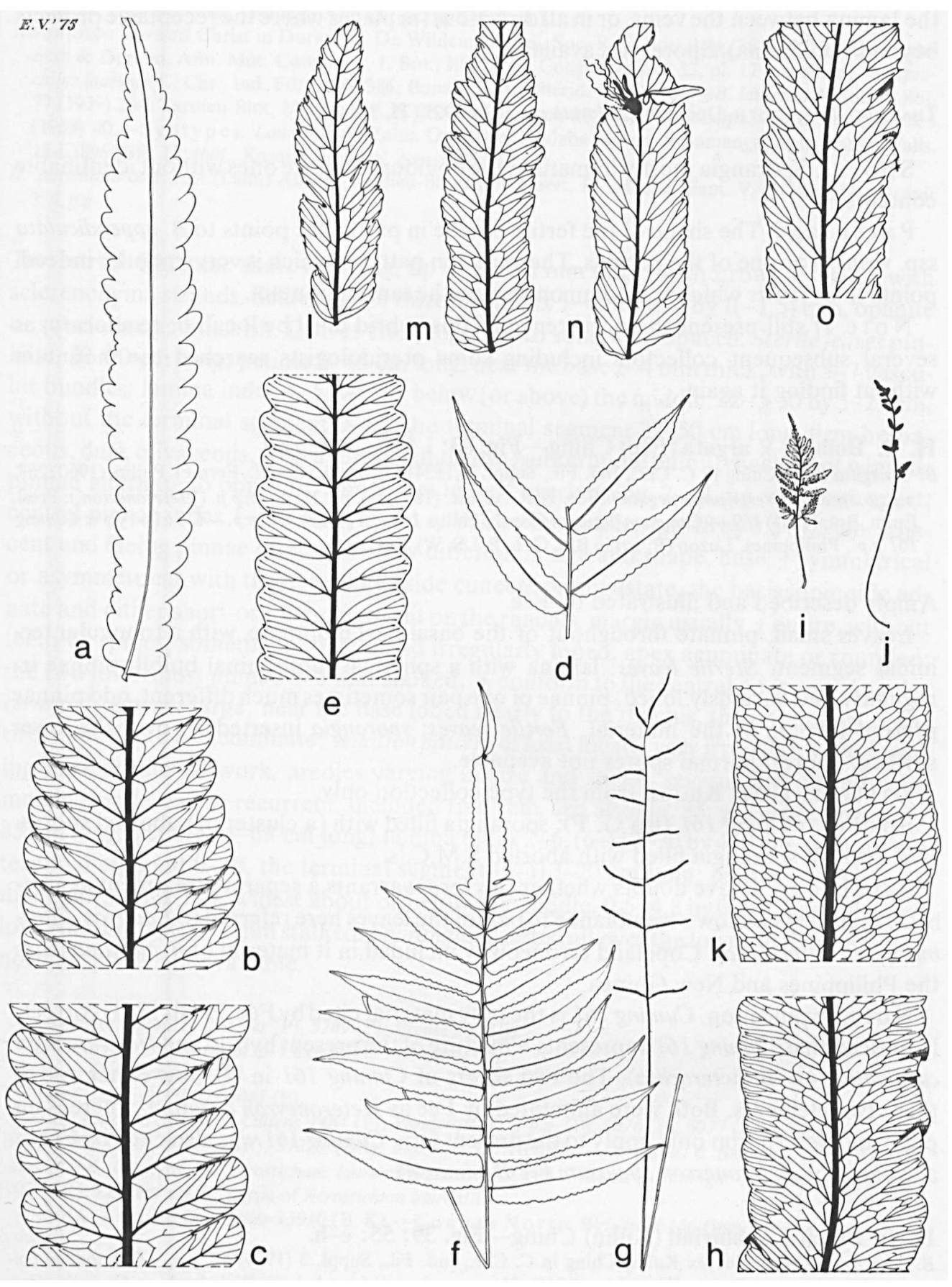


pinnae. Sporangia mainly near the margin, inserted either beneath on both the veins and the lamina between the veins, or in all directions (in places where the receptacle projects beyond the lamina). Spores not available.

ThaILAND. N or th ern. Doi Suthep: Hayata s.n., 16-x-1921 (TI, 2 sh.).

S p ores. Sporangia mostly immature, the obviously mature ones without identifiable contents.

Pa r entage. The shape of the fertile pinnae in particular points to $B$. appendiculata ssp. vivipara as one of the parents. The venation pattern, which is very irregular indeed, points to $B$. virens which is a common fern on the same mountain.

Note. If still present the occurrence of this hybrid must be local, or temporary, as several subsequent collectors including some pteridologists searched the mountain without finding it again.

H. 4. Bolbitis $x$ arguta (Fée) Ching-Fig. 83: i, j.

B. $\times$ arguta (Fée) Ching in C. Chr., Ind. Fil., Suppl. 3 (1934) 47, p. spec.; Copel., Fern Fl. Philip. (1960) 263, p.p., p.spec.-Heteroneuron argutum Fee, Hist. Acrost. (1845) 96, pl. 25; fig. 2, p.p. ('Heteronevron'); Presl, Epim. Bot. (185I) 169.—Campium argutum Copel., Philip. J.Sc. 37(1928) 376,p.p.—L ect o ty pe: Cuming 161 p.p., Philippines, Luzon (P; iso in BM, G, L, P, US, W). See note.

Amply described and illustrated by Fée.

Leaves small, pinnate throughout or the basal part bipinnate, with a triangular terminal segment. Sterile leaves: lamina with a spherical subterminal bulbil; pinnae irregularly and variously lobed, pinnae of one pair sometimes much different, odd pinnae present in part of the material. Fertile leaves: sporangia inserted all over the lower surface. Spores: normal spores not available.

Distribution. Known from the type collection only.

S p or es. Cuming 161 (iso G, P): sporangia filled with (a cluster of) aborted spores. Ibid. (iso L): sporangia filled with aborted S.M.C.'s.

Parentage. I have doubts whether this fern warrants a separate treatment as a hybrid, as its leaves show resemblance to part of the leaves here referred tc Edanyoa difformis $(=B$. heteroclita $)$. Copeland erroneously included in it material of $B$. quoyana from the Philippines and New Guinea.

Note. Typification. Cuming 161 is the only material cited by Fée. Presl(1851) correctly noticed that Cuming 161 represents a mixture of the present hybrid and Heteroneuron cuspidatum $(=B$. heteroclita). The two sheets of Cuming 161 in W show either one of these two elements. Both were annotated by Fée as Heteronevron argutum, whereas his plate and description only apply to the present fern. Cuming $16 \mathrm{l}$ was also cited by Fée as a syntype of Heteronevron sinuosum $(=B$. sinuosa).

H. 5. Bolbitis $\times$ boivinii (Kuhn) Ching-Fig. 39; 55: e-h.

B. $\times$ boivinii (Mettenius ex Kuhn) Ching in C. Chr., Ind. Fil., Suppl. 3 (1934) 47, p.p.-Chrysodium boivinii Mettenius ex Kuhn, Fil. Afric. (1868) 51, p.p.-Acrostichum boivinii Welwitsch ex Carruth., Cat. Afr. PI. Welwitsch 2, 2 (1901) 276, p.p., non Mettenius ex Kuhn (1868) (= Elaphoglossum spec.).-Leptochilus boivinii C. Chr., Ind. Fil. (1906) 384, p.p._Gymnopteris boivinii Engler, Veg. Erde 9, 2 (1908) 
16, p.p.-Campium boivinii Copel., Philip. J. Sc. 37 (1928) 378, p.p.-Lect otype: Welwitsch 156, Angola, Cuanza Norte, Golungo Alto (K; iso in BM, 2 sh.).

Acrostichum laurentii Christ in Durand \& De Wildeman, Bull. Soc. Roy. Bot. Belg. 36 (1897) 94; De Wildeman \& Durand, Ann. Mus. Congo Ser. I. Bot., Illustr. Fl. Congo 1 (1898) 33, pl. 17 (excellent).-Leptochilus laurentii C. Chr., Ind. Fil. (1906) 386; Bonap., Notes Ptérid. 1 (1915) 51.-B. laurentii Alston, J. Bot. 77 (1939) 284; Tardieu-Blot, Mém. I.F.A.N. 28 (1953) 110, pl. 18: fig. 2, 3; Schelpe, Contr. Bolus Herb. 1 (1969) 40.-S y nt y pes: Laurent s.n., Zaire, Orientale, Lualaba-Congo at Bamanga, near Stanley Falls, 18-i-1896 (BR, L); ibid., Kasai, Lusambo, 2-xii-1895 (BR, P).

B. auriculata auct. non (Lam.) Alston: Tardieu-Blot in Humbert, Fl. Madag. Fam. V, 2 (1960) 60, pl. xvi: $3,4, p . p$.

Terrestrial. Rhizome short-creeping, up to $7(-10) \mathrm{mm}$ thick, with 2 rows of leaves, with sclerenchyma strands. Scales of the rhizome: index 1-4, up to 5 by $1(-1.5) \mathrm{mm}$, opaque or subclathrate, blackish. Leaves close together to somewhat spaced. Sterile leaves pinnate, 30-90 cm long; petiole 8-45 cm long, near the base 2-4 mm thick, with 5-7 vascular bundles; lamina index $1-5$, widest below (or above) the middle, $22->50$ by $5-25 \mathrm{~cm}$, without the terminal segment $0-17$, the terminal segment $22-50 \mathrm{~cm}$ long, firm-herbaceous, dark olivaceous, with a (primordium of a) subterminal bulbil; rhachis not winged; pinnae alternate to opposite, sometimes in part overlapping, 2-14, up to $35 \mathrm{~mm}$ apart; central pinnae index $1-6$, widest about or below the middle, $0.5-18$ by $0.5-4.5 \mathrm{~cm}$, adjacent and facing pinnae often markedly different in size and shape, base \pm symmetrical or asymmetrical with the acroscopic side cuneate or angustate, the basiscopic side adnate and either short-or long-decurrent on the rhachis, margin usually \pm entire, without teeth or spines, sometimes slightly and irregularly lobed, apex acuminate or rounded; the two lowermost pinnae $0-3 \mathrm{~mm}$ stalked, \pm conform to the central ones; \pm triangular terminal segment large, near the base lobed nearly to the rhachis, the upper part \pm entire, apex acute or acuminate; venation pattern at least locally very irregular, veins forming an intricate network, areoles varying in size and shape, several with usually one mostly simple, ex- or recurrent, included free vein, see also fig. 55: g. Fertile leaves 25$85 \mathrm{~cm}$ long; petiole $14-63 \mathrm{~cm}$ long; lamina index $2-6,(6-) 17-30$ by $4-8 \mathrm{~cm}$, without the terminal segment $0-10$, the terminal segment (6-)13-20 cm long; pinnae 2-8, up to 35 $\mathrm{mm}$ apart, index $1-4$, widest about or below the middle, $0.5-4.8$ by $0.5-1.8 \mathrm{~cm}$; the two lowermost pinnae 0-2 $\mathrm{mm}$ stalked. Sporangia inserted all over the lower surface. Spores: normal spores not available.

Cameroun. Letouzey 2935bis (P), 3289 (P); Preuss 205 (B).

Central African Republic. Lake Babli: Anonymus (P).

TCHAD. Chevalier 6252 (P).

GABON. Hallé \& Villiers 5043 (P).

Zalre. Le o poldvill e: Callens 1484 (P); Vanderijst 6075 p.p. (P), 6076 (P), 6077 (BR), 8083 (P), 30587 (BR), 30902 (BR), 30923 (BR). 31229 (BR): Flamigni 187A (BR).-Ori en tale: Bequaert 1514 (BR. L): Laurent s.n. (syntype of Acrostichum laurentii); Seret 48 (BR).-Kas a i: Flamigni 50 (BR); Gentil s.n., 1902 (BR); Laurent s.n. (syntype of Acrostichum laurentii).

Angola. L unda: Gossweiler 13910 (B, K).-Cu anza Nort e: Welwitsch 156 (lectotype of Chrysodium boivinii).

$\mathrm{H}$ a bit a t. Forest; several times reported from forest along streams. Altitude: $700 \mathrm{~m}$ (Gossweiler 13910). 
Spores. Welwitsch 156 (lectotype of Chrysodium boivinii; all the specimens). Laurent s.n., 2-xii-1897 (syntype of Acrostichum laurentii, BR), Callens 2818 (BM), Germani 8605 (BM): sporangia filled with aborted S.M.C.'s and/or aborted spores. In all the other collections the spores were studied with the dissecting microscope; the sporangia contained aborted S.M.C.'s and/or aborted spores.

Parentage. In outline the leaf is intermediate between a simple and a pinnate one. The only two African species with simple leaves are B. fluviatilis and B. gaboonensis. Because of the properties of the venation pattern and the texture of the hybrid, B. gaboonensis seems a better choice than B. fuviatilis. Besides, the data on the label indicate that $B . \times$ boivinii is frequently found growing together with $B$. gaboonensis. There is little doubt that $B$. gaboonensis is one of the parents. The other parent is $B$. auriculata, $B$. acrostichoides, or $B$. gemmifera. Specimens of these three species have been collected from the same place as from where specimens belonging to the present hybrid were taken. The geographical distribution of the hybrid largely excludes the Congo Basin. As B. gaboonensis is common throughout the Basin, it seems possible that the geographical distribution of the second parent also largely excludes the Congo Basin. If so, $B$. acrostichoides seems a better choice for the other parent than both $B$. auriculata and $B$. gemmifera.

$\mathrm{N}$ ot e s. 1. The structural irregularities as seen in the shape of the pinnae and in the venation pattern, together with the evidence from the spores, are decisive for the hybrid status assigned by me to this form. The outline of the leaves and the venation pattern of the present hybrid are strikingly similar to these features in part of $B$. semipinnatifida.

2. Copeland (1928), without studying any specimen of this, placed it in 'the group of B. quoyana' (Asia and the Pacific).

3. In a few collections tufts of small scales were found in the axils of the lowermost pinnae and the rhachis. These tufts might indicate primordia of axillar bulbils (known from ser. Alienae and ser. Euryostichae).

\section{H.6. Bolbitis fluviatilis $x$ heudelotii-Fig. 83: $\mathbf{k}$.}

Rhizome not seen. Sterile leaf pinnate, $50 \mathrm{~cm}$ long; petiole $13 \mathrm{~cm}$ long; lamina $37 \mathrm{by}$ $22 \mathrm{~cm}$, widest somewhat below the middle, without the terminal segment $4 \mathrm{~cm}$ long, herbaceous, dark olivaceous, without a bulbil; pinnae alternate, $4,15 \mathrm{~mm}$ apart, the largest one 16 by $3.3 \mathrm{~cm}$, widest somewhat below the middle, base cuneate, margin entire, without teeth or spines, apex acuminate; the two lowermost pinnae almost sessile, conform to the two other ones; terminal segment triangular, large, deeply lobed nearly to the rhachis throughout, lateral lobes 12 , resembling the pinnae, terminal lobe with a smaller lobe at the base; venation pattern: veins anastomosing into an intricate network of areoles varying in size and shape, several with one simple, ex- or recurrent included free vein. Fertile leaf $57 \mathrm{~cm}$ long; petiole $25 \mathrm{~cm}$ long; lamina 32 by $13 \mathrm{~cm}$, without the terminal segment $10 \mathrm{~cm}$ long; pinnae 6 , the largest one 11 by $1.5 \mathrm{~cm}$; terminal segment with several small lobes near the base. Sporangia inserted all over the lower surface. Spores: no normal spores available. 
ZAIRE. L e o p oldvill e. Kwango, Kambangu: Callens 3183 (BM).

$S$ p o r e s. The far greater part of the sporangia immature, a minority filled with aborted S.M.C.'s and/or aborted spores.

$\mathrm{P}$ a r e $\mathrm{n} \mathrm{t}$ a $\mathrm{g}$ e. The shape of the leaves, that of the pinnae. and the venation pattern are regarded to be intermediate between those of $B$. heudelotii and $B$. fluviatilis. The present fern grew together with B.acrostichoidek/(Callens 3185), B. fluviatilis (Callens 3188). and B. heudelotii (Callens 3184).

$\mathrm{N}$ ot $\mathrm{e}$. The venation pattern is reminiscent of that of $B$. humblotii.

H.7. Bolbitis heteroclita $\times$ rhizophylla-Fig. 83: 1-0.

Rhizome short-creeping, $3.5 \mathrm{~mm}$ thick, with 2 rows of leaves. Scales of the rhizome: up to $3(-4)$ by $1(-1.5) \mathrm{mm}$, opaque to subclathrate, brownish, sometimes with a paler marginal strip. Leaves rather close together to spaced (to $15 \mathrm{~mm}$ ). Sterile leaves ( $5 \mathrm{com}$ plete leaves available) pinnate, $38-43 \mathrm{~cm}$ long; petiole $13-16 \mathrm{~cm}$ long; lamina index 2-4, widest about the middle, $24-28$ by 7-10 cm, without the terminal segment $16-20$, the terminal segment 6-12 cm long, with a spherical subterminal bulbil; rhachis with or without narrow wing; pinnae \pm symmetrical, alternate to opposite, $16-24,15-30 \mathrm{~mm}$ apart; central pinnae index 3-5, widest about or somewhat below the middle, 4.5-6 by $1.2-1.6 \mathrm{~cm}$, base truncate to cuneate, margin usually \pm entire, sometimes irregularly and finely serrate or with a few incisions about halfway towards the costa, with distinct spines, apex acute or acuminate; the two lowermost pinnae 0-1 mm stalked, conform to the central ones; terminal segment triangular, deeply lobed near the base, apex acute or acuminate; venation pattern irregular: veins forming a costal areole (rarely lacking), sometimes also one or a few smaller distal areoles, see also fig. 83: 1-o. Fertile leaves 41,42 , and $54 \mathrm{~cm}$ long; petioles 25,28 , and $29 \mathrm{~cm}$ long resp.; laminas: (1) 16 by 4.5 , the terminal segment $3 \mathrm{~cm}$ long and with 16 pinnae, (2) 14 by $4 \mathrm{~cm}$, the terminal segment $4 \mathrm{~cm}$ long and with 18 pinnae, (3) 25 by $7.5 \mathrm{~cm}$, the terminal segment $4 \mathrm{~cm}$ long and with 19 pinnae; central pinnae index $2-5,1.6-4$ by $0.6-0.8 \mathrm{~cm}$; the two lowermost pinnae c. $2 \mathrm{~mm}$ stalked. Sporangia inserted mainly on the veins. Spores: normal spores not available.

Chromosomes. 2 n $=82$; at meiosis univalents only.

Philuppines. L u z o n. Mt. Maquiling. Copeland s.n. (MICH, herb. Copeland, 20358); Price 163 (CAHUP, L).

H a bit a t. A shady place in forest. Altitude: $350 \mathrm{~m}$ (Price 163), $400 \mathrm{~m}$ (Copeland s.n.).

$\mathrm{S}$ p o r e s. Copeland s.n., Price 163 (L): sporangia filled with aborted S.M.C.'s, sometimes also in part with aborted spores.

$\mathrm{P}$ a r e $\mathrm{n} \mathrm{ta}$ e. The venation pattern indicates that one of the parents is free-veined. Because of the shape of the sterile pinnae which is \pm symmetrical, and the insertion of the sporangia which is mainly on the veins, $B$. rhizophylla almost certainly takes part. 
The shape of the rhizome, the relative position of the leaves on the rhizome, the margin of the sterile pinnae, the number of pinnae to a leaf, and the venation pattern point to $B$. heteroclita rather than to $B$. repanda as the other parent. The shape of the pinnae from the largest leaves cultivated comes very near to that of those of $B . \times$ singaporensis. $B$. heteroclita and $B$. rhizophylla were found growing together with the present hybrid (Mr. Price, in litt.).

$\mathrm{N}$ o t e. Cultivation. The living material of this hybrid, sent by Mr. Price to me in June 1969 , formed small-sized sterile leaves which became loaded with small plants grown from the bulbils on the terminal and lateral segments. After a sunny period in early summer 1971 the newly formed sterile leaves attained a much larger size (to $55 \mathrm{~cm}$ long); several large-sized fertile leaves were formed later in the same year.

\section{H. 8. Bolbitis $\times$ lancea (Copel.) Ching-Fig. 84: a-g.}

B. $\times$ lancea (Copel.) Ching in C. Chr., Ind. Fil., Suppl. 3 (1934) 48, p. spec.-Campium lanceum Copel., Philip. J. Sc. 37 (1928) 380, fig. 32, pl. 25.-T y p e: Gamble 15921, India, Madras, Rumpa Hills, 900 $\mathrm{m}$ alt. (K).

B. kanarensis Nayar \& Chandra, Amer. Fern J. 54 (1964) 9, fig. 1-9; Nayar \& Kaur, Bull. Nat. Bot. Gard. Lucknow 88 (1964) 48, fig. 88.-T y pe: Chandra LWG 95146, India, Mysore, N. Kanara, Gudkewadi, Castle Rock, $400 \mathrm{~m}$ alt. (LWG, n.v.).

Heteroneuron scalpturatum auct. non Fée: Fée, Hist. Acrost. (1845) 95, pl. 56, p.p.

Amply described by Copeland (1928), Nayar \& Chandra (1964), and Nayar \& Kaur (1964).

Sterile leaves. Shape of the pinnae rather as in B. angustipinna; terminal segment \pm triangular, either much prolonged or flagelloid; venation pattern irregular, see fig. 84: b-g. Fertile leaves with narrow pinnae. Spores: the normally shaped spores with a smooth variously shaped perispore.

INDIA. Southern part: d'Almeida 299 (BLAT); Anonymus (?Nuddie) 912 (G); Beddome s.n. (K): Chandra LWG 77718 (LWG); Fairbank 1501 (MO, UC); Gamble 15921 (type of Campium lanceum); Hooker \& Thomson s.n. ('105', BM); Hügel $2161,2165,4140,4974$ (all at W, all paratypes of Heteroneuron scalpturatum Fée); Law s.n. (BM, P); Saldanha 6012 (C).

H a bi t a t. In forest. Altitude: $900 \mathrm{~m}$ (Gamble 15921, Mooney 677), $1140 \mathrm{~m}$ (Beddome s.n.).

Spores. d'Almeida 299, Chandra LWG 77718: the majority abnormal. Gamble 15921: nearly all abnormal, the normally shaped spores with a densely folded and minutely spiny perispore. Mooney 677: all abnormal, the perispore of the normally shaped spores verrucate-undulate.

Parentage of $B . \times$ lancea and $B . \times$ prolifera. Plants with part of their features very irregularly expressed, but otherwise obviously related to $B$. angustipinna, $B$. subcrenata, and $B$. semicordata (all three belonging to ser. Bolbitianae), are common in Peninsular India. The venation pattern of the hybrids in particular shows much variation; also when comparing that of pinnae of different parts of the same leaf, or 
when comparing that of adjacent areas of one pinna. All plants are provided with distinct bulbils. Vegetative propagation seems dominant, also as the spores of the collections studied are largely abnormal.

It appeared possible to divide this heterogeneity into two rather distinct groups, one composed of plants which are as to size and venation pattern intermediate between $B$. angustipinna and $B$. subcrenata $(B . \times$ lancea $)$ and one intermediate between $B$. angustipinna and $B$. semicordata $(B . \times$ prolifera). Plants intermediate between $B$. subcrenat $a$ and $B$. semicordata were not recognized, although hybridization between these two species is likely to occur. In the venation pattern of $B . \times$ lancea areoles along the distal parts of the secondary veins are few or absent; the ultimate veins usually run about parallel and are usually not connected by tertiary veins. In the venation pattern of $B . \times$ prolifera areoles are usually present also along the distal parts of the secondary veins, whereas the ultimate veins generally do not run parallel being often connected by tertiary veins. $B . \times$ lancea and $B . \times$ prolifera deserve detailed experimental study.

N o t e s. 1. Copeland (1928) regarded Campium lanceum 'really near' to C. undulatum $(=B$. virens $\mathrm{f}$. undulata $)$, keeping the latter separate because of differences in the shape of the fertile pinnae only.

2. Mooney $677(\mathrm{~K})$ is morphologically near to $B . \times$ lancea. It originates from Orissa from a place where $B$. virens var. deltigera and $B$. sinensis also occur. The Orissa ferns need further investigation.

H. 9. Bolbitis nicotianifolia $\times$ portoricensis-Fig. 84: $\mathbf{h}$, i.

Rhizome absent. Sterile leaf pinnate, $>64 \mathrm{~cm}$ long; petiole $>20 \mathrm{~cm}$ long; lamina 44 by $28 \mathrm{~cm}$, widest about the middle, without the terminal segment $16 \mathrm{~cm}$ long, herbaceous, greenish, with a large, axillar, spherical bulbil at $11 \mathrm{~cm}$ from the base of the lamina; rhachis not winged; pinnae \pm opposite, 8 , up to $60 \mathrm{~mm}$ apart; central pinnae: shape irregular, widest about the middle, the largest one 16 by $3.2 \mathrm{~cm}$, base asymmetrical, its acroscopic side \pm cuneate, its basiscopic side adnate to the rhachis, (abruptly) decurrent, margin \pm entire or slightly obliquely lobed in the basal half, without teeth or spines, apex acuminate; the two lowermost pinnae with a large basal basiscopic lobe; terminal segment triangular, near the base pinnatipartite, tapering towards the proportionally large entire terminal lobe; venation pattern intricate; veins forming a dense network, areoles varying in size and shape, some with mostly one simple or forked, ex- or recurrent included free vein, see also fig. 84: i. Fertile leaf $120 \mathrm{~cm}$ long; petiole $66 \mathrm{~cm}$ long; lamina $20 \mathrm{~cm}$ wide, without the terminal segment $33 \mathrm{~cm}$ long; pinnae 14. Sporangia situated all over the lower surface. Spores aborted. Otherwise like the fertile leaves of $B$. portoricensis.

GUATEMALA. I z a b a l: Jungle at ruins of Quirigua, Weatherwax 248 (MO).

Parentage. The shape of the sterile pinnae is irregular, but \pm intermediate be- 

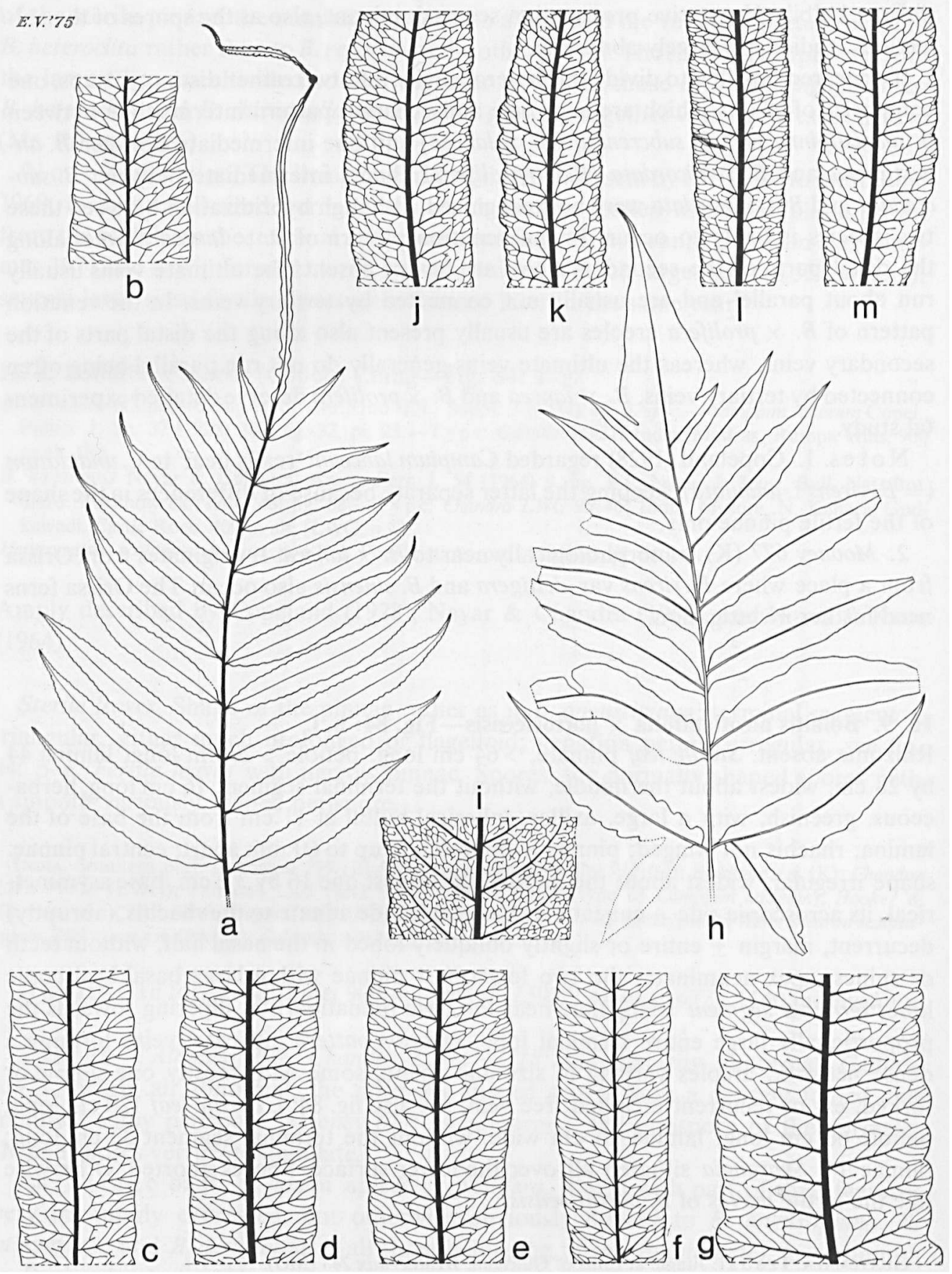
tween that of $B$. portoricensis and $B$. nicotianifolia. The latter two species are also known from Quirigua. The presence of an axillar bulbil in the lower half of the lamina is of interest. The bulbils in $B$. portoricensis are subterminal, whereas they are not known from B. nicotianifolia. Bulbils are axillar in some other species of ser. Euryostichae. The sporangia are all filled with aborted spores. The fact that aborted S.M.C.'s were never found may be significant for the relationship between ser. Portoricensis and ser. Euryostichae.

H. 10. Bolbitis $x$ prolifera (Bory) C. Chr. \& Tardieu-Blot-Fig. 84: j-m.

B. $X$ prolifera (Bory) C. Chr. \& Tardieu-Blot in Tardieu-Blot \& C. Chr., Not. Syst. 7 (1938) 102, p. spec., quoad nomen solum.-Polybotrya prolifera Bory in Bélanger, Voy. Ind. Or. Bot. 2 (1833) 18, p. spec., non (Hooker) Mettenius (1856) (= B. subcrenata). - Heteroneuron proliferum Fee, Hist. Acrost. (1845) 92, pl. 55: fig. 1 ('Heteronevron').-Poecilopteris prolifera Presl, Epim. Bot. (1851) 172.-Leptochilus prolifer C. Chr., Ind. Fil. (1906) 387; v.A.v.R., Handb. Malayan Ferns (1908) 738, p.p.; Brause, Bot. Jahrb. 56 (1920) 119, p.p.-T y pe: Bélanger s.n., India, Madras, Madura ('Madurai'), Dendigàl ('Dindigul') (P).

B. virens auct. non (Hooker \& Grev.) Ching c. syn. homot.: auct. p.p.

Poecilopteris repanda auct. non (Bl.) Presl: Bedd., Ferns S. Ind. (1864) 68, pl. 202.

Gymnopteris contaminans Bedd., Ferns Br. Ind., Suppl. (1876) 27, p.p., nom. superfl.; Handb. Ferns Br. Ind. (1883) 435, fig. 264, ibid.; Handb. Ferns Br. Ind., Suppl. (1892) 105, ibid. - L e ct ot y pe: Beddome, Ferns S. India (1864) pl. 202 ('Poecilopteris repanda auct. non (BI.) Presl').

Acrostichum terminans Wall., Cat. (1830) no. 2168, nom. nud.-T y p e: Wallich 2168 (Wight leg.), South India, Courtallum (K, herb. Wallich; iso in $\mathrm{K}$, herb. Hooker).

Sterile leaves. The shape of the leaves and the pinnae is usually rather as in B. semicordata; terminal segment usually conform to the central pinnae, sometimes triangular and mostly prolonged; rhachis generally with a narrow wing; venation pattern irregular, see also fig. 84: j-m. Fertile leaves usually with narrow pinnae. Spores: the normally shaped spores with a smooth, usually undulate, sometimes verrucate perispore.

INDIA. Sou th: Abraham 1105 (US), 1141 (US), 1143 (US); Bates s.n. (K); Beddome s.n. (K); Bélanger (type of Polybotrya prolifera); Bole s.n. (BLAT); Cockburn 101 (BM); Van Hardeveld \& Van der Werff 323 (L); Hooker \& Thomson s.n. (BM, '105', '107'; K, P, W); Hooper \& Ramaswami 39418 (CAL); Law s.n. (K); Nayar \& party LWG 44621 (LWG), 45058 (LWG); Noyes s.n. (US, 299693); Wallich 2168/l (K, herb. Hooker); Wight 63 (= Pen. Ind. Or. 3178; B, K, L, W), (Pen. Ind. Or.?) 3175 (B), s.n. (W).

H a bit a t. Forest. Altitude: $500-1200 \mathrm{~m}$.

S p o r es. Bélanger s.n. (type of Polybotrya prolifera), Van Hardeveld \& Van der Werff 323 (L), Wallich 2168/1 (K, herb. Hooker): all abnormal.

Parentage. See H. 8. Bolbitis $\times$ lancea.

Fig. 84. a-g. Bolbitis $\times$ lancea. a. sterile leaf, $\times 1 / 5 ; b-g$. venation pattern of sterile pinnae, $\times 4 / 5$ (a, c. Hügel 4140, W, syntype of Heteroneuron scalpturatum; b. Gamble 15921, K, holotype of $B$. $\times$ lancea; $d$. D'Almeida 299, BLAT, loc. class. of B. kanarensis; e. Fairbanks s.n., UC 811487; f. Chandra LWG 77718, LWG; g. Nuddie $912, \mathrm{G}) .-\mathrm{h}$, i. Bolbitis nicotianifolia $\times$ portoricensis. h. sterile leaf, $\times 1 / 5$; $i$. venation pattern of sterile pinna, $\times 4 / 5(\mathrm{~h}$, i. Weatherwax $248, \mathrm{MO}) .-\mathrm{j}-\mathrm{m}$. Bolbitis $\times$ prolifera, venation pattern of sterile pinnae, $\times 4 / 5$ (j. Wight Pen. Ind. Or. 3178, B; k. Anonymus s.n. in herb. Hooker \& Thomson, Mysore, B; 1. Hooper \& Ramaswasmi 39418, CAL; m. Anonymus s.n. in herb. Hooker \& Thomson, Mysore, W). 
Note. Beddome (1876) described Gymnopteris contaminans to accommodate the material he earlier (1864, 68, pl. 202) referred to Poecilopteris repanda auct. non (Bl.) Presl, and (Ferns Br. India, 1868, 270, pl. 270) to Poecilopteris semicordata (Baker) Bedd. His species also included 'Acrostichum virens and crispatulum'. I have selected pl. 202 (1864) as the lectotype, as Beddome (1868, l.c.) - when enumerating Poecilopteris semicordata - expressed the opinion (though with misgivings) that the fern he (1864) named Poecilopteris repanda should be renamed 'Poecilopteris contaminans (Wall.)'. The venation pattern as figured by Beddome is very irregular; his species easily fits in the hybrid as here construed.

\section{H. 11. Bolbitis $x$ singaporensis Holttum-Fig. 85: a-d.}

B. $\times$ singaporensis Holttum, Gard. Bull. S. S. 11 (1947) 271, p. spec.; Ferns Malaya (1954) 467, fig. 274, p. spec.-T y p e: Hullett s.n., Singapore, Bt. Timah, iii-1882 (SING, 20947; iso in BM, K, SING).

B. quoyana auct. non (Gaudich.) C. Chr.: Holttum, Gard. Bull. S. S. 9 (1937) 122.

Rhizome short-creeping, 3-6 mm thick, with 2 rows of leaves. Leaves approximate to somewhat spaced. Sterile leaves pinnate, $35-70 \mathrm{~cm}$ long; petiole $16-30 \mathrm{~cm}$ long; lamina index $1-3$, widest somewhat below the middle, $22-45$ by $10-25 \mathrm{~cm}$, without the terminal segment $11-29$, the terminal segment $10-19 \mathrm{~cm}$ long, firm-herbaceous, bright green to olivaceous, with a spherical subterminal bulbil; rhachis not winged; pinnae opposite or alternate, 14-27, 15-35 mm apart; central pinnae index 3-5, widest below (to about) the middle, $5.5-12.5$ by $1.5-3 \mathrm{~cm}$, base varying from \pm symmetrical, subcordate, cuneate or angustate, to (strongly) oblique with its acroscopic side much better developed and provided with a distinct basal acroscopic lobe (or auricle), margin entire to lobed to $1 / 7(-1 / 4)$ towards the costa, with a more or less distinct spine in (some of) the sinuses, apex acute or orbicular; the two lowermost pinnae 0-1 mm stalked, conform to the central pinnae; terminal segment triangular, deeply lobed near the base, gradually narrowing towards the acute or acuminate apex; venation pattern very irregular: veins forming a costal areole (rarely lacking), with or without few to several smaller distal areoles varying in size and shape, the areoles with or without usually one excurrent included free vein, see also fig. 85: a-d. Fertile leaves $50-70 \mathrm{~cm}$ long; petiole $37->46 \mathrm{~cm}$ long; lamina index $2-5,14-34$ by $3-12 \mathrm{~cm}$, without the terminal segment $10-26$, the terminal segment 4-8 cm long; pinnae 16-22, 25-50 mm apart; central pinnae index $4-8,2-6.5$ by $0.4-1.2 \mathrm{~cm}$; the two lowermost pinnae $0-1$

Fig. 85. a-d. Bolbitis $\times$ singaporensis, venation pattern of sterile pinnae, $\times 4 / 5$ (a. Anonymus s.n., SING 20950; b. Holttum SF 19799, C; c, d. Holttum s.n., SING).-e-p. Bolbitis $\times$ sinuosa.-e, f. nm. sinuosa. e. sterile leaf, $\times 1 / 5$; f. venation pattern of sterile pinna, $\times 4 / 5 .-g-p$. nm. foxii. g. sterile leaf, $\times 1 / 5$; $h, j, 1, n, p$. venation pattern of sterile pinnae, $\times 4 / 5 ; i, k, m, 0$. shape of sterile pinnae, $\times 1 / 5(e$. Cuming 105, W, holotype of $B$. $\times$ sinuosa; f. ibid., BM, isotype; g, h. Fox PNH 8904, PNH, isotype of B. $\times$ foxii; i-p. Price $763, \mathrm{~L}) .-\mathrm{q}-\mathrm{w}$. Leptochilus $\times$ trifidus. $\mathrm{q}-\mathrm{u}$. sterile and fertile leaves, $\times 1 / 5$; v, w. venation pattern of sterile laminas, $\times 4 / 5$, the asterisks indicate isolated veins within areoles (q. $r$. Brooks 403.S., BM; s, t. Van Alderwerelt van Rosenburgh s.n., BO, holotype of Leptochilus $\times$ trifidus; u. ibid., P; v. ibid., L; w. Matthew s.n., K). 


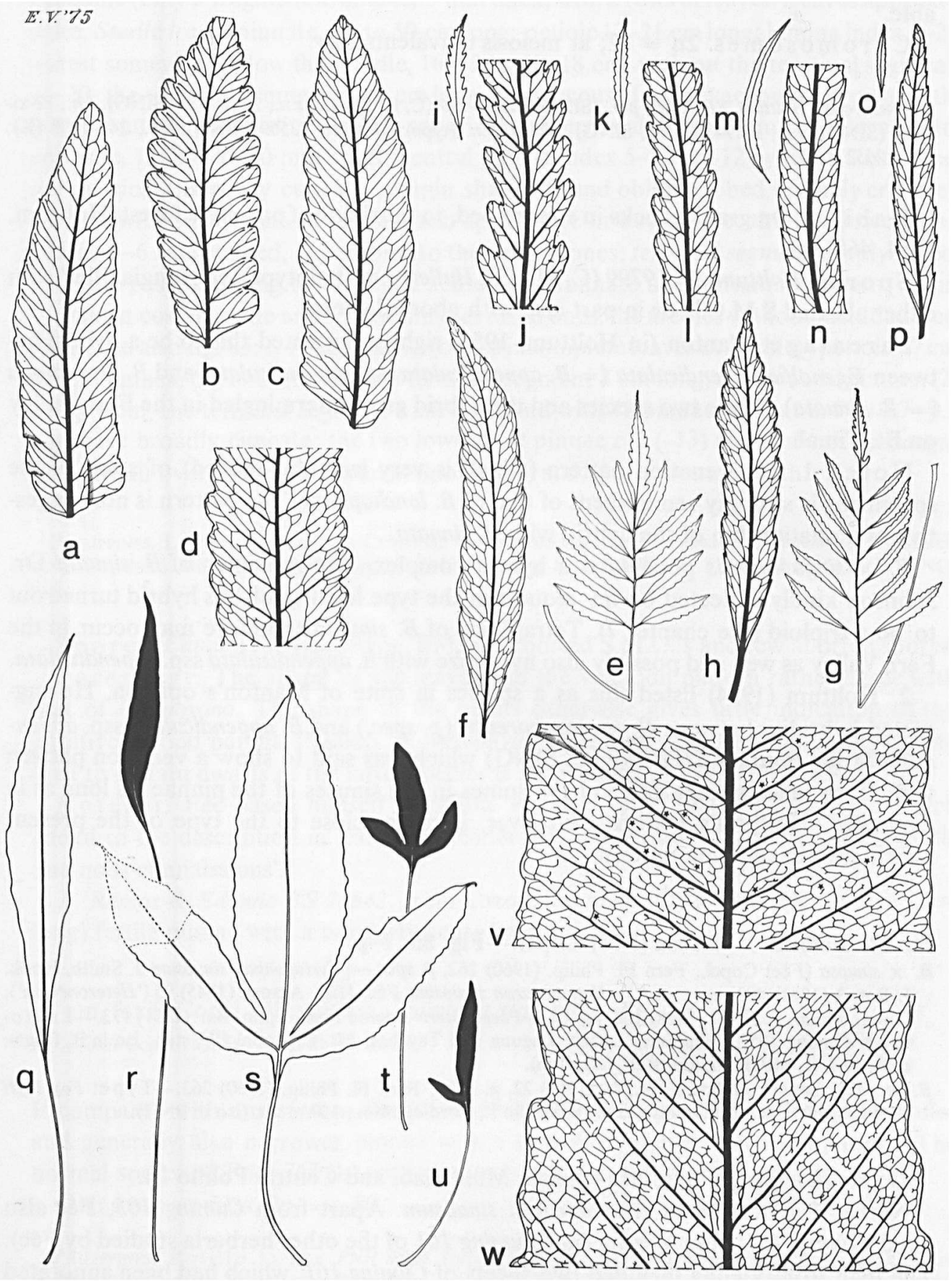


mm stalked. Sporangia inserted mainly on the veins. Spores: normal spores not available.

Chrom os omes. $2 \mathrm{n}=82$; at meiosis univalents only.

SingaPORE, Holttum SF 10479 p.p. (SING), SF 19799 (C, K, SING), s.n., 27-ii-1923 (SING), s.n., 12-xi1928 (SING), s.n., ii-1943 (K, L, SING); Hullett s.n. (type); Matthew 12569 (SING), s.n., 24-i-1908 (K); Allen 2052 (US).

$\mathrm{H}$ a bit a t. On granite rocks in stream-bed, in the shade of primitive forest (Holttum, 1954, 468).

Sp or es. Holttum SF 19799 (C, SING), Hullett s.n. (holotype): sporangia filled with either aborted S.M.C.'s or in part also with aborted spores.

Parentage. Manton (in Holttum, 1954) rightly suggested this to be a hybrid between Egenolfia appendiculata $(=B$. appendiculata ssp. appendiculata $)$ and $B$. diversifolia $(=B$. sinuata). These two species and the hybrid grow intermingled in the Fern Valley on Bt. Timah.

Notes. 1. The venation pattern (which is very irregular indeed) of some of the specimens is strongly reminiscent of that of $B$. lonchophora. The pattern is not suggestive of a relationship of the hybrid with $B$. sinuata.

$B$. $X$ singaporensis possibly is a hybrid complex. The specimen of $B$. sinuata Dr. Johnson kindly collected on my request in the type locality of this hybrid turned out to be a triploid (see chapter 7). Tetraploids of $B$. sinuata therefore may occur in the Fern Valley as well and possibly also hybridize with $B$. appendiculata ssp. appendiculata.

2. Holttum (1954) listed this as a species in spite of Manton's opinion. He suggested hybridity between $B . \times$ singaporensis ( $p$. spec.) and $B$. appendiculata ssp. appendiculata for Holttum SF 19799 (C, SING) which was said to show a venation pattern with fewer anastomoses and to have spines in the sinuses of the pinnae 'as long as in Egenolfia'. Holttum's material, however. is rather close to the type of the present hybrid also in venation pattern.

H. 12. Bolbitis $x$ sinuosa (Fée) Copel.-Fig. 85: e-p.

B. $\times$ simuosa (Fée) Copel., Fern Fl. Philip. (1960) 262, p. spec.-[Cyrtogonium sinuosum J. Smith, Hook. J. Bot. 3 (1841) 403, nom. nud.] - Heteroneuron sinuosum Fee, Hist. Acrost. (1845) 95 ('Heteronevron'); Copel., Philip. J. Sc. 37 (1928) 368, fig. 21. -Poecilopteris sinuosa Presl, Epim. Bot. (1851) 173. - Lec toty pe: Cuming 105, Philippines, Luzon, Laguna and Tayabas, Mt. Cristobal (W, ster.; iso in B, fragm, ster., BM, K, MICH, fragm. ster., US, ster.).

B. $\times$ foxii Copel., Philip. J. Sc. 81 (1952) 22, p. spec.; Fern Fl. Philip. (1960) 263.-Ty pe: Fox PNH 8904 (Fieldno. 328), Philippines, Central Polillo I., Bordios Mun., $150 \mathrm{~m}$ alt. (iso in PNH).

D istribution. Philipines (Luzon, Mindanao, and Central Polillo I.).

Note. Typification of Heteroneurori sinuosum. Apart from Cuming 105, Fée also cited Cuming 161 from Vienna (not Cuming 161 of the other herbaria studied by Fée). The loan from Vienna included two sheets of Cuming 161, which had been annotated by Fée as 'Heteronevron argutum' and which represent $B$. heteroclita. 
a. nm. sinuosa-Fig. 85: e. f.

Rhizome (only a fragment available) $5 \mathrm{~mm}$ thick, with 2 rows of leaves. Leaves approximate. Sterile leaves pinnate, up to $50 \mathrm{~cm}$ long; petiole $12-21 \mathrm{~cm}$ long; lamina ind ex 1-2, widest somewhat below the middle, 16-32 by 10-18 cm, without the terminal segment 11-20, the terminal segment 8-12 cm long, herbaceous, dark olivaceous or brown, with or without (a primordium of) a spherical subterminal bulbil; pinnae alternate to opposite, 13-20, 20-30 mm apart; central pinnae index $5-7,7.5-12$ by $1.1-1.8 \mathrm{~cm}$, base symmetrical, narrowly cuneate, margin shallowly and oblique lobed or finely crenateserrate, with small teeth in the sinuses, apex acute or acuminate; the two lowermost pinnae 3-6 mm stalked, \pm conform to the central ones; terminal segment deeply lobed near the base, tapering towards the acute or acuminate apex; venation pattern: veins forming a costal areole and several smaller distal ones, the areoles without included free veins, see also fig. 85: f. Fertile leaves (only 3 incomplete leaves available); petiole $27 \mathrm{~cm}$ long; laminas: (1) 19 by $16 \mathrm{~cm}$, the terminal segment $8 \mathrm{~cm}$ long, and with 10 pinnae, (2) 21 by $6 \mathrm{~cm}$, the terminal segment $7 \mathrm{~cm}$ long, and with 12 pinnae; pinnae c. 5 by $0.7-0.9$ $\mathrm{cm}$, base broadly cuneate; the two lowermost pinnae c. $2(-13) \mathrm{mm}$ stalked. Sporangia inserted all over the lower surface. Spores: normal spores not met with. -

PhilipPINes. Lu z o n. Laguna, Mt. Cristobal: Cuming 105 (lectotype); Pagsanjan: Copeland s.n. (MICH, herb. Copeland 10191, p.p., ster.); Tayabas, Mt. Binuang: Ramos \& Edaño BS 28842 (BO, K, L, SING, US).

Sp ores. Cuming 105 (lectotype, BM, K): aborted S.M.C.'s and few aborted spores.

$P$ arentage. The shape of the leaves and the venation pattern rather agree with that of $B$. quoyana. The shape of the plants otherwise gives little indication of the putative second parent. Possibly $B$. rhizophylla or $B$. heteroclita. The similarity of nm. foxii to certain dwarfs of the latter species is noteworthy.

Notes. 1. Fée based himself on sterile material only. In the phrases in French added to the description in Latin he erroneously spoke about fertile leaves 'les seuls que nous connaissions'.

2. Ramos \& Edanño $B S 28842$, from Luzon, has markedly petiolulate (up to $13 \mathrm{~mm}$ long) fertile pinnae with a narrowly acute base.

b. nm. foxii (Copel.) Hennipman, comb. et stat. nov. -Fig. 85: g-p.

B. $\times$ foxii Copel., Philip. J. Sc. 81 (1952) 22.

If compared with mn. sinuosa the plants are of the same size or smaller, with smaller and generally also narrower pinnae with a somewhat simpler venation pattern. The normal spores of Price 763 (L) with a smooth cristate perispore; crests low.

Chromosomes. $2 \mathrm{n}=c .80$.

Philippines. Polillo I.: Fox PNH 8904 (type of $B$. $\times$ foxii).-Luzon. Quezon, Llavac, Univ. Philip. Land Grant, $250 \mathrm{~m}$ alt:: Price 763 (L, 6 sh.).-Mindan a o. Agusan del Sur Prov., Esperanza, $125^{\circ} 30^{\prime}$ E, $8^{\circ} 45^{\prime}$ N: Price 2805 (L). 
H a bi t a t. 'On rocks in stream' (type); 'in partial shade along riverbank' (Price 763).

S pores. Fox 8904 (iso PNH), Price 763 (L, sh. 2 \& 3): all abnormal. Price 763 (L, sh. 4): the greater part normal.

P a rent age. See nm. sinuosa.

Note. Price 763 is in cultivation at Leiden since early 1970 . The plants grow rather well; as yet (1976) they have not produced fertile leaves.

\section{Hybridae incertae sedis}

\section{H. 13. Leptochilus $x$ trifidus v.A.v.R.-Fig. 85: $q-w$.}

L. $\times$ trifidus v.A.v.R., Bull. Dép. Agr. I. N. 18 (1908) 26, p. spec.-T y p e: Van Alderwerelt van Rosenburgh s.n., 1907, cult. (BO; iso in L, ster., P).

Hemigramma latifolia auct. non Copel.: Copel., Philip. J. Sc. 37 (1928) 404, p.p.

Rhizome short-creeping, up to $10 \mathrm{~cm}$ long, up to $5 \mathrm{~mm}$ thick, with 2 rows of leaves, without sclerenchyma strands. Scales of the rhizome: index 2-6, up to 5 by $1.5 \mathrm{~mm}$, pseudo-peltate, or attached with the central part of the base, triangular, opaque to subclathrate, blackish, margin entire, apex acuminate. Leaves rather close together to somewhat spaced. Sterile leaves simple, either entire or trifid, $30-60 \mathrm{~cm}$ long. Entire leaves 30-52 cm long; petiole 8-20 cm long, near the base 1-3 mm thick; lamina index $3-6$, widest about the middle, $22-32$ by $4-9 \mathrm{~cm}$, firm-herbaceous to subcoriaceous, brownish, with a small but conspicuous spherical subterminal bulbil, base narrowly cuneate or angustate, gradually decurrent on the petiole, margin entire or slightly sinuate, apex acuminate. Trifid leaves $41-60 \mathrm{~cm}$ long; petiole $10-25 \mathrm{~cm}$ long, near the base $2-4 \mathrm{~mm}$ thick; lamina index 1-1.5, widest below the middle, 24-35 by $17-30 \mathrm{~cm}$, base \pm abruptly decurrent on the petiole; lateral lobes somewhat falcate, widest about the middle, the basiscopic side somewhat larger than the acroscopic one, $11.5-20$ by $2.5-8 \mathrm{~cm}$; terminal lobe symmetrical, widest about or below the middle, $20-30$ by $5.5-12 \mathrm{~cm}$, at the base $2.5-4.5 \mathrm{~cm}$ wide; otherwise like the entire leaves; venation pattern rather irregular but otherwise like that found in Ser. Euryostichae, see also fig. 85: v, w. - Fertile leaves. Entire leaves $27-50 \mathrm{~cm}$ long; petiole $18-30 \mathrm{~cm}$ long; lamina index $5-10,8-20$ by $1.5-3.5 \mathrm{~cm}$. Trifid leaves $25-40 \mathrm{~cm}$ long; petiole $16-27 \mathrm{~cm}$ long; lamina index $1-2,6-13$ by $5-10 \mathrm{~cm}$; lateral lobes $2-5.5$ by $0.7-2 \mathrm{~cm}$; terminal lobe $5-11$ by $1.2-3.5 \mathrm{~cm}$, at the base $0.4-1.2 \mathrm{~cm}$ wide. Sporangia inserted all over the lower surface. Spores: normally shaped spores with a smooth, (imperfectly developed) cristate-undulate or cristate perispore.

Sumatra. W est C o as t. Padangpandjang, 'Anai Kloof': Matthew s.n. (K.)-E as t C o as t. Sibolangit Forest Reserve: Lörzing 12404 (BO), 12405 (BO, K, L, p.p.).-B encoolen. Lebong Tandai: Brooks 44.S. (BM), 61.S. (BM), 73.S. (BM, MICH, P), 75.S. (BM), 403.S. (BM), s.n. (BM).

$\mathrm{H}$ a bit a t. Forest; reported from rocks either in streams or on stream banks. Altitude: 450-500 m (Lörzing's collections). 
S pores. Van Alderwerelt van Rosenburgh s.n. (BO, holo): all abnormal, a few normally shaped with an (imperfectly developed) undulate-cristate perispore. Brooks s.n. (BM), Lörzing $12405(\mathrm{~K})$ : all abnormal, a few normally shaped with an imperfectly developed cristate perispore. Lörzing 12404 (BO): sporangia filled with either (a cluster of) abnormal spores, or in part with (apogamous?) normal spores with a cristate perispore.

P a rentage. A specimen of the present fern was once found mounted together with a specimen of Leptochilus decurrens (also dimorphic). Lörzing (annotations to his collections), Copeland (annotation to Brooks 73.S., MICH), and Van Alderwerelt van Rosenburgh (1908) thought it related to this species. Copeland (1928) included it in Hemigramma latifolia Copel. which is, however, different.

The rhizome. The arrangement of the vascular bundles in the rhizome is \pm radially symmetric; there is one slightly larger ventral bundle; roots are given off in all directions and arise from all bundles; leaves occur in 2 rows dorsolaterally. In Leptochilus decurrens this arrangement is \pm radially symmetric; cross-sections show a number of \pm equally wide bundles in a \pm circular arrangement; roots are formed in 2 rows ventrolaterally; leaves occur in 2 rows dorsolaterally. The arrangement in Bolbitis sinuata is dorsiventral (see the general chapters). Sclerenchyma strands of the ground tissue of the rhizome are absent in Leptochilus $\times$ trifidus, present or absent in B. sinuata, and present in Leptochilus decurrens. A sheath of sclerenchyma somewhat below the epidermis which is present in B. sinuata is absent in Leptochilus $\times$ trifidus and L.decurrens. Aerophores are absent in $L$. decurrens, absent or not distinctly developed in $L$. $\times$ trifidus, and present in B. sinuata.

The anatomical data of Leptochilus $\times$ trifidus were obtained from Lörzing 12405 (K) and Brooks 73.S. (P).

The petiole. The leaves of all three plants are joined to the rhizome. The sheath of sclerenchyma as seen from cross-sections in the basal part of the petiole: in Leptochilus decurrens the sheath is situated directly below the epidermis and not interrupted by aerophores. In Leptochilus $\times$ trifidus the sheath is situated somewhat below the epidermis and not interrupted by aerophores. In Bolbitis sinuata the sheath is situated somewhat below the epidermis and interrupted by aerophores.

The scales. The shape of the scales is similar in all three. Leptochilus decurrens has clathrate scales without or with but few glandular cells which are situated terminally on (incisions of) the scales, or on short, stout, glandular hairs. Leptochilus $\times$ trifidus has subclathrate or opaque scales with few, stout, glandular cells. Bolbitis sinuata has subclathrate scales with slender glandular hairs.

The stomata are polocytic in all three.

A bulbil is absent in Leptochilus decurrens and present in L. $\times$ trifidus and Bolbitis sinuata.

The spores. The spores are monolete in all three.

The perispore. It is generally believed that the spores of Leptochilus species lack a perispore. This proved to be incorrect as a rather well developed minutely spiny perispore was found in several Leptochilus species (L. axillaris, decurrens, lanceolatus, 
and minor). The perispore of the normally shaped spores of Leptochilus $\times$ trifidus is imperfectly cristate or cristate-undulate, that of $B$. sinuata is either cristate or cristateundulate.

The gametophyte of Leptochilus decurrens is of the ribbon-like type, and bears onecelled papillate hairs (Nayar \& Kaur, Bot. Rev. 37, 1971, 368), those of $L . \times$ trifidus and $B$. sinuata are unknown.

In conclusion, the shape, texture, and venation pattern of Leptochilus $\times$ trifidus I consider intermediate between that of $L$. decurrens and $B$. sinuata. The presence of a bulbil, the shape of the perispore, and the texture of the scales rather agree with these properties of $B$. sinuata; the absence of aerophores on the petiole and the rhizome, the absence of a sclerenchyma sheath in the rhizome, and to some extent also the vascularization in the rhizome, point towards a fern like Leptochilus decurrens.

In spite of this I have refrained of creating a new hybrid genus as the chromosome number of Bolbitis $(\mathrm{n}=41)$ differs from that of Leptochilus $(\mathrm{n}=36$, see Manton \& Sledge, Phil. Trans. R. Soc. Lond. ser. B, 238, 1954, 171), and as the spore output does not reflect this difference (aborted S.M.C.'s are not found). This very interesting hybrid which possibly questions the relationships of the polypodioid genera Leptochilus and Colysis (which are likely to be congeneric, see Sledge, Ann. Mag. Nat. Hist. ser. $12,9,1956)$ needs to be studied for its karyology.

Notes. 1. The type collection and Brooks s.n. (BM) consist of sterile and fertile leaves which are either entire, or trifid, or 'bifid' (with but one lateral lobe, see the illustration).

2. The specimens with entire leaves have a similar habit as certain specimens with simple leaves of ser. Euryostichae.

TAXA ATQUE NOMINA DUBIA

\section{Species dubiae}

D. 1. Bolbitis cadieri (Christ) Ching-Fig. 86: a-d.

B. cadieri (Christ) Ching in C. Chr., Ind. Fil., Suppl. 3 (1934) 47; Tardieu-Blot \& C. Chr., Not. Syst. 7 (1938) 100; in Lecomte, Fl. Gén. I.-C. 7 (1941) 432.-Gymnopteris cadieri Christ, J. de Bot. 19 (1905) 126; Copel., Philip. J. Sc. 37 (1928) 358.-Leptochilus cadieri C. Chr., Ind. Fil. (1906) 384.-T y p e: Cadière 146, Vietnam, Annam, Thanh Tan (= Thua Thien), $100-200 \mathrm{~m}$ alt. (P).

Rhizome (but 2 available) short-creeping, 5 and $15 \mathrm{~mm}$ thick, with 2 and 3 rows of leaves resp., with sclerenchyma strands. Scales: index 3-8, up to 5 by $1 \mathrm{~mm}$, subclathrate, with or without a paler margin, brownish. Leaves tufted. Sterile leaves pinnate, 56->84 cm long; petiole $24-50 \mathrm{~cm}$ long; lamina index 1-2, widest below or about the middle, $30->46$ by $24-30 \mathrm{~cm}$, without the terminal segment $10-26$, the terminal segment $17-25 \mathrm{~cm}$ long, subcoriaceous, with a spherical subterminal bulbil on terminal (and lateral). segments; rhachis not winged; pinnae alternate or opposite, 6-10, 20- 
$60 \mathrm{~mm}$ apart; central pinnae index 4-6, widest somewhat below the middle, 12-24 by 2.7-6 cm, base symmetrical or asymmetrical with its basiscopic side rounded, the acroscopic side broadly attenuate, margin entire or sinuate, apex acute to long-acuminate; the two lowermost pinnae up to $4 \mathrm{~mm}$ stalked, conform to the central pinnae; terminal segment conform to the pinnae or with an odd lobe near the base; venation pattern resembling that of $B$. heteroclita but part of the areoles with mostly one simple, excurrent, included free vein ending in a hydathode, see also fig. 86: b-d. Fertile leaves $65-105 \mathrm{~cm}$ long; petiole $50-70 \mathrm{~cm}$ long; lamina index $2-4,15-40$ by $6-12 \mathrm{~cm}$, without the terminal segment $11-31$, the terminal segment 4-9 cm long; pinnae 8-12, up to $55 \mathrm{~mm}$ apart; central pinnae index $5-6,4.5-10$ by $0.8-2 \mathrm{~cm}$; the two lowermost pinnae 6-12 mm stalked. Sporangia all over the lower surface. Spores with a smooth, cristate or cristate-spiny perispore.

Vietnam. Tonkin. Lang Met: Pételot s.n. (BM. P). -A n n a m. Thua Thien: Cadière 123 (BM, P, both ster.), 146 (type), 169 (P, ster.), 196 (P); Mt. Bah-na (near Danang): Cadière 91 (C, ster., P), 129 (P); Clemens s.n. (UC, 339924); Sallet s.n. (C, L, P, all ster.).

$\mathrm{H}$ a b i t a t. Forest; terrestrial (near streams). Altitude: 100-200 m (type), 800-1500 m (Mt. Bah-na).

S p or es. Cadière $196(\mathrm{P})$ : the greater part normal. Cadière 146 (type): the greater part abnormal. Pételot s.n. (P): sporangia filled with aborted S.M.C.'s and aborted spores.

Notes. 1. The venation pattern and leaf architecture are intermediate between that of $B$. heteroclita and B. virens (or B. subcordata?). B. cadieri is likely to be of hybrid derivation.

2. Christensen (annotations to Campium juglandifolium C. Chr. ined., based on Pételot's collection from Lang Met) compared this fern with Campium costatum $(=B$. costata) which is quite different.

3. Copeland, who did not study any material of this fern, doubted it to be a Campium (= Bolbitis), since Christ reported a venation pattern with clavate free veinlets, 'a characteristic of Hemigramma'.

4. Collections rather intermediate between the present fern and B. subcordata are known from Vietnam; they include: Eberhardt s.n. (P), Hayata s.n. (TI), Pételot 3955 (Colani leg., MICH, P), Tsang 29432 (A, C), all from Tonkin, and Cadière 101 (P) from Annam.

\section{2. Bolbitis christensenii (Ching) Ching-Fig. 86: e, f.}

B. christensenii (Ching) Ching in C. Chr., Ind. Fil., Suppl. 3 (1934) 47; Tardieu-Blot \& C. Chr. in Lecomte, Fl. Gén. I.-C. 7 (1941) 437.-Campium christensenii Ching, Bull. Fan Mem. Inst. Biol. 2 (1931) 214, pl. 31.-T y p e: Esquirol 2672, China, Kweichow, Ta-iong, iii-1911 (n.v.).

Rhizome (but 2 available) c. $10 \mathrm{~mm}$ thick, with 3 rows of leaves, without sclerenchyma strands. Scales: index 3-6, up to 4.5 by $1.5 \mathrm{~mm}$, subclathrate, blackish, the old ones with a paler margin. Leaves tufted. Sterile leaves pinnate, 49,73 , and $86 \mathrm{~cm}$ long resp.; 


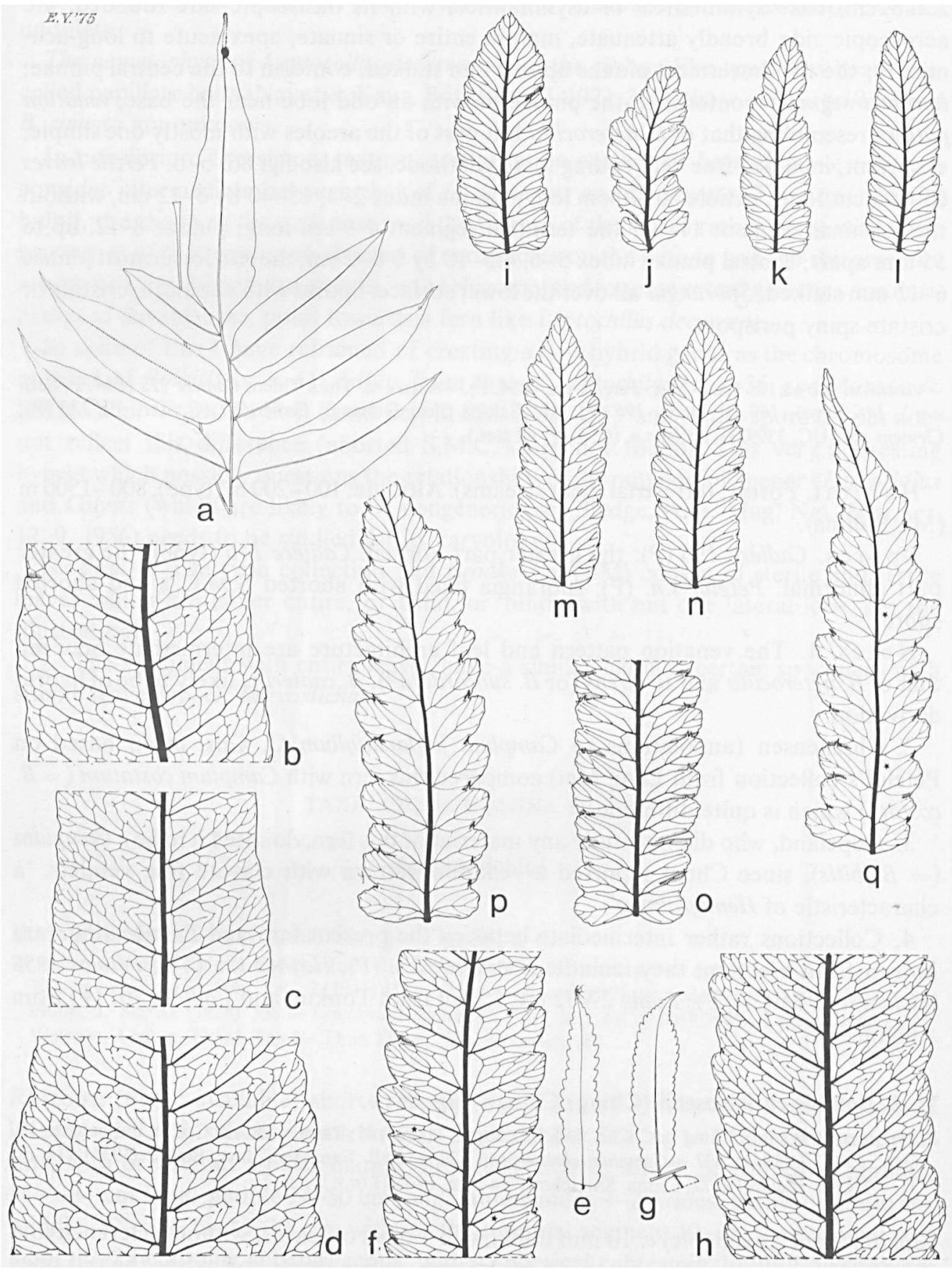


petiole 7, 25, and $33 \mathrm{~cm}$ long resp.; laminas widest about the middle, (1) 42 by $22 \mathrm{~cm}$, the terminal segment $22 \mathrm{~cm}$ long, with 11 pinnae, (2) 48 by $22 \mathrm{~cm}$, the terminal segment $21 \mathrm{~cm}$ long, with 18 pinnae, (3) 53 by $25 \mathrm{~cm}$, the terminal segment $18 \mathrm{~cm}$ long, with 20 pinnae, resp.; herbaceous, olivaceous, with a spherical subterminal bulbil quite near the apex (also of pinnae); rhachis not winged; pinnae alternate or opposite, up to $60 \mathrm{~mm}$ apart; central pinnae index 4-7, widest about the middle, $13-15$ by $2.3-3.2 \mathrm{~cm}$, base \pm symmetrical, \pm truncate to subcordate, margin lobed to $1 / 5$ towards the costa; sometimes with small teeth in the sinuses, apex acute to acuminate; the two lowermost pinnae conform to the central ones, shortly petiolulate; uppermost pinnae: their basiscopic side adnate, decurrent on the rhachis; terminal segment triangular, pinnatipartite near the base, tapering towards the apex; venation pattern: veins forming a regular network similar to that sometimes found in $B$. angustipinna, aeroles without included free veins, see also fig. 86: f. Fertile leaves 38, 82, and $>87 \mathrm{~cm}$ long resp.; petioles 25,40 , and $47 \mathrm{~cm}$ long resp.; laminas widest below the middle, (1) 13 by $9 \mathrm{~cm}$, the terminal segment $5 \mathrm{~cm}$ long, with 12 pinnae, (2) 42 by $12 \mathrm{~cm}$, the terminal segment $10 \mathrm{~cm}$ long, with 19 pinnae, (3) $>40$ by $10 \mathrm{~cm}$, without the terminal segment $>37 \mathrm{~cm}$ long with $>20$ pinnae; pinnae up to $70 \mathrm{~mm}$ apart; central pinnae index $4-7$, widest about or below the middle, $4-6.5$ by $0.9-1.2 \mathrm{~cm}$. Sporangia mainly inserted on the veins. Spores with a cristate-undulate perispore.

China. Kw ei ch ow. Ta-iong: Esquirol 2672 (type, n.v.).

VIETNAM. Tonkin. Than-moi: Balansa 150 (= 95); Dong-son: Balansa 151 (= 94); Chobo: Balansa 1951 (P).

$\mathrm{H}$ a bit a t. Forest, along streams. Once reported from limestone.

S p o res. Balansa 1951: normal. Balansa 151: in part normal. Balansa 150: sporangia filled with aborted spores and/or aborted S.M.C.'s.

Notes. 1. A very interesting fern as the venation pattern of the sterile pinnae suggests it to be intimately related to B. angustipinna which has, however, quite a different leaf architecture and an undulate perine. I doubt the relationship to $B$. subcordata as suggested by Ching, as the venation pattern does not show included free veins. The sporangia are mainly inserted on the veins. This, and the habit of the fertile leaves are also found in $B$. sinensis. The venation pattern of the fertile pinnae shows many irregularities, being rather similar to that of $B$. angustipinna $\times$ sinensis.

Fig. 86. a-d. Bolbitis cadieri. a. sterile leaf, $\times 1 / 5$; b-d. venation pattern of sterile pinnae, $\times 4 / 5(a, b$. Cadière 146, P, holotype of B. cadieri; c. Clemens s.n., UC 339924; d. Cadière 91, C).-e, f. Bolbitis christensenii. e. sterile pinna, $\times 1 / 5$; f. venation pattern of sterile pinna, $\times 4 / 5$; asterisks indicate asymmetry in the venation pattern (e, f. Balansa $151, \mathrm{P}) .-\mathrm{g}$. h. Bolbitis interlineata. g. sterile pinna, $\mathrm{x}$ $1 / 5$; h. venation pattern of sterile pinna, $\times 4 / 5(\mathrm{~g}, \mathrm{~h}$. Brooks $12, \mathrm{MICH}$, holotype of $B$. interlineata). i-q. Bolbitis laxireticulata, venation pattern of sterile pinnae, $\times 4 / 5$; asterisks indicate local differences in venation pattern (i, j. Tagawa \& Iwatsuki 4605 , L, both from one leaf; k, l. ibid., L; m-o. ibid., US; p, q. Iwatsuki 4884, L). 
The fern possibly has a hybrid origin; it is likely that a fern like B. angustipinna takes part. The other parent is likely to have a cristate or cristate-undulate perispore; I have no well-founded suggestions.

2. The shape of the leaves and the pinnae, and the venation pattern are surprisingly similar to that of $B$. quoyana. The venation pattern of $B$. quoyana is symmetrical, whereas it is usually asymmetrical in B. angustipinna.

3. The bulbil of the sterile leaf of Balansa 151 bears two small sterile leaves composed of a large triangular, shallowly lobed terminal segment and two \pm orbicular pinnae. The venation pattern of the terminal segment is similar to that found in the adults; it is free in part of the basal pinnae.

\section{3. Bolbitis interlineata (Copel.) Ching-Fig. 86: g, h.}

B. interlineata (Copel.) Ching in C. Chr., Ind. Fil., Suppl. 3 (1934) 48; Ito, J. Jap. Bot. 14 (1938) 439, quoad nomen solum. - Campium interlineatum Copel., Philip. J. Sc. 37 (1928) 370, fig. 24, pl. 17.-T y p e: Brooks 12, Borneo, Sarawak, Bungo Range, iv-1909 (MICH; iso in BM).

Rhizome (only fragments available) $5 \mathrm{~mm}$ thick, with many sclerenchyma strands. Scales up to 4 by $1 \mathrm{~mm}$, subclathrate, blackish. Leaves close together. Sterile leaves pinnate, $60-75 \mathrm{~cm}$ long; petiole $30-35 \mathrm{~cm}$ long; lamina index $1-2$, widest about or below the middle, $30-40$ by $20-24 \mathrm{~cm}$, without the terminal segment $10-28$, the terminal segment $12-20 \mathrm{~cm}$ long, herbaceous, with a spherical subterminal bulbil; rhachis not winged; pinnae alternate or opposite, $8-16,20-35 \mathrm{~mm}$ apart; central pinnae index $3-5$, widest above or below the middle, $13.5-15$ by $3-4 \mathrm{~cm}$, base either \pm symmetrical, broadly cuneate to subcordate, or asymmetrical with its basiscopic side longer and/or wider than its acroscopic side, margin entire or (in part) slightly sinuate, apex acute to acuminate; the two lowermost pinnae up to $4 \mathrm{~mm}$ stalked, conform to the central ones; terminal segment \pm conform to the pinnae or triangular with 1-2 basal lobes; venation pattern very intricate, reminiscent of that of $B$. heteroclita but part of the areoles with usually one excurrent included free vein, see also fig. 86: h. Fertile leaves $60-85 \mathrm{~cm}$ long; petiole $44-50 \mathrm{~cm}$ long; lamina index $2-4,17-35$ by $8-10 \mathrm{~cm}$, without the terminal segment 13-30, the terminal segment 4-9 cm long; pinnae 10-18, up to $40 \mathrm{~mm}$ apart; central pinnae index $5-8,5-6$ by $0.7-1 \mathrm{~cm}$, the two lowermost pinnae up to $5 \mathrm{~mm}$ stalked. Sporangia all over the lower surface. Spores with a smooth cristate perispore.

Borneo. S a rawak. Southern part. Bungo Range: Brooks 12 (type); Mt. Matang: Allen 3145 (K, ster.); Matthew s.n. (K, ster., juv.); Mt. Penrissen: Brooks s.n. (BM).

H a bi t a t. Forest; on rocks in streams. Altitude: $300-690 \mathrm{~m}$.

S p ores. Brooks s.n. (BM): in part normal. Brooks 12 (holotype of Campium interlineatum): the greater part abnormal. Brooks 12 (iso in BM): all abnormal.

Note. A distinct fern easily characterized by leaf architecture and venation pattern which resembles that of $B$. cadieri (spec. dub.). Possibly of hybrid origin and related to $B$. heteroclita.(venation pattern!) and to either B. scalpturata or B. repanda. 
D. 4. Bolbitis laxireticulata Iwatsuki-Fig. 86: i-q.

B. laxireticulata Iwatsuki, Acta Phytotax. Geobot. 18 (1959) 50, fig. 7 \& 8.-Egenolfia laxireticulata Kuo in Fl. Taiwan 1 (1975) 352.-T y pe: Tagawa \& Iwatsuki 2918, Japan, Ryukyu, Amami-Ooshima, Mt. Yuwan-dake, $500 \mathrm{~m}$ alt. (KYO, n.v., L, phot.; iso in KYO, n.v., L, phot., L, ster., US).

Rhizome $c .4 \mathrm{~mm}$ thick, with 2 rows of leaves, with sclerenchyma strands. Scales up to $3(-5)$ by $1.5(-2) \mathrm{mm}$, subclathrate, brown-blackish. Leaves \pm approximate. Sterile leaves pinnate, $30-75 \mathrm{~cm}$ long; petiole $10-34 \mathrm{~cm}$ long; lamina index $2-4$, widest below or about the middle, $20-42$ by $10-15 \mathrm{~cm}$, without the terminal segment $14-35$, the terminal segment $4-11 \mathrm{~cm}$ long, papyraceous, with a spherical subterminal bulbil; rhachis (in the upper half) with a narrow wing; pinnae alternate or opposite, 18-31, up to $35 \mathrm{~mm}$ apart; central pinnae index 3-7, widest about or below the middle, 4.5-9.5 by $1.3-2.3 \mathrm{~cm}$, base sometimes symmetrical, variously cuneate, usually asymmetrical, its acroscopic side broader than its basiscopic side and sometimes with a basal lobe, margin \pm entire to lobed to $2 / 5$ towards the costa, lobes finely crenate-serrate, with a distinct spine in the sinuses, apex acute to acuminate; the two lowermost pinnae up to $1.5 \mathrm{~mm}$ stalked, conform to the central pinnae, base of pinnae of the.upper half of the lamina asymmetrical; terminal segment triangular; venation pattern (very) irregular, veins forming a costal areole at least in part of the pinnae of one plant, otherwise varying, see also fig. 86: i-q. Fertile leaves (only 2 immature leaves available); pinnae 12 and 17. Sporangia inserted mainly on the veins. Spores not available.

JAPAN. R y u k y u Is. Amami-Ooshima: Iwatsuki 4884 (L, US, ster.); Serizawa 11713 (= Pterid. Jap. exsicc. 48) (L, U, both ster.); Tagawa \& Iwatsuki 2918 (type). Iriomote: Tagawa \& Iwatsuki 4605 (L, US, both ster.). Also reported from Okinawa (Iwatsuki, 1959).

$\mathrm{H} \mathrm{abitat}$. Terrestrial in moist places in forest. Altitude: $500-550 \mathrm{~m}$.

Notes. 1. The venation pattern, the shape of the pinnae, and the presence of a very narrow wing along the rhachis may be regarded as intermediate between those of $B$. appendiculata ssp. appendiculata and $B$. subcordata. The venation pattern is structurally irregular: in many pinnae it does not decrease in complexity towards the apex, the venation pattern in the basal part of a certain pinna being less complex than that in the middle and apical part of the same pinna. Iwatsuki himself judged the venation pattern 'quite indefinite' and found it to be different in every individual. He also noted the enormous variation of other features.

Iwatsuki did not describe the spores. The sporangia available to me are all immature. $B$. laxireticulata seems to be of hybrid derivation.

Iwatsuki related the present fern to $B$. sinensis and $B . \times$ singaporensis (p. spec.). The differential characters he gave do not hold. His concept of $B$. sinensis is that of Christensen which is heterogeneous (see the notes on $B$. sinensis). He suggested hybridity between B. laxireticulata and B. appendiculata and referred to Holttum (Ferns of Malaya, 1954) who found plants intermediate between $B$. $\times$ singaporensis ( $p$. spec.) and $B$. appendiculata. See also $B . \times$ singaporensis note 2 and sect. Lysidictyon Iwatsuki $(=$ sect. dub.). 
2. The shape of the pinnae and the venation pattern (only incidental anastomoses) of Tagawa \& Iwatsuki 4605 (ster.) resemble that of B. rhizophylla.

\section{5. Bolbitis longiflagellata (Bonap.) Ching-Fig. 87.}

B. Iongifagellata (Bonap.) Ching in C. Chr., Ind. Fil., Suppl. 3 (1934) 49; Tardieu-Blot in Humbert, F. Madag. Fam. V, 2 (1960) 57, fig. xvi: 5, 6. -Leptochilus longifagellatus Bonap., Notes Ptérid. 4 (1917) 68.-Campium longiffagellatum C. Chr. in Perrier de la Bâthie, Cat. Pl. Madag., Pterid. (1932) 32; Dansk Bot. Ark. 7 (1932) 70, pl. 22: fig. 5.-T y pe: Perrier de la Bâthie 7490 p.p., Madagascar, Sambirano, Manongarivo Massif ( $\mathrm{P}$; iso in $\mathrm{P}, 6$ sh., one mixed with $B$. humblotii, $\mathrm{BM}$, fragm.).

Acrostichum virens auct. non Hooker \& Grev.: auct. p.p.

Campium humblotii auct. non (Baker) Copel.: Copel., Philip. J. Sc. 37 (1928) 395, fig. 45.

B. acrostichoides auct. non (Swartz) C. Chr.: Alston, J. Bot. 77 (1939) 286, p.p.; in Exell, Cat. Vasc. Pl. S. Tomé (1944) 68, p.p.; Tardieu-Blot, Mém. I.F.A.N. 28 (1953) 115, p.p.; in Aubrév., Fl. Gabon 8 (1964) 190, p.p.; in Aubrév., Fl. Cameroun 3 (1964) 320, pl. 51: fig. 4, p.p.; Schelpe, Contr. Bolus Herb. 1 (1969) 42, p.p.

Rhizome short-creeping, up to $8 \mathrm{~mm}$ thick, with 2 rows of leaves, with sclerenchyma strands. Scales of the rhizome: index $1-8$, up to 12 by $1.5 \mathrm{~mm}$, subclathrate, rather firm, blackish or brownish, at least the basal part with a paler marginal strip. Leaves \pm

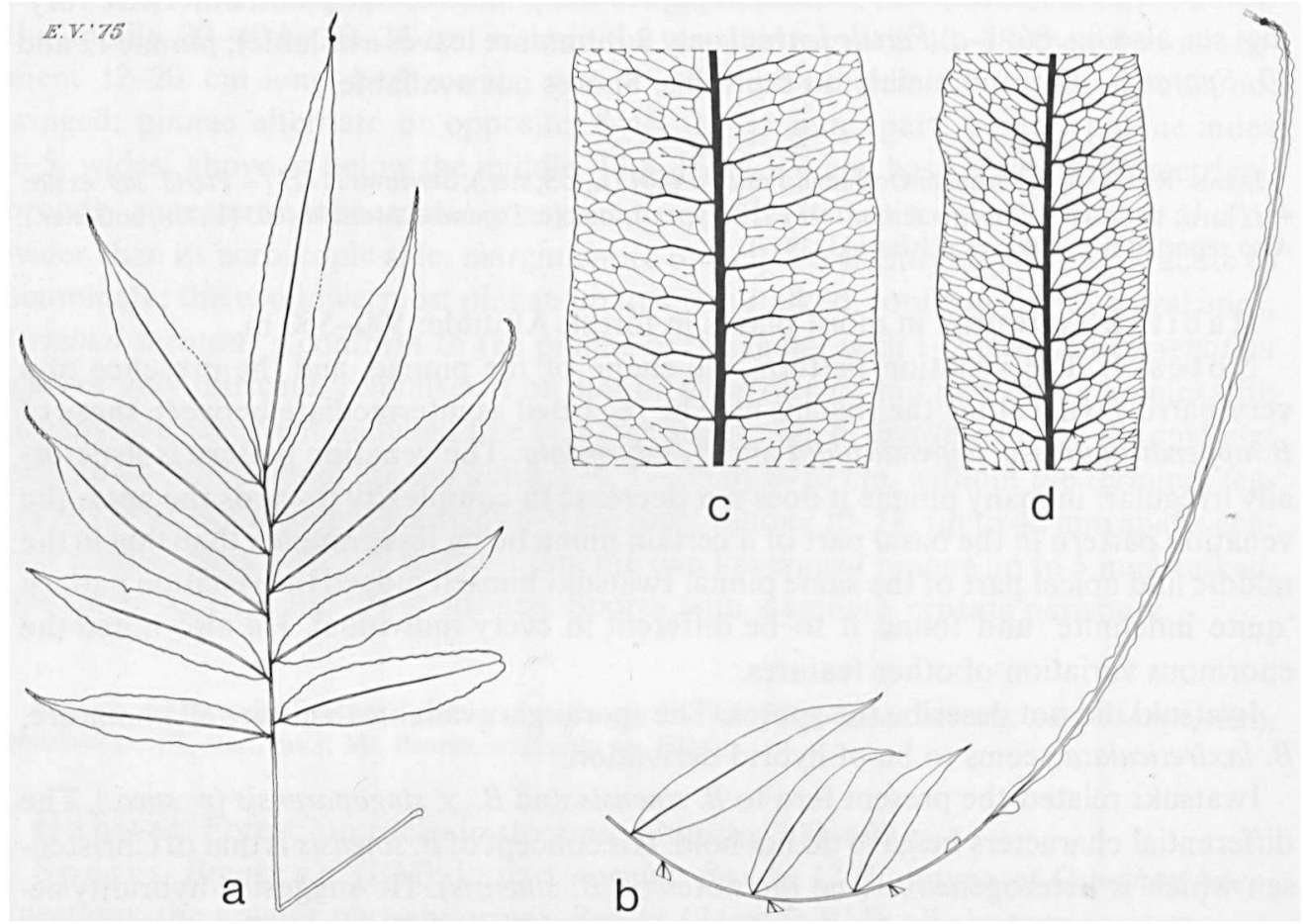

Fig. 87. Bolbitis longiflagellata. a. sterile leaf, $\times 1 / 5$; b. terminal part of a sterile leaf, $\times 1 / 5$; c, $d$. venation pattern of sterile pinnae, $\times 4 / 5$ (a-c. Perrier de la Bâthie $7490, \mathrm{P}$, type of B. longiflagellata; d. Last s.n., K). 
approximate. Sterile leaves pinnate, $48->110 \mathrm{~cm}$ long; petiole $12-28 \mathrm{~cm}$ long, near the base 2-3 mm thick, with 5-7 vascular bundles; lamina index 1-4, widest below or about the middle, $30-92$ by $15-30 \mathrm{~cm}$, without the terminal segment $9-32$, the terminal segment $20-60 \mathrm{~cm}$ long, herbaceous, dark green, with a spherical subterminal bulbil (situated rather close to the apex); rhachis not winged; pinnae \pm alternate, 9-17, 20-45 $\mathrm{mm}$ apart; central pinnae index 4-7, widest about the middle, $11.5-22$ by $1.8-3.8 \mathrm{~cm}$, base symmetrical, cuneate, margin \pm entire, without teeth or spines, apex acute to caudate; the two lowermost pinnae $0-3 \mathrm{~mm}$ stalked, conform to the central pinnae; terminal segment \pm conform to the central pinnae or flagelloid, subarticulate to the rhachis; venation pattern: veins \pm irregularly reticulate, aeroles decreasing in size towards the margin, otherwise varying in size and shape, especially the larger areoles with mostly one simple, excurrent, included free vein, see also fig. 87: c, d. Fertile leaves (but 3 complete ones available) 51, 63, and $84 \mathrm{~cm}$ long; petiole $27-51 \mathrm{~cm}$ long; laminas: 20,24 , and $33 \mathrm{~cm}$ long resp., c. $7 \mathrm{~cm}$ wide, the terminal segment 5.5, 6, and $7.5 \mathrm{~cm}$ long resp.; pinnae 9-15, 30-60 mm apart; central pinnae index $5-7$, widest about the middle, $4-4.5$ by $c .0 .8 \mathrm{~cm}$; the two lowermost pinnae 1-3 mm stalked. Sporangia inserted all over the lower surface. Spores: normal spores not available.

Madagascar. Northwestern. Sambirano, Manongarivo Massif: Perrier de la Bâthie 7490 p.p. (type); Be Kilus Mts.: Last s.n. (BM, K); Bealanana Dist.: Dufournet s.n. (P).

H a bit a t. 'Bois rocailleux au-dessous de $500 \mathrm{~m}$ d'alt.' (type).

S p o r es. Last s.n. (K): all abnormal, shrivelled; perispore very strongly cristate (or folded?). Perrier de la Bâthie 7490 (P): all three fertile leaves with immature sporangia only.

Notes. 1. A distinct fern because of its scales, leaf architecture, and venation pattern. The one fertile leaf available with \pm mature sporangia shows abnormal spores only. The venation pattern may well be intermediate between that of $B$. humblotii (with which it shares the large and firm scales, the shape of the terminal segment, and the subterminal bulbil) and B. auriculata (with which it shares the shape of the pinnae and particulars of the venation pattern).

Bonaparte and Copeland related $B$. longiflagellata to $B$. heteroclita because of the flagelloid leaves. Christensen and Tardieu-Blot related it to, or included it in B. acrostichoides because of supposed similarities in the venation pattern which is, however, much different when studied in detail.

The identity of $B$. longiflagellata is not sufficiently clear to me; it possibly represents a hybrid between $B$. humblotii (with which it was found to grow together in two of the three localities from which it is known) and $B$. auriculata (obviously locally common in Madagascar).

2. The type material shows two types of sterile leaves: one (with which the fertile leaves occur in association) with the terminal segment conform to the pinnae, the other with the terminal segment long-flagelloid. 
3. Copeland's remarks on Campium humblotii were based on material of B. longiflagellata. He studied the collection of J. F. Last ('J. B. Last'), from N.W. ('N.E.') Madagascar collected in 1890 ('1900'), and overlooked Bonaparte's publication of Leptochilus longiflagellatus.

\section{6. Bolbitis sagenioides (Kuhn) Ching}

B. sagenioides (Kuhn) Ching in C. Chr., Ind. Fil., Suppl. 3 (1934) 50.-Chrysodium sagenioides Kuhn, Linnaea 36 (1869) 63; Verh. Zool. Bot. Ges. Wien 19 (1869) 570; Copel., Philip. J. Sc. 37 (1928) 393.Leptochilus sagenioides C. Chr., Ind. Fil. (1906) 307.-T y p e: Milne 337, Melanesia, New Hebrides, Aneityum (iso in K).

Campium samoense Copel., Philip. J. Sc. 37 (1928) 372, fig. 26, pl. 19.-B. samoensis C. Chr., Ind. Fil., Suppl. 3 (1934) 50.-T y pe: Powell s.n., Melanesia, Samoa Is. (GH, 'Kew Distr. no. 1173'; iso in BM). Acrostichum repandum auct. non $\mathrm{Bl}$. : auct. p.p.

B. lonchophora auct. non (Kunze) C. Chr.: C. Chr., Bull. Bish. Mus. 117 (1943) 104, p.p.

Rhizome unknown. Sterile leaves pinnate (only one complete leaf available), $60 \mathrm{~cm}$ long; petiole $25 \mathrm{~cm}$ long; lamina index 1-3, widest somewhat below the middle, 35$40 \mathrm{~cm}$ long, without the terminal segment $15-21 \mathrm{~cm}$, the terminal segment $15-25 \mathrm{~cm}$ long; pinnae 10-17, otherwise intermediate between those of B. quoyana and B. rivularis; terminal segment triangular. Fertile leaf $85 \mathrm{~cm}$ long (only one complete leaf available); petiole $50 \mathrm{~cm}$ long; lamina index $1-4,33-42 \mathrm{~cm}$ long, without the terminal segment $25-$ 35 , the terminal segment $7-10 \mathrm{~cm}$ long; pinnae 12-23. Sporangia inserted all over the lower surface. Spores with a smooth cristate perispore.

Solomon Is. Gu a d a l c a n a l: Braithwaite RSS 4090 (K, L, ster.).

New Hebrides. A n e ity u m: Herus 107 (B, BM, G, P); Kajewski 893 (BISH, BO, GH, US); Milne 337 (type of Chrysodium sagenioides); Morrison s.n. (K).

SAMOA Is. Powell s.n. (type of Campium samoense); Reinecke 5a (P).

$\mathrm{H}$ a b i t a t. 'On steep rocky slope by river' (Braithwaite RSS 4090); 'mountain woods' (Herus 107).

S p o r es. Kajewski 893 (BO, GH, US), Powell s.n. (holotype of Campium samoense): the greater part normal. Herus 107 (B): the greater part normal, several sporangia filled with abnormal spores only. Herus 107 (BM), Powell s.n. (isotype of Campium samoense, BM), Kajewski 893 (BISH): all or the greater part abnormal, or in part also with aborted S.M.C.'s.

Note. The mostly incomplete specimens are intermediate between those of $B$. quoyana and $B$. rivularis and likely to have sprung from hybridization. Copeland(1928) thought Campium samoense 'more particularly related to Campium lonchophorum' $(=B$. lonchophora). He later (Bull. Bish. Mus. 93, 1932, 15) thought the type specimen an aberrant individual 'better regarded a form of Campium lonchophorum', with which Christensen (1943) agreed.

D. 7. Bolbitis tonkinensis (C. Chr.) Iwatsuki

B. tonkinensis (C. Chr.) Iwatsuki, Acta Phytotax. Geobot. 18 (1959) 49; Tagawa \& Iwatsuki, Acta Phytotax. Geobot. 25 (1971) 18. - Egenolfa tonkinensis C. Chr. in Ching., Bull. Fan Mem. Inst. Biol. 2 
1931) 306; Tardieu-Blot \& C. Chr., Not. Syst. 7 (1938) 99; in Lecomte, Fl. Gén. I.-C. 7 (1941) 424, p.p.-T y p e: Herb. École Sup. Agric. \& Sylvic. Hanoi 3396 (Colani leg.), Vietnam, Tonkin, Lang-Son (BM; iso in BM, P, UC, US).

Spores with a smooth cristate perispore. Amply described by Christensen.

Vietnam. Tonkin. Long-Son: Herb. Ecole Sup. Agric. \& Sylvic. Hanoi 3396 (type). Gia-hoa: Herb. Ecole Sup. Agric. \& Sylvic. Hanoi 3412 (BM, C, UC, all ster.).

ThaILAND. N or th ern. Chiengrai: Winit 1022 (= E. Smith 1674)(K).

$\mathrm{H}$ a bit a t. Moist forest. Altitudinal records: $100 \mathrm{~m}$ (type), 550-590 m (Winit 1022).

Spores. Isotype (US): the greater part normal. Holotype: the greater part abnormal, mostly shrivelled. Winit 1022 (K): all abnormal.

Note. I agree with Christensen that the present species, based on two rather different collections one of which sterile, is closely related to B. sinensis. Christensen gave several differential characters of which the following more or less hold. Petiole and rhachis-the costa far less so-covered with \pm permanent scales; the dark colour of the dried leaves which is unlike that of $B$. sinensis agreeing with that sometimes found in B. appendiculata ssp. appendiculata; the shape of the lobes of the sterile pinnae, which is \pm subfalcate with an acute apex (usually straight and rounded in $B$. sinensis). The latter feature varies considerably when different parts of the type material are compared. The perispore is cristate whereas it is cristate-undulate in B. sinensis. A cristate but reticulate perispore is found in $B$. appendiculata. Here possibly also belong the specimens enumerated under $B$. sinensis, note $2 \mathrm{c}$.

\section{8. Acrostichum flabellifolium Link}

A. flabellifolium Link, Fil. Spec. Hort. Berol. (1841) 165; C. Chr., Ind. Fil. (1906) 8. - T y p e: a specimen cultivated at Berlin (n.v.).

Note. The fern was described from sterile material; the description may cover $B$. heteroclita. The name 'Acrostichum flabelliforme Link' (sphalm.) was found written on several sheets of mostly cultivated material of $B$. heteroclita.

\section{9. Asplenium ? setosum Presl}

A. $?$ setosum Presl, Rel. Haenk. 1 (1825) 42; C. Chr., Ind. Fil. (1906) 132; Holttum, Nov. Bot. Univ. Carol. Prag. (1968) 11.-T y p e: Haenke s.n., Philippines, Luzon (n.v.).

Christensen (1906) thought it a Diplazium. Copeland (Fern Fl. Philip., 1960, 418) regarded it as a synonym of Asplenium serrulatum Presl and suggested that the latter species might be an Egenolfia. Holttum identified the type of $A$. serrulatum as Diplazium esculentum (Retz.) Swartz. He further considered Asplenium setosum to be an Egenolfia. From the description given by Presl I judge $A$. setosum to represent Bolbitis rhizophylla (Kaulf.) Hennipman. 


\author{
D. 10. Campium punctulatum Presl \\ C. punctulatum Presl, Tent. Pterid. (1836) 239, pl. 10: fig. 22.-T y pe: unknown. \\ Heteroneuron preslianum auct. non Fée, Hist. Acrost. (1845) 92, pl. 39: i('Heteronevron'): auct.p.p.
}

In the original description Presl referred to Acrostichum punctulatum auct. non Swartz: Presl, Rel. Haenk. 1 (1825) 16 ('Acrostichum punctulatum Presl nec Lin.'). The name Campium punctulatum the epithet of which is regarded as new in the genus Campium, was validly published as it so happened that Presl gave data about its anatomy and venation pattern in the generic description. However, these data do not permit to ascertain its identity.

The drawing of the venation pattern does not apply to material of a species known from the Philippines, and therefore could not have been taken from Haenke's specimens upon which Presl based Acrostichum punctulatum auct. non Swartz. The drawing said to represent the venation pattern of part of a fertile leaf comes nearest to that of a sterile leaf of $B$. presliana (with which it agrees most), B. subcrenata, $B . \times$ lancea, or $B$. $\times$ prolifera. The anatomical data given are too general as to give further clues to its identity.

Prof. Holttum kindly searched for the type when studying Presl's herbarium (Holttum, Nov. Bot. Univ. Carol., Praq., 1968, 8). He traced a sheet with three sterile leaves the largest of which is similar to one of the leaves illustrated by Fée (1845) as Heteroneuron preslianum. The sheet is annotated as 'Campium punctulatum Presl' and 'In Luzon ad portum Sorzogon legit Haenke'. As B. presliana is endemic to southern India, the locality as well as the collector are erroneous. I have correlated the details of the venation pattern as given in Presl's drawing with that of the sterile leaves mentioned above as well as with sterile and fertile leaves of the type of Heteroneuron preslianum, assuming that Hügel's collection was available to Presl as early as 1836 . Presl's rather schematical drawing does not match the venation patterns of any part of any of the leaves in sufficient detail.

\title{
D. 11. [Panna Mara-maravara, Rheede, Hort. Malab. 12 (1703) 39, pl. 19.]
}

In the original description of Acrostichum flagelliferum ( $=$ B. subcrenata), Hooker \& Greville referred to Rheede. The identity of Rheede's plant cannot be ascertained as an herbarium of Rheede is unknown (Stafleu, Taxonomic Literature, 1967, 390), and the illustration rather poor. The plant represents either $B$. subcrenata var.prolifera, to which it was also referred by Beddome (Handb. Ferns Br. India, 1883, 437) (as Gymnopteris subcrenata), or $B$. heteroclita which is not indigenous to southern India but cultivated. The name is given here because of the medicinal use attributed to this fern by Rheede: 'Cum saccharo in syruptum redacta, tussim, morbum endemium; omnia pulmonum vitia, dispnaeam, morbum lunaticum, phthysin curat'.

\section{12. Leptochilus stolonifer Christ}

L. stolonifer Christ, Bull. Herb. Boiss. II, 6 (1906) 1004; v.A.v.R., Handb. Malayan Ferns (1908) 739.T y p e: Loher s.n., Philippines, Angilog. iii-1906 (n.v.). = Bolbitis spec. 
In the original description Christ referred this fern to Leptochilus cuspidatus (Presl) C. Chr. (=B. repanda). This fern may be synonymous to $B$. heteroclita, but the venation pattern as given by Christ is deviating.

\section{Sectio dubiosa}

D. 13. Sect. Lysidictyon Iwatsuki

Sect. Lysidictyon Iwatsuki, Acta Phytotax. Geobot. 18 (1959) 47.-T y p e: Bolbitis laxireticulata Iwatsuki. $(=$ spec. $d u b$.$) .$

Iwatsuki included three species in Sect. Lysidictyon, but mentioned the type species only. The only two other taxa mentioned in the discussion are $B . \times$ singaporensis (p. spec.) and B. sinenis (ser. Egenolfianae).

\section{Nomen dubium}

D. 14. [Bolbitis labrusca (C. Chr.) Ching]

[B. labrusca (Christ ex C. Chr.) Ching in C. Chr., Ind. Fil., Suppl. 3 (1934) 48, nom. nud._Leptochilus labrusca C. Chr., Ind. Fil. (1906) 386, nom. nud.].

Christensen (1906) referred to Acrostichum labrusca Christ, Ann. Mus. Congo Bot. V, 1 (1903) 10 (which is not listed in the Index), where it is not found as already stated by Copeland (Philip. J. Sc. 37, 1928, 402). As becomes clear from the preface of Christensen's Index, Christ assisted Christensen in various ways during the preparation of the MS of the Index. Christensen obviously included the name on the authority of Christ who expunged the species later.

\section{EXCLUDED}

E. 1. Bolbitis guianensis (Aublet) Kramer, Acta Bot. Neerl. 3 (1954) 486, comb. invalid. (Art. 33); Vareschi in Lasser, Fl. Venezuela 1 (1969) 376.-Polypodium guianense Aublet, Hist. Pl. Guian. (1775) 962.-Acrostichum guianense Baker, Fl. Brasil. 1 (1870) 591.-Gymnopteris guianensis Christ, Bull. Herb. Boiss. II, 1 (1901) 70.Leptochilus guianensis C. Chr., Bot. Tidsskr. 26 (1904) 288, fig. 1.-T y p e: $n . v .=$ Lomagramma guianensis (Aublet) Ching, Amer. Fern J. 22(1932) 17.

For heterotypic synonyms see C. Chr. (op. cit.) and Morton (Contr. U.S. Nat. Herb. $38,1967,65)$.

Note. With the otherwise Asian representatives of the genus Lomagramma the present species agrees as to the scandent rhizome, the articulated pinnae, the venation pattern, and the presence of paraphyses between the sporangia. It differs from the other species in the pinnatifid (not entire) terminal segment which is continuous (not artic- 
ulate) with the rhachis, and in the well-developed cristate perispore (scarcely visible or absent in the other species). According to Ching (op. cit.) it was Christensen's opinion to segregate the present fern as a distinct genus. Holttum (Gard. Bull. S. S. 9, 1937, 196) excluded this fern from Lomagramma and suggested this to be a rather unusually aberrant species of Bolbitis only. More recently Holttum (in litt.) agreed with Christensen. As the leaf shape can be regarded a retention of a juvenile condition as found in the bathyphylls of Asian species, the assignment of the present species to Lomagramma seems the best solution.

E. 2. Bolbitis laciniata (Hooker) Abeywickr., Ceylon J. Sc. sect. A, Bot. 13 (1956) 22, nom. invalid. (Art. 33). -Acrostichum variabile Hooker var. laciniatum Hooker, Spec. Fil. 5 (1864) 277.-Campium laciniatum Copel., Philip. J. Sc. 37 (1928) 354, pl. 7.Leptochilus laciniatus Ching, Bull. Fan. Mem. Inst. Biol. 4 (1933) 344. = Leptochilus decurrens Bl., see Sledge, Ann. Mag. Nat. Hist. ser. 12, 9 (1956) 865-877.

E. 3. Bolbitis macrophylla (Kunze) Maxon \& Morton, Bull. Torr. Bot. Cl. 65 (1938) 375.-Meniscium macrophyllum Kunze, Flora 22 (1839) Beibl. 44.-Phegopteris macrophylla Mettenius, Fil. Lechl. 2 (1859), 24, quoad nomen solum?-Nephrodium macrophyllum Keyserling, Polyp. \& Cyath. Herb. Bung. (1873) 48, comb. illeg., non Baker in Hooker \& Baker, Syn. Fil.(1868) 300.-Dryopteris macrophylla C. Chr., Ind. Fil., Suppl. 1 (1913) 35. - T y p e: n.v. = Thelypteris macrophylla (Kunze) Morton, Amer. Fern J. 61 (1971) 17.

N o te. The main argument for Maxon \& Morton to exclude this dimorphic fern from Dryopteris (s.l., including the thelypteroid ferns) was that the sporangia are not borne on a definite receptacle (as in all other species of this genus) but are regularly distributed over the whole surface. The thelypteroid identity of this fern became irrefutable after the study of the anatomy, the indument, and the spores. After my information (in litt.) to Mr. Morton, the fern got its proper place.

E. 4. Bolbitis mollis (Copel.) Ching in C. Chr., Ind. Fil., Suppl. 3 (1934) 49.-Campium molle Copel., Philip. J. Sc. 37 (1928) 390, fig. 41, pl. 29.-T y p e: G. Gardner s.n., Brazil ('Ceylon') (K). = Cyclodium meniscioides (Willd.) Presl.

Notes. 1. Hooker (Spec. Fil. 5, 1864, 262) referred the collection of Gardner to Acrostichum virens ( $=$ Bolbitis virens (Hooker \& Grev.) Schott). Hooker supplied a short description of the specimen and thought it 'probably a distinct species'. Copeland newly described this common American fern in a genus which at that time he confined to the Old World.

2. The specimen has been mislabelled; G. Gardner collected in both Brazil and Ceylon (Britten \& Boulger, A biogeographical index etc., 2nd ed., 1931, 119). 
E. 5. Bolbitis neglecta (F. M. Bailey) Morton, Contr. U.S. Nat. Herb. 38 (1973) 215.for complete synonymy see Hennipman, Blumea 13 (1966) 397. = Pteridoblechnum neglectum (F. M. Bailey) Hennipman, 1.c.

E. 6. Bolbitis ovata (Copel.) Ching in C. Chr., Ind., Fil., Suppl. 3(1934) 49.-Leptochilus ovatus Copel., Philip. J. Sc. 9 (1914) Bot. 229._Campium ovatum Copel., Philip. J. Sc. 28 (1937) 354, fig. 9, pl. 6.-Paraleptochilus ovatus Copel., Gen. Fil. (1947) 198.T y p e: C. J. Brooks 155.S., Sumatra, Bencoolen, Lebong Tandai, ii-1913 (iso in BM). $=$ Colysis $c f$. pedunculata (Hooker \& Grev.) Ching.

E. 7. Bolbitis rawsonii (Baker) Ching in C. Chr., Ind. Fil., Suppl. 3 (1934) 49.-Acrostichum rawsonii Baker, Ann. Bot. 5 (1891) 496.-Campium rawsonii Copel., Philip. J. Sc. 37 (1928) 400.-T y p e: Sir Rawson W. Rawson s.n., Mauritius, Grand River $(\mathrm{K} ?, n . v$.$) . = Species incertae sedis.$

Note. Apart from the description nothing is known of this fern. Copeland copied Baker's original description verbatim without comment. Alston (J. Bot. 77, 1939, 283-286) did not mention it. The type specimen which should be at Kew was not traced neither by me nor by Dr. F. M. Jarrett (in litt.). I exclude it from Bolbitis as according to the description the rootstock is erect.

\section{Excluded fossil:}

E. 8. Bolbitis coloradica R. W. Brown, U.S. Geol. Surv. Prof. Paper 221-D (1950) 49, pl. 12: fig. 6, 7.-T y p e: not indicated. = Astralopteris coloradica (R. W. Brown) Reveal, Tidwell \& Rushford, Bringham Young Univ. Geol. Stud. 14 (1967) 239, pl. 1-6.

Note. Brown placed this Cretaceous fern in Bolbitis because of certain resemblances of this fern to B. macrophylla (Kunze) Maxon \& Morton (= Thelypteris macrophylla (Kunze) Morton). Reveal et al. regarded it related to Drynaria (Polypodiaceae) because of properties of the venation pattern. This relationship seems doubtful in view of the derived character of Drynaria and the aspecific occurrence of the drynarioid venation within modern leptosporangiate ferns.

\section{INDEX OF COLLECTIONS}

The collections are referred to the number of the taxon in the Taxonomic Part. For mixed collections all indentifications are listed. Cultivated material and unnumbered collections are not included.

Specimens with institutional serial numbers are cited under these series. The collectors, who collected in such a series, are mentioned in the list with a cross-reference to the pertaining series.

Type specimens are marked with (T); they are not necessarily the type of the accepted species, but may belong to one of its synonyms. 
des Abbayes 403: 5; 438: 2; 593: 27; 798: 9; 2146: 2-Abbott 822: 22; 1388: 1; 1568a, 1627: 31; 2575, 2603: 1; 2607: 31; 2703a: 22; 2703c: 31-Abraham 649, 650: 16.a; 1065: 41; 1105, 1141: H.10; 1142: 11; 1143: H.10Achmad 447: 25-Achten 685A,B: 27-Adam 2747, 3225: 5; 3236: 9; 3248: 2; 4758: 9; 4762, 4763, 4768: 27; 4901: 5, 5185: 27; 5315: 9; 5387, 7425: 5; 7481: 2; 7800, 7861, 8023: 5-Adams 1064: 11-C.D. Adams 331 (= 2635), 415: 2; 427: 5, 518: 27; 1509: 5, 1892: 2; 1978 (= 3877): 9, 5327: 36_Addison, see SFAdelbert 23: 41-Aet (exp. Lundquist) 93: 25; 274: 32-Alcasid \& Edaño, see PNH-Alfaro 16535, 17179, 17180: 31-Allard 21558, 21672: 1; 22359: 23; 22367: 1-H. A. Allard 368: 36; 388: 5-B. E. G. Molesworth Allen 1377, 1434: 25; 1486: 26; 1514: 25; 1515: 26; 1538: 16.a; 1631: 25; 1696: 26; 1743: 25; 1783: 16.a; 1785 , 1813, 1870: 25; 1891, 2012: 26; 2052: 16.a \& H.11; 2069: 26; 2163: 19; 2164: 10; 2646, 2781: 16.a; 3005: 25; 3145; D.3; 3179, 3384 (T), 3405, 3419: 26; 3449: 25; 3489, 4149, 4365, 4556, 4557: 26; 4558: 25; 4731: 26; 4915: 25-P.H. Allen 2132: 22-Almeida 299: H.8; 311: 40; 870, 929: 14.b-Alston 5826: 31; 5830: $13 ; 6161: 31 ; 7654: 13 ; 7836: 28 ; 12755: 41 ; 13756,14266,14488,15375: 25 ; 15766: 32 ; 15774,16231: 41 ; 16462$, 16504: 25; 16656: 32-Amano 6735: 16.a-Amdjah 120p.p., 121p.p., 122, 127p.p., 284, 310: 26; 443, 444: 25; 445, 448, 449p.p., 480, 522, 526p.p.: 26; 562: 25; 563, 565, 577p.p.: 26; 600, 670: 25-Amdjah \& Koningsberger 31, 32: 16.b.2-Ames 41: 35; 93, (P) 180: 31-J. W. Anderson 88: 26-André 1093: 13; 1297 bis: 35-C. W. Andrews 90, 126:25-F. W. Andrews A1478: 5; A1627:2; A1717:5-Annet 6: 2; 219:3; 222: 2; 298: 5; 347: 3; 446: 20; 1384: 2-Antun-Gupffert 4: 36; 11: 5; 33: 27; 62: 2-Appun 882: 22-Arcutt 5207: 31-Aristeguieta 2776: 31-Aristeguieta \& Pannier 831, 1885: 24-Arsin HB 19443: 25-Asdat 54, 55: 25-Ashton S 18162: 25-Austen 54: 7; 60, 84: 16.a-Aviles 64: 31; 67: 22.

Bacani, see FB-Backer 138: 41; 523: 10; 649: 41; 1632: 25;6065: 41; 6103: 25; 7036: 41; 11846: 25; 13941: 41; 14467: 26; 21289, 21290, 21291: 25; 23045: 16.a; 23740, 25542, 26358: 25-Baker 2158: 18; 2185: 31Bákhuizen van den Brink 1433, 3035: 41; 3212: 25; 3558: 41; 4744: 16.a; 4954: 41; 6291, 6412: 26-Bakshi 204: 36-Balansa 150 (=95), 151 (=94): D.2; 798, 1561: 38; 1887: 25; 1891: 16.a; 1951: D.2; 2852 (T), 2853 (T): 13-Baldwin Jr 6096: 27; 6709: 2; 7064, 9482: 5; 9660: 36; 13763: 9-van Balgooy 1753: 38-Ballard 1525: 14.a-Barker 1158: 5; 1170: 2-Barnard 28: 25-Barnes 545: 11-Barrington 403: 29-Barter 1451: 2-Bartlett 6659, 6793, 7225: 25; 7721: 26; 8144, 15282: 25-Bartsch 230: 18; 307, 313, 405, 438, 445: 25; 528, 538: 18-Bates 369: 2-Bateson 71, 127: 32-Baudon 1053: 2; 1745: 5; 2244: 27-Baudouin 306, 869: 38-Baumann 535: 27-Beard 17, 18: 31-Beccari 587: 16.a-Beckett 8770: 6-hb. Beddome 783: 31-Beguin 1012: 32-Bélanger 4: 31; 491: 16.b.1; s.n. (hb. Cosson 2150) (T): 16.b.1.-Belcher 610: 16.b.1.-Bell 1988: 26-Belshaw 3624: 28-Bennett 17: 31; 18: 13-Benson 135A: 2-Bequaert 1133; 2; 1514: H.5; 1785: 2; 2096: 5; 2531: 2; 2912, 4831: 27; 6593: 5-Bernoulli \& Cario 361: 31; 382 (T):35-Berrie s.n. (Univ. Coll. Herb. Ibadan 761): 5-Betche 6: 38-Beumée 1108: 41-van Beusekom 83, 163: 16.a; 164: 15.a.1; 293: 25; 550: 26; 814: 16.a; 2602: 19; 2606: 15.a.1; 2303: 16.a; 3204: 8.b-Beyrich 3438: 13-Biolley 11, 2016, 10213, 17391: 31-Bischler 2418: 31; 2609: 28-Biswas 5083: 16.b.1-BKF Series (Forest Herbarium, Bangkok) 1368 (= Winit 927): 7; 1370 (= Winit 929): 25; 2785 (= Dee Boon Peng 332), 5785 (= Smitinand 950), 5825 (= Smitinand 1049), 6157 (= Smitinand 132): 16.a;6753 (= Smitinand 1352): 25; 8951 (= Phloenchit 365), 9899 (= Phloenchit 769) 16.a; 12728 (Smitinand): 15.a.1; 15333 (= Bunak 391), 16032 (= Bunak 743): 16.a; 16143 (= Dee 1031): 6; 19124 (= Smitinand 4114): 16.a; 24438 (= Smitinand et al. 6093): 8.b; 24724 (= Smitinand et al. 6214), 24743 (= ibid. 6616), 24785 (= ibid. 6648): 19; 28301 (= Phloenchit 2030), 29670 (= Phengkhlai 294): 16.a; 30207 (= Hambananda 357): 15.a.1-Blauner 303: 22; 303A: 24Bloembergen 4800: 25-Blomquist 13130: 1-Boivin 1581/2: 37; 2847: 2-Boldingh 440B: 1-Bole 1531: 40-Bolema 74: 36-Bolster 74: 18; 244: 25; 244A: 26; 355: 32-Bonati 88 ser B, 490: 38-Boom 1847: 41Bornmüller 463: 5-van Borssum Waalkes 603(T): 16.b.2-Bouillod 32, 33,46: 16.a-Bourgeau 2008: 35, 2147 : 31-Bowie \& Cunningham 26: 13-Box 381: 31; 2068: 36; 2068A: 2; 2082, 2083: 27; 2431, 2478: 2; 2853, 2854: 5; 2857: 2; 2859, 2864, 2867: 5; 2883: 36; 2889: 2; 2892: 27; 2906: 5; 2910: 2-Brade \& Brade 366 (= Ros. Fil. Costaric. exsicc. 166): 31; 367p.p. (T): 23; 369: 22; 370 (T): 24; 371 (= Ros. Fil. Costaric. exsicc. 315): 28; 443: 35; 552 (T): 22; 4308, 8256 (= Ros. Fil. Bras. exsicc. II.224), 8438 (= ibid. II.223): 13-Bradford 166: 16.a; ?1954 (T): 14.a-Braithwaite (RSS) 4090: D.6; 4130: 32; 4356, 4442: 25-Brandis 211: 15.a.1; 277: 16.b.1-Brass 5621, 12833: 32; 13242: 25; 13473: 32; 13833, 13856: 33; 13857: 32; 13902: 25; 20273: 34; 23417: 33; 23616: 32; 23627, 23629: 33; 24411: 32; 24986: 39; 25931, 27162: 32; 27916, 28008 (T): 39; 31692: 33-Brauer 10, 11, 12: 4-Braun 763: 2; 1894 (T): 36-Breedlove 34191: 24-Breedlove \& Raven 13554: 31-Brenez 6490, 19215, 20298: 31-Breteler 957: 36; 1019: 2; 1053, 1076: 5-Breteler, de Wilde \& Leeuwenberg 2425: 36-de Briey 1006: 9-Brinkman 425: 16.a-Britton et al. 634: 22; 899, 1910: 31; 2256: 21; 5217: 24; 5218: 31; 5258: 24-Broadway 2006, 4398: 31; 4676 p.p.: 1; 5825: 21; 6897, 9295 : 31-Brooke 8387, 9257: 26; 9818: 25-Brooks 12 (T), 13C D.3; 44.S, 61.S, 73.S, 75.S: H.13; 95.S, 99.S: 25; 142.S: 26; 145.S: 16.a; 202.S: 26; 226.S, 260.S (T): 25; 364.S: 26; 403.S: H.13;7924: 25—hb. Brousmiche 
'102': 38-Bruggeman 793: 41-BS Series (Bureau of Science, Manila) 68 (Foxworthy) (T): 25; 1823 (Ramos), 1897 (Foxworthy): 18; 3912 (Fénix): 25; 5309 (Ramos), 7155 (ibid.): 18; 7522 (ibid.): 32; 7529 (ibid.): 25; 7536 (ibid.): 26; 7542 (ibid.): 25; 7764 (ibid.), 8011 (ibid.): 18; 8142 (ibid.): 25; 9490 (Robinson): 26; 12984 (Fénix): 18; 13049 (Ramos), 13884 (ibid.): 25; 13955 (ibid.): 18; 13994 (ibid.): 26; 14816 (ibid.) (T): 25; 14871 (ibid.): 32; 15205 (Kienholz): 25; 17556 (ibid.): 32; 17676 (Otanes): 18; 19383 (Ramos), 22085 (ibid.): 25; 24825 (Edaño), 24829 (ibid.): 26; 24868 (ibid.): 32; 24968 (Fénix): 25; 26798 (Edaño), 27244 (Ramos): 18; 28088 (Fénix): 26; 28225 (ibid.): 32; 28542 (Ramos \& Edaño): 26; 28842 (ibid.): H.12.a; 28843 (ibid.): 25; 30031 (Fénix): 18; 30311 (Ramos), 30797 (Ramos \& Edaño): 26; 30847 (ibid.): 32; 31309 (ibid.): 26; 34103 (ibid.): 18; 36640 (ibid.), 36971 (ibid.), 37029 (ibid.): 26; 38756 (ibid.): 25; 39793 (Ramos): 18; 44024 (Ramos \& Edaño): 25; 46986 (ibid.) (T), 48855 (Edaño): 18; 76512 (ibid.): 16.a; 77179 (Ramos), 77186 (ibid.): 18; 78686 (Edaño), 78711 (ibid.), 78718 (ibid.), 78724 (ibid.): 25; 79653 (ibid.): 26; 80160 (Ramos), 80351 (ibid.): 25-BSIP Series (Forestry Department, Honiara, British Solomon Islands Protectorate) 2120 (Whitmore \& Grubb): 25; 17502 (Wall \& Nakisi): 32-Buchanan 118: 25 \& 38-Buchtien 273: 23; 1002: 1; 2131: 13; 2220 (= Ros. Fil. Boliv. exsicc. 85): 28; 2221, 2223: 13-Buchwald 357 (T), 358 (T): 36-Bües 1756: 22-Bunak 391 (= BKF 15333), 743 (= BKF 16032): 16.a-Bünnemeijer 3263, 8502: 26; 10914: 25-Burch 4481: 22-Burchell 3086: 13-Burger \& Stolze 4842: 31-Busse 3534: 5Büttner 57: 5; 184, 374: 27; 514: 2; 600: 27-Buwalda 2848: 25; 2857: 41; 5078: 32-Buysman hb.anal. 170: 41; 199: 25; 2021: 41; 2882: 16.a; 2885: 7-Bijhouwer 228: 24.

Cadière 36: 16.a; 38, 38bis, 69, 77, 77bis: 25; 91: D.1; 101: see D.1; 104: 16.a; 121: 25; 123: D.1; 127: 16.a; 129, 146 (T): D.1; 149 (T): 25; 169: D.1; 583: 43; 1116: 25; 1146: 19-Caha 92: 9-Caille 6: 5-Calderón 2010: 31 -Callens 222: $36 ; 656: 27 ; 843: 20 ; 1484:$ H.5; 1823: 27; 2219: 5 ; 2221: 3;2233: 5; 2810: 27; 2818: H.5; 2819: 20; 3183: H.6; 3184: 27; 3185bis: 5; 3188: 3; 4045: 5; 4087: 36-Campbell 4: 31-Cäpurro 217, 271 : 13-Cárdenas 2996: 13-Carr 11362: 32; 11362A: 25-Carrick \& Enoch JC/345: 26-Castro \& Añonuevo, see PNH-Cavalerie 3402, 3872: 25; 4230: 19: 7018, 7059, 7645: 25-CCC Series (Canton Christian College, Canton) 8687 (McClure): 16.a; 9346 (ibid.), 9436 (ibid.) (T): 43-Chandra et al. (all LWG nos.) 77703: 16.a; 77712: 40; 77718: H.8; 81111: 7; 81152: 6; 81154: 16.a; 81171, 83037: 19; 83038: 8.a; 89090: 25; 94132: 19; 95102: 14.b; $95145(\mathrm{~T})$ : 11-Charvet 79: $5 ; 160,184: 20$-Chase 3100 (= Govt.Herb.Salisb. 31808), 6638, 6638A, 7715: 27-Cheeseman 49: 25; 122: 32; 789: 38-Chevalier 529, 756: 27; 2541: 16.b.1; 2544: 15.b; 3760: 16.a; 4167: 43; 5776, 5786: 5; 5801, 6048: 27; 6252: H.5; 6253, 6887, 7348, 12779: 5; 14335: 2; 14797, 16669, 16675: 5; 17536: 2; 17545: 9; 20575: 2; 20617: 5; 20780: 2; 20798: 27; 20798bis: 9; 22682: 5; 24171 : 27; 29851: 8.b; 29889, 29903, 31791: 16.a-Chillou 2237: 5-R.C. Ching 8238: 16.a-Christophersen 221: 38-Chrysler \& Roever 4847: 31; 5151: 24-N.K. Chun \& C.L. Tso 44026: 16.a-Claessens 1242: 27Clarke 5464: 19, 5554: 6; 8244: 25; 8292, 8293: 7; 8294: 6; 8743: 16.a; 9090: 15.c: 11904, $11964: 25$; 13794: 7; 15213: 6; 16228, 18252: 19; 18494, 19362, 19366: 8.a; 19370: 19; 19398; 16.a; 19621: 17; 19653, 19707: 7; 19798, 19799, 19803: 25; 19840: 15.a.1; 1984l: 15.c; 19845: 15 (ster.); 19881: 16.b.1; 19887, 19888: 7; 19948: 16.b.1; 21839: 19; 27332: 15.c; 27604: 25; 31730: 7; 35127, 35148, 35547: 25; 35938, 35947: 6; 36180, 36582: 15.c; 36876: 19; 40868: 7; 40880: 19; 42452, 42858A: 8.a; 42976: 16.b.1; 42977: 15.c; 43003: 7, 44979: 19; 45156, 45231: 8.a-Clemens, N. Borneo: 4364: 16.a; 21469, 22001: 25; 22070, 27653, 28234: 26; Philippines: 552 (T): 25; New Guinea: 7990: 32-Clément 649: 31; 914: 24; 1322: 1; 2416: 24-Clute 283: 24-Cockburn 63: 11; 101: H.10; 109: 14.b-Colani 3396 (T), 3412: D.7; 4078: 19-Collie 95B: 38-Commerson 97: 18-Compton 1281, 1910: 38-Conover 1048, 1165: 43; 1751: 16.a; 1775:43-Conrau 33:2-Contreras 2260, 2455: 35; 3387: 31; 4245: 35-Cook 30: 5; 31: 9; 34: $2 ; 65: 27 ; 66: 9$ \& 27; 67: 5 \& 27; 138: 2; 163: 9; 167: 2; 452: 5-Cook \& Doyle 238: 31-hb. Copeland 245: 18; 249: 10; 251 (T): 25; 286 (= Brooks 12) (T): D.3; 290 (T), 1543: 25; 1565 (T): 26; 1580: 25; 1968: 18; 1969: 25; 1983: 18: 2029: 26; 2030: 25; 2116 : 18; 4342: 16.a; 4344: 18; 4351: 16.a; 10138: 7; 10157, 10158, 10161 (=Clemens 552)(T), 10163, 10167, 10174, 25; 10191: H.12.a; 10196, 10197, 10198: 32; 10199: 41; 10202: 25; 10207, 10212, 10214, 10215: 26; 11538 (T): $25 ; 11539$ (T), 11540 (T): 32; 13502: 26; 13580: 25; 17067: 16.a; 20299, 20304: 25; 20358: H.7; 20375, 20409, P.P.Exs. 33: 25; P.P.Exs. 85: 26; P.P.Exs. 176: 18; P.P.Exs. 278, 279: 25-Corner, see SF-Cornman 623, 644 (= Killip 2778, T): 22 -Cours 3410: 37; 3412: 2-Couzatti \& Gonzáles 596: 31 -Craib 350: 19_ Cremers 306, 307: 5-Cribbs 449: 38-Croizat 793: 42-Crüger 38: 31; 173: 21; 221: 1-Cuatrecasas 13254: 1; 16243: 22; Cuatrecasas \& Llano 24199: 22-Cudjoe 13: 27; 19: 2; 45: 36-Cuming 5 (T), 32: 25; 47 (T): 18; 104: 41; 105 (T): H.12.a; 152: 32; 157: 22; 161p.p.: 25; 161p.p. (T): H.4; 225 (T): 26; 225p.p.: 32; 269 (T): 18; 294 (T): 32; 1416 (T): 38-Cummins 10 or 231: $2 ; 70: 2$-Cunningham \& Bowie 1814: 13Curran, see FB-Curtis 35: 25; 612 p.p.: 16.a; 615: 26; 628: 15.b; 3371: 26.

Dahl 174: 32-Dale 40: 22-Dalziel 73: 27; 242: 5-Daniëls \& Jonker 820, 1245: 42-Daramola 40466: 5-Darko WACRI Herb. 893: 2-Dawe 534: 2-Day 334: 31-Deam 465, 6196: 31-Decary 18306: 
37-Dee 1031 (= BKF 16143): 6-Dee Boon Peng 332 (= BKF 2785): 16.a-Degener 14351: 38; 14410: 33; 14411: 38-Deighton 1057: 5; 1696: 27; 3028: 36; 4811: 27; 6019: 36-Deistel 115: 27-Demange 3547: 27-Demeuse 20: 36-Descoings 11319: 5; 11374: 20-Desvaux 4: 22-Devred 2734: 36-hb. Deyrolle 336: 22-Dickason 6621, 6657: 19; 6711: 15.a.1; 6789, 6849, 6867: 16.a; 7014: 15.a.2; 7029: 15.c; 7120: 16.b.1; 7766: 15.c; 9140: 19-Dinklage 2455: 27-Djamhari 562: 25-Docters van Leeuwen \& Docters van Leeuwen-Reynvaan 929: 25; 1121: 41; 1621: 10; 2007: 25; 2194: 16.a; 9135: 26; 9495, 10698: 32-Dodge \& Allen 17054, 17306, 17329: 31-Don 32847: 5-Donald 18: 2-Donis 1499: 2-Donk 6, 54, P92, 106: 41-Donnell Smith 2420, 2425, 2721, 6940: 31-Duchassaing 177: 22-Dümmer 698 (T): 36; 4471: 2Dusen 5040, 10136: 13-Duss 869: 31; 870, 1699: 22; 1705, 4059, 4144: 31; 4145: 22-Duthie 10654: 17; 26036: 6-Dwyer, Elias \& Maxwell 567: 31-Dyer 227, 230: 31.

Eames 122: 38-Eberhardt 394: 25; 409: 16.a; 427, 1953: 25; 3023: 16.a; 3726bis: 25; 5194: 16.a; 5233: 25-Edaño, see BS and PNH-Edelfeldt 53, 111: 32-Eggers 2755, 2755b, 4915: 24; 5223: 1-Ekman, P1. It. Regnel. III 1970: 24; 2753: 31 ; 2766, 3323: 24; 5049: 31; 7018: 1; 7242, H.18512: 31; Pl. Ind. Occ. H. 3793: 31; 3794, 4379: 24; 4380: 31; 4408: 1; 8987, 10257: 24; 10326: 31; 11197: 22; 11436; 1; 14292, 14375, 14951: 24; 15195: 22; 15282: 1; 15296: 31-Elliott 31: 5-Elmer 6457: 18; 6703: 25; 6705: 18; 7629, 7842, 9041: 26; 9705, 10041, 11209: 25; 13468, 13468a: 32; 15899: 18; 16281: 26; 16793: 18; 17554: 25; 17753 : 18; 18022: 26; 18068, 22303: 25; 22333: 18-Elsener H 127: 26-Endert 2949: 26-Endres 19: 23; 44: 31Engel 14: 31-Engler 619 (T): 36; 3382: 27-Englesing 83: 1; 296: 24-Everett 281: 41-Evrard 655: 25-Exell 572: 2-Eyerdam \& Beetle 22762, 22765: 13.

Faber 1042: 25-Fairbank 1500: 14.b; 1501: H.8; 1502: 7-Fanshawe 3834 (field no. 1098), 3881 (field no. 1145), 4822 (field no. 2086): 42-Farron 4115: 2-Faurie 279: 18; 281 (T): 6; 282, 637: 43; 650: 16.a; 4562: 43-FB Series (Forestry Bureau, Manila) 8982 (Curran): 18; 16663 (Bacani): 25; 19588 (Curran): 18-Fée 20: 22-Félix 41: 2; 41 a: 5; 42: 3; 933 (T): 27; 1002: 5; 1147 (T): 36; 1753: 9; 7505: 5-Fendler 63: 1; 63y: 31; 101 (T): 21; 311: 13; 336: 31-Fénix, see BS-Ferguson 207: 16.a; 210: 14.a; 213: 16.aFiebrig 91, 96: 13-Finck 110, 198: 31-Finsch 18: 25-Fischer 1937: 16.a-Flamigni 7: 27; 35: 2 \& 36; 50: H.5; 61, 71: 2; 72: 9; 80: 20; 120: 27; 141: 3; 187: 20; 187A: H.5-Fleischmann 7: 13-Fleury 29:2-Floyd 3503, 5553: 32-Foote 2: 36-D. M. Forbes 157: 31-H. O. Forbes 549: 16.a; 551: 43; 1223: 32; $1226: 26$ Fosberg 11471: 38: 37296: 43; 37801: 25-Fox, see PNH-Foxworthy, see BS-Franc 490: 38-Franck 37: 32-Friedrichsthal 1044: 31-Fuertes 740:31; 772: 22; 1210: 1; 1439: 24; 1570: 1-Fung 20146: 43.

Galathea Exp. 2691: 26; 2858: 16.b.2; 2859: 26; 2900: 16.b.2-Galdermans 26c: 36-Galeotti 6572: 31-Gallatly 64: 25; 67: 16.b.1; 296: 16.a-Gamble 8556: 17; 10571: 16.a; 14815: 14.b; 15458: 16.a; 15921 (T): H.8; 18326: 14.b-Gammie 272: 25; 275: 15.c-Gardner 15, 16: 16.a; 217: 13; 1160: 16.a; 1161: 6; 1234, 1314: 16.a-Garnier A 1202, A 1204, 1448: 31-Garrett 223: 25; 594: 19-Gascoyne-Cecil 22, 27, 31 , 88: 28-Gaudichaud 23, 24 (T): 16.a; 157: 13-Gentle 9164: 35-R. Germain 29, 39: 16.a-R. G. A. Germain 4410: 5; 4592, 5239: 2; 8604: 20; 8605: H.5; 8786: 36-Geijskes 1008: 42-Ghiesbrecht 370: 29; 407: 31; 409: 1 \& 13-Gillespie 2606: 38; 2622: 33-Gillet 824: 2; 2309: 36; 2317: 20-Gironcourt 404, 461: 2-Glaziou 369, 953 (T), 1671, 2422 (T): 13; 15715: 22-Gledhill UIH 10475: 27-Glynne 242: 27-Gocker 122: 2-Godefroy (exp. Harmand) 852: 16.a-Goméz 2308: 31-Goodby 26: 32Gossweiler 58: 27; 6591: 2; 7647: 20; 7695: 27; 9037: 36; 13910: H.5; 14069: 5-Gough 60: 11; 61: 14.bGoy 436: 34-Graeffe 108: 33; 1045: 38-Graive 36: 38-M. L. Grant 3579, 3967: 38; 10463, 10899: 13-V. Grant 1010: 31-de Granville 1219, 1499: 13-Greenway 2810, 4154: 27-Gregory 243D: 2Grether 3601: 32-Grether \& Wagner 4036, 4218, 4583: 32-Griffith 16: 16.a: 40: 25; 93: 16.a; 188: 25; 2820: 6-Grote 5790: 27-Grubb et al. 1651: 28; 1710A: 12-Guinea 416, 420, 855: 3; 972, 1222: 2Guppy 98: 33; 99: 32-Gutierrez, see PNH.

Hahn 10: 22; 1186: 31-Hallberg 1597: 29-Halle 96, 279: 36, 417, 1929: 2; 2300: 5; 3906: 3; 3919, 4009: 9; 4030, 4231: 2-Hallé \& Cours 5920: 2-Hallé \& LeThomas 221: 20-Hallé \& Villiers 5043: 20 \& H.5-Hallier 4136: 26; 4128: 18-Hambananda 357 (=BKF 30207): 15.a.1-Hance 23: 43-Hancock 10: 25; 42: 16.a; 56, 176: 25-Haniff \& Nur, see SF-Hansen, Seidenfaden \& Smitinand 11300: 8.b-Hansen \& Smitinand 11816: 16.a; 11836: 15.b; 12205: 16.a; 12967: 15.a.1-Hara et al. 6305227: 25-van Hardeveld \& van der Werff 322: 16.a; 323: H.10; 323A, B: 11-Harley F12: 5; F15: 36; F56: 5; F76, F76A: 2; F77: 5; F86: 36; F111, F114, F114A: 2; F120: 36; F152, F152A: 2; F159: 9; F168: 5; F180: 2-Harmand (exp.) 96 (T): 16.a-Harris 7411: 24-Hart 63: 31; 333: 22; 345: 1; 6255: 21-Hartley 9873: 32-Hartmann 88: 32-Hassler 662, 10377, 11633: 13-Haswell 115: 5-Hatschbach 10755: 13-Hattori 29: 43-Hatusima 20117, 20153: 16.a-Haught 1322: 1; 1432: 22; 1774: 13; 2037: 22; 4293, 4629:31-Hayes 2: 31 - Heinson 14: 2-Helion 100: 31-Hellwig 366: 32-Helms 1230: 34-Henderson, see SF-Hennipman 3049: 25; 3119: 16.b.1; 3136: 15.a.1; 3140, 3158: 19; 3159: 15.a.1; 3229, 3229A, 3318: 19; 3320: 16.b.1; 3353: 19; 3361: 6; 
3389: 19; 3488: 16.b.1; 3527: 16.a; 3532: 15.a.1; 3536: 6; 3624: 19; 3632: 8.b; 3637: 6; 3688: 10; 3721: 16.a; 3800: 25; 3802, 3835: 16.a; 3836, 3983: 25-Henry 81, 81A: 18; 10762, 10825: 25; 12494 (T): 19; 12907 : 25-Hepper 1476, 1496, 1722: 5; 1826: 27-H. M. S. Herald 573: 32-van Hermann 3203: 24-Herminier 9: $22 ; 10,12: 31 ; 14: 22 ; 19: 31 ; 20$ (=Fée 20): 22; 21: 1; 22: 31-Herre 294: 32-Herter 3993, 4008: 13Herus 107: D.6-Heudelot 702: 5; 805 (T): 27-Heyde \& Lux 4694, 4695, 6290: 31-Hieram 2444: 24; 6477: 31-Hildebrandt 1803: 2-Hill 54: 34-Hillebrand 334: 31-Hinton et al. 11050, 14679, 16305: 31 - Hodge 6512, 7044, 7072: 28-Hohenacker Pl.Ind.Or. 605: 14.b-Holst 2277 (T), 4268 (T): 36-Holstvoogd 793: 26; 809, 873: 19-Holttum, see SF-Hombersley 41: 1; 61a,b: 21; 254, 404: 31-Hoogland 4156: 32-Hooker \& Thomson 104: 11; 108: 6; 110: 7; 329: 16.b.1; 598: 25-Hooper \& Ramaswami 39418: H.10-Hope 60p.p.: 16.a-Horich, Univ. Calif. Bot. Gard. Acc. no. 58544: 31-Horne 22: 38; 166: 4; 814 : 33-Hose 159 (T), 211: 25-Hosokawa 7901: 41-Hosseus 217: 19-Howard et al. 15553: 22-Hoyle 704: 5-H. H. Hu 156: 43-Hügel 2161 (T), 2165 (T): H.8; 2424: 16.a; 3854 (T): 40; 4140 (T): H.8; 4636: 18 ; 4974 (T): H.8-Humbert \& Cours 22971: 37; 22972: 2-Humblot 258: 2; 300 (T): 37-Hume 7306: 26; 8170: 25; 8459, 8683, 8685, 8738: 26; 8946: 25-Hunnewell 19543: 35-Hürlimann 450: 38-Hutchinson \& Wright 3712: 23-Hyland AFO 2671: 34.

Iboet 531: 26-Idrobo 478: 13-Idrobo \& Schultes 1113: 23-Irvine 1179: 5; 2615: 2-Ito 232: 43Iwatsuki 2940, 4022, 4114, 4648: 43; 4769, 4835: 16.a; 4884: D.4; 4904: 16.a; 4928, 4989: 43; 5027: 16.aIwatsuki \& Fukuoka T3427: 19; T3959: 15.a.1; T5486: 16.a; T7169: 19; T10915: 25.

Jacobs 7950: 25-Jaeger 632: 9-Jans 503: 20-Jarrett 600: 14-Jarrett, Saldanha \& Ramamoorthy HFP 840: 11; 929: 14.b; 990: 11-Jate 75: 31-Jaurequi 58: 31-Jefford, Juniper \& Newbould 2720: 5-Jeffrey 313: 2; 584, 759: 4-Jenman 26: 31; 1463: 42; 2142: 22-Jentle 3301: 31; 9164: 35-Jermy 2638, 2639, 2640, 2641, 2642: 21; 2875: 1; 2906, 2907: 21; 3101: 1; 3710, 3740: 33; 3741: 32; 3742: 33; 4431, 4445, 4492, 4530, 4945 (= T9615/6), 4992, 4993: 32_Jiménez 1014: 24; 2372, 3303, 3878: 31-Jochems 579: 26Johnson 883: 35; 1165: 31-H.H. Johnston 28: 5-I.M. Johnston 406: 22; 482, 1321: 31-Johnstone 9: 14.b; 89: 27; 450: 22-de Joncheere BCO 23: 36-A.P.D. Jones \& Onochie FHI 16953: 5; 17514: 20; 18601: 27; 18744: 5; 18931: 9-E.W. Jones 3965: 5-T.S. Jones FB11: 5; 295: 27; 321, 348: 5; 352: 9Jordan 876: 5-Junghuhn 56: 16.a.

Kairo \& Emos, see NGF-Kajewski 893: 33 \& D.6; 1763 (T): 33-Kalbreyer 24: 2; 160: 5; 970: 23-Kao 6748: 16.a-Kappler 8: 22-Kari 183: 19-Karigone 69 (T): 25-Karsten (Crypt. no.) 165 (T): 1Kassner 3132: 2; 3409: 36-Katsumatra Hongkong hb. 6692: 43-Kaulback 250: 25-Keay FHI 22253, 25481: 5; 28286: 27-Keay \& Onochie FHI 21549: 5; 21730: 2-Keith 470: 25-Kennedy 2673: 2Kenoyer 37: 31; 43: 22-Kerr 2268: 16.b.1; 2587: 25; 6850: 16.a; 8218: 8.b; 8515: 16.a; 8655: 6; 8903: 25; 9751: 10; 10502, 12013: 16.a-Kersting 701: 27-Keysser 57: 25; 151: 32-Kiah, see SFKienholz, see BS-Killip 2753, 2778 (T): 22; 2804: 31; 2846: 22; 2863: 24; 2893, 2918, 35028: 22; 39980: 31-Killip \& Garcia 33530: 22-Killip \& Smith 22626, 22995, 23612, 23722: 13; 25184: 28; 25283: 13; 26039: 30; 26174, 26769: 23; 27797: 13; 27815: 22; 28495: 12; 28919: 13-C. King 42: 25; 83, 144 (T): 32; 265: 25; 269: 32; 284: 25-King's Collector 335: 16.a; 354: 26; 1097: 25; 1322: 16.a; 3084, 5797, 8398: 26; 8660: 25; 10933: 16.a-Kirk (Livingstone exp.) 20: 5-Kjellberg 408: 25; 2506 (T), 3635 (T): $32-K l e i n 912$ (T): 16.a-Kleinhoonte 635: 16.a-Kleinschmidt 165, 166: 38-Kloss 6496, 6531, 6741: 16.a; 14805: 26Klotsch? 66: 13-Klug 1873: 23-Knight P-29208, P-29302: 31-S.P. Ko 56007: 25-Koechlin 5272: 27; 8110 (811C?): 9-Kondo \& Edaño, see PNH-Koorders 119: 16.b(ster.); 17112, 17118, 17121: 25-Korthals 153: 25; 205: 41-Kostermans 2673, 2909: 32; 9518: 25; 18206: 41-Kostermans \& van Woerden 181: 25C. C. Kou \& M. T. Kao 4302: 16.a-Kramer \& Hekking 3119: 42-Krukoff 10228: 13-Kuhn 5448,6261, 6530: 1-Kunkel F-32: 27; F-34: 36; F-51: 2; F-56: 9; F-57: 2; F-132 (T): 5; F-200 (T): 2-Kuntze 5017: 16.b.2-Kupper 337: 23; 493, 739: 31; 1623: 28-Kurata 946, 5883: 43-Kurita \& Nakaike 1113 (= Pterid, Jap. exsicc. 52): 43.

Lace 4508: 16.b.1; 4900: 19; 5210: 7; 5025: 16.b.1-Lahaie 766: 32-Lamont 989: 43-Langlassé 705: 31Lanjouw \& Lindeman 2317, 2374, 2383: 42-Lankester 583: 24; 588: 28; 606: 31; 811: 24; 835: 23; 949: 22Larsen 8908, 8909, 8910, 8911: 16.a; 8912: 15 (ster.); 9588: 25-Larsen, Smitinand \& Warnecke 560: 19S. K. Lau 2389, 28113: 16.a-Laurent 446: 2; 646: 36; 1557: 2-Lauterbach 66: 32-Lebrun 4383: 2Lechler 2305: 13; 2373: 22-Lecomte \& Finet 1796, 1813: 8.b-Ledermann 689, 914: 2; 1158, 2125, 2863: 5; 7840: 33; 8574, 10487a: 32; 12278, 13176, 13279, 13765: 25-Ledoux \& Huyghe 23: 2-Leeuwenberg 3834, 5409, 5831: 36; 6088, 6681: 2; 6741: 36; 6797: 2; 7091: 36-Leeuwenberg \& Voorhoeve 4781: 2; 4813: 27-Lefevre 586: 16.a-Lehmann 48: 24; 8927: 22-Leland, Chase \& Tilden 87: 38-Lellinger 912: 22Lemos Fróes 21474: 42-Lent 292: 24; 2215: 23-A. Leonard 297: 36-E. C. Leonard 8582: 24-E. C. Leonard \& G. M. Leonard 14322, 14364: 31-Lépine 92: 38-LePrieur 17: 22; 61: 42; 62: 22; 184a: 42- 
LeRoy A18, 61: 2-Lescrauwaet 213: 27-LeTestu 1543: 20; 1547: 9; 1550, 1564: 2; 1570 (T): 3; 1589 (T): 5; 2184: $3 ; 2533$ : 5; 3075: 2 ; 3076: 5-Letouzey 2396: 5; 2923: 36; 2925: 20; 2925bis: H.5; 3081: 2; 3099: 36; 3289: 20 \& H.5; 3292, 3383: $2 ; 3451: 5 ; 3452: 27 ; 4081,5096,5409: 2 ; 5410: 5 ; 5423: 36 ; 5758: 5 ; 9031: 3$-Levy 301: $31 ; 472: 35 ; 1458: 31 ; 1460: 24$-Lewis et al. 2634, 2649,3242:31-H. Y. Liang 62714,63187:16.a-Liben 3223: 27-Liebmann PI. Mex. 2446 (T): 29; Pl. Mex. 2689(T): 44-Lienchow Hongkong hb. 5075: 25; 5113: 16.aLindemann LBB 12080: 42-Linden 1573, 1751:31; 1759: 1;2117:24-Linder 968:5;976: 36; 1150:9; 1154:27; 258 (T): 28-Lindley 1837: 16.a-Lindman A 2337, A 3061 (T): 13-Lister 36: 19; 93: 16.b.1-Lobb 200, 203: 16.b.2-Lockhart 64: 31-Löfgren \& Edwah 4498: 13-Loher 813: 18; 816: 10 \& 25; 890, 891: 25; 1262: 18; 14785, 14792, 14794: 25-Longfield 13: 2; 16: 36-Loret 752bis: 2; 1161 : 9-Lörzing 4482: 26; 5322: 16.a; 5386b: 26; 5527, 5895: 25; 6993: 16.a; 7365, 11923: 25; 12365: 16.a; 12404, 12405: H.13-Lötschert 149: 31-Louis 298: 2 ; 589, 6775, 7141, 7491: 36; 10864, 11830: 20; 12196: 2; 12196A, 13443: 36; 13595: 20; 13979: 36; 14065: 2; 14379: 36-Lovi 1, 11, 19, 20: 2; 23: 5-Ludwigs 7: 5-Luetzelburg 929, 7180(T), 18962: 13; 21294: 42-Luja 17: 27; 23: 5; 216: 20; 421, 456: 27-Lundell 17667: 31-Lütjeharms 3598, 3944, 4040: 25 .

MacDaniels 1279, 1620: 38-Macedo 2437: 13-MacGillivray 166: 13-MacLeay 227, 429: 5-Maguire 24744 (T): 13; 24814 (T): 42-Maitland 667: 2-Malhotra 137: 25-Mangubat 388: 18-Mann 133: 2; 133? (T): 9; 442 (T): 3; 1049 (T): 20-Marie 5: 22; 151, 188: 2-Matthew 1796: 28-C. G. Matthew 28: 19; 34: 15.b; 614: 41-Matthews 1107: 28-Matuda 3379: 31; 18605: 35-Maxon 3908, 4164: 24; 4685: 31; 5745: 28; 8561: 24; 9316, 9324: 22; 9327, 9350: 1; 9357: 24-Maxon, Harvey \& Valentine 6814, 7378, 7524, 7811: 31-Maxon \& Killip 40: 22; 550: 24-Mazé 92: 31; 336, 366bis: 22-McClure, see CCC-McGregor 659: 18; 662: 25-Mclvor 4 (T): 11; 7: 16.a-McKee 1612: 32; 6109, P8261: 16.b.1-McLean et al. 222: 32Meebold 5646: 25; 6225: 19; 7734: 40; 7753: 14.b; 10905: 11; 10906: 14.b; 12200: 11; 17038: 15.a.2; 17039: 25; 17040, 17041: 16.a-Melinon 364: 22; 367, 369: 42-Mell \& Mell 207: 22-Melville 3695: 34-Melville \& Hooker 61, 377A: 5; 377B: 9; 455: 36; 630: 5-Mendoza 1368 (T): 13-Mendoza \& Convocar, see PNHMendoza \& Steiner, see PNH-Merrill 1236: 18; 1391, 1397, 3128 (T): 25; 5062: 18; 5883, 7259, 7266: 25; 7267: 10; 8285: 25; 9243: 18; 10469: 26-Messmer P6854, P6856, P6932: 34-Metcalf 7380: 43-Meijer 545, 2972: 26-Mexia 1233, 1287: 31; 6186a: 22; 7260: 28; 9186a: 31-Mickel 1308: 31; 2785: 22; 2849: 35; 3120: 23-Miers 22: 13-Mildbraed 2215, 2330: 5; 2332: 2; 3134: 27; 4196: 2; 5980, 6286: 3; 6421, 6944, 7292: 2; 7364: 5; 7380: 27; 9132: 5; 9404 (T): 27-Millar \& Holttum, see NGF-Miller 1226: 22-Milne 337 (T): D.6-Milne-Redhead 4081: 27-Mjöberg 15, 49, 53: 26-Moewis 34: 16.a-Molino 6719: 31; et al. 17319: 22; 17639: 31-Möller 66: 2; 67: 5-Monod 12137: 3-Monod de Froideville 654: 16.a-Moon 58: 16.a-Mooney 675: 15.c; 677: H.8; 1468, 4097: 19-Moore \& MaxCetto 6237: 31-Moritz 129, 447: 13-C.V. Morton et al. 2540, 3322: 31; 3334: 24; 3336: 31; 3336: $1 ; 4396: 31 ; 7587 ; 7635: 35 ; 7740: 31 ; 7757: 35 ; 7828$ : 31-F. Morton 302, 395, 469:31-J. K. Morton SL 1266: 5; A 3973: 27-Mosén 75 (T), 2258, 2534: 13-Motley 427: 25-Mousset 107: 41; 169: 10; 170, 199: 41; 227, 562: 10; 873: 19 \& 41-Mugnier-Serand B2, 3: 5Mullenders 2248: 36-Munch 19 (T): 13-Murray 25: 31-Murton 10: 15.b.

Namen, see SF-Naumann 763: 43-Nayar et al. LWG 44621, 45058: H.10; 45552: 16.a; 50905: 15 (ster.); 50913: 19; 50931: 25; 50932: 7; 50936: 16.a; 63414: 8.a; 63417: 16.b.1; 63419: 19;63421: 6; 63434: 25; 63446: 16.b.1; 77002, 77005: 16.a; 77006: 19-Nere 961, 2207: 2; 2226: 20-Newbould \& Harley 4447: 5-Newbould \& Jefford 1287: 27-NGF Series (New Guinea Forces/Forests, Lae) 15887(Millar \& Holttum), 18792(ibid.), 18793 (ibid.), 30981 (Kairo \& Emos): 32-Nickles 2: 5; 91: 27; 92, 93: 3-Nielsen 1667: 5-Nishida hb. Chib. 78, 304: 43; 503: 16.a; 676: 25-Nooteboom 681: 15.a.1-Norris 46: 16.a-Norton 1058, 1059: 43-Nur, see SF-Nyman 403b: 25; 1074: 32 .

Oberwinkler 14678: 21 \& 24-Ochioni 14: 13-Ogata 127: 25-OHBA 662254:43-Onochie FHI 36405: 27-van Ooststroom 13644: 41-Ostenfeld 147: 38-Othner 160: 13; 167: 21; 168:1-Otto-Surbeck 397: 16.a.

Pabst 4302 (= hb. Bradeanum 4359): 13-Palmer 9, 10: 38-W. Palmer \& Bryant 129, 300: 41; 555, 667: 26; 716: 25; 847, 1067: 41; 1268: 16.b; 1346: 41-Pancher 3: 38-Panigrahi 19543: 19-Paniza, see PNHParish 60(T): 19; 63: 15.a.1; 69: 16.a \& 19; 70, 71: 19; 96: 16.b.1-Parks \& Parks 20528, 22043, 22153, 22508: 38-Paroisse 24: 2-Parrett 10: 38-Parry 504: 19; 589, 600, 603: 16.b.1-Peekel 14: 32-Perkins 1159:1Perrier de la Bâthie 1004, 7488: 2; 7489: 37; 7490 (T): D.5; 7979: 2-Perrottet 1473: 11-Pervillé 74: 4Pételot 25, 2716, 2886: 25; 3955: see D.1; 4083: 16.a; 4204: 25; 4205, 6270: 16.a-Peter 10301, 14039: 27; 16901: 36; 18129: 5 \& 36; 18921: 2; 21508: 27; 21872: 36; 21879: 2_Phengkhlai 294 (= BKF 29670): 16.aW. R. Philipson 10130: 38-W. R. Philipson \& Idrobo 1781: 1; 1796: 35; 2055: 31-Phloenchit 365 (= BKF 8951), 769 (= BKF 9899), 2030 (= BKF 28301): 16.a-Pichi Sermolli 1970, 1971, 2523: 27; 5240:5-Pierre 166: 16.a; 1269: 16.b.1; 1300: 16.a; 1300bis: 8.b; 5723:25-Pinto \& Bischler 223: 12-Pittier 361, 3096, 3279, 
3545: $31 ; 4225: 28 ; 5162: 31 ; 5336: 13 ; 5607: 31 ; 5608,6066: 13 ; 6554,7517: 31 ; 7632(T): 35 ; 8016,9108: 24$; 10614, 11975: 31-Pleyte 9: 41; 452: 32-Ploem 16455: 16.a-Plowes 2051: 27-Plowman 2132: 23Plumier '26': 31-PNH Series (Philippine National Herbarium, Manila) 510 (Edaño), 511 (ibid.): 25 ; 524 (ibid.), 532 (ibid.): 26; 790 (ibid.), 3846 (ibid.), 3866 (ibid.): 25; 3867 (ibid.): 26; 3909 (ibid.), 3914 (ibid.), 3945 (Fox), 4529 (Edaño): 25; 4530 (ibid.): 18; 4531 (ibid.), 4541 (ibid.), 4555 (ibid.): 25; 4748 (Alcasid \& Edaño): 26; 5060 (Fox), 5213 (Edaño) (T): 25; 5360 (Alcasid \& Edaño): 18; 5644 (ibid.): 25; 5971 (Castro \& Añonuevo), 6626 (Edaño): 26; 8593 (Quisumbing): 25; 8608 (Mendoza \& Convocar): 32; 8811 (Edaño) (T): 25; 8904 (Fox) (T): H.12.b; 9243 (Paniza): 25; 11176 (Edaño), 11226 (ibid.): 26; 11238 (ibid.), 11252 (ibid.): 32; 12577 (ibid.), 13904 (ibid.): 25; 15182 (ibid.): 26; 16588 (ibid.), 16693 (Sulit \& Conklin): 18; 21374 (Edaño): 25; 21388 (ibid.): 32; 35867 (ibid.), 38631 (Kondo \& Edaño), 38696 (ibid.): 25; 41373 (Mendoza \& Steiner): 18; 41800 (Sinclair \& Edaño): 25; 55302 (ibid.): 26; 78327 (Gutierrez): 25; 93809 (Edaño): 26Pobéguin $8(=2227): 5$; 37: 27; 46: 20; 50, 50bis: 2 ; 2227 (=8): 5; 2256: 27-Pogge 1620 (T): 36-Poilane 2439: 16.a; 2482: 25; 2544: 15.b; 2564: 8.b; 3314: 25; 3409, 3640: 16.a; 6364: 25; 7484: 16.a; 7593: 25; 7603, 9544: 16.a; 10572: 25; 11388: 16.b.1; 18179 (T): 16.a; 19467: 25; 21668 (T), 23686, 23787: 16.a; 23888, 24408: 19; 25142: 25; 25144: 16.a; 27770: 25; 28761: 19; 28825: 8.b-Pollard \& Palmer 147: 1; 164: 24-Popta 145: 41-Posthumus 606: 16.a; 1103: 10; 1106, 1112: 25; 1126: 10; 1128: 25; 1331, 1369, 1554:41; 1611: 25; 1677: $41 ; 1912: 10 ; 2280: 32,2323,2331: 25 ; 2715,3373,3440,3704: 41 ; 3704$ A: 19; 3708: 10;3806: 25; 3828: 33; 3891: 32; 4049: 16.a; 4093: 41-Pourret 28: 2-Powell 66 (= Kew 1173): 38-Preuss 132: 20; 133, 179: 2; 205: H.5; 206: 20; 315, 358b: 2; 363: 27; 882: 3; 1112: 2-Price 163: H.7; 254, 255: 18; 259, 265: 25; 305, 306, 307, 308: $26 ; 351,452,479,487: 25 ; 531$ : 18; 532: 41; 551, 604: 26; 623: 18; 673: 25; 680: 26; 763: H.12.b; 873: 41; 884: 16.a; 1078: 25; 1993: 10; 2120: 41; 2518A,B: 25; 2805: H.12.b-Proctor 4127: 24; 4280: 22; 19262: 31; 21481: 24; 21482: 22; 21869: 24; 21999: 1; 22105: 24; 22475: 21; 27455:31-Pterid. Jap. exsicc. 48(= Serizawa 11713): D.4; 52 (= Kurita \& Nakaike 1113): 43-Purpus 4354, 4355, 4371, 4471: 44; 6865, 6871: 31; 7246: 35; 11142, 12082: 44; 16600: 31; 16723: 44-Purseglove 4530, 5242: 26-Pynaert 78: 20; 90: 36.

Quayle 140, 2228: 38-Questel 2831: 31-Quintas F1. Afric. exsicc. 34: 2; 66, 68 (T): 3-Quiros 675: 31-Quisumbing, see PNH.

Raap 862: 25-Raciborsky 127, 153: 41-Rahmat si Boeea 5374, 5577: 25-Ramos, see BS-Ramos \& Edaño, see BS-Rant 388: 32; 1036, 1042: 25-le Rat 2887: 38-Rawson \& Rawson 890: 4; 1008: 18; 1058: 2; 1059: 4; 1061: 2; 1301: 26; 2999: 2; 3180: 31; 3350: 16.a; 3410: 1-Ray 2139: 22-Raynal 9880: 20; 10030: 5; 10264: 27; 10678: 5; 10705: 3; 12981: 27-Rechinger 626: 32; 783; 32 \& 38; 1064, 1606: 38; 3650: 32-Regnell ser. III 1439: 13-Reimschüsel 10: 32-Reinecke 5, 5a: 38; 5b: 32 \& 38; 5c, 5i, 104: 38-Rensch 52, 576:41; 630: 19; 636 (T): 10; 1225: 41-Reynolds 744: 24-Ribourt 111: 38-Richards 756: 42; 2233: 25; 3242, 3316, 3393 : 2; 3735: 5; 3881, 3942: 2; 4020: 27; 5171: 2; 5249, 13201:27-Riddell 7:6-Ridley 177: 25; 1682: 15.b;6559: 16.a; 12510, 12538: 25; 12569 (Matthew leg.): H.11; 15703: 16.a; 15707:15.b-Ridoutt 11658:28; 11710:13Roberty 10651, 17294: 5-C. B. Robinson, see BS-H. C. Robinson 21: 16.a; 26: 6; 6261: 16.a; 6358: 15.b-Rock 229: 15.a.1; 759(T): 16.a; 823: 16.b.1; 1503: 15.a.1; 2427: 6; 2636: H.1; 2657, 2913:19-Rojas 98: 23; 537: 31-Rose 410: 3-Rosenstock Fil. Austro-brasil. exsicc. 5, 218, 219: 13-Rosenstock Fil. Boliv. exsicc. 85 (= Buchtien 2220): 28-Rosenstock Fil. Bras. exsicc. II 223 (= Brade \& Brade 8438), II 224 (= ibid. 8256): 13-Rosenstock Fil. Costaric. exsicc. 166 (= Brade \& Brade 366): 31; 315 (= Brade 371): 28-Rosenstock Fil. Formos. exsicc. 197: 18; 198: 6-Rosenstock Fil. Jav. Or. exsicc. 107: 32; 146: 10Rosenstock Fil. Nov. Caled. exsicc. 31: 38-Rosenstock Fil. Nov. Guin. exsicc. 11: 25 \& 32-Rosenstock Fil. Sumatr. exsicc. 51: 25-Ross 669: 44; 671a, 1101, 1136: 31-Rostrup 130: 16.a-Rusby \& Squires 380: 42-Rusby \& White 552: 13.

Safford 6, 973, 974: 38-Saldanha 6012: H.8; 16020: 14.b; 16247, 16349: 16.a; 56012: 6-Salvius \& Godman 280: 31-Samat b. Abdullah 218: 25-Sandeman 3446: 28; 4967: 13; 5006: 28-Sandwitch 1484: 42-Sangkhachand 391, 1511: 16.a-Sangster 87p.p.: 2; 190: 5; 192, 292: 2; 293: 5-Santapau 1240: 14.bSanyal A 2639: 15.c-Sarasin 1130: 25; 1193 : 10-Savatier 743: 38-Savory \& Keay FHI 25012, 25137: 27 Scaëtta 3236, 3239: 5-Scamman 5979, 5980, 5981, 7146, 7147B, 7477: 31; 7479: 22; 7683: 23; 7684, 7999: 31-Schipp 526: 31; S-764: 24; S-766: 35-Schlechter 12754p.p.: 2; 12874: 3; 13697: 32; 14442: 26; 16163 (T), 16236: 32; 17207, 17903: 25-Schlieben 2875: 2-Schlim 1032: 31-Schmalz 101: 13-Schmid 1080, 1151: 16.a-Schmidt 131, 349: 16.a; 777p.p.: 25; 891: 10-Schmutz 42, 56, 84: 41; 3677a: 10-Schnell 159, $213,473,544,550: 5$; 768: 27 ; 778: 5 ; 830: 27; 831: 36; 832: 2; 1057: 36; 1085, 1105: 2, 1160: 5; 1323: 27; 1402 , 1464: $2 ; 2609,2617: 9 ; 2666,2667: 27 ; 2787: 9 ; 2807,2815: 27 ; 2825,2839: 5 ; 4910: 36 ; 7367,7690: 5-$ Scholes 67, 257: 2-Schottmüller 59: 13; 217p.p.: 43-Schroeder 157: 27-Schultes \& Cabrera 12929: 42Schulz 8922, 10252: 22; 10257a: 13-Schulz-Varela 5179: 13-Schunke 161, A 215: 13; 333: 28; 372: 22; 846, 854, 14076: 28-Schweichert 2408: 27-Schweinfurth 2235, 2236, 3043: 5; 3102: 2-Scolnik 877: 13- 
Scortechini 1670: 25-Scott Elliot 4048: 5; 7096: 2-Seaverns 14(a), 14(c), 48: 31-Seemann 233: 24; 368: 31-Seidenfaden 2123: 10 \& 15.b; 2626: 16.a-Seimund 59: 25-Seler 4978, 4995: 31-Selinck 150: 38-Selliers 25: 13-Seret 48: H.5; 452bis: 2; 615: 20; 649: 2; 878: 20-Serizawa 11713 (= Pterid. Jap. exsicc. 48): D.4-Setchell \& Parks 24, 295, 435, 521: 38-SF Series (Singapore Field numbers) 2087: (Haniff \& Nur): 16.a; 3830 (ibid.): 15.b; 7140 (ibid.): 16.a; 9476 (Sinclair \& Edaño), 9522 (ibid.): 18; 9540 (Holttum): 26; 9541 (ibid.): 16.a; 9547 (Sinclair \& Edaño), 9605 (Holttum), 9874 (ibid.): 26; 10354 (Haniff \& Nur): 25; 10478 (Holttum): 26; 10479p.p. (ibid.): 16.a \& H.11; 10956 (ibid.): 26; 11489 (ibid.): 25; 11845 (Nur), 11846 (ibid.): 26; 11848 (ibid.): 16.a; 12756 (Holttum): 25; 14116,17440 (ibid.): 26; 17700 (ibid.), 17977 (Henderson) (T): 25; 18589 (Henderson) (T): 26; 18590 (ibid.): 25; 18614 (ibid.), 19307 (Holttum), 19578 (Henderson): 16.a; 19799 (Holttum): H.11; 19800 (ibid.), 20021 (ibid.): 26; 20799 (ibid.): 16.a; 21625 (ibid.), 23690 (ibid.): 25; 24257 (Kiah): 16.a; 24699 (Holttum), 24786 (ibid.), 24793 (ibid.): 25; 25141 (ibid.), 25352 (ibid.): 26; 25585 (ibid.): 41; 29082 (Henderson): 16.A. 29084 (ibid.): 15.b; 30251 (Corner): 26; 31338 (Holttum), 31352 (ibid.): 25; 31920 (Kiah): 16.a; 34196 (Nur), 34303 (ibid.), 37211 (Addison): 26; 38015 (Namen): 25; 38811 (Sinclair \& Kiah): 16.a; 40127 (Sinclair): 26-Shafer 8803: 24-Shafer \& Leon 13698: 31-Shank \& Molina 4873: 22-Shattuck 281: 31-Sherring 75: 22-Shimek 60-104: 31-Shimizu et al. 11381: 19-Sidney 53: 25-Sieber Syn. Fil. 24 (T): 2-Sigafoos 56: 32-Sillitoe 97, 98: 5; 107:2S. S. Sin \& K. K. Whang 714: 43-Sinclair \& Edaño, see PNH and SF-Sintenis 2839: 1; 3115, 4245, 5110b: 24; 5166: 1 \& 31; 5448: 1; 5464b: 31; 5946, 6031b, 6135: 24; 6261: 31; 6268: 22; 6464: 1; 6530: 31; 6534: 24Sita 228: 20; 783: 36; 833: 20-Skutch 2577: 31; 2972: 23; 3084: 13; 4050: 31; 5389: 22-Sledge 1278: 6-van Slooten 128, 517: 41-Austin Smith 48/312: 23-C. L. Smith 2106, 2127: 31-E. Smith 537: 16.a; 541: 26; 722: 16.a; 841: 26; 886: 25; 1066: 15.a.1; 1108: 16.b.1; 1109, 1110, 1111: 19; 1112, 1113: 15.a.1; 1639, 1640: 16.a; 1641: 43; 1696: 25; 2019: 26, 2823, 2824: 41; 2825, 2826: 16.a; 2827, 2828: 16.b.1; 2832: 25; 2837: 6-H. Smith 16: 13-H. H. Smith 1054: 31; 2522: 13; 2695: 24-H. M. Smith 204: 16.a; 298, 302 (T): 8.b; 306: 16.b. 1; 595, 604, 610: 16.a-L. B. Smith 1214, 2265: 13; 3359: 24-L. S. Smith N. G. 39: 32-Smitinand 132 (= BKF 6157), 950 (= BKF 5785), 1049 (= BKF 5825): 16.a; 1352 (= BKF6753), 3452: 25;4114(= BKF 19124): 16.a; (et al.) 5173: 15.a.1; 6093 (BKF 24438): 8.b; 6214 (= BKF 24724), 6616 (= BKF 24743), 6648 (= BKF 24785): 19-Sonohara, Tawada \& Amano 6287: 43-Sørensen, Larsen \& Hansen 488a: 16.a; 543: 25; 672: 16.a; 1308: 19; 2914: 15.a.1; 3887: 16.a; 3960: 19; 5159: 16.a \& 16.b.1-Spruce 28: 22; 577, 2121 (T): 42; 3013: 1; 4123 (T): 12; 4636 (T): 22; 4736: 13; 4737 (T): 23; 4741 (T), 5684 (T): 30-Squires 845: 8.b-Srivastava et al. LWG 79581: 16.b.2-Stahel \& Gonggrijp 76 (= BW 2933): 13-Standley (et al.) 2604: 31; 7034: 24; 20275, 21643: 31; 24202: 22; 24203: 31; 26217: 24; 28413, 29946, 30584, 31363, 31708: 31; 33952: 23; 37054: 22; 40154, 40915, 44291: 31; 44743: 35; 51023: 23; 52821: 1; 52943: 22; 53866: 31; 53996: 35; 54164: 22; 54831, 64977: 31; 68309: 35; 68729, 78211, 78946, 87323, 87421, 89518: 31; 91689: 35-Staudt 449: 20; 463 (T): 5; 806: 27-van Steenis 953, 3632: 25-Steeves \& Ray 402: 31; 490: 35Steinbach 1093 (hb. Inst. Miq. Lillo 20768): 13; 3003, 7143, 7163: 13-Steinheil 205: 31-Stern 2072: 25Steyermark 33337, 33338: 35; 33837: 31; 37225, 37336: 35; 39150: 31; 44369, 44416: 35; 44982, 45379: 24; 45390: 31; 49365: 22; 49632, 61142: 31; 61144: 13; (\& Agostini) 91193, (\& Rabe) 96119: 21-St. John 16654, 16768: 38-St. John \& Fosberg 14130, 15331: 38-Stoffers 3631/3635: 31-Stokes 45, 110, 209, 330, 336B: 38-Stolz 1003: '27-Stork 479: 28-Stübel 658: 1; 673, 690, 695: 13; 906 (T): 23; 929: 28; 929a: 35;-Stuhlmann 394 (T): 36-Sulit \& Conklin, see PNH-Surbeck 651: 26; 660: 25-Suvanakoseś BKF 9072: 16.a-Swynnerton 833, 834: 27-Sydow 64: 31 .

Taam 221: 43; 1471: 16.a; 1473, 1845: 43-Tagawa 235: 16.a; 884, 984(T), 7704: 43; 11789: 16.a-Tagawa \& Iwatsuki 1181, 2733: 43; 2918 (T): D.4; 4061, 4128: 43; 4514: 25; 4605: D.4; 4690: 25-Tagawa, Iwatsuki \& Fukuoka T372: 15.a.1; T380: 6; T932, T1086: 15.a.1; T1094: 16.a; T1100: 19; T1909: 6; T1910: 8.b; T2103: 25; T2108: 19; T2110: 15.c; T2665, T4214: 19; T4671: 16.a; T5289: 25; T6802 (T): 15.b; T9243: 15.a.1; T9986: 19-Tagawa \& Yamada T43: 19; T44, T45: 15.a.1; T210: 16.a; T211: 25; T213: 26; T214: 15.bTakamatsu 531, 953: 25-Tang Siu Ging 13580: 43-Taylor 1895: 22-van Telborg B80: 3; B81 (T): 5Terry \& Terry 1618: 31-Tessmann 4001, 4415: 28-Thieme 5668: 31-Thollon 719: 20; 4099: 27-Thomas 1344: 36; 1390: 2; 1527, 1742: 5; 4549: 36-Thomasset 113: 4-Thomson 102: 16.b.1-Thorel 2480: 8.bim Thurn 103: 38-Thwaites CP 1313: 14.a; 1314: 16.a; 3075: 6-Tilden 474: 38-Tisserant 223, 418: 2; 419, 1241, 1429: 5; 2200: 27; 2635: 2; 2716: 20-Tonduz, in Pittier 4842, in hb. Inst. Phys.-geogr. Costaric. 6554, 9477: 31; 12855: $28 \& 31 ; 13353: 24$-Toppin 4348: 25; 6079: 16.a; 6115: 16.b.1 -LeRoy Topping 340: $18 ; 386,444: 25 ; 632,655: 18 ; 656: 25 ; 674: 18 ; 683,718,832,844: 25 ; 920,930$ : 18-Toussaint 2053: 2Trinidad Bot. Gard. Herb. 260, 1387: 31-Troupin 2995: 2-Tryon \& Tryon 5234: 13; 5281: 1; 6489: 3-H. T. Tsai 60522: 25-Wai-tak Tsang 382: 25; 758, 16355 (16255?): 16.a; 21228: 43; 23204, 26823: 16.a; 29432: see D.1; 29700: 43-K. K. Tsoong 2842: 25-von Türckheim II 422: 24; II 1077: 22; II 1492: 35; 2648: 24; 
2728: 1 \& 31; 2822: 22; 4034: 35; 8045: 24; 8829: 22; 8830 (T), 8831 (T): 35-Turnau 894: 25.

Ule 9138: 28-Underwood 1426 (T): 24.

Vaid 23376: 16.a-de Vaillant 4: 24-Valerio 46, 284: 31-Valeur 340: 24; 535: 1-Vanderijst 2791, 2792, 2796: 2 ; 4020: 27 ; 4643, 4645, 4645bis: $20 ; 4908: 36 ; 5650: 5 ; 5764,5772: 27$; 6038: 20; 6039: 2; 6074: 36; 6075: $20 ; 6076,6077$ : H.2; 6221: $2 ; 7507,7509: 27 ; 7514: 36 ; 7536,7538: 27 ; 7564,7565: 20 ; 7582: 2 ; 8019$, 8078: $20 ; 8083:$ H. $5 ; 8263,8264: 2 ; 8401: 36 ; 8424: 2 ; 8629,8828: 36 ; 9078: 2 ; 9532: 36 ; 9863: 2 ; 11278: 36 ; 12274$ : 2; 12512: 36; 12523: 20; 13920, 13944: 36, 14610: 20; 15957:5; 16166:27; 16241:3; 16247:2; 16296, 17278, 17305: $5 ; 17581 ; 2 ; 17591 ; 27 ; 22409: 2 ; 23795: 36 ; 25474,26088: 2 ; 26618: 9 ; 28807,29197: 2 ; 29202,30462: 20$; 30587: H.5; 30588, 30590: 5; 30593: 36; 30593bis: 5 \& 36; 30596: 9; 30902, 30923: H.5; 31224: 2; 31229: H.5; 31231: 5; 31241, 31322: 20; 31409: 5; 31639: 2; 31677: 9; 31682: 5; 32134: 27; 32412: 20; 40294: 36-Vanoverbergh 2600: 18-Vareschi \& Pannier 2619: 31-Vasro 1561: 38-Vaupel 28: 38-Verdcourt 90: 5; 92: 2-Vermoesen 1751: 20; 1752, 1843: 2-Vervoorst 631: 13-Vesterdal 427, 473: 16.a- Vidal 1905: 18-Vieillard 2, 12, 61, 2134: 38-Viets 24: 33-Vigne 2713: 2; 4104: 5; 4333: 27-Vink (BW) 17580: 25.

Wagner 76: 13-W. H. Wagner Jr 3277: 32-Wagner \& Grether 4229: 25-Wakefield 1037: 32; 1101: 33; 1156: 25 ; 1161: 32; 1212: 33; 1213: 25; 1214: 32-E. H. Walker \& Tawada 6545: 25; 6713: 16.a-T. G. Walker T2728: 24; T7882: 32; T7983, T8207: 33; T9019, T9033, T9067, T9068: 32; T9127, T9128: 33; T9300, T9603, T9604, T9616, T10052, T10053, T10054, T10055: 32; T10491, T10492, T10493, T10495, T10496, T10497, T10498, T10520, T10586: 31-Wall 10562: 14.a-Wall \& Nakisi, see BSIP-Wallich 10: 15.a.2; 22 (T): 6; 24p.p. (T): 8.a; 25: 25; 26 (T): 7; 28 (T): 16.a; 29 (T): 16.b.1; 30: 16.a; 59(T): 15.c; $140(\mathrm{~T})$ : 15.a.2; 141, 161: 15.a.1; 171: 16.a; 1033 (T): 15.a.1; 2162: 26; 2163: 16.a; 2168: H.10; 2685 (T): 19-C. W. Wang 74857: 25; 75301: 19; 77117, 77910: 25; 78750: 19; 78807: 15.a.1; 78894: 25; 79027: 19; 80051: 25一 Warburg 988: 15.c; 11602, 11622: 25; 11978, 12508, 12748: 18; 19322: 32-Ward, F. Kingdon 21651: 25Warnecke 339: 2-Wasscevicz 33: 31-Waterhouse 47: 32; 411B: 25-G. Watt 5705: 25; 5867: 6-M. D. Watt 118: 31; 124: 24-Wawra 1057: 16.a-C. Weber 6162: 24; 6167: 31-C. M. Weber 1190, 1553: 25Webster 4158: 24-Weddel 649: 13-Wellens 306: 36; 314, 316: 2; 327: 9-Welwitsch 58: 2; 63b, 134: 5; 153: $2 ; 154: 5 ; 155: 20 ; 156$ (T): H.5; $157(\mathrm{~T}), 157 \mathrm{~b}(\mathrm{~T}): 36 ; 193: 5$-Wendland $298: 31 ; 507,576: 28 ; 787: 22$ Wenger 91: 25; 110: 16.b.1; 159: 19; 160, 513: 16.b.1-Wercklé 17099: 31-van der Werff 671: 12-Werner 8: 16.a; 41: 32; 43: 25 \& 32-West-Skinn 95, 109: 2; 176: 36; 249: 27; 265: 2-Wetmore \& Woodworth 127: 31-Wettstein \& Schiffner 5642: 13-Wheatherwax 248: H.9-White 558: 25-Whitford 1124: 25Whitmee 66, 151: 38-Whitmore \& Grubb, see BSIP-K. K. Whong, Sun Yatsen Univ. 140: 16.a; 714 : 43-Wight 46, 60, 61, 62: 16.a; 63 (= P.I.O. 3178): 11 \& H.10; 64 (Kew 3175): 14.b \& H.10-Wigman Jr 6; 32-J.J.F.E. de Wilde (et al.) 630: 5; 3878: 9-W.J.J.O. de Wilde (et al.) 1687, 4323: 5-Wilder 1027, 1090: 38-Wildt \& Chase 5569: 27-Wilford 478: 43-Wilkes (U.S. Expl. Exp.) 5: 13-L. Williams 3240, 3416, 3418: 28; 9508: 35; 10803: 31; 11438: 13 \& 31-L. L. Williams \& Alston 200: 31-L. O. Williams \& Molina 17766: 31; 17771: 1; 17784: 31; 24274: 22-R. S. Williams 23: 25; 30: 10; 203, 204: 25; 233: 18; 234: 10; 881, 1187: 13; 1293: 23; 2055: 26; 3114: 25-Williams \& Smitinand 17231, 17261: 16.a-K. A. Wilson \& Webster 531: 24-N. Wilson 500: 1; 1496: 24-Winckel 1201B: 41; 1520B: 26; 1566B: 16.a; 1609B: 26-Winit 53a, 53b: 16.a; 54: 16.b.1; 55: 19; 58: 7; 59: 15.c; 60: 25; 922a: 16.a; 923: 16.b.1; 924: 19; 927 (BKF 1368): 7; 928: 15.a.1; 929 (BKF 1370): 25; 1022: D.7; 1026: 8.b; 1034: 15.a.2 \& 15.c; 1126: 6Hub. Winkler 2225: 25-Wirawan 323: 25-Wirkura 1925: 18-Wirtgen 509: 18-Wisse 72: 32; 98: 25de Wit 7026: 27; 7028: 2; 7030: 9; 7049: 2-de Wit \& Morton 617: 5; 625:2-Witte 4267: 27-Womersley \& Millar 8545: 32-G. H. S. Wood 788: 36-Wray 3374: 25; 3505, 3512: 26-Wright 104: 1; 783(A): 1 \& 31; 784: 16.a; 788: 24-Wullschlagel 1209: 24-Wurdack 1896: 23; 1897: 30; 2033: 1; 2110: 12-Wurdack \& Monachino 41313: 42-Wydler 420: 24.

Yapp 210, 460: 25, 572: 16.a; 576: 26-Young 547: 5-Yuncker et al. 8825: 31; 8827: 35.

Zahn 312: 5; 406: 27-Zenker (\& Staudt) 81, 87: 27; 294: 2; 530: 27; 627: 2; 1467: 27; 1598 (T): 2; 2456: 20; 2751: 2; 3806: 20; 4043: 5; 4044: 2; 4119: 3; 4120: 5-Zippel 207: 32-Zollinger 884 (T): 25; 884x: 41; 1441 (T): 25; 1293 (T), 1499: 26; 2162: $10 \&$ 14.a; 2634: 25. 


\section{INDEX TO TAXONOMIC NAMES}

Accepted names in roman type; new taxa and combinations in bold type; synonyms in italics. The numbers refer to the number of the accepted taxon. H.: hybrids (p. 284 onwards); D.: taxa atque nomina dubia (p.302 onwards); E.: excluded (p. 313 onwards).

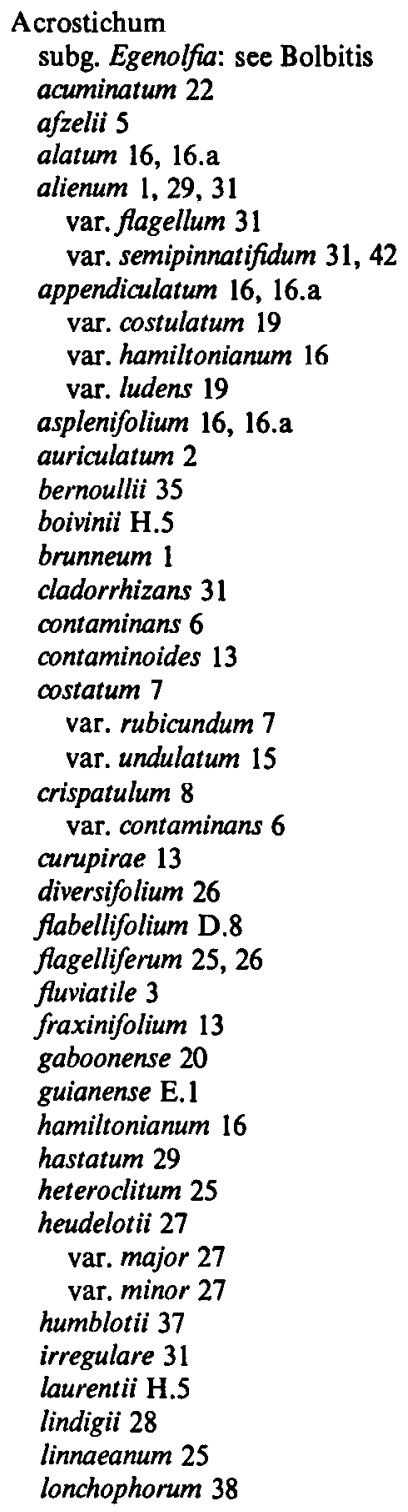

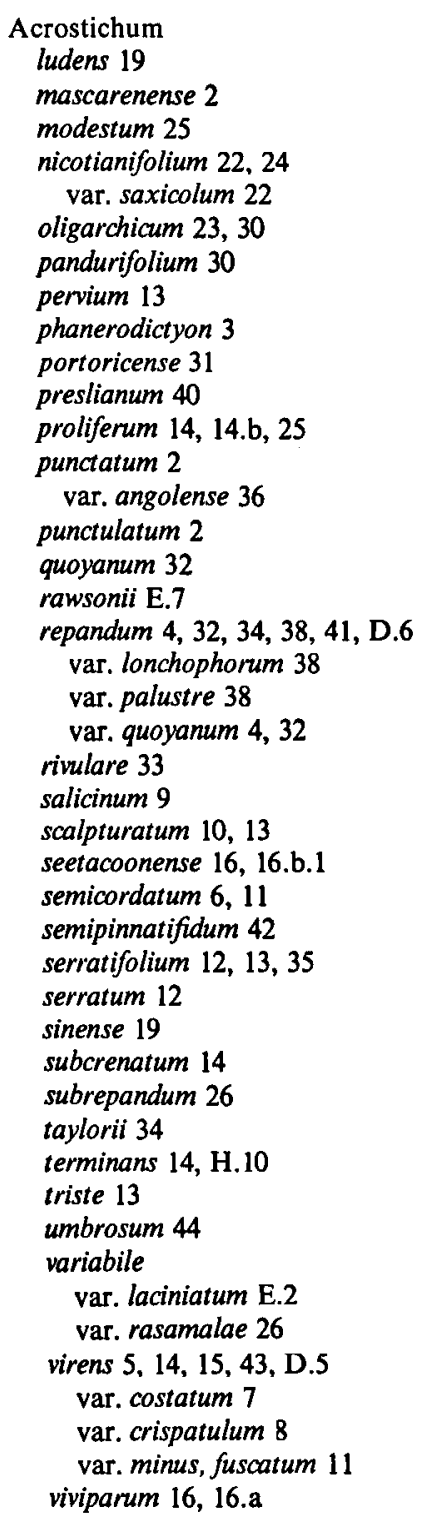


Acrostichum

zollingeri 26

Anapausia

subg. Euryostichum: see Bolbitis acuminata 22

aliena 1

heudelotii 27

nicotianifolia 22

portoricensis 31

zollingeri 26

Aspidium novo-pommeranicum 32

Asplenium? setosum D.9

Astralopteris coloradica E.8

Bolbitis p. 123

sect. Granulina: see Bolbitis

sect. Lysidictyon D.13

sect. Lysidictyon: see Bolbitis

ser. Alienae p. 132

ser. Bipinnatifidae p. 144

ser. Bolbitianae p. 147

ser. Egenolfianae p. 185

ser. Euryostichae p. 206

ser. Heteroclitae p. 220

ser. Heudelotianae p. 235

ser. Lindigianae p. 240

ser. Portoricenses p. 242

ser. Quoyanae p. 250

acrostichoides 5

acrostichoides D.5

aliena 1

aliena 42

angustipinna 6

angustipinna $\times$ sinensis $H .1$

annamensis 25

appendiculata 16

ssp. appendiculata 16. a

ssp. vivipara $16 . b$

var. neglecta 16. b. 2

var. vivipara 16.b.1

ssp. vivipara $\times$ virens $H .3$

appendiculata $\times$ sinensis $\mathbf{H . 2}$

$x$ arguta $\mathrm{H} .4$

asplenifolia 16

auriculata 2

var. undulato-crenata 2

auriculata H.5

bernoullii 35

bipinnatifida 4

bipinnatifida 19

$\times$ boivinii $\mathrm{H} .5$

boninensis 32

bradeorum 22, 23

bradfordii 14

cadieri D.1

christensenii D.2
Bolbitis

cladorrhizans 31

coloradica E.8

contaminans 6

copelandii 8, 8.b, 18

f. viridis 8

costata 7

crenata 13

crispatula 8

var. copelandii $8 . \mathrm{b}$

var. crispatula $8 . a$

curupirae 13

cuspidata 25

deltigera 15

dentata 42

diversibasis 5

diversifolia 25, 26

donnell-smithii 35

edanyoi 25

enormis 32

felixii 27

flagellifera 25

fluviatilis 3

fluviatilis 2

var. crenatis 2,3

fluviatilis $\times$ heudelotii H.6

formosana 43

$\times$ foxii H.12, H.12.b

foxworthyi 25

gaboonensis 20

gemmifera 36

grossedentata 3

guianensis E.1

guianensis 36

hastata 29

helferiana 16

hemiotis 21

heteroclita 25

heteroclita $\times$ rhizophylla $\mathrm{H} .7$

heudelotii 27

var. angustifolia 27

hookeriana 16

humblotii 37

hydrophylla 26

inconstans 25

interlineata D.3

interlineata 25

intermedia 18

kanarensis $\mathrm{H} .8$

killipii 22

koidzumii 25

labrusca D.14

laciniata $\mathrm{E} .2$

$\times$ lancea H.8

laurentii $\mathrm{H} .5$

laxireticulata D.4, D.13 


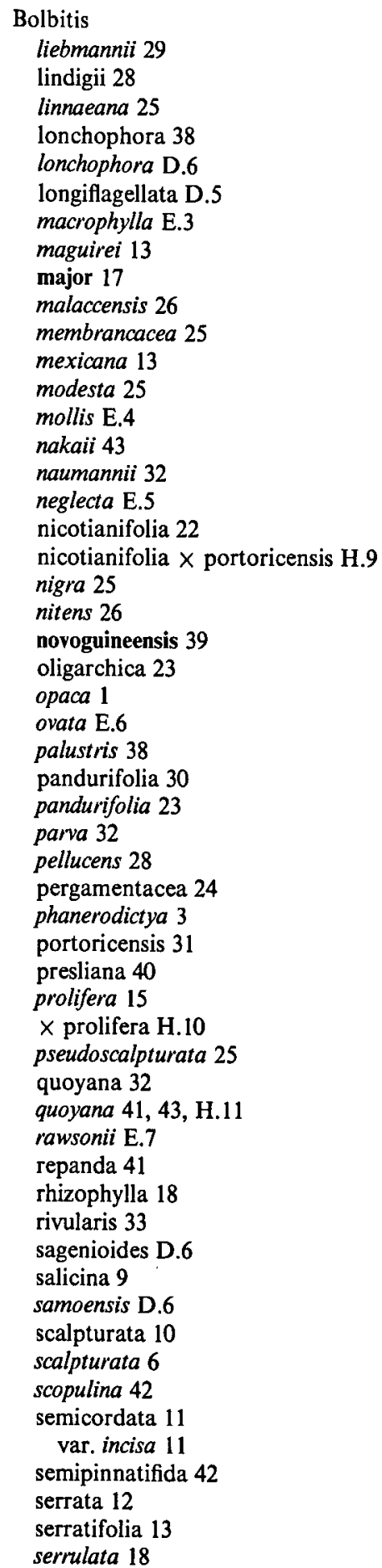

Bolbitis

simplicifolia 25

sinensis 19

var. costulata 19

$X$ singaporensis $\mathbf{H} .11$

sinuata 26

$x$ sinuosa H.12

$\mathrm{nm}$. foxii H.12.b

nm. sinuosa H.12.a

stenophylla 25

stuebelii 23

subcordata 43

subcrenata 14

var. prolifera 14.b

var. subcrenata 14 .a

subsimplex 25,26

taylorii 34

tenuissima 25

tonkinensis D.7

turrialbae 24

umbrosa 44

undulata 15

virens $\mathrm{H} .10$

virens 15

var. compacta $15 . \mathrm{b}$

var. deltigera $15 . \mathrm{c}$

var. virens $15 . a$

f. undulata 15.a.2

f. virens 15.a.1

vivipara 32

Campium: see Bolbitis

sect. Heteroneuron: see Bolbitis

acrostichoides 5

angustifolium 27

angustipinnum 6

argutum $\mathrm{H} .4$

auriculatum 2

bipinnatifidum 4

boivinii H.5

bradfordii 14, 14.a

christensenii D.2

costatum 7

crispatulum 8

cuspidatum 25

deltigerum 15

diversifolium 26

enorme 32

feeianum 40

fluviatile 3

foxworthyi 25

gaboonense 20

gemmiferum 5, 36

heteroclitum 25

var. eurybasis 25

heudelotii 27 


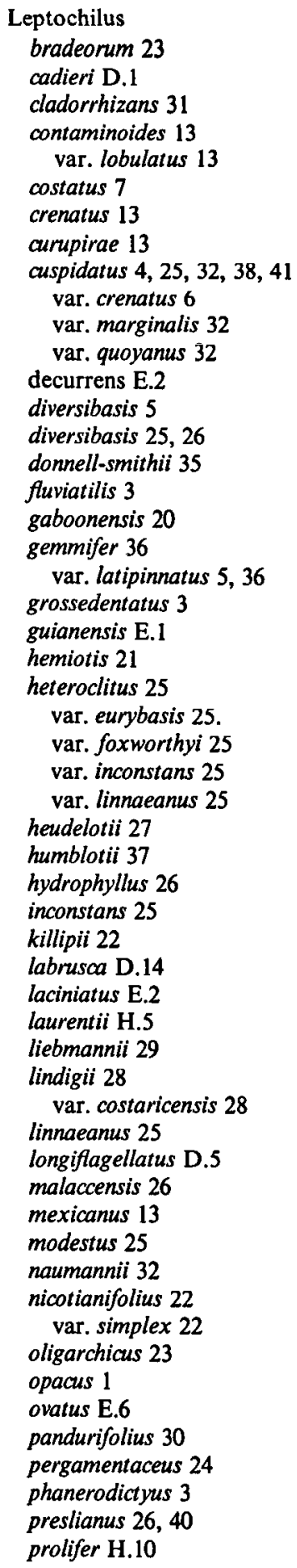

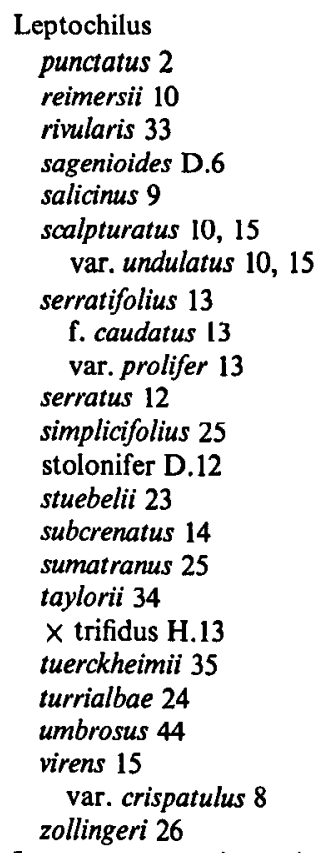

Lomagramma guianensis E.1

Lomaria bredemeyeriana 13

Lomariopsis undulata 15

Meniscium deltigerum 15 macrophyllum E.3

Nephrodium cuspidatum 25 macrophyllum E.3

Neurocallis rivularis 33

Notholaena undulata 15

Panna Mara-maravara D.11

Paraleptochilus ovatus E.6

Phegopteris macrophylla E.3

Photinopteris acuminata 22

Poecilopteris: see Bolbitis brunnea 1

contaminans 6

costata 7

var. deltigera 15, 15.c

var. rubicunda 7

var. undulata 15

crenata 13

crispatula 8

diversifolia 26

donnell-smithii 35

flagellifera 25

fluviatilis 3

fraxinifolia 13

hookeriana 14 
Poecilopteris

lobulosa 31

presliana 40

prolifera $\mathrm{H} .10$

punctulata 2

quoyana 32

repanda 41, H.10

var. lonchophora 38

rivularis 33

semicordata 11

sinuosa $\mathrm{H} .12$

stenophylla 25

subcrenata 14

subrepanda 26

terminans $14,14.6$

virens 15

Polybotrya

sect. Egenolfia: see Bolbitis subg. Ectoneura: see Bolbitis subg. Egenolfia: see Bolbitis acrostichoides 5

appendiculata 16, 19

var. asplenifolia 16

var. costulata 19

subvar. petiolulata 19

var. hamiltoniana 16, 16.b.2

var. helferiana 16, 16.a
Polybotrya appendiculata

var. ludens 19

var. major 17

var. marginata 16

var. rhizophylla 16.b.2, 18

var. subintegra 16, 16.b.1

asplenifolia 16

duplicato-serrata 18

exaltata 18

fraxinifolia 13

hamiltoniana 16, 16.b.1

helferiana 16, 16.a

intermedia 18

marginata $16,16 . a$

neglecta 18

nodiflora 16, 16.b.1

prolifera $14, \mathrm{H} .10$

rhizophylla 18

serrulata 8

vivipara 16, 16.b.1

Polypodium guianense E.1

sinuatum 26

Polystichum cuspidatum 25

Pteridoblechnum neglectum E.5

Stenosemia dimorpha 32

Thelypteris macrophylla E.3 